\title{
Palladium-Catalyzed Regioselective Arylation of Unprotected Allylamines
}

Vinod G. Landge, Justin M. Maxwell, Pratibha Chand-Thakuri, Mohit Kapoor, Evan T. Diemler, and Michael C. Young*

Department of Chemistry and Biochemistry, School of Green Chemistry and Engineering, University of Toledo,

Toledo, OH, 43606.

\section{Supporting Information}

\section{Table of Contents}

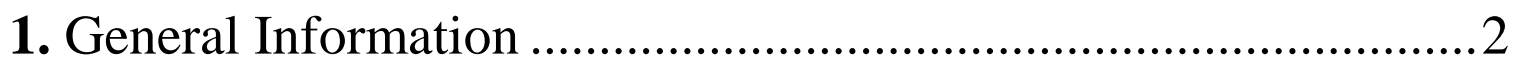

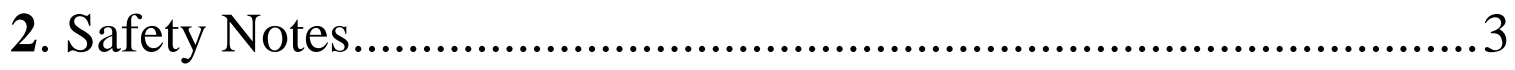

3. Synthesis of Amine Substrates ...................................................

4. Synthesis of Iodoarenes.......................................................... 18

5. Optimization and Synthesis of $\gamma$-Arylated Cinnamylamines..... 19

6. Optimization and Synthesis of $\gamma, \gamma$-Diarylated Allylamines ......44

7. Directing Group Experiments ....................................................49

8. Outlier Substrate Experiments ...................................................55

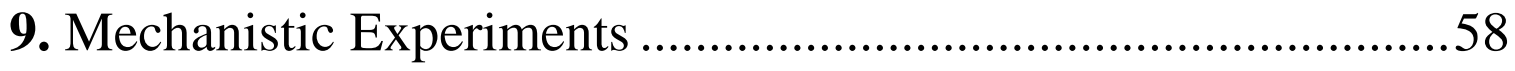

10. ${ }^{1} \mathrm{H}$ and ${ }^{13} \mathrm{C}$ Spectra of New Compounds ..................................68

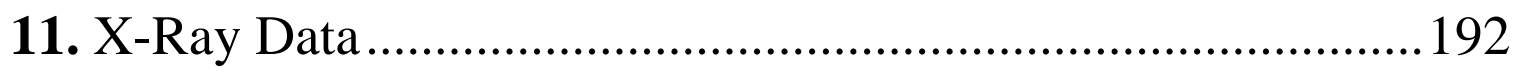

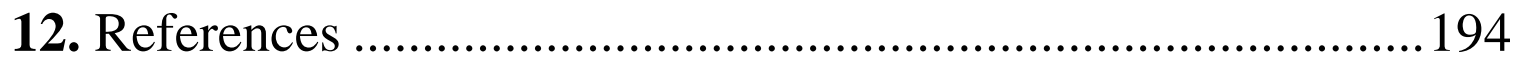




\section{General Information}

${ }^{1} \mathrm{H},{ }^{13} \mathrm{C}$, and ${ }^{19} \mathrm{~F}$ spectra were recorded on either a Varian Inova $400 \mathrm{MHz}$ NMR spectrometer or a Varian Inova 600 MHz NMR spectrometer, and were processed using MestReNova by Mestrelab Research S.L. Proton $\left({ }^{1} \mathrm{H}\right)$ chemical shifts are reported in parts per million $(\delta)$ with respect to tetramethylsilane (TMS, $\delta=0$ ), and referenced internally with respect to the protio solvent impurity. ${ }^{1}$ Carbon $\left({ }^{13} \mathrm{C}\right)$ chemical shifts are reported in parts per million $(\delta)$ with respect to tetramethylsilane (TMS, $\delta=0$ ), and referenced internally with respect to the carbon signal of the solvent. Fluorine $\left({ }^{19} \mathrm{~F}\right)$ chemical shifts are reported in parts per million $(\delta)$ and referenced internally with respect to hexafluorobenzene included in an insert tube $\left(\mathrm{C}_{6} \mathrm{~F}_{6}, \delta=-164.9\right)$. Mass spectra were recorded on an Agilent $6530 \mathrm{LC}$ Q-TOF mass spectrometer using electrospray ionization with fragmentation voltage set at $115 \mathrm{~V}$ or lower for sensitive substrates, and processed with an Agilent MassHunter Operating System, or on a Waters SYNAPT G2-Si High Definition Mass Spectrometer. For limited cases where ESI data was not obtained, an Agilent 5975C Inert XL MSD with Triple-Axis Detector were used, and data processed using 5975C MSD Data Analysis software. Deuterated NMR solvents were obtained from Cambridge Isotope Laboratories, Inc., Andover, MA, and used without further purification. All other materials were obtained from Sigma-Aldrich Chemical Company, St. Louis, MO, Combi-Blocks, San Diego, CA, Oakwood Chemical, Estill, SC, Alfa Aesar, Ward Hill, MA, Acros Organic, Geel, Belgium, or TCI, Tokyo, Japan, and were used as received. 


\section{Safety Notes}

During our work, we found that standard 2 dram vials from Qorpak could be set-up with the necessary reagents, $1 \mathrm{~mL}$ of solvent, and $100 \mathrm{mg}$ of dry ice, followed by immediately sealing. In our experience, the vials never failed, even upon heating to $100^{\circ} \mathrm{C}$. Although we did not have any lab incidents, we advise that all reactions should be performed behind appropriate shielding and should not be heated until such engineering controls (blast shield and/or hood sash) are in place. It is worth noting that a similar strategy has been used frequently to allow the use of liquid $\mathrm{CO}_{2}$ as a solvent for thin layer chromatography, which may provide additional guidelines for the safe handling of $\mathrm{CO}_{2}$ pressure in non-standard sealed systems. ${ }^{2}$ Alternatively, one may consider using a pressure tube for these reactions (e.g. Ace Glassware, 8648-03). When it is time to work-up the reactions, we first always cool the reactions to room temperature. The caps are slowly opened and allowed to vent. Sometimes, depending on the level of precipitate at the bottom of the flask, the reaction mixtures bubble vigorously, which necessitates performing this operation multiple times to slowly bleed off the carbon dioxide. Although the pressures observed have never caused any incidents in our hands using the published conditions, we recommend this operation be performed in the fume hood, and that all appropriate PPE, especially goggles, are worn at all times. When using a pressure tube, we will treat the top much like a champagne cork, and as a precaution will secure a towel over the cap to ensure it does not go flying off and subsequently bouncing around the hood. Although this has never happened to us, we do recommend this precaution when using enough dry ice to generate the maximum recommended pressure for the pressure tubes. We have also published a visual demonstration to assist with the safe handling of this procedure. ${ }^{3}$ 


\section{Synthesis of Amine Substrates}

A) General Procedure for Synthesis of $N$-Cinnamylamine Substrates from Cinnamaldehydes (Method A)

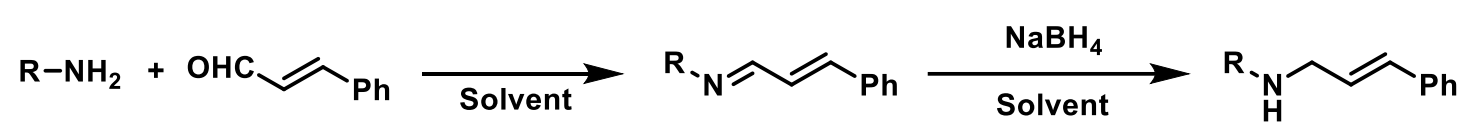

Typical procedure for a reductive amination: To a 50-mL round bottom flask charged with a stir bar was added the primary amine ( 1 equiv), cinnamaldehyde ( 1 equiv) and $15 \mathrm{~mL}$ of chloroform. The reaction was stirred at $60{ }^{\circ} \mathrm{C}$ for $4 \mathrm{~h}$, and filtered over $\mathrm{Na}_{2} \mathrm{SO}_{4}$. The filtrate was concentrated in vacuo, followed by dissolution in methanol (10 $\mathrm{mL}$ ). Sodium borohydride ( 2 equiv) was added portion wise, followed by stirring for $12 \mathrm{~h}$. After stirring the reaction was quenched with aq. $\mathrm{NaOH}$ solution. The aqueous layer was extracted with diethyl ether $(2 \times 25 \mathrm{~mL})$, dried over $\mathrm{Na}_{2} \mathrm{SO}_{4}$, followed by concentration in vacuo. The residue was then acidified using $1.2 \mathrm{M}$ hydrochloric acid, followed by subsequent washing with diethyl ether $(2 \times 50 \mathrm{~mL})$. The aqueous layer was then made basic with ammonium hydroxide and extracted with DCM $(2 \times 50 \mathrm{~mL})$. After drying over $\mathrm{Na}_{2} \mathrm{SO}_{4}$, the organic layer was concentrated in vacuo to give the secondary amine product.

\section{B) General Procedure for Synthesis of $\mathrm{N}$-Cinnamylamine Substrates from Cinnamylamine (Method B)

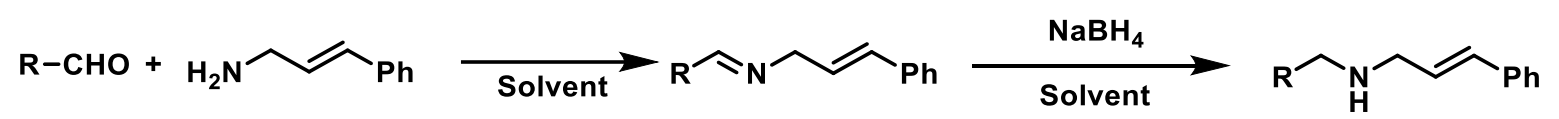

Typical procedure for a reductive amination: To a 50-mL round bottom flask charged with a stir bar was added cinnamylamine ( 1 equiv), cinnamaldehyde ( 1 equiv) and $15 \mathrm{~mL}$ of chloroform. The reaction was stirred at $60{ }^{\circ} \mathrm{C}$ for $4 \mathrm{~h}$, and filtered over $\mathrm{Na}_{2} \mathrm{SO}_{4}$. The filtrate was concentrated in vacuo, followed by dissolution in methanol (10 $\left.\mathrm{mL}\right)$. Sodium borohydride ( 2 equiv) was added portion wise, followed by stirring for $12 \mathrm{~h}$. After stirring the reaction was quenched with aq. $\mathrm{NaOH}$ solution. The aqueous layer was extracted with diethyl ether $(2 \times 25 \mathrm{~mL})$, dried over $\mathrm{Na}_{2} \mathrm{SO}_{4}$, followed by concentration in vacuo. The residue was then acidified using $1.2 \mathrm{M}$ hydrochloric acid, followed by subsequent washing with diethyl ether $(2 \times 50 \mathrm{~mL})$. The aqueous layer was then made basic with ammonium hydroxide and extracted with DCM $(2 \times 50 \mathrm{~mL})$. After drying over $\mathrm{Na}_{2} \mathrm{SO}_{4}$, the organic layer was concentrated in vacuo to give the secondary amine product.

\section{C) General Procedure for Synthesis of Secondary Amines via Amine Alkylation (Method C)}

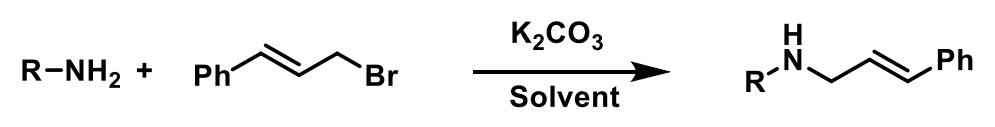

Amine ( 1 equiv) was combined with (E)-(3-bromoprop-1-en-1-yl)benzene (1 equiv), followed by addition of $\mathrm{K}_{2} \mathrm{CO}_{3}$ (2 equiv) and $\mathrm{MeCN}(10 \mathrm{~mL})$. The reaction was heated to $80^{\circ} \mathrm{C}$ for $14 \mathrm{~h}$, followed by cooling, and then all volatiles were removed in vacuo. The resulting residue was treated with brine, followed by extracting with dichloromethane ( 3 x $25 \mathrm{~mL}$ ). The combined organic fractions were dried over $\mathrm{Na}_{2} \mathrm{SO}_{4}$, followed by evaporation in vacuo and purification by flash chromatography over silica to give the desired product. 
D) General Procedure for Synthesis of Secondary Amines via Amine Alkylation:

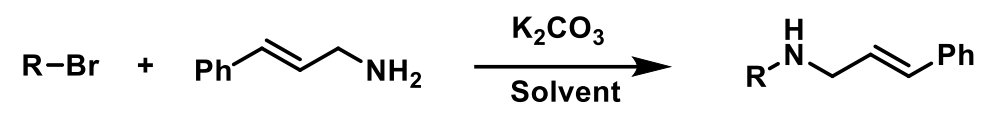

Alkyl bromide (1 equiv) was combined with (E)-3-phenylprop-2-en-1-amine (1equiv), followed by addition of $\mathrm{K}_{2} \mathrm{CO}_{3}$ (2 equiv) and $\mathrm{MeCN}(10 \mathrm{~mL})$. The reaction was heated to $80^{\circ} \mathrm{C}$ for $14 \mathrm{~h}$, then the reaction was cooled, and all volatiles removed in vacuo. The resulting residue was treated with brine, followed by extracting with dichloromethane $(3 \times 25$ $\mathrm{mL}$ ). The combined organic fractions were dried over $\mathrm{Na}_{2} \mathrm{SO}_{4}$, followed by evaporation in vacuo and purification by flash chromatography over silica to give the desired product.

\section{C) Characterization Data}

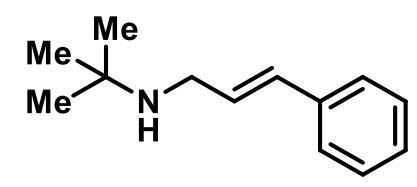

(E)- $N$-(tert-Butyl)-3-phenylprop-2-en-1-amine ( $S$-1): Method A used. 2-methylpropan-2-amine (500 mg) was used. Product recovered as a yellow oil (1.08 g, 84\% yield). Spectral data was consistent with that in the literature. ${ }^{4}{ }^{1} \mathrm{H}$ NMR $\left(600 \mathrm{MHz}, \mathrm{CDCl}_{3}\right) \delta 7.36(\mathrm{dd}, J=8.1,0.9 \mathrm{~Hz}, 2 \mathrm{H}), 7.29(\mathrm{t}, J=7.7 \mathrm{~Hz}, 2 \mathrm{H}), 7.24-7.16(\mathrm{tt}, \mathrm{t}, J=7.3 \mathrm{~Hz}, 1 \mathrm{H}), 6.52$ $(\mathrm{d}, J=15.8 \mathrm{~Hz}, 1 \mathrm{H}), 6.34(\mathrm{dt}, J=15.8,6.4 \mathrm{~Hz}, 1 \mathrm{H}), 3.37(\mathrm{dd}, J=6.4,1.4 \mathrm{~Hz}, 2 \mathrm{H}), 1.16(\mathrm{~s}, 9 \mathrm{H}) .{ }^{13} \mathrm{C}$ NMR $(151 \mathrm{MHz}$, $\left.\mathrm{CDCl}_{3}\right) \delta=137.3,130.9,129.5,128.6,127.3,126.3,50.6,45.3,29.2$.

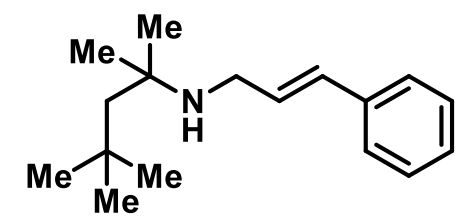

$N$-Cinnamyl-2,4,4-trimethylpentan-2-amine ( $S$-2): Method A used. 2,4,4-trimethylpentan-2-amine (500 mg) was used. Product recovered as a yellow oil (663 mg, 70\% yield). ${ }^{1} \mathrm{H}$ NMR (400 MHz, $\left.\mathrm{CDCl}_{3}\right) \delta 7.37(\mathrm{~d}, J=7.7 \mathrm{~Hz}, 2 \mathrm{H})$, $7.35-7.24$ (t, 2H), $7.25-7.15$ (tt, 1H), 6.53 (d, $J=15.9 \mathrm{~Hz}, 1 \mathrm{H}), 6.33$ (dt, $J=15.8,6.3 \mathrm{~Hz}, 1 \mathrm{H}), 3.37$ (dd, $J=6.3$, $1.1 \mathrm{~Hz}, 2 \mathrm{H}), 1.50$ (s, 2H), $1.21(\mathrm{~s}, 6 \mathrm{H}), 1.04(\mathrm{~s}, 9 \mathrm{H}) .{ }^{13} \mathrm{C} \mathrm{NMR}\left(101 \mathrm{MHz}, \mathrm{CDCl}_{3}\right) \delta=137.3,130.8,129.3,128.5$, 127.3, 126.3, 54.5, 52.7, 44.7, 31.8, 31.7, 29.1. LRMS (EI - MS): calcd. $245.2\left[\right.$ [M] $^{+}$Found: 245.2.

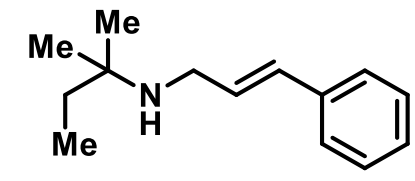

$\mathrm{N}$-Cinnamyl-2methylbutan-2-amine (S-3): Method A used. 2-methylbutan-2-amine (500 $\mathrm{mg})$ was used. Product recovered as a yellow oil (816 mg, 75\% yield). ${ }^{1} \mathrm{H}$ NMR (400 MHz, $\left.\mathrm{CDCl}_{3}\right) \delta 7.37(\mathrm{~d}, J=7.7 \mathrm{~Hz}, 2 \mathrm{H}), 7.29(\mathrm{t}, J=$ $7.6 \mathrm{~Hz}, 2 \mathrm{H}), 7.21(\mathrm{t}, J=7.2 \mathrm{~Hz}, 1 \mathrm{H}), 6.52(\mathrm{~d}, J=15.8 \mathrm{~Hz}, 1 \mathrm{H}), 6.34(\mathrm{dt}, J=15.8,6.4 \mathrm{~Hz}, 1 \mathrm{H}), 3.31(\mathrm{~d}, J=6.3 \mathrm{~Hz}$, 2H), $1.46(\mathrm{q}, J=7.5 \mathrm{~Hz}, 2 \mathrm{H}), 1.09$ (s, 6H), 0.87 (t, $J=7.5 \mathrm{~Hz}, 3 \mathrm{H}) .{ }^{13} \mathrm{C} \mathrm{NMR}\left(101 \mathrm{MHz}, \mathrm{CDCl}_{3}\right) \delta=137.3,130.8$, 129.5, 128.6, 127.3, 126.3, 52.7, 44.8, 33.2, 26.8, 8.4. HRMS (ESI - MS): calcd. 201.1747 [M+H] ${ }^{+}$Found: 204.1748. 


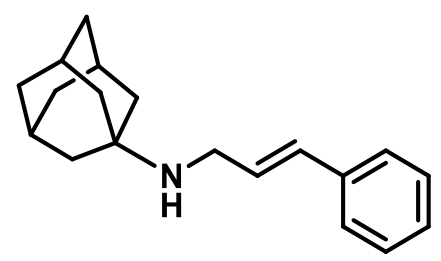

$(\mathbf{1 s}, 3 \mathrm{~s})$ - $\mathrm{N}$-Cinnamyladamantan-1-amine $(\boldsymbol{S}-4)$ : Method A used. 1-Adamantylamine (300 mg) was used. Product recovered as a yellow oil (344 mg, 65\% yield). Spectral data was consistent with that in the literature. ${ }^{5}{ }^{1} \mathrm{H}$ NMR (400 $\left.\mathrm{MHz}, \mathrm{CDCl}_{3}\right) \delta 7.34(\mathrm{~d}, J=7.3 \mathrm{~Hz}, 2 \mathrm{H}), 7.30-7.21(\mathrm{t}, J=7.2 \mathrm{~Hz}, 2 \mathrm{H}), 7.17(\mathrm{t}, J=7.2 \mathrm{~Hz}, 1 \mathrm{H}), 6.48(\mathrm{~d}, J=15.8 \mathrm{~Hz}$, $1 \mathrm{H}), 6.30(\mathrm{dt}, J=15.8,6.4 \mathrm{~Hz}, 1 \mathrm{H}), 3.37(\mathrm{dd}, J=6.4,1.1 \mathrm{~Hz}, 2 \mathrm{H}), 2.06(\mathrm{~s}, 3 \mathrm{H}), 1.62(\mathrm{dd}, J=24.0,7.3 \mathrm{~Hz}, 12 \mathrm{H}) .{ }^{13} \mathrm{C}$ $\operatorname{NMR}\left(101 \mathrm{MHz}, \mathrm{CDCl}_{3}\right) \delta=137.1,131.7,128.7,128.6,128.0,127.5,126.4,51.9,49.4,31.9,29.9,27.1,22.7,14.2$.

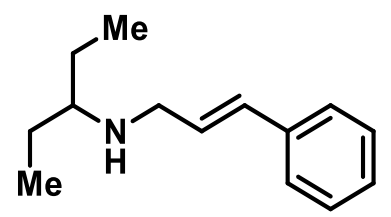

$\mathrm{N}$-Cinnamylpentan-3-amine ( $\mathrm{S}$-5): Method A used. Pentan-3-amine $(300 \mathrm{mg})$ was used. Product recovered as a yellow oil (573 mg, 82\% yield). ${ }^{1} \mathrm{H}$ NMR (400 MHz, $\left.\mathrm{CDCl}_{3}\right) \delta 7.37(\mathrm{~d}, J=7.3 \mathrm{~Hz}, 2 \mathrm{H}), 7.30(\mathrm{t}, J=7.6 \mathrm{~Hz}, 2 \mathrm{H}), 7.22$ (t, $J=7.2 \mathrm{~Hz}, 1 \mathrm{H}), 6.53(\mathrm{~d}, J=15.9 \mathrm{~Hz}, 1 \mathrm{H}), 6.32(\mathrm{dt}, J=15.8,6.3 \mathrm{~Hz}, 1 \mathrm{H}), 3.39$ (dd, $J=6.3,1.0 \mathrm{~Hz}, 2 \mathrm{H}), 2.47$ (p, $J=5.9 \mathrm{~Hz}, 1 \mathrm{H}), 1.53-1.38(\mathrm{~m}, 4 \mathrm{H}), 0.90(\mathrm{t}, J=7.5 \mathrm{~Hz}, 6 \mathrm{H}) .{ }^{13} \mathrm{C} \mathrm{NMR}\left(101 \mathrm{MHz}, \mathrm{CDCl}_{3}\right) \delta 136.8,131.0,128.2$, 128.0 127.0, 125.9, 58.8, 48.7, 25.1, 9.5. HRMS (ESI - MS): calcd. 204.1747 [M+H] ${ }^{+}$Found: 204.1753.

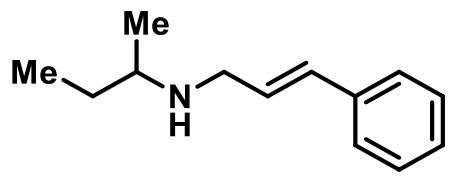

$\boldsymbol{N}$-Cinnamylbutan-2-amine (S-6): Method A used. Butan-2-amine (300 $\mathrm{mg}$ ) was used. Product recovered as a yellow oil (746 mg, 96\% yield). ${ }^{1} \mathrm{H}$ NMR (400 MHz, $\left.\mathrm{CDCl}_{3}\right) \delta 7.36-7.29$ (d, 2H), 7.24 (tt, $\left.J=9.5,4.8,0.8 \mathrm{~Hz}, 2 \mathrm{H}\right), 7.15$ (tt, $J=7.4,2.1,1.0 \mathrm{~Hz}, 1 \mathrm{H}), 6.46(\mathrm{~d}, J=15.8 \mathrm{~Hz}, 1 \mathrm{H}), 6.36-6.18(\mathrm{~m}, 1 \mathrm{H}), 3.46-3.24(\mathrm{~m}, 2 \mathrm{H}), 2.67-2.52(\mathrm{~m}, 1 \mathrm{H})$, $1.76(\mathrm{~s}, 1 \mathrm{H}), 1.55-1.40(\mathrm{~m}, 1 \mathrm{H}), 1.40-1.21(\mathrm{~m}, 1 \mathrm{H}), 1.01(\mathrm{dd}, J=6.3,1.9 \mathrm{~Hz}, 3 \mathrm{H}), 0.85(\mathrm{td}, J=7.1,3.5 \mathrm{~Hz}, 3 \mathrm{H})$. ${ }^{13} \mathrm{C} \mathrm{NMR}\left(101 \mathrm{MHz}, \mathrm{CDCl}_{3}\right) \delta=137.0,130.9,128.6,128.4,127.2,126.1,53.8,49.2,29.4,19.6,10.1$. HRMS (ESI MS): calcd. $190.1590[\mathrm{M}+\mathrm{H}]^{+}$Found: 190.1594.<smiles>CC(C)NCC=Cc1ccccc1</smiles>

(E)- $\boldsymbol{N}$-Isopropyl-3-phenylprop-2-en-1-amine $(\boldsymbol{S}$-7): Method A used. Propan-2-amine (300 $\mathrm{mg})$ was used. Product recovered as a yellow oil (654 mg, 82\% yield). ${ }^{1} \mathrm{H} \mathrm{NMR}\left(600 \mathrm{MHz}, \mathrm{CDCl}_{3}\right) \delta 7.37$ (dd, $\left.J=8.1,0.9 \mathrm{~Hz}, 2 \mathrm{H}\right), 7.30$ (tt, $J=7.7 \mathrm{~Hz}, 2 \mathrm{H}), 7.24-7.19$ (tt, 1H), $6.53(\mathrm{~d}, J=15.9 \mathrm{~Hz}, 1 \mathrm{H}), 6.32(\mathrm{dt}, J=15.8,6.4 \mathrm{~Hz}, 1 \mathrm{H}), 3.42$ (dd, $J=6.4,1.4$ 
$\mathrm{Hz}, 2 \mathrm{H}), 2.90(\mathrm{sept}, J=6.3 \mathrm{~Hz}, 1 \mathrm{H}), 1.10(\mathrm{~d}, J=6.3 \mathrm{~Hz}, 6 \mathrm{H}) .{ }^{13} \mathrm{C} \mathrm{NMR}\left(151 \mathrm{MHz}, \mathrm{CDCl}_{3}\right) \delta=137.3,131.2,128.8$, 128.7, 127.4, 126.4, 49.6, 48.3, 23.1. HRMS (ESI - MS): calcd. 176.1434 [M+H] ${ }^{+}$Found: 176.1437.

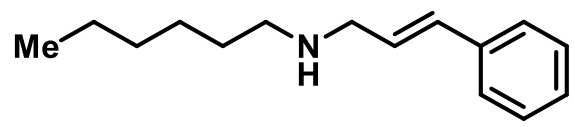

$\boldsymbol{N}$-Cinnamylhexan-1-amine $(\boldsymbol{S - 8})$ : Method A used. Hexan-1-amine (300 $\mathrm{mg}$ ) was used. Product recovered as a yellow oil (547mg, 85\% yield). Spectral data was consistent with that in the literature. ${ }^{4} \mathrm{H} \mathrm{NMR}^{1}\left(400 \mathrm{MHz}^{\mathrm{CDCl}}{ }_{3}\right)$ $\delta 7.34(\mathrm{~d}, J=7.4 \mathrm{~Hz}, 2 \mathrm{H}), 7.26(\mathrm{t}, J=7.5 \mathrm{~Hz}, 2 \mathrm{H}), 7.17(\mathrm{t}, J=7.2 \mathrm{~Hz}, 1 \mathrm{H}), 6.48(\mathrm{~d}, J=15.9 \mathrm{~Hz}, 1 \mathrm{H}), 6.27(\mathrm{dt}, J=$ $15.8,6.3 \mathrm{~Hz}, 1 \mathrm{H}), 3.36(\mathrm{~d}, J=6.3 \mathrm{~Hz}, 2 \mathrm{H}), 2.75-2.45(\mathrm{~m}, 2 \mathrm{H}), 1.92(\mathrm{~s}, 1 \mathrm{H}), 1.47(\mathrm{~m}, 2 \mathrm{H}), 1.40-1.18(\mathrm{~m}, 6 \mathrm{H}), 0.87$ $(\mathrm{t}, J=6.7 \mathrm{~Hz}, 3 \mathrm{H}) .{ }^{13} \mathrm{C} \mathrm{NMR}\left(101 \mathrm{MHz}, \mathrm{CDCl}_{3}\right) \delta=137.1,131.1,128.5,128.4,127.3,126.2,51.9,49.5,31.8,30.0$, $27.1,22.6,14.1$.

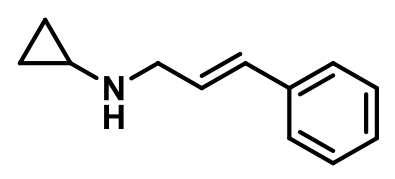

N-Cinnamylcyclopropanamine (S-9): Method A used. Cyclopropanamine (500 mg) was used. Product recovered as a yellow oil (1.13 g, 75\% yield). ${ }^{1} \mathrm{H}$ NMR (400 $\left.\mathrm{MHz} \mathrm{CDCl}_{3}\right) \delta 7.43-7.35(\mathrm{~d}, J=8.2 \mathrm{~Hz}, 2 \mathrm{H}), 7.31(\mathrm{t}, J=7.2 \mathrm{~Hz}$, $2 \mathrm{H}), 7.24(\mathrm{~m}, 1 \mathrm{H}), 6.53(\mathrm{~d}, J=15.9 \mathrm{~Hz}, 1 \mathrm{H}), 6.32(\mathrm{dt}, J=15.8,6.4 \mathrm{~Hz}, 1 \mathrm{H}), 3.48(\mathrm{~d}, J=6.4 \mathrm{~Hz}, 2 \mathrm{H}), 2.22(\mathrm{tt}, J=6.9$, $3.5 \mathrm{~Hz}, 1 \mathrm{H}), 1.79(\mathrm{~s}, 1 \mathrm{H}), 0.58-0.38(\mathrm{~m}, 4 \mathrm{H}) .{ }^{13} \mathrm{C} \mathrm{NMR}\left(101 \mathrm{MHz}, \mathrm{CDCl}_{3}\right) \delta=137.3,131.2,128.7,128.6,127.4$, 126.3, 51.8, 30.2, 6.5. HRMS (ESI - MS): calcd. 174.1277 [M+H] ${ }^{+}$Found: 174.1279.

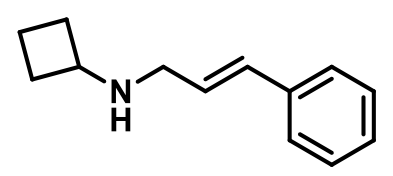

$\boldsymbol{N}$-Cinnamylcyclobutanamine ( $\boldsymbol{S}$-10): Method A used. Cyclobutanamine (300 $\mathrm{mg}$ ) was used. Product recovered as a yellow oil (703 mg, 89\% yield). ${ }^{1} \mathrm{H} \mathrm{NMR}\left(400 \mathrm{MHz}, \mathrm{CDCl}_{3}\right) \delta 7.40-7.34(\mathrm{~m}, 2 \mathrm{H}), 7.30(\mathrm{tt}, J=7.5,1.3 \mathrm{~Hz}, 2 \mathrm{H})$, $7.22(\mathrm{tt}, J=7.2,3.81 \mathrm{H}), 6.51(\mathrm{~d}, J=15.9 \mathrm{~Hz}, 1 \mathrm{H}), 6.29(\mathrm{dt}, J=15.8,6.4 \mathrm{~Hz}, 1 \mathrm{H}), 3.34(\mathrm{dd}, J=6.3,1.3 \mathrm{~Hz}, 2 \mathrm{H}), 2.25$ $(\mathrm{ddd}, J=12.2,6.2,3.6 \mathrm{~Hz}, 2 \mathrm{H}), 1.76-1.62(\mathrm{~m}, 4 \mathrm{H}) .{ }^{13} \mathrm{C} \mathrm{NMR}\left(101 \mathrm{MHz}, \mathrm{CDCl}_{3}\right) \delta=137.3,131.1,128.8,128.7$, 128.6, 127.4, 126.3, 53.8, 49.2, 31.4, 14.9. HRMS (ESI - MS): calcd. 188.1434 [M+H] $]^{+}$Found: 188.1438.

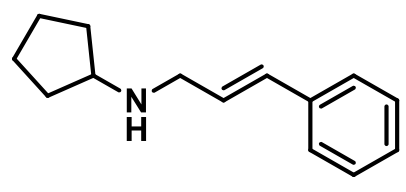

$\boldsymbol{N}$-Cinnamylcyclopentanamine (S-11): Method A used. Cyclopentanamine (300 mg) was used. Product recovered as a yellow oil $\left(603 \mathrm{mg}, 82 \%\right.$ yield). Spectral data was consistent with that in the literature. ${ }^{6}{ }^{1} \mathrm{H} \mathrm{NMR}(600 \mathrm{MHz}$, $\left.\mathrm{CDCl}_{3}\right) \delta 7.37(\mathrm{~d}, J=7.4 \mathrm{~Hz}, 2 \mathrm{H}), 7.30(\mathrm{t}, J=7.7 \mathrm{~Hz}, 2 \mathrm{H}), 7.22(\mathrm{t}, J=7.3 \mathrm{~Hz}, 1 \mathrm{H}), 6.52(\mathrm{~d}, J=15.9 \mathrm{~Hz}, 1 \mathrm{H}), 6.33$ $(\mathrm{dt}, J=15.8,6.4 \mathrm{~Hz}, 1 \mathrm{H}), 3.41(\mathrm{dd}, J=6.4,1.4 \mathrm{~Hz}, 2 \mathrm{H}), 3.15(\mathrm{p}, J=6.8 \mathrm{~Hz}, 1 \mathrm{H}), 1.92-1.85(\mathrm{~m}, 2 \mathrm{H}), 1.70(\mathrm{tdd}, J=$ 7.3, 5.5, $1.6 \mathrm{~Hz}, 2 \mathrm{H}), 1.55(\mathrm{ddd}, J=12.6,7.2,3.2 \mathrm{~Hz}, 2 \mathrm{H}), 1.40-1.32(\mathrm{~m}, 2 \mathrm{H}) .{ }^{13} \mathrm{C} \mathrm{NMR}\left(151 \mathrm{MHz}, \mathrm{CDCl}_{3}\right) \delta=$ $137.3,131.2,128.9,128.7,127.4,126.4,59.5,50.9,33.4,24.2$. 


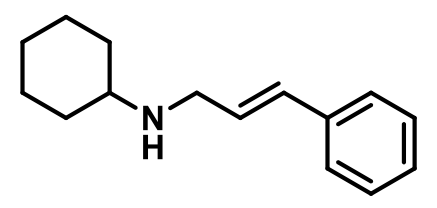

$\boldsymbol{N}$-Cinnamylcyclohexanamine ( $\boldsymbol{S}$-12): Method A used. Cyclohexanamine $(300 \mathrm{mg}$ ) was used. Product recovered as a yellow oil (559 mg, 86\% yield). Spectral data was consistent with that in the literature. ${ }^{4}{ }^{1} \mathrm{H} \mathrm{NMR}\left(400 \mathrm{MHz}, \mathrm{CDCl}_{3}\right)$ $\delta 7.43-7.34(\mathrm{~m}, 2 \mathrm{H}), 7.30$ (t, $J=7.6 \mathrm{~Hz}, 2 \mathrm{H}), 7.21(\mathrm{t}, J=7.2 \mathrm{~Hz}, 1 \mathrm{H}), 6.51(\mathrm{~d}, J=15.9 \mathrm{~Hz}, 1 \mathrm{H}), 6.32(\mathrm{dt}, J=15.8$, $6.3 \mathrm{~Hz}, 1 \mathrm{H}), 3.44(\mathrm{dd}, J=6.3,1.2 \mathrm{~Hz}, 2 \mathrm{H}), 2.50(\mathrm{tt}, J=10.4,3.7 \mathrm{~Hz}, 1 \mathrm{H}), 2.01-1.86(\mathrm{~m}, 2 \mathrm{H}), 1.86-1.68(\mathrm{~m}, 2 \mathrm{H})$, $1.63(\mathrm{~d}, J=12.0 \mathrm{~Hz}, 1 \mathrm{H}), 1.34-1.03(\mathrm{~m}, 5 \mathrm{H}) .{ }^{13} \mathrm{C} \mathrm{NMR}\left(101 \mathrm{MHz}, \mathrm{CDCl}_{3}\right) \delta=137.3,130.9,129.2,128.6,127.4$, $126.3,56.3,49.1,33.8,26.3,25.2$.

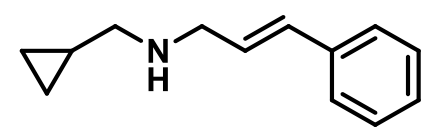

(E)- $N$-(Cyclopropylmethyl)-3-phenylprop-2-en-1-amine ( $S$-13): Method A used. Cyclopropylmethanamine (500 $\mathrm{mg}$ ) was used. Product recovered as a yellow oil $\left(671 \mathrm{mg}, 51 \%\right.$ yield). ${ }^{1} \mathrm{H} \mathrm{NMR}\left(600 \mathrm{MHz}, \mathrm{CDCl}_{3}\right) \delta 7.37$ (d, $J=7.3$ $\mathrm{Hz}, 2 \mathrm{H}), 7.30$ (t, $J=7.7 \mathrm{~Hz}, 2 \mathrm{H}), 7.22(\mathrm{t}, J=7.3 \mathrm{~Hz}, 1 \mathrm{H}), 6.53(\mathrm{~d}, J=15.9 \mathrm{~Hz}, 1 \mathrm{H}), 6.31$ (dt, $J=15.9,6.3 \mathrm{~Hz}, 1 \mathrm{H})$, $3.44(\mathrm{dd}, J=6.3,1.3 \mathrm{~Hz}, 2 \mathrm{H}), 2.52(\mathrm{~d}, J=6.9 \mathrm{~Hz}, 2 \mathrm{H}), 1.54(\mathrm{~s}, 1 \mathrm{H}), 0.98(\mathrm{~s}, 1 \mathrm{H}), 0.53-0.39$ (m, 2H), $0.20-0.08$ $(\mathrm{m}, 2 \mathrm{H}) .{ }^{13} \mathrm{C}$ NMR $\left(151 \mathrm{MHz}, \mathrm{CDCl}_{3}\right) \delta=137.3,131.3,128.7,128.6,127.4,126.4,54.7,51.9,11.4,3.5$. HRMS (ESI - MS): calcd. $188.1434[\mathrm{M}+\mathrm{H}]^{+}$Found: 188.1439.

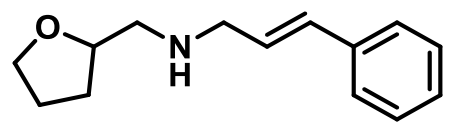

(E)-3-Phenyl- $N$-((tetrahydrofuran-2-yl)methyl)prop-2-en-1-amine ( $S$-14): Method A used. (tetrahydrofuran-2yl)methanamine $\left(300 \mathrm{mg}\right.$ ) was used. Product recovered as a yellow oil $\left(483 \mathrm{mg}, 75 \%\right.$ yield). ${ }^{1} \mathrm{H} \mathrm{NMR}(400 \mathrm{MHz}$, $\left.\mathrm{CDCl}_{3}\right) \delta 7.37(\mathrm{~d}, J=7.2 \mathrm{~Hz}, 1 \mathrm{H}), 7.30(\mathrm{t}, J=7.5 \mathrm{~Hz}, 2 \mathrm{H}), 7.22(\mathrm{t}, J=7.2 \mathrm{~Hz}, 1 \mathrm{H}), 6.54(\mathrm{~d}, J=15.9 \mathrm{~Hz}, 1 \mathrm{H}), 6.30$ $(\mathrm{dt}, J=15.9,6.3 \mathrm{~Hz}, 1 \mathrm{H}), 4.04(\mathrm{ddd}, J=14.6,7.4,3.8 \mathrm{~Hz}, 1 \mathrm{H}), 3.94-3.82(\mathrm{~m}, 1 \mathrm{H}), 3.76(\mathrm{dd}, J=14.4,7.5 \mathrm{~Hz}, 1 \mathrm{H})$, $3.45(\mathrm{dd}, J=6.3,1.3 \mathrm{~Hz}, 2 \mathrm{H}), 2.72(\mathrm{ddd}, J=19.8,12.0,5.8 \mathrm{~Hz}, 2 \mathrm{H}), 2.06-1.95(\mathrm{~m}, 1 \mathrm{H}), 1.91(\mathrm{dt}, J=13.7,7.7 \mathrm{~Hz}$, 2H), $1.64-1.43(\mathrm{~m}, 1 \mathrm{H}) .{ }^{13} \mathrm{C}$ NMR $\left(101 \mathrm{MHz}, \mathrm{CDCl}_{3}\right) \delta=137.2,131.5,128.7,128.4,127.5,126.4,78.4,68.1,53.9$, 52.1, 29.5, 26.0. HRMS (ESI - MS): calcd. $218.1539[\mathrm{M}+\mathrm{H}]^{+}$Found: 218.1548.

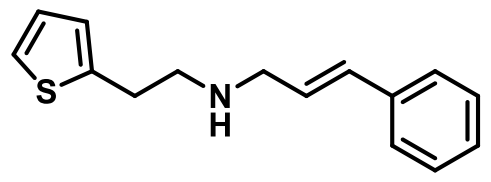

(E)-3-Phenyl- $N$-(2-(thiophen-2-yl)ethyl)prop-2-en-1-amine ( $\boldsymbol{S}$-15): Method A used. 2-(thiophen-2-yl)ethan-1amine (300 mg) was used. Product recovered as a yellow oil $\left(470 \mathrm{mg}, 82 \%\right.$ yield). ${ }^{1} \mathrm{H}$ NMR $\left(400 \mathrm{MHz}, \mathrm{CDCl}_{3}\right) \delta(\mathrm{d}$, $J=7.1 \mathrm{~Hz}, 2 \mathrm{H}), 7.30(\mathrm{tt}, J=9.7,5.3 \mathrm{~Hz}, 2 \mathrm{H}), 7.25-7.19(\mathrm{tt}, J=5.8,3.6 \mathrm{~Hz}, 1 \mathrm{H}), 7.15(\mathrm{dd}, J=5.1,1.1 \mathrm{~Hz}, 1 \mathrm{H}), 6.53$ (d, $J=15.9 \mathrm{~Hz}, 1 \mathrm{H}), 6.29(\mathrm{dt}, J=15.9,6.4 \mathrm{~Hz}, 1 \mathrm{H}), 3.47(\mathrm{dd}, J=6.4,1.3 \mathrm{~Hz}, 2 \mathrm{H}), 3.09$ (t, $J=6.8 \mathrm{~Hz}, 2 \mathrm{H}), 2.98(\mathrm{dd}$, $J=10.5,3.7 \mathrm{~Hz}, 2 \mathrm{H}), 2.00$ (s, 1H). ${ }^{13} \mathrm{C}$ NMR $\left(151 \mathrm{MHz}, \mathrm{CDCl}_{3}\right) \delta=142.6,137.2,131.5,128.7,128.4,127.5,127.0$, 126.4, 125.2, 123.7, 51.8, 50.7, 30.6. HRMS (ESI - MS): calcd. $244.1154[\mathrm{M}+\mathrm{H}]^{+}$Found: 244.1163. 


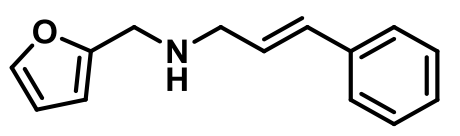

(E)-N-(Furan-2-ylmethyl)-3-phenylprop-2-en-1-amine ( $\boldsymbol{S}$-16): Method A used. Furfurylamine (500 mg) was used. Product recovered as a yellow oil $\left(548 \mathrm{mg}, 50 \%\right.$ yield). Spectral data was consistent with that in the literature. ${ }^{7} \mathrm{H}$ NMR (600 MHz, $\left.\mathrm{CDCl}_{3}\right) \delta 7.41-7.34(\mathrm{~m}, 3 \mathrm{H}), 7.31(\mathrm{t}, J=7.7 \mathrm{~Hz}, 2 \mathrm{H}), 7.23(\mathrm{t}, J=7.3 \mathrm{~Hz}, 1 \mathrm{H}), 6.54(\mathrm{~d}, J=15.9$ $\mathrm{Hz}, 1 \mathrm{H}), 6.33(\mathrm{dd}, J=3.1,1.9 \mathrm{~Hz}, 1 \mathrm{H}), 6.30(\mathrm{dt}, J=15.9,6.4 \mathrm{~Hz}, 1 \mathrm{H}), 6.20(\mathrm{~d}, J=3.1 \mathrm{~Hz}, 1 \mathrm{H}), 3.83(\mathrm{~s}, 2 \mathrm{H}), 3.43$ $(\mathrm{dd}, J=6.4,1.4 \mathrm{~Hz}, 2 \mathrm{H}), 1.66(\mathrm{~s}, 1 \mathrm{H}) .{ }^{13} \mathrm{C} \mathrm{NMR}\left(151 \mathrm{MHz}, \mathrm{CDCl}_{3}\right) \delta=153.8,142.0,137.1,131.8,128.7,128.1$, $127.5,126.4,110.2,107.2,51.0,45.5$.<smiles>C(=C/c1ccccc1)\CNCc1ccccc1</smiles>

(E)-N-Benzyl-3-phenylprop-2-en-1-amine ( $\boldsymbol{S}$-17): Method A used. Benzyl amine (500 mg) was used. Product recovered as a yellow oil $\left(855 \mathrm{mg}, 82 \%\right.$ yield). Spectral data was consistent with that in the literature. ${ }^{8}{ }^{1} \mathrm{H}$ NMR $(600$ $\left.\mathrm{MHz} \mathrm{CDCl}_{3}\right) \delta 7.40(\mathrm{dd}, J=8.2,0.9 \mathrm{~Hz}, 2 \mathrm{H}), 7.38-7.35(\mathrm{~m}, 4 \mathrm{H}), 7.33(\mathrm{t}, J=7.7 \mathrm{~Hz}, 2 \mathrm{H}), 7.31-7.27(\mathrm{~m}, 1 \mathrm{H}), 7.25$ $(\mathrm{ddd}, J=9.0,5.2,1.6 \mathrm{~Hz}, 1 \mathrm{H}), 6.56(\mathrm{~d}, J=15.9 \mathrm{~Hz}, 1), 6.35(\mathrm{dt}, J=15.9,6.3 \mathrm{~Hz}, 1 \mathrm{H}), 3.86(\mathrm{~s}, 2 \mathrm{H}), 3.46(\mathrm{dd}, J=6.3$, $1.4 \mathrm{~Hz}, 2 \mathrm{H}), 1.61(\mathrm{~s}, 1 \mathrm{H}) .{ }^{13} \mathrm{C} \mathrm{NMR}\left(151 \mathrm{MHz} \mathrm{CDCl}_{3}\right) \delta=140.3,137.2,131.5,128.7,128.6,128.5,128.3,127.5$, $127.1,126.4,53.5,51.3$.

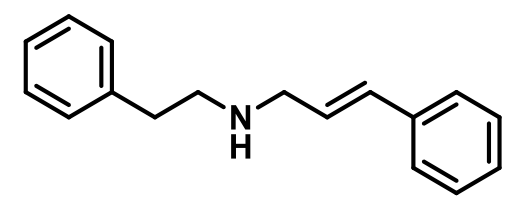

(E)-N-Phenethyl-3-phenylprop-2-en-1-amine ( $\boldsymbol{S}$-18): Method A used. 2-phenylethan-1-amine (500 mg) was used. Product recovered as a yellow oil $\left(822 \mathrm{mg}, 84 \%\right.$ yield). ${ }^{1} \mathrm{H}$ NMR (400 $\left.\mathrm{MHz}, \mathrm{CDCl}_{3}\right) \delta 7.37(\mathrm{~d}, J=7.7 \mathrm{~Hz}, 2 \mathrm{H}), 7.34$ $-7.28(\mathrm{~m}, 4 \mathrm{H}), 7.26-7.20(\mathrm{~m}, 4 \mathrm{H}), 6.51(\mathrm{~d}, J=15.9 \mathrm{~Hz}, 1 \mathrm{H}), 6.29(\mathrm{dt}, J=15.8,6.3 \mathrm{~Hz}, 1 \mathrm{H}), 3.44(\mathrm{dd}, J=6.3,1.3$ $\mathrm{Hz}, 2 \mathrm{H}), 2.95(\mathrm{dd}, J=10.9,4.0 \mathrm{~Hz}, 2 \mathrm{H}), 2.85(\mathrm{t}, J=7.1 \mathrm{~Hz}, 2 \mathrm{H}) .{ }^{13} \mathrm{C}$ NMR $\left(101 \mathrm{MHz}, \mathrm{CDCl}_{3}\right) \delta=140.1,137.2$, 131.4, 128.9, 128.6, 128.6, 128.5, 127.5, 126.4, 126.3, 51.9, 50.8, 36.6. HRMS (ESI - MS): calcd. 238.1590 [M+H] ${ }^{+}$ Found: 238.1600 .

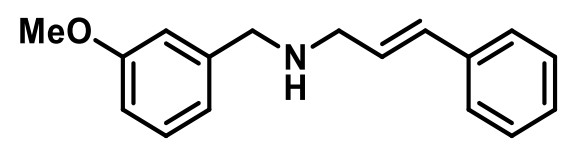

(E)-N-(3-Methoxybenzyl)-3-phenylprop-2-en-1-amine ( $\boldsymbol{S}$-19): Method A used. (3-methoxyphenyl)methanamine $(500 \mathrm{mg})$ was used. Product recovered as a yellow oil $\left(738 \mathrm{mg}, 80 \%\right.$ yield). ${ }^{1} \mathrm{H}$ NMR $\left(600 \mathrm{MHz}, \mathrm{CDCl}_{3}\right) \delta 7.39(\mathrm{dd}, J$ $=8.2,1.0 \mathrm{~Hz}, 2 \mathrm{H}), 7.32(\mathrm{t}, J=7.7 \mathrm{~Hz}, 2 \mathrm{H}), 7.27(\mathrm{~d}, J=7.8 \mathrm{~Hz}, 1 \mathrm{H}), 7.25-7.22(\mathrm{~m}, 1 \mathrm{H}), 6.96-6.92(\mathrm{~m}, 2 \mathrm{H}), 6.86-$ $6.79(\mathrm{~m}, 1 \mathrm{H}), 6.56(\mathrm{~d}, J=15.9 \mathrm{~Hz}, 1 \mathrm{H}), 6.34(\mathrm{dt}, J=15.9,6.3 \mathrm{~Hz}, 1 \mathrm{H}), 3.83(\mathrm{~s}, 2 \mathrm{H}), 3.82(\mathrm{~s}, 3 \mathrm{H}), 3.45(\mathrm{dd}, J=6.3$, $1.4 \mathrm{~Hz}, 2 \mathrm{H}), 1.65$ (bs, 1H). ${ }^{13} \mathrm{C} \mathrm{NMR}\left(151 \mathrm{MHz}, \mathrm{CDCl}_{3}\right) \delta=159.9,142.0,137.2,131.6,129.5,128.7,128.5,127.5$, 126.4, 120.6, 113.7, 112.7, 55.3, 53.4, 51.3. HRMS (ESI - MS): calcd. 254.1539 [M+H] $]^{+}$Found: 254.1549. 


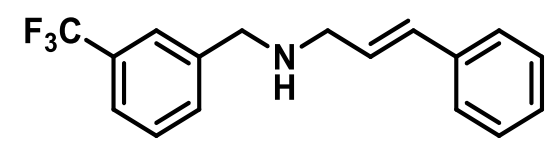

(E)-3-Phenyl- $N$-(3-(trifluoromethyl)benzyl)prop-2-en-1-amine $\quad(\boldsymbol{S}-20): \quad$ Method $\quad$ A $\quad$ used. 3(trifluoromethyl)benzylamine (500 mg) was used. Product recovered as a yellow oil (274 mg, 33\% yield). ${ }^{1} \mathrm{H}$ NMR $\left(600 \mathrm{MHz}, \mathrm{CDCl}_{3}\right) \delta 7.63(\mathrm{~s}, 1 \mathrm{H}), 7.56(\mathrm{~d}, J=7.7 \mathrm{~Hz}, 1 \mathrm{H}), 7.53(\mathrm{~d}, J=7.8 \mathrm{~Hz}, 1 \mathrm{H}), 7.46(\mathrm{t}, J=7.7 \mathrm{~Hz}, 1 \mathrm{H}), 7.38(\mathrm{~d}$, $J=7.6 \mathrm{~Hz}, 2 \mathrm{H}), 7.32(\mathrm{dd}, J=10.5,4.8 \mathrm{~Hz}, 2 \mathrm{H}), 7.24(\mathrm{t}, J=7.3 \mathrm{~Hz}, 1 \mathrm{H}), 6.55(\mathrm{~d}, J=15.9 \mathrm{~Hz}, 1 \mathrm{H}), 6.32(\mathrm{dt}, J=15.9$, $6.4 \mathrm{~Hz}, 1 \mathrm{H}), 3.91(\mathrm{~s}, 2 \mathrm{H}), 3.46(\mathrm{dd}, J=6.4,1.3 \mathrm{~Hz}, 2 \mathrm{H}), 1.95(\mathrm{~s}, 1 \mathrm{H}) .{ }^{13} \mathrm{C} \mathrm{NMR}\left(151 \mathrm{MHz}, \mathrm{CDCl}_{3}\right) \delta=140.9,137.0$, 132.2, 131.8, 130.9 (q, $J=32.0 \mathrm{~Hz}), 129.0,128.7,127.7,127.7,126.5,125.1$ (q, $J=3.7 \mathrm{~Hz}), 124.1$ (q, $J=3.8 \mathrm{~Hz})$, $124.3(\mathrm{~d}, J=272.3 \mathrm{~Hz}), 52.7,51.2 .{ }^{19} \mathrm{~F}$ NMR $\left(376 \mathrm{MHz}, \mathrm{CDCl}_{3}\right) \delta=-62.9$. HRMS (ESI - MS): calcd. 292.1308 $[\mathrm{M}+\mathrm{H}]^{+}$Found: 292.1317.

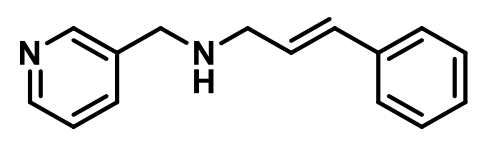

(E)-3-Phenyl- $N$-(pyridine-3-ylmethyl)prop-2-en-1-amine (S-21): Method A used. 3-(Aminomethyl)pyridine (500 $\mathrm{mg}$ ) was used. Product recovered as a yellow oil $\left(539 \mathrm{mg}, 52 \%\right.$ yield). ${ }^{1} \mathrm{H}$ NMR $\left(600 \mathrm{MHz}, \mathrm{CDCl}_{3}\right) \delta 8.54(\mathrm{~s}, 1 \mathrm{H})$, $8.53-8.43(\mathrm{~m}, 1 \mathrm{H}), 7.66(\mathrm{~d}, J=7.7 \mathrm{~Hz}, 1 \mathrm{H}), 7.34(\mathrm{~d}, J=7.3 \mathrm{~Hz}, 2 \mathrm{H}), 7.27$ (t, $J=7.6 \mathrm{~Hz}, 2 \mathrm{H}), 7.25-7.21(\mathrm{~m}, 1 \mathrm{H})$, $7.19(\mathrm{t}, J=7.3 \mathrm{~Hz}, 1 \mathrm{H}), 6.51(\mathrm{~d}, J=15.9 \mathrm{~Hz}, 1 \mathrm{H}), 6.32(\mathrm{dt}, J=15.9,6.3 \mathrm{~Hz}, 1 \mathrm{H}), 3.81(\mathrm{~s}, 2 \mathrm{H}), 3.48-3.30(\mathrm{~m}, 2 \mathrm{H})$, $1.72(\mathrm{~s}, 1 \mathrm{H}) .{ }^{13} \mathrm{C} \mathrm{NMR}\left(151 \mathrm{MHz}, \mathrm{CDCl}_{3}\right) \delta=149.9,148.7,137.0,136.0,135.6,131.9,128.7,128.0,127.6,126.4$, 123.6, 51.3, 50.6. HRMS (ESI - MS): calcd. 225.1386 [M+H]+ Found: 225.1395.

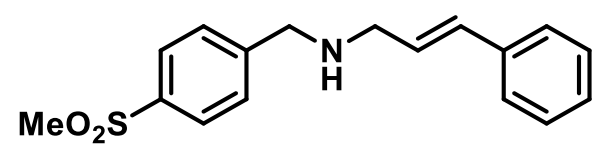

(E)- $N$-(4-(Methylsulfonyl)benzyl)-3-phenylprop-2-en-1-amine $\quad(S-22): \quad$ Method $\quad$ A $\quad$ used. (4(methylsulfonyl)phenyl)methanamine (500 mg) was used. Product recovered as a yellow oil (488 $\mathrm{mg}, 60 \%$ yield). ${ }^{1} \mathrm{H}$ NMR $\left(600 \mathrm{MHz}, \mathrm{CDCl}_{3}\right) \delta 7.91(\mathrm{~d}, J=8.3 \mathrm{~Hz}, 2 \mathrm{H}), 7.57(\mathrm{~d}, J=8.2 \mathrm{~Hz}, 2 \mathrm{H}), 7.37(\mathrm{~d}, J=7.7 \mathrm{~Hz}, 2 \mathrm{H}), 7.31(\mathrm{t}, J=7.6$ $\mathrm{Hz}, 2 \mathrm{H}), 7.24(\mathrm{t}, J=7.3 \mathrm{~Hz}, 1 \mathrm{H}), 6.54(\mathrm{~d}, J=15.9 \mathrm{~Hz}, 1 \mathrm{H}), 6.29(\mathrm{dt}, J=15.8,6.3 \mathrm{~Hz}, 1 \mathrm{H}), 3.94(\mathrm{~s}, 2 \mathrm{H}), 3.45(\mathrm{dd}, J$ $=6.4,1.3 \mathrm{~Hz}, 2 \mathrm{H}), 3.04(\mathrm{~s}, 3 \mathrm{H}), 1.62(\mathrm{~s}, 1 \mathrm{H}) .{ }^{13} \mathrm{C} \mathrm{NMR}\left(151 \mathrm{MHz}, \mathrm{CDCl}_{3}\right) \delta=147.0,139.2,137.0,132.0,129.1$, 128.7, 127.9, 127.7, 126.4, 52.7, 51.4, 44.7. HRMS (ESI - MS): calcd. 302.1209 [M+H] ${ }^{+}$Found: 302.1219.

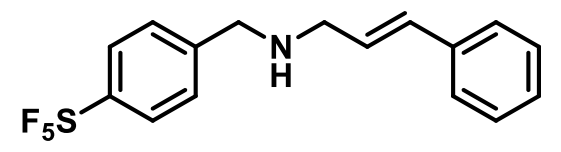

$(\boldsymbol{E})-\boldsymbol{N}$-(4-(Pentafluoro- $\boldsymbol{\lambda}^{6}$-sulfaneyl)benzyl)-3-phenylprop-2-en-1-amine $\quad(\boldsymbol{S}-23)$ : $\quad$ Method $\quad$ B $\quad$ used. $\quad 4$ (Pentafluorosulfanyl)benzaldehyde $\left(500 \mathrm{mg}\right.$ ) was used. Product recovered as a yellow oil (586 mg, 78\% yield). ${ }^{1} \mathrm{H}$ NMR (600 MHz, $\left.\mathrm{CDCl}_{3}\right) \delta 7.75-7.69(\mathrm{~m}, 2 \mathrm{H}), 7.46(\mathrm{~d}, J=8.4 \mathrm{~Hz}, 2 \mathrm{H}), 7.37(\mathrm{dd}, J=8.2,1.0 \mathrm{~Hz}, 2 \mathrm{H}), 7.32(\mathrm{dd}, J=$ 10.5, $4.9 \mathrm{~Hz}, 2 \mathrm{H}), 7.26-7.22(\mathrm{~m}, 1 \mathrm{H}), 6.55$ (d, $J=15.9 \mathrm{~Hz}, 1 \mathrm{H}), 6.30$ (dt, $J=15.9,6.4 \mathrm{~Hz}, 1 \mathrm{H}), 3.90(\mathrm{~s}, 2 \mathrm{H}), 3.44$ $(\mathrm{dd}, J=6.4,1.4 \mathrm{~Hz}, 2 \mathrm{H}), 1.72$ (bs, 1H). ${ }^{13} \mathrm{C} \mathrm{NMR}\left(151 \mathrm{MHz}, \mathrm{CDCl}_{3}\right) \delta=152.8$ (quint, $J=17.1 \mathrm{~Hz}$ ), 144.3, 137.0, 
132.0, 128.7, 128.4, 127.9, 127.7, 126.4, 126.2 (quint, $J=4 \mathrm{~Hz}), 77.4,76.9,52.3,51.3 .{ }^{19} \mathrm{~F}$ NMR $\left(376 \mathrm{MHz}, \mathrm{CDCl}_{3}\right)$ $\delta=84.5$ (quint), 62.6 (d). HRMS (ESI - MS): calcd. $350.0996[\mathrm{M}+\mathrm{H}]^{+}$Found: 350.1009.

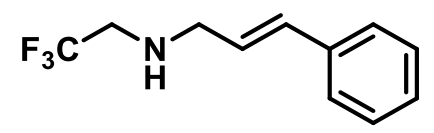

(E)-3-Phenyl- $\boldsymbol{N}$-(2,2,2-trifluoroethyl)prop-2-en-1-amine ( $\boldsymbol{S}$-24): Method A used. 4 2,2,2-trifluoroethan-1-amine $(500 \mathrm{mg})$ was used. Product recovered as a yellow oil $\left(705 \mathrm{mg}, 65 \%\right.$ yield). ${ }^{1} \mathrm{H}$ NMR $\left(600 \mathrm{MHz}, \mathrm{CDCl}_{3}\right) \delta 7.40(\mathrm{~d}, J$ $=7.7 \mathrm{~Hz}, 2 \mathrm{H}), 7.34(\mathrm{t}, J=7.6 \mathrm{~Hz}, 2 \mathrm{H}), 7.26(\mathrm{q}, J=7.2 \mathrm{~Hz}, 1 \mathrm{H}), 6.57(\mathrm{~d}, J=15.9 \mathrm{~Hz}, 1 \mathrm{H}), 6.24(\mathrm{dt}, J=15.9,6.4 \mathrm{~Hz}$, $1 \mathrm{H}), 3.53(\mathrm{~d}, J=6.4 \mathrm{~Hz}, 2 \mathrm{H}), 3.24(\mathrm{q}, J=9.4 \mathrm{~Hz}, 2 \mathrm{H}), 1.51(\mathrm{~s}, 1 \mathrm{H}) .{ }^{13} \mathrm{C} \mathrm{NMR}\left(151 \mathrm{MHz}, \mathrm{CDCl}_{3}\right) \delta=136.8,132.4$, 128.7, 128.5, 127.8, 127.2, 126.7, $125.7(\mathrm{q}, J=279 \mathrm{~Hz}), 49.8(\mathrm{q}, J=31.4 \mathrm{~Hz}), 48.1 .{ }^{19} \mathrm{~F}$ NMR $\left(376 \mathrm{MHz}, \mathrm{CDCl}_{3}\right)$ $\delta=-71.9(\mathrm{t})$. HRMS (ESI - MS): calcd. 216.0995 [M+H] $]^{+}$Found: 216.0999.

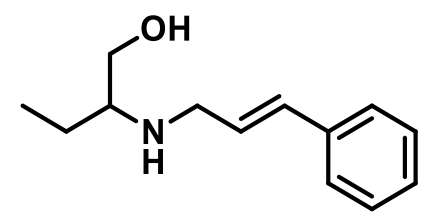

2-(Cinnamylamino)butan-1-ol (S-25): Method A used. 2-aminobutan-1-ol (500 mg) was used. Product recovered as a yellow oil (633 mg, 55\% yield). ${ }^{1} \mathrm{H}$ NMR (600 MHz, $\left.\mathrm{CDCl}_{3}\right) \delta 7.37(\mathrm{dd}, J=8.2,1.0 \mathrm{~Hz}, 2 \mathrm{H}), 7.34-7.29(\mathrm{~m}, 2 \mathrm{H})$, $7.25-7.20(\mathrm{~m}, 1 \mathrm{H}), 6.53(\mathrm{~d}, J=15.9 \mathrm{~Hz}, 1 \mathrm{H}), 6.28(\mathrm{dt}, J=15.8,6.3 \mathrm{~Hz}, 1 \mathrm{H}), 3.66(\mathrm{dd}, J=10.6,4.0 \mathrm{~Hz}, 1 \mathrm{H}), 3.47$ (ddd, $J=13.9,6.5,1.4 \mathrm{~Hz}, 1 \mathrm{H}), 3.40$ (ddd, $J=13.9,6.1,1.2 \mathrm{~Hz}, 1 \mathrm{H}), 3.34(\mathrm{dd}, J=10.6,6.4 \mathrm{~Hz}, 1 \mathrm{H}), 2.68-2.57$ (m, $1 \mathrm{H}), 2.11(\mathrm{bs}, 1 \mathrm{H}), 1.61-1.51(\mathrm{~m}, 1 \mathrm{H}), 1.51-1.38(\mathrm{~m}, 1 \mathrm{H}), 0.94(\mathrm{t}, J=7.5 \mathrm{~Hz}, 3 \mathrm{H}) .{ }^{13} \mathrm{C} \mathrm{NMR}\left(151 \mathrm{MHz}, \mathrm{CDCl}_{3}\right) \delta$ $=137.1,131.3,128.7,128.6,127.5,126.4,62.7,59.7,49.1,24.5,10.5$. HRMS (ESI - MS): calcd. 206.1539 [M+H] ${ }^{+}$ Found: 206.1546.

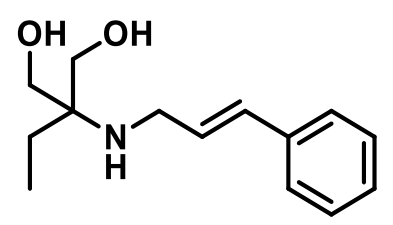

2-(Cinnamylamino)-2-ethylpropane-1,3-diol ( $\boldsymbol{S}$-26): Method A used. Pentan-3-amine (500 $\mathrm{mg}$ ) was used. Product recovered as a yellow oil $\left(375 \mathrm{mg}, 38 \%\right.$ yield). ${ }^{1} \mathrm{H}$ NMR $\left(600 \mathrm{MHz}, \mathrm{CDCl}_{3}\right) \delta 7.36(\mathrm{dd}, J=8.2,1.0 \mathrm{~Hz}, 2 \mathrm{H}), 7.30$ (t, $J=7.7 \mathrm{~Hz}, 2 \mathrm{H}), 7.25-7.17(\mathrm{~m}, 1 \mathrm{H}), 6.56(\mathrm{~d}, J=15.9 \mathrm{~Hz}, 1 \mathrm{H}), 6.30(\mathrm{dt}, J=15.8,6.4 \mathrm{~Hz}, 1 \mathrm{H}), 3.55(\mathrm{q}, J=11.0 \mathrm{~Hz}$, $4 \mathrm{H}), 3.32(\mathrm{dd}, J=6.4,1.1 \mathrm{~Hz}, 2 \mathrm{H}), 3.00(\mathrm{~s}, 3 \mathrm{H}), 1.43(\mathrm{q}, J=7.5 \mathrm{~Hz}, 2 \mathrm{H}), 0.87$ (t, $J=7.6 \mathrm{~Hz}, 3 \mathrm{H}) .{ }^{13} \mathrm{C}$ NMR $(151$ $\left.\mathrm{MHz}, \mathrm{CDCl}_{3}\right) \delta=137.0,131.9,128.7,127.8,127.7,126.4,64.7,59.4,43.5,23.6,7.4$. HRMS (ESI - MS): calcd. $236.1645[\mathrm{M}+\mathrm{H}]^{+}$Found: 236.1651.

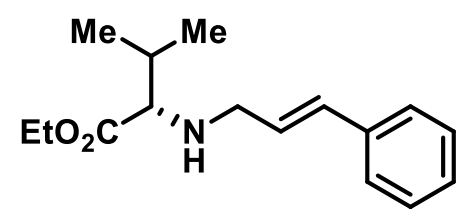


Ethyl cinnamyl- $L$-valinate $(S$-27): Method A used. Ethyl $L$-valinate $(500 \mathrm{mg})$ was used. Product recovered as a yellow oil (728 mg, 81\% yield). ${ }^{1} \mathrm{H}$ NMR (600 MHz, $\left.\mathrm{CDCl}_{3}\right) \delta 7.40-7.32(\mathrm{~m}, 2 \mathrm{H}), 7.33-7.27(\mathrm{~m}, 2 \mathrm{H}), 7.24-7.18$ (m, 1H), $6.51(\mathrm{~d}, J=15.9 \mathrm{~Hz}, 1 \mathrm{H}), 6.24(\mathrm{dt}, J=15.8,6.4 \mathrm{~Hz}, 1 \mathrm{H}), 4.30-4.07(\mathrm{~m}, 2 \mathrm{H}), 3.43$ (ddd, $J=13.8,6.4,1.5$ $\mathrm{Hz}, 1 \mathrm{H}), 3.26(\mathrm{ddd}, J=13.8,6.4,1.4 \mathrm{~Hz}, 1 \mathrm{H}), 3.05(\mathrm{~d}, J=5.9 \mathrm{~Hz}, 1 \mathrm{H}), 2.02-1.86(\mathrm{~m}, 1 \mathrm{H}), 1.74(\mathrm{~s}, 1 \mathrm{H}), 1.26(\mathrm{t}, J=$ $7.1 \mathrm{~Hz}, 3 \mathrm{H}), 0.97(\mathrm{~d}, J=6.9 \mathrm{~Hz}, 3 \mathrm{H}), 0.96(\mathrm{~d}, J=6.8 \mathrm{~Hz}, 3 \mathrm{H}) .{ }^{13} \mathrm{C} \mathrm{NMR}\left(151 \mathrm{MHz}, \mathrm{CDCl}_{3}\right) \delta=175.4,137.2,131.7$, 128.6, 128.4, 127.5, 126.4 66.5, 60.5, 50.8, 31.8, 19.3, 18.8, 14.5. HRMS (ESI - MS): calcd. 262.1802 [M+H] $]^{+}$Found: 262.1811 .

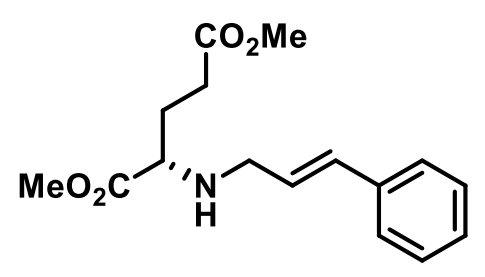

Dimethyl cinnamyl- $\boldsymbol{L}$-glutamate $(\boldsymbol{S}$-28): Method A used. Dimethyl $L$-glutamate $(500 \mathrm{mg}$ ) was used. Product recovered as a yellow oil (374 mg, 45\% yield). ${ }^{1} \mathrm{H} \mathrm{NMR}\left(600 \mathrm{MHz}, \mathrm{CDCl}_{3}\right) \delta 7.33(\mathrm{~d}, J=7.2 \mathrm{~Hz}, 2 \mathrm{H}), 7.27(\mathrm{t}, J=$ $7.7 \mathrm{~Hz}, 1 \mathrm{H}), 7.18$ (t, $J=7.3 \mathrm{~Hz}, 1 \mathrm{H}), 6.47(\mathrm{~d}, J=15.9 \mathrm{~Hz}, 1 \mathrm{H}), 6.18$ (dt, $J=15.9,6.3 \mathrm{~Hz}, 1 \mathrm{H}), 3.67$ (s, 3H), $3.62(\mathrm{~s}$, $3 \mathrm{H}), 3.38(\mathrm{ddd}, J=13.9,6.5,1.4 \mathrm{~Hz}, 1 \mathrm{H}), 3.29$ (dd, $J=8.1,5.6 \mathrm{~Hz}, 1 \mathrm{H}), 3.24(\mathrm{ddd}, J=13.9,6.2,1.4 \mathrm{~Hz}, 1 \mathrm{H}), 2.44$ $(\mathrm{t}, J=7.4 \mathrm{~Hz}, 2 \mathrm{H}), 2.02-1.94(\mathrm{~m}, 1 \mathrm{H}), 1.90-1.84(\mathrm{~m}, 1 \mathrm{H}), 1.71(\mathrm{~s}, 1 \mathrm{H}) \cdot{ }^{13} \mathrm{C} \mathrm{NMR}\left(151 \mathrm{MHz}, \mathrm{CDCl}_{3}\right) \delta=175.4$, 173.5, 136.9, 131.5, 128.5, 127.9, 127.3, 126.2, 59.6, 51.8, 51.5, 50.1, 30.4, 28.3. HRMS (ESI - MS): calcd. 292.1543 $[\mathrm{M}+\mathrm{H}]^{+}$Found: 292.1548.

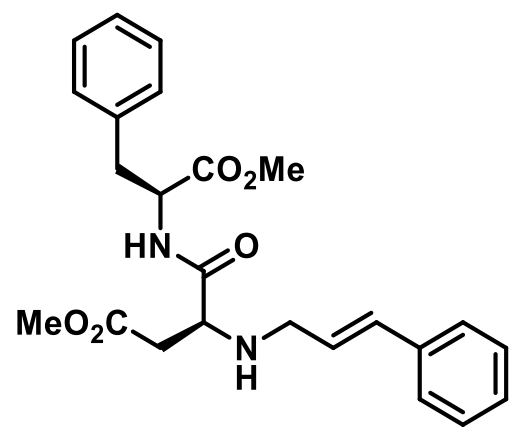

Methyl (S)-3-(cinnamylamino)-4-(((S)-1-methoxy-1-oxo-3-phenylpropan--yl)amino)-4-oxobutanoate $(S$-29): Method C used. Methyl (S)-3-amino-4-(((S)-1-methoxy-1-oxo-3-phenylpropan-2-yl)amino)-4-oxobutanoate (500 $\mathrm{mg}$ ) was used. Product recovered as a yellow oil $\left(502 \mathrm{mg}, 73 \%\right.$ yield). ${ }^{1} \mathrm{H}$ NMR $\left(600 \mathrm{MHz}, \mathrm{CDCl}_{3}\right) \delta 7.78(\mathrm{~d}, J=8.5$ $\mathrm{Hz}, 1 \mathrm{H}), 7.36-7.27(\mathrm{~m}, 6 \mathrm{H}), 7.23(\mathrm{~m}, 2 \mathrm{H}), 7.15(\mathrm{~d}, J=7.0 \mathrm{~Hz}, 2 \mathrm{H}), 6.47(\mathrm{~d}, J=15.9 \mathrm{~Hz}, 1 \mathrm{H}), 6.15(\mathrm{dt}, J=15.9,6.3$ $\mathrm{Hz}, 1 \mathrm{H}), 4.87$ (dt, $J=8.5,6.2 \mathrm{~Hz}, 1 \mathrm{H}), 3.72(\mathrm{~s}, 3 \mathrm{H}), 3.68(\mathrm{~s}, 3 \mathrm{H}), 3.50(\mathrm{dd}, J=8.1,4.1 \mathrm{~Hz}, 1 \mathrm{H}), 3.32(\mathrm{dd}, J=6.3,1.4$ $\mathrm{Hz}, 2 \mathrm{H}), 3.16(\mathrm{dd}, J=13.9,5.7 \mathrm{~Hz}, 1 \mathrm{H}), 3.10(\mathrm{dd}, J=13.9,6.6 \mathrm{~Hz}, 1 \mathrm{H}), 2.76(\mathrm{dd}, J=16.6,4.1 \mathrm{~Hz}, 1 \mathrm{H}), 2.58(\mathrm{dd}, J$ $=16.6,8.1 \mathrm{~Hz}, 1 \mathrm{H}), 1.90$ (bs, $1 \mathrm{H}) .{ }^{13} \mathrm{C}$ NMR $\left(151 \mathrm{MHz}, \mathrm{CDCl}_{3}\right) \delta=172.5,172.2,172.0,136.9,136.1,132.0,129.4$, 128.7, 128.7, 127.7, 127.4, 127.3, 126.5, 77.4, 76.9, 58.2, 52.8, 52.4, 52.1, 50.0, 38.1, 36.0. HRMS (ESI - MS): calcd. 425.2071 [M+H] $]^{+}$Found: 425.2069 . 


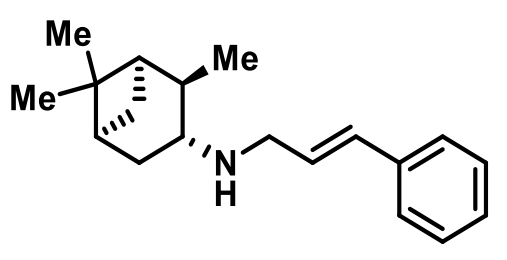

$(1 R, 2 R, 3 R, 5 S)$ - $N$-Cinnamyl-2,6,6-trimethylbicyclo[3.1.1]heptan-3-amine ( $S$-30): Method A used. $(1 R, 2 S, 3 R, 5 S)$ 3,6,6-trimethylbicyclo[3.1.1] heptan-2-amine (500 mg) was used. Product recovered as a yellow oil (483 $\mathrm{mg}, 55 \%$ yield). ${ }^{1} \mathrm{H} \mathrm{NMR}\left(600 \mathrm{MHz}, \mathrm{CDCl}_{3}\right) \delta 7.38(\mathrm{~d}, J=7.3 \mathrm{~Hz}, 2 \mathrm{H}), 7.31(\mathrm{dd}, J=10.5,4.8 \mathrm{~Hz}, 2 \mathrm{H}), 7.22(\mathrm{t}, J=7.3 \mathrm{~Hz}$, $1 \mathrm{H}), 6.55(\mathrm{~d}, J=15.9 \mathrm{~Hz}, 1 \mathrm{H}), 6.35$ (dt, $J=15.8,6.4 \mathrm{~Hz}, 1 \mathrm{H}), 3.49$ (ddd, $J=13.6,6.1,1.4 \mathrm{~Hz}, 1 \mathrm{H}), 3.39$ (ddd, $J=$ 13.7, 6.8, 1.2 Hz, 1H), $2.94(\mathrm{dt}, J=9.3,5.5 \mathrm{~Hz}, 1 \mathrm{H}), 2.45-2.37(\mathrm{~m}, 1 \mathrm{H}), 2.37-2.29(\mathrm{~m}, 1 \mathrm{H}), 1.96(\mathrm{dt}, J=5.6,2.7$ $\mathrm{Hz}, 1 \mathrm{H}), 1.89-1.76(\mathrm{~m}, 2 \mathrm{H}), 1.63(\mathrm{ddd}, J=13.5,5.1,2.6 \mathrm{~Hz}, 1 \mathrm{H}), 1.22(\mathrm{~s}, 3 \mathrm{H}), 1.13$ (d, $J=7.2 \mathrm{~Hz}, 3 \mathrm{H}), 0.99-0.96$ (m, 4H). ${ }^{13} \mathrm{C}$ NMR $\left(151 \mathrm{MHz}, \mathrm{CDCl}_{3}\right) \delta=137.3,131.3,128.9,128.7,127.4,126.4$, , 56.4, 50.2, 48.1, 45.3, 41.9, 38.7, 36.9, 33.9, 27.9, 23.6, 21.7. HRMS (ESI - MS): calcd. $270.2216[\mathrm{M}+\mathrm{H}]^{+}$Found: 270.2225.

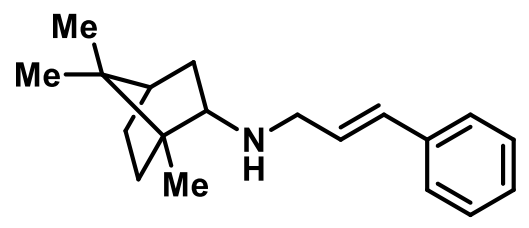

(2S)-N-Cinnamyl-1,7,7-trimethylbicyclo[2.2.1]heptan-2-amine $\quad(\boldsymbol{S}$-31): $\quad$ Method $\quad$ A $\quad$ used. $\quad(2 S)-1,7,7-$ trimethylbicyclo[2.2.1]heptan-2-amine (500 mg) was used. Product recovered as a yellow oil (527 mg, 60\% yield). ${ }^{1} \mathrm{H}$ NMR (600 MHz, $\left.\mathrm{CDCl}_{3}\right) \delta 7.39(\mathrm{dd}, J=8.2,1.0 \mathrm{~Hz}, 2 \mathrm{H}), 7.34-7.29(\mathrm{t}, J=7.7 \mathrm{~Hz}, 2 \mathrm{H}), 7.25-7.20(\mathrm{~m}, 1 \mathrm{H})$, $6.53(\mathrm{~d}, J=15.9 \mathrm{~Hz}, 1 \mathrm{H}), 6.33(\mathrm{dt}, J=15.8,6.3 \mathrm{~Hz}, 1 \mathrm{H}), 3.47$ (ddd, $J=13.9,6.0,1.5 \mathrm{~Hz}, 1 \mathrm{H}), 3.38$ (ddd, $J=13.9$, 6.6, $1.3 \mathrm{~Hz}, 1 \mathrm{H}), 2.90$ (ddd, $J=10.0,4.1,2.0 \mathrm{~Hz}, 1 \mathrm{H}), 2.20$ (dddd, $J=13.0,10.0,4.7,3.3 \mathrm{~Hz}, 1 \mathrm{H}), 1.86-1.77$ (m, $1 \mathrm{H}), 1.77-1.68(\mathrm{~m}, 1 \mathrm{H}), 1.64(\mathrm{t}, J=4.6 \mathrm{~Hz}, 1 \mathrm{H}), 1.31$ (tdd, $J=12.9,4.6,2.0 \mathrm{~Hz}, 1 \mathrm{H}), 1.21$ (ddd, $J=12.5,9.5,4.6$ $\mathrm{Hz}, 1 \mathrm{H}), 0.89$ (s, 3H), 0.88 (s, 3H), 0.88 (s, 3H). ${ }^{13} \mathrm{C}$ NMR $\left(151 \mathrm{MHz}, \mathrm{CDCl}_{3}\right) \delta=137.4,130.8,129.6,128.6,127.3$, 126.4, 63.0, 51.5, 48.9, 48.5, 45.1, 38.5, 28.6, 27.5, 20.0, 18.8, 14.5. HRMS (ESI - MS): calcd. 270.2216 [M+H] ${ }^{+}$ Found: 270.2222 .
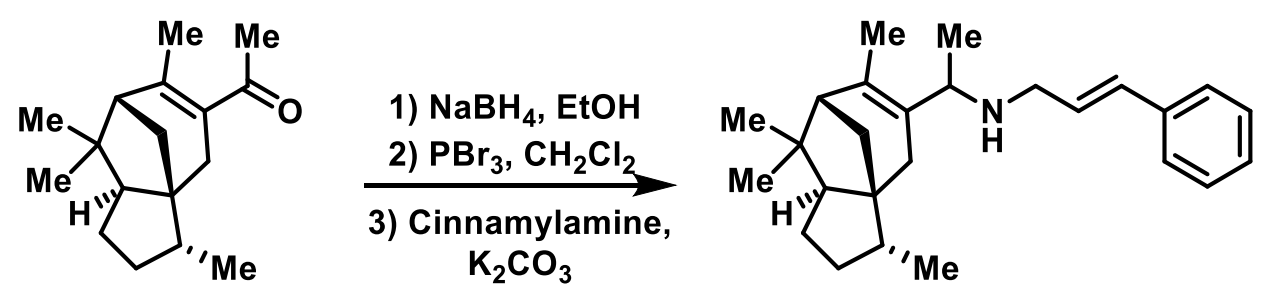

(E)-3-Phenyl- $N$-(1-((3R,3a $R, 7 R, 8 \mathrm{a} S)$-3,6,8,8-tetramethyl-2,3,4,7,8,8a-hexahydro-1H-3a,7methanoazulen-5yl)ethyl)prop-2-en-1-amine ( $\boldsymbol{S - 3 2})$ : To a solution of methyl cedryl ketone (4.0 mmol, 1.0 equiv.) in EtOH (10 $\mathrm{mL})$ was added $\mathrm{NaBH}_{4}$ (1.2 equiv.) at $0{ }^{\circ} \mathrm{C}$ and the reaction mixture was stirred at room temperature for $2 \mathrm{~h}$. After completion of the reaction (monitored by TLC), the reaction was quenched with deionized water, diluted with $\mathrm{CH}_{2} \mathrm{Cl}_{2}$, and extracted with $\mathrm{CH}_{2} \mathrm{Cl}_{2}$ three times. The combined organic layer was washed with brine, dried over $\mathrm{Na}_{2} \mathrm{SO}_{4}$, filtered, and concentrated in vacuo to afford the corresponding alcohol, which was directly used in the next step without further purification. To a solution of the residue obtained above in anhydrous $\mathrm{CH}_{2} \mathrm{Cl}_{2}(10.0 \mathrm{~mL})$ was added 
$\mathrm{PBr}_{3}$ (0.70 equiv.) under an anhydrous nitrogen atmosphere at $0{ }^{\circ} \mathrm{C}$, and the resulting reaction mixture was stirred at room temperature. After completion of reaction (monitored by TLC), the mixture was quenched with water and extracted with $\mathrm{CH}_{2} \mathrm{Cl}_{2}$ three times. The combined organic phase was washed with brine, and concentrated in vacuo to afford the corresponding crude alkyl bromide, which was directly used in the next step without further purification. Then, Method D used. Product was purified over silica gel in hexanes/EtOAc (80:20) and recovered as a light yellow oil $\left(581 \mathrm{mg}, 40 \%\right.$ yield). ${ }^{1} \mathrm{H}$ NMR $\left(600 \mathrm{MHz}, \mathrm{CDCl}_{3}\right) \delta 7.37(\mathrm{~d}, J=7.8 \mathrm{~Hz}, 2 \mathrm{H}), 7.30(\mathrm{t}, J=7.7 \mathrm{~Hz}, 2 \mathrm{H}), 7.23-7.19$ $(\mathrm{m}, 1 \mathrm{H}), 6.49(\mathrm{~d}, J=15.8 \mathrm{~Hz}, 1 \mathrm{H}), 6.32(\mathrm{dt}, J=15.8,6.4 \mathrm{~Hz}, 1 \mathrm{H}), 3.78(\mathrm{q}, J=6.6 \mathrm{~Hz}, 1 \mathrm{H}), 3.30(\mathrm{dd}, J=13.5,5.7$ $\mathrm{Hz}, 1 \mathrm{H}), 3.20(\mathrm{ddd}, J=13.8,6.8,1.0 \mathrm{~Hz}, 1 \mathrm{H}), 2.10(\mathrm{dd}, J=16.2,1.9 \mathrm{~Hz}, 1 \mathrm{H}), 1.91-1.83(\mathrm{~m}, 1 \mathrm{H}), 1.80(\mathrm{dd}, J=11.2$, $5.4 \mathrm{~Hz}, 3 \mathrm{H}), 1.68(\mathrm{t}, J=1.7 \mathrm{~Hz}, 3 \mathrm{H}), 1.59(\mathrm{~d}, J=6.5 \mathrm{~Hz}, 2 \mathrm{H}), 1.42-1.34(\mathrm{~m}, 4 \mathrm{H}), 1.13(\mathrm{~d}, J=6.7 \mathrm{~Hz}, 3 \mathrm{H}), 0.96(\mathrm{~d}$, $J=13.5 \mathrm{~Hz}, 6 \mathrm{H}), 0.90(\mathrm{~d}, J=7.2 \mathrm{~Hz}, 3 \mathrm{H}) .{ }^{13} \mathrm{C} \mathrm{NMR}\left(151 \mathrm{MHz}, \mathrm{CDCl}_{3}\right) \delta=137.4,135.1,131.3,129.9,129.1,128.6$, 127.4, 126.5, 126.4, 58.9, 57.2, 53.9, 52.2, 49.6, 48.3, 41.8, 40.8, 36.1, 35.6, 28.0, 25.8, 24.8, 20.7, 20.2, 15.6. HRMS (ESI - MS): calcd. $364.2999[\mathrm{M}+\mathrm{H}]^{+}$Found: 364.3002.
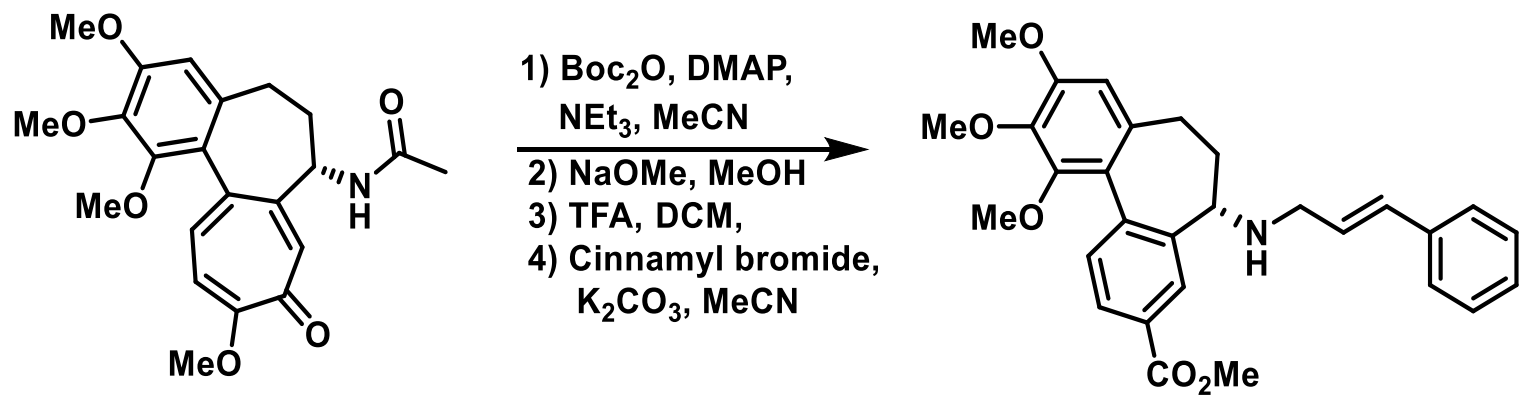

Methyl (5S)-5-(cinnamylamino)-9,10,11-trimethoxy-6,7-dihydro-5H-dibenzo[a,c][7]annulene-3-carboxylate (S-33): Based on a modification of a literature procedure, ${ }^{9}$ a mixture of colchicine $(2.6 \mathrm{~g}, 6.5 \mathrm{mmol}), \mathrm{Boc}_{2} \mathrm{O}(6.0 \mathrm{~g}$, $27.5 \mathrm{mmol})$, DMAP (790 mg, $7.0 \mathrm{mmol})$ and trimethylamine $(1.75 \mathrm{~mL}, 12.6 \mathrm{mmol})$ were combined in $25 \mathrm{~mL} \mathrm{CH}_{3} \mathrm{CN}_{\text {, }}$ followed by heating under reflux for $20 \mathrm{~h}$. After cooling the mixture was extracted with $\mathrm{CHCl}_{3}$, washed with $10 \%$ citric acid, followed by brine. Concentration in vacuo gave the crude product, which was treated with $\mathrm{NaOMe}(1.2 \mathrm{~g}, 22.2$ $\mathrm{mmol}$ ) and $35 \mathrm{~mL} \mathrm{MeOH}$ at $0^{\circ} \mathrm{C}$ and stirred for $12 \mathrm{~h}$ at $50^{\circ} \mathrm{C}$. The mixture was extracted with EtOAc and concentrated in vасио to give boc protected desacetylallocolchicine. The product was mixed with $15 \mathrm{~mL}$ trifluoroacetic acid and $30 \mathrm{~mL} \mathrm{DCM}$ and stirred at room temperature for $3 \mathrm{~h}$. Aqueous $1 \mathrm{~N} \mathrm{NaOH}$ was then added into the mixture until the $\mathrm{pH}$ reached 10. The reaction mixture was then extracted with DCM (3 x $25 \mathrm{~mL})$ and concentrated in vacuo give crude product (809 mg, 35\% yield), which was directly used in the next step without further purification. Method C was then used. Product was purified over silica gel in hexanes/EtOAc (50:50) and recovered as a light yellow oil (428 mg, $40 \%$ yield). ${ }^{1} \mathrm{H}$ NMR $\left(600 \mathrm{MHz}, \mathrm{CDCl}_{3}\right) \delta 8.26(\mathrm{~s}, 1 \mathrm{H}), 7.97(\mathrm{~d}, J=7.9 \mathrm{~Hz}, 1 \mathrm{H}), 7.52(\mathrm{~d}, J=8.0 \mathrm{~Hz}, 1 \mathrm{H}), 7.27-7.23$ $(\mathrm{m}, 4 \mathrm{H}), 7.16(\mathrm{~s}, 1 \mathrm{H}), 6.55(\mathrm{~s}, 1 \mathrm{H}), 6.38(\mathrm{~d}, J=15.8 \mathrm{~Hz}, 1 \mathrm{H}), 6.31-6.16(\mathrm{~m}, 1 \mathrm{H}), 3.92(\mathrm{~s}, 3 \mathrm{H}), 3.87(\mathrm{~d}, J=11.9 \mathrm{~Hz}$, $6 \mathrm{H}), 3.60(\mathrm{~s}, 1 \mathrm{H}), 3.51(\mathrm{~s}, 3 \mathrm{H}), 3.46-3.33(\mathrm{~m}, 1 \mathrm{H}), 3.22(\mathrm{~d}, J=6.1 \mathrm{~Hz}, 1 \mathrm{H}), 2.39(\mathrm{~d}, J=6.0 \mathrm{~Hz}, 2 \mathrm{H}), 2.17(\mathrm{dd}, J=$ 18.5, $11.3 \mathrm{~Hz}, 1 \mathrm{H}), 1.78(\mathrm{~s}, 1 \mathrm{H}) .{ }^{13} \mathrm{C} \mathrm{NMR}\left(151 \mathrm{MHz}, \mathrm{CDCl}_{3}\right) \delta=167.6,153.3,151.0,141.1,140.5,137.1,135.9$, 132.1, 128.9, 128.6, 127.5, 126.4, 125.2, 107.7, 77.4, 76.9, 61.3, 61.1, 57.1, 56.1, 52.3, 50.3, 31.0, 14.3. HRMS (ESI - MS): calcd. $474.2275[\mathrm{M}+\mathrm{H}]^{+}$Found: 474.2283. 


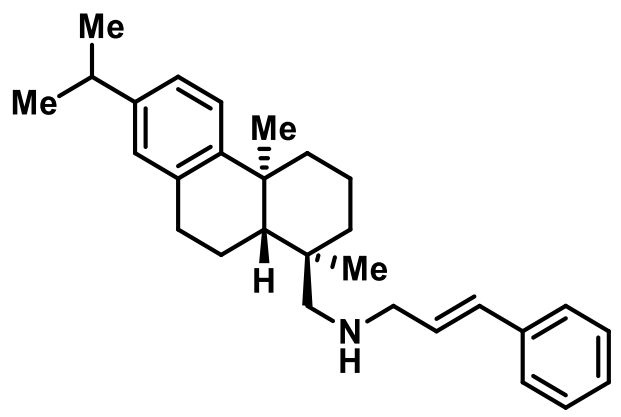

(E)- $N$-(((1R,4aS,10aR)-7-Isopropyl-1,4a-dimethyl-1,2,3,4,4a,9,10,10a-octahydrophenanthren-1-yl)methyl)-3-

phenylprop-2-en-1-amine (S-34): Method C used. (+)-Dehydroabietylamine (1 gm) was used. Product recovered as a yellow oil (885 mg, 63\% yield). ${ }^{1} \mathrm{H}$ NMR $\left(600 \mathrm{MHz}, \mathrm{CDCl}_{3}\right) \delta 7.46(\mathrm{~d}, J=7.5 \mathrm{~Hz}, 2 \mathrm{H}), 7.38(\mathrm{t}, J=7.6 \mathrm{~Hz}, 2 \mathrm{H})$, $7.28(\mathrm{ddd}, J=11.5,10.5,3.8 \mathrm{~Hz}, 2 \mathrm{H}), 7.08(\mathrm{dd}, J=8.1,1.6 \mathrm{~Hz}, 1 \mathrm{H}), 6.98(\mathrm{~s}, 1 \mathrm{H}), 6.60(\mathrm{~d}, J=15.9 \mathrm{~Hz}, 1 \mathrm{H}), 6.37$ (dd, $J=14.2,7.9 \mathrm{~Hz}, 1 \mathrm{H}), 3.47(\mathrm{~d}, J=5.9 \mathrm{~Hz}, 2 \mathrm{H}), 3.01-2.86(\mathrm{~m}, 2 \mathrm{H}), 2.65(\mathrm{~d}, J=11.8 \mathrm{~Hz}, 2 \mathrm{H}), 2.48-2.33(\mathrm{~m}, 2 \mathrm{H})$, $1.91-1.82(\mathrm{~m}, 2 \mathrm{H}), 1.80-1.67(\mathrm{~m}, 3 \mathrm{H}), 1.63-1.46(\mathrm{~m}, 4 \mathrm{H}), 1.32(\mathrm{~d}, J=7.2 \mathrm{~Hz}, 6 \mathrm{H}), 1.09-1.07$ (m, 1H), 1.03 (s, 2H), $0.99-0.92(\mathrm{~m}, 2 \mathrm{H}) .{ }^{13} \mathrm{C}$ NMR $\left(151 \mathrm{MHz}, \mathrm{CDCl}_{3}\right) \delta=147.6,145.5,137.3,134.9,130.9,129.2,128.6,127.3$, 126.8, 126.3, 124.3, 123.8, 61.2, 52.9, 45.5, 38.6, 37.5, 37.1, 36.3, 33.5, 30.4, 25.4, 24.1, 24.1, 19.3, 19.0, 18.9. HRMS (ESI - MS): calcd. $402.3155[\mathrm{M}+\mathrm{H}]^{+}$Found: 402.3163.
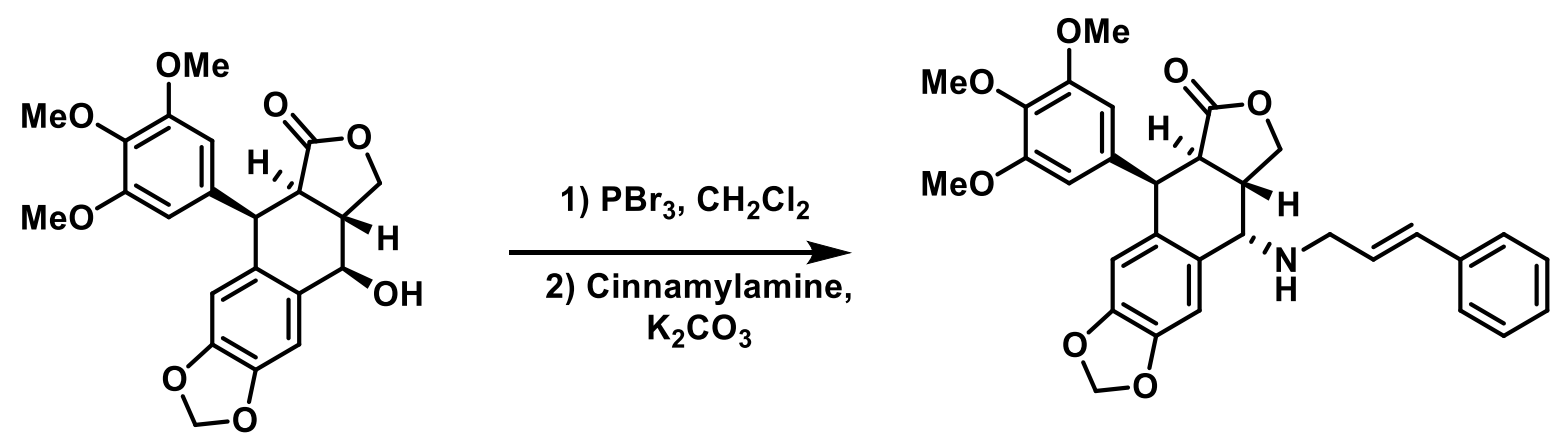

(5R,5aR,8aS,9S)-9-(Cinnamylamino)-5-(3,4,5-trimethoxyphenyl)-5,8,8a,9-

tetrahydrofuro[3',3':6,7]naphtha(2,3-d] $[\mathbf{1 , 3}]$ dioxol-6(5aH)-one (S-35): To a solution of podophyllotoxin $(1 \mathrm{~g}, 2.4$ mmol, 1 equiv.) in dry $\mathrm{CH}_{2} \mathrm{Cl}_{2}(10 \mathrm{~mL})$ was added $\mathrm{PBr}_{3}(457 \mathrm{mg}, 1.7 \mathrm{mmol}, 0.70$ equiv.) under argon atmosphere at $0{ }^{\circ} \mathrm{C}$ and the resulting reaction mixture was stirred at room temperature. After completion of reaction (monitored by TLC), the mixture was quenched by water and extracted with $\mathrm{CH}_{2} \mathrm{Cl}_{2}$ three times. The combined organic phase was washed by brine, and concentrated under reduced pressure to afford the corresponding crude alkyl bromide, which was directly used in the next step without further purification. Then, method D was used. Product was purified over silica gel in hexanes/EtOAc (50:50) and recovered as a light yellow oil (383 $\mathrm{mg}, 30 \%$ yield). Unexpected stereochemistry of the amine center was determined through coupling constant analysis and comparison with similar structures in the literature, ${ }^{10}$ and through 2-D NMR analysis. ${ }^{1} \mathrm{H}$ NMR $\left(600 \mathrm{MHz}, \mathrm{CDCl}_{3}\right) \delta 7.39(\mathrm{~d}, J=7.2 \mathrm{~Hz}, 1 \mathrm{H})$, $7.33(\mathrm{t}, J=7.5 \mathrm{~Hz}, 1 \mathrm{H}), 7.24(\mathrm{t}, J=7.3 \mathrm{~Hz}, 1 \mathrm{H}), 6.79(\mathrm{~s}, 1 \mathrm{H}), 6.58(\mathrm{~d}, J=15.8 \mathrm{~Hz}, 1 \mathrm{H}), 6.48(\mathrm{~s}, 1 \mathrm{H}), 6.33-6.23(\mathrm{~m}$, 3H), $5.94(\mathrm{dd}, J=10.3,1.2 \mathrm{~Hz}, 2 \mathrm{H}), 4.54(\mathrm{~d}, J=5.3 \mathrm{~Hz}, 1 \mathrm{H}), 4.33(\mathrm{~d}, J=9.0 \mathrm{~Hz}, 2 \mathrm{H}), 3.94(\mathrm{~d}, J=3.5 \mathrm{~Hz}, 1 \mathrm{H}), 3.79$ (s, 3H), 3.73 (s, 6H), 3.54 (ddd, $J=14.0,7.2,0.8 \mathrm{~Hz}, 1 \mathrm{H}), 3.44$ (dd, $J=13.9,5.2 \mathrm{~Hz}, 1 \mathrm{H}), 3.33$ (dd, $J=13.8,5.3 \mathrm{~Hz}$, 
1H), $2.88-2.72(\mathrm{~m}, 1 \mathrm{H}) .{ }^{13} \mathrm{C}$ NMR $\left(151 \mathrm{MHz} \mathrm{CDCL}_{3}\right) \delta=175.5,152.5,147.8,147.4,137.1,136.7,135.8,132.7$, 132.3, 131.6, 128.7, 127.8, 127.5, 126.4, 110.3, 108.5, 108.3, 101.5, 68.7, 60.8, 56.2, 55.5, 52.7, 43.8, 41.4, 38.7. HRMS (ESI - MS): calcd. $530.2173[\mathrm{M}+\mathrm{H}]^{+}$Found: 530.2180.<smiles>C=CCNCc1ccc(Cl)cc1Cl</smiles>

N-(2,4-Dichlrobenzyl)prop-2-en-1-amine ( $\boldsymbol{S}$-36): Method A used. Allylamine (500 mg) was used. Product recovered as a yellow oil $(1.5 \mathrm{~g}, 80 \%$ yield $) .{ }^{1} \mathrm{H}$ NMR $\left(600 \mathrm{MHz}, \mathrm{CDCl}_{3}\right) \delta 7.36(\mathrm{~d}, J=2.1 \mathrm{~Hz}, 1 \mathrm{H}), 7.33(\mathrm{~d}, J=8.2$ $\mathrm{Hz}, 1 \mathrm{H}), 7.21(\mathrm{dd}, J=8.2,2.1 \mathrm{~Hz}, 1 \mathrm{H}), 5.91(\mathrm{ddt}, J=22.4,10.3,6.0 \mathrm{~Hz}, 1 \mathrm{H}), 5.20(\mathrm{dq}, J=17.2,1.6 \mathrm{~Hz}, 1 \mathrm{H}), 5.12$ $(\mathrm{dq}, J=10.3,1.3 \mathrm{~Hz}, 1 \mathrm{H}), 3.83(\mathrm{~s}, 2 \mathrm{H}), 3.25(\mathrm{dt}, J=5.9,1.4 \mathrm{~Hz}, 2 \mathrm{H}), 1.45(\mathrm{br}, 1 \mathrm{H}) .{ }^{13} \mathrm{C} \mathrm{NMR}(151 \mathrm{MHz}, \mathrm{CDCl}) \delta$ 136.6, 136.4, 134.4, 133.3, 133.3, 131.0, 131.0, 129.4, 129.3, 127.1, 116.4, 51.7, 50.0. HRMS (ESI - MS): calcd. $216.0341[\mathrm{M}+\mathrm{H}]^{+}$Found: 216.0343 .

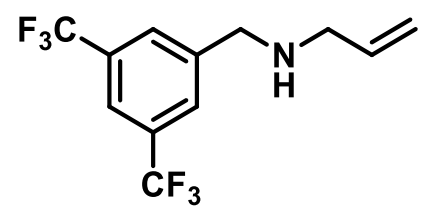

$\boldsymbol{N}$-(3,5-bis(Trifluoromethyl)benzyl)prop-2-en-1-amine ( $\boldsymbol{S}$-37): Method A used. Allylamine (500 mg) was used. Product recovered as a yellow oil (2.0 g, 80\% yield). Spectral data was consistent with that in the literature. ${ }^{11}{ }^{1} \mathrm{H}$ NMR $\left(600 \mathrm{MHz}, \mathrm{CDCl}_{3}\right) \delta 7.82(\mathrm{~s}, 2 \mathrm{H}), 7.76(\mathrm{~s}, 1 \mathrm{H}), 5.91(\mathrm{ddt}, J=17.1,10.2,6.0 \mathrm{~Hz}, 1 \mathrm{H}), 5.21(\mathrm{dq}, J=17.2,1.6 \mathrm{~Hz}, 1 \mathrm{H})$, $5.15(\mathrm{dq}, J=10.2,1.5 \mathrm{~Hz}, 1 \mathrm{H}), 3.92(\mathrm{~s}, 2 \mathrm{H}), 3.29(\mathrm{dt}, J=6.0,1.4 \mathrm{~Hz}, 2 \mathrm{H}), 1.43(\mathrm{br} \mathrm{s}, 1 \mathrm{H}) .{ }^{13} \mathrm{C} \mathrm{NMR}(151 \mathrm{MHz}$, $\left.\mathrm{CDCl}_{3}\right) \delta 143.2,131.7(\mathrm{q}, J=33.1 \mathrm{~Hz}), 128.3(\mathrm{q}, J=2.8 \mathrm{~Hz}), 123.6(\mathrm{q}, J=272.6 \mathrm{~Hz}), 121.1(\mathrm{sept}, J=3.6 \mathrm{~Hz}), 116.7$, 52.2, 51.9. ${ }^{19} \mathrm{~F}$ NMR (376 MHz, $\left.\mathrm{CDCl}_{3}\right) \delta=-61.3$. HRMS (ESI - MS): calcd. $284.0868[\mathrm{M}+\mathrm{H}]^{+}$Found: 284.0876.

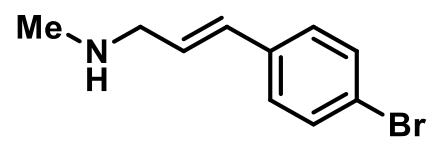

(E)-3-(4-Bromophenyl)- $\boldsymbol{N}$-methylprop-2-en-1-amine ( $\boldsymbol{S}$-38): Method A used. Methylamine hydrochloride (159 $\mathrm{mg}),(E)$-3-(4-bromophenyl)acrylaldehyde $(584 \mathrm{mg})$ and triethylamine $(241 \mathrm{mg})$ were used. Product recovered as a yellow oil (187 mg, 35\% yield). ${ }^{1} \mathrm{H}$ NMR (400 MHz, $\left.\mathrm{CDCl}_{3}\right) \delta 7.41(\mathrm{~d}, J=7.1 \mathrm{~Hz}, 2 \mathrm{H}), 7.22(\mathrm{~d}, J=8.2 \mathrm{~Hz}, 2 \mathrm{H}), 6.49$ $(\mathrm{d}, J=16.0 \mathrm{~Hz}, 1 \mathrm{H}), 6.34-6.20(\mathrm{~m}, 1 \mathrm{H}), 4.47(\mathrm{bs}, 1 \mathrm{H}), 3.39(\mathrm{~d}, J=6.3 \mathrm{~Hz}, 2 \mathrm{H}), 2.47(\mathrm{~d}, J=1.2 \mathrm{~Hz}, 3 \mathrm{H}) .{ }^{13} \mathrm{C} \mathrm{NMR}$ $\left(151 \mathrm{MHz} \mathrm{CDCl}_{3}\right) \delta 136.2,131.8,130.3,129.2,127.9,121.2,53.8,36.1$. HRMS (ESI - MS): calcd. 226.0226 [M+H] ${ }^{+}$ Found: 226.0226.

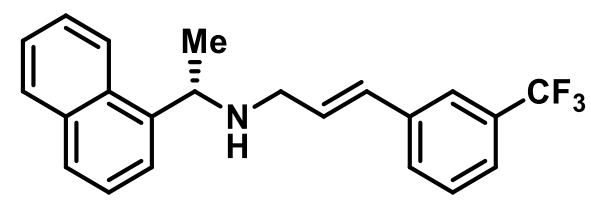

$(S, E)-N$-(1-(Napthalen-1-yl)ethyl)-3-(3-(trifluoromethyl)phenyl)prop-2-en-1-amine (S-39): Method A used. (S)1-(naphthalen-1-yl)ethan-1-amine (500 mg) and (E)-3-(3-(trifluoromethyl)phenyl)acrylaldehyde (584 mg) were used. 
Product recovered as a yellow oil $\left(778 \mathrm{mg}, 75 \%\right.$ yield). Spectral data was consistent with that in the literature. ${ }^{12} \mathrm{H}$ $\operatorname{NMR}\left(600 \mathrm{MHz}, \mathrm{CDCl}_{3}\right) \delta 8.21(\mathrm{~d}, J=8.2 \mathrm{~Hz}, 1 \mathrm{H}), 7.99-7.85(\mathrm{~m}, 1 \mathrm{H}), 7.79(\mathrm{~d}, J=8.2 \mathrm{~Hz}, 1 \mathrm{H}), 7.72(\mathrm{~d}, J=7.0$ Hz, 1H), 7.59 (s, 1H), 7.51 (ddd, $J=19.0,16.1,9.4 \mathrm{~Hz}, 5 \mathrm{H}), 7.41$ (t, $J=7.7 \mathrm{~Hz}, 1 \mathrm{H}), 6.50(\mathrm{~d}, J=15.9 \mathrm{~Hz}, 1 \mathrm{H}), 6.41$ $(\mathrm{dd}, J=14.0,8.0 \mathrm{~Hz}, 1 \mathrm{H}), 4.76(\mathrm{q}, J=6.6 \mathrm{~Hz}, 1 \mathrm{H}), 3.47-3.22(\mathrm{~m}, 2 \mathrm{H}), 1.56(\mathrm{~d}, J=6.6 \mathrm{~Hz}, 3 \mathrm{H}) .{ }^{13} \mathrm{C} \mathrm{NMR}(151 \mathrm{MHz}$, $\left.\mathrm{CDCl}_{3}\right) \delta=141.0,138.1,134.1,131.0(\mathrm{q}, J=32 \mathrm{~Hz}), 130.9,129.8,129.5,129.1,129.1,127.4,126.0,125.5,124.28$ $(\mathrm{d}, J=272 \mathrm{~Hz}), 123.9$ (q, $J=4 \mathrm{~Hz}), 123.4,123.1(\mathrm{q}, J=4 \mathrm{~Hz}), 122.9,77.4,76.9,53.0,49.7,23.8 .{ }^{19} \mathrm{~F}$ NMR $(376$ $\left.\mathrm{MHz}, \mathrm{CDCl}_{3}\right) \delta=-63.0$. 


\section{Synthesis of Iodoaromatics}

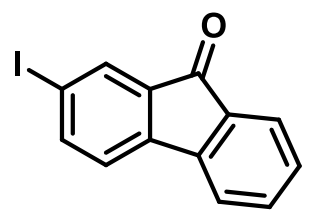

2-Iodo-9H-fluoren-9-one $\left(\boldsymbol{S}\right.$-40): Following a modification of a procedure for benzylic oxidation, ${ }^{13}$ sodium dodecyl sulfate $(127 \mathrm{mg}, 0.44 \mathrm{mmol})$ and iron chloride hexahydrate (143 $\mathrm{mg}, 0.53 \mathrm{mmol})$ were combined in $30 \mathrm{~mL}$ deionized $\mathrm{H}_{2} \mathrm{O}$, followed by 2-iodofluorene (1.00 g, $\left.3.42 \mathrm{mmol}\right)$ and tert-butylhydrogen peroxide $(10 \mathrm{~mL}, 70 \%$ solution in water), followed by heating the reaction under air for $15 \mathrm{~h}$ at $60^{\circ} \mathrm{C}$. After cooling, the reaction mixture was filtered and dried, followed by recrystallization from 95\% ethanol to give the desired product as a yellow solid (590 mg, 56\%) Spectral data was consistent with that in the literature. ${ }^{14}{ }^{1} \mathrm{H}$ NMR $\left(600 \mathrm{MHz}, \mathrm{CDCl}_{3}\right) \delta 7.96(\mathrm{~s}, 1 \mathrm{H}), 7.82(\mathrm{dd}, J=7.8$, $1.2 \mathrm{~Hz}, 1 \mathrm{H}), 7.65(\mathrm{~d}, J=7.4 \mathrm{~Hz}, 1 \mathrm{H}), 7.53-7.48(\mathrm{~m}, 2 \mathrm{H}), 7.35-7.31(\mathrm{~m}, 1 \mathrm{H}), 7.28(\mathrm{~d}, J=7.8 \mathrm{~Hz}, 1 \mathrm{H})$.<smiles>CC(C)(COC(=O)c1cccc(I)c1)COC(=O)c1cccc(I)c1</smiles>

2,2-Dimethylpropane-1,3-diyl bis(3-iodobenzoate) (S-41): 3-Iodobenzoic acid (1.98 g, $8.0 \mathrm{mmol}$ ) DMAP (10 mg, $0.080 \mathrm{mmol})$, and dicyclohexylcarbodiimide $(1.65 \mathrm{~g}, 8.0 \mathrm{mmol})$ were added to anhydrous dichloromethane $(40 \mathrm{~mL})$, followed by 2,2-dimethyl-1,3-propanediol ( $416 \mathrm{mg}, 4.0 \mathrm{mmol}$ ). Reaction was stirred for 16h, followed by filtration through celite and evaporation in vacuo. The reaction mixture was then chromatographed over silica gel using $10 \%$ EtOAc/hexanes to give the desired product as a colorless oil $(722 \mathrm{mg}, 32 \%) .{ }^{1} \mathrm{H} \mathrm{NMR}\left(600 \mathrm{MHz}, \mathrm{CDCl}_{3}\right) \delta 8.33(\mathrm{t}, J$ $=1.6 \mathrm{~Hz}, 2 \mathrm{H}), 7.97(\mathrm{dt}, J=\mathrm{Hz}, 2 \mathrm{H}), 7.86(\mathrm{ddd}, J=7.9,1.7,1.1 \mathrm{~Hz}, 2 \mathrm{H}), 7.15(\mathrm{t}, J=7.8 \mathrm{~Hz}, 2 \mathrm{H}), 4.23(\mathrm{~s}, 4 \mathrm{H}), 1.15$ (s, 6H). ${ }^{13} \mathrm{C}$ NMR $\left(151 \mathrm{MHz}, \mathrm{CDCl}_{3}\right) \delta 164.9,142.0,138.5,131.9,130.2,128.7,94.0,70.2,35.4,22.1$. HRMS (ESI MS): calcd. $564.9367[\mathrm{M}+\mathrm{H}]^{+}$Found: 564.9361. 


\section{Optimization and Synthesis of $\boldsymbol{\gamma}$-Arylated Cinnamylamines}

A. Typical procedure for optimization experiments for the $\gamma$-arylation of cinnamylamines:

\begin{tabular}{|c|c|c|c|}
\hline (0.30 $\mathrm{mmol})$ & 2 equiv. & $\begin{array}{c}\mathrm{Pd}(\mathrm{OAc})_{2}(10 \mathrm{~mol} \%) \\
\stackrel{\mathrm{AgOAc}\left(1.5 \text { equiv. }^{\circ}\right)}{\underset{\mathrm{CO}_{2}(7 \text { equiv. })}{\mathrm{H}_{2} \mathrm{O}(5 \text { equiv. })}} \\
\text { TFA }(0.30 \mathrm{M}), 40^{\circ} \mathrm{C}, 14 \mathrm{~h}\end{array}$ & \\
\hline Entry & \multicolumn{2}{|c|}{ Modification to Reaction Conditions } & NMR Yield (\%) \\
\hline 1 & \multicolumn{2}{|l|}{ None } & 65 \\
\hline 2 & \multicolumn{2}{|c|}{$\mathrm{PdCl}_{2}$ instead of $\mathrm{Pd}(\mathrm{OAc})_{2}$} & 30 \\
\hline 3 & \multicolumn{2}{|c|}{$\mathrm{Pd}(\mathrm{TFA})_{2}$ instead of $\mathrm{Pd}(\mathrm{OAc})_{2}$} & 55 \\
\hline 4 & \multicolumn{2}{|c|}{$\mathrm{Pd}_{2}(\mathrm{dba})_{3}$ instead of $\mathrm{Pd}(\mathrm{OAc})_{2}$} & 10 \\
\hline 5 & \multicolumn{2}{|c|}{$\mathrm{Pd}(\mathrm{dba})_{2}$ instead of $\mathrm{Pd}(\mathrm{OAc})_{2}$} & 30 \\
\hline 6 & \multicolumn{2}{|c|}{$\mathrm{NiCl}_{2}\left(\mathrm{PPh}_{3}\right)_{2}$ instead of $\mathrm{Pd}(\mathrm{OAc})_{2}$} & $<4$ \\
\hline 7 & \multicolumn{2}{|c|}{$\mathrm{Ni}(\mathrm{dppp}) \mathrm{Cl}_{2}$ instead of $\mathrm{Pd}(\mathrm{OAc})_{2}$} & $<4$ \\
\hline 8 & \multicolumn{2}{|c|}{$\mathrm{Co}(\mathrm{OAc})_{2}$ instead of $\mathrm{Pd}(\mathrm{OAc})_{2}$} & $<4$ \\
\hline 9 & \multicolumn{2}{|c|}{$\left.\mathrm{Cp}^{*} \mathrm{RhCl}_{2}\right]_{2}$ instead of $\mathrm{Pd}(\mathrm{OAc})_{2}$} & $<4$ \\
\hline 10 & \multicolumn{2}{|c|}{$\mathrm{Pd}(\mathrm{OAc})_{2}$ omitted } & $<4$ \\
\hline 11 & \multicolumn{2}{|c|}{ AgOAc omitted } & $<4$ \\
\hline 12 & \multicolumn{2}{|c|}{ Dry lce omitted } & 40 \\
\hline 13 & \multicolumn{2}{|c|}{ Reaction Performed at $30^{\circ} \mathrm{C}$} & $<4$ \\
\hline 14 & \multicolumn{2}{|c|}{ AgTFA instead of AgOAC } & 61 \\
\hline 15 & \multicolumn{2}{|c|}{$\mathrm{Ag}_{2} \mathrm{CO}_{3}$ instead of $\mathrm{AgOAc}$} & 20 \\
\hline 16 & \multicolumn{2}{|c|}{ KOAc instead of AgOAc } & 10 \\
\hline 17 & \multicolumn{2}{|c|}{$\mathrm{NaOAc}$ instead of $\mathrm{AgOAc}$} & 6 \\
\hline 18 & \multicolumn{2}{|c|}{ LiTFA instead of AgOAc } & 9 \\
\hline 19 & \multicolumn{2}{|c|}{ NaTFA instead of AgOAc } & 5 \\
\hline 20 & \multicolumn{2}{|c|}{$\mathrm{Me}_{4} \mathrm{NCl}$ instead of $\mathrm{AgOAC}$} & 8 \\
\hline 21 & \multicolumn{2}{|c|}{ p-Benzoquinone } & 8 \\
\hline 22 & \multicolumn{2}{|c|}{$1: 1 \mathrm{AcOH} / \mathrm{HFIP}$ as solvent at $100^{\circ} \mathrm{C}$} & 60 \\
\hline 23 & \multicolumn{2}{|c|}{$\mathrm{AcOH}$ as solvent } & $<4$ \\
\hline $24^{a}$ & \multicolumn{2}{|c|}{$\mathrm{AcOH}$ as solvent, Reaction Performed at $100^{\circ} \mathrm{C}$} & 72 \\
\hline 25 & \multicolumn{2}{|c|}{$\mathrm{CO}_{2}$ (3 equiv.) } & 41 \\
\hline 26 & \multicolumn{2}{|c|}{$\mathrm{CO}_{2}$ (7 equiv.) } & 65 \\
\hline 27 & \multicolumn{2}{|c|}{$\mathrm{CO}_{2}$ (15 equiv.) } & 49 \\
\hline 28 & \multicolumn{2}{|l|}{$\mathrm{H}_{2} \mathrm{O}$ omitted } & 49 \\
\hline 29 & \multicolumn{2}{|c|}{$\mathrm{H}_{2} \mathrm{O}$ (3 equiv.) } & 53 \\
\hline 30 & Phl (1 equiv. & & 34 \\
\hline 31 & Phl (1.5 equ & & 47 \\
\hline 32 & $\mathrm{AgOAc}(1 \mathrm{ec}$ & (iv.) & 61 \\
\hline 33 & $\mathrm{AgOAc}(2 \mathrm{ec}$ & iv.) & 43 \\
\hline
\end{tabular}

$a=$ AgTFA was used

Table $S$-1. Optimization of $\gamma$-Arylated of $1^{\circ}$ Amines. 


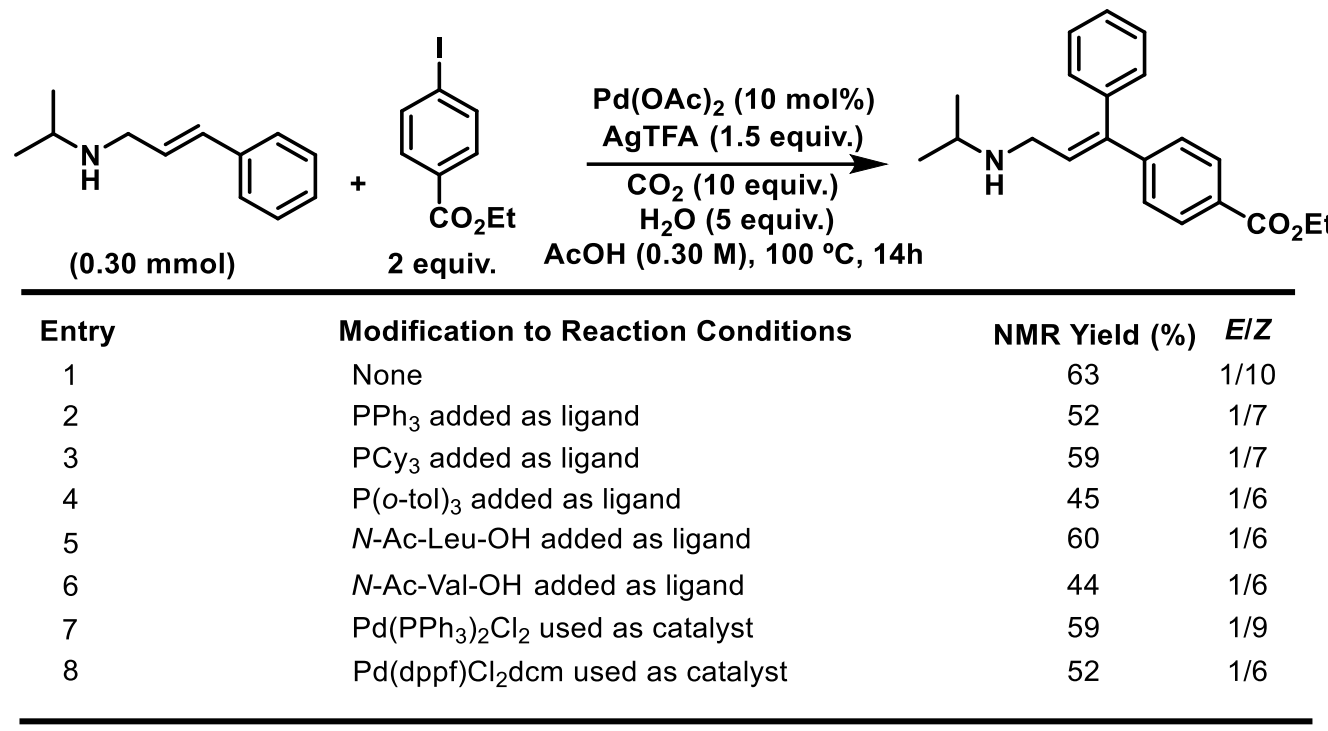

Table $S$-2. Optimization of trans selective $\gamma$-Arylated of $2^{\circ}$ Amines.

Optimization Procedure: A $7.5 \mathrm{~mL}$ vial was charged with Pd catalyst $(0.03 \mathrm{mmol}, 0.10$ equiv), silver salt $(0.45$ mmol, 1.5 equiv), organohalide ( $0.45 \mathrm{mmol}, 1.5$ equiv), amine ( $0.30 \mathrm{mmol}, 1.0$ equiv), solvent $(1.0 \mathrm{~mL})$, and water (21.7 $\mu \mathrm{L}, 1.5 \mathrm{mmol}, 5$ equiv), followed by the addition of dry ice as $\mathrm{CO}_{2}$ source. The vial was quickly sealed with a PTFE lined cap and stirred at RT for 15 min. After 15 min of stirring, the reaction mixture was heated in a pie-block at the desired reaction temperature under stirring for $14 \mathrm{~h}$. After cooling to RT, the reaction mixture was concentrated in vacuo. Crude ${ }^{1} \mathrm{H}$ NMR was performed on the reaction mixture using 1,1,2,2-tetrachloroethane as an internal standard. Note: While addition of $\mathrm{CO}_{2}$ was found critical to the reproducible success of the reaction, the background reaction was found to be highly variable, with yields from anywhere between $40 \%$ as determined by NMR (Entry 7). We rationalize that this is a consequence of the background reaction occurring from pre-adsorption of $\mathrm{CO}_{2}$ into both the solvent and potentially the amine. ${ }^{15}$

B. Procedure for Substrate Scope Study (Using AcOH, Method E).
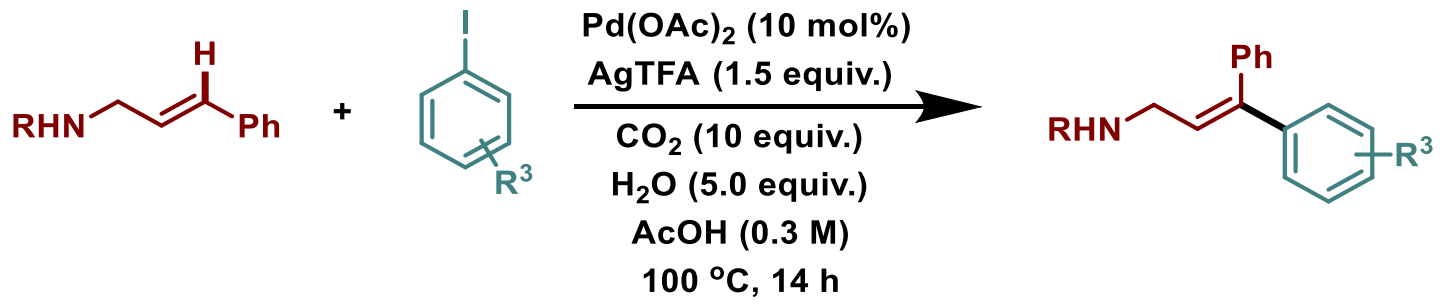

A $7.5 \mathrm{~mL}$ vial was charged with $\mathrm{Pd}(\mathrm{OAc})_{2}(6.7 \mathrm{mg}, 0.03 \mathrm{mmol}, 0.10$ equiv), silver trifluoroacetate $(99.9 \mathrm{mg}, 0.45$ $\mathrm{mmol}, 1.5$ equiv), organohalide ( $0.60 \mathrm{mmol}, 2.0$ equiv), amine ( $0.30 \mathrm{mmol}, 1.0$ equiv), acetic acid $(1.0 \mathrm{~mL})$, and water ( $27 \mu \mathrm{L}, 1.5 \mathrm{mmol}, 5.0$ equiv) followed by the addition of dry ice as ( $132 \mathrm{mg}, 3.0 \mathrm{mmol}, 10$ equiv) $\mathrm{CO}_{2}$ source. . The vial was sealed with a PTFE lined cap and stirred at room temperature for $15 \mathrm{~min}$. After $15 \mathrm{~min}$ of stirring, the reaction mixture was heated in a pie-block at $100{ }^{\circ} \mathrm{C}$ under stirring for $14 \mathrm{~h}$. After cooling to room temperature, the reaction 
mixture was made basic with ammonium hydroxide solution up to $\mathrm{pH}=8$. The reaction mixture was stirred for 15 minutes followed by extraction with dichloromethane $(3 \times 10 \mathrm{~mL})$. The organic layer was washed with brine and dried over $\mathrm{Na}_{2} \mathrm{SO}_{4}$. The filtrate was concentrated in vacuo and further purified by flash column chromatography over silica to give the $\mathrm{C}-\mathrm{H}$ arylation product.

C. Procedure for Substrate Scope Study (Using TFA, Method F).

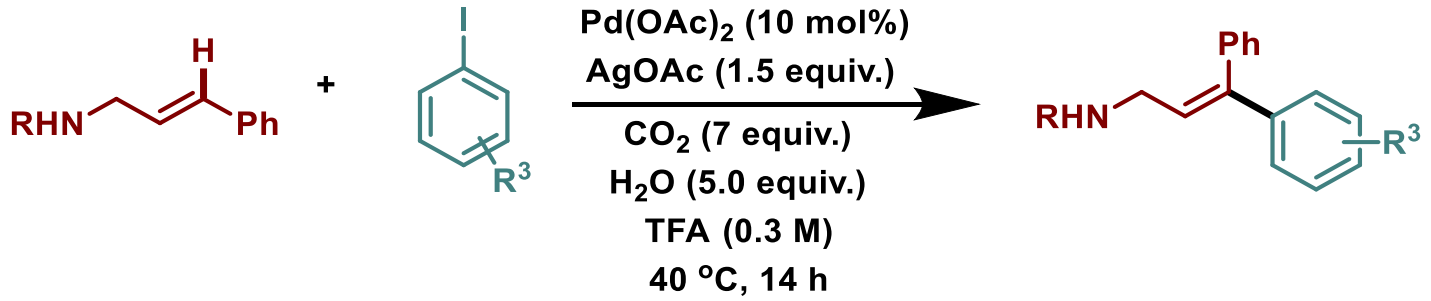

A $7.5 \mathrm{~mL}$ vial was charged with $\mathrm{Pd}(\mathrm{OAc})_{2}(6.7 \mathrm{mg}, 0.03 \mathrm{mmol}, 0.10$ equiv), silver acetate $(75.1 \mathrm{mg}, 0.45 \mathrm{mmol}, 1.5$ equiv), organohalide ( $0.60 \mathrm{mmol}, 2.0$ equiv), amine $(0.30 \mathrm{mmol}, 1.0$ equiv), trifluoroacetic acid $(1.0 \mathrm{~mL})$, and water ( $27 \mu \mathrm{L}, 1.5 \mathrm{mmol}, 5.0$ equiv) followed by the addition of dry ice as ( $\sim 92 \mathrm{mg}, 2.1 \mathrm{mmol}, 7$ equiv) $\mathrm{CO}_{2}$ source. The vial was sealed with a PTFE lined cap and stirred at room temperature for $15 \mathrm{~min}$. After $15 \mathrm{~min}$ of stirring, the reaction mixture was heated in a pie-block at $40{ }^{\circ} \mathrm{C}$ under stirring for $14 \mathrm{~h}$. After cooling to room temperature, the reaction mixture was made basic with ammonium hydroxide solution up to $\mathrm{pH}=8$. The reaction mixture was stirred for 15 minutes followed by extraction with dichloromethane $(3 \times 10 \mathrm{~mL})$. The organic layer was washed with brine and dried over $\mathrm{Na}_{2} \mathrm{SO}_{4}$. The filtrate was concentrated in vacuo and further purified by flash column chromatography over silica to give the $\mathrm{C}-\mathrm{H}$ arylation product.

D. Characterization data

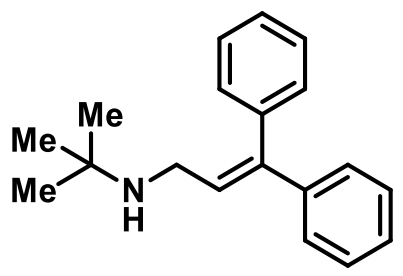

$N$-(tert-Butyl)-3,3-diphenylprop-2-en-1-amine (1a): Method D used. Reaction time 14h, purified by EtOAc:MeOH (95:5). Product recovered as yellow oil (42.9 mg, 54\% yield). $\mathrm{R}_{f}=0.1$ (EtOAc/MeOH=1:1). ${ }^{1} \mathrm{H} \mathrm{NMR}(600 \mathrm{MHz}$, $\left.\left.\mathrm{CDCl}_{3}\right) \delta 7.38(\mathrm{tt}, J=7.5,6.4,1.2 \mathrm{~Hz}, 2 \mathrm{H}), 7.35-7.30(\mathrm{tt}, J=7.3,6.4,1.2 \mathrm{~Hz}, 1 \mathrm{H}),\right), 7.26-7.22(\mathrm{~m}, 5 \mathrm{H}), 7.20(\mathrm{dd}$, $J=5.1,3.2 \mathrm{~Hz}, 2 \mathrm{H}), 6.23(\mathrm{t}, J=7.0 \mathrm{~Hz}, 1 \mathrm{H}), 3.28(\mathrm{~d}, J=7.0 \mathrm{~Hz}, 2 \mathrm{H}), 1.06(\mathrm{~s}, 9 \mathrm{H}){ }^{13} \mathrm{C} \mathrm{NMR}\left(101 \mathrm{MHz}, \mathrm{CDCl}_{3}\right) \delta$ 143.4, 142.2, 139.7, 129.8, 128.3, 128.2, 127.5, 127.4, 127.3, 51.2, 42.0, 28.9. HRMS (ESI - MS): calcd. 266.1903 $[\mathrm{M}+\mathrm{H}]^{+}$Found: 266.1905. 


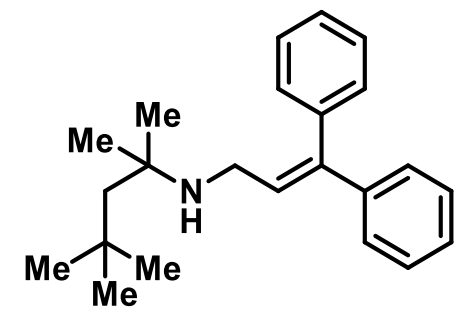

$N$-(3,3-Diphenylallyl)-2,4,4-trimethylpentan-2-amine (1b): Method D used. Reaction time 14h, purified by EtOAc:MeOH (98:2). Product recovered as yellow oil (64.5 mg, 67\% yield). $\mathrm{R}_{f}=0.1$ (EtOAc/MeOH=95:5). ${ }^{1} \mathrm{H}$ NMR $\left(400 \mathrm{MHz}, \mathrm{CDCl}_{3}\right) \delta 7.37(\mathrm{~d}, J=7.6 \mathrm{~Hz}, 2 \mathrm{H}), 7.33(\mathrm{~d}, J=7.1 \mathrm{~Hz}, 1 \mathrm{H}), 7.28-7.23(\mathrm{~m}, 5 \mathrm{H}), 7.22-7.17(\mathrm{~m}, 2 \mathrm{H}), 6.19$ $(\mathrm{t}, J=7.0 \mathrm{~Hz}, 1 \mathrm{H}), 3.24(\mathrm{~d}, J=7.0 \mathrm{~Hz}, 2 \mathrm{H}), 1.36(\mathrm{~s}, 2 \mathrm{H}), 1.08(\mathrm{~s}, 6 \mathrm{H}), 0.96(\mathrm{~s}, 9 \mathrm{H}) .{ }^{13} \mathrm{C} \mathrm{NMR}\left(101 \mathrm{MHz}, \mathrm{CDCl}_{3}\right) \delta$ $=142.9,142.3,139.8,129.8,128.5,128.2$, 128.1, 127.4, 127.3, 127.2, 54.6, 52.9, 41.5, 31.8, 31.7, 29.1. HRMS (ESI - MS): calcd. $322.2529[\mathrm{M}+\mathrm{H}]^{+}$Found: 322.2528.

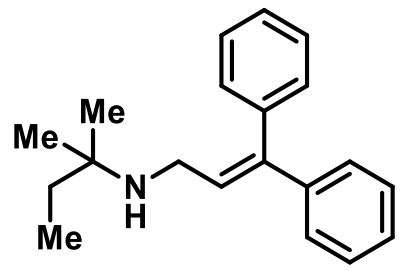

$N$-(3,3-Diphenylallyl)-2-methylbutan-2-amine (1c): Method D used. Reaction time 14h, purified by toluene:acetone (90:10). Product recovered as yellow oil (51.0 mg, $61 \%$ yield). $\mathrm{R}_{f}=0.1$ (toluene/acetone $\left.=8: 2\right) .{ }^{1} \mathrm{H}$ NMR (400 MHz, $\left.\mathrm{CDCl}_{3}\right) \delta 7.41-7.31(\mathrm{~m}, 3 \mathrm{H}), 7.30-7.21(\mathrm{~m}, 5 \mathrm{H}), 7.21-7.15(\mathrm{~m}, 2 \mathrm{H}), 6.22(\mathrm{t}, J=7.0 \mathrm{~Hz}, 1 \mathrm{H}), 3.20(\mathrm{~d}, J=7.0 \mathrm{~Hz}$, $2 \mathrm{H}), 1.33(\mathrm{q}, J=7.5 \mathrm{~Hz}, 2 \mathrm{H}), 0.98(\mathrm{~s}, 6 \mathrm{H}), 0.76(\mathrm{dd}, J=8.0,6.9 \mathrm{~Hz}, 3 \mathrm{H}) .{ }^{13} \mathrm{C}$ NMR $\left(151 \mathrm{MHz}, \mathrm{CDCl}_{3}\right) \delta=143.1$, 142.2, 139.8, 129.8, 128.3, 128.2, 127.4, 127.3, 41.4, 33.1, 26.6, 8.3. HRMS (ESI - MS): calcd. 280.2060 [M+H] ${ }^{+}$ Found: 280.2064.

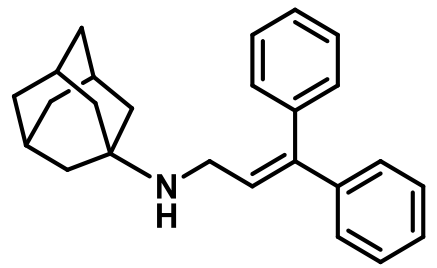

(1s,3s)- $N$-(3,3-Diphenylallyl)adamantan-1-amine (1d): Method D used. Reaction time 14h, purified by toluene:acetone (95:5). Product recovered as yellow oil (64.8 mg, 63\% yield). $\mathrm{R}_{f}=0.1$ (toluene/acetone=9:1). ${ }^{1} \mathrm{H}$ NMR $\left(400 \mathrm{MHz}, \mathrm{CDCl}_{3}\right) \delta 7.36(\mathrm{t}, J=7.2 \mathrm{~Hz}, 2 \mathrm{H}), 7.31(\mathrm{~d}, J=7.2 \mathrm{~Hz}, 1 \mathrm{H}), 7.28-7.19(\mathrm{~m}, 5 \mathrm{H}), 7.19-7.12(\mathrm{~m}, 2 \mathrm{H}), 6.19$ $(\mathrm{t}, J=7.0 \mathrm{~Hz}, 1 \mathrm{H}), 3.28(\mathrm{~d}, J=7.0 \mathrm{~Hz}, 2 \mathrm{H}), 2.01(\mathrm{~s}, 3 \mathrm{H}), 1.68-1.49(\mathrm{~m}, 12 \mathrm{H}) \cdot{ }^{13} \mathrm{C} \mathrm{NMR}\left(101 \mathrm{MHz}, \mathrm{CDCl}_{3}\right) \delta=$ 142.9, 142.3, 139.7, 129.8, 128.5, 128.2, 128.1, 127.4, 127.3, 127.2, 51.0, 42.7, 40.0, 36.7, 29.6. HRMS (ESI - MS): calcd. $344.2373[\mathrm{M}+\mathrm{H}]^{+}$Found: 344.2367. 


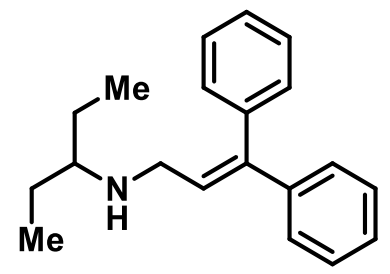

$N$-(3,3-Diphenylallyl)pentan-3-amine (1e): Method D used. Reaction time 14h, purified by toluene:acetone (95:5). Product recovered as yellow oil (49.4 mg, 59\% yield). $\mathrm{R}_{f}=0.1$ (toluene/acetone $\left.=9: 1\right) .{ }^{1} \mathrm{H} \mathrm{NMR}\left(600 \mathrm{MHz}, \mathrm{CDCl}_{3}\right) \delta$ $7.35(\mathrm{t}, J=7.4 \mathrm{~Hz}, 2 \mathrm{H}), 7.30(\mathrm{dd}, J=8.4,6.2 \mathrm{~Hz}, 1 \mathrm{H}), 7.27-7.20(\mathrm{~m}, 5 \mathrm{H}), 7.16(\mathrm{~d}, J=7.1 \mathrm{~Hz}, 2 \mathrm{H}), 6.18(\mathrm{t}, J=6.9$ $\mathrm{Hz}, 1 \mathrm{H}), 3.26(\mathrm{~d}, J=6.9 \mathrm{~Hz}, 2 \mathrm{H}), 2.42-2.29(\mathrm{~m}, 1 \mathrm{H}), 1.33$ (ddd, $J=13.3,9.3,5.8 \mathrm{~Hz}, 4 \mathrm{H}), 0.82(\mathrm{t}, J=7.5 \mathrm{~Hz}, 6 \mathrm{H})$. ${ }^{13} \mathrm{C}$ NMR $\left(151 \mathrm{MHz}, \mathrm{CDCl}_{3}\right) \delta=143.2,142.3,139.8,129.8,128.3,128.2,127.5,127.3,59.6,46.0,25.8$, 9.9. HRMS (ESI - MS): calcd. $280.2060[\mathrm{M}+\mathrm{H}]^{+}$Found: 280.2065.

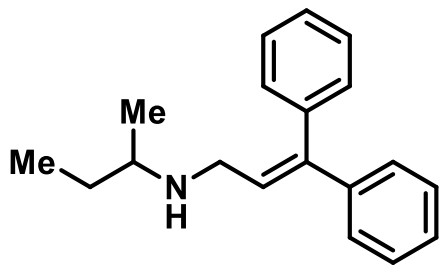

$N$-(3,3-Diphenylallyl)butan-2-amine (1f): Method D used. Reaction time 14h, purified by toluene:acetone (90:10). Product recovered as yellow oil (50.1 mg, 63\% yield). $\mathrm{R}_{f}=0.1$ (toluene/acetone $\left.=7: 3\right) .{ }^{1} \mathrm{H} \mathrm{NMR}\left(600 \mathrm{MHz}, \mathrm{CDCl}_{3}\right) \delta$ $7.40(\mathrm{tt}, J=8.1,1.6 \mathrm{~Hz}, 2 \mathrm{H}), 7.35(\mathrm{tt}, J=7.4,4.3 \mathrm{~Hz}, 1 \mathrm{H}), 7.30-7.25(\mathrm{~m}, 5 \mathrm{H}), 7.22-7.17(\mathrm{~m}, 2 \mathrm{H}), 6.22(\mathrm{t}, J=6.9$ $\mathrm{Hz}, 1 \mathrm{H}), 3.34$ (ddd, $J=33.3,13.8,6.9 \mathrm{~Hz}, 2 \mathrm{H}), 2.64-2.54(\mathrm{~m}, 1 \mathrm{H}), 1.45(\mathrm{dd}, J=7.5,5.3 \mathrm{~Hz}, 1 \mathrm{H}), 1.29$ (m, $1 \mathrm{H}), 1.00$ $(\mathrm{d}, J=6.3 \mathrm{~Hz}, 3 \mathrm{H}), 0.88(\mathrm{t}, J=7.5 \mathrm{~Hz}, 3 \mathrm{H}) .{ }^{13} \mathrm{C} \mathrm{NMR}\left(151 \mathrm{MHz}, \mathrm{CDCl}_{3}\right) \delta=143.3,142.2,139.7,129.8,128.3,128.2$, 128.0, 127.5, 127.4, 127.3, 54.2, 46.1, 29.5, 19.8, 10.3. HRMS (ESI - MS): calcd. 266.1903 [M+H] ${ }^{+}$Found: 266.1896.

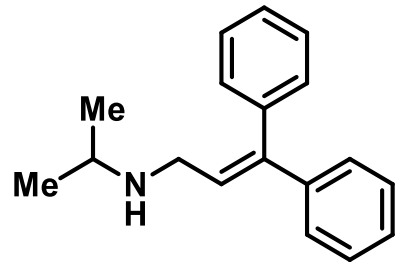

$N$-Isopropyl-3,3-diphenylprop-2-en-1-amine (1g): Method D used. Reaction time 14h, purified by toluene:acetone (80:20). Product recovered as yellow oil $\left(50.1 \mathrm{mg}, 63 \%\right.$ yield). $\mathrm{R}_{f}=0.1$ (toluene/acetone $\left.=7: 3\right) .{ }^{1} \mathrm{H} \mathrm{NMR}(400 \mathrm{MHz}$, $\left.\mathrm{CDCl}_{3}\right) \delta 7.34(\mathrm{~m}, 3 \mathrm{H}), 7.26-7.18(\mathrm{~m}, 5 \mathrm{H}), 7.15(\mathrm{dd}, J=7.8 \mathrm{~Hz}, 2 \mathrm{H}), 6.16(\mathrm{t}, J=6.8 \mathrm{~Hz}, 1 \mathrm{H}), 3.29(\mathrm{~d}, J=6.8 \mathrm{~Hz}$, 2H), 2.78 (dt, $J=12.4,6.2 \mathrm{~Hz}, 1 \mathrm{H}), 0.98(\mathrm{~d}, J=6.2 \mathrm{~Hz}, 6 \mathrm{H}) .{ }^{13} \mathrm{C} \mathrm{NMR}\left(101 \mathrm{MHz}, \mathrm{CDCl}_{3}\right) \delta=147.2,141.0,138.5$, 129.6, 128.6, 128.3, 128.1, 128.0, 127.6, 120.4, 48.8, 43.9, 20.0. HRMS (ESI - MS): calcd. $252.1747[\mathrm{M}+\mathrm{H}]^{+}$Found: 252.1758 . 


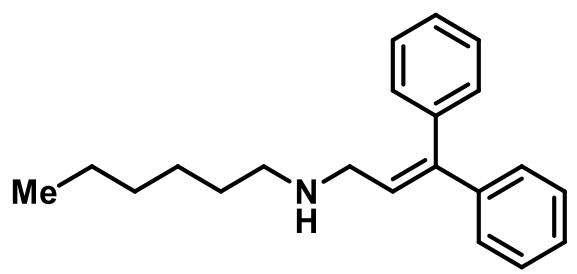

$N$-(3,3-Diphenylallyl)hexan-1-amine (1h): Method D used. Reaction time 14h, purified by toluene:acetone (80:20).

Product recovered as yellow oil $\left(44.8 \mathrm{mg}, 51 \%\right.$ yield). $\mathrm{R}_{f}=0.1$ (toluene/acetone=7:3). ${ }^{1} \mathrm{H} \mathrm{NMR}(400 \mathrm{MHz} \mathrm{CDCl}) \delta$ $7.42-7.33(\mathrm{~m}, 3 \mathrm{H}), 7.32-7.22(\mathrm{~m}, 5 \mathrm{H}), 7.16(\mathrm{~d}, J=6.6 \mathrm{~Hz}, 2 \mathrm{H}), 6.42-6.11(\mathrm{~m}, 1 \mathrm{H}), 3.42(\mathrm{~d}, J=6.9 \mathrm{~Hz}, 2 \mathrm{H}), 2.64$ $(\mathrm{t}, J=7.4 \mathrm{~Hz}, 2 \mathrm{H}), 1.51(\mathrm{~d}, J=6.8 \mathrm{~Hz}, 2 \mathrm{H}), 1.25(\mathrm{bs}, 6 \mathrm{H}), 0.87(\mathrm{dd}, J=6.7,5.5 \mathrm{~Hz}, 3 \mathrm{H}) .{ }^{13} \mathrm{C} \mathrm{NMR}\left(100 \mathrm{MHz}^{\mathrm{N}} \mathrm{CDCl}\right)^{2}$ $\delta=145.3,141.6,139.2,129.8,128.5,128.3,128.3,127.7,127.6,124.5,48.6,47.7,31.7,28.7,26.9,22.7,14.2$. HRMS (ESI - MS): calcd. $294.2216[\mathrm{M}+\mathrm{H}]^{+}$Found: 294.2211.

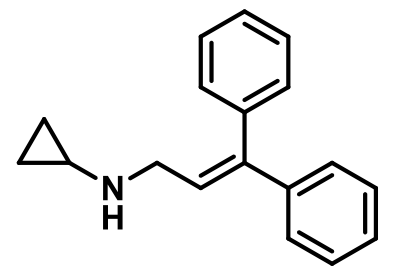

N-(3,3-Diphenylallyl)cyclopropanamine (1i): Method E used. Reaction time 14h, purified by hexanes:EtOAc (50:50). Product recovered as yellow oil $\left(56.0 \mathrm{mg}, 75 \%\right.$ yield). $\mathrm{R}_{f}=0.3$ (hexanes/EtOAc=4:6). ${ }^{1} \mathrm{H} \mathrm{NMR}(600 \mathrm{MHz}$, $\left.\mathrm{CDCl}_{3}\right) \delta 7.45-7.38(\mathrm{~m}, 2 \mathrm{H}), 7.36(\mathrm{ddd}, J=8.5,4.5,1.3 \mathrm{~Hz}, 1 \mathrm{H}), 7.31-7.26(\mathrm{~m}, 5 \mathrm{H}), 7.23-7.20(\mathrm{~m}, 2 \mathrm{H}), 6.22(\mathrm{t}$, $J=6.9 \mathrm{~Hz}, 1 \mathrm{H}), 3.42(\mathrm{~d}, J=6.9 \mathrm{~Hz}, 2 \mathrm{H}), 2.19-2.13(\mathrm{~m}, 1 \mathrm{H}), 0.44(\mathrm{td}, J=6.4,4.6 \mathrm{~Hz}, 2 \mathrm{H}), 0.37(\mathrm{dq}, J=5.2,3.8 \mathrm{~Hz}$, 2H). ${ }^{13} \mathrm{C} \mathrm{NMR}\left(151 \mathrm{MHz}, \mathrm{CDCl}_{3}\right) \delta=143.3,142.3,139.8,129.9,128.3,128.2,127.9,127.5,127.3,48.5,30.4,6.6$. HRMS (ESI - MS): calcd. 250.1590 [M+H] ${ }^{+}$Found: 250.1582.

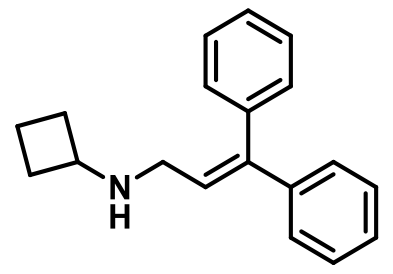

N-(3,3-Diphenylallyl)cyclobutanamine (1j): Method E used. Reaction time 14h, purified by DCM:MeOH (98:2). Product recovered as yellow oil $\left(51.3 \mathrm{mg}, 65 \%\right.$ yield). $\mathrm{R}_{f}=0.12(\mathrm{DCM} / \mathrm{MeOH}=9: 1) .{ }^{1} \mathrm{H} \mathrm{NMR}\left(400 \mathrm{MHz}^{\mathrm{CDCl}}{ }_{3}\right) \delta$ $7.45-7.33(\mathrm{~m}, 4 \mathrm{H}), 7.26(\mathrm{q}, J=6.1 \mathrm{~Hz}, 6 \mathrm{H}), 7.20(\mathrm{t}, J=10.9 \mathrm{~Hz}, 4 \mathrm{H}), 6.24(\mathrm{t}, J=7.0 \mathrm{~Hz}, 1 \mathrm{H}), 3.42-3.25(\mathrm{~m}, 4 \mathrm{H})$, $2.23-2.07(\mathrm{~m}, 2 \mathrm{H}), 1.94-1.79(\mathrm{~m}, 2 \mathrm{H}), 1.73(\mathrm{~d}, J=9.7 \mathrm{~Hz}, 1 \mathrm{H}), 1.64(\mathrm{~s}, 2 \mathrm{H}) .{ }^{13} \mathrm{C} \mathrm{NMR}\left(100 \mathrm{MHz}, \mathrm{CDCl}_{3}\right) \delta=$ 146.1, 141.7, 139.2, 129.8, 128.4, 128.3, 127.9, 127.7, 127.6, 127.5, 124.7, 77.5, 76.8, 53.0, 45.3, 29.9, 15.1 . HRMS (ESI - MS): calcd. 264.1747 [M+H] ${ }^{+}$Found: 264.1739. 


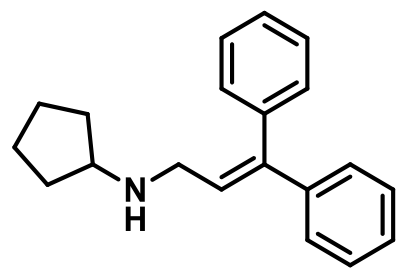

$N$-(3,3-Diphenylallyl)cyclopentanamine (1k): Method D used. Reaction time 14h, purified by toluene:acetone (80:20). Product recovered as yellow oil (50.5 mg, 64\% yield). $\mathrm{R}_{f}=0.17$ (toluene/acetone $\left.=7: 3\right) .{ }^{1} \mathrm{H} \mathrm{NMR}$ (400 MHz, $\left.\mathrm{CDCl}_{3}\right) \delta 7.43-7.34(\mathrm{~m}, 3 \mathrm{H}), 7.28-7.23(\mathrm{~m}, 5 \mathrm{H}), 7.19-7.14(\mathrm{~m}, 2 \mathrm{H}), 6.22(\mathrm{t}, J=7.1 \mathrm{~Hz}, 1 \mathrm{H}), 3.50(\mathrm{~d}, J=7.1 \mathrm{~Hz}$, 2H), $3.34-3.18(\mathrm{~m}, 1 \mathrm{H}), 1.79(\mathrm{dt}, J=11.9,6.1 \mathrm{~Hz}, 2 \mathrm{H}), 1.73-1.58(\mathrm{~m}, 2 \mathrm{H}), 1.48(\mathrm{ddd}, J=20.6,12.6,5.6 \mathrm{~Hz}, 4 \mathrm{H})$. ${ }^{13} \mathrm{C} \mathrm{NMR}\left(100 \mathrm{MHz}, \mathrm{CDCl}_{3}\right) \delta=147.0,141.2,138.6,129.6,128.6,128.3,128.0,127.9,127.6,121.0,58.7,46.1,30.8$, 24.0. HRMS (ESI - MS): calcd. $278.1903[\mathrm{M}+\mathrm{H}]^{+}$Found: 278.1893.

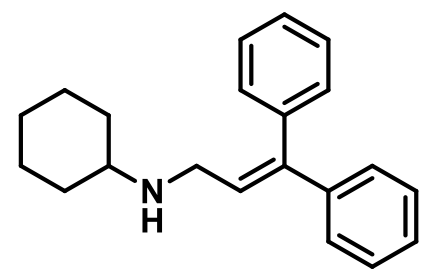

$N$-(3,3-Diphenylallyl)cyclohexanamine (11): Method D used. Reaction time 14h, purified by toluene:acetone (80:20). Product recovered as yellow oil $\left(56.7 \mathrm{mg}, 65 \%\right.$ yield). $\mathrm{R}_{f}=0.2$ (toluene/acetone $\left.=7: 3\right) .{ }^{1} \mathrm{H} \mathrm{NMR}(600 \mathrm{MHz}$, $\left.\mathrm{CDCl}_{3}\right) \delta 7.35-7.30(\mathrm{~m}, 2 \mathrm{H}), 7.30-7.25(\mathrm{~m}, 1 \mathrm{H}), 7.23-7.17(\mathrm{~m}, 5 \mathrm{H}), 7.15-7.10(\mathrm{~m}, 2 \mathrm{H}), 6.15(\mathrm{td}, J=6.8,1.4$ $\mathrm{Hz}, 1 \mathrm{H}), 3.30$ (dd, $J=6.9,1.4 \mathrm{~Hz}, 2 \mathrm{H}), 2.38(\mathrm{t}, J=10.5 \mathrm{~Hz}, 1 \mathrm{H}), 1.74(\mathrm{~d}, J=11.9 \mathrm{~Hz}, 2 \mathrm{H}), 1.63(\mathrm{~d}, J=12.7 \mathrm{~Hz}, 2 \mathrm{H})$, $1.53(\mathrm{~d}, J=12.2 \mathrm{~Hz}, 1 \mathrm{H}), 1.20-1.04(\mathrm{~m}, 3 \mathrm{H}), 0.98(\mathrm{q}, J=11.8 \mathrm{~Hz}, 2 \mathrm{H}) .{ }^{13} \mathrm{C}$ NMR $\left(151 \mathrm{MHz}, \mathrm{CDCl}_{3}\right) \delta=142.2$, 139.7, 129.8, 128.3, 128.2, 127.7, 127.5, 127.4, 56.3, 45.6, 33.4, 26.2, 25.1. HRMS (ESI - MS): calcd. 292.2060 $[\mathrm{M}+\mathrm{H}]^{+}$Found: 292.2067.

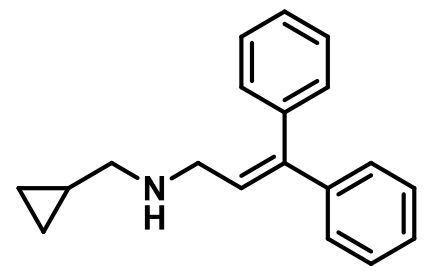

$N$-(Cyclopropylmethyl)-3,3-diphenylprop-2-en-1-amine (1m): Method D used. Reaction time 14h, purified by toluene:acetone (80:20). Product recovered as yellow oil ( $43.4 \mathrm{mg}, 55 \%$ yield). $\mathrm{R}_{f}=0.15$ (toluene/acetone $=7: 3$ ). ${ }^{1} \mathrm{H}$ $\operatorname{NMR}\left(400 \mathrm{MHz}, \mathrm{CDCl}_{3}\right) \delta 7.37(\mathrm{t}, J=7.3 \mathrm{~Hz}, 3 \mathrm{H}), 7.28-7.22(\mathrm{~m}, 5 \mathrm{H}), 7.16(\mathrm{~d}, J=7.1 \mathrm{~Hz}, 2 \mathrm{H}), 6.21(\mathrm{t}, J=6.8 \mathrm{~Hz}$, $1 \mathrm{H}), 3.36(\mathrm{~d}, J=6.9 \mathrm{~Hz}, 2 \mathrm{H}), 2.45(\mathrm{~d}, J=6.9 \mathrm{~Hz}, 2 \mathrm{H}), 1.01-0.79(\mathrm{~m}, 1 \mathrm{H}), 0.45(\mathrm{~d}, J=8.0 \mathrm{~Hz}, 2 \mathrm{H}), 0.08(\mathrm{~d}, J=4.9$ $\mathrm{Hz}, 2 \mathrm{H}) .{ }^{13} \mathrm{C} \mathrm{NMR}\left(151 \mathrm{MHz}, \mathrm{CDCl}_{3}\right) \delta=143.7,142.2,139.7,129.9,129.2,128.3,128.3,127.5,127.4,127.4,125.4$, 54.4, 48.3, 31.1, 11.1, 3.6. HRMS (ESI - MS): calcd. 264.1747 [M+H] $]^{+}$Found: 264.1738. 


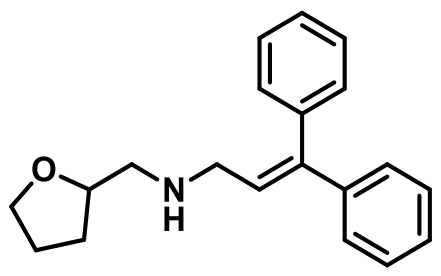

3,3-Diphenyl- $N$-((tetrahydrofuran-2-yl)methyl)prop-2-en-1-amine (1n): Method D used. Reaction time 14h, purified by toluene:acetone (70:30). Product recovered as yellow oil $\left(63.3 \mathrm{mg}, 72 \%\right.$ yield). $\mathrm{R}_{f}=0.1$ (toluene/acetone=7:3). ${ }^{1} \mathrm{H}$ NMR $\left(600 \mathrm{MHz}, \mathrm{CDCl}_{3}\right) \delta 7.38(\mathrm{dd}, J=8.0,6.6 \mathrm{~Hz}, 2 \mathrm{H}), 7.34(\mathrm{~d}, J=7.4 \mathrm{~Hz}, 1 \mathrm{H}), 7.29-$ $7.24(\mathrm{~m}, 5 \mathrm{H}), 7.18(\mathrm{dd}, J=8.1,1.3 \mathrm{~Hz}, 2 \mathrm{H}), 6.20(\mathrm{t}, J=6.8 \mathrm{~Hz}, 1 \mathrm{H}), 3.97(\mathrm{qd}, J=7.4,3.8 \mathrm{~Hz}, 1 \mathrm{H}), 3.84(\mathrm{dt}, J=8.2$, $6.7 \mathrm{~Hz}, 1 \mathrm{H}), 3.77-3.70(\mathrm{~m}, 1 \mathrm{H}), 3.36(\mathrm{~d}, J=6.8 \mathrm{~Hz}, 2 \mathrm{H}), 2.68(\mathrm{dd}, J=12.0,3.7 \mathrm{~Hz}, 1 \mathrm{H}), 2.62(\mathrm{dd}, J=12.0,7.8 \mathrm{~Hz}$, 1H), $1.98-1.91(\mathrm{~m}, 1 \mathrm{H}), 1.90-1.84(\mathrm{~m}, 2 \mathrm{H}), 1.56-1.47(\mathrm{~m}, 1 \mathrm{H}) .{ }^{13} \mathrm{C} \mathrm{NMR}\left(151 \mathrm{MHz}, \mathrm{CDCl}_{3}\right) \delta=143.4,142.2$, 139.7, 129.9, 128.3, 128.2, 127.8, 127.5, 127.3, 127.3, 78.3, 77.4, 76.9, 68.0, 53.9, 48.7, 29.4, 25.9. HRMS (ESI MS): calcd. $294.1852[\mathrm{M}+\mathrm{H}]^{+}$Found: 294.1852.

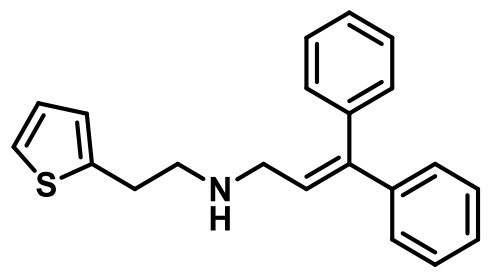

3,3-Diphenyl- $N$-(2-(thiophen-2-yl)ethyl)prop-2-en-1-amine (1o): Method E used. Reaction time 14h, purified by toluene:acetone (80:20). Product recovered as yellow oil $\left(50.7 \mathrm{mg}, 53 \%\right.$ yield). $\mathrm{R}_{f}=0.3$ (toluene/acetone $\left.=7: 3\right) .{ }^{1} \mathrm{H}$ $\operatorname{NMR}\left(600 \mathrm{MHz}, \mathrm{CDCl}_{3}\right) \delta 7.42-7.32(\mathrm{~m}, 3 \mathrm{H}), 7.30-7.27(\mathrm{~m}, 3 \mathrm{H}), 7.26-7.24(\mathrm{~m}, 2 \mathrm{H}), 7.18-7.15(\mathrm{~m}, 3 \mathrm{H}), 6.95$ $(\mathrm{dd}, J=5.1,3.4 \mathrm{~Hz}, 1 \mathrm{H}), 6.83-6.78(\mathrm{~m}, 1 \mathrm{H}), 6.18(\mathrm{t}, J=6.9 \mathrm{~Hz}, 1 \mathrm{H}), 3.36(\mathrm{~d}, J=6.9 \mathrm{~Hz}, 2 \mathrm{H}), 3.00(\mathrm{t}, J=6.8 \mathrm{~Hz}$, 2H), $2.91(\mathrm{t}, J=6.7 \mathrm{~Hz}, 2 \mathrm{H}) .{ }^{13} \mathrm{C} \mathrm{NMR}\left(151 \mathrm{MHz}, \mathrm{CDCl}_{3}\right) \delta=143.7,142.5,142.1,139.7,129.9,128.4,128.3,127.5$, 127.4, 127.4, 127.0, 125.2, 123.7, 77.4, 76.9, 50.6, 48.3, 30.4. HRMS (ESI - MS): calcd. $320.1467[\mathrm{M}+\mathrm{H}]^{+}$Found: 320.1456 .

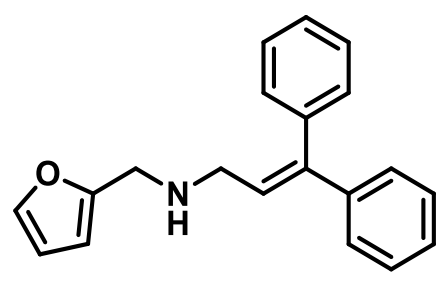

$N$-(Furan-2-ylmethyl)-3-phenylprop-2-en-1-amine (1p): Method E used. Reaction time 14h, purified by hexanes:EtOAc (60:40). Product recovered as yellow oil (58.9 mg, 68\% yield). $\mathrm{R}_{f}=0.29$ (hexanes/EtOAc $\left.=1: 1\right) .{ }^{1} \mathrm{H}$ NMR $\left(600 \mathrm{MHz}, \mathrm{CDCl}_{3}\right) \delta 7.42-7.35(\mathrm{~m}, 3 \mathrm{H}), 7.31(\mathrm{dd}, J=10.5,4.8 \mathrm{~Hz}, 2 \mathrm{H}), 7.23(\mathrm{t}, J=7.3 \mathrm{~Hz}, 1 \mathrm{H}), 6.54(\mathrm{~d}, J=$ $15.9 \mathrm{~Hz}, 1 \mathrm{H}), 6.36-6.24(\mathrm{~m}, 2 \mathrm{H}), 6.20(\mathrm{~d}, J=3.1 \mathrm{~Hz}, 1 \mathrm{H}), 3.83(\mathrm{~s}, 2 \mathrm{H}), 3.43(\mathrm{dd}, J=6.4,1.4 \mathrm{~Hz}, 2 \mathrm{H}) .{ }^{13} \mathrm{C} \mathrm{NMR}$ $\left(151 \mathrm{MHz}, \mathrm{CDCl}_{3}\right) \delta=153.8,142.0,137.1,131.8,128.7,128.1,127.5,126.4,110.2,107.2,, 51.0,45.5$. HRMS (ESI MS): calcd. 290.1539 [M+H] $]^{+}$Found: 290.1538. 


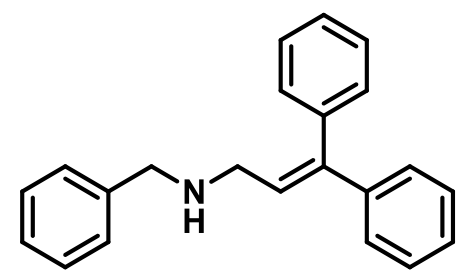

$N$-Benzyl-3,3-diphenylprop-2-en-1-amine (1q): Method E used. Reaction time 14h, purified by hexanes:EtOAc (60:40). Product recovered as yellow oil $\left(64.6 \mathrm{mg}, 72 \%\right.$ yield). $\mathrm{R}_{f}=0.25$ (hexanes/EtOAc $=1: 1$ ). Spectral data was consistent with that in the literature. ${ }^{16}{ }^{1} \mathrm{H}$ NMR $\left(400 \mathrm{MHz}, \mathrm{CDCl}_{3}\right) \delta 7.39-7.30(\mathrm{~m}, 3 \mathrm{H}), 7.29-7.21(\mathrm{~m}, 10 \mathrm{H}), 7.18$ $-7.12(\mathrm{~m}, 2 \mathrm{H}), 6.20(\mathrm{t}, J=6.8 \mathrm{~Hz}, 1 \mathrm{H}), 3.74(\mathrm{~s}, 2 \mathrm{H}), 3.35(\mathrm{~d}, J=6.7 \mathrm{~Hz}, 2 \mathrm{H}) .{ }^{13} \mathrm{C} \mathrm{NMR}\left(101 \mathrm{MHz}, \mathrm{CDCl}_{3}\right) \delta=143.7$, $142.2,140.1,139.7,129.9,128.5,128.3,128.2,127.6,127.5,127.4,127.4,127.1,53.5$, 48.1. HRMS (ESI - MS): calcd. $300.1747[\mathrm{M}+\mathrm{H}]^{+}$Found: 300.1743.

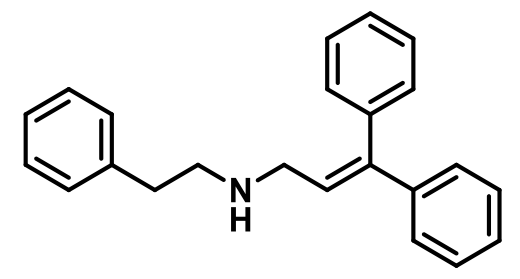

$N$-Phenethyl-3,3-diphenylprop-2-en-1-amine (1r): Method E used. Reaction time 14h, purified by hexanes:EtOAc (60:40). Product recovered as yellow oil ( $57.3 \mathrm{mg}, 61 \%$ yield). $\mathrm{R}_{f}=0.23$ (hexanes/EtOAc $\left.=1: 1\right) .{ }^{1} \mathrm{H} \mathrm{NMR}(600 \mathrm{MHz}$, $\left.\mathrm{CDCl}_{3}\right) \delta 7.30(\mathrm{tdd}, J=9.1,7.2,2.9 \mathrm{~Hz}, 3 \mathrm{H}), 7.25-7.16(\mathrm{~m}, 8 \mathrm{H}), 7.13-7.06(\mathrm{~m}, 4 \mathrm{H}), 6.11(\mathrm{t}, J=6.7 \mathrm{~Hz}, 1 \mathrm{H}), 3.32$ -3.25 (m, 2H), $2.86-2.78(\mathrm{~m}, 2 \mathrm{H}), 2.76-2.68(\mathrm{~m}, 2 \mathrm{H}) .{ }^{13} \mathrm{C} \mathrm{NMR}\left(101 \mathrm{MHz}, \mathrm{CDCl}_{3}\right) \delta=143.7,142.1,139.9,139.6$, 129.8, 128.8, 128.6, 128.3, 128.2, 127.5, 127.4, 127.4, 126.3, 50.6, 48.4, 36.3. HRMS (ESI - MS): calcd. 314.1903 $[\mathrm{M}+\mathrm{H}]^{+}$Found: 314.1910 .

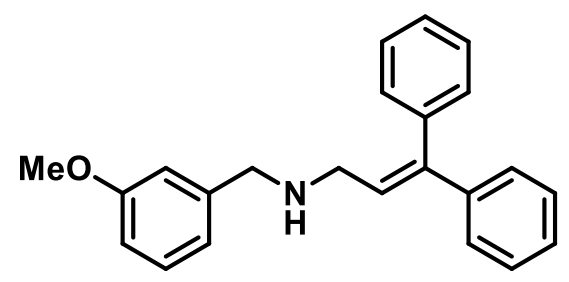

$N$-(3-Methoxybenzyl)-3,3-diphenylprop-2-en-1-amine (1s): Method E used. Reaction time 14h, purified by hexanes:EtOAc (60:40). Product recovered as yellow oil (59.2 mg, 60\% yield). $\mathrm{R}_{f}=0.2$ (hexanes/EtOAc $\left.=1: 1\right) .{ }^{1} \mathrm{H}$ NMR $\left(600 \mathrm{MHz}, \mathrm{CDCl}_{3}\right) \delta 7.32(\mathrm{tdd}, J=8.6,4.4,3.0 \mathrm{~Hz}, 3 \mathrm{H}), 7.26-7.19(\mathrm{~m}, 6 \mathrm{H}), 7.17-7.12(\mathrm{~m}, 2 \mathrm{H}), 6.82(\mathrm{~d}, J=$ $6.6 \mathrm{~Hz}, 2 \mathrm{H}), 6.80-6.70(\mathrm{~m}, 1 \mathrm{H}), 6.19(\mathrm{t}, J=6.8 \mathrm{~Hz}, 1 \mathrm{H}), 3.76(\mathrm{~s}, 3 \mathrm{H}), 3.71(\mathrm{~s}, 2 \mathrm{H}), 3.34(\mathrm{t}, J=5.4 \mathrm{~Hz}, 2 \mathrm{H}) .{ }^{13} \mathrm{C}$ $\operatorname{NMR}\left(151 \mathrm{MHz}, \mathrm{CDCl}_{3}\right) \delta=159.8,143.6,142.3,142.0,139.7,129.9,129.5,129.5,128.3,128.2,127.8,127.5,127.4$, 127.3, 120.5, 113.6, 112.7, 55.3, 53.6, 48.2. HRMS (ESI - MS): calcd. 330.1852 [M+H] ${ }^{+}$Found: 330.1857. 


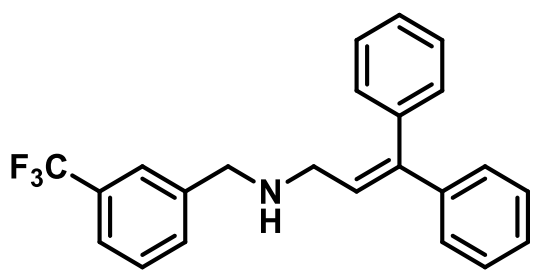

3,3-Diphenyl- $N$-(3-(trifluoromethyl)benzyl)prop-2-en-1-amine (1t): Method E used. Reaction time 14h, purified by hexanes:EtOAc (60:40). Product recovered as yellow oil $\left(60.5 \mathrm{mg}, 55 \%\right.$ yield). $\mathrm{R}_{f}=0.3($ hexanes/EtOAc $=1: 1) .{ }^{1} \mathrm{H}$ $\left.\operatorname{NMR}\left(600 \mathrm{MHz}, \mathrm{CDCl}_{3}\right)\right):{ }^{1} \mathrm{H} \mathrm{NMR}\left(600 \mathrm{MHz}, \mathrm{CDCl}_{3}\right) \delta 7.58(\mathrm{~s}, 1 \mathrm{H}), 7.51(\mathrm{~d}, J=7.6 \mathrm{~Hz}, 1 \mathrm{H}), 7.47(\mathrm{~d}, J=7.7 \mathrm{~Hz}$, $1 \mathrm{H}), 7.42(\mathrm{~d}, J=7.7 \mathrm{~Hz}, 1 \mathrm{H}), 7.39-7.32(\mathrm{~m}, 3 \mathrm{H}), 7.32-7.23(\mathrm{~m}, 6 \mathrm{H}), 7.19-7.14(\mathrm{~m}, 2 \mathrm{H}), 6.22(\mathrm{t}, J=6.9 \mathrm{~Hz}, 1 \mathrm{H})$, $3.83(\mathrm{~s}, 2 \mathrm{H}), 3.37$ (d, $J=6.9 \mathrm{~Hz}, 2 \mathrm{H}), 1.49$ (bs, 1H). ${ }^{13} \mathrm{C} \mathrm{NMR}\left(151 \mathrm{MHz}, \mathrm{CDCl}_{3}\right) \delta=144.1,142.1,141.1,139.5$, 131.6, $131.0(\mathrm{q}, J=32 \mathrm{~Hz}), 129.8,128.9,128.3,127.5,127.4,127.1,125.0(\mathrm{q}, J=3 \mathrm{~Hz}), 124.3$ (q, $J=272 \mathrm{~Hz}), 124.0$ $(\mathrm{q}, J=4 \mathrm{~Hz}), 52.9,48.1 .{ }^{19} \mathrm{~F} \mathrm{NMR}\left(376 \mathrm{MHz}, \mathrm{CDCl}_{3}\right) \delta=-62.9$. HRMS (ESI - MS): calcd. $368.1621[\mathrm{M}+\mathrm{H}]^{+} \mathrm{Found}$ : 368.1625 .

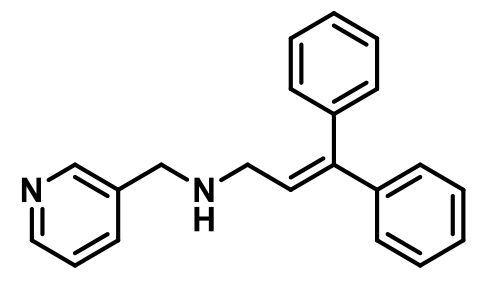

3,3-Diphenyl- $N$-(pyridine-3-ylmethyl)prop-2-en-1-amine (1u): Method E used. Reaction time 14h, purified by toluene:acetone (60:40). Product recovered as yellow oil $\left(22.5 \mathrm{mg}, 25 \%\right.$ yield). $\mathrm{R}_{f}=0.1$ (toluene/acetone $\left.=1: 1\right)$. ${ }^{1} \mathrm{H}$ $\operatorname{NMR}\left(600 \mathrm{MHz}, \mathrm{CDCl}_{3}\right) \delta 8.46(\mathrm{dd}, J=13.1,2.5 \mathrm{~Hz}, 2 \mathrm{H}), 7.58(\mathrm{~d}, J=7.8 \mathrm{~Hz}, 1 \mathrm{H}), 7.37-7.28(\mathrm{~m}, 3 \mathrm{H}), 7.28-7.16$ $(\mathrm{m}, 6 \mathrm{H}), 7.13(\mathrm{~d}, J=6.9 \mathrm{~Hz}, 2 \mathrm{H}), 6.17(\mathrm{t}, J=6.9 \mathrm{~Hz}, 1 \mathrm{H}), 3.73(\mathrm{~s}, 2 \mathrm{H}), 3.33(\mathrm{~d}, J=6.9 \mathrm{~Hz}, 2 \mathrm{H}) .{ }^{13} \mathrm{C} \mathrm{NMR}(151 \mathrm{MHz}$, $\left.\mathrm{CDCl}_{3}\right) \delta=149.8,148.6,144.1,142.1,139.5,135.9,135.5,129.8,128.3,128.3,127.5,127.5,127.5,127.2,123.4$ 50.8, 48.1. HRMS (ESI - MS): calcd. $301.1699[\mathrm{M}+\mathrm{H}]^{+}$Found: 301.1706.

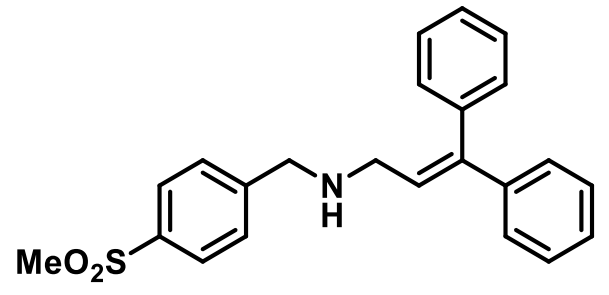

$N$-(4-(Methylsulfonyl)benzyl)-3,3-diphenylprop-2-en-1-amine (1v): Method E used. Reaction time 14h, purified by hexanes:EtOAc (60:40). Product recovered as yellow oil $\left(84.8 \mathrm{mg}, 75 \%\right.$ yield). $\mathrm{R}_{f}=0.25$ (hexanes/EtOAc $\left.=1: 1\right)$. ${ }^{1} \mathrm{H} \mathrm{NMR}\left(600 \mathrm{MHz}, \mathrm{CDCl}_{3}\right) \delta 7.86(\mathrm{~d}, J=8.2 \mathrm{~Hz}, 2 \mathrm{H}), 7.48(\mathrm{~d}, J=8.1 \mathrm{~Hz}, 2 \mathrm{H}), 7.39-7.31(\mathrm{~m}, 3 \mathrm{H}), 7.30-7.23(\mathrm{~m}$, $5 \mathrm{H}), 7.16(\mathrm{~d}, J=6.7 \mathrm{~Hz}, 2 \mathrm{H}), 6.20(\mathrm{t}, J=6.9 \mathrm{~Hz}, 1 \mathrm{H}), 3.86(\mathrm{~s}, 2 \mathrm{H}), 3.35(\mathrm{~d}, J=6.9 \mathrm{~Hz}, 2 \mathrm{H}), 3.04(\mathrm{~s}, 3 \mathrm{H}) .{ }^{13} \mathrm{C} \mathrm{NMR}$ $\left(151 \mathrm{MHz} \mathrm{CDCl}_{3}\right) \delta=147.0,144.2,142.0,139.5,139.1,129.8,129.0,128.3,128.3,127.6,127.5,127.1,52.8,48.0$, 44.7. HRMS (ESI - MS): calcd. 378.1522 [M+H] ${ }^{+}$Found: 378.1527. 


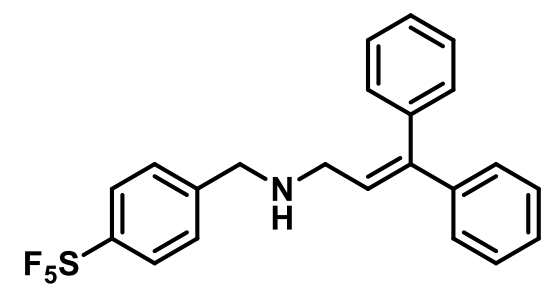

$N$-(4-(Pentafluoro- $\lambda^{6}$-sulfaneyl)benzyl)-3,3-diphenylprop-2-en-1-amine (1w): Method E used. Reaction time 14h, purified by hexanes:EtOAc (60:40). Product recovered as yellow oil ( $99.4 \mathrm{mg}, 78 \%$ yield). $\mathrm{R}_{f}=0.3$ (hexanes/EtOAc = 1:1). ${ }^{1} \mathrm{H}$ NMR (600 MHz, $\left.\mathrm{CDCl}_{3}\right) \delta 7.67(\mathrm{~d}, J=8.7 \mathrm{~Hz}, 2 \mathrm{H}), 7.39-7.34(\mathrm{~m}, 5 \mathrm{H}), 7.30-7.26(\mathrm{~m}, 3 \mathrm{H}), 7.25-7.21$ $(\mathrm{m}, 2 \mathrm{H}), 7.17-7.12(\mathrm{~m}, 2 \mathrm{H}), 6.19(\mathrm{t}, J=6.9 \mathrm{~Hz}, 1 \mathrm{H}), 3.81(\mathrm{~s}, 2), 3.35(\mathrm{~d}, J=6.9 \mathrm{~Hz}, 2 \mathrm{H}) .{ }^{13} \mathrm{C} \mathrm{NMR}(151 \mathrm{MHz}$, $\mathrm{CDCl}_{3}$ ) $\delta=152.7$ (qunit, $J=16 \mathrm{~Hz}$ ), 144.4, 144.2, 142.1, 139.5, 129.8, 128.3, 128.3, 127.5, 127.5, 127.5, 127.2, 126.1 (qunit, $J=4 \mathrm{~Hz}$ ), 64.0, 52.5, 47.9. ${ }^{19} \mathrm{~F} \mathrm{NMR} \mathrm{(376} \mathrm{MHz,} \mathrm{CDCl}_{3}$ ) $\delta=84.5$ (quint), 62.6 (d). HRMS (ESI - MS): calcd. $426.1309[\mathrm{M}+\mathrm{H}]^{+}$Found: 426.1310.

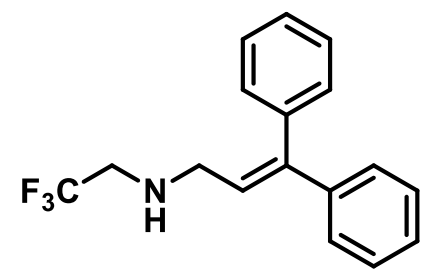

3,3-Diphenyl- $N$-(2,2,2-trifluoroethyl)prop-2-en-1-amine (1x): Method E used. Reaction time $14 \mathrm{~h}$, purified by hexanes:EtOAc (99:5). Product recovered as yellow oil ( $48.0 \mathrm{mg}, 55 \%$ yield). $\mathrm{R}_{f}=0.35$ (hexanes/EtOAc $=90: 10$ ). ${ }^{1} \mathrm{H}$ $\operatorname{NMR}\left(600 \mathrm{MHz}, \mathrm{CDCl}_{3}\right) \delta 7.38(\mathrm{tt}, J=7.9,1.7 \mathrm{~Hz}, 2 \mathrm{H}), 7.34(\mathrm{tt}, J=7.3,3.71 \mathrm{H}), 7.29-7.23(\mathrm{~m}, 6 \mathrm{H}), 7.18-7.14$ $(\mathrm{m}, 2 \mathrm{H}), 6.14(\mathrm{t}, J=6.9 \mathrm{~Hz}, 1 \mathrm{H}), 3.43(\mathrm{~d}, J=6.9 \mathrm{~Hz}, 2 \mathrm{H}), 3.15(\mathrm{q}, J=9.4 \mathrm{~Hz}, 2 \mathrm{H}), 1.69(\mathrm{~s}, 1 \mathrm{H}) .{ }^{13} \mathrm{C} \mathrm{NMR}(151 \mathrm{MHz}$, $\left.\mathrm{CDCl}_{3}\right) \delta=144.8,141.9,139.3,129.8,128.4,128.3,127.7,127.6,127.5,126.2,125.4$ (q, $\left.J=279 \mathrm{~Hz}\right), 77.4,76.9$, $49.8(\mathrm{q}, J=31.4 \mathrm{~Hz}), 48.1 .{ }^{19} \mathrm{~F}$ NMR $\left(376 \mathrm{MHz}, \mathrm{CDCl}_{3}\right) \delta=-62.9$ (t). HRMS (ESI - MS): calcd. 292.1308 [M+H] ${ }^{+}$ Found: 292.1304 .

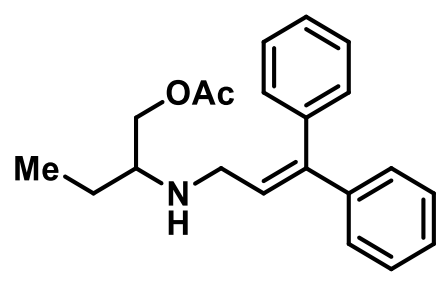

2-((3,3-Diphenylallyl)amino)butyl acetate (1y): Method D used. Reaction time 14h, purified by hexanes:EtOAc (60:40). Product recovered as yellow oil (51.4 mg, 53\% yield). $\mathrm{R}_{f}=0.3$ (hexanes/EtOAc $\left.=1: 1\right) .{ }^{1} \mathrm{H} \mathrm{NMR}(400 \mathrm{MHz}$, $\left.\mathrm{CDCl}_{3}\right) \delta 7.42-7.29(\mathrm{~m}, 3 \mathrm{H}), 7.29-7.19(\mathrm{~m}, 5 \mathrm{H}), 7.18-7.11(\mathrm{~m}, 2 \mathrm{H}), 6.16(\mathrm{t}, J=6.9 \mathrm{~Hz}, 1 \mathrm{H}), 4.03(\mathrm{dd}, J=11.2$, $4.3 \mathrm{~Hz}, 1 \mathrm{H}), 3.92(\mathrm{dd}, J=11.2,6.1 \mathrm{~Hz}, 1 \mathrm{H}), 3.33(\mathrm{~d}, J=6.9 \mathrm{~Hz}, 2 \mathrm{H}), 2.68(\mathrm{dd}, J=6.2,4.4 \mathrm{~Hz}, 1 \mathrm{H}), 2.02(\mathrm{~s}, 3 \mathrm{H}), 1.46$ $-1.28(\mathrm{~m}, 2 \mathrm{H}), 0.88(\mathrm{t}, J=7.5 \mathrm{~Hz}, 3 \mathrm{H}) .{ }^{13} \mathrm{C} \mathrm{NMR}\left(101 \mathrm{MHz}, \mathrm{CDCl}_{3}\right) \delta=171.2,143.7,142.1,139.6,129.8,128.3$, 128.3, 127.5, 127.5, 127.4, 66.1, 57.3, 46.2, 24.4, 21.1, 10.3. HRMS (ESI - MS): calcd. $324.1958[\mathrm{M}+\mathrm{H}]^{+}$Found: 324.1965 . 


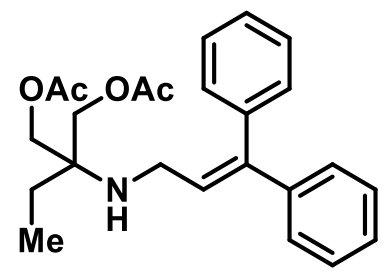

2-((3,3-Diphenylallyl)amino)2-ethylpropane-1,3-diyl diacetate (1z): Method D used. Reaction time 14h, purified by hexanes:EtOAc (90:10). Product recovered as yellow oil ( $45.0 \mathrm{mg}, 38 \%$ yield). $\mathrm{R}_{f}=0.45$ (hexanes/EtOAc $=8: 2$ ). ${ }^{1} \mathrm{H}$ NMR $\left(400 \mathrm{MHz}, \mathrm{CDCl}_{3}\right) \delta 7.35(\mathrm{ddd}, J=16.1,6.7,3.4 \mathrm{~Hz}, 3 \mathrm{H}), 7.28-7.22(\mathrm{~m}, 5 \mathrm{H}), 7.17(\mathrm{dd}, J=7.8,1.4 \mathrm{~Hz}$, 2H), $6.13(\mathrm{t}, J=7.0 \mathrm{~Hz}, 1 \mathrm{H}), 3.94(\mathrm{q}, J=11.3 \mathrm{~Hz}, 4 \mathrm{H}), 3.20(\mathrm{~d}, J=7.0 \mathrm{~Hz}, 2 \mathrm{H}), 2.03(\mathrm{~s}, 6 \mathrm{H}), 1.41$ (dd, $J=14.9,7.4$ $\mathrm{Hz}, 3 \mathrm{H}), 0.84(\mathrm{t}, J=7.5 \mathrm{~Hz}, 3 \mathrm{H}) .{ }^{13} \mathrm{C} \mathrm{NMR}\left(151 \mathrm{MHz}, \mathrm{CDCl}_{3}\right) \delta=170.9,143.7,142.1,139.5,129.7,128.4,128.3$, 127.5, 127.5, 127.5, 127.3, 65.0, 56.8, 40.7, 23.9, 21.0, 6.9. HRMS (ESI - MS): calcd. 396.2169 [M+H] $]^{+}$Found: 396.2177.

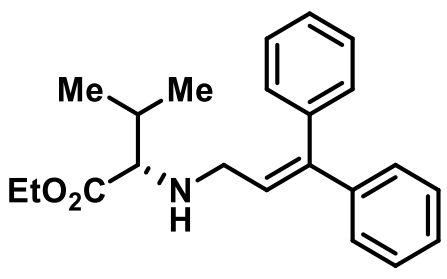

Ethyl (3,3-diphenylallyl)- $L$-valinate (1aa): Method D used. Reaction time 14h, purified by hexanes:EtOAc (90:10). Product recovered as yellow oil (63.7 mg, 63\% yield). $\mathrm{R}_{f}=0.4$ (hexanes/EtOAc $\left.=9: 1\right) .{ }^{1} \mathrm{H}$ NMR $\left(400 \mathrm{MHz}, \mathrm{CDCl}_{3}\right)$ $\delta 7.39-7.29$ (m, 3H), 7.25 (ddd, $J=7.9,4.6,2.4 \mathrm{~Hz}, 5 \mathrm{H}), 7.16(\mathrm{dd}, J=5.1,3.1 \mathrm{~Hz}, 2 \mathrm{H}), 6.13(\mathrm{t}, J=6.9 \mathrm{~Hz}, 1 \mathrm{H})$, $4.17-3.95(\mathrm{~m}, 2 \mathrm{H}), 3.31(\mathrm{dd}, J=13.7,6.7 \mathrm{~Hz}, 1 \mathrm{H}), 3.18(\mathrm{dd}, J=13.7,7.2 \mathrm{~Hz}, 1 \mathrm{H}), 2.96(\mathrm{~d}, J=5.8 \mathrm{~Hz}, 1 \mathrm{H}), 1.90$ $(\mathrm{dd}, J=13.0,6.6 \mathrm{~Hz}, 1 \mathrm{H}), 1.18(\mathrm{t}, J=7.1 \mathrm{~Hz}, 3 \mathrm{H}), 0.94(\mathrm{dd}, J=6.8,1.5 \mathrm{~Hz}, 6 \mathrm{H}) .{ }^{13} \mathrm{C}$ NMR $\left(151 \mathrm{MHz}, \mathrm{CDCl}_{3}\right) \delta=$ 175.2, 143.8, 142.2, 139.6, 129.9, 128.2, 127.5, 127.4, 127.3, 77.4, 76.9, 66.7, 60.5, 47.6, 31.8, 19.3, 18.8, 14.4. HRMS (ESI - MS): calcd. $338.2115[\mathrm{M}+\mathrm{H}]^{+}$Found: 338.2118.

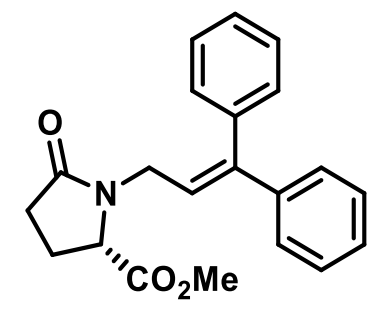

Methyl (S)-1-(3,3-diphenylallyl)-5-oxopyrrolidine-2-carboxylate (1ab): Method D used. Reaction time 14h, purified by hexanes:EtOAc (70:30). Product recovered as yellow oil (60.7 mg, $58 \%$ yield). $\mathrm{R}_{f}=0.3$ (hexanes/EtOAc = 7:3). ${ }^{1} \mathrm{H}$ NMR (400 MHz, $\left.\mathrm{CDCl}_{3}\right) \delta 7.35(\mathrm{ddd}, J=8.6,7.6,6.1 \mathrm{~Hz}, 3 \mathrm{H}), 7.29-7.22(\mathrm{~m}, 3 \mathrm{H}), 7.22-7.17(\mathrm{~m}, 2 \mathrm{H})$, $7.17-7.08(\mathrm{~m}, 2 \mathrm{H}), 5.97(\mathrm{dd}, J=8.4,6.0 \mathrm{~Hz}, 1 \mathrm{H}), 4.31(\mathrm{dd}, J=15.3,6.0 \mathrm{~Hz}, 1 \mathrm{H}), 4.13(\mathrm{dd}, J=9.0,2.9 \mathrm{~Hz}, 1 \mathrm{H})$, $3.74(\mathrm{dd}, J=15.3,8.5 \mathrm{~Hz}, 1 \mathrm{H}), 3.57$ (s, 3H), 2.48 (dd, $J=17.1,8.0 \mathrm{~Hz}, 1 \mathrm{H}), 2.42-2.16(\mathrm{~m}, 2 \mathrm{H}), 2.08-1.94(\mathrm{~m}, 1 \mathrm{H})$. ${ }^{13} \mathrm{C} \mathrm{NMR}\left(100 \mathrm{MHz}, \mathrm{CDCl}_{3}\right) \delta=175.0,172.6,146.0,141.3,138.6,129.7,128.4,128.3,127.8,127.6,127.3,122.4$, 59.1, 52.4, 40.8, 29.5, 23.1. HRMS (ESI - MS): calcd. 336.1594 [M+H] $]^{+}$Found: 336.1593. 


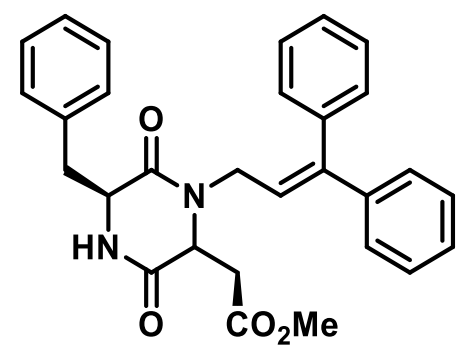

Methyl 2-((5S)-5-benzyl-1-(3,3-diphenylallyl)-3,6-dioxopiperazin-2-yl)acetate (1ac): Method D used. Reaction time 14h, purified by hexanes:EtOAc (60:40). Product recovered as yellow oil (42.1 mg, 30\% yield). $\mathrm{R}_{f}=0.1$ (hexanes/EtOAc = 1:1). ${ }^{1} \mathrm{H}$ NMR $\left(600 \mathrm{MHz} \mathrm{CDCl}_{3}\right) \delta 7.42(\mathrm{t}, J=7.4 \mathrm{~Hz}, 2 \mathrm{H}), 7.37(\mathrm{~m}, 3 \mathrm{H}), 7.29(\mathrm{~m}, 6 \mathrm{H}), 7.20(\mathrm{~d}$, $J=7.2 \mathrm{~Hz}, 4 \mathrm{H}), 6.00(\mathrm{t}, J=6.7 \mathrm{~Hz}, 1 \mathrm{H}), 5.94(\mathrm{~s}, 1 \mathrm{H}), 4.29(\mathrm{dd}, J=15.5,6.3 \mathrm{~Hz}, 1 \mathrm{H}), 4.21(\mathrm{~d}, J=9.4 \mathrm{~Hz}, 1 \mathrm{H}), 4.15$ - $4.08(\mathrm{~m}, 1 \mathrm{H}), 3.98(\mathrm{q}, J=7.1 \mathrm{~Hz}, 1 \mathrm{H}), 3.60$ (s, 3H), $3.38-3.34(\mathrm{~m}, 1 \mathrm{H}), 3.24(\mathrm{dd}, J=13.4,9.7 \mathrm{~Hz}, 1 \mathrm{H}), 2.58(\mathrm{dd}$, $J=16.9,3.7 \mathrm{~Hz}, 1 \mathrm{H}), 2.17(\mathrm{dd}, J=16.9,5.8 \mathrm{~Hz}, 1 \mathrm{H}) .{ }^{13} \mathrm{C} \mathrm{NMR}\left(151 \mathrm{MHz}, \mathrm{CDCl}_{3}\right) \delta=170.1,166.2,165.2,145.9$, $141.1,138.6,136.2,130.0,129.8,129.3,128.7,128.4,128.1,128.0,127.6,127.5,122.4,57.0,56.2,52.1,43.9$, 40.6, 35.5. HRMS (ESI - MS): calcd. 469.2122 [M+H] $]^{+}$Found: 469.2135.

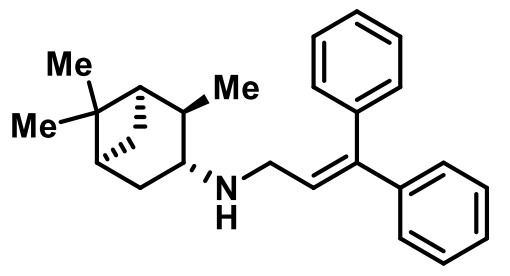

$(1 R, 2 R, 3 R, 5 S)-N$-(3,3-Diphenylallyl)-2,6,6-trimethylbicyclo[3.1.1]heptan-3-amine (1ad): Method D used. Reaction time 14h, purified by hexanes:EtOAc (60:40). Product recovered as yellow oil $(58.0 \mathrm{mg}, 56 \% \mathrm{yield})$. $\mathrm{R}_{f}=$ 0.15 (hexanes/EtOAc = 1:1). ${ }^{1} \mathrm{H}$ NMR $\left(600 \mathrm{MHz} \mathrm{CDCl}_{3}\right) \delta 7.41(\mathrm{ddd}, J=7.5,4.5,1.3 \mathrm{~Hz}, 2 \mathrm{H}), 7.36(\mathrm{dt}, J=4.7,1.9$ $\mathrm{Hz}, 1 \mathrm{H}), 7.32-7.25(\mathrm{~m}, 5 \mathrm{H}), 7.24-7.21(\mathrm{~m}, 2 \mathrm{H}), 6.25(\mathrm{dd}, J=7.4,6.4 \mathrm{~Hz}, 1 \mathrm{H}), 3.40(\mathrm{dd}, J=13.7,6.3 \mathrm{~Hz}, 1 \mathrm{H}), 3.31$ $(\mathrm{dd}, J=13.7,7.5 \mathrm{~Hz}, 1 \mathrm{H}), 2.85(\mathrm{dt}, J=9.2,5.6 \mathrm{~Hz}, 1 \mathrm{H}), 2.38-2.28(\mathrm{~m}, 1 \mathrm{H}), 2.27-2.17(\mathrm{~m}, 1 \mathrm{H}), 1.92(\mathrm{dt}, J=5.5$, $2.6 \mathrm{~Hz}, 1 \mathrm{H}), 1.85-1.70(\mathrm{~m}, 2 \mathrm{H}), 1.50(\mathrm{ddd}, J=13.5,5.2,2.6 \mathrm{~Hz}, 1 \mathrm{H}), 1.22(\mathrm{~s}, 3 \mathrm{H}), 1.09(\mathrm{~d}, J=7.2 \mathrm{~Hz}, 3 \mathrm{H}), 0.95(\mathrm{~d}$, $J=11.5 \mathrm{~Hz}, 4 \mathrm{H}) .{ }^{13} \mathrm{C} \operatorname{NMR}\left(151 \mathrm{MHz}, \mathrm{CDCl}_{3}\right) \delta=143.5,142.3,139.8,129.9,128.3,128.2,128.0,127.5,127.3$, 127.3, 56.3, 48.0, 46.7, 45.2, 41.8, 38.7, 36.7, 33.9, 27.9, 23.5, 21.6. HRMS (ESI - MS): calcd. 346.2529 [M+H] ${ }^{+}$ Found: 346.2540 .

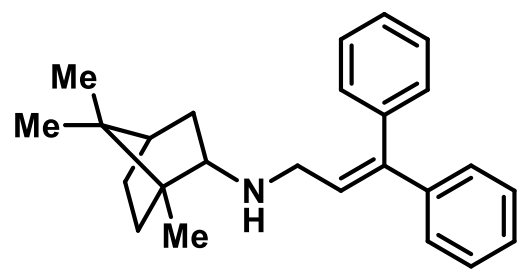

(2S)-N-(3,3-Diphenylallyl)-1,7,7-trimethylbicyclo[2.2.1]heptan-2-amine (1ae): Method D used. Reaction time 14h, purified by toluene:acetone (90:10). Product recovered as yellow oil (52.8 $\mathrm{mg}, 51 \%$ yield). $\mathrm{R}_{f}=0.1$ (hexanes/EtOAc = 8:2). ${ }^{1} \mathrm{H}$ NMR $\left(400 \mathrm{MHz}, \mathrm{CDCl}_{3}\right) \delta 7.41-7.31(\mathrm{~m}, 3 \mathrm{H}), 7.29-7.22(\mathrm{~m}, 5 \mathrm{H}), 7.19(\mathrm{dd}, J=8.0$, $1.3 \mathrm{~Hz}, 2 \mathrm{H}), 6.22(\mathrm{t}, J=6.9 \mathrm{~Hz}, 1 \mathrm{H}), 3.30(\mathrm{ddd}, J=35.1,13.7,7.0 \mathrm{~Hz}, 2 \mathrm{H}), 2.87-2.70(\mathrm{~m}, 1 \mathrm{H}), 2.11-1.92(\mathrm{~m}, 1 \mathrm{H})$, 
$1.71(\mathrm{tdd}, J=12.0,8.8,4.0 \mathrm{~Hz}, 2 \mathrm{H}), 1.57(\mathrm{t}, J=4.3 \mathrm{~Hz}, 1 \mathrm{H}), 1.31-1.21(\mathrm{~m}, 1 \mathrm{H}), 1.21-1.07(\mathrm{~m}, 1 \mathrm{H}), 0.89-0.77$ $(\mathrm{m}, 8 \mathrm{H}), 0.71(\mathrm{dd}, J=12.8,4.0 \mathrm{~Hz}, 1 \mathrm{H}) .{ }^{13} \mathrm{C} \mathrm{NMR}\left(101 \mathrm{MHz}, \mathrm{CDCl}_{3}\right) \delta=143.3,142.3,139.8,129.9,128.4,128.3$, $128.2,127.5,127.3,77.5,76.8,63.0,48.8,48.4,48.0,45.1,38.3,28.5,27.5,20.0,18.8,14.3$. HRMS (ESI - MS): calcd. $346.2529[\mathrm{M}+\mathrm{H}]^{+}$Found: 346.2534 .

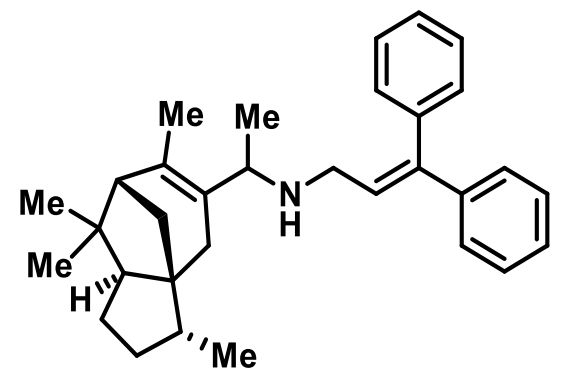

3,3-Diphenyl- $N$-(1-((3R,3a $R, 7 R, 8 \mathrm{a} S)-3,6,8,8$-tetramethyl-2,3,4,7,8,8a-hexahydro-1H-3a,7methanoazulen-5yl)ethyl)prop-2-en-1-amine (1af): Method D used. Reaction time 14h, purified by hexanes:EtOAc (70:30). Product recovered as yellow oil $\left(64.5 \mathrm{mg}, 49 \%\right.$ yield). $\mathrm{R}_{f}=0.27$ (hexanes/EtOAc $\left.=6: 4\right) .{ }^{1} \mathrm{H} \mathrm{NMR}\left(600 \mathrm{MHz}, \mathrm{CDCl}_{3}\right) \delta 7.36$ $-7.33(\mathrm{~m}, 2 \mathrm{H}), 7.31(\mathrm{dd}, J=5.1,3.7 \mathrm{~Hz}, 1 \mathrm{H}), 7.27-7.24(\mathrm{~m}, 5 \mathrm{H}), 7.15(\mathrm{dd}, J=8.1,1.3 \mathrm{~Hz}, 2 \mathrm{H}), 6.23(\mathrm{t}, J=7.0 \mathrm{~Hz}$, $1 \mathrm{H}), 3.72(\mathrm{q}, J=6.4 \mathrm{~Hz}, 1 \mathrm{H}), 3.21(\mathrm{dd}, J=13.5,7.1 \mathrm{~Hz}, 1 \mathrm{H}), 3.10(\mathrm{dd}, J=13.7,6.8 \mathrm{~Hz}, 1 \mathrm{H}), 1.86-1.81(\mathrm{~m}, 2 \mathrm{H})$, $1.69-1.67(\mathrm{~m}, 2 \mathrm{H}), 1.61(\mathrm{t}, J=1.8 \mathrm{~Hz}, 3 \mathrm{H}), 1.51-1.48(\mathrm{~m}, 2 \mathrm{H}), 1.37-1.33(\mathrm{~m}, 2 \mathrm{H}), 1.07(\mathrm{~d}, J=6.6 \mathrm{~Hz}, 3 \mathrm{H}), 1.01$ $-0.95(\mathrm{~m}, 2 \mathrm{H}), 0.91(\mathrm{~d}, J=9.4 \mathrm{~Hz}, 6 \mathrm{H}), 0.84(\mathrm{~d}, J=7.2 \mathrm{~Hz}, 3 \mathrm{H}) .{ }^{13} \mathrm{C} \mathrm{NMR}\left(151 \mathrm{MHz}, \mathrm{CDCl}_{3}\right) \delta=143.2,142.2$, 139.6, 135.0, 129.8, 128.1, 127.4, 127.2, 58.4, 56.9, 53.8, 52.3, 48.2, 46.0, 41.4, 40.3, 35.6, 35.2, 27.9, 25.6, 24.5, 20.5, 20.0, 15.5. HRMS (ESI - MS): calcd. $440.3312[\mathrm{M}+\mathrm{H}]^{+}$Found: 440.3326.

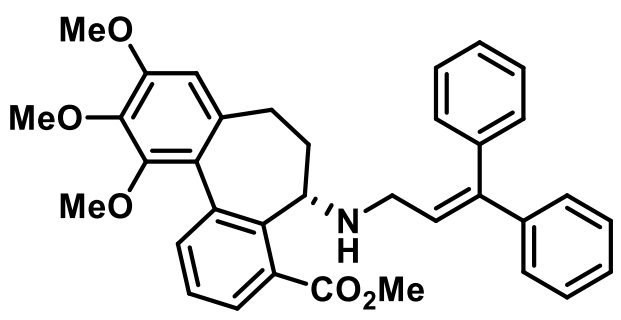

\section{Methyl (5S)-5-((3,3-diphenylallyl)amino)-9,10,11-trimethoxy-6,7-dihydro-5H-dibenzo[ $[a, c][7]$ annulene-4-} carboxylate (1ag): Method D used. Reaction time 14h, purified by hexanes:EtOAc (50:50). Product recovered as yellow oil (69.2 mg, $42 \%$ yield). $\mathrm{R}_{f}=0.25$ (hexanes/EtOAc $\left.=4: 6\right) .{ }^{1} \mathrm{H}$ NMR $\left(600 \mathrm{MHz}, \mathrm{CDCl}_{3}\right) \delta 8.26(\mathrm{~d}, J=1.7 \mathrm{~Hz}$, 1H), $7.99(\mathrm{dd}, J=8.0,1.8 \mathrm{~Hz}, 1 \mathrm{H}), 7.52(\mathrm{~d}, J=8.0 \mathrm{~Hz}, 1 \mathrm{H}), 7.26-7.19(\mathrm{~m}, 6 \mathrm{H}), 7.17-7.14(\mathrm{~m}, 2 \mathrm{H}), 7.03-6.97$ (m, 2H), $6.57(\mathrm{~s}, 1 \mathrm{H}), 6.15(\mathrm{t}, J=6.8 \mathrm{~Hz}, 1 \mathrm{H}), 3.94(\mathrm{~s}, 3 \mathrm{H}), 3.90(\mathrm{~s}, 3 \mathrm{H}), 3.88(\mathrm{~s}, 3 \mathrm{H}), 3.58(\mathrm{~s}, 3 \mathrm{H}), 3.53(\mathrm{dd}, J=6.8$, $4.5 \mathrm{~Hz}, 1 \mathrm{H}), 3.30$ (dd, $J=14.0,6.3 \mathrm{~Hz}, 1 \mathrm{H}), 3.19$ (dd, $J=14.0,7.4 \mathrm{~Hz}, 1 \mathrm{H}), 2.40$ (dd, $J=12.8,6.2 \mathrm{~Hz}, 1 \mathrm{H}), 2.34(\mathrm{td}$, $J=12.2,6.4 \mathrm{~Hz}, 1 \mathrm{H}), 2.19(\mathrm{td}, J=12.7,7.3 \mathrm{~Hz}, 1 \mathrm{H}), 1.75(\mathrm{~d}, J=7.3 \mathrm{~Hz}, 1 \mathrm{H}) \cdot{ }^{13} \mathrm{C} \mathrm{NMR}\left(151 \mathrm{MHz}, \mathrm{CDCl}_{3}\right) \delta=$ 167.6, 153.3, 150.9, 143.4, 142.3, 141.1, 140.3, 139.5, 135.9, 130.3, 129.7, 128.9, 128.2, 128.2, 127.9, 127.5, 127.4, 127.2, 125.3, 124.2, 107.6, 61.2, 61.2, 57.1, 56.1, 52.2, 47.3, 40.6, 31.0. HRMS (ESI - MS): calcd. 550.2588 [M+H] ${ }^{+}$ Found: 550.2588 . 


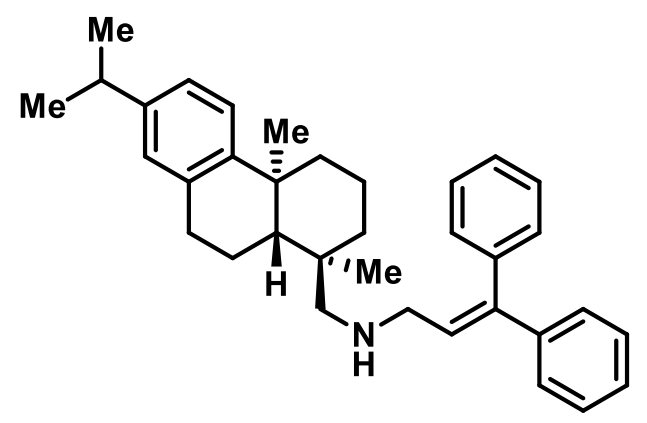

(E)- $N$-(((1R,4aS,10aR)-7-Isopropyl-1,4a-dimethyl-1,2,3,4,4a,9,10,10a-octahydrophenanthren-1-yl)methyl)-3,3diphenylprop-2-en-1-amine (1ah): Method D used. Reaction time 14h, purified by hexanes:EtOAc (80:20). Product recovered as yellow oil $\left(90.2 \mathrm{mg}, 63 \%\right.$ yield). $\mathrm{R}_{f}=0.3$ (hexanes/EtOAc $\left.=7: 3\right) .{ }^{1} \mathrm{H} \mathrm{NMR}\left(600 \mathrm{MHz}, \mathrm{CDCl}_{3}\right) \delta 7.42-$ $7.39(\mathrm{~m}, 2 \mathrm{H}), 7.37-7.35(\mathrm{~m}, 1 \mathrm{H}), 7.32-7.26(\mathrm{~m}, 6 \mathrm{H}), 7.24-7.20(\mathrm{~m}, 4 \mathrm{H}), 7.03(\mathrm{dd}, J=8.2,1.7 \mathrm{~Hz}, 1 \mathrm{H}), 6.93(\mathrm{~d}, J$ $=1.3 \mathrm{~Hz}, 1 \mathrm{H}), 6.21(\mathrm{t}, J=6.8 \mathrm{~Hz}, 1 \mathrm{H}), 3.32(\mathrm{~d}, J=6.8 \mathrm{~Hz}, 2 \mathrm{H}), 2.94-2.84(\mathrm{~m}, 3 \mathrm{H}), 2.51(\mathrm{~d}, J=11.8 \mathrm{~Hz}, 1 \mathrm{H}), 2.31$ $(\mathrm{d}, J=11.9 \mathrm{~Hz}, 2 \mathrm{H}), 1.83-1.76(\mathrm{~m}, 2 \mathrm{H}), 1.76-1.67(\mathrm{~m}, 2 \mathrm{H}), 1.63(\mathrm{dd}, J=11.9,2.6 \mathrm{~Hz}, 2 \mathrm{H}), 1.45(\mathrm{dd}, J=13.2,4.4$ $\mathrm{Hz}, 3 \mathrm{H}), 1.7(\mathrm{~d}, J=6.9 \mathrm{~Hz}, 6 \mathrm{H}), 1.25(\mathrm{~s}, 3 \mathrm{H}), 0.95(\mathrm{~s}, 3 \mathrm{H}) .{ }^{13} \mathrm{C} \mathrm{NMR}\left(151 \mathrm{MHz}, \mathrm{CDCl}_{3}\right) \delta=147.7,145.6,143.2$, 142.4, 139.9, 134.9, 129.9, 128.3, 128.2, 127.5, 127.3, 127.3, 126.9, 124.4, 123.9, 77.4, 76.9, 61.4, 49.6, 45.5, 38.6, 37.5, 37.1, 36.4, 33.6, 30.4, 25.5, 24.2, 24.1, 19.4, 19.0, 18.9. HRMS (ESI - MS): calcd. $478.3468[\mathrm{M}+\mathrm{H}]^{+}$Found: 478.3478 .

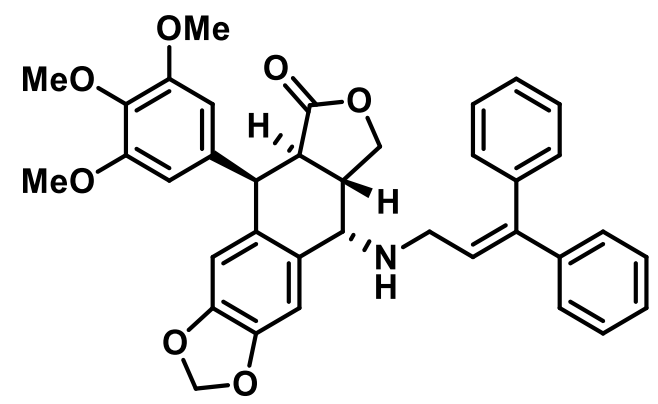

\section{(5R,5aR,8aS,9S)-9-((3,3-Diphenylallyl)amino)-5-(3,4,5-trimethoxyphenyl)-5,8,8a,9-}

tetrahydrofuro $\left[3^{\prime}, 3\right.$ ':6,7]naphtha(2,3-d][1,3]dioxol-6(5aH)-one (1ai): Method E used. Reaction time 14h, purified by hexanes:EtOAc (60:40). Product recovered as yellow oil (118.0 mg, 65\% yield). $\mathrm{R}_{f}=0.28$ (hexanes/EtOAc $=1: 1$ ). ${ }^{1} \mathrm{H}$ NMR (600 MHz, $\left.\mathrm{CDCl}_{3}\right) \delta 7.44-7.37$ (m, 3H), 7.33 - $7.26(\mathrm{~m}, 5 \mathrm{H}), 7.22-7.16(\mathrm{~m}, 2 \mathrm{H}), 6.63(\mathrm{~s}, 1 \mathrm{H}), 6.46$ (s, $1 \mathrm{H}), 6.25(\mathrm{~s}, 2 \mathrm{H}), 6.21(\mathrm{dd}, J=11.8,4.9 \mathrm{~Hz}, 1 \mathrm{H}), 5.96(\mathrm{~d}, J=4.3 \mathrm{~Hz}, 2 \mathrm{H}), 4.51(\mathrm{~d}, J=5.1 \mathrm{~Hz}, 1 \mathrm{H}), 4.18-4.08(\mathrm{~m}$, 1H), 4.07 (d, $J=7.2 \mathrm{~Hz}, 1 \mathrm{H}), 3.79$ (s, 3H), $3.72(\mathrm{~s}, 6 \mathrm{H}), 3.72-3.69(\mathrm{~m}, 1 \mathrm{H}), 3.42(\mathrm{dd}, J=13.4,8.1 \mathrm{~Hz}, 1 \mathrm{H}), 3.29$ $(\mathrm{dd}, J=13.2,4.8 \mathrm{~Hz}, 2 \mathrm{H}), 2.73-2.56(\mathrm{~m}, 1 \mathrm{H}) .{ }^{13} \mathrm{C} \mathrm{NMR}\left(151 \mathrm{MHz}, \mathrm{CDCl}_{3}\right) \delta=175.5,152.5,147.8,147.4,144.9$, 141.5, 139.3, 137.1, 135.8, 132.7, 131.6, 129.7, 128.5, 128.4, 127.7, 127.7, 127.3, 126.1, 110.2, 108.5, 108.3, 101.5, 68.6, 60.8, 56.3, 55.7, 49.1, 43.8, 41.4, 38.7. HRMS (ESI - MS): calcd. 606.2486 [M+H] $]^{+}$Found: 606.2485. 


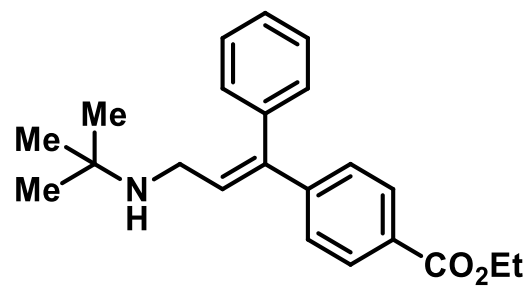

Ethyl 4-(3-(tert-butylamino)-1-phenylprop-1-en-1-yl)benzoate (2a): Method D used. Reaction time 14h, purified by toluene:acetone (80:20). Product recovered as yellow oil $\left(65.7 \mathrm{mg}, 65 \%\right.$ yield). $\mathrm{R}_{f}=0.10$ (toluene/acetone $\left.=7: 3\right)$ and the Z/E ratio was determined as 10:1 from the crude NMR. ${ }^{1} \mathrm{H}$ NMR $\left(600 \mathrm{MHz}, \mathrm{CDCl}_{3}\right) \delta 7.88(\mathrm{~d}, J=8.3 \mathrm{~Hz}$, $2 \mathrm{H}), 7.35(\mathrm{t}, J=7.3 \mathrm{~Hz}, 2 \mathrm{H}), 7.31(\mathrm{~d}, J=7.0 \mathrm{~Hz}, 1 \mathrm{H}), 7.27-7.23(\mathrm{~m}, 2 \mathrm{H}), 7.13(\mathrm{~d}, J=7.0 \mathrm{~Hz}, 2 \mathrm{H}), 6.27(\mathrm{t}, J=6.9$ $\mathrm{Hz}, 1 \mathrm{H}), 4.31(\mathrm{q}, J=7.1 \mathrm{~Hz}, 2 \mathrm{H}), 3.24(\mathrm{~d}, J=6.9 \mathrm{~Hz}, 2 \mathrm{H}), 1.33(\mathrm{t}, J=7.1 \mathrm{~Hz}, 3 \mathrm{H}), 1.01(\mathrm{~s}, 9 \mathrm{H}) .{ }^{13} \mathrm{C} \mathrm{NMR}(151 \mathrm{MHz}$, $\left.\mathrm{CDCl}_{3}\right) \delta=166.6,146.6,142.4,139.0,129.9,129.7,129.6,129.5,129.1,128.5,128.3,127.6,127.4,127.3,61.0,42.0$, 29.0, 14.5. HRMS (ESI - MS): calcd. 338.2115 [M+H] $]^{+}$Found: 338.2123.

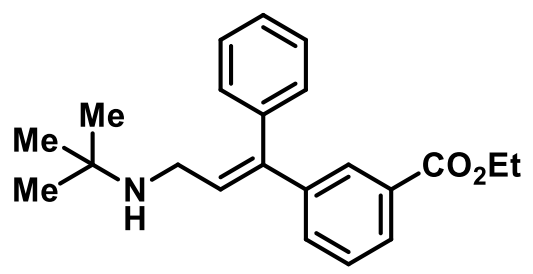

Ethyl 3-(3-tert-butylamino)-1-phenylprop-1-en-1-yl)benzoate (2b): Method D used. Reaction time 14h, purified by toluene:acetone (80:20). Product recovered as yellow oil $\left(55.6 \mathrm{mg}, 55 \%\right.$ yield). $\mathrm{R}_{f}=0.10$ (toluene/acetone $\left.=7: 3\right)$ and the Z/E ratio was determined as 5:1 from the crude NMR. ${ }^{1} \mathrm{H}$ NMR $\left(600 \mathrm{MHz}, \mathrm{CDCl}_{3}\right) \delta 7.99(\mathrm{~d}, J=1.3 \mathrm{~Hz}, 1 \mathrm{H})$, $7.95-7.83(\mathrm{~m}, 1 \mathrm{H}), 7.39-7.33(\mathrm{~m}, 2 \mathrm{H}), 7.32-7.23(\mathrm{~m}, 3 \mathrm{H}), 7.19-7.09(\mathrm{~m}, 2 \mathrm{H}), 6.22(\mathrm{dd}, J=9.0,4.8 \mathrm{~Hz}, 1 \mathrm{H})$, $4.40-4.09(\mathrm{~m}, 2 \mathrm{H}), 3.24(\mathrm{~d}, J=6.9 \mathrm{~Hz}, 2 \mathrm{H}), 1.33(\mathrm{dd}, J=11.2,4.0 \mathrm{~Hz}, 3 \mathrm{H}), 1.02(\mathrm{~s}, 9 \mathrm{H}) .{ }^{13} \mathrm{C} \mathrm{NMR}(151 \mathrm{MHz}$, $\left.\mathrm{CDCl}_{3}\right) \delta=166.7,142.6,142.3,139.2,134.2,132.1,130.5,129.7,129.3,128.4,128.3,128.2,128.1,127.5,127.4$, 61.1, 50.8, 41.9, 29.0, 14.4. HRMS (ESI - MS): calcd. 338.2115 [M+H] ${ }^{+}$Found: 338.2126.

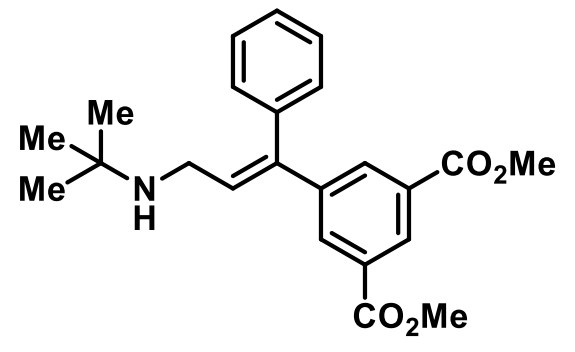

Dimethyl 5-(3-(tert-butylamino)-1-phenylprop-1-en-1-yl)isophthalate (2c): Method D used. Reaction time 14h, purified by toluene:acetone (70:40). Product recovered as yellow oil (50.3 mg, 44\% yield). $\mathrm{R}_{f}=0.10$ (toluene/acetone $=6: 4)$ and the Z/E ratio was determined as 5:1 from the crude NMR. ${ }^{1} \mathrm{H}$ NMR $\left(400 \mathrm{MHz}, \mathrm{CDCl}_{3}\right) \delta 8.53(\mathrm{~d}, J=0.9$ $\mathrm{Hz}, 1 \mathrm{H}), 8.11-8.07$ (m, 2H), $7.42-7.34$ (m, 3H), 7.17 (d, J=7.7 Hz, 2H), 6.29 (t, J=6.8 Hz, $1 \mathrm{H}), 3.90$ (s, $6 \mathrm{H}), 3.31$ $(\mathrm{d}, J=6.9 \mathrm{~Hz}, 2 \mathrm{H}), 1.07(\mathrm{~s}, 9 \mathrm{H}) .{ }^{13} \mathrm{C} \mathrm{NMR}\left(151 \mathrm{MHz}, \mathrm{CDCl}_{3}\right) \delta=166.4,143.3,138.5,135.0,132.7,130.7,129.6$, 129.5, 128.7, 128.5, 128.0, 127.5, 52.5, 41.9, 28.8. HRMS (ESI - MS): calcd. 382.2013 [M+H] Found: 382.2021. 


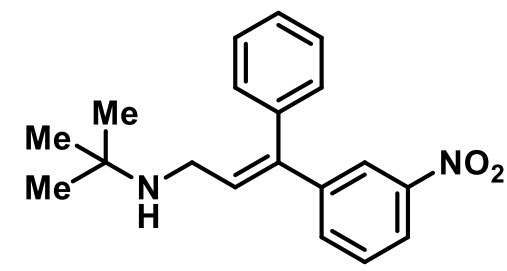

$N$-(tert-Butyl)-3-(3-nitrophenyl)-3-phenylprop-2-en-1-amine (2d): Method D used. Reaction time 14h, purified by toluene:acetone (70:30). Product recovered as yellow oil ( $41.8 \mathrm{mg}, 45 \%$ yield). $\mathrm{R}_{f}=0.15$ (toluene/acetone $=6: 4$ ). and the Z/E ratio was determined as 5:1 from the crude NMR. ${ }^{1} \mathrm{H}$ NMR $\left(400 \mathrm{MHz}, \mathrm{CDCl}_{3}\right) \delta 8.11(\mathrm{~s}, 1 \mathrm{H}), 8.06(\mathrm{~d}, J=8.0$ $\mathrm{Hz}, 1 \mathrm{H}), 7.51(\mathrm{~d}, J=7.5 \mathrm{~Hz}, 1 \mathrm{H}), 7.48-7.35(\mathrm{~m}, 4 \mathrm{H}), 7.16(\mathrm{~d}, J=7.2 \mathrm{~Hz}, 2 \mathrm{H}), 6.34(\mathrm{t}, J=6.7 \mathrm{~Hz}, 1 \mathrm{H}), 3.33(\mathrm{~d}, J=$ $6.7 \mathrm{~Hz}, 2 \mathrm{H}), 1.08(\mathrm{~s}, 9 \mathrm{H}) .{ }^{13} \mathrm{C} \mathrm{NMR}\left(151 \mathrm{MHz}, \mathrm{CDCl}_{3}\right) \delta=148.4,144.0,141.4,138.3,133.4,129.6,129.1,128.8$, 128.1, 127.9, 122.2, 122.1, 51.5, 41.9, 28.8. HRMS (ESI - MS): calcd. 311.1754 [M+H] ${ }^{+}$Found: 311.1758.

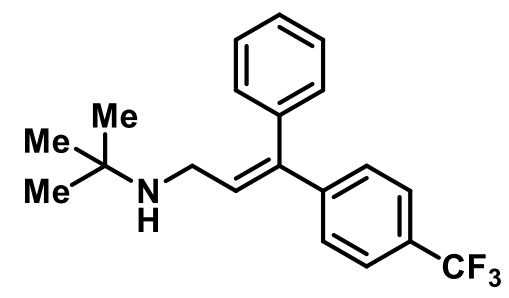

$N$-(tert-Butyl)-3-phenyl-3-(4-(trifluoromethyl)phenyl)prop-2-en-1-amine (2e): Method D used. Reaction time $14 \mathrm{~h}$, purified by toluene:acetone (80:20). Product recovered as yellow oil $(52.9 \mathrm{mg}, 53 \%$ yield $) . \mathrm{R}_{f}=0.10$ (toluene/acetone $=7: 3$ ) and the $\mathrm{Z} / \mathrm{E}$ ratio was determined as 7:1 from the crude NMR. ${ }^{1} \mathrm{H} \mathrm{NMR}\left(600 \mathrm{MHz}, \mathrm{CDCl}_{3}\right) \delta$ $7.50(\mathrm{~d}, J=8.2 \mathrm{~Hz}, 2 \mathrm{H}), 7.39(\mathrm{ddd}, J=7.5,6.3,1.3 \mathrm{~Hz}, 2 \mathrm{H}), 7.35(\mathrm{dt}, J=11.1,5.1 \mathrm{~Hz}, 3 \mathrm{H}), 7.19-7.12(\mathrm{~m}, 2 \mathrm{H}), 6.28$ $(\mathrm{t}, J=6.9 \mathrm{~Hz}, 1 \mathrm{H}), 3.29(\mathrm{~d}, J=6.9 \mathrm{~Hz}, 2 \mathrm{H}), 1.05(\mathrm{~s}, 9 \mathrm{H}) .{ }^{13} \mathrm{C} \mathrm{NMR}\left(151 \mathrm{MHz}, \mathrm{CDCl}_{3}\right) \delta=145.7,142.0,138.9,130.4$, 130.2, 129.7, 129.2 (q, $J=33 \mathrm{~Hz}), 128.5,128.4,127.7,127.5,125.2$ (q, $J=3 \mathrm{~Hz}), 124.3$ (d, $J=272 \mathrm{~Hz}), 51.0,42.0$, 29.0. ${ }^{19} \mathrm{~F}$ NMR $\left(376 \mathrm{MHz}, \mathrm{CDCl}_{3}\right) \delta=-62.8$. HRMS (ESI - MS): calcd. $334.1777[\mathrm{M}+\mathrm{H}]^{+}$Found: 334.1790.

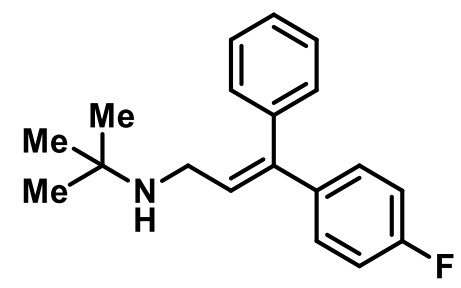

$N$-(tert-Butyl)-3-(4-fluorophenyl)-3-phenylprop-2-en-1-amine (2f): Method D used. Reaction time 14h, purified by toluene:acetone (80:20). Product recovered as yellow oil (51.8 $\mathrm{mg}, 61 \%$ yield). $\mathrm{R}_{f}=0.10$ (toluene/acetone $\left.=7: 3\right)$ and the Z/E ratio was determined as 8:1 from the crude NMR. ${ }^{1} \mathrm{H}$ NMR $\left(400 \mathrm{MHz}, \mathrm{CDCl}_{3}\right) \delta 7.41-7.30(\mathrm{~m}, 3 \mathrm{H}), 7.24-$ $7.12(\mathrm{~m}, 5 \mathrm{H}), 6.92(\mathrm{t}, J=8.7 \mathrm{~Hz}, 2 \mathrm{H}), 6.17(\mathrm{~s}, 1 \mathrm{H}), 3.28(\mathrm{~d}, J=6.9 \mathrm{~Hz}, 2 \mathrm{H}), 1.07(\mathrm{~s}, 9 \mathrm{H}) .{ }^{13} \mathrm{C} \mathrm{NMR}\left(151 \mathrm{MHz}, \mathrm{CDCl}_{3}\right)$ $\delta=162.3(\mathrm{~d}, J=246 \mathrm{~Hz}), 139.5,138.3,131.5,131.4,129.7,129.6,129.1,129.0,128.4,128.3$, 127.8, 127.5, $115.2(\mathrm{~d}$, $J=21 \mathrm{~Hz}), 114.9,41.9,28.8 .{ }^{19} \mathrm{~F}$ NMR (376 $\left.\mathrm{MHz}, \mathrm{CDCl}_{3}\right) \delta=-115.9(\mathrm{~m})$. HRMS (ESI - MS): calcd. 284.1809 $[\mathrm{M}+\mathrm{H}]^{+}$Found: 284.1818. 


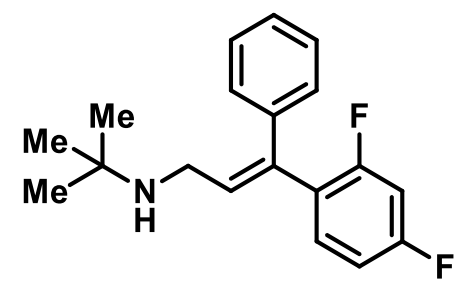

$N$-(tert-Butyl)-3-(2,4-difluorophenyl)-3-phenylprop-2-en-1-amine (2g): Method D used. Reaction time 14h, purified by toluene:acetone (80:20). Product recovered as yellow oil ( $45.1 \mathrm{mg}, 50 \%$ yield). $\mathrm{R}_{f}=0.10$ (toluene/acetone $=8: 2)$ and the Z/E ratio was determined as 5:1 from the crude NMR. ${ }^{1} \mathrm{H}$ NMR $\left(600 \mathrm{MHz}, \mathrm{CDCl}_{3}\right) \delta 7.37$ (ddd, $J=$ 7.5, 4.5, $1.3 \mathrm{~Hz}, 2 \mathrm{H}), 7.34-7.31(\mathrm{~m}, 1 \mathrm{H}), 7.25(\mathrm{dd}, J=6.5,1.7 \mathrm{~Hz}, 1 \mathrm{H}), 7.22-7.16(\mathrm{~m}, 3 \mathrm{H}), 6.84-6.73(\mathrm{~m}, 2 \mathrm{H})$, $6.09(\mathrm{t}, J=6.9 \mathrm{~Hz}, 1 \mathrm{H}), 3.38(\mathrm{~d}, J=7.0 \mathrm{~Hz}, 2 \mathrm{H}), 1.10(\mathrm{~s}, 8 \mathrm{H}) \cdot{ }^{13} \mathrm{C} \mathrm{NMR}\left(151 \mathrm{MHz}, \mathrm{CDCl}_{3}\right) \delta=162.2(\mathrm{dd}, J=248,12$ $\mathrm{Hz}), 160.3(\mathrm{dd}, J=251,12 \mathrm{~Hz}), 140.8,139.4,137.0,132.3,132.0$ (q, $J=5 \mathrm{~Hz}), 129.1,128.4,128.3,127.5,126.7,111$. (dd, $J=16,4 \mathrm{~Hz}), 104.2$ (t, $J=25 \mathrm{~Hz}), 41.7,29.0 .{ }^{19} \mathrm{~F}$ NMR (376 MHz, $\left.\mathrm{CDCl}_{3}\right) \delta=-111.6(\mathrm{~m}),-109.7(\mathrm{~m})$. HRMS (ESI - MS): calcd. $302.1715[\mathrm{M}+\mathrm{H}]^{+}$Found: 302.1723.

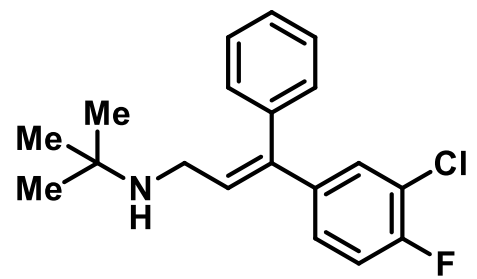

$N$-(tert-Butyl)-3-(3-chloro-4-fluorophenyl)-3-phenylprop-2-en-1-amine (2h): Method D used. Reaction time 14h, purified by EtOAc:MeOH (95:5). Product recovered as yellow oil $\left(57.0 \mathrm{mg}, 60 \%\right.$ yield). $\mathrm{R}_{f}=0.10$ (EtOAc/MeOH $=$ 8:2) and the Z/E ratio was determined as 6:1 from the crude NMR. ${ }^{1} \mathrm{H}$ NMR $\left(400 \mathrm{MHz}, \mathrm{CDCl}_{3}\right) \delta 7.43-7.32(\mathrm{~m}$, $3 \mathrm{H}), 7.28-7.24(\mathrm{~m}, 1 \mathrm{H}), 7.17-7.12(\mathrm{~m}, 2 \mathrm{H}), 7.07(\mathrm{dd}, J=4.7,2.2 \mathrm{~Hz}, 1 \mathrm{H}), 7.01(\mathrm{~d}, J=8.7 \mathrm{~Hz}, 1 \mathrm{H}), 6.14(\mathrm{t}, J=6.9$ $\mathrm{Hz}, 1 \mathrm{H}), 3.25(\mathrm{~d}, J=6.9 \mathrm{~Hz}, 2 \mathrm{H}), 1.05(\mathrm{~s}, 9 \mathrm{H}) .{ }^{13} \mathrm{C} \mathrm{NMR}\left(151 \mathrm{MHz}, \mathrm{CDCl}_{3}\right) \delta=157.5(\mathrm{~d}, J=249.2 \mathrm{~Hz}), 141.3$, $139.5(\mathrm{~d}, J=3.8 \mathrm{~Hz}), 138.8,129.6,129.5,128.6,127.8,127.1(\mathrm{~d}, J=7.0 \mathrm{~Hz}), 120.8(\mathrm{~d}, J=17.8 \mathrm{~Hz}), 116.2(\mathrm{~d}, J=$ 21.1 Hz), 77.4, 76.9, 51.2, 41.9, 28.9. ${ }^{19} \mathrm{~F}$ NMR (376 MHz, $\left.\mathrm{CDCl}_{3}\right) \delta=-118.2$. HRMS (ESI - MS): calcd. 318.1419 $[\mathrm{M}+\mathrm{H}]^{+}$Found: 318.1428.

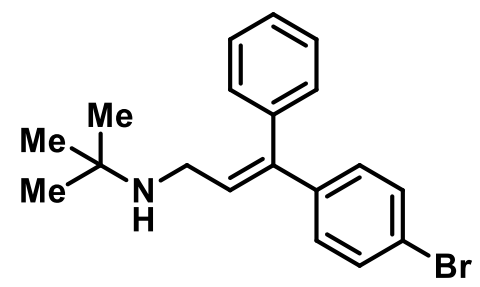

$N$-(tert-Butyl)-3-(4-bromophenyl)-3-phenylprop-2-en-1-amine (2i): Method D used. Reaction time 14h, purified by toluene:acetone (80:20). Product recovered as yellow oil $\left(56.6 \mathrm{mg}, 55 \%\right.$ yield). $\mathrm{R}_{f}=0.13$ (toluene/acetone $=8: 2$ ) and the Z/E ratio was determined as 8:1 from the crude NMR. ${ }^{1} \mathrm{H}$ NMR $\left(600 \mathrm{MHz}, \mathrm{CDCl}_{3}\right) \delta 7.41-7.32(\mathrm{~m}, 5 \mathrm{H})$, $7.15(\mathrm{dd}, J=8.1,1.3 \mathrm{~Hz}, 2 \mathrm{H}), 7.09(\mathrm{~d}, J=8.6 \mathrm{~Hz}, 2 \mathrm{H}), 6.21(\mathrm{t}, J=6.9 \mathrm{~Hz}, 1 \mathrm{H}), 3.26(\mathrm{~d}, J=7.0 \mathrm{~Hz}, 2 \mathrm{H}), 1.07$ (s, 2H), 1.06 (s, 9H). ${ }^{13} \mathrm{C}$ NMR $\left(151 \mathrm{MHz}, \mathrm{CDCl}_{3}\right) \delta=142.2,141.2,139.1,131.6,131.5,131.3,129.7,129.1,128.4,128.3$, 127.6, 127.5, 121.4, 77.4, 76.9, 51.2, 41.9, 28.9. HRMS (ESI - MS): calcd. 344.1008 [M+H] ${ }^{+}$Found: 344.1004. 


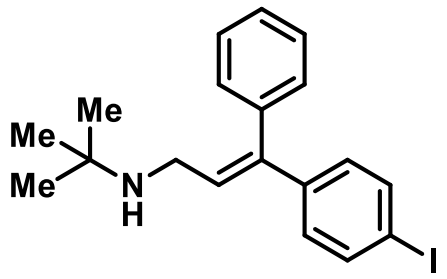

$N$-(tert-Butyl)-3-(4-iodophenyl)-3-phenylprop-2-en-1-amine (2j): Method D used. Reaction time 14h, purified by toluene:acetone (80:20). Product recovered as yellow oil $\left(72.7 \mathrm{mg}, 62 \%\right.$ yield). $\mathrm{R}_{f}=0.10$ (toluene/acetone $\left.=7: 3\right)$ and the Z/E ratio was determined as 6:1 from the crude NMR. ${ }^{1} \mathrm{H}$ NMR $\left(600 \mathrm{MHz}, \mathrm{CDCl}_{3}\right) \delta 7.59-7.54(\mathrm{~m}, 2 \mathrm{H}), 7.38(\mathrm{t}$, $J=4.2 \mathrm{~Hz}, 2 \mathrm{H}), 7.33(\mathrm{~d}, J=7.3 \mathrm{~Hz}, 1 \mathrm{H}), 7.15(\mathrm{~d}, J=6.9 \mathrm{~Hz}, 2 \mathrm{H}), 6.97(\mathrm{~d}, J=8.6 \mathrm{~Hz}, 2 \mathrm{H}), 6.21(\mathrm{t}, J=6.8 \mathrm{~Hz}, 1 \mathrm{H})$, $3.25(\mathrm{~d}, J=6.9 \mathrm{~Hz}, 2 \mathrm{H}), 1.05(\mathrm{~s}, 9 \mathrm{H}){ }^{13} \mathrm{C}$ NMR $\left(151 \mathrm{MHz}, \mathrm{CDCl}_{3}\right) \delta=141.8,139.0,137.5,137.3,131.8,129.7,129.4$, 128.5, 128.3, 127.6, 127.5, 93.0, 77.4, 76.9, 51.3, 42.0, 28.9. HRMS (ESI - MS): calcd. $392.0870[\mathrm{M}+\mathrm{H}]^{+}$Found: 392.0871.

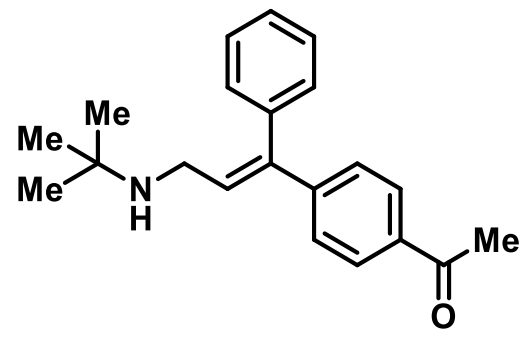

1-(4-(3-(tert-Butylamino)-1-phenylprop-1-en-1-yl)phenyl)ethan-1-one (2k): Method D used. Reaction time 14h, purified by toluene:acetone (80:20). Product recovered as yellow oil ( $50.6 \mathrm{mg}, 55 \%$ yield). $\mathrm{R}_{f}=0.10$ (toluene/acetone $=7: 3)$ and the Z/E ratio was determined as 10:1 from the crude NMR. ${ }^{1} \mathrm{H}$ NMR (400 MHz, $\left.\mathrm{CDCl}_{3}\right) \delta 7.84(\mathrm{~d}, J=8.4$ $\mathrm{Hz}, 2 \mathrm{H}), 7.42-7.36(\mathrm{~m}, 2 \mathrm{H}), 7.34-7.30(\mathrm{~m}, 2 \mathrm{H}), 7.16$ (dd, $J=6.6,1.4 \mathrm{~Hz}, 2 \mathrm{H}), 6.32$ (t, $J=6.9 \mathrm{~Hz}, 1 \mathrm{H}), 3.28(\mathrm{~d}, J$ $=6.9 \mathrm{~Hz}, 2 \mathrm{H}), 2.56(\mathrm{~d}, J=1.2 \mathrm{~Hz}, 3 \mathrm{H}), 1.05(\mathrm{~s}, 9 \mathrm{H}) .{ }^{13} \mathrm{C} \mathrm{NMR}\left(151 \mathrm{MHz}, \mathrm{CDCl}_{3}\right) \delta 197.8(\mathrm{~s}), 146.1,138.2,136.1$, 129.6, 128.8, 128.5, 128.4, 128.1, 127.8, 54.3, 41.6, 27.4, 26.8. HRMS (ESI - MS): calcd. 308.2009 [M+H] $]^{+}$Found: 308.2014 .

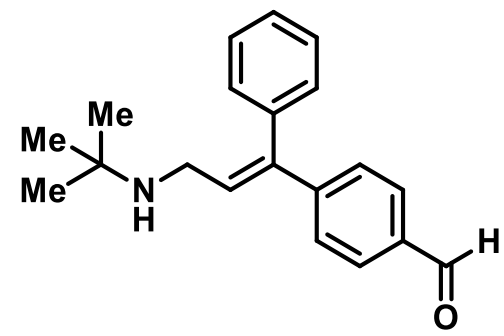

4-(3-(tert-Butylamino)-1-phenylprop-1-en-1-yl)benzaldehyde (2l): Method D used. Reaction time 14h, purified by EtOAc:MeOH (95:5). Product recovered as yellow oil (39.5 mg, 45\% yield). $\mathrm{R}_{f}=0.10$ (EtOAc/MeOH $=9: 1$ ) and the Z/E ratio was determined as 10:1 from the crude NMR. ${ }^{1} \mathrm{H}$ NMR $\left(400 \mathrm{MHz}, \mathrm{CDCl}_{3}\right) \delta 9.96(\mathrm{~s}, 1 \mathrm{H}), 7.76(\mathrm{~d}, J=8.3$ $\mathrm{Hz}, 2 \mathrm{H}), 7.43-7.33(\mathrm{~m}, 5 \mathrm{H}), 7.17$ (d, $J=6.6 \mathrm{~Hz}, 2 \mathrm{H}), 6.35$ (t, $J=6.9 \mathrm{~Hz}, 1 \mathrm{H}), 3.29$ (d, $J=6.9 \mathrm{~Hz}, 24 \mathrm{H}), 1.05$ (s, 9H). ${ }^{13} \mathrm{C} \mathrm{NMR}\left(151 \mathrm{MHz}, \mathrm{CDCl}_{3}\right) \delta=192.0,148.2,142.2,138.8,135.2,131.2,129.8,129.7,128.6,127.9,127.8$, 51.1, 42.0, 29.0. HRMS (ESI - MS): calcd. 294.1852 [M+H] $]^{+}$Found: 294.1863. 


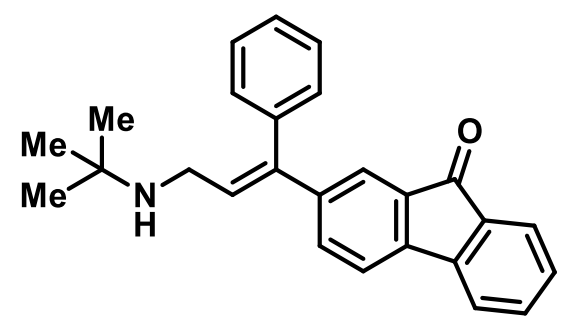

2-(3-(tert-Butylamino)-1-phenylprop-1-en-1-yl)-9H-fluoren-9-one (2m): Method D used. Reaction time 14h, purified by toluene:acetone (80:20). Product recovered as yellow oil (56.1 mg, 58\% yield). $\mathrm{R}_{f}=0.21$ (toluene/acetone $=7: 3)$ and the $\mathrm{Z} / \mathrm{E}$ ratio was determined as 10:1 from the crude $\mathrm{NMR} .{ }^{1} \mathrm{H}$ NMR $\left(600 \mathrm{MHz}, \mathrm{CDCl}_{3}\right) \delta 7.61(\mathrm{dd}, J=$ 7.3, 0.6 Hz, 1H), 7.52 (s, 1H), $7.48-7.44(\mathrm{~m}, 2 \mathrm{H}), 7.42-7.34(\mathrm{~m}, 5 \mathrm{H}), 7.25$ (ddd, J = 7.5, 3.8, 2.1 Hz, 2H), 7.21 $7.15(\mathrm{~m}, 2 \mathrm{H}), 6.29(\mathrm{t}, J=6.9 \mathrm{~Hz}, 1 \mathrm{H}), 3.30(\mathrm{~d}, J=6.9 \mathrm{~Hz}, 2 \mathrm{H}), 1.08(\mathrm{~s}, 9 \mathrm{H}) .{ }^{13} \mathrm{C} \mathrm{NMR}\left(151 \mathrm{MHz}, \mathrm{CDCl}_{3}\right) \delta=194.0$, $144.4,143.5,143.3,138.8,134.9,134.6,134.4,133.7,130.4,129.7,129.1,128.6,127.8,124.4,123.4,120.4,120.2$, 77.4, 76.9, 51.6, 41.9, 29.8, 28.8. HRMS (ESI - MS): calcd. 368.2009 [M+H]+ Found: 368.2016.

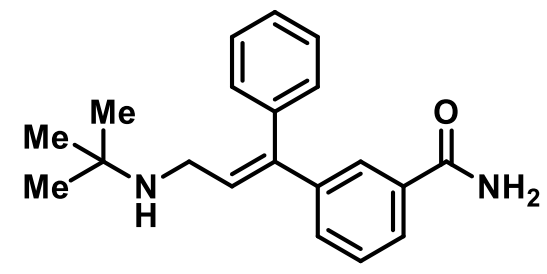

3-(3-(tert-Butylamino)-1-phenylprop-1-en-1yl)benzamide (2n): Method D used. Starting arene was 3iodobenzonitrile. Reaction time 14h, purified by EtOAc:MeOH (80:20). Product recovered as yellow oil (47.1 mg, $51 \%$ yield). $\mathrm{R}_{f}=0.15(\mathrm{EtOAc} / \mathrm{MeOH}=7: 3)$ and the $\mathrm{Z} / \mathrm{E}$ ratio was determined as 12:1 from the crude NMR. ${ }^{1} \mathrm{H}$ NMR $\left(600 \mathrm{MHz}, \mathrm{CD}_{3} \mathrm{OD}\right) \delta 7.83(\mathrm{~s}, 1 \mathrm{H}), 7.77-7.70(\mathrm{~m}, 1 \mathrm{H}), 7.44-7.40(\mathrm{~m}, 2 \mathrm{H}), 7.39-7.30(\mathrm{~m}, 3 \mathrm{H}), 7.23-7.15(\mathrm{~m}$, $2 \mathrm{H}), 6.19(\mathrm{t}, J=7.0 \mathrm{~Hz}, 1 \mathrm{H}), 3.52(\mathrm{~d}, J=7.1 \mathrm{~Hz}, 2 \mathrm{H}), 1.85(\mathrm{~s}, 2 \mathrm{H}), 1.17(\mathrm{~s}, 9 \mathrm{H}) .{ }^{13} \mathrm{C} \mathrm{NMR}\left(151 \mathrm{MHz}, \mathrm{CD}{ }_{3} \mathrm{OD}\right) \delta=$ $172.0,147.7,143.1,139.4,135.3,132.1,130.7,129.9,129.6,129.4,128.1,127.9,127.8,123.1,55.9,42.2,26.7$. HRMS (ESI - MS): calcd. 309.1961 [M+H] $]^{+}$Found: 309.1968.

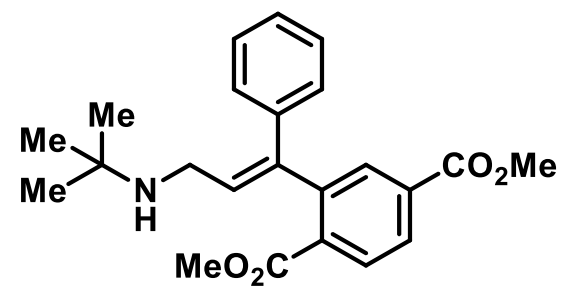

2-(3-(tert-Butylamino)-1-phenylprop-1-en-1-yl)terephthalate (2o): Method D used. Reaction time 14h, purified by toluene:acetone (80:20). Product recovered as yellow oil $\left(51.4 \mathrm{mg}, 45 \%\right.$ yield). $\mathrm{R}_{f}=0.25$ (toluene/acetone $\left.=7: 3\right)$ and the $\mathrm{Z} / \mathrm{E}$ ratio was determined as $6: 1$ from the crude $\mathrm{NMR} .{ }^{1} \mathrm{H} \mathrm{NMR}\left(600 \mathrm{MHz}, \mathrm{CDCl}_{3}\right) \delta 8.05(\mathrm{~d}, J=1.4 \mathrm{~Hz}, 1 \mathrm{H})$, $7.98(\mathrm{dd}, J=8.0,1.6 \mathrm{~Hz}, 1 \mathrm{H}), 7.70(\mathrm{~d}, J=8.0 \mathrm{~Hz}, 1 \mathrm{H}), 7.30(\mathrm{t}, J=7.3 \mathrm{~Hz}, 2 \mathrm{H}), 7.26(\mathrm{~m}, 1 \mathrm{H}), 7.17(\mathrm{~d}, J=7.2 \mathrm{~Hz}$, 2H), $5.98(\mathrm{~s}, 1 \mathrm{H}), 3.94-3.91(\mathrm{~m}, 3 \mathrm{H}), 3.60(\mathrm{~s}, 3 \mathrm{H}), 3.56-3.50(\mathrm{~m}, 2 \mathrm{H}), 1.14(\mathrm{~s}, 9 \mathrm{H}) .{ }^{13} \mathrm{C} \mathrm{NMR}\left(151 \mathrm{MHz}, \mathrm{CDCl}_{3}\right)$ $\delta=168.0,166.3,143.7,138.5,135.4,132.5,132.4,132.1,130.5,129.8,129.7,128.6,128.4,128.1,127.8,126.9,52.7$, 52.4, 41.5, 28.1. HRMS (ESI - MS): calcd. $382.2013[\mathrm{M}+\mathrm{H}]^{+}$Found: 382.2020. 


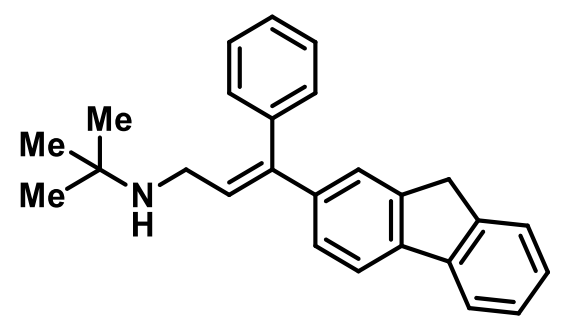

$N$-(tert-Butyl)-3-(9H-fluoren-2-yl)-3-phenylprop-2-en-1-amine (2p): Method D used. Reaction time 14h, purified by toluene:acetone (80:20). Product recovered as yellow oil ( $61.4 \mathrm{mg}, 58 \%$ yield). $\mathrm{R}_{f}=0.27$ (toluene/acetone $=8: 2$ ) and the $\mathrm{Z} / \mathrm{E}$ ratio was determined as 5:1 from the crude NMR. ${ }^{1} \mathrm{H}$ NMR $\left(600 \mathrm{MHz}, \mathrm{CDCl}_{3}\right) \delta 7.76(\mathrm{~d}, J=7.6 \mathrm{~Hz}, 1 \mathrm{H})$, $7.68(\mathrm{~d}, J=8.0 \mathrm{~Hz}, 1 \mathrm{H}), 7.53(\mathrm{~d}, J=7.4 \mathrm{~Hz}, 1 \mathrm{H}), 7.45-7.41(\mathrm{~m}, 3 \mathrm{H}), 7.39-7.36(\mathrm{~m}, 2 \mathrm{H}), 7.32-7.29$ (m, 2H), 7.27 (dd, $J=7.8,0.9 \mathrm{~Hz}, 2 \mathrm{H}), 6.33(\mathrm{t}, J=7.0 \mathrm{~Hz}, 1 \mathrm{H}), 3.84(\mathrm{~s}, 2 \mathrm{H}), 3.34(\mathrm{~d}, J=7.0 \mathrm{~Hz}, 2 \mathrm{H}), 1.11(\mathrm{~s}, 9 \mathrm{H}) .{ }^{13} \mathrm{C}$ NMR $(151$ $\left.\mathrm{MHz}, \mathrm{CDCl}_{3}\right) \delta=143.7,143.3,141.5,141.0,140.9,139.9,129.8,128.5,128.4,128.2,127.6,127.4,127.4,126.9$, 126.9, 126.8, 126.7, 126.4, 125.2, 125.1, 124.2, 120.0, 119.7, 119.6, 51.5, 42.0, 37.0, 28.8. HRMS (ESI - MS): calcd. 354.2216 [M+H] $]^{+}$Found: 354.2211 .

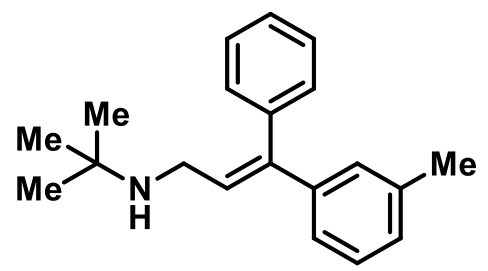

$N$-(tert-Butyl)-3-phenyl-3-(m-tolyl)prop-2-en-1-amine (2q): Method D used. Reaction time 14h, purified by toluene:acetone (90:10). Product recovered as yellow oil (51.9 mg, 62\% yield). $\mathrm{R}_{f}=0.21$ (toluene/acetone $\left.=8: 2\right)$ and the Z/E ratio was determined as 10:1 from the crude NMR. ${ }^{1} \mathrm{H} \mathrm{NMR}\left(600 \mathrm{MHz}, \mathrm{CDCl}_{3}\right) \delta 7.37$ (tt, $J=8.0,1.5 \mathrm{~Hz}$, $2 \mathrm{H}), 7.34-7.30(\mathrm{~m}, 1 \mathrm{H}), 7.20-7.18(\mathrm{~m}, 2 \mathrm{H}), 7.14(\mathrm{t}, J=7.6 \mathrm{~Hz}, 1 \mathrm{H}), 7.08(\mathrm{~s}, 1 \mathrm{H}), 7.06-6.98(\mathrm{~m}, 2 \mathrm{H}), 6.21(\mathrm{t}, J=$ $7.0 \mathrm{~Hz}, 1 \mathrm{H}), 3.27$ (d, J=7.0 Hz, 2H), $2.28(\mathrm{~s}, 3 \mathrm{H}), 1.06(\mathrm{~s}, 9 \mathrm{H}) .{ }^{13} \mathrm{C} \mathrm{NMR}\left(151 \mathrm{MHz}, \mathrm{CDCl}_{3}\right) \delta=143.5,137.7,129.8$, 128.3, 128.2, 128.1, 128.1, 127.3, 124.8, 51.3, 42.0, 28.9, 21.6. HRMS (ESI - MS): calcd. 280.2060 [M+H] ${ }^{+}$Found: 280.2060 .

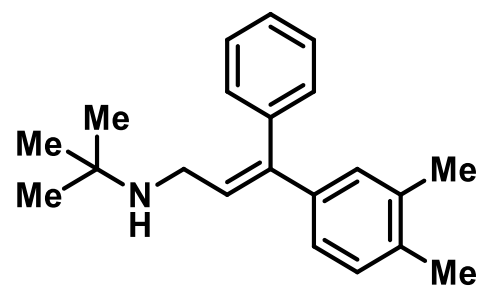

$N$-(tert-Butyl)-3-(3,4-dimethylphenyl)-3-phenylprop-2-en-1-amine (2r): Method D used. Reaction time 14h, purified by toluene:acetone (80:20). Product recovered as yellow oil ( $48.3 \mathrm{mg}, 55 \%$ yield). $\mathrm{R}_{f}=0.21$ (toluene/acetone $=7: 3$ ) and the $\mathrm{Z} / \mathrm{E}$ ratio was determined as $6: 1$ from the crude NMR. ${ }^{1} \mathrm{H}$ NMR (400 MHz, $\left.\mathrm{CDCl}_{3}\right) \delta 7.36$ (dd, $J=7.8$, $1.6 \mathrm{~Hz}, 2 \mathrm{H}), 7.32(\mathrm{~d}, J=6.3 \mathrm{~Hz}, 1 \mathrm{H}), 7.29-7.23(\mathrm{~m}, 1 \mathrm{H}), 7.21-7.16(\mathrm{~m}, 2 \mathrm{H}), 7.08-6.99(\mathrm{~m}, 2 \mathrm{H}), 6.95(\mathrm{~d}, J=7.7$ $\mathrm{Hz}, 1 \mathrm{H}), 6.18(\mathrm{t}, J=7.0 \mathrm{~Hz}, 1 \mathrm{H}) 3.25(\mathrm{dd}, J=6.9,2.0 \mathrm{~Hz}, 2 \mathrm{H}), 2.23$ (s, 3H), $2.20(\mathrm{~s}, 3 \mathrm{H}), 1.05(\mathrm{~s}, 9 \mathrm{H}) .{ }^{13} \mathrm{C}$ NMR $(151$ $\left.\mathrm{MHz}, \mathrm{CDCl}_{3}\right) \delta=143.2,140.0,139.9,136.3,135.8,130.9,129.8,129.5,128.7,128.2,128.1,127.6,127.2,125.0$, 42.0, 29.0, 19.9, 19.6. HRMS (ESI - MS): calcd. $294.2216[\mathrm{M}+\mathrm{H}]^{+}$Found: 294.2221. 


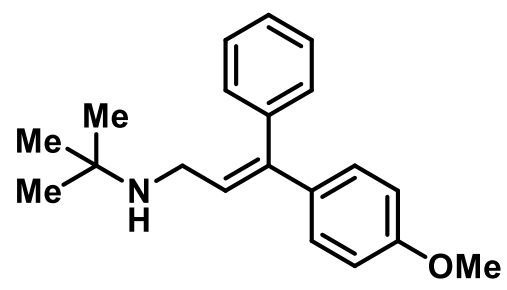

$N$-(tert-Butyl)-3-(4-methoxyphenyl)-3-phenylprop-2-en-1-amine (2s): Method D used. Reaction time 14h, purified by toluene:acetone (80:20). Product recovered as yellow oil ( $42.5 \mathrm{mg}, 48 \%$ yield). $\mathrm{R}_{f}=0.21$ (toluene/acetone $=7: 3$ ) and the $\mathrm{Z} / \mathrm{E}$ ratio was determined as 2:1 from the crude NMR. Notably, product was recovered as a mixture of $\mathrm{Z}$ and E isomers. ${ }^{1} \mathrm{H}$ NMR $\left(600 \mathrm{MHz}, \mathrm{CDCl}_{3}\right) \delta 7.39-7.37(\mathrm{~m}, 2 \mathrm{H}), 7.37-7.31(\mathrm{~m}, 1 \mathrm{H}), 7.28(\mathrm{~d}, J=11.7 \mathrm{~Hz}, 2 \mathrm{H}), 7.22-$ $7.17(\mathrm{~m}, 3 \mathrm{H}), 7.13(\mathrm{~d}, J=8.7 \mathrm{~Hz}, 2 \mathrm{H}), 6.93(\mathrm{~d}, J=8.7 \mathrm{~Hz}, 2 \mathrm{H}), 6.85-6.74(\mathrm{~m}, 3 \mathrm{H}), 6.25-6.11(\mathrm{~m}, 1 \mathrm{H}), 3.86(\mathrm{~s}$, $3 \mathrm{H}), 3.83-3.74(\mathrm{~m}, 5 \mathrm{H}), 3.34(\mathrm{~d}, J=6.9 \mathrm{~Hz}, 2 \mathrm{H}), 3.28(\mathrm{~d}, J=7.0 \mathrm{~Hz}, 3 \mathrm{H}), 1.11(\mathrm{~s}, 9 \mathrm{H}), 1.08(\mathrm{~d}, J=8.9 \mathrm{~Hz}, 15 \mathrm{H})$. ${ }^{13} \mathrm{C}$ NMR $\left(151 \mathrm{MHz}, \mathrm{CDCl}_{3}\right) \delta=159.1,158.9,142.6,139.9,134.8,132.0,131.0,129.8,129.6,128.6,128.6,128.3$, 128.2, 127.8, 127.7, 127.6, 127.3, 127.3, 127.1, 113.7, 113.6, , 55.4, 51.4, 42.0, 41.9, 28.8. HRMS (ESI - MS): calcd. $296.2009[\mathrm{M}+\mathrm{H}]^{+}$Found: 296.2007.

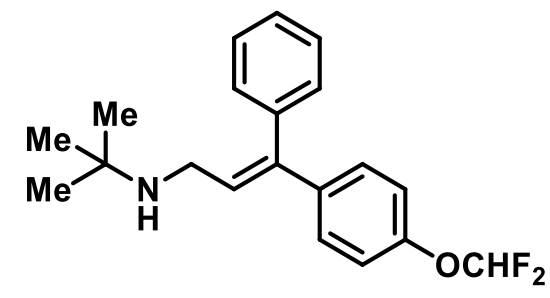

$N$-(tert-Butyl)-3-(4-(difluoromethoxy)phenyl)-3-phenylprop-2-en-1-amine (2t): Method D used. Reaction time $14 \mathrm{~h}$, purified by toluene:acetone (80:20). Product recovered as yellow oil $\left(42.2 \mathrm{mg}, 45 \%\right.$ yield). $\mathrm{R}_{f}=0.25$ (toluene/acetone $=7: 3$ ) and the $\mathrm{Z} / \mathrm{E}$ ratio was determined as 10:1 from the crude NMR. ${ }^{1} \mathrm{H} \mathrm{NMR}\left(400 \mathrm{MHz}, \mathrm{CDCl}_{3}\right)$ $\delta 7.48-7.29(\mathrm{~m}, 3 \mathrm{H}), 7.25-7.21(\mathrm{~m}, 2 \mathrm{H}), 7.20-7.15(\mathrm{~m}, 2 \mathrm{H}), 7.06-6.88(\mathrm{~m}, 2 \mathrm{H}), 6.49(\mathrm{dd}, J=75.5,73.9 \mathrm{~Hz}, 1 \mathrm{H})$, $6.19(\mathrm{t}, J=5.3 \mathrm{~Hz}, 1 \mathrm{H}), 3.26(\mathrm{~d}, J=8.0 \mathrm{~Hz}, 2 \mathrm{H}), 1.06(\mathrm{~s}, 9 \mathrm{H}) .{ }^{13} \mathrm{C} \mathrm{NMR}\left(101 \mathrm{MHz}, \mathrm{CDCl}_{3}\right) \delta=150.5,142.1,139.6$, 139.4, 131.3, 129.7, 128.8, 128.4, 128.3, 127.6, 127.5, 119.2, 119.1, $116.1(\mathrm{t}, J=259 \mathrm{~Hz}), 51.0,42.0,29.0 .{ }^{19} \mathrm{~F}$ NMR $\left(376 \mathrm{MHz}, \mathrm{CDCl}_{3}\right) \delta=-81.0$ (d). HRMS (ESI - MS): calcd. $332.1820[\mathrm{M}+\mathrm{H}]^{+}$Found: 332.1831.

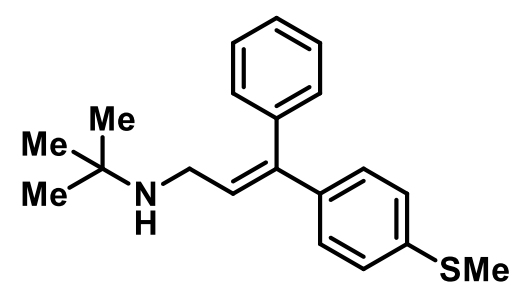

$N$-(tert-Butyl)-3-(4-(methylthio)phenyl)-3-phenylprop-2-en-1-amine (2u): Method D used. Reaction time 14h, purified by toluene:acetone (80:20). Product recovered as yellow oil ( $45.7 \mathrm{mg}, 49 \%$ yield). $\mathrm{R}_{f}=0.25$ (toluene/acetone $=7: 3)$ and the Z/E ratio was determined as 6:1 from the crude NMR. ${ }^{1} \mathrm{H}$ NMR $\left(400 \mathrm{MHz}, \mathrm{CDCl}_{3}\right) \delta 7.45-7.31(\mathrm{~m}$, 3H), $7.30-7.20$ (m, 1H), $7.23-7.06(\mathrm{~m}, 5 \mathrm{H}), 6.19$ (dd, $J=11.7,4.7 \mathrm{~Hz}, 1 \mathrm{H}), 3.24$ (d, $J=6.9 \mathrm{~Hz}, 2 \mathrm{H}), 2.45$ (s, 3H), 
1.04 (s, 9H). ${ }^{13} \mathrm{C}$ NMR (101 MHz, $\left.\mathrm{CDCl}_{3}\right) \delta=142.6,139.5,139.0,137.4,130.3,129.7,128.3,128.2,127.8,127.6$, 127.4, 126.2 126.1 123.5, 51.0, 41.9, 29.0, 15.9. HRMS (ESI - MS): calcd. 312.1780 [M+H] $]^{+}$Found: 312.1786.

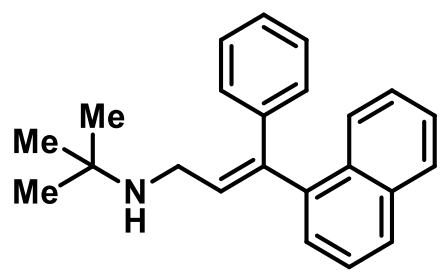

$N$-(tert-Butyl)-3-(naphthalene-1-yl)-3-phenylprop-2-en-1-amine (2v): Method D used. Reaction time 14h, purified by toluene:acetone (80:20). Product recovered as yellow oil $\left(46.3 \mathrm{mg}, 49 \%\right.$ yield). $\mathrm{R}_{f}=0.2$ (toluene/acetone $\left.=8: 2\right)$ and the Z/E ratio was determined as 10:1 from the crude NMR. ${ }^{1} \mathrm{H}$ NMR $\left(600 \mathrm{MHz}, \mathrm{CDCl}_{3}\right) \delta 7.90(\mathrm{~d}, J=8.4 \mathrm{~Hz}$, $1 \mathrm{H}), 7.81(\mathrm{~d}, J=8.1 \mathrm{~Hz}, 1 \mathrm{H}), 7.78(\mathrm{dd}, J=7.1,2.2 \mathrm{~Hz}, 1 \mathrm{H}), 7.43(\mathrm{dd}, J=7.2,5.3 \mathrm{~Hz}, 2 \mathrm{H}), 7.42-7.36(\mathrm{~m}, 1 \mathrm{H}), 7.36$ $-7.31(\mathrm{~m}, 1 \mathrm{H}), 7.31-7.27(\mathrm{~m}, 4 \mathrm{H}), 7.25-7.21(\mathrm{~m}, 1 \mathrm{H}), 5.99(\mathrm{t}, J=6.9 \mathrm{~Hz}, 1 \mathrm{H}), 3.59(\mathrm{~d}, J=6.9 \mathrm{~Hz}, 2 \mathrm{H}), 1.13(\mathrm{~s}$, 9H). ${ }^{13} \mathrm{C}$ NMR $\left(151 \mathrm{MHz}, \mathrm{CDCl}_{3}\right) \delta=142.2,133.9,131.9,129.1,128.3,128.2,127.8,127.5,127.3,126.4,125.9$, 125.6, 125.4, 51.3, 41.8, 29.0. HRMS (ESI - MS): calcd. 316.2060 [M+H] ${ }^{+}$Found: 316.2064.

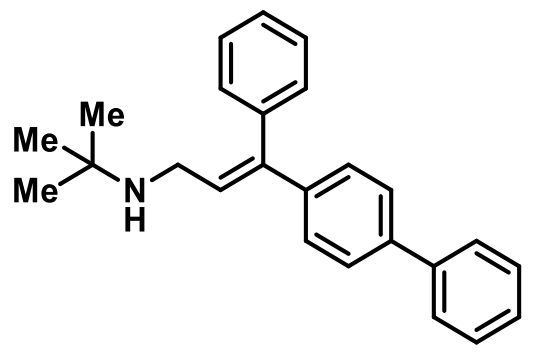

3-([1,1'-Biphenyl]-4-yl)- $N$-(tert-Butyl)-3-phenylprop-2-en-1-amine (2w): Method D used. Reaction time 14h, purified by toluene:acetone (80:20). Product recovered as yellow oil (62.4 mg, 61\% yield). $\mathrm{R}_{f}=0.25$ (toluene/acetone $=7: 3)$ and the $\mathrm{Z} / \mathrm{E}$ ratio was determined as 6:1 from the crude NMR. ${ }^{1} \mathrm{H}$ NMR $\left(400 \mathrm{MHz}, \mathrm{CDCl}_{3}\right) \delta 7.58(\mathrm{~d}, J=8.2$ $\mathrm{Hz}, 2 \mathrm{H}), 7.49(\mathrm{~d}, J=8.1 \mathrm{~Hz}, 2 \mathrm{H}), 7.41(\mathrm{dd}, J=15.6,7.7 \mathrm{~Hz}, 4 \mathrm{H}), 7.33(\mathrm{t}, J=7.3 \mathrm{~Hz}, 4 \mathrm{H}), 7.23(\mathrm{~d}, J=7.8 \mathrm{~Hz}, 2 \mathrm{H})$, $6.30(\mathrm{t}, J=6.9 \mathrm{~Hz}, 1 \mathrm{H}), 3.30(\mathrm{~d}, J=6.9 \mathrm{~Hz}, 2 \mathrm{H}), 1.07(\mathrm{~s}, 9 \mathrm{H}) .{ }^{13} \mathrm{C} \mathrm{NMR}\left(151 \mathrm{MHz}, \mathrm{CDCl}_{3}\right) \delta=143.1,141.0,140.8$, $140.1,139.6,130.3,129.8,129.7,128.9,128.9,128.4,128.3,127.9,127.7,127.5,127.4,127.2,127.1,127.0,126.9$, 51.5, 41.9, 28.7. HRMS (ESI - MS): calcd. 342.2216 [M+H] ${ }^{+}$Found: 342.2224.

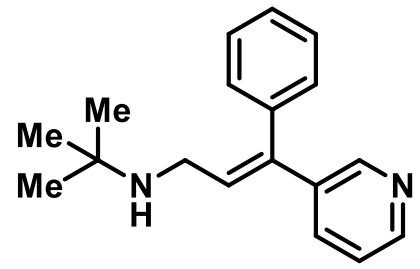

$N$-(tert-Butyl)-3-phenyl-3-(pyridin-3-yl)prop-2-en-1-amine (2x): Method E used, although reaction was performed at $70^{\circ} \mathrm{C}$. Reaction time 14h, purified by EtOAc: $\mathrm{MeOH}(80: 20)$. Product recovered as yellow oil (49.5 mg, $62 \%$ yield). $\mathrm{R}_{f}=0.25(\mathrm{EtOAc} / \mathrm{MeOH}=7: 3)$ and the $\mathrm{Z} / \mathrm{E}$ ratio was determined as 10:1 from the crude NMR. ${ }^{1} \mathrm{H} \mathrm{NMR}(600 \mathrm{MHz}$, $\left.\mathrm{CDCl}_{3}\right) \delta 8.55-8.49(\mathrm{~m}, 1 \mathrm{H}), 8.44(\mathrm{dd}, J=4.8,1.6 \mathrm{~Hz}, 1 \mathrm{H}), 7.51-7.45(\mathrm{~m}, 1 \mathrm{H}), 7.38(\mathrm{ddd}, J=7.6,4.5,1.3 \mathrm{~Hz}, 2 \mathrm{H})$, $7.33(\mathrm{dt}, J=9.6,4.4 \mathrm{~Hz}, 1 \mathrm{H}), 7.18(\mathrm{dd}, J=5.2,3.2 \mathrm{~Hz}, 2 \mathrm{H}), 7.14(\mathrm{ddd}, J=8.0,4.8,0.7 \mathrm{~Hz}, 1 \mathrm{H}), 6.26(\mathrm{t}, J=7.0 \mathrm{~Hz}$, 
$1 \mathrm{H}), 3.31(\mathrm{~d}, J=7.0 \mathrm{~Hz}, 2 \mathrm{H}), 1.06(\mathrm{~s}, 9 \mathrm{H}) .{ }^{13} \mathrm{C} \mathrm{NMR}\left(151 \mathrm{MHz}, \mathrm{CDCl}_{3}\right) \delta=148.6,148.4,140.6,138.4,137.7,134.7$, 129.6, 128.6, 127.8, 123.0, 51.6, 41.8, 28.7. HRMS (ESI - MS): calcd. 267.1856 [M+H] ${ }^{+}$Found: 267.1865.<smiles>CNCC=C(c1ccc(Br)cc1)c1cccnc1</smiles>

3-(-4-Bromophenyl)- $N$-methyl-3-(pyridin-3-yl)prop-2-en-1-amine (2y): Method E used although reaction was performed at $70{ }^{\circ} \mathrm{C}$. Reaction time 14h, purified by EtOAc:MeOH (80:20). Product recovered as yellow oil (69.7 mg, $77 \%$ yield). $\mathrm{R}_{f}=0.25$ (EtOAc/MeOH $=7: 3$ ) and the $\mathrm{Z} / \mathrm{E}$ ratio was determined as 10:1 from the crude NMR. Spectral data was consistent with that in the literature. ${ }^{17}{ }^{1} \mathrm{H}$ NMR $\left(400 \mathrm{MHz}, \mathrm{CDCl}_{3}\right) \delta 8.51(\mathrm{~d}, J=2.0 \mathrm{~Hz}, 1 \mathrm{H}), 8.47(\mathrm{dd}, J=$ 4.8, $1.5 \mathrm{~Hz}, 1 \mathrm{H}), 7.52(\mathrm{~d}, J=8.4 \mathrm{~Hz}, 2 \mathrm{H}), 7.47-7.43(\mathrm{~m}, 1 \mathrm{H}), 7.18(\mathrm{dd}, J=8.0,4.3 \mathrm{~Hz}, 1 \mathrm{H}), 7.03(\mathrm{~d}, J=8.4 \mathrm{~Hz}$, $2 \mathrm{H}), 6.20(\mathrm{t}, J=6.8 \mathrm{~Hz}, 1 \mathrm{H}), 3.29(\mathrm{~d}, J=6.8 \mathrm{~Hz}, 2 \mathrm{H}), 2.41(\mathrm{~s}, 3 \mathrm{H}), 1.56(\mathrm{bs}, 1 \mathrm{H}) .{ }^{13} \mathrm{C} \mathrm{NMR}\left(151 \mathrm{MHz}, \mathrm{CDCl}_{3}\right) \delta=$ 148.7, 148.6, 139.5, 137.4, 137.3, 134.7, 131.8, 131.6, 131.4, 130.0, 129.0, 123.2, 122.0, 50.4, 36.2.<smiles>Cc1ccc(/C(=C/CN[C@H](C)c2cccc3ccccc23)c2cccc(C(F)(F)F)c2)cc1C</smiles>

\section{(S)-3-(3,4-Dimethylphenyl)- $N$-(1-(naphthalen-1-yl)ethyl)-3-(3-(trifluoromethyl)phenyl)prop-2-en-1-amine}

(2z): Method D used. Reaction time 14h, purified by hexanes:EtOAc (50:50). Product recovered as yellow oil (79.9 $\mathrm{mg}, 58 \%$ yield). $\mathrm{R}_{f}=0.25$ (hexanes/EtOAC $=1: 1$ ) and the $\mathrm{Z} / \mathrm{E}$ ratio was determined as 3:1 from the crude NMR. ${ }^{1} \mathrm{H}$ NMR (400 MHz, $\left.\mathrm{CDCl}_{3}\right) \delta 8.18-8.12(\mathrm{~m}, 1 \mathrm{H}), 7.92-7.85(\mathrm{~m}, 1 \mathrm{H}), 7.78-7.71(\mathrm{~m}, 1 \mathrm{H}), 7.63(\mathrm{~d}, J=7.1 \mathrm{~Hz}, 1 \mathrm{H})$, $7.54-7.43(\mathrm{~m}, 5 \mathrm{H}), 7.35-7.30(\mathrm{~m}, 2 \mathrm{H}), 7.08-7.02(\mathrm{~m}, 1 \mathrm{H}), 6.98(\mathrm{~s}, 1 \mathrm{H}), 6.80(\mathrm{~s}, 1 \mathrm{H}), 6.24(\mathrm{t}, J=7.4 \mathrm{~Hz}, 1 \mathrm{H})$, $4.65(\mathrm{dd}, J=6.4,4.3 \mathrm{~Hz}, 1 \mathrm{H}), 3.36(\mathrm{dd}, J=6.9,1.2 \mathrm{~Hz}, 1 \mathrm{H}), 3.26(\mathrm{dd}, J=7.0,4.6 \mathrm{~Hz}, 1 \mathrm{H}), 2.28(\mathrm{~s}, 3 \mathrm{H}), 2.18(\mathrm{~s}, 3 \mathrm{H})$, 1.60 (br s, $1 \mathrm{H}), 1.49$ (dd, $J=6.6,2.4 \mathrm{~Hz}, 3 \mathrm{H}) .{ }^{13} \mathrm{C} \mathrm{NMR}\left(101 \mathrm{MHz}, \mathrm{CDCl}_{3}\right) \delta 143.5,142.4,140.9,140.6,139.2,136.9$, 136.2, 136.2, 136.0, 134.1, 133.2, 131.3, 131.1, 131.0, 130.7, 130.5 (q, $J=33 \mathrm{~Hz}), 129.7,129.6,129.3,129.1,128.6$, 128.0, 127.3, 127.3, 127.2, 125.9, 125.8, 125.7, 125.4, 125.0, 124.4 (q, $J=272 \mathrm{~Hz}), 124.0(\mathrm{q}, J=3.7 \mathrm{~Hz}), 123.8(\mathrm{q}, J$ $=3.6 \mathrm{~Hz}), 123.0,122.8,53.3,46.9,23.7,19.8,19.6 .{ }^{19} \mathrm{~F} \mathrm{NMR}\left(376 \mathrm{MHz}, \mathrm{CDCl}_{3}\right) \delta=-62.8$. HRMS (ESI - MS): calcd. $460.2247[\mathrm{M}+\mathrm{H}]^{+}$Found: 460.2260 .<smiles>C[C@H](NCC=C(c1cccc(C(F)(F)F)c1)c1cccc(C(F)(F)F)c1)c1cccc2ccccc12</smiles> 
(S)-N-(1-(naphthalen-1-yl)ethyl)-3,3-bis(3-(trifluoromethyl)phenyl)prop-2-en-1-amine (2aa): Method D used. Reaction time $14 \mathrm{~h}$, purified by hexanes:EtOAc (60:40). Product recovered as yellow oil (94.3 $\mathrm{mg}, 63 \%$ yield). $\mathrm{R}_{f}=$ 0.3 (hexanes/EtOAC $=1: 1) .{ }^{1} \mathrm{H}$ NMR $\left(600 \mathrm{MHz}, \mathrm{CDCl}_{3}\right) \delta 8.15(\mathrm{~d}, J=7.9 \mathrm{~Hz}, 1 \mathrm{H}), 7.87(\mathrm{dd}, J=7.2,2.3 \mathrm{~Hz}, 1 \mathrm{H})$, $7.74(\mathrm{~d}, J=8.1 \mathrm{~Hz}, 1 \mathrm{H}), 7.59(\mathrm{~d}, J=7.0 \mathrm{~Hz}, 1 \mathrm{H}), 7.57-7.54(\mathrm{~m}, 1 \mathrm{H}), 7.53-7.46(\mathrm{~m}, 4 \mathrm{H}), 7.46-7.41(\mathrm{~m}, 1 \mathrm{H}), 7.39$ $-7.31(\mathrm{~m}, 3 \mathrm{H}), 7.21(\mathrm{~d}, J=7.9 \mathrm{~Hz}, 1 \mathrm{H}), 7.15(\mathrm{~d}, J=7.7 \mathrm{~Hz}, 1 \mathrm{H}), 6.32(\mathrm{t}, J=7.0 \mathrm{~Hz}, 1 \mathrm{H}), 4.63(\mathrm{~d}, J=6.6 \mathrm{~Hz}, 1 \mathrm{H})$, $3.29(\mathrm{dd}, J=7.0,2.4 \mathrm{~Hz}, 2 \mathrm{H}), 1.49(\mathrm{~d}, J=6.6 \mathrm{~Hz}, 3 \mathrm{H}) .{ }^{13} \mathrm{C} \mathrm{NMR}\left(151 \mathrm{MHz}, \mathrm{CDCl}_{3}\right) \delta=142.4,140.9,140.6,139.5$, $134.1,133.2,131.3,131.1,130.9(\mathrm{q}, J=32.1 \mathrm{~Hz}), 130.9(\mathrm{q}, J=32.2 \mathrm{~Hz}), 129.2,128.9,128.9,127.5,126.4(\mathrm{q}, J=4$ $\mathrm{Hz}), 126.0,125.8,125.5,125.7,125.5,124.6(\mathrm{q}, J=4 \mathrm{~Hz}), 124.3(\mathrm{q}, J=3 \mathrm{~Hz}), 124.2(\mathrm{q}, J=272 \mathrm{~Hz}), 124.1(\mathrm{q}, J=$ $272 \mathrm{~Hz}), 123.9$ (q, $J=4 \mathrm{~Hz}), 122.9,122.9,53.6,46.8,23.7 .{ }^{19} \mathrm{~F}$ NMR $\left(376 \mathrm{MHz}, \mathrm{CDCl}_{3}\right) \delta=-62.9$. HRMS (ESI MS): calcd. $500.1807[\mathrm{M}+\mathrm{H}]^{+}$Found: 500.1824. 


\section{Optimization and Synthesis of $\gamma, \gamma$-Diarylated Allylamines}

A. Typical procedure for optimization experiments for the $\gamma$-arylation of terminal allylamines:

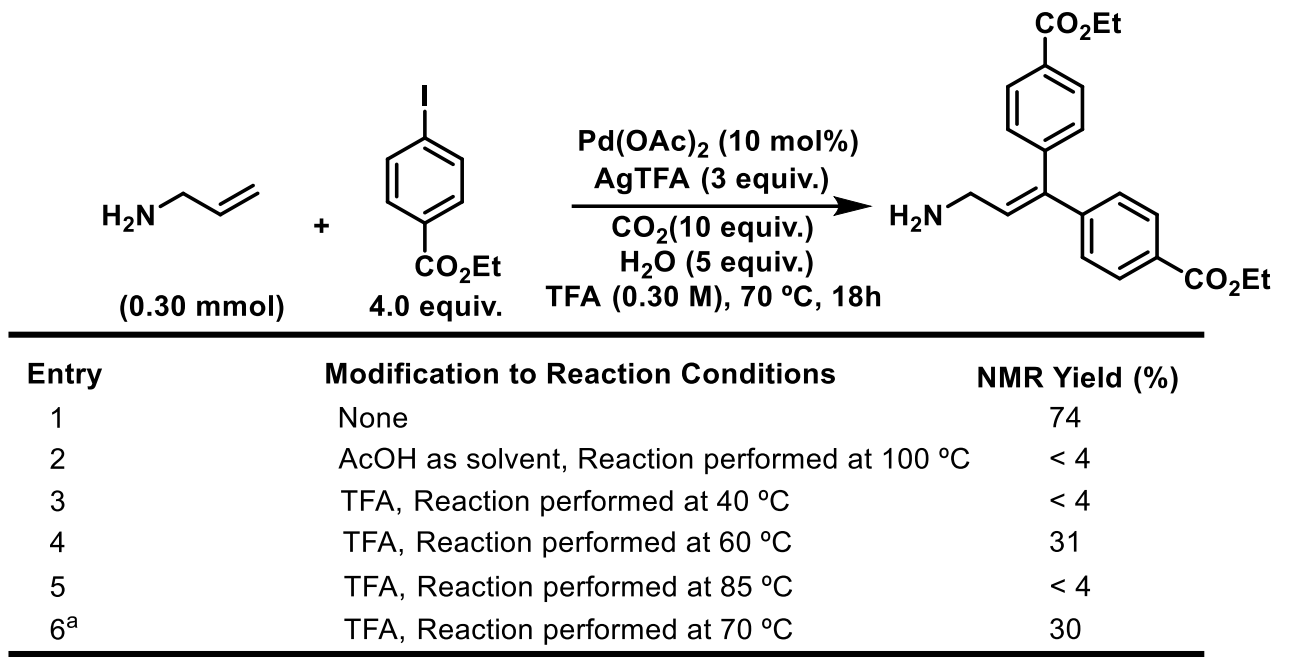

Table $S$-3. Optimization of Diarylation of Terminal Amines. ${ }^{\text {a }} 0.6 \mathrm{mmol}$ of amine were used, remaining amounts left unchanged, reaction also was found to give $30 \%$ yield of mono-arylated product.

Optimization Procedure: A $7.5 \mathrm{~mL}$ vial was charged with Pd catalyst (0.03 mmol, 0.10 equiv), silver salt (0.9 mmol, 3 equiv), organohalide (1.2 mmol, 4 equiv), amine ( $0.30 \mathrm{mmol}, 1$ equiv), solvent (1.0 mL), and water (21.7 $\mu \mathrm{L}, 1.5$ mmol, 5 equiv), followed by the addition of dry ice as $\mathrm{CO}_{2}$ source. The vial was quickly sealed with a PTFE lined cap and stirred at RT for $15 \mathrm{~min}$. After $15 \mathrm{~min}$ of stirring, the reaction mixture was heated in a pie-block at the desired reaction temperature under stirring for $18 \mathrm{~h}$. After cooling to RT, the reaction mixture was concentrated in vacuo. Crude ${ }^{1} \mathrm{H}$ NMR was performed on the reaction mixture using 1,1,2,2-tetrachloroethane as an internal standard.

B. Typical procedure for substrate scope study
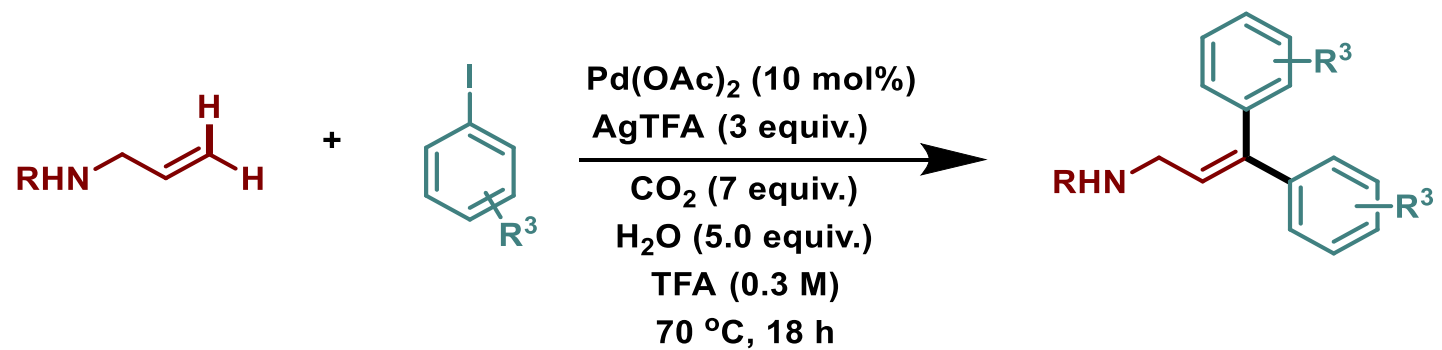

A $7.5 \mathrm{~mL}$ vial was charged with $\mathrm{Pd}(\mathrm{OAc})_{2}(6.7 \mathrm{mg}, 0.03 \mathrm{mmol}, 0.10$ equiv), silver trifluoroacetate $(198.9 \mathrm{mg}, 0.45$ mmol, 3 equiv), organohalide ( $1.2 \mathrm{mmol}, 4$ equiv for primary/secondary allylamine and 2.4 mmol 12 equiv for diallyl amine $)$, amine $(0.30 \mathrm{mmol}, 1.0$ equiv), trifluoroacetic acid $(1.0 \mathrm{~mL})$, and water $(27 \mu \mathrm{L}, 1.5 \mathrm{mmol}, 5.0$ equiv) followed by the addition of dry ice as ( 132 mg, $3.0 \mathrm{mmol}, 10$ equiv) $\mathrm{CO}_{2}$ source. The vial was immediately sealed with a PTFE lined cap and stirred at room temperature for $15 \mathrm{~min}$. After $15 \mathrm{~min}$ of stirring, the reaction mixture was heated in a pie-block at $70{ }^{\circ} \mathrm{C}$ under stirring for $18 \mathrm{~h}$. After cooling to room temperature, the reaction mixture was made basic 
with ammonium hydroxide solution up to $\mathrm{pH}=8$. The reaction mixture was stirred for 15 minutes followed by extraction with dichloromethane $(3 \times 10 \mathrm{~mL})$. The organic layer was washed with brine and dried over $\mathrm{Na}_{2} \mathrm{SO}_{4}$. The filtrate was concentrated in vacuo and further purified by flash column chromatography over silica to give the $\mathrm{C}-\mathrm{H}$ arylation product.

C. Characterization data

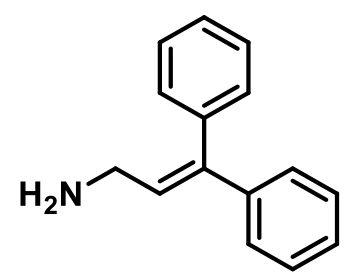

3,3-Diphenylprop-2-en-1-amine (3a): Reaction time 18h, purified by EtOAc:MeOH (90:10). Product recovered as yellow oil (33.2 mg, 53\% yield). $\mathrm{R}_{f}=0.10(\mathrm{EtOAc} / \mathrm{MeOH}=8: 2)$. Spectral data was consistent with that in the literature. ${ }^{18}{ }^{1} \mathrm{H} \mathrm{NMR}\left(600 \mathrm{MHz}, \mathrm{CDCl}_{3}\right) \delta 7.36(\mathrm{t}, J=7.3 \mathrm{~Hz}, 2 \mathrm{H}), 7.34-7.31(\mathrm{~m}, 1 \mathrm{H}), 7.24(\mathrm{~d}, J=2.8 \mathrm{~Hz}, 5 \mathrm{H}), 7.17$ $(\mathrm{d}, J=7.1 \mathrm{~Hz}, 2 \mathrm{H}), 6.18(\mathrm{t}, J=7.0 \mathrm{~Hz}, 1 \mathrm{H}), 5.05(\mathrm{~s}, 2 \mathrm{H}), 3.47(\mathrm{~d}, J=7.0 \mathrm{~Hz}, 2 \mathrm{H}) .{ }^{13} \mathrm{C}$ NMR $\left(151 \mathrm{MHz}, \mathrm{CDCl}_{3}\right) \delta=$ 145.0, 141.6, 139.0, 129.8, 128.5, 128.3, 127.8, 127.7, 127.7, 125.3, 77.4, 76.9, 40.3.

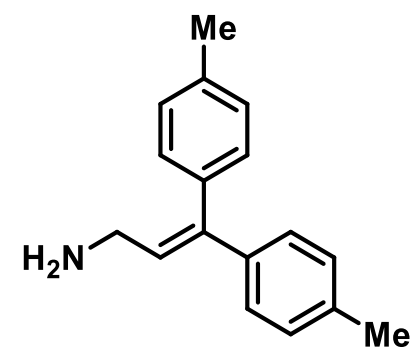

3,3-Di-p-tolylprop-2-en-1-amine (3b): Reaction time 18h, purified by EtOAc:MeOH (90:10). Product recovered as yellow oil (39.13 mg, 55\% yield). $\mathrm{R}_{f}=0.15(\mathrm{EtOAc} / \mathrm{MeOH}=8: 2) .{ }^{1} \mathrm{H} \mathrm{NMR}\left(600 \mathrm{MHz}, \mathrm{CDCl}_{3}\right) \delta 7.14(\mathrm{~d}, J=7.8 \mathrm{~Hz}$, 2H), $7.08(\mathrm{~d}, J=8.1 \mathrm{~Hz}, 2 \mathrm{H}), 6.91(\mathrm{~s}, 2 \mathrm{H}), 6.87(\mathrm{~d}, J=8.2 \mathrm{~Hz}, 2 \mathrm{H}), 6.50(\mathrm{~s}, 1 \mathrm{H}), 3.63(\mathrm{~d}, J=1.3 \mathrm{~Hz}, 2 \mathrm{H}), 2.36(\mathrm{~s}$, 3H), 2.24 (s,3H), 1.59 (bs, 2H). ${ }^{13} \mathrm{C}$ NMR (151 MHz, $\left.\mathrm{CDCl}_{3}\right) \delta=143.2,137.0,136.8,136.3,134.2,129.6,129.1$, 128.8, 128.7, 125.2, 50.9, 21.4, 21.3. HRMS (ESI - MS): calcd. 238.1591 [M+H] $]^{+}$Found: 238.1585.<smiles>NCC=C(c1cccc(C(F)(F)F)c1)c1cccc(C(F)(F)F)c1</smiles>

3,3-bis(3-(Trifluoromethyl)phenyl)prop-2-en-1-amine (3c): Reaction time 18h, purified by EtOAc:MeOH (90:10). Product recovered as yellow oil (79.7 mg, 75\% yield). $\mathrm{R}_{f}=0.20(\mathrm{EtOAc} / \mathrm{MeOH}=8: 2) .{ }^{1} \mathrm{H} \mathrm{NMR}\left(600 \mathrm{MHz}, \mathrm{CDCl}_{3}\right)$ $\delta 7.63(\mathrm{~d}, J=7.5 \mathrm{~Hz}, 1 \mathrm{H}), 7.54-7.52(\mathrm{~m}, 2 \mathrm{H}), 7.40(\mathrm{dd}, J=13.9,4.7 \mathrm{~Hz}, 2 \mathrm{H}), 7.35(\mathrm{~d}, J=7.5 \mathrm{~Hz}, 1 \mathrm{H}), 7.31(\mathrm{~d}, J=$ $7.7 \mathrm{~Hz}, 1 \mathrm{H}), 7.27-7.26(\mathrm{~m}, 1 \mathrm{H}), 6.26(\mathrm{t}, J=6.9 \mathrm{~Hz}, 1 \mathrm{H}), 3.39(\mathrm{~d}, J=6.9 \mathrm{~Hz}, 2 \mathrm{H}), 1.76(\mathrm{~s}, 2 \mathrm{H}) .{ }^{13} \mathrm{C}$ NMR $(151 \mathrm{MHz}$, $\left.\mathrm{CDCl}_{3}\right) \delta=142.3,140.2,139.5,133.2,132.8,131.1(\mathrm{q}, J=32.5 \mathrm{~Hz}), 131.0(\mathrm{q}, J=32 \mathrm{~Hz}), 130.9,129.2,129.0,126.5$ 
$(\mathrm{q}, J=3 \mathrm{~Hz}), 124.8(\mathrm{q}, J=3 \mathrm{~Hz}), 124.5,(\mathrm{q}, J=3 \mathrm{~Hz}), 124.2(\mathrm{q}, J=273), 124.1(\mathrm{q}, J=272 \mathrm{~Hz}), 124.0,(\mathrm{q}, J=4 \mathrm{~Hz})$, 41.1. ${ }^{19} \mathrm{~F}$ NMR (376 MHz, $\left.\mathrm{CDCl}_{3}\right) \delta=-63.2$ (m). HRMS (ESI - MS): calcd. 344.0868 [M-H] $]^{+}$Found: 344.0879.

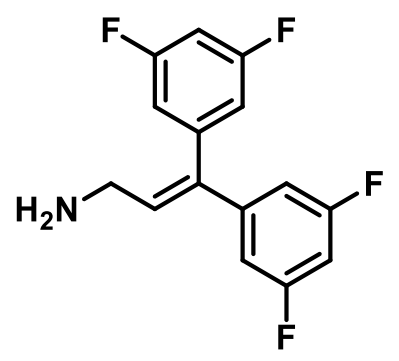

3,3-bis(3,5-Difluorophenyl)prop-2-en-1-amine (3d): Reaction time 18h, purified by EtOAc:MeOH (90:10). Product recovered as yellow oil $(49.7 \mathrm{mg}, 59 \%$ yield $) . \mathrm{R}_{f}=0.22(\mathrm{EtOAc} / \mathrm{MeOH}=8: 2) .{ }^{1} \mathrm{H} \mathrm{NMR}\left(600 \mathrm{MHz} \mathrm{CDCl}_{3}\right) \delta 6.87-$ $6.78(\mathrm{~m}, 1 \mathrm{H}), 6.71(\mathrm{dd}, J=6.8,4.6 \mathrm{~Hz}, 3 \mathrm{H}), 6.68(\mathrm{dd}, J=7.9,2.2 \mathrm{~Hz}, 2 \mathrm{H}), 6.21(\mathrm{t}, J=6.9 \mathrm{~Hz}, 1 \mathrm{H}), 3.35(\mathrm{~d}, J=6.9$ $\mathrm{Hz}, 2 \mathrm{H}), 1.39$ (s, 2H). ${ }^{13} \mathrm{C} \mathrm{NMR}\left(151 \mathrm{MHz}, \mathrm{CDCl}_{3}\right) \delta 163.9(\mathrm{dd}, J=12.9,10.6 \mathrm{~Hz}), 162.3(\mathrm{dd}, J=13.0,8.8 \mathrm{~Hz}), 114.2$ $(\mathrm{t}, J=9.2 \mathrm{~Hz}), 141.6(\mathrm{t}, J=9.3 \mathrm{~Hz}), 138.4(\mathrm{~m}), 133.6,112.8(\mathrm{dd}, J=19.8,5.3 \mathrm{~Hz}), 110.2(20.6,5.3 \mathrm{~Hz}), 103.6(\mathrm{t}, J=$ $25.2 \mathrm{~Hz}), 103.1(\mathrm{t}, J=25.6 \mathrm{~Hz}), 41.1 .{ }^{19} \mathrm{~F} \mathrm{NMR}\left(376 \mathrm{MHz}, \mathrm{CDCl}_{3}\right) \delta=-109.2(\mathrm{~m})$. HRMS (ESI - MS): calcd. 282.0900 $[\mathrm{M}+\mathrm{H}]^{+}$Found: 282.0903 .<smiles>CC(C)(COC(=O)c1cccc(I)c1)COC(=O)c1cccc(C(=O)OCC(C)(C)COC(=O)c2cccc(I)c2)c1</smiles>

Bis(3-((3-Iodobenzoyl)oxy)-2,2-dimethylpropyl) 3,3'-(3-aminoprop-1-ene (3e): After 14h and cooling to room temperature, the reaction mixture was concentrated in vacuo. Aq. $\mathrm{HCl}(10 \mathrm{~mL}, 1.2 \mathrm{M})$ was added to the residual reaction mixture and stirred for $15 \mathrm{~min}$. The mixture was extracted with diethyl ether $(8.0 \mathrm{~mL})$ and the organic layer was discarded. The aqueous layer was made basic with ammonium hydroxide solution up to $\mathrm{pH}=8$, followed by extraction with dichloromethane $(2 \times 10 \mathrm{~mL})$. The organic layer was washed with water $(5.0 \mathrm{~mL})$, followed by brine, and dried over $\mathrm{Na}_{2} \mathrm{SO}_{4}$.afford the pure product as a yellow oil (119.8 mg, 43\% yield). ${ }^{1} \mathrm{H} \mathrm{NMR}\left(400 \mathrm{MHz}, \mathrm{CDCl}_{3}\right) \delta$ $8.40-8.29(\mathrm{~m}, 2 \mathrm{H}), 7.97(\mathrm{~m}, 4 \mathrm{H}), 7.90-7.84(\mathrm{~m}, 4 \mathrm{H}), 7.47-7.39(\mathrm{~m}, 2 \mathrm{H}), 7.34-7.30(\mathrm{~m}, 2 \mathrm{H}), 7.16(\mathrm{td}, J=7.8$, $3.4 \mathrm{~Hz}, 2 \mathrm{H}), 6.24(\mathrm{t}, J=6.8 \mathrm{~Hz}, 1 \mathrm{H}), 4.24-4.17(\mathrm{~m}, 8 \mathrm{H}), 3.35(\mathrm{~d}, J=6.3 \mathrm{~Hz}, 2 \mathrm{H}), 1.17-1.09(\mathrm{~m}, 12 \mathrm{H}) .{ }^{13} \mathrm{C} \mathrm{NMR}$ $\left(151 \mathrm{MHz} \mathrm{CDCl}_{3}\right) \delta 166.3,166.2,142.0,140.25,139.4,138.5,134.5,132.3,132.1,132.0,131.9,130.9,130.2,128.8$, 128.5, 94.0, 70.2, 69.9, 69.8, 41.2, 35.4, 22.1. HRMS (ESI - MS): calcd. 930.0994 [M+H] ${ }^{+}$Found: 930.0982. 


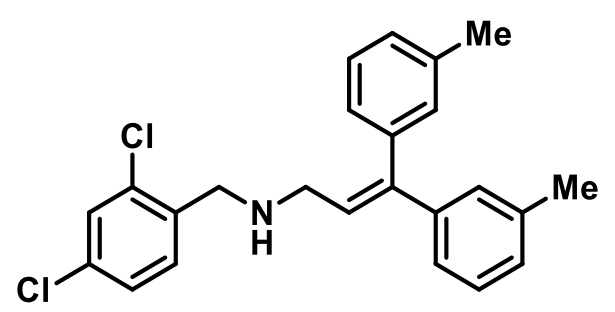

$N$-(2,4-Dichlorobenzyl)3,3,-di-m-tolylprop-2-en-1-amine (3f): Reaction time 14h, purified by Hexane:EtOAc (80:20). Product recovered as a white solid (73.7 mg, 62\% yield). $\mathrm{R}_{f}=0.7$ (Hexane:EtOAc (80:20). ${ }^{1} \mathrm{H}$ NMR (600 $\left.\mathrm{MHz}, \mathrm{CDCl}_{3}\right) \delta 7.34(\mathrm{~d}, J=2.1 \mathrm{~Hz}, 1 \mathrm{H}), 7.23-7.08(\mathrm{~m}, 10 \mathrm{H}), 5.92(\mathrm{t}, J=6.9 \mathrm{~Hz}, 1 \mathrm{H}), 3.79(\mathrm{~s}, 2 \mathrm{H}), 3.26(\mathrm{~d}, J=6.9$ $\mathrm{Hz}, 2 \mathrm{H}), 2.27$ (s, 3H), 2.09 (s, 3H), 1.52 (br s, 1H). ${ }^{13} \mathrm{C} \mathrm{NMR}\left(151 \mathrm{MHz}, \mathrm{CDCl}_{3}\right) \delta 143.0,142.0,139.5,136.3,136.3$, 135.6, 134.3, 133.8, 131.3, 130.9, 130.9, 130.4, 130.4, 130.0, 129.3, 127.4, 127.1, 125.7, 125.4, 50.2, 47.8, 21.2, 20.2. HRMS (ESI - MS): calcd. $396.1280[\mathrm{M}+\mathrm{H}]^{+}$Found: 396.1290.

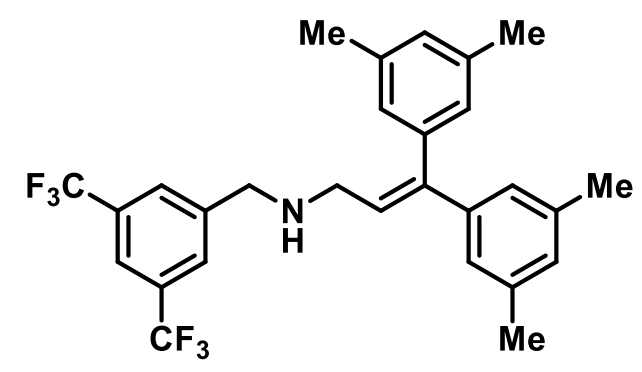

$N$-(3,5-bis(Trifluoromethyl)benzyl)-3,3-bis(3,5-dimethylphenyl)prop-2-en-1-amine (3g): Reaction time 14h, purified by Hexane:EtOAc (80:20). Product recovered as a yellow oil (75.2 mg, $51 \%$ yield). $R_{f}=0.75$ (Hexane:EtOAc (80:20). ${ }^{1} \mathrm{H}$ NMR (600 MHz, CDCl $) \delta 7.78$ (s, 2H), $7.76(\mathrm{~s}, 1 \mathrm{H}), 6.95(\mathrm{t}, J=0.5 \mathrm{~Hz}, 1 \mathrm{H}), 6.85(\mathrm{~s}, 2 \mathrm{H}), 6.76(\mathrm{~s}, 1 \mathrm{H})$, $6.62(\mathrm{~s}, 2 \mathrm{H}), 6.43(\mathrm{~s}, 1 \mathrm{H}), 3.91(\mathrm{~s}, 2 \mathrm{H}), 3.60(\mathrm{~d}, J=1.0 \mathrm{~Hz}), 2.28(\mathrm{~s}, 6 \mathrm{H}), 2.13(\mathrm{~s}, 6 \mathrm{H}) .{ }^{13} \mathrm{C} \mathrm{NMR}\left(151 \mathrm{MHz}, \mathrm{CDCl}_{3}\right)$ $\delta 143.2,139.8,139.4,138.4,137.4,136.4,131.6$ (q, $J=33 \mathrm{~Hz}), 129.2,128.6,128.5$ (q, $2.8 \mathrm{~Hz}), 128.2,127.2,126.2$, 123.6 (q, $J=272 \mathrm{~Hz}), 121.0$ (sept, $J=3.9 \mathrm{~Hz}), 57.4,51.3,21.4 .{ }^{19} \mathrm{~F} \mathrm{NMR}\left(376 \mathrm{MHz}, \mathrm{CDCl}_{3}\right) \delta=-61.1$. HRMS (ESI - MS): calcd. $492.2120[\mathrm{M}+\mathrm{H}]^{+}$Found: 492.2132.<smiles>C(CNCC=C(c1ccccc1)c1ccccc1)=C(c1ccccc1)c1ccccc1</smiles>

bis(3,3-Diphenylallyl)amine (3h): Reaction used 12.0 equiv of the aryl halide and 4.0 equiv of AgTFA. Reaction time 18h, purified by EtOAc:MeOH (90:10). Product recovered as yellow oil (50.5 mg, $42 \%$ yield). $\mathrm{R}_{f}=0.3$ $(\mathrm{EtOAc} / \mathrm{MeOH}=8: 2) .{ }^{1} \mathrm{H}$ NMR $\left(600 \mathrm{MHz}, \mathrm{CDCl}_{3}\right) \delta 7.38-7.30(\mathrm{~m}, 6 \mathrm{H}), 7.26(\mathrm{~m}, 6 \mathrm{H}), 7.22-7.17(\mathrm{~m}, 4 \mathrm{H}), 7.16-$ $7.11(\mathrm{~m}, 4 \mathrm{H}), 6.15(\mathrm{t}, J=6.9 \mathrm{~Hz}, 2 \mathrm{H}), 3.32(\mathrm{~d}, J=6.9 \mathrm{~Hz}, 4 \mathrm{H}), 2.23(\mathrm{br} \mathrm{s}, 1 \mathrm{H}) .{ }^{13} \mathrm{C} \mathrm{NMR}\left(151 \mathrm{MHz}, \mathrm{CDCl}_{3}\right) \delta=$ 144.0, 142.1, 139.6, 129.8, 128.3, 128.2, 127.5, 127.4, 127.4, 127.0, 48.0. HRMS (ESI - MS): calcd. 402.2216 [M+H] ${ }^{+}$ Found: 402.2208 . 


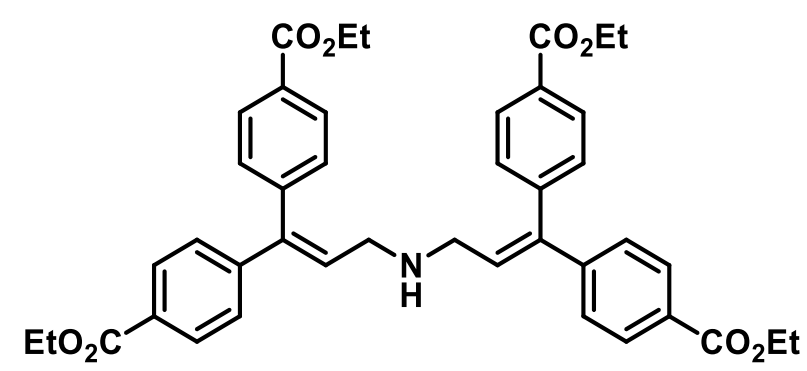

Tetraethyl 4,4',4',4"',-(azanediylbis(prop-1-en-3,1,1,-triyl))tetrabenzoate (3i): Reaction used 12.0 equiv of the aryl halide and 4.0 equiv of AgTFA. Reaction time 18h, purified by EtOAc:MeOH (90:10). Product recovered as yellow oil (78.5 mg, 38\% yield). $\mathrm{R}_{f}=0.20(\mathrm{EtOAc} / \mathrm{MeOH}=8: 2) .{ }^{1} \mathrm{H} \mathrm{NMR}\left(600 \mathrm{MHz}, \mathrm{CDCl}_{3}\right) \delta 8.11-7.99(\mathrm{~m}, 2 \mathrm{H})$, $7.96-7.89(\mathrm{~m}, 2 \mathrm{H}), 7.21-7.17(\mathrm{~m}, 4 \mathrm{H}), 6.23(\mathrm{t}, J=6.8 \mathrm{~Hz}, 1 \mathrm{H}), 4.39(\mathrm{q}, J=7.1 \mathrm{~Hz}, 2 \mathrm{H}), 4.35(\mathrm{q}, J=7.1 \mathrm{~Hz}, 2 \mathrm{H})$, $3.28(\mathrm{~d}, J=6.8 \mathrm{~Hz}, 2 \mathrm{H}), 1.40(\mathrm{t}, J=7.2 \mathrm{~Hz}, 3 \mathrm{H}), 1.37(\mathrm{t}, J=7.1 \mathrm{~Hz}, 3 \mathrm{H}) .{ }^{13} \mathrm{C} \mathrm{NMR}\left(151 \mathrm{MHz}, \mathrm{CDCl}_{3}\right) \delta=166.4$, 166.3, 145.6, 143.6, 142.3, 137.7, 131.1, 130.1, 129.9, 129.8, 129.7, 129.6, 129.6, 127.3, 77.4, 76.9, 61.2, 61.1, 48.1, 14.4. HRMS (ESI - MS): calcd. $690.3061[\mathrm{M}+\mathrm{H}]^{+}$Found: 690.3024.

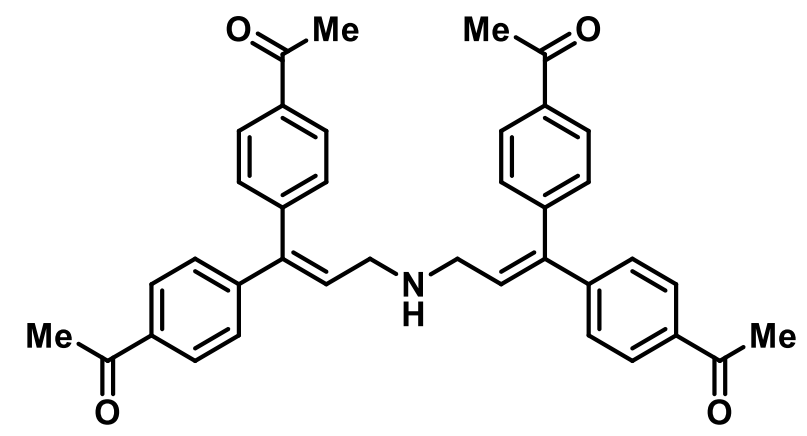

1,1',1",1','-(Azanediylbis(prop-1-en-3,1,1,-triyl))tetrakis(benzene-4,1-diyl))tetrakis(ethan-1-one)

(3j):

Reaction used 12.0 equiv of the aryl halide and 4.0 equiv of AgTFA. Reaction time 18h, purified by EtOAc:MeOH (90:10). Product recovered as yellow oil (68.3 mg, 40\% yield). $\mathrm{R}_{f}=0.27(\mathrm{EtOAc} / \mathrm{MeOH}=8: 2) .{ }^{1} \mathrm{H} \mathrm{NMR}(400 \mathrm{MHz}$, $\left.\mathrm{CDCl}_{3}\right) \delta 7.95(\mathrm{~d}, J=8.1 \mathrm{~Hz}, 4 \mathrm{H}), 7.84(\mathrm{~d}, J=8.3 \mathrm{~Hz}, 4 \mathrm{H}), 7.20(\mathrm{dd}, J=8.0,6.3 \mathrm{~Hz}, 8 \mathrm{H}), 6.27(\mathrm{t}, J=6.9 \mathrm{~Hz}, 2 \mathrm{H})$, $3.34(\mathrm{~d}, J=6.8 \mathrm{~Hz}, 4 \mathrm{H}), 2.61(\mathrm{~s}, 6 \mathrm{H}), 2.57(\mathrm{~s}, 6 \mathrm{H}) .{ }^{13} \mathrm{C} \mathrm{NMR}\left(151 \mathrm{MHz}, \mathrm{CDCl}_{3}\right) \delta=197.7,197.7,145.5,143.6,142.9$, 136.6, 136.4, 130.0, 129.0, 128.7, 128.6, 127.6, 77.4, 76.9, 47.7, 26.8, 26.8. LRMS (ESI - MS): calcd. 570.3 [M+H] Found: 570.3 . 


\section{Directing Group Experiments}

A. Summary of Results for Other Amine Substrates

a) Other Directing Groups for Regioselective $\gamma$-Arylation<smiles>CCN(CC)CC=C(c1ccccc1)c1ccccc1</smiles>

b) Directing Group for $\beta, \gamma$-Diarylation<smiles>O=C1c2ccccc2C(=O)N1CC(=C(c1ccccc1)c1ccccc1)c1ccccc1</smiles>

c) Unsuccessful Directing Groups for $\gamma$-Arylation (Due to Substrate Decomposition)<smiles>CC[N+](CC)(CC)C/C=C/c1ccccc1</smiles>

B. Synthesis and Experiments with an $N$-Boc Cinnamylamine Substrate<smiles>COC(=O)N(C/C=C/c1ccccc1)C(C)(C)C</smiles>

Methyl tert-butyl(cinnamyl)carbamate (4): $N$-tert-Butylcinnamylamine (830 mg, $4.38 \mathrm{mmol}$ ) was dissolved in anhydrous $\mathrm{MeCN}(10 \mathrm{~mL})$ along with potassium carbonate $(1.21 \mathrm{~g}, 8.76 \mathrm{mmol})$, followed by slow addition of methyl chloroformate $(510 \mu \mathrm{L}, 6.58 \mathrm{mmol})$, leading to a significant exotherm. Reaction was stirred at room temperature for $24 \mathrm{~h}$, followed by pouring into $150 \mathrm{~mL}$ of deionized $\mathrm{H}_{2} \mathrm{O}$, followed by extraction with dichloromethane $(3 \mathrm{x} 15 \mathrm{~mL})$. The combined organic phase was dried over $\mathrm{Na}_{2} \mathrm{SO}_{4}$, followed by evaporation in vacuo to give the desired product as a yellow oil $(863 \mathrm{mg}, 80 \%) . \mathrm{R}_{f}=0.2($ Hexanes/EtOAc $=9: 1) .{ }^{1} \mathrm{H} \mathrm{NMR}\left(600 \mathrm{MHz}, \mathrm{CDCl}_{3}\right) \delta 7.36(\mathrm{~d}, J=7.6 \mathrm{~Hz}, 2 \mathrm{H})$, $7.31(\mathrm{t}, J=7.6 \mathrm{~Hz}, 2 \mathrm{H}), 7.23(\mathrm{t}, J=7.2 \mathrm{~Hz}, 1 \mathrm{H}), 6.44(\mathrm{~d}, J=16.0 \mathrm{~Hz}, 1 \mathrm{H}), 6.19$ (dt, $J=16.0,5.7 \mathrm{~Hz}, 1 \mathrm{H}), 4.13(\mathrm{~d}, J$ $=5.6 \mathrm{~Hz}, 2 \mathrm{H}), 3.70(\mathrm{~s}, 3 \mathrm{H}), 1.44(\mathrm{~s}, 9 \mathrm{H}) .{ }^{13} \mathrm{C} \mathrm{NMR}\left(151 \mathrm{MHz}, \mathrm{CDCl}_{3}\right) \delta 156.8,137.1,130.5,128.7,128.5,127.5$, 126.4, 56.3, 52.1, 46.9, 29.4. HRMS (ESI - MS): calcd. $248.1645[\mathrm{M}+\mathrm{H}]^{+}$Found: 248.1650. 

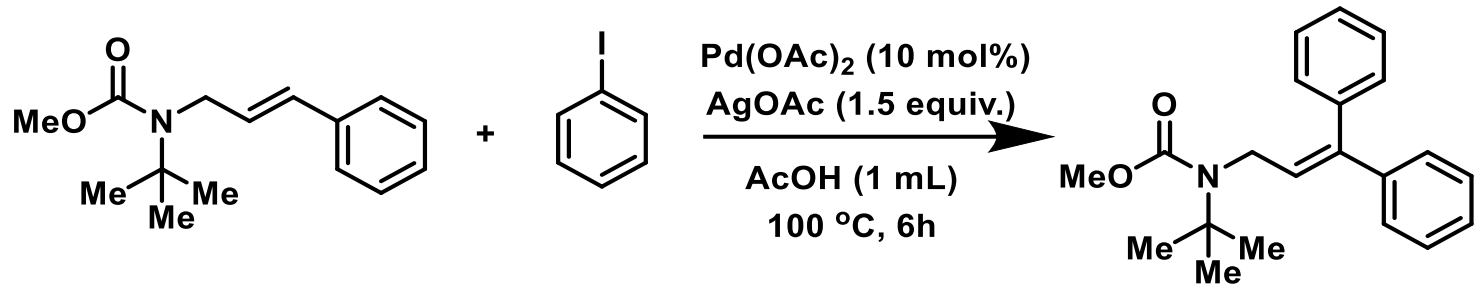

Methyl tert-butyl(3,3-diphenylallyl)carbamate (5): A $7.5 \mathrm{~mL}$ vial was charged with $\mathrm{Pd}(\mathrm{OAc})_{2}(6.7 \mathrm{mg}, 0.03 \mathrm{mmol}$, 0.10 equiv), silver trifluoroacetate ( $99.9 \mathrm{mg}, 0.45 \mathrm{mmol}, 1.5$ equiv), phenyl iodide ( $122.5 \mathrm{mg}, 0.60 \mathrm{mmol}, 2.0$ equiv), carbamate $(74.2 \mathrm{mg}, 0.30 \mathrm{mmol}, 1.0$ equiv), acetic acid $(1.0 \mathrm{~mL})$, followed by sealing with a PTFE lined cap and stirring at room temperature for $15 \mathrm{~min}$. After $15 \mathrm{~min}$ of stirring, the reaction vial was heated in a pie-block at $100{ }^{\circ} \mathrm{C}$ under stirring for $6 \mathrm{~h}$. After cooling to room temperature, the reaction mixture was poured into $1.2 \mathrm{M} \mathrm{HCl}_{(\mathrm{aq})}(75 \mathrm{~mL})$, followed by extraction with dichloromethane $(3 \times 10 \mathrm{~mL})$. The combined organic phase was dried over $\mathrm{Na}_{2} \mathrm{SO}_{4}$, followed by evaporating in vacuo. The resulting oil was columned over silica (10\% EtOAc/hexanes) to give the desired product as a yellow oil $(38.7 \mathrm{mg}, 40 \%)$. Notably, increasing the reaction time led to a decrease in the isolated yield. $\mathrm{R}_{f}=0.2(\mathrm{Hexanes} / \mathrm{EtOAc}=9: 1) . \operatorname{NMR}\left(600 \mathrm{MHz}, \mathrm{CDCl}_{3}\right) \delta 7.40(\mathrm{dd}, J=7.9,6.6 \mathrm{~Hz}, 2 \mathrm{H}), 7.35(\mathrm{t}, J=7.4 \mathrm{~Hz}, 1 \mathrm{H})$, 7.29 (dt, $J=7.4,5.0 \mathrm{~Hz}, 3 \mathrm{H}), 7.23(\mathrm{dd}, J=8.2,1.4 \mathrm{~Hz}, 2 \mathrm{H}), 7.19-7.15(\mathrm{~m}, 2 \mathrm{H}), 6.02(\mathrm{t}, J=6.0 \mathrm{~Hz}, 1 \mathrm{H}), 4.06(\mathrm{~d}, J$ $=6.0 \mathrm{~Hz}, 2 \mathrm{H}), 3.68(\mathrm{~s}, 3 \mathrm{H}), 1.32(\mathrm{~s}, 9 \mathrm{H}) .{ }^{13} \mathrm{C} \mathrm{NMR}\left(151 \mathrm{MHz}, \mathrm{CDCl}_{3}\right) \delta 156.7,142.1,141.7,139.3,129.9,128.9$, 128.3, 127.60, 127.4, 56.1, 52.1, 44.5, 29.4. HRMS (ESI - MS): calcd. 346.1778 [M+H]+ Found: 346.1790.

C. Synthesis and Experiments with an $N$-Cinnamylphthalimide Substrate

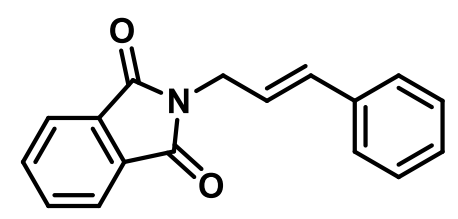

2-Cinnamylisoindoline-1,3-dione (6): To a solution of phtalimide (373 mg, $2.5 \mathrm{mmol}$ ) in DMF (4 mL) were added potassium carbonate $(350 \mathrm{mg}, 2.5 \mathrm{mmol}$ ) and cinnamyl bromide $(500 \mathrm{mg}, 8.82 \mathrm{~mL}, 2.5 \mathrm{mmol})$. The reaction was stirred at $50{ }^{\circ} \mathrm{C}$ for $18 \mathrm{~h}$. After cooling to room temperature, the reaction mixture was poured into $10 \mathrm{~mL}$ of deionized water, followed by extraction with dichloromethane $(4 \times 15 \mathrm{~mL})$. The combined organic phase was dried over $\mathrm{Na}_{2} \mathrm{SO}_{4}$, followed by evaporation in vасио to give the desired product as a yellow oil that solidified upon standing (519 $\mathrm{mg}$, $79 \%$ ), and matched spectra reported in the literature. ${ }^{19} \mathrm{R}_{f}=0.2($ Hexanes/EtOAc $=8: 2) .{ }^{1} \mathrm{H} \mathrm{NMR}\left(600 \mathrm{MHz}, \mathrm{CDCl}_{3}\right)$ $\delta 7.87(\mathrm{dd}, J=5.3,3.1 \mathrm{~Hz}, 2 \mathrm{H}), 7.72(\mathrm{dd}, J=5.4,3.0 \mathrm{~Hz}, 2 \mathrm{H}), 7.35(\mathrm{~d}, J=7.5 \mathrm{~Hz}, 2 \mathrm{H}), 7.28(\mathrm{t}, J=7.6 \mathrm{~Hz}, 2 \mathrm{H}), 7.22$ $(\mathrm{t}, J=7.3 \mathrm{~Hz}, 1 \mathrm{H}), 6.66(\mathrm{~d}, J=15.8 \mathrm{~Hz}, 1 \mathrm{H}), 6.26(\mathrm{dt}, J=15.8 \mathrm{~Hz}, 1 \mathrm{H}), 4.45(\mathrm{~d}, J=6.5 \mathrm{~Hz}, 2 \mathrm{H}) .{ }^{13} \mathrm{C}$ NMR $(151$ $\left.\mathrm{MHz}, \mathrm{CDCl}_{3}\right) \delta 168.13,136.4,134.1,133.9,132.3,128.7,128.0,126.7,123.5,122.8,39.8$. 

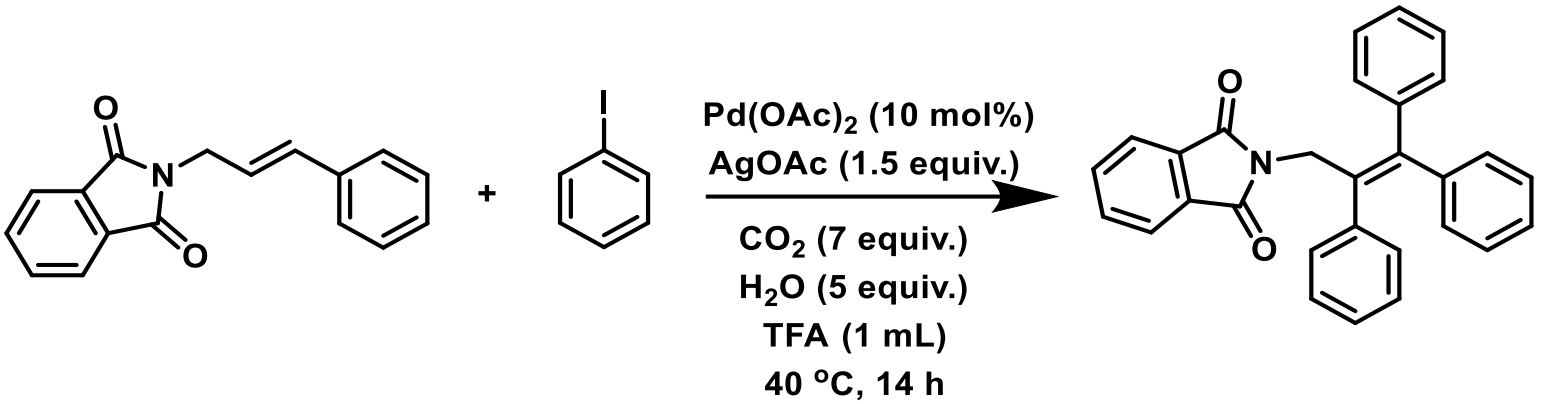

$40^{\circ} \mathrm{C}, 14 \mathrm{~h}$

2-(2,3,3-Triphenylallyl)isoindoline-1,3-dione (7): A $7.5 \mathrm{~mL}$ vial was charged with $\mathrm{Pd}(\mathrm{OAc})_{2}(6.7 \mathrm{mg}, 0.03 \mathrm{mmol}$, 0.10 equiv), silver acetate $(75.1 \mathrm{mg}, 0.45 \mathrm{mmol}, 1.5$ equiv), phenyl iodide (122.5 mg, $0.60 \mathrm{mmol}, 2.0$ equiv), cinnamylisoindoline-1,3-dione ( $0.30 \mathrm{mmol}, 1.0$ equiv), trifluoroacetic acid $(1.0 \mathrm{~mL})$, and water $(27 \square \mathrm{L}, 1.5 \mathrm{mmol}$, 5.0 equiv) followed by the addition of dry ice as $\left(\sim 92 \mathrm{mg}, 2.1 \mathrm{mmol}, 7\right.$ equiv) $\mathrm{CO}_{2}$ source. The vial was sealed with a PTFE lined cap and stirred at room temperature for $15 \mathrm{~min}$. After $15 \mathrm{~min}$ of stirring, the reaction mixture was heated in a pie-block at $40{ }^{\circ} \mathrm{C}$ under stirring for $14 \mathrm{~h}$. After cooling to room temperature, the reaction mixture was made basic with ammonium hydroxide solution up to $\mathrm{pH}=8$. The reaction mixture was stirred for 15 minutes followed by extraction with dichloromethane $(3 \times 10 \mathrm{~mL})$. The organic layer was washed with brine and dried over $\mathrm{Na}_{2} \mathrm{SO}_{4}$. followed by evaporating in vacuo. The resulting oil was columned over silica (15\% EtOAc/hexanes) to give the desired product as a yellow oil $(18.6 \mathrm{mg}, 15 \%) . \mathrm{R}_{f}=0.2($ Hexanes/EtOAc $=8: 2)$, which matched spectral data reported in the literature. ${ }^{20}{ }^{1} \mathrm{H} \mathrm{NMR}\left(600 \mathrm{MHz}, \mathrm{CDCl}_{3}\right) \delta 7.66(\mathrm{dd}, J=5.5,3.0 \mathrm{~Hz}, 2 \mathrm{H}), 7.61-7.55(\mathrm{~m}, 4 \mathrm{H}), 7.36(\mathrm{t}, J=7.7 \mathrm{~Hz}, 2 \mathrm{H})$, 7.25 (s, 1H), $7.15-7.10(\mathrm{~m}, 2 \mathrm{H}), 7.05-6.93(\mathrm{~m}, 8 \mathrm{H}), 4.77$ (s, 2H). ${ }^{13} \mathrm{C}$ NMR (151 MHz, $\left.\mathrm{CDCl}_{3}\right) \delta 168.1,142.4$, $142.3142 .0,138.8,134.4,133.8,131.8,130.4,129.9,129.6,128.6,127.9,127.6,127.1,126.9,126.4,123.0,42.3$

D. Synthesis and Experiments with a $3^{\circ}$ Cinnamylamine Substrate

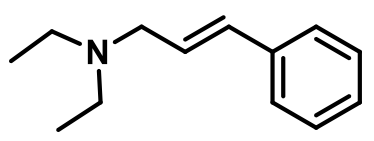

(E)- $N, N$-Diethyl-3-phenylprop-2-en-1-amine (8): Cinnamyl chloride (1.5 mL, $10.8 \mathrm{mmol})$ was added to diethyl amine $(10 \mathrm{~mL})$. Notably, the diethyl amine was relatively old and was not purified prior to use, though this didn't seem to hinder the reaction. The reaction was heated under reflux for $18 \mathrm{~h}$, followed by cooling and pouring into $1 \mathrm{M}$ $\mathrm{NaOH}$. The reaction mixture was extracted with dichloromethane $(3 \times 15 \mathrm{~mL})$, followed by drying over sodium sulfate and evaporating in vacuo to give the product as a brown oil (1.91 g, 93\%), which was found to match the spectroscopic data in the literature. ${ }^{21} \mathrm{R}_{f}=0.2(\mathrm{EtOAc} / \mathrm{MeOH}=9: 1) .{ }^{1} \mathrm{H} \mathrm{NMR}\left(400 \mathrm{MHz}, \mathrm{CDCl}_{3}\right) \delta 7.39(\mathrm{~d}, J=7.2 \mathrm{~Hz}, 2 \mathrm{H}), 7.32$ $(\mathrm{t}, J=7.7 \mathrm{~Hz}, 2 \mathrm{H}), 7.22(\mathrm{t}, J=7.2 \mathrm{~Hz}), 6.53(\mathrm{~d}, J=15.9 \mathrm{~Hz}, 1 \mathrm{H}), 6.32(\mathrm{dt}, J=15.8,6.7 \mathrm{~Hz}, 1 \mathrm{H}), 3.28(\mathrm{dd}, J=6.7$, $1.1 \mathrm{~Hz}, 2 \mathrm{H}), 2.60(\mathrm{q}, J=7.1 \mathrm{~Hz}, 4 \mathrm{H}), 1.09$ (t, $J=7.1 \mathrm{~Hz}, 6 \mathrm{H}) .{ }^{13} \mathrm{C} \mathrm{NMR}\left(100 \mathrm{MHz}, \mathrm{CDCl}_{3}\right) \delta 137.1,132.2,128.5$, $127.5,127.3,126.2,55.6,46.7,11.7$. 


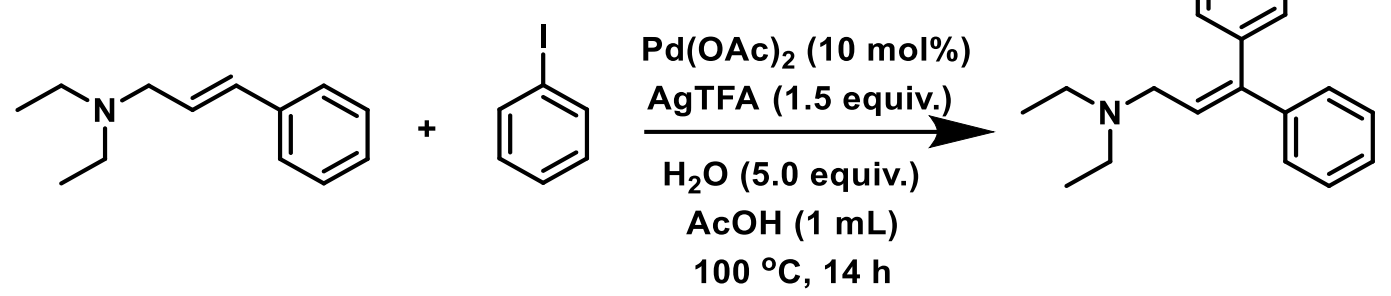

$N, N$-Diethyl-3,3-diphenylprop-2-en-1-amine (9): A $7.5 \mathrm{~mL}$ vial was charged with $\mathrm{Pd}(\mathrm{OAc})_{2}(6.7 \mathrm{mg}, 0.03 \mathrm{mmol}$, 0.10 equiv), silver trifluoroacetate ( $99.9 \mathrm{mg}, 0.45 \mathrm{mmol}, 1.5$ equiv), phenyl iodide (122.5 mg, $0.60 \mathrm{mmol}, 2.0$ equiv), amine (56.7 mg, $0.30 \mathrm{mmol}, 1.0$ equiv), acetic acid ( $1.0 \mathrm{~mL})$ and water $(21.7 \mu \mathrm{L}, 1.5 \mathrm{mmol}, 5$ equiv), followed by sealing with a PTFE lined cap and stirring at room temperature for $15 \mathrm{~min}$. After $15 \mathrm{~min}$ of stirring, the reaction vial was heated in a pie-block at $100{ }^{\circ} \mathrm{C}$ under stirring for $14 \mathrm{~h}$. After cooling to room temperature, the reaction mixture was made basic with ammonium hydroxide solution up to $\mathrm{pH}=8$. The reaction mixture was stirred for 15 minutes followed by extraction with dichloromethane $(3 \times 10 \mathrm{~mL})$. The combined organic phase was dried over $\mathrm{Na}_{2} \mathrm{SO}_{4}$, followed by evaporating in vacuo. The resulting oil was columned over silica ( $2 \% \mathrm{MeOH} / \mathrm{EtOAc})$ to give the desired product as a yellow oil $(21.4 \mathrm{mg}, 27 \%) . \mathrm{R}_{f}=0.2(\mathrm{MeOH} / \mathrm{EtOAc}=1: 9) .{ }^{1} \mathrm{H} \mathrm{NMR}\left(600 \mathrm{MHz}, \mathrm{CDCl}_{3}\right) \delta 7.38(\mathrm{t}, J=7.3$ $\mathrm{Hz}, 2 \mathrm{H}), 7.33(\mathrm{t}, J=7.3 \mathrm{~Hz}, 1 \mathrm{H}), 7.27-7.23(\mathrm{~m}, 5 \mathrm{H}), 7.16(\mathrm{~d}, J=7.0 \mathrm{~Hz}, 2 \mathrm{H}), 6.25(\mathrm{t}, J=6.8 \mathrm{~Hz}, 1 \mathrm{H}), 3.21(\mathrm{~d}, J=$ $6.6 \mathrm{~Hz}, 2 \mathrm{H}), 2.58(\mathrm{q}, J=6.9 \mathrm{~Hz}, 4 \mathrm{H}), 0.99(\mathrm{t}, J=7.1 \mathrm{~Hz}, 6 \mathrm{H}) .{ }^{13} \mathrm{C} \mathrm{NMR}\left(151 \mathrm{MHz}, \mathrm{CDCl}_{3}\right) \delta 144.3,142.2,139.7$, 129.9, 128.3, 128.2, 127.4, 127.3, 51.6, 47.0, 11.6. HRMS (ESI - MS): calcd. 266.1903 [M+H] ${ }^{+}$Found: 266.1906.
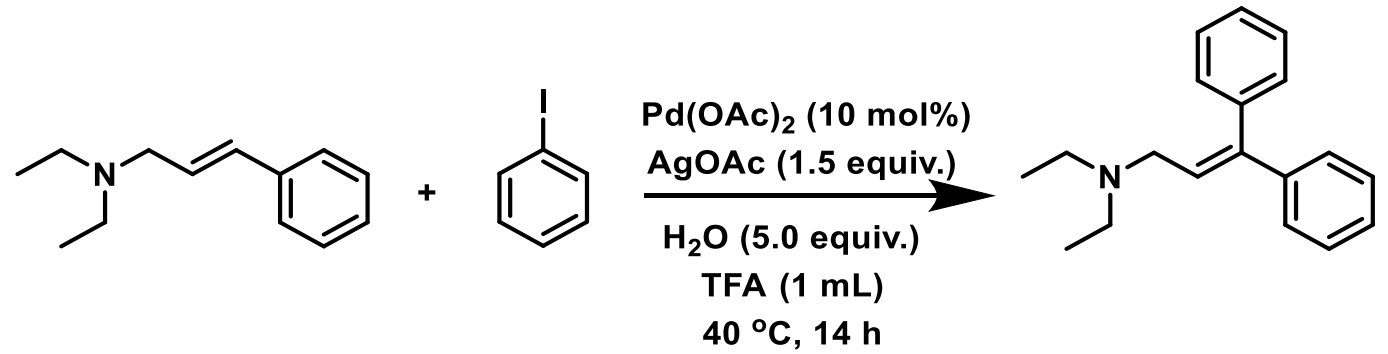

$N, N$-Diethyl-3,3-diphenylprop-2-en-1-amine (9): A $7.5 \mathrm{~mL}$ vial was charged with $\mathrm{Pd}(\mathrm{OAc})_{2}(6.7 \mathrm{mg}, 0.03 \mathrm{mmol}$, 0.10 equiv), silver acetate ( $75.1 \mathrm{mg}, 0.45 \mathrm{mmol}, 1.5$ equiv), phenyl iodide ( $122.5 \mathrm{mg}, 0.60 \mathrm{mmol}, 2.0$ equiv), amine (56.7 $\mathrm{mg}, 0.30 \mathrm{mmol}, 1.0$ equiv), trifluoroacetic acid $(1.0 \mathrm{~mL})$ and water $(21.7 \mu \mathrm{L}, 1.5 \mathrm{mmol}, 5$ equiv), followed by sealing with a PTFE lined cap and stirring at room temperature for $15 \mathrm{~min}$. After $15 \mathrm{~min}$ of stirring, the reaction vial was heated in a pie-block at $40{ }^{\circ} \mathrm{C}$ under stirring for $14 \mathrm{~h}$. After cooling to room temperature, the reaction mixture was made basic with ammonium hydroxide solution up to $\mathrm{pH}=8$. The reaction mixture was stirred for 15 minutes followed by extraction with dichloromethane $(3 \times 10 \mathrm{~mL})$. The combined organic phase was dried over $\mathrm{Na}_{2} \mathrm{SO}_{4}$, followed by evaporating in vacuo. The resulting oil was columned over silica $(2 \% \mathrm{MeOH} / \mathrm{EtOAc})$ to give the desired product as a yellow oil $(15.9 \mathrm{mg}, 20 \%) . \mathrm{R}_{f}=0.2(\mathrm{MeOH} / \mathrm{EtOAc}=1: 9)$. 


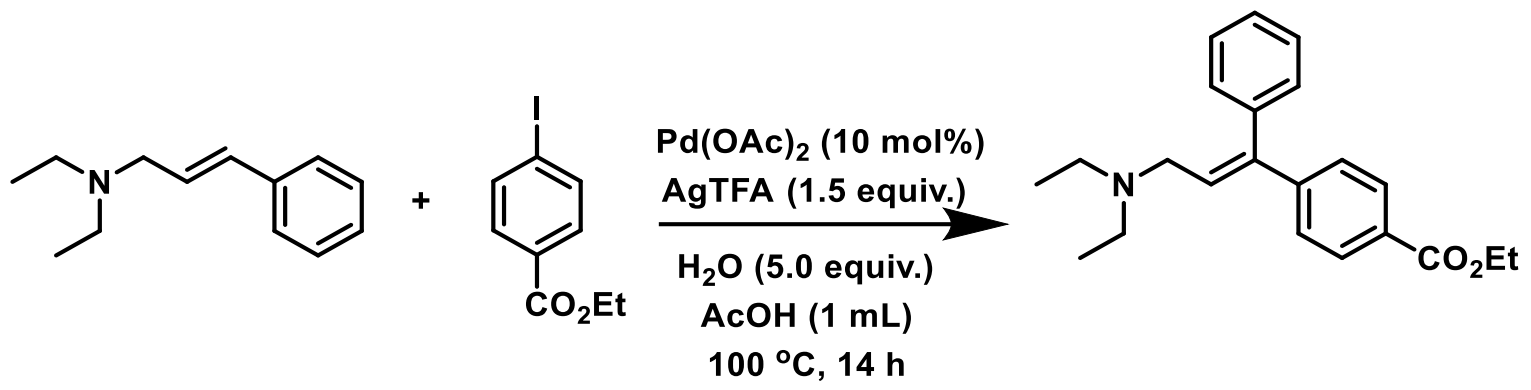

Ethyl (Z)-4-(3-(diethylamino)-1-phenylprop-1-en-1-yl)benzoate (10): A $7.5 \mathrm{~mL}$ vial was charged with $\mathrm{Pd}(\mathrm{OAc})_{2}$ (6.7 mg, $0.03 \mathrm{mmol}, 0.10$ equiv), silver trifluoroacetate ( $99.9 \mathrm{mg}, 0.45 \mathrm{mmol}, 1.5$ equiv), ethyl 4 -iodobenzoate (165.5 $\mathrm{mg}, 0.60 \mathrm{mmol}, 2.0$ equiv), amine (56.7 mg, $0.30 \mathrm{mmol}, 1.0$ equiv), acetic acid (1.0 mL) and water (21.7 $\mu \mathrm{L}, 1.5$ mmol, 5 equiv), followed by sealing with a PTFE lined cap and stirring at room temperature for $15 \mathrm{~min}$. After $15 \mathrm{~min}$ of stirring, the reaction vial was heated in a pie-block at $100{ }^{\circ} \mathrm{C}$ under stirring for $14 \mathrm{~h}$. After cooling to room temperature, the reaction mixture was made basic with ammonium hydroxide solution up to $\mathrm{pH}=8$. The reaction mixture was stirred for 15 minutes followed by extraction with dichloromethane $(3 \times 10 \mathrm{~mL})$. The combined organic phase was dried over $\mathrm{Na}_{2} \mathrm{SO}_{4}$, followed by evaporating in vacuo. The resulting oil was columned over silica (2\% $\mathrm{MeOH} / \mathrm{EtOAc})$ to give the desired product as a yellow oil $(42.5 \mathrm{mg}, 42 \%) . \mathrm{R}_{f}=0.2(\mathrm{MeOH} / \mathrm{EtOAc}=1: 9) .{ }^{1} \mathrm{H} \mathrm{NMR}$ $\left(600 \mathrm{MHz}, \mathrm{CDCl}_{3}\right) \delta 7.94(\mathrm{~d}, J=8.3 \mathrm{~Hz}, 2 \mathrm{H}), 7.39(\mathrm{~m}, 3 \mathrm{H}), 7.29(\mathrm{~d}, J=8.3 \mathrm{~Hz}, 2 \mathrm{H}), 7.12(\mathrm{~d}, J=7.6 \mathrm{~Hz}, 2 \mathrm{H}), 6.36$ (t, $J=6.9 \mathrm{~Hz}, 1 \mathrm{H}), 4.35(\mathrm{q}, J=7.1 \mathrm{~Hz}, 2 \mathrm{H}), 3.42(\mathrm{~d}, J=6.9 \mathrm{~Hz}, 2 \mathrm{H}), 2.77(\mathrm{q}, J=7.2 \mathrm{~Hz}, 4 \mathrm{H}), 1.37$ (t, $J=7.1 \mathrm{~Hz}$, $3 \mathrm{H}), 1.04(\mathrm{t}, J=7.2 \mathrm{~Hz}, 6 \mathrm{H}) .{ }^{13} \mathrm{C} \mathrm{NMR}\left(151 \mathrm{MHz}, \mathrm{CDCl}_{3}\right) \delta 166.4,145.7,138.4,129.7,129.6,128.7,128.1,127.4$, 61.1, 50.8, 46.5, 14.4, 10.3 HRMS (ESI - MS): calcd. $338.2115[\mathrm{M}+\mathrm{H}]^{+}$Found: 338.2117.

E. Synthesis and Experiments with a $4^{\circ}$ Cinnamylammonium Substrate

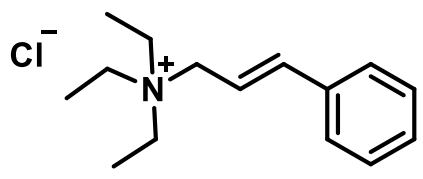

(E)-N,N,N-Triethyl-3-phenylprop-2-en-1-ammonium Chloride (11): Following a modified procedure for the synthesis of quaternary cinnamylammonium chlorides,${ }^{22}$ cinnamyl chloride $(1.5 \mathrm{~mL}, 10.8 \mathrm{mmol})$ was added to triethyl amine $(1.83 \mathrm{~mL})$ in $\mathrm{MeCN}(10 \mathrm{~mL})$. The reaction was heated under reflux for $24 \mathrm{~h}$, followed by cooling and evaporation in vacuo. The resulting mixture was triturated in EtOAc, and filtered to give the product as a white solid $(2.31 \mathrm{~g}, 93 \%)$. Notably, the solid was quickly stored under an inert atmosphere, otherwise it would turn into a puddle we assume due to significant hygroscopicity. ${ }^{1} \mathrm{H}$ NMR (600 MHz, $\left.\mathrm{CDCl}_{3}\right) \delta 7.44(\mathrm{~d}, J=6.3 \mathrm{~Hz}, 2 \mathrm{H}), 7.34-7.27$ (m, $3 \mathrm{H}), 7.02$ (d, $J=14.5 \mathrm{~Hz}, 1 \mathrm{H}), 6.22$ (br s, 1H), 4.32 (br s, 2H), 3.47 (br s, 6H), 1.38 (br s, 9H). ${ }^{13} \mathrm{C} \mathrm{NMR} \mathrm{(100} \mathrm{MHz,}$ $\left.\mathrm{CDCl}_{3}\right) \delta 143.0,134.7,129.5,128.9,127.4,114.0,60.2,53.4$, 8.4. HRMS (ESI - MS): calcd. $218.1904[\mathrm{M}]^{+}$Found: 218.1903. 

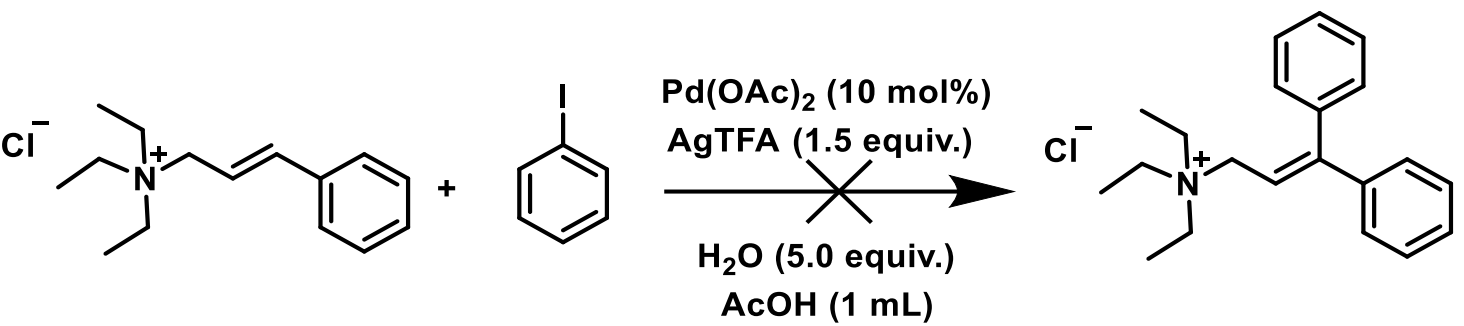

$100^{\circ} \mathrm{C}, 14 \mathrm{~h}$

Attempted Synthesis of $N, N$-Diethyl-3,3-diphenylprop-2-en-1-amine : A $7.5 \mathrm{~mL}$ vial was charged with $\mathrm{Pd}(\mathrm{OAc})_{2}$ (6.7 mg, $0.03 \mathrm{mmol}, 0.10$ equiv), silver trifluoroacetate ( $99.9 \mathrm{mg}, 0.45 \mathrm{mmol}, 1.5$ equiv), phenyl iodide (122.5 mg, $0.60 \mathrm{mmol}, 2.0$ equiv), amine ( $75.9 \mathrm{mg}, 0.30 \mathrm{mmol}, 1.0$ equiv), acetic acid $(1.0 \mathrm{~mL})$ and water $(21.7 \mu \mathrm{L}, 1.5 \mathrm{mmol}, 5$ equiv), followed by sealing with a PTFE lined cap and stirring at room temperature for $15 \mathrm{~min}$. After $15 \mathrm{~min}$ of stirring, the reaction vial was heated in a pie-block at $100{ }^{\circ} \mathrm{C}$ under stirring for $14 \mathrm{~h}$. After cooling to RT, the reaction mixture was concentrated in vacuo. Crude ${ }^{1} \mathrm{H}$ NMR was performed on the reaction mixture using 1,1,2,2-tetrachloroethane as an internal standard. The expected product was not observed with no recovery of starting material.

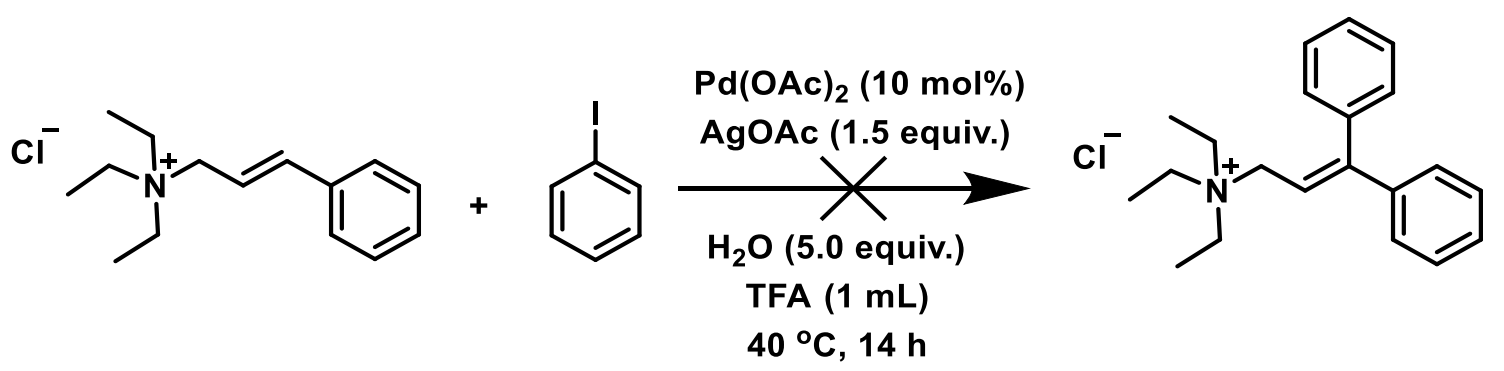

Attempted Synthesis of $N, N$-Diethyl-3,3-diphenylprop-2-en-1-amine: A $7.5 \mathrm{~mL}$ vial was charged with $\operatorname{Pd}(\mathrm{OAc})_{2}$ ( $6.7 \mathrm{mg}, 0.03 \mathrm{mmol}, 0.10$ equiv), silver acetate $(75.1 \mathrm{mg}, 0.45 \mathrm{mmol}, 1.5$ equiv), phenyl iodide ( $122.5 \mathrm{mg}, 0.60 \mathrm{mmol}$, 2.0 equiv), amine (75.9 mg, $0.30 \mathrm{mmol}, 1.0$ equiv), trifluoroacetic acid $(1.0 \mathrm{~mL})$ and water $(21.7 \mu \mathrm{L}, 1.5 \mathrm{mmol}, 5$ equiv), followed by sealing with a PTFE lined cap and stirring at room temperature for $15 \mathrm{~min}$. After $15 \mathrm{~min}$ of stirring, the reaction vial was heated in a pie-block at $40{ }^{\circ} \mathrm{C}$ under stirring for $14 \mathrm{~h}$. After cooling to $\mathrm{RT}$, the reaction mixture was concentrated in vacuo. Crude ${ }^{1} \mathrm{H}$ NMR was performed on the reaction mixture using 1,1,2,2-tetrachloroethane as an internal standard. The expected product was not observed and 38\% NMR yield of the starting material was recovered. 


\section{Outlier Substrate Experiments}

A. Synthesis and Experiments with a $\beta$-Methylcinnamylamine Substrate<smiles>CC(=Cc1ccccc1)CNC(C)(C)C</smiles>

(E)- $N$-Isopropyl-2-methyl-3-phenylprop-2-en-1-amine (12): Method A used. tert-Butylamine (3.0 mL, $28.7 \mathrm{mmol})$ and (E)-2-methyl-3-phenylacrylaldehyde $(2.0 \mathrm{~mL}, 14.4 \mathrm{mmol})$ were used. Product recovered as a colorless oil (2.06 g, 70\% yield). ${ }^{1} \mathrm{H} \mathrm{NMR}\left(600 \mathrm{MHz}, \mathrm{CDCl}_{3}\right) \delta 7.32(\mathrm{t}, J=7.6 \mathrm{~Hz}, 2 \mathrm{H}), 7.28(\mathrm{~d}, J=7.1 \mathrm{~Hz}, 2 \mathrm{H}), 7.20(\mathrm{t}, J=7.3 \mathrm{~Hz}$, $1 \mathrm{H}), 6.47(\mathrm{~s}, 1 \mathrm{H}), 3.28(\mathrm{~d}, J=0.7 \mathrm{~Hz}, 2 \mathrm{H}), 1.93(\mathrm{~d}, J=1.3 \mathrm{~Hz}, 3 \mathrm{H}), 1.17(\mathrm{~s}, 9 \mathrm{H}) .{ }^{13} \mathrm{C}$ NMR $\left(151 \mathrm{MHz}, \mathrm{CDCl}_{3}\right) \delta$ 138.4, 138.3, 128.9, 128.1, 126.1, 124.9, 51.4, 50.5, 29.3. HRMS (ESI - MS): calcd. 204.1747 [M+H] $]^{+}$Found: 204.1747.
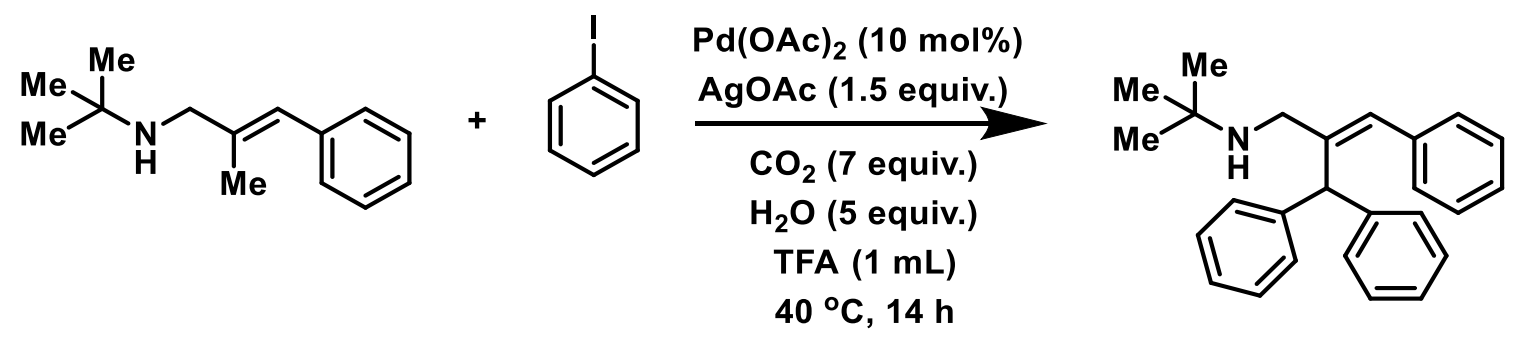

(E)-2-Benzhydryl- $N$-Isopropyl-3-phenylprop-2-en-1-amine (13): A $7.5 \mathrm{~mL}$ vial was charged with $\mathrm{Pd}(\mathrm{OAc})_{2}(6.7$ $\mathrm{mg}, 0.03 \mathrm{mmol}, 0.10$ equiv), silver acetate $(75.0 \mathrm{mg}, 0.45 \mathrm{mmol}, 1.5$ equiv), phenyl iodide ( $122.5 \mathrm{mg}, 0.60 \mathrm{mmol}, 2.0$ equiv), amine ( $60.9 \mathrm{mg}, 0.30 \mathrm{mmol}, 1.0$ equiv), trifluoroacetic acid $(1.0 \mathrm{~mL})$ and water ( $21.7 \mu \mathrm{L}, 1.5 \mathrm{mmol}, 5$ equiv), followed by the addition of dry ice as ( $92 \mathrm{mg}, 2.1 \mathrm{mmol}, 7$ equiv) $\mathrm{CO}_{2}$ source. The vial was sealed with a PTFE lined cap and stirred at room temperature for $15 \mathrm{~min}$. After $15 \mathrm{~min}$ of stirring, the reaction vial was heated in a pie-block at $40{ }^{\circ} \mathrm{C}$ under stirring for $14 \mathrm{~h}$. After cooling to room temperature, the reaction mixture was made basic with ammonium hydroxide solution up to $\mathrm{pH}=8$. The reaction mixture was stirred for 15 minutes followed by extraction with dichloromethane $(3 \times 10 \mathrm{~mL})$. The combined organic phase was dried over $\mathrm{Na}_{2} \mathrm{SO}_{4}$, followed by evaporation in vacuo to give the desired product as a yellow oil $(27.7 \mathrm{mg}, 26 \%) . \mathrm{R}_{f}=0.2($ Hexanes/EtOAc $=9: 1) .{ }^{1} \mathrm{H}$ NMR $(600 \mathrm{MHz}$, $\left.\mathrm{CDCl}_{3}\right) \delta 7.34(\mathrm{~m}, 8 \mathrm{H}), 7.28-7.22(\mathrm{~m}, 7 \mathrm{H}), 6.16(\mathrm{~s}, 1 \mathrm{H}), 5.35(\mathrm{~s}, 1 \mathrm{H}), 3.29(\mathrm{~s}, 2 \mathrm{H}), 0.99(\mathrm{~s}, 9 \mathrm{H}) .{ }^{13} \mathrm{C} \mathrm{NMR}(151 \mathrm{MHz}$, $\left.\mathrm{CDCl}_{3}\right) \delta 144.6,142.6,137.7,131.4,129.8,128.8,128.4,128.2,126.7,126.5,56.6,50.8,42.7,28.9$. HRMS (ESI MS): calcd. $356.2373[\mathrm{M}+\mathrm{H}]^{+}$Found: 356.2377.

B. Synthesis and Experiments with a $\beta$-Methyloxycarbonyl cinnamylamine Substrate

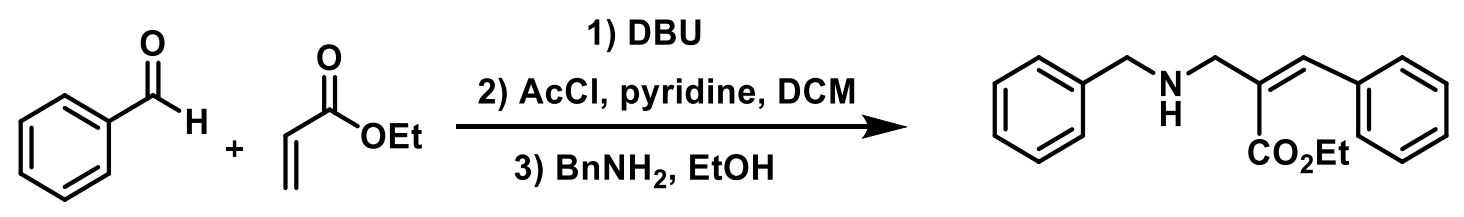


Ethyl (Z)-2-((benzylamino)methyl)-3-phenylacrylate (14): To a stirred mixture of ethyl acrylate (1.51 g, 15.1 mmol) and benzaldehyde ( $2 \mathrm{gm} 15.1 \mathrm{mmol})$ at room temperature under nitrogen was added DBU ( $2.3 \mathrm{~g}, 15.1 \mathrm{mmol})$. After $18 \mathrm{~h}$ the reaction was stopped by diluting with ether $(30 \mathrm{ml})$ and washed with $\mathrm{HCl}(2 \mathrm{M}, 20 \mathrm{ml})$ followed by water $(20 \mathrm{ml})$. After drying over $\mathrm{Na}_{2} \mathrm{SO}_{4}$, filtration and evaporation, concentrated in vacuo to give the crude BaylisHillman alcohol product, which was used for the next step without any further purification. ${ }^{23}$ To a well stirred solution of Baylis-Hilmann adduct (5-7) $(10 \mathrm{mmol})$ in dichloromethane $(30 \mathrm{~mL})$ at ${ }^{\circ} \mathrm{C}$ under argon atmosphere was added pyridine $(15 \mathrm{mmol})$ followed by acetyl chloride $(11 \mathrm{mmol})$ and allowed to stir at room temperature for $6 \mathrm{~h}$. Upon completion, it was diluted with $\mathrm{H}_{2} \mathrm{O}(15 \mathrm{~mL})$ and extracted with dichloromethane $(2 \times 25 \mathrm{~mL})$. The combined organic layers were washed with $\mathrm{HCl}(2 \mathrm{M}, 20 \mathrm{ml})$ until pyridine removed, and then organic layer was dried over $\mathrm{Na}_{2} \mathrm{SO}_{4}$, filtered and concentrated under reduced pressure. The resulting residue was subjected to column chromatography (ethyl acetate/hexane 1: 15) to afford pure compounds acetylated Baylis- Hillman product. ${ }^{24}$ The mixture of BaylisHillman acetate ( $1 \mathrm{~g}, 4.03 \mathrm{mmol}, 1$ equiv.) and amine ( $862 \mathrm{mg}, 8.06 \mathrm{mmol}, 2$ equiv) in EtOH (15 mL) was stirred at room temperature for $18 \mathrm{~h}$. The reaction mixture was concentrated under reduced pressure then diluted with $\mathrm{H}_{2} \mathrm{O}$ (20 $\mathrm{mL})$ and extracted with dichloromethane $(3 \times 15 \mathrm{~mL})$. The combined organic phase was dried over $\mathrm{Na}_{2} \mathrm{SO}_{4}$, followed by evaporation in vacuo to give the desired product as a yellow oil ( $808 \mathrm{mg}, 68 \%)$, with spectroscopy consistent with that reported in the literature. ${ }^{2525} \mathrm{R}_{f}=0.2$ (Hexanes/EtOAc $=8: 2$ ). ${ }^{1} \mathrm{H}$ NMR $\left(600 \mathrm{MHz}, \mathrm{CDCl}_{3}\right) \delta 7.77(\mathrm{~s}, 1 \mathrm{H}), 7.39$ $(\mathrm{dd}, J=6.4,2.8 \mathrm{~Hz}, 2 \mathrm{H}), 7.32-7.25(\mathrm{~m}, 7 \mathrm{H}), 7.21(\mathrm{~d}, J=3.2 \mathrm{~Hz}, 1 \mathrm{H}), 4.24(\mathrm{q}, J=7.1 \mathrm{~Hz}, 2 \mathrm{H}), 3.77$ (s, 2H), 3.55 (s, $2 \mathrm{H}), 1.80(\mathrm{br} \mathrm{s}, 1 \mathrm{H}), 1.31(\mathrm{t}, J=7.1 \mathrm{~Hz}, 3 \mathrm{H}) .{ }^{13} \mathrm{C} \mathrm{NMR}\left(151 \mathrm{MHz}, \mathrm{CDCl}_{3}\right) \delta 168.2,141.8,140.2,135.2,130.9,129.7$, $128.9,128.4,128.5,127.0,61.0,53.9,45.5,14.4$.
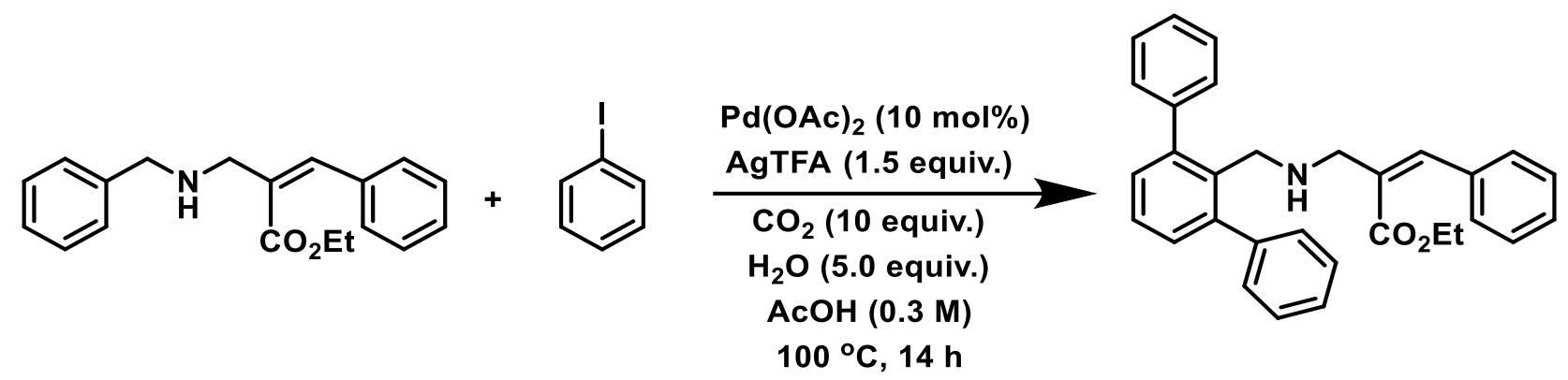

Methyl (Z)-2-((([1,1':3',1'-terphenyl]-2'-ylmethyl)amino)methyl)-3-phenylacrylate (15): A $7.5 \mathrm{~mL}$ vial was charged with $\mathrm{Pd}(\mathrm{OAc})_{2}(6.7 \mathrm{mg}, 0.03 \mathrm{mmol}, 0.10$ equiv), silver trifluoroacetate ( $99.9 \mathrm{mg}, 0.45 \mathrm{mmol}, 1.5$ equiv), phenyl iodide (125.5 mg, $0.60 \mathrm{mmol}, 2.0$ equiv), amine ( $88.5 .9 \mathrm{mg}, 0.30 \mathrm{mmol}, 1.0$ equiv), trifluoroacetic acid (1.0 $\mathrm{mL}$ ) and water ( $21.7 \mu \mathrm{L}, 1.5 \mathrm{mmol}, 5$ equiv), followed by the addition of dry ice as ( $132 \mathrm{mg}, 3 \mathrm{mmol}, 10$ equiv) $\mathrm{CO}_{2}$ source. The vial was sealed with a PTFE lined cap and stirred at room temperature for $15 \mathrm{~min}$. After $15 \mathrm{~min}$ of stirring, the reaction vial was heated in a pie-block at $40{ }^{\circ} \mathrm{C}$ under stirring for $14 \mathrm{~h}$. After cooling to room temperature, the reaction mixture was made basic with ammonium hydroxide solution up to $\mathrm{pH}=8$. The reaction mixture was stirred for 15 minutes followed by extraction with dichloromethane $(3 \times 10 \mathrm{~mL})$. The combined organic phase was dried over $\mathrm{Na}_{2} \mathrm{SO}_{4}$, followed by evaporation in vacuo to give the desired product as a yellow oil $(28.1 \mathrm{mg}, 21 \%)$. $\mathrm{R}_{f}=0.2$ $\left(\right.$ Hexanes/EtOAc = 9:1). ${ }^{1} \mathrm{H}$ NMR $\left(600 \mathrm{MHz}, \mathrm{CDCl}_{3}\right) \delta 7.63(\mathrm{~s}, 1 \mathrm{H}), 7.52-7.48(\mathrm{~m}, 4 \mathrm{H}), 7.43-7.36(\mathrm{~m}, 6 \mathrm{H}), 7.34$ $(\mathrm{dd}, J=8.0,7.1 \mathrm{~Hz}, 1 \mathrm{H}), 7.29(\mathrm{dd}, J=8.3,6.3 \mathrm{~Hz}, 1 \mathrm{H}), 7.27-7.23(\mathrm{~m}, 4 \mathrm{H}), 7.20(\mathrm{~d}, J=7.4 \mathrm{~Hz}, 2 \mathrm{H}), 4.19(\mathrm{q}, J=7.1$ 
$\mathrm{Hz}, 2 \mathrm{H}), 3.61$ (s, 2H), 3.18 (s, 2H), $1.28(\mathrm{t}, J=7.1 \mathrm{~Hz}, 3 \mathrm{H}) .{ }^{13} \mathrm{C} \mathrm{NMR}\left(151 \mathrm{MHz}, \mathrm{CDCl}_{3}\right) \delta 167.9,143.4,141.8,141.3$, 135.3, 135.2, 131.1, 129.8, 129.6, 129.5, 128.7, 128.6, 128.2, 127.1, 126.8, 60.8, 48.1, 45.6, 14.5. HRMS (ESI - MS): calcd. $448.2271[\mathrm{M}+\mathrm{H}]^{+}$Found: 448.2275. 


\section{Mechanistic Experiments}

\section{A. Isomerization Experiment}
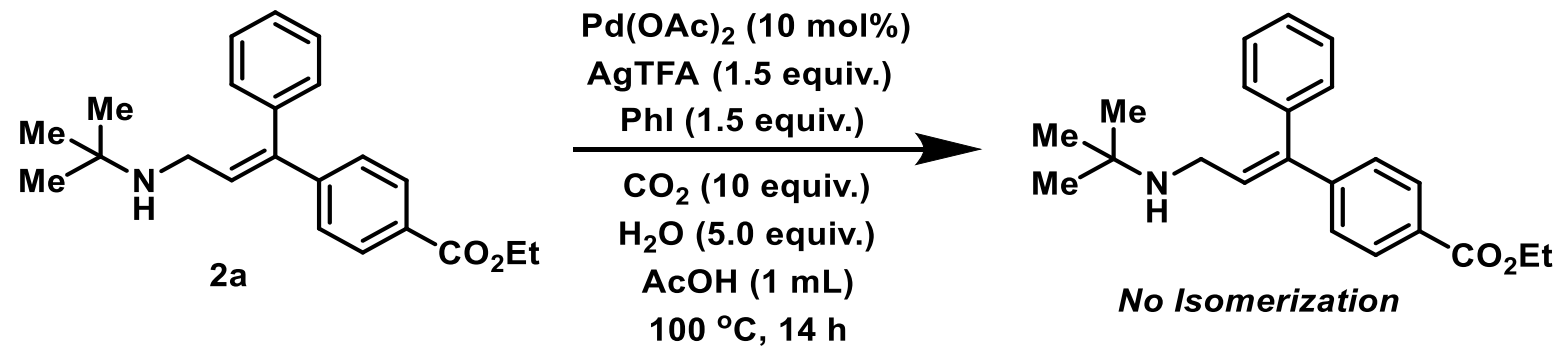

No Isomerization

A $7.5 \mathrm{~mL}$ vial was charged with $\mathrm{Pd}(\mathrm{OAc})_{2}(6.7 \mathrm{mg}, 0.03 \mathrm{mmol}, 0.10$ equiv), silver trifluoroacetate $(99.9 \mathrm{mg}, 0.45$ mmol, 1.5 equiv), organohalide ( $0.60 \mathrm{mmol}, 2.0$ equiv), amine ( $0.30 \mathrm{mmol}, 1.0$ equiv), acetic acid $(1.0 \mathrm{~mL})$, and water (27 $\mu \mathrm{L}, 1.5 \mathrm{mmol}, 5.0$ equiv) followed by the addition of dry ice as ( $132 \mathrm{mg}, 3.0 \mathrm{mmol}, 10$ equiv) $\mathrm{CO}_{2}$ source. The vial was sealed with a PTFE lined cap and stirred at room temperature for $15 \mathrm{~min}$. After $15 \mathrm{~min}$ of stirring, the reaction mixture was heated in a pie-block at $100{ }^{\circ} \mathrm{C}$ under stirring for $14 \mathrm{~h}$. After cooling to room temperature, the reaction mixture was made basic with ammonium hydroxide solution up to $\mathrm{pH}=8$. The reaction mixture was stirred for 15 minutes followed by extraction with dichloromethane $(3 \times 10 \mathrm{~mL})$. The organic layer was washed with brine and dried over $\mathrm{Na}_{2} \mathrm{SO}_{4}$. The filtrate was concentrated in vacuo and further purified by flash column chromatography over silica to give the $\mathrm{C}-\mathrm{H}$ arylation product in $30 \%$ yield (average of two reactions). NMR showed no sign of the isomerization product.

\section{B. Reaction Scale-Up}
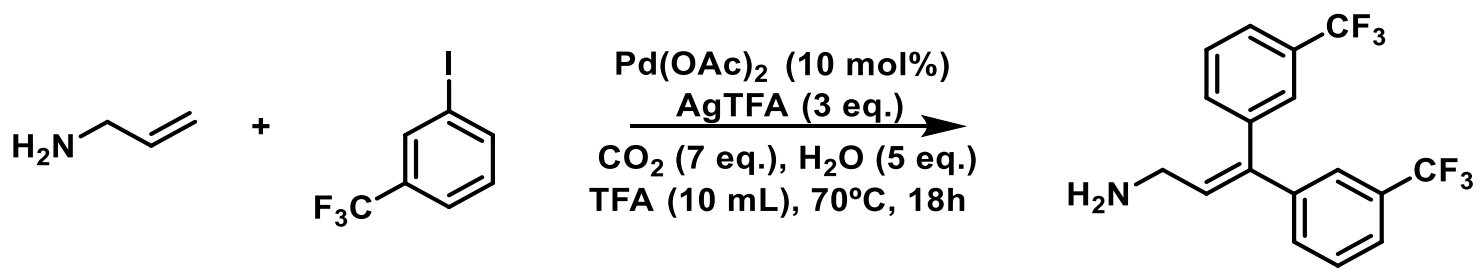

Procedure: A $40 \mathrm{~mL}$ pressure tube (Ace Glassware) was charged with $\mathrm{Pd}(\mathrm{OAc})_{2}(67 \mathrm{mg}, 0.30 \mathrm{mmol}, 0.10$ equiv), silver trifluoroacetate (1.99 g, $9.0 \mathrm{mmol}, 3.0$ equiv), 3-iodobenzotrifluoride (1.73 mL, $12.0 \mathrm{mmol}, 4.0$ equiv), allylamine ( $225 \mu \mathrm{L}, 3.0 \mathrm{mmol}, 1.0$ equiv), trifluoroacetic acid $(10 \mathrm{~mL})$, and water ( $270 \mu \mathrm{L}, 15 \mathrm{mmol}, 5.0$ equiv) followed by the addition of dry ice as $\left(\sim 1.32 \mathrm{~g}, 30.0 \mathrm{mmol}, 10\right.$ equiv) $\mathrm{CO}_{2}$ source. The tube was immediately sealed with a PTFE stopper and stirred at room temperature for $15 \mathrm{~min}$. After $15 \mathrm{~min}$ of stirring, the reaction mixture was heated in a pie-block at $70{ }^{\circ} \mathrm{C}$ under stirring for $18 \mathrm{~h}$. After cooling to room temperature, the reaction mixture was made basic with ammonium hydroxide solution up to $\mathrm{pH}=8$. The reaction mixture was stirred for 15 minutes followed by extraction with dichloromethane $(3 \times 25 \mathrm{~mL})$. The organic layer was washed with brine and dried over $\mathrm{Na}_{2} \mathrm{SO}_{4}$. The filtrate was concentrated in vacuo and further purified by flash column chromatography over silica to give the $\mathrm{C}-\mathrm{H}$ arylation product. Vide supra for characterization data.

C. Synthesis and Experiments with an Acyclic 1,2-Disubstituted Aliphatic 


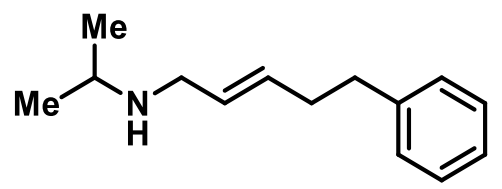

(E)- $N$-Isopropyl-5-phenylprop-2-en-1-amine (16): Method A used. Isopropylamine (258 mg) and (E)-5phenylpent-2-enal (700 mg) were used. Product recovered as a colorless oil (631 mg, 71\% yield). ${ }^{1} \mathrm{H} \mathrm{NMR} \mathrm{(600} \mathrm{MHz,}$ $\left.\mathrm{CDCl}_{3}\right) \delta 7.30-7.26(\mathrm{~m}, 2 \mathrm{H}), 7.18(\mathrm{t}, J=7.0 \mathrm{~Hz}, 3 \mathrm{H}), 5.65(\mathrm{~m}, 1 \mathrm{H}), 5.60-5.54(\mathrm{~m}, 1 \mathrm{H}), 3.21(\mathrm{dd}, J=6.3,0.8 \mathrm{~Hz}$, $2 \mathrm{H}), 2.81(\mathrm{dt}, J=12.6,6.3 \mathrm{~Hz}, 1 \mathrm{H}), 2.72-2.66(\mathrm{~m}, 2 \mathrm{H}), 2.35(\mathrm{td}, J=7.5,0.8 \mathrm{~Hz}, 2 \mathrm{H}), 1.08(\mathrm{~d}, J=6.3 \mathrm{~Hz}, 6 \mathrm{H}) .{ }^{13} \mathrm{C}$ $\operatorname{NMR}\left(151 \mathrm{MHz}, \mathrm{CDCl}_{3}\right) \delta 141.9,132.5,128.5,128.4,128.3,125.9,48.9,48.1,35.7,34.3,22.6$. HRMS (ESI - MS): calcd. 204.1747 [M+H] $]^{+}$Found: 204.1747.

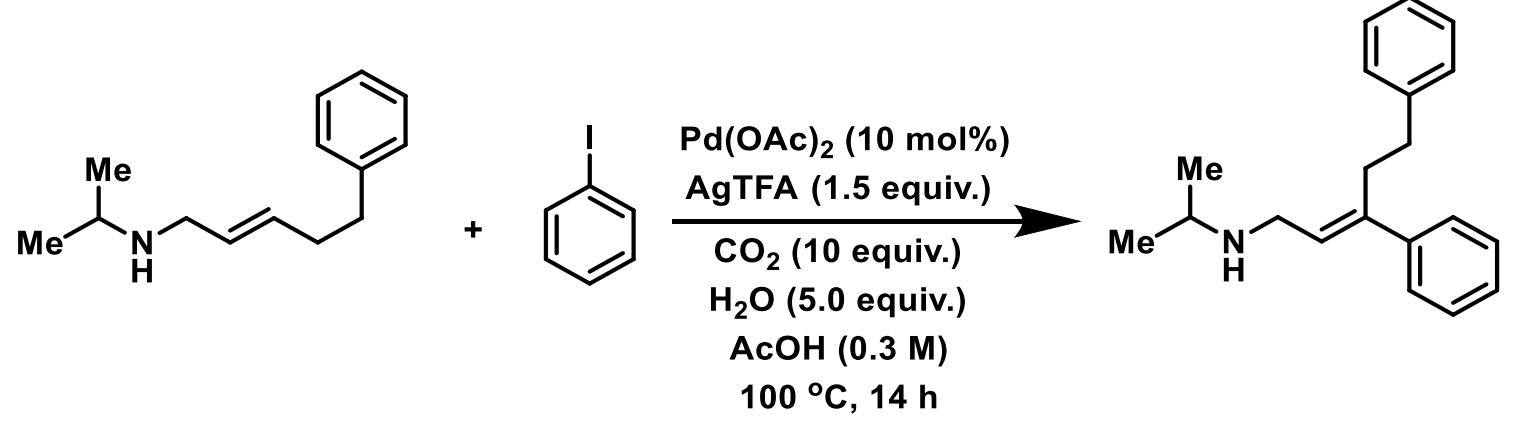

(E)-2-Benzhydryl- $N$-Isopropyl-3-phenylprop-2-en-1-amine (17): A $7.5 \mathrm{~mL}$ vial was charged with $\mathrm{Pd}(\mathrm{OAc})_{2}(6.7$ $\mathrm{mg}, 0.03 \mathrm{mmol}, 0.10$ equiv), silver trifluoroacetate $(99.9 \mathrm{mg}, 0.45 \mathrm{mmol}, 1.5$ equiv), phenyl iodide (122.5 $\mathrm{mg}, 0.60$ mmol, 2.0 equiv), amine (60.9 mg, $0.30 \mathrm{mmol}, 1.0$ equiv), trifluoroacetic acid (1.0 mL) and water $(21.7 \mu \mathrm{L}, 1.5 \mathrm{mmol}$, 5 equiv), followed by the addition of dry ice as ( $\sim 2 \mathrm{mg}, 2.1 \mathrm{mmol}, 7$ equiv) $\mathrm{CO}_{2}$ source. The vial was sealed with a PTFE lined cap and stirred at room temperature for $15 \mathrm{~min}$. After $15 \mathrm{~min}$ of stirring, the reaction vial was heated in a pie-block at $40{ }^{\circ} \mathrm{C}$ under stirring for $14 \mathrm{~h}$. After cooling to room temperature, the reaction mixture was made basic with ammonium hydroxide solution up to $\mathrm{pH}=8$. The reaction mixture was stirred for 15 minutes followed by extraction with dichloromethane $(3 \times 10 \mathrm{~mL})$. The combined organic phase was dried over $\mathrm{Na}_{2} \mathrm{SO}_{4}$, followed by evaporation in vacuo to give the desired product as a yellow oil $(27.7 \mathrm{mg}, 41 \%)$ and the $\mathrm{E} / \mathrm{Z}$ ratio was determined as 10:1 from the crude NMR. $\mathrm{R}_{f}=0.2(\mathrm{EtOAc} / \mathrm{MeOH}=95: 5) .{ }^{1} \mathrm{H} \mathrm{NMR}\left(600 \mathrm{MHz}, \mathrm{CDCl}_{3}\right) \delta 7.41(\mathrm{~d}, J=7.6 \mathrm{~Hz}, 2 \mathrm{H})$, $7.35(\mathrm{t}, J=7.6 \mathrm{~Hz}, 2 \mathrm{H}), 7.28(\mathrm{~d}, J=7.6 \mathrm{~Hz}, 2 \mathrm{H}), 7.19(\mathrm{t}, J=7.1 \mathrm{~Hz}, 2 \mathrm{H}), 7.12(\mathrm{~d}, J=7.5 \mathrm{~Hz}, 2 \mathrm{H}), 5.78(\mathrm{t}, J=6.9 \mathrm{~Hz}$, $1 \mathrm{H}), 3.17(\mathrm{~d}, J=6.9 \mathrm{~Hz}, 2 \mathrm{H}), 2.83(\mathrm{t}, J=7.5 \mathrm{~Hz}, 2 \mathrm{H}), 2.78(\mathrm{~m}, J=6.3 \mathrm{~Hz}, 1 \mathrm{H}), 2.63(\mathrm{t}, J=7.5 \mathrm{~Hz}, 2 \mathrm{H}), 1.04(\mathrm{~d}, J=$ $6.3 \mathrm{~Hz}, 6 \mathrm{H}) .{ }^{13} \mathrm{C} \mathrm{NMR}\left(151 \mathrm{MHz}, \mathrm{CDCl}_{3}\right) \delta 142.2,141.8,141.2,128.8,128.5,128.4,127.3,126.7,126.1,48.6,44.9$, 34.7, 32.1, 22.5. HRMS (ESI - MS): calcd. 280.2060 [M+H] $]^{+}$Found: 280.2061.

D. Competition Between Ethyl Acrylate and $N$-Isopropylcinnamyl Amine for Arylation Reaction
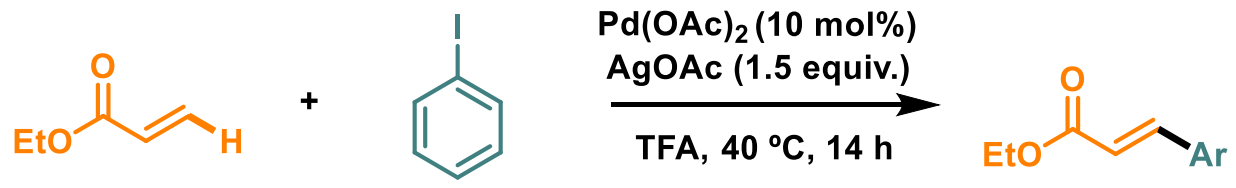
Procedure: A $7.5 \mathrm{~mL}$ vial was charged with $\mathrm{Pd}(\mathrm{OAc})_{2}(6.7 \mathrm{mg}, 0.03 \mathrm{mmol}, 0.10$ equiv), silver acetate $(75.1 \mathrm{mg}, 0.45$ mmol, 1.5 equiv), iodobenzene ( $0.30 \mathrm{mmol}, 1.0$ equiv), ethyl acrylate $(0.30 \mathrm{mmol}, 1.0$ equiv), acetic acid $(1.0 \mathrm{~mL})$, and water ( $27 \mu \mathrm{L}, 1.5 \mathrm{mmol}, 5.0$ equiv) followed by the addition of dry ice as ( $132 \mathrm{mg}, 3.0 \mathrm{mmol}, 10$ equiv) $\mathrm{CO}_{2}$ source. The vial was sealed with a PTFE lined cap and stirred at room temperature for $15 \mathrm{~min}$. After $15 \mathrm{~min}$ of stirring, the reaction mixture was heated in a pie-block at $40{ }^{\circ} \mathrm{C}$ under stirring for $14 \mathrm{~h}$. The reaction was then filtered through celite, evaporated in vacuo, and the ${ }^{1} \mathrm{H}$ NMR taken using 1,1,2,2-tetrachloroethane as an internal standard. 8\% NMR yield of the ethyl cinnamate product was observed as compared with an authentic sample, in addition to other side products and residual iodobenzene.

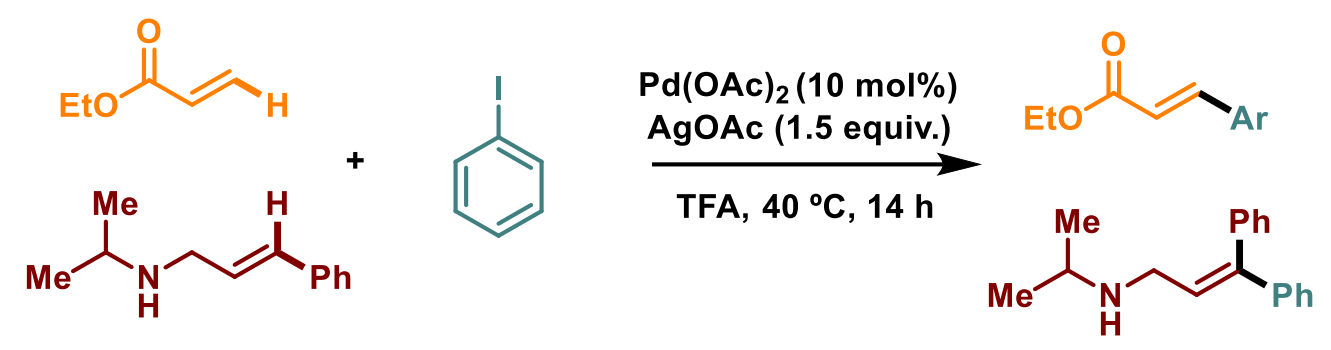

Procedure: A $7.5 \mathrm{~mL}$ vial was charged with $\mathrm{Pd}(\mathrm{OAc})_{2}(6.7 \mathrm{mg}, 0.03 \mathrm{mmol}, 0.10$ equiv), silver acetate $(75.1 \mathrm{mg}, 0.45$ mmol, 1.5 equiv), iodobenzene ( $0.30 \mathrm{mmol}, 1.0$ equiv), ethyl acrylate $(0.30 \mathrm{mmol}, 1.0$ equiv), amine, $(0.30 \mathrm{mmol}, 1.0$ equiv.), acetic acid (1.0 mL), and water $(27 \mu \mathrm{L}, 1.5 \mathrm{mmol}, 5.0$ equiv) followed by the addition of dry ice as ( 132 $\mathrm{mg}$, 3.0 mmol, 10 equiv) $\mathrm{CO}_{2}$ source. The vial was sealed with a PTFE lined cap and stirred at room temperature for 15 min. After $15 \mathrm{~min}$ of stirring, the reaction mixture was heated in a pie-block at $40{ }^{\circ} \mathrm{C}$ under stirring for $14 \mathrm{~h}$. The reaction was then filtered through celite, evaporated in vacuo, and the ${ }^{1} \mathrm{H}$ NMR taken using 1,1,2,2-tetrachloroethane as an internal standard. 57\% NMR yield of the ethyl cinnamate product was observed as compared with an authentic sample, in addition to other side products, with $76 \%$ recovery of the $N$-isopropylcinnamylamine starting material and no significant resonances observed for the diarylamine product.

\section{E. Following Reaction Progress In Situ Using NMR}

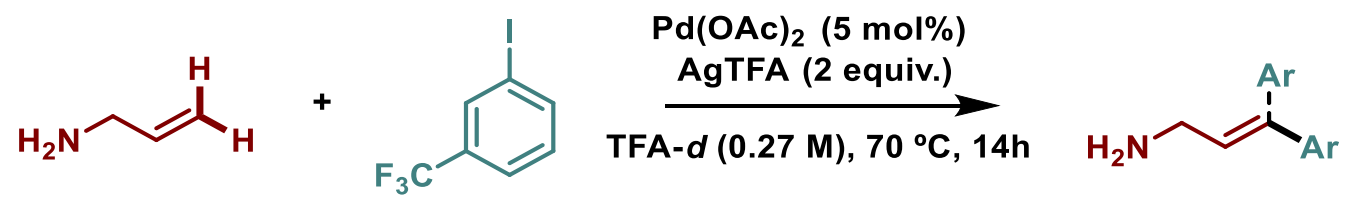

Procedure: A standard NMR tube was charged with TFA- $d$ in the glovebox. For $\mathrm{CO}_{2}$ free reactions, the remaining reagents were added, the tube capped, then added to the NMR at $70{ }^{\circ} \mathrm{C}$. For reactions with $\mathrm{CO}_{2}, \mathrm{D}_{2} \mathrm{O}(20 \mu \mathrm{L})$ was added to the NMR tube, followed by purging with $\sim 200 \mathrm{mg}$ of dry ice in five portions. Remaining reagents were added, followed by an additional purge of $\sim 100 \mathrm{mg}$ of dry ice in three portions. See $S$-241 and S-242 for data.

\section{F. Deuteration Experiments}




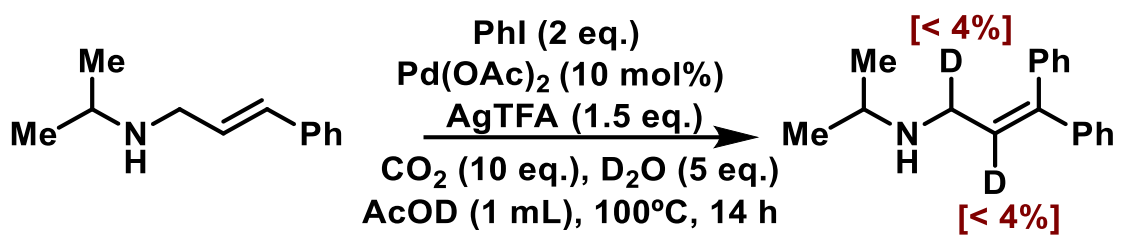

Effects of Deuterated Solvent: A $7.5 \mathrm{~mL}$ vial was charged with $\mathrm{Pd}(\mathrm{OAc})_{2}(6.7 \mathrm{mg}, 0.03 \mathrm{mmol}, 0.10$ equiv), silver trifluoroacetate $(99.9 \mathrm{mg}, 0.45 \mathrm{mmol}, 1.5$ equiv), organohalide ( $0.60 \mathrm{mmol}, 2.0$ equiv), amine (0.30 mmol, 1.0 equiv), acetic acid- $d(1.0 \mathrm{~mL})$, and water $(27 \mu \mathrm{L}, 1.5 \mathrm{mmol}, 5.0$ equiv) followed by the addition of dry ice as $(\sim 132 \mathrm{mg}, 3.0$ mmol, 10 equiv) $\mathrm{CO}_{2}$ source. The vial was sealed with a PTFE lined cap and stirred at room temperature for 15 min. After 15 min of stirring, the reaction mixture was heated in a pie-block at $100{ }^{\circ} \mathrm{C}$ under stirring for $14 \mathrm{~h}$. After cooling to room temperature, the reaction mixture was made basic with ammonium hydroxide solution up to $\mathrm{pH}=8$. The reaction mixture was stirred for 15 minutes followed by extraction with dichloromethane $(3 \times 25 \mathrm{~mL})$. The organic layer was washed with brine and dried over $\mathrm{Na}_{2} \mathrm{SO}_{4}$. The filtrate was concentrated in vacuo and further purified by flash column chromatography over silica to give the $\mathrm{C}-\mathrm{H}$ arylation product in $51 \%$ yield (average of three reactions). Relative peak intensities were compared against resonances that were not expected to undergo deuteration, and no significant level of deuteration was observed.

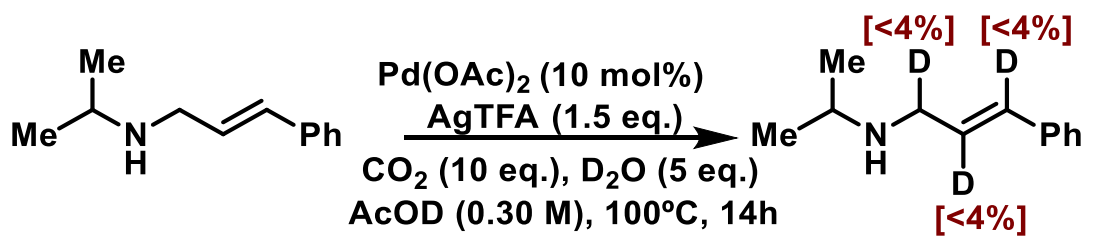

Effects of Deuterated Solvent without Phenyl iodide: A $7.5 \mathrm{~mL}$ vial was charged with $\mathrm{Pd}(\mathrm{OAc})_{2}(6.7 \mathrm{mg}, 0.03$ mmol, 0.10 equiv), silver trifluoroacetate $(99.9 \mathrm{mg}, 0.45 \mathrm{mmol}, 1.5$ equiv), amine ( $0.30 \mathrm{mmol}, 1.0$ equiv), acetic acid$d(1.0 \mathrm{~mL})$, and water $(27 \mu \mathrm{L}, 1.5 \mathrm{mmol}, 5.0$ equiv) followed by the addition of dry ice as $(\sim 132 \mathrm{mg}, 3.0 \mathrm{mmol}, 10$ equiv) $\mathrm{CO}_{2}$ source. The vial was sealed with a PTFE lined cap and stirred at room temperature for 15 min. After 15 min of stirring, the reaction mixture was heated in a pie-block at $100{ }^{\circ} \mathrm{C}$ under stirring for $14 \mathrm{~h}$. After cooling to room temperature, the reaction mixture was evaporated in vacuo, and the ${ }^{1} \mathrm{H} \mathrm{NMR}$ spectrum obtained in $\mathrm{CDCl}_{3}$, using 1,1,2,2-tetrachloroethane as an internal standard. The starting material was recovered in $25 \%$ yield, and relative peak intensities were compared against resonance that were not expected to undergo deuteration, showing no significant level of deuteration.

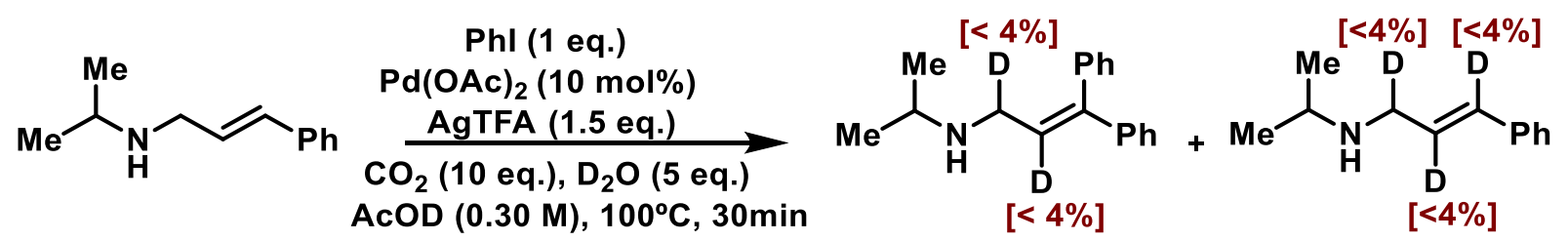

Investigating Deutetaration Mid-Reaction: A $7.5 \mathrm{~mL}$ vial was charged with $\mathrm{Pd}(\mathrm{OAc})_{2}(6.7 \mathrm{mg}, 0.03 \mathrm{mmol}, 0.10$ equiv), silver trifluoroacetate ( $99.9 \mathrm{mg}, 0.45 \mathrm{mmol}, 1.5$ equiv), ), organohalide ( $0.30 \mathrm{mmol}, 1.0$ equiv), amine ( 0.30 
mmol, 1.0 equiv), acetic acid- $d(1.0 \mathrm{~mL})$, and water ( $27 \mu \mathrm{L}, 1.5 \mathrm{mmol}, 5.0$ equiv) followed by the addition of dry ice as ( $132 \mathrm{mg}, 3.0 \mathrm{mmol}, 10$ equiv) $\mathrm{CO}_{2}$ source. The vial was sealed with a PTFE lined cap and stirred at room temperature for $15 \mathrm{~min}$. After $15 \mathrm{~min}$ of stirring, the reaction mixture was heated in a pie-block at $100{ }^{\circ} \mathrm{C}$ under stirring for $30 \mathrm{~min}$. After cooling to room temperature, the reaction mixture was made basic with ammonium hydroxide solution up to $\mathrm{pH}=8$. The reaction mixture was stirred for 15 minutes followed by extraction with dichloromethane $(3 \times 25 \mathrm{~mL})$. The organic layer was washed with brine and dried over $\mathrm{Na}_{2} \mathrm{SO}_{4}$. The filtrate was concentrated in vacuo and further purified by flash column chromatography over silica to give the $\mathrm{C}-\mathrm{H}$ arylation product in $43 \%$ yield and the starting material was recovered in $25 \%$ yield (average of three reactions). Relative peak intensities were compared against resonances that were not expected to undergo deuteration, and no significant level of deuteration was observed.

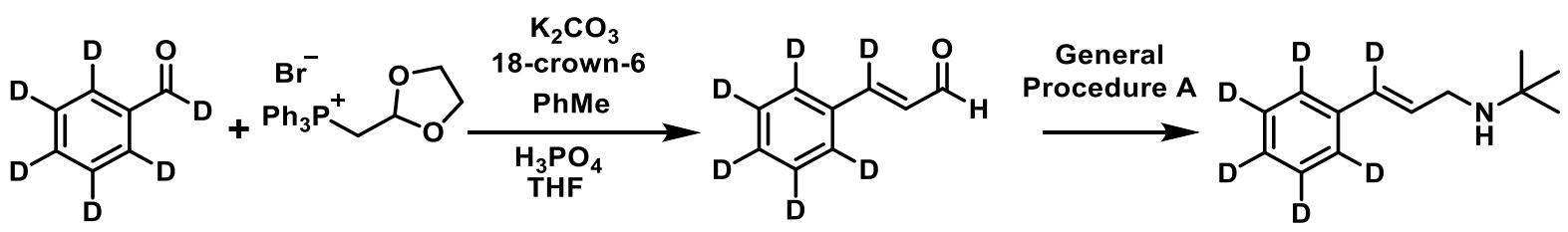

Synthesis of Deuterated Substrate $(E)-N$-(tert-Butyl)-3-phenylprop-2-en-1-amine- $d_{6}\left(S-1-d_{6}\right)$ : A $40 \mathrm{~mL}$ vial was charged with benzldehyde- $d_{6} \quad(1 \mathrm{~g}, \quad 1.0$ equiv.), 18-crown-6 (47 mg, 2 mol\%), (1,3-dioxolan-2ylmethyl)triphenylphosphonium bromide ( $7.64 \mathrm{~g}, 2$ equiv.), $\mathrm{K}_{2} \mathrm{CO}_{3}(2.46 \mathrm{~g}, 2$ equiv.), and toluene $(15 \mathrm{~mL})$. The vial was sealed with a PTFE lined cap and the reaction mixture was heated in a pie-block at $100{ }^{\circ} \mathrm{C}$ with stirring for $18 \mathrm{~h}$. After cooling to room temperature, the solvent was evaporated in vacuo. The reaction mixture was then diluted with deionized water, followed by extraction with dichloromethane $(2 \times 20 \mathrm{~mL})$. The combined organic phases were dried over $\mathrm{Na}_{2} \mathrm{SO}_{4}$, then concentrated in vacuo to give the crude acetal product ( $\left.4.5 \mathrm{~g}\right)$, which was used in the next step without any further purification.

The crude acetal $(4.5 \mathrm{~g})$ was added to tetrahydrofuran $(20 \mathrm{~mL})$, followed by $2 \mathrm{~mL}$ of $85 \% \mathrm{H}_{3} \mathrm{PO}_{4}$. The reaction was then heated at $80{ }^{\circ} \mathrm{C}$ for $16 \mathrm{~h}$. After cooling to room temperature, the solvent was evaporated in vacuo. The reaction mixture was diluted with deionized water, followed by extraction with dichloromethane $(2 \times 20 \mathrm{~mL})$. The combined organic phases were dried over $\mathrm{Na}_{2} \mathrm{SO}_{4}$, then concentrated in vacuo. The crude reaction mixture was then purified by flash chromatography over silica to give the desired cinnamaldehyde product ( $850 \mathrm{mg}, 69 \%$ ).

Then Method A used. tert-Butylamine $(500 \mathrm{mg})$ and cinnamaldehyde- $d_{6}(945 \mathrm{mg})$ were used. Product recovered as a yellow oil (1.08 g, 81\% yield). ${ }^{1} \mathrm{H}$ NMR (600 MHz, $\left.\mathrm{CDCl}_{3}\right) \delta 6.43-6.25(\mathrm{~m}, 1 \mathrm{H}), 3.37(\mathrm{~d}, J=6.5 \mathrm{~Hz}, 2 \mathrm{H}), 1.16$ (s, 9H). ${ }^{13} \mathrm{C} \mathrm{NMR}\left(151 \mathrm{MHz}, \mathrm{CDCl}_{3}\right) \delta 137.1,130.6(\mathrm{t}, J=23.2 \mathrm{~Hz}), 129.3,128.1$ (t, $\left.J=24.3 \mathrm{~Hz}\right), 126.9(\mathrm{t}, J=24.1$ $\mathrm{Hz}), 125.9$ (t, $J=23.9 \mathrm{~Hz}), 50.6,45.3,29.2$. 

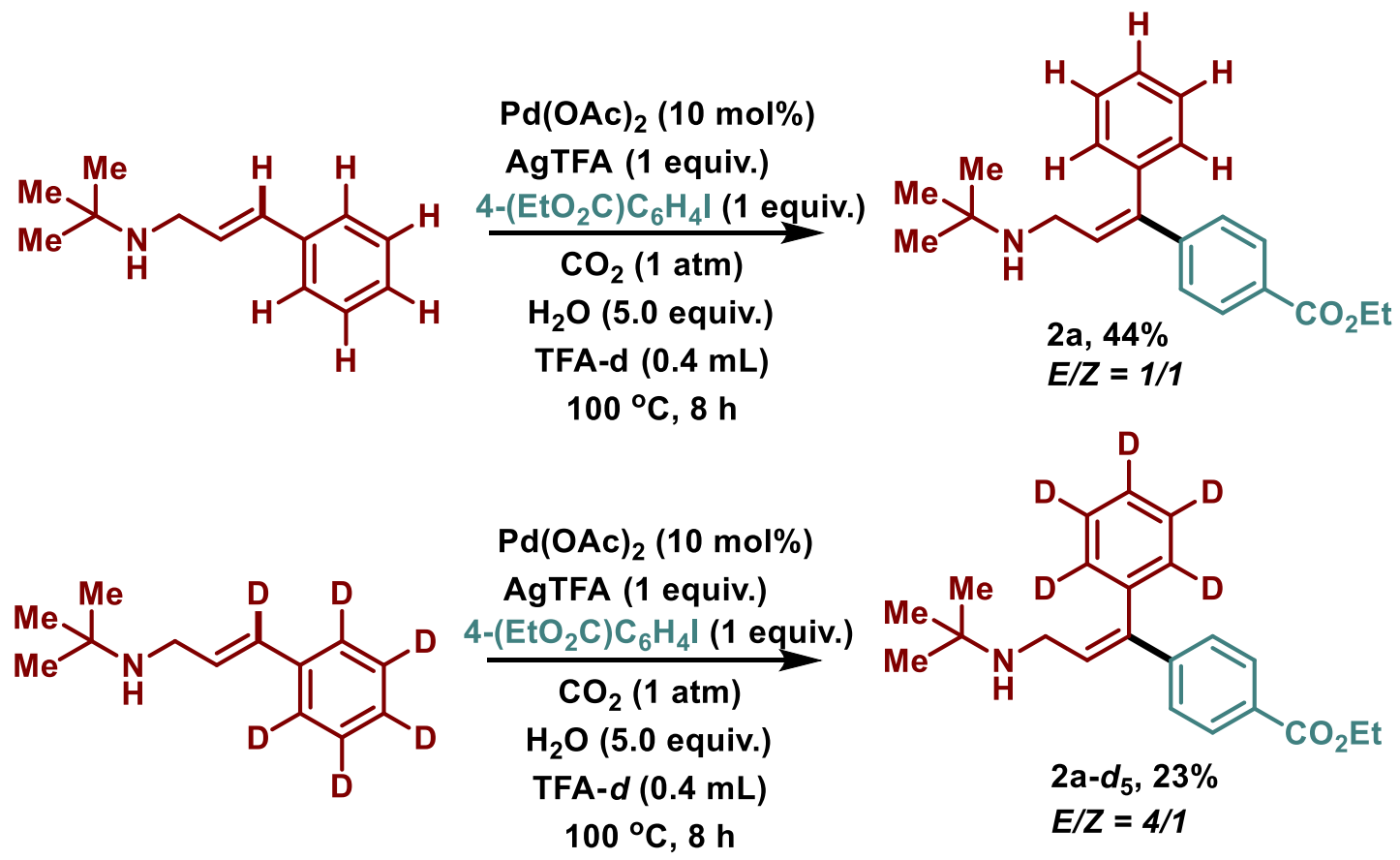

Kinetic Isotope Effect Study: An NMR tube was charged with $\mathrm{Pd}(\mathrm{OAc})_{2}(3.4 \mathrm{mg}, 0.15 \mathrm{mmol}, 0.10$ equiv), silver trifluoroacetate ( $33.1 \mathrm{mg}, 0.15 \mathrm{mmol}, 1$ equiv), ethyl 4-iodobenzoate ( $41.3 \mathrm{mg}, 0.15 \mathrm{mmol}, 1.0$ equiv), amine or deuterated amine ( $0.30 \mathrm{mmol}, 1.0$ equiv), trifluoroacetic acid $-d(0.6 \mathrm{~mL})$, water $(13 \mu \mathrm{L}, 0.75 \mathrm{mmol}, 5.0$ equiv) and 1,1,2,2-tetrachloroethane as internal standard followed by the slow addition of dry ice as ( 92 $\mathrm{mg}, 2.1 \mathrm{mmol}, 7$ equiv) $\mathrm{CO}_{2}$ source. The tube was immediately sealed with a standard NMR cap and shaken at room temperature for 15 min. The reaction mixture was heated in an oil bath at $100{ }^{\circ} \mathrm{C}$ for $8 \mathrm{~h}$. After cooling to room temperature, crude ${ }^{1} \mathrm{H}$ NMR was performed on the reaction mixture.

\section{G. Observing Carbamate Formation}

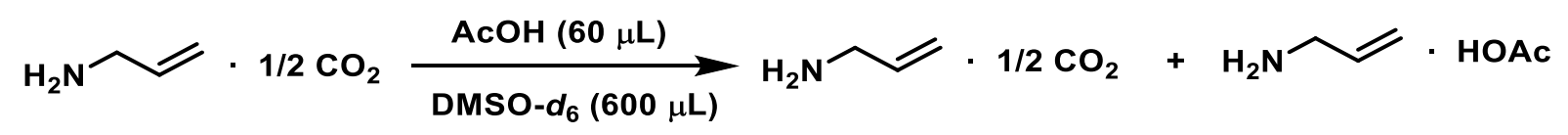

Procedure: Allylamine was added to a standard $20 \mathrm{~mL}$ scintillation vial, and dry ice (powdered) was added and stirred until fully solidified. The mixture was stirred, and then left to sit until all of the dry ice had sublimed. The carbamate salt was observed to be stable, although it evaporated/sublimed when left out overnight. Approximately $25 \mathrm{mg}$ of the salt was added to DMSO- $d_{6}$, and the ${ }^{1} \mathrm{H}$ and ${ }^{13} \mathrm{C}$ NMR data were collected. The flask was then treated with AcOH (60 $\mu \mathrm{L}$ ), followed by retaking of the NMR spectra. Together these data show that even in the presence of $\sim 3$ equivalents of $\mathrm{AcOH}$, the carbamate of the allylamine is still accessible, even at only ambient pressure.

\section{H. Filtration test}



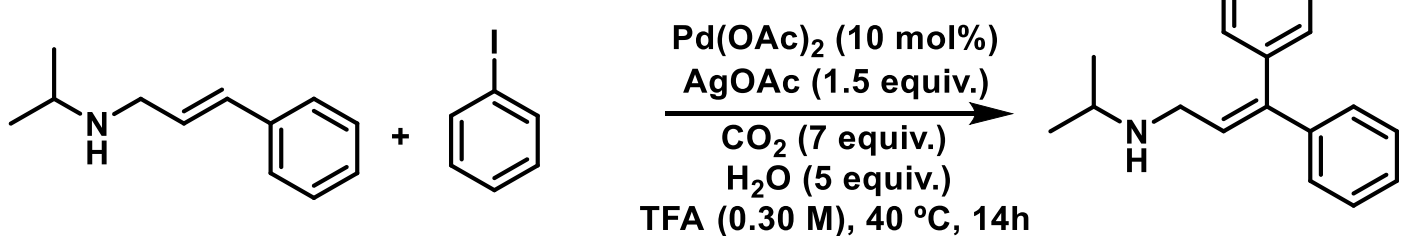

A $7.5 \mathrm{~mL}$ vial was charged with $\mathrm{Pd}(\mathrm{OAc})_{2}(6.7 \mathrm{mg}, 0.03 \mathrm{mmol}, 0.10$ equiv), silver acetate $(75.1 \mathrm{mg}, 0.45 \mathrm{mmol}, 1.5$ equiv), organohalide ( $0.60 \mathrm{mmol}, 2.0$ equiv), amine $(0.30 \mathrm{mmol}, 1.0$ equiv), trifluoroacetic acid $(1.0 \mathrm{~mL})$, and water ( $27 \mu \mathrm{L}, 1.5 \mathrm{mmol}, 5.0$ equiv) followed by the addition of dry ice as ( $92 \mathrm{mg}, 2.1 \mathrm{mmol}, 7$ equiv) $\mathrm{CO}_{2}$ source. The vial was sealed with a PTFE lined cap and stirred at room temperature for $15 \mathrm{~min}$. After 15 min of stirring, the reaction mixture was heated in a pie-block at $40{ }^{\circ} \mathrm{C}$ under stirring for $4 \mathrm{~h}$. After cooling to room temperature, the reaction was then filtered through celite, washed with trifluoroacetic acid, evaporated in vacuo, and the ${ }^{1} \mathrm{H}$ NMR taken using dimethyl isophthalate as an internal standard. 12\% NMR yield (average of three reactions) of the $\mathrm{C}-\mathrm{H}$ arylation product was observed. Filtrate and solid residue were then used for further reactions.

Reaction with filtrate - A $7.5 \mathrm{~mL}$ vial was charged with above filtrate, silver acetate $(75.1 \mathrm{mg}, 0.45 \mathrm{mmol}, 1.5$ equiv), organohalide ( $0.60 \mathrm{mmol}, 2.0$ equiv), amine $(0.30 \mathrm{mmol}, 1.0$ equiv), trifluoroacetic acid $(1.0 \mathrm{~mL})$, and water $(27 \mu \mathrm{L}$, $1.5 \mathrm{mmol}, 5.0$ equiv) followed by the addition of dry ice as ( $\sim 2 \mathrm{mg}, 2.1 \mathrm{mmol}, 7$ equiv) $\mathrm{CO}_{2}$ source. The vial was sealed with a PTFE lined cap and stirred at room temperature for $15 \mathrm{~min}$. After $15 \mathrm{~min}$ of stirring, the reaction mixture was heated in a pie-block at $40{ }^{\circ} \mathrm{C}$ under stirring for $14 \mathrm{~h}$. After cooling to room temperature, the solvent was evaporated in vacuo and ${ }^{1} \mathrm{H}$ NMR taken using 1,1,2,2-tetrachloroethane as an internal standard. 18\% NMR yield of the $\mathrm{C}-\mathrm{H}$ arylation product was observed.

Reaction with residue - A $7.5 \mathrm{~mL}$ vial was charged with above residue, silver acetate $(75.1 \mathrm{mg}, 0.45 \mathrm{mmol}, 1.5$ equiv), organohalide $(0.60 \mathrm{mmol}, 2.0$ equiv), amine $(0.30 \mathrm{mmol}, 1.0$ equiv), trifluoroacetic acid $(1.0 \mathrm{~mL})$, and water ( $27 \mu \mathrm{L}, 1.5 \mathrm{mmol}, 5.0$ equiv) followed by the addition of dry ice as ( $92 \mathrm{mg}, 2.1 \mathrm{mmol}, 7$ equiv) $\mathrm{CO}_{2}$ source. The vial was sealed with a PTFE lined cap and stirred at room temperature for $15 \mathrm{~min}$. After $15 \mathrm{~min}$ of stirring, the reaction mixture was heated in a pie-block at $40{ }^{\circ} \mathrm{C}$ under stirring for $14 \mathrm{~h}$. After cooling to room temperature, the solvent was evaporated in vacuo and ${ }^{1} \mathrm{H}$ NMR taken using 1,1,2,2-tetrachloroethane as an internal standard, no $\mathrm{C}-\mathrm{H}$ arylation product was observed.

\section{Mercury test}
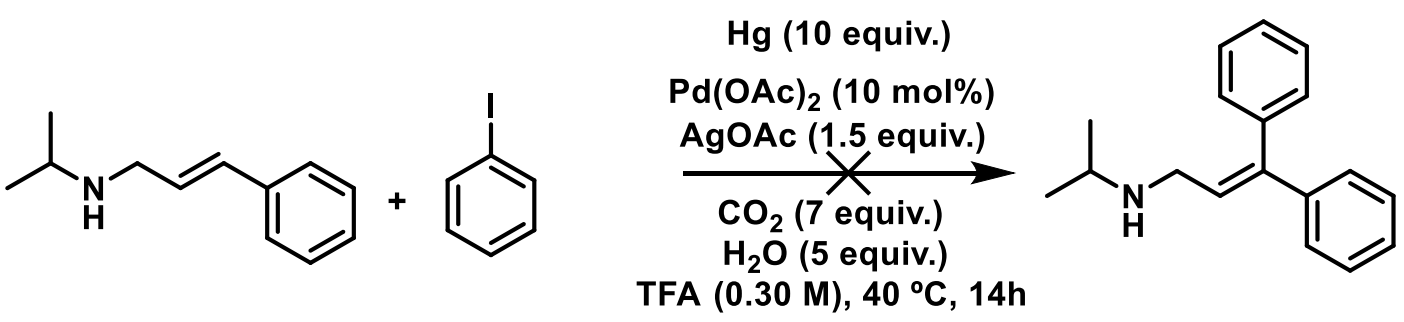
1) A $7.5 \mathrm{~mL}$ vial was charged with $\mathrm{Pd}(\mathrm{OAc})_{2}(6.7 \mathrm{mg}, 0.03 \mathrm{mmol}, 0.10$ equiv), silver acetate $(75.1 \mathrm{mg}, 0.45 \mathrm{mmol}$, 1.5 equiv), organohalide ( $0.60 \mathrm{mmol}, 2.0$ equiv), amine ( $0.30 \mathrm{mmol}, 1.0$ equiv), trifluoroacetic acid $(1.0 \mathrm{~mL})$, and water ( $27 \mu \mathrm{L}, 1.5 \mathrm{mmol}, 5.0$ equiv) followed by the addition of dry ice as ( $\sim 92 \mathrm{mg}, 2.1 \mathrm{mmol}, 7$ equiv) $\mathrm{CO}_{2}$ source. The vial was sealed with a PTFE lined cap and stirred at room temperature for $10 \mathrm{~min}$. Then $\mathrm{Hg}$ ( $3 \mathrm{mmol}, 10$ equiv) and dry ice as ( $\sim 92 \mathrm{mg}, 2.1 \mathrm{mmol}, 7$ equiv) $\mathrm{CO}_{2}$ source was added. After $15 \mathrm{~min}$ of stirring, the reaction mixture was heated in a pie-block at $40{ }^{\circ} \mathrm{C}$ under stirring for $14 \mathrm{~h}$. After cooling to room temperature, the solvent was evaporated in vacuo and ${ }^{1} \mathrm{H}$ NMR taken using 1,1,2,2-tetrachloroethane as an internal standard, no $\mathrm{C}-\mathrm{H}$ arylation product was observed.

2) A $7.5 \mathrm{~mL}$ vial was charged with $\mathrm{Pd}(\mathrm{OAc})_{2}(6.7 \mathrm{mg}, 0.03 \mathrm{mmol}, 0.10$ equiv), silver acetate $(75.1 \mathrm{mg}, 0.45 \mathrm{mmol}$, 1.5 equiv), organohalide ( $0.60 \mathrm{mmol}, 2.0$ equiv), amine ( $0.30 \mathrm{mmol}, 1.0$ equiv), trifluoroacetic acid $(1.0 \mathrm{~mL}), \mathrm{Hg}(3$ mmol, 10 equiv) and water ( $27 \mu \mathrm{L}, 1.5 \mathrm{mmol}, 5.0$ equiv) followed by the addition of dry ice as ( 92 mg, $2.1 \mathrm{mmol}, 7$ equiv) $\mathrm{CO}_{2}$ source. The vial was sealed with a PTFE lined cap and stirred at room temperature for 15 min. After 15 min of stirring, the reaction mixture was heated in a pie-block at $40{ }^{\circ} \mathrm{C}$ under stirring for $14 \mathrm{~h}$. After cooling to room temperature, the solvent was evaporated in vacuo and ${ }^{1} \mathrm{H}$ NMR taken using 1,1,2,2-tetrachloroethane as an internal standard, no $\mathrm{C}-\mathrm{H}$ arylation product was observed.

\section{J. Nanoparticle-Based Catalysis}

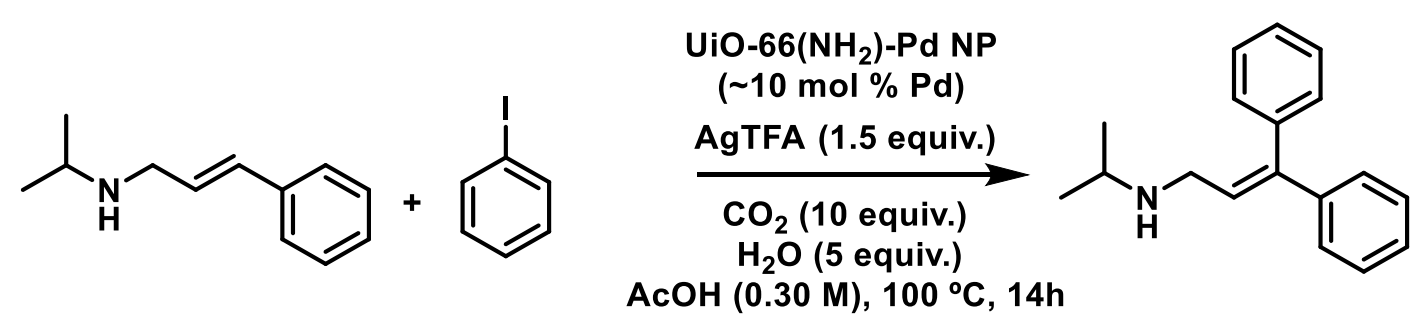

Preparation of UiO-66( $\left.\mathrm{NH}_{2}\right)$-Supported Palladium Nanoparticles: Based on a literature procedure, ${ }^{26}$ UiO$66\left(\mathrm{NH}_{2}\right)(60 \mathrm{mg})$ prepared by a known method ${ }^{27}$ was combined with $\mathrm{Pd}(\mathrm{OAc})_{2}(13.4 \mathrm{mg}, 0.06 \mathrm{mmol})$ in a plastic centrifuge tube in methanol $(5 \mathrm{~mL})$, and the suspension was placed in an ultrasonification bath for $2 \mathrm{~h}$. Sodium borohydride (30 mg, $0.79 \mathrm{mmol}$ ) was dissolved in $5 \mathrm{~mL} \mathrm{MeOH}$, and added dropwise, giving an immediate black color to the solution. This was then centrifuged, the solvent decanted, and three cycles of $\mathrm{MeOH}$ addition, $1 \mathrm{~h}$ incubation, and decanting of the solvent were performed. The subsequently dried $\mathrm{UiO}-66\left(\mathrm{NH}_{2}\right)-\mathrm{Pd}$ nanoparticle mixture was then used in the next step.

Preparation of UiO-66( $\left.\mathrm{NH}_{2}\right)$-Supported Palladium Nanoparticles: The Pd nanoparticle-functionalized metalorganic framework above prepared was suspended in $\mathrm{AcOH}(2 \mathrm{~mL})$, followed by adding to two separate $7.5 \mathrm{~mL}$ vials, which were subsequently charged with silver trifluoroacetate $(99.4 \mathrm{mg}, 0.45 \mathrm{mmol}, 1.5$ equiv), iodobenzene (122.5 $\mathrm{mg}, 0.60 \mathrm{mmol}, 2.0$ equiv), $N$-isopropyl cinnamylamine ( $52.6 \mathrm{mg}, 0.30 \mathrm{mmol}, 1.0$ equiv), water ( $27 \mu \mathrm{L}, 1.5 \mathrm{mmol}$, 5.0 equiv) followed by the addition of dry ice ( $132 \mathrm{mg}, 3.0 \mathrm{mmol}, 10$ equiv). The vials were sealed with a PTFE lined cap and stirred at room temperature for $15 \mathrm{~min}$. After $15 \mathrm{~min}$ of stirring, the reaction mixtures were heated in a pieblock at $100{ }^{\circ} \mathrm{C}$ under stirring for $14 \mathrm{~h}$. After cooling to room temperature, the solvent was evaporated in vacuo and 
${ }^{1} \mathrm{H}$ NMR taken using 1,1,2,2-tetrachloroethane as an internal standard, showing an average of $48 \%$ yield of product and $14 \%$ recovered starting material between the two reactions.

K. Investigation of Allylamine $\gamma$-Heterodiarylation

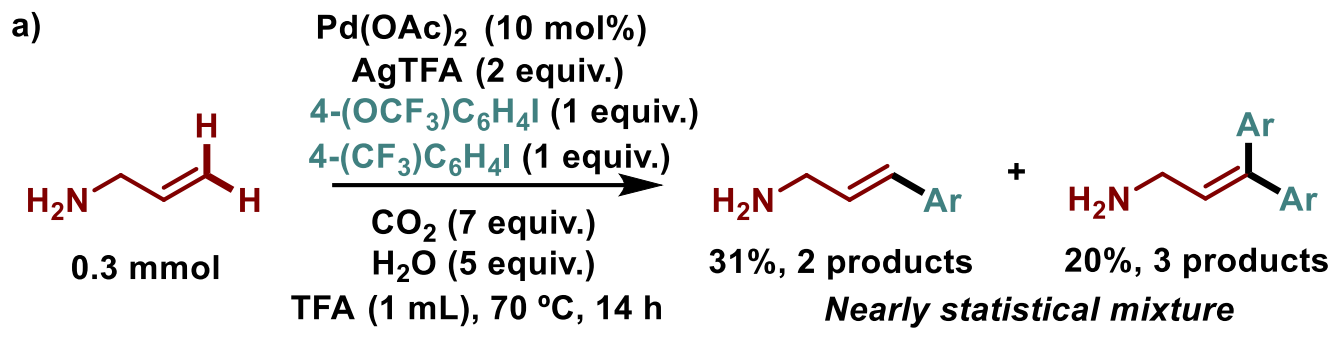

Heterodiarylation via Synchronous Addition: A $7.5 \mathrm{~mL}$ vial was charged with $\mathrm{Pd}(\mathrm{OAc})_{2}(6.7 \mathrm{mg}, 0.03 \mathrm{mmol}, 0.10$ equiv), silver trifluoroacetate ( $132.5 \mathrm{mg}, 0.6 \mathrm{mmol}, 2$ equiv), 1-iodo-4-(trifluoromethyl)benzene $(81.5 \mathrm{mg}, 0.30 \mathrm{mmol}$, 1.0 equiv), 1-iodo-4-(trifluoromethoxy)benzene ( $86.3 \mathrm{mg}, 0.30 \mathrm{mmol}, 1.0$ equiv), allyl amine ( $0.30 \mathrm{mmol}, 1.0$ equiv), trifluoroacetic acid $(1.0 \mathrm{~mL})$, and water $(27 \mu \mathrm{L}, 1.5 \mathrm{mmol}, 5.0$ equiv) followed by the addition of dry ice as ( $92 \mathrm{mg}$, $2.1 \mathrm{mmol}, 7$ equiv) $\mathrm{CO}_{2}$ source. The vial was immediately sealed with a PTFE lined cap and stirred at room temperature for $15 \mathrm{~min}$. After $15 \mathrm{~min}$ of stirring, the reaction mixture was heated in a pie-block at $70{ }^{\circ} \mathrm{C}$ under stirring for $18 \mathrm{~h}$. After cooling to room temperature, the reaction mixture was made basic with ammonium hydroxide solution up to $\mathrm{pH}$ $=8$. The reaction mixture was stirred for 15 minutes followed by extraction with dichloromethane $(3 \times 10 \mathrm{~mL})$. The organic layer was washed with brine and dried over $\mathrm{Na}_{2} \mathrm{SO}_{4}$. The filtrate was concentrated in vacuo and crude ${ }^{1} \mathrm{H}$ NMR was performed on the reaction mixture using 1,1,2,2-tetrachloroethane as an internal standard. A mixture of mono and diarylation products were observed. The majority of the recovery was actually the monoarylation products, while a complex mixture of diarylation products were observed.

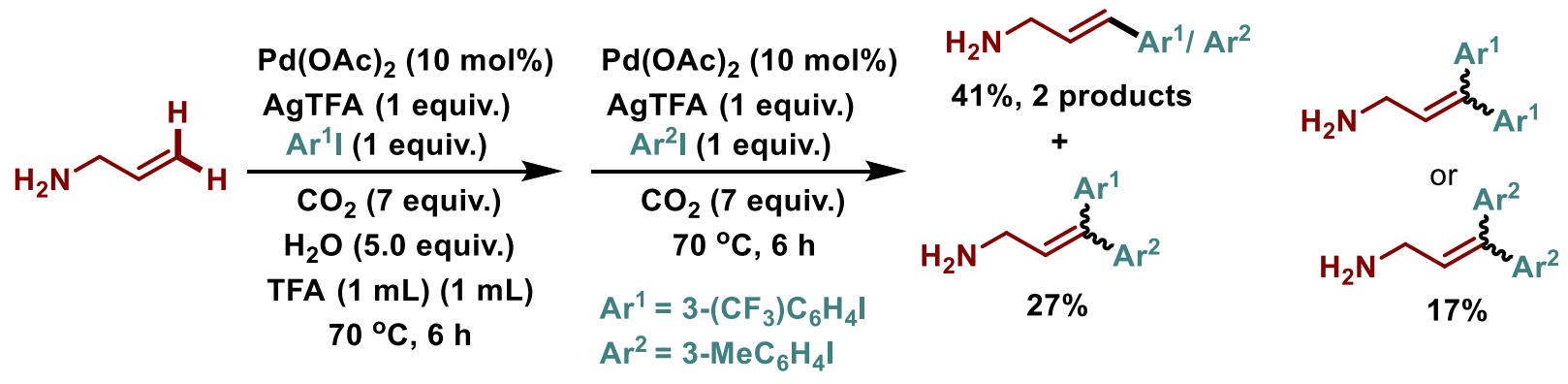

Heterodiarylation via Sequential Addition: A $7.5 \mathrm{~mL}$ vial was charged with $\mathrm{Pd}(\mathrm{OAc})_{2}(6.7 \mathrm{mg}, 0.03 \mathrm{mmol}, 0.10$ equiv), silver trifluoroacetate (66.2 mg, $0.3 \mathrm{mmol}, 1$ equiv), 1-iodo-3-(trifluoromethyl)benzene ( $81.5 \mathrm{mg}, 0.30 \mathrm{mmol}$, 1.0 equiv), allyl amine ( $0.30 \mathrm{mmol}, 1.0$ equiv), trifluoroacetic acid $(1.0 \mathrm{~mL})$, and water ( $27 \mu \mathrm{L}, 1.5 \mathrm{mmol}, 5.0$ equiv) followed by the addition of dry ice as ( $\sim 2 \mathrm{mg}, 2.1 \mathrm{mmol}, 7$ equiv) $\mathrm{CO}_{2}$ source. The vial was immediately sealed with a PTFE lined cap and stirred at room temperature for $15 \mathrm{~min}$. After $15 \mathrm{~min}$ of stirring, the reaction mixture was heated in a pie-block at $70{ }^{\circ} \mathrm{C}$ under stirring for $6 \mathrm{~h}$. After cooling to room temperature, vial was charged with $\mathrm{Pd}(\mathrm{OAc})_{2}(6.7$ $\mathrm{mg}, 0.03 \mathrm{mmol}, 0.10$ equiv), silver trifluoroacetate $(66.2 \mathrm{mg}, 0.6 \mathrm{mmol}, 1$ equiv), 1 -iodo-3-methylbenzene ( $65.3 \mathrm{mg}$, 
$0.30 \mathrm{mmol}, 1.0$ equiv), followed by the addition of dry ice as ( $\sim 2 \mathrm{mg}, 2.1 \mathrm{mmol}, 7$ equiv) $\mathrm{CO}_{2}$ source. The vial was immediately sealed with a PTFE lined cap and stirred at room temperature for $15 \mathrm{~min}$. After $15 \mathrm{~min}$ of stirring, the reaction mixture was heated in a pie-block at $70{ }^{\circ} \mathrm{C}$ under stirring for $6 \mathrm{~h}$. After cooling to room temperature, the reaction mixture was made basic with ammonium hydroxide solution up to $\mathrm{pH}=8$. The reaction mixture was stirred for 15 minutes followed by extraction with dichloromethane $(3 \times 10 \mathrm{~mL})$. The organic layer was washed with brine and dried over $\mathrm{Na}_{2} \mathrm{SO}_{4}$. The filtrate was concentrated in vacuo and crude ${ }^{1} \mathrm{H}$ NMR was performed on the reaction mixture using 1,1,2,2-tetrachloroethane as an internal standard. The majority of the recovery was actually the monoarylation products, while a complex mixture of diarylation products were observed. 
10. ${ }^{1} \mathrm{H}$ and ${ }^{13} \mathrm{C}$ Spectra of New Compounds

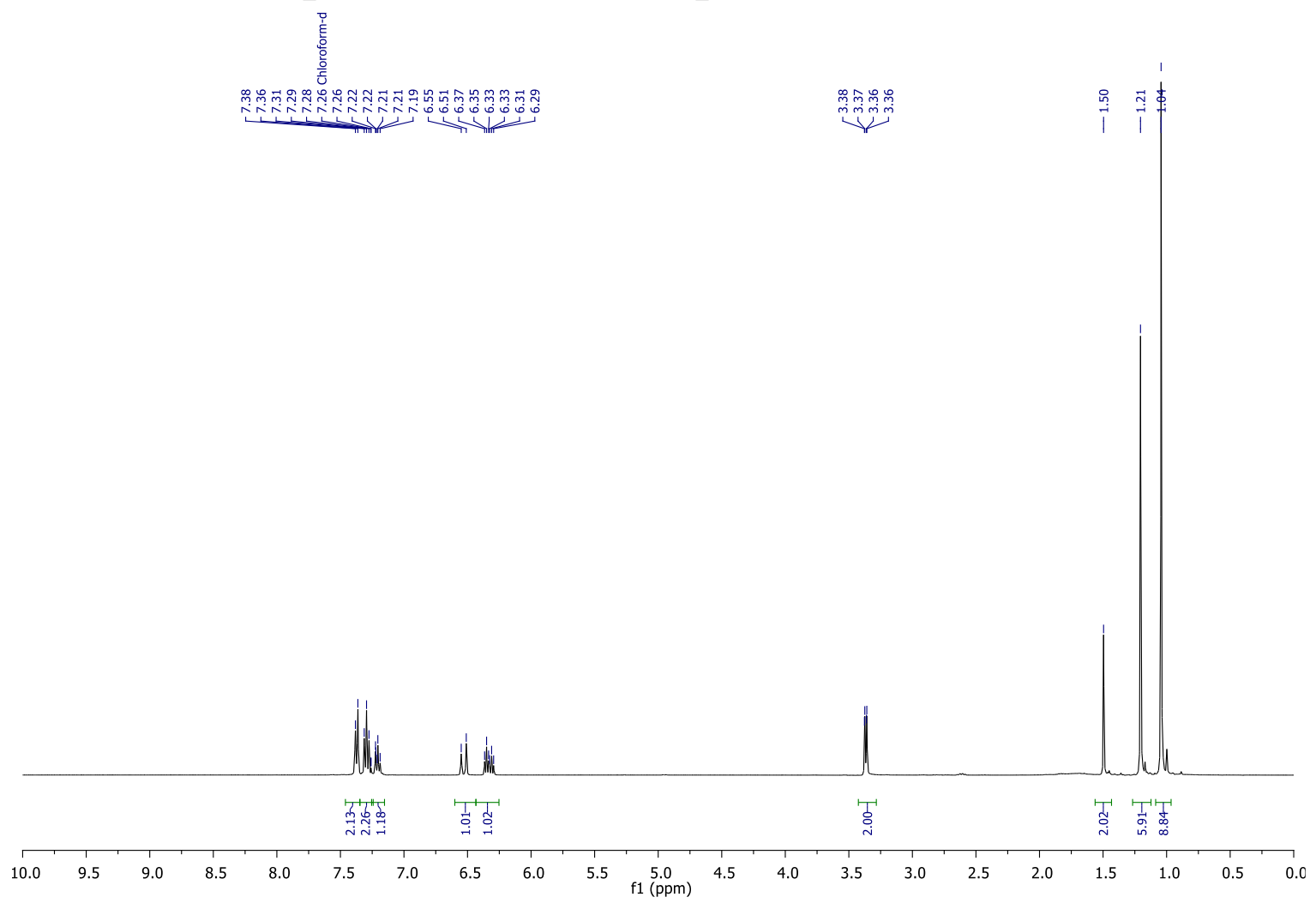

Figure S-1. ${ }^{1} \mathrm{H}$ NMR spectrum of $\mathbf{S - 2}\left(\mathrm{CDCl}_{3}, 400 \mathrm{MHz}, 298 \mathrm{~K}\right)$

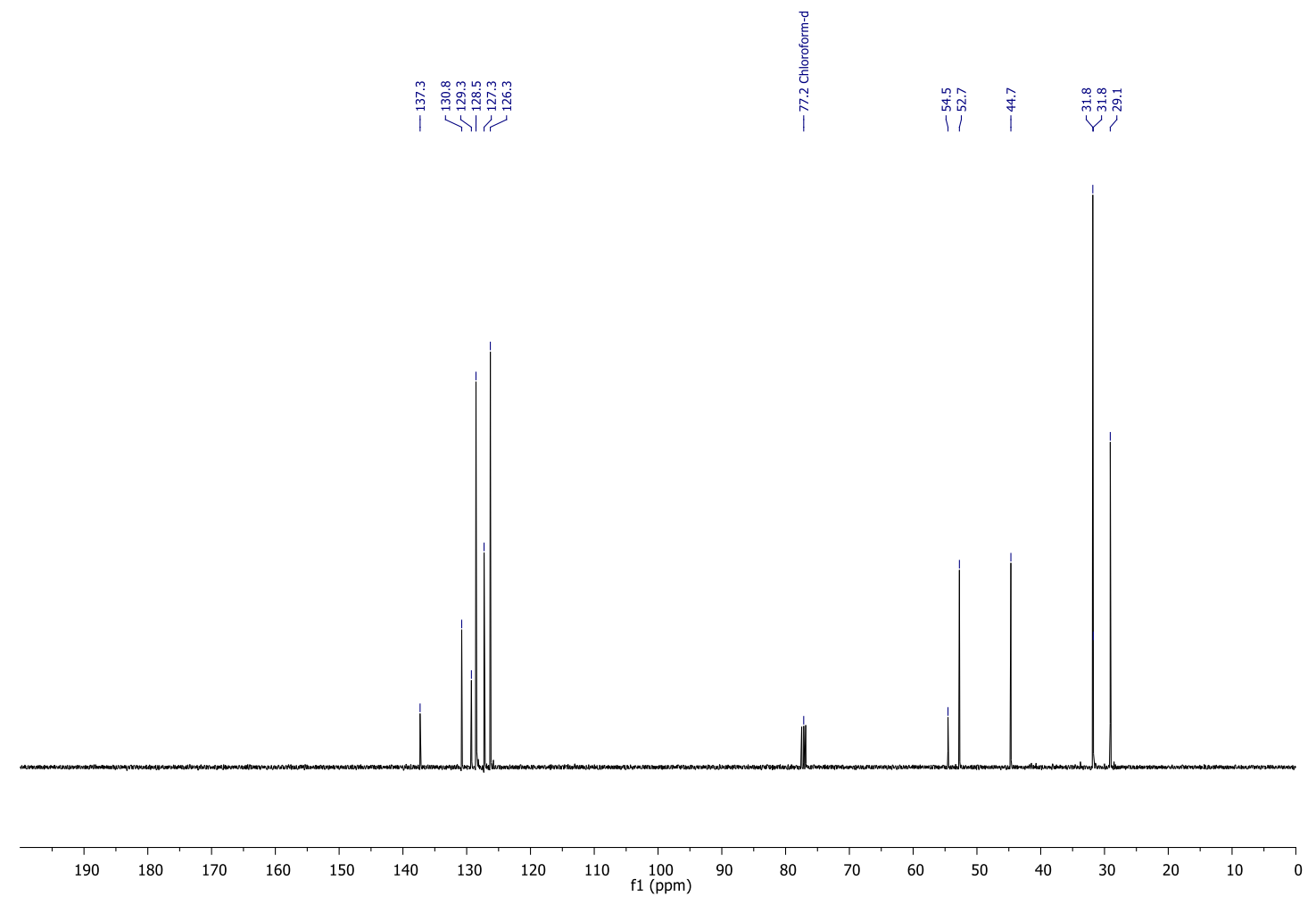

Figure S-2. ${ }^{13} \mathrm{C}$ NMR spectrum of $\mathbf{S}-2\left(\mathrm{CDCl}_{3}, 101 \mathrm{MHz}, 298 \mathrm{~K}\right)$ 


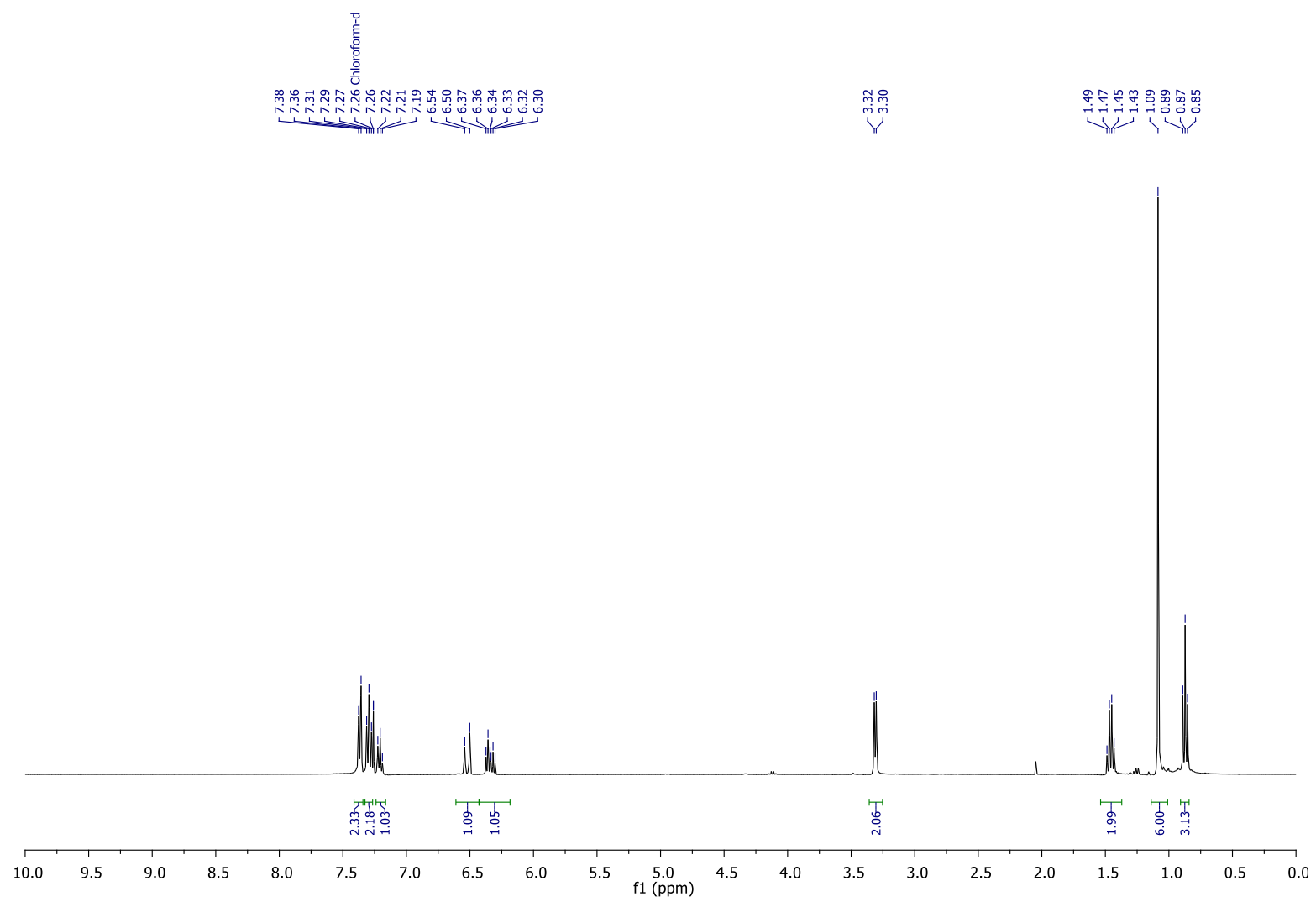

Figure S-3. ${ }^{1} \mathrm{H}$ NMR spectrum of $\mathbf{S - 3}\left(\mathrm{CDCl}_{3}, 600 \mathrm{MHz}, 298 \mathrm{~K}\right)$
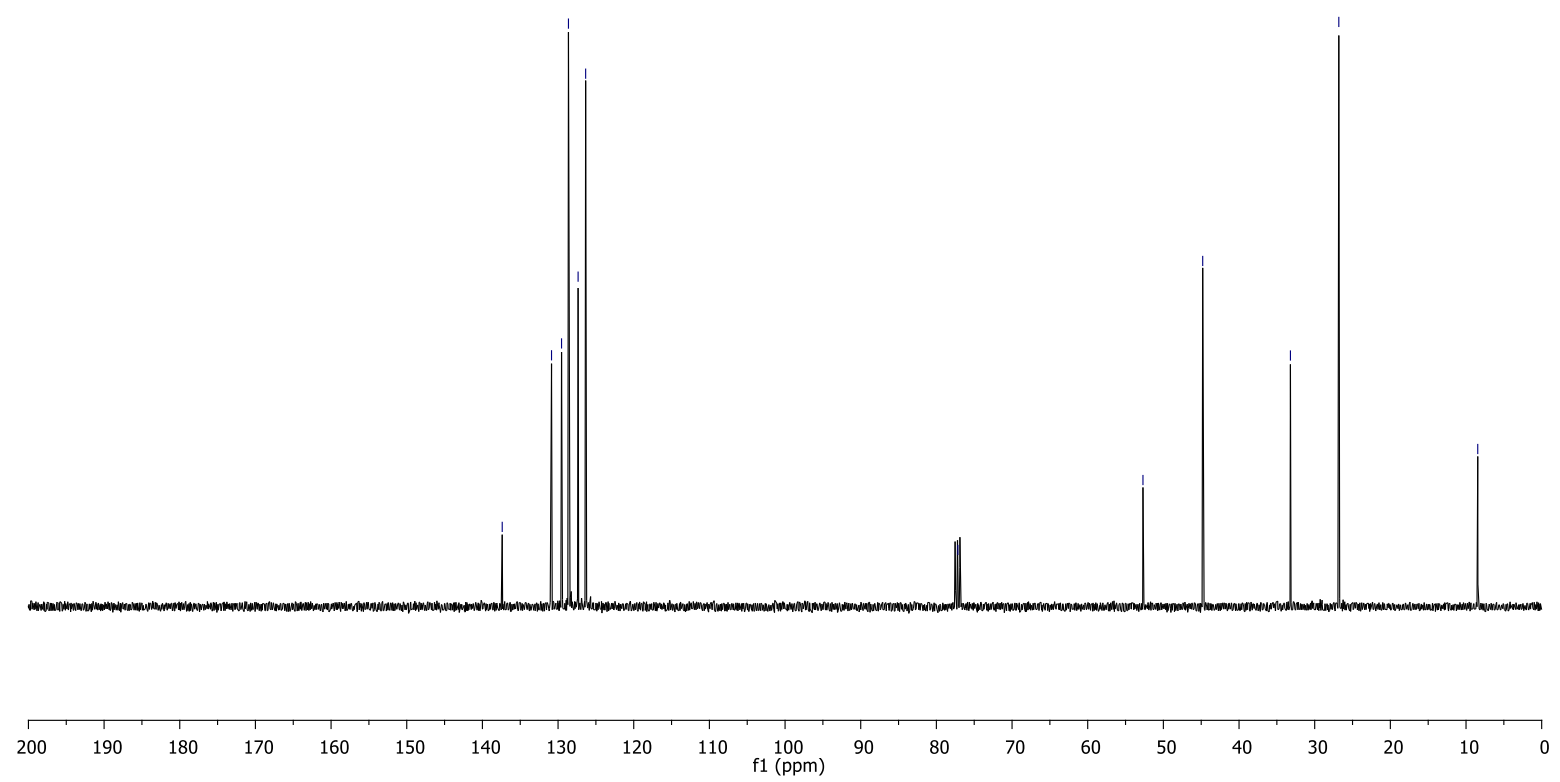

Figure S-4. ${ }^{13} \mathrm{C}$ NMR spectrum of $\mathbf{S - 3}\left(\mathrm{CDCl}_{3}, 151 \mathrm{MHz}, 298 \mathrm{~K}\right)$ 


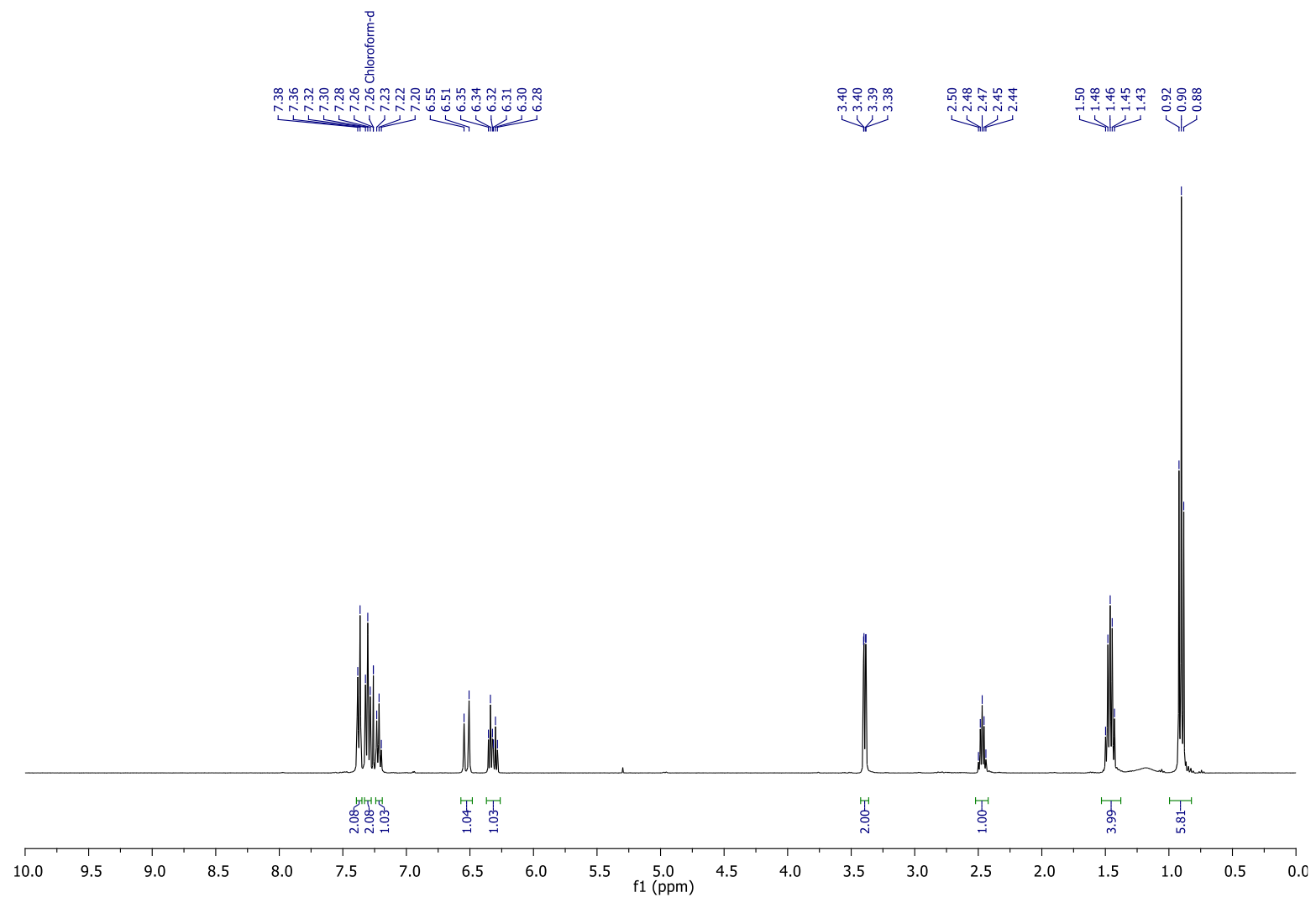

Figure S-5. ${ }^{1} \mathrm{H}$ NMR spectrum of $\mathbf{S - 5}\left(\mathrm{CDCl}_{3}, 400 \mathrm{MHz}, 298 \mathrm{~K}\right)$
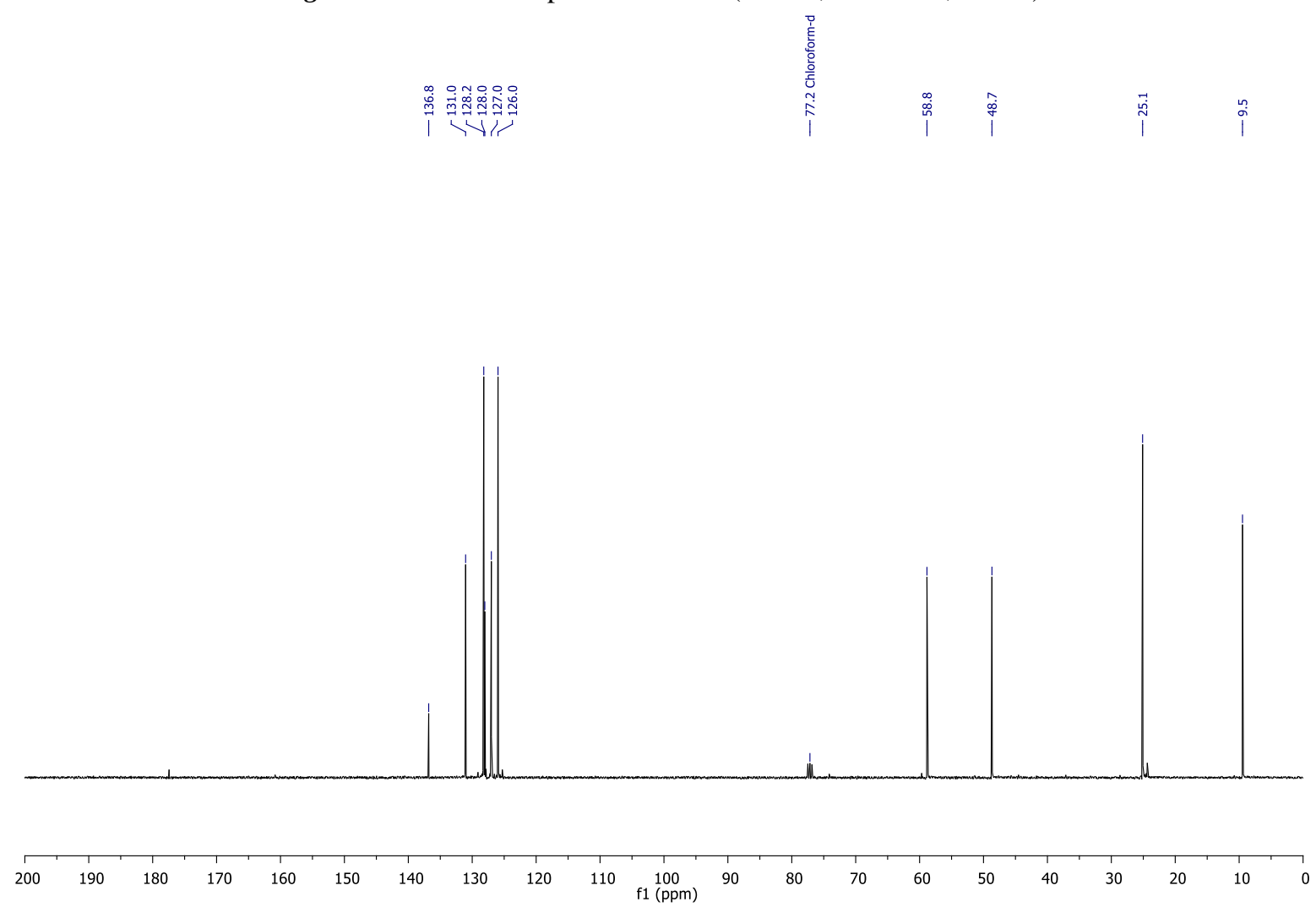

Figure S-6. ${ }^{13} \mathrm{C}$ NMR spectrum of $\mathbf{S - 5}\left(\mathrm{CDCl}_{3}, 101 \mathrm{MHz}, 298 \mathrm{~K}\right)$ 

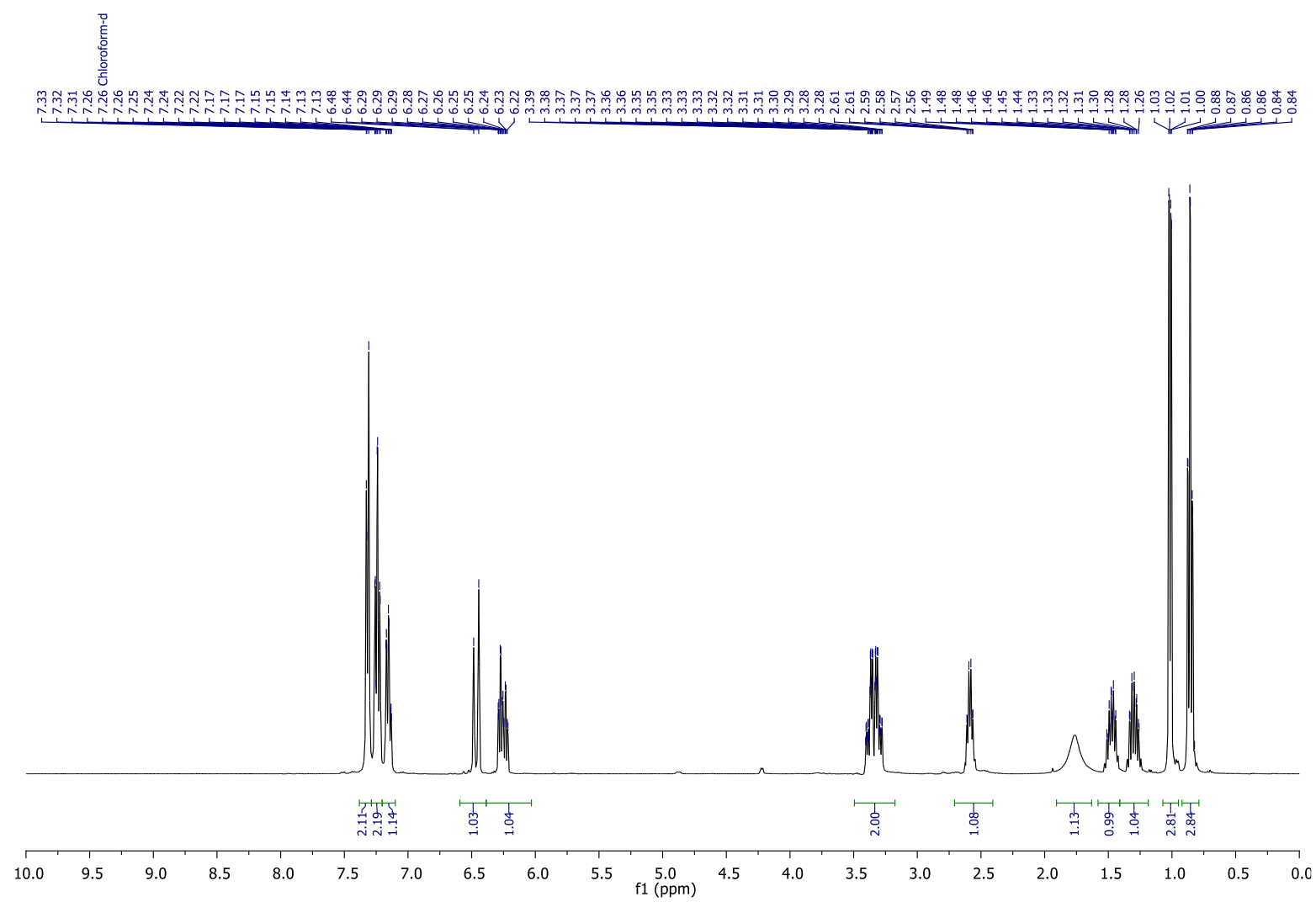

Figure S-7. ${ }^{1} \mathrm{H}$ NMR spectrum of $\mathbf{S - 6}\left(\mathrm{CDCl}_{3}, 400 \mathrm{MHz}, 298 \mathrm{~K}\right)$

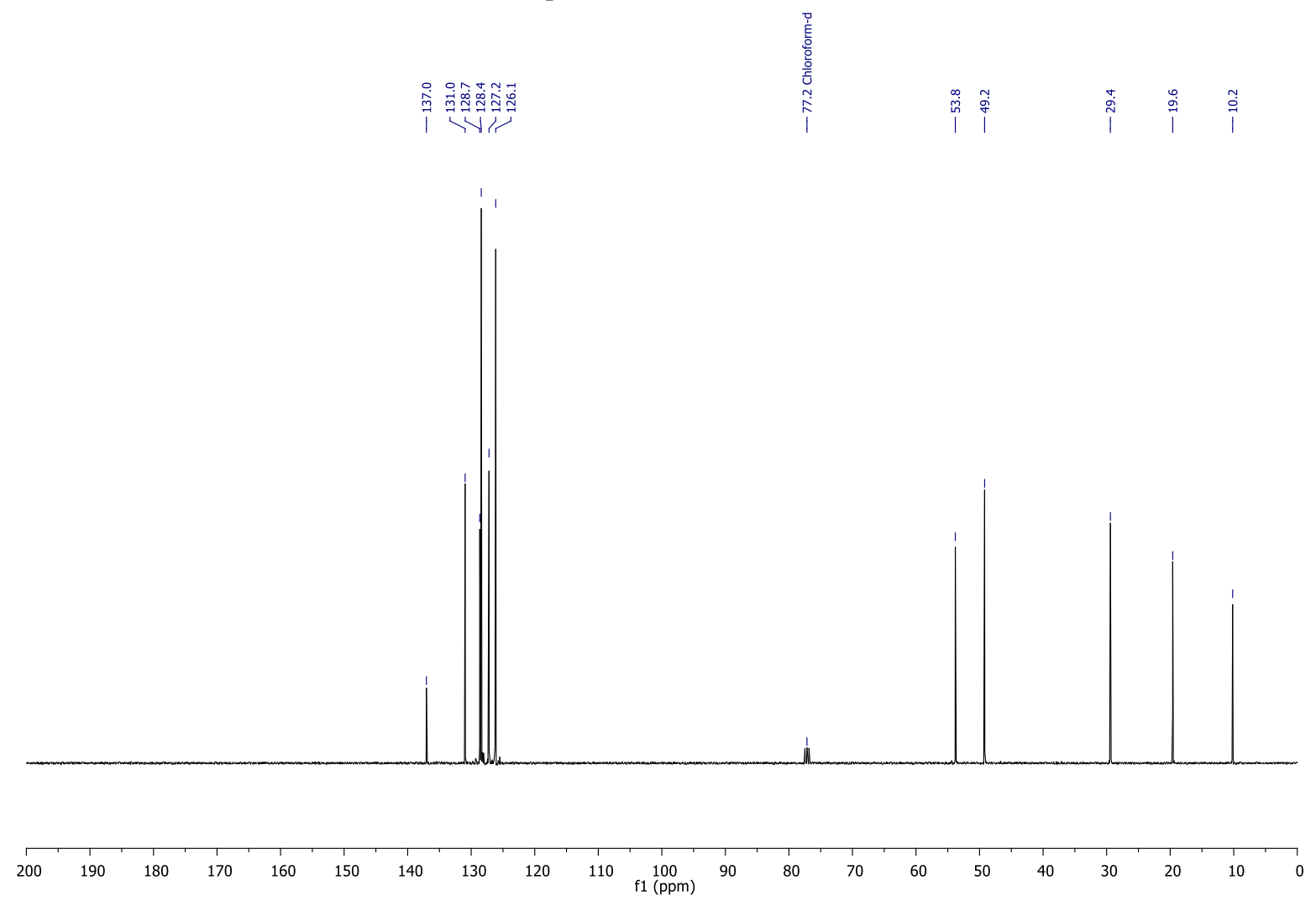

Figure S-8. ${ }^{13} \mathrm{C}$ NMR spectrum of $\mathbf{S - 6}\left(\mathrm{CDCl}_{3}, 101 \mathrm{MHz}, 298 \mathrm{~K}\right)$ 


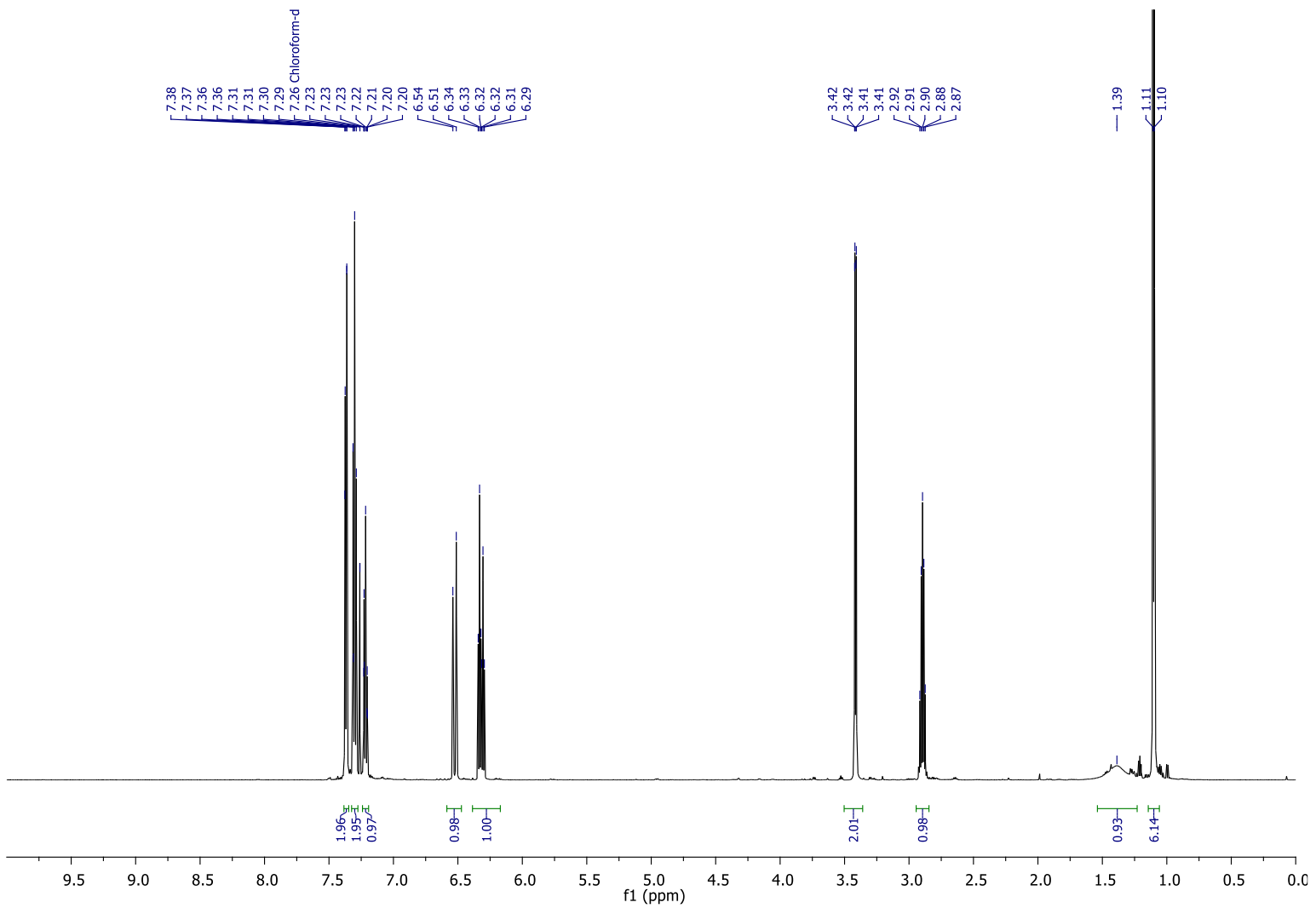

Figure S-9. ${ }^{1} \mathrm{H}$ NMR spectrum of $\mathbf{S}-\mathbf{7}\left(\mathrm{CDCl}_{3}, 600 \mathrm{MHz}, 298 \mathrm{~K}\right)$
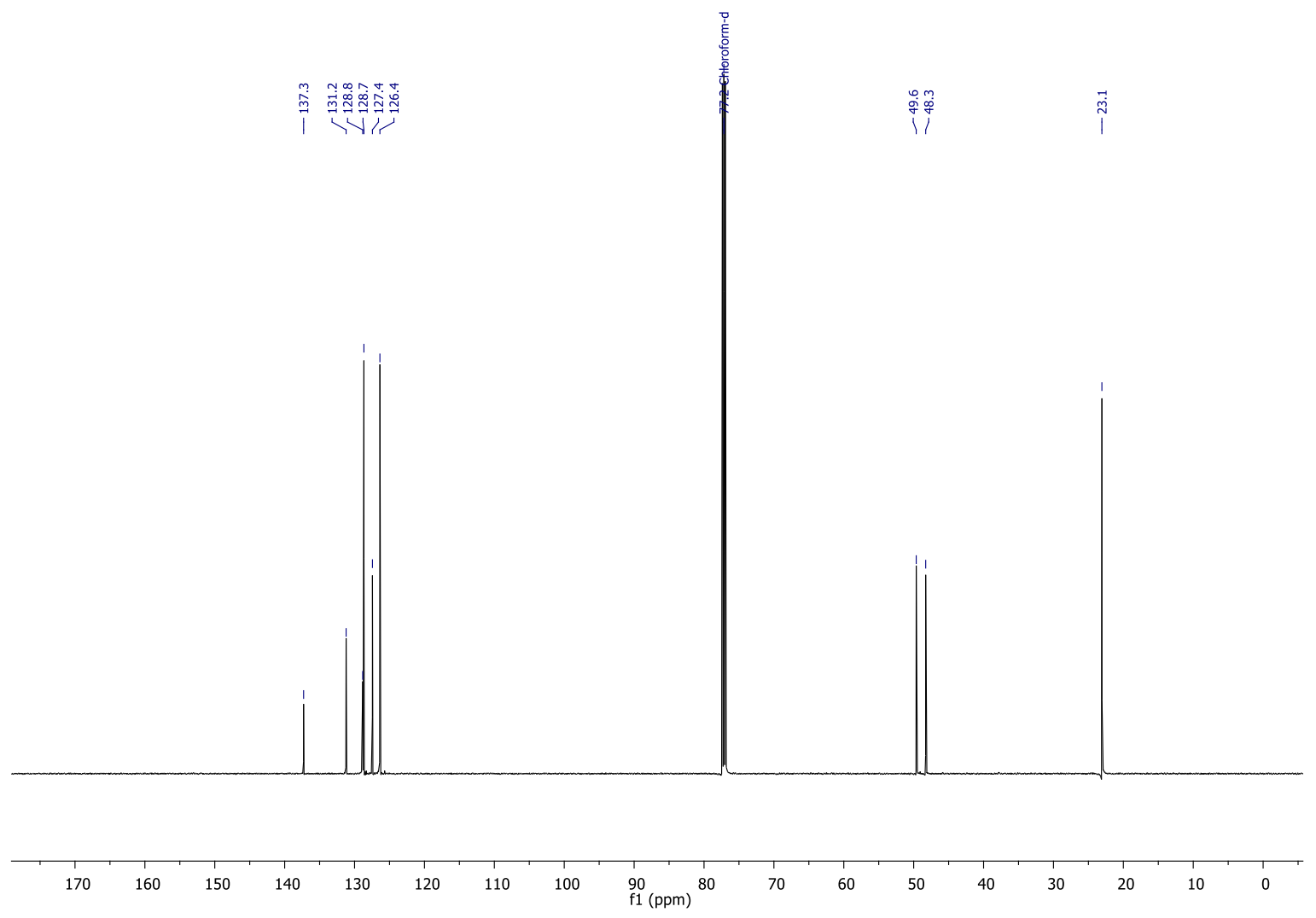

Figure S-10. ${ }^{13} \mathrm{C}$ NMR spectrum of $\mathbf{S - 7}\left(\mathrm{CDCl}_{3}, 151 \mathrm{MHz}, 298 \mathrm{~K}\right)$ 


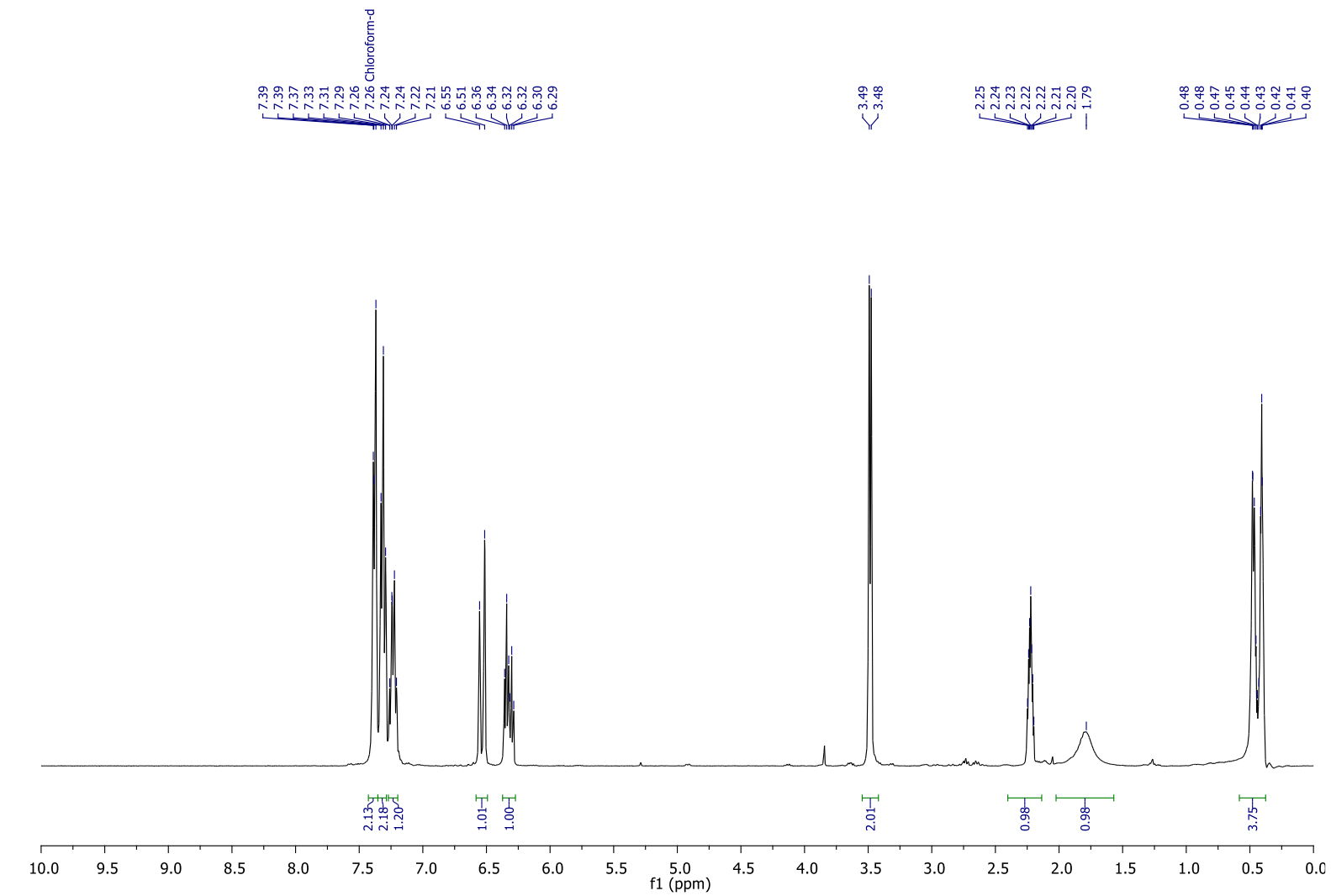

Figure S-11. ${ }^{1} \mathrm{H}$ NMR spectrum of $\mathbf{S - 9}\left(\mathrm{CDCl}_{3}, 400 \mathrm{MHz}, 298 \mathrm{~K}\right)$
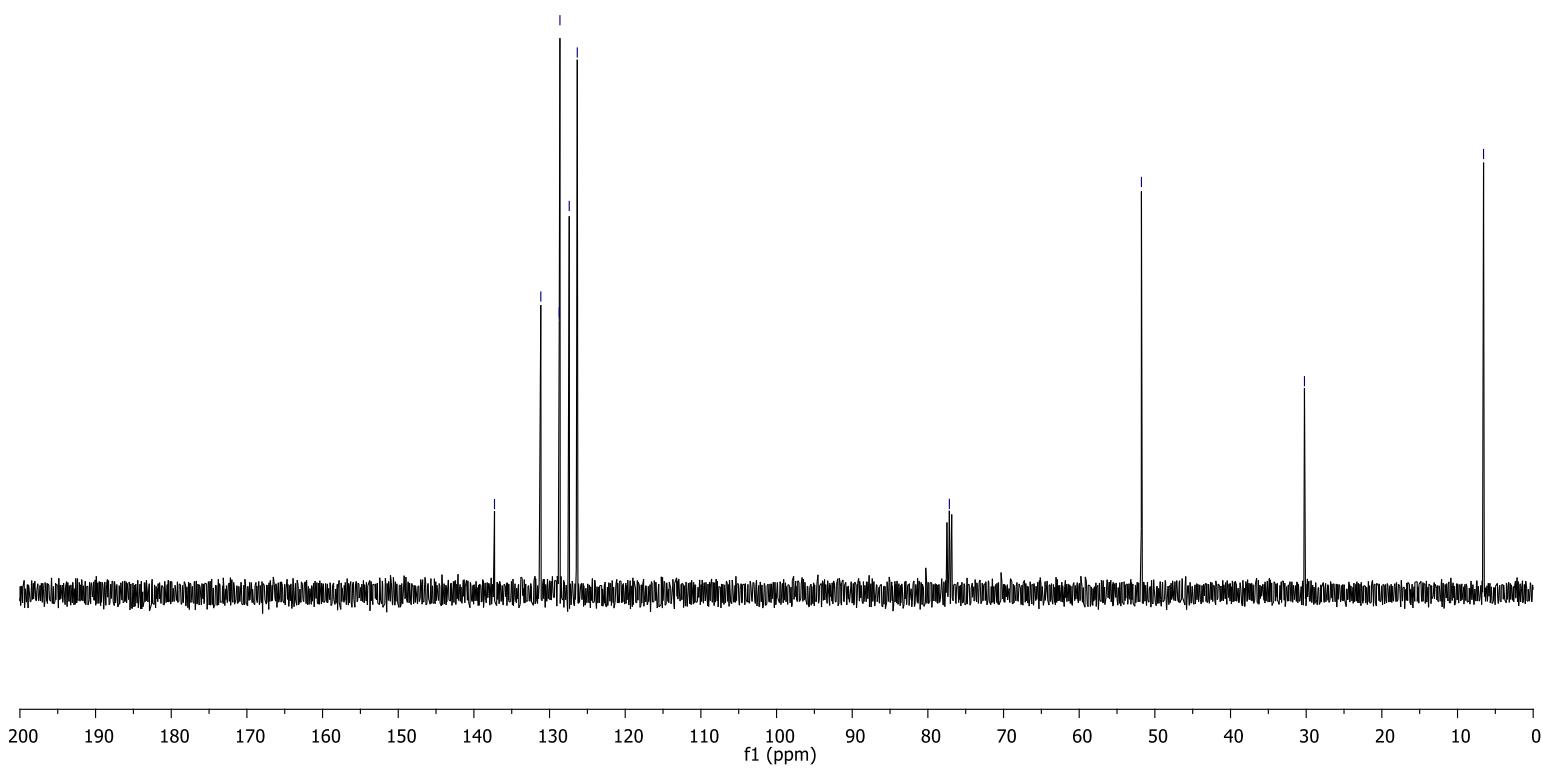

Figure $\mathbf{S}$-12. ${ }^{13} \mathrm{C}$ NMR spectrum of $\mathbf{S - 9}\left(\mathrm{CDCl}_{3}, 151 \mathrm{MHz}, 298 \mathrm{~K}\right)$ 

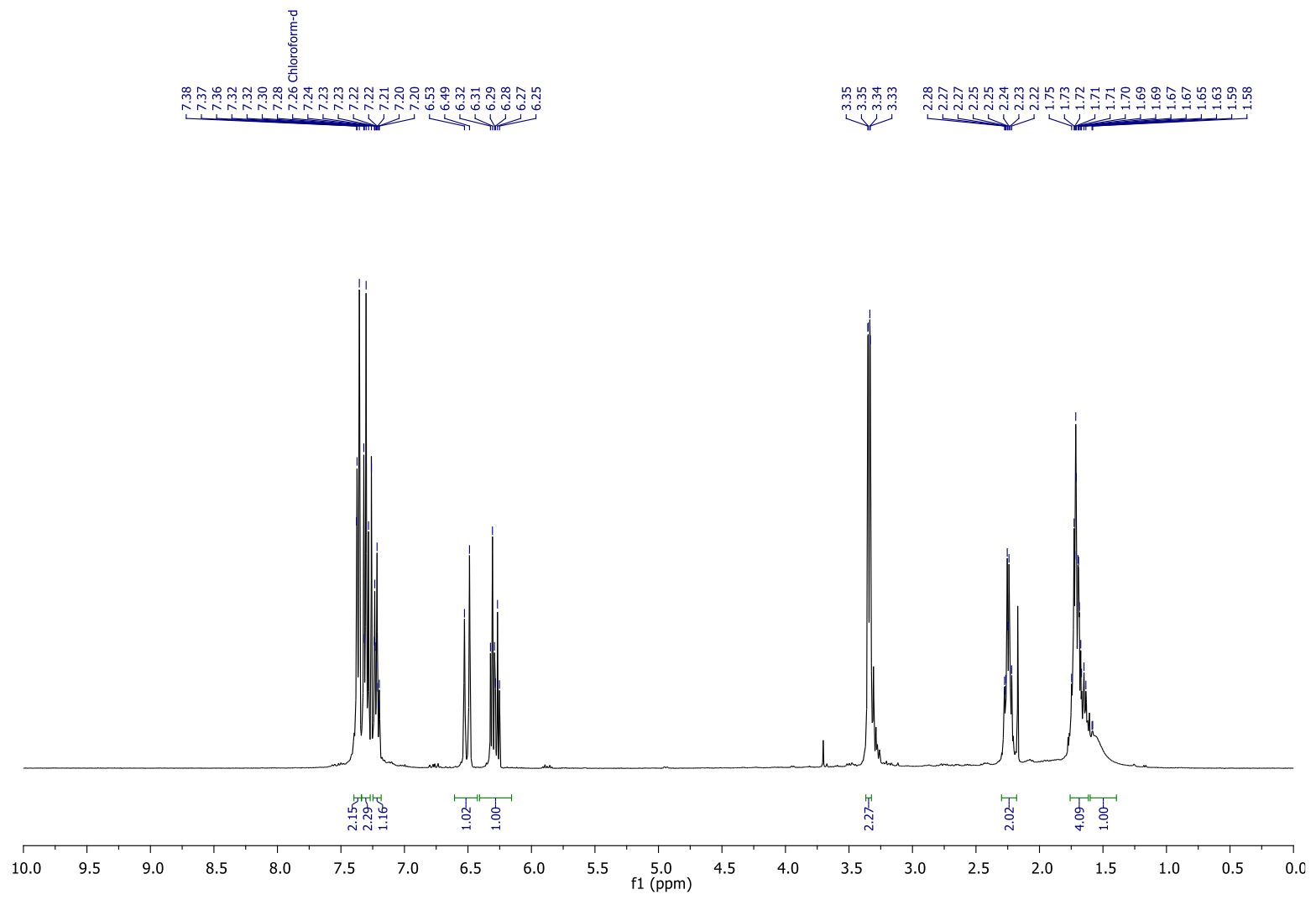

Figure S-13. ${ }^{1} \mathrm{H}$ NMR spectrum of $\mathbf{S - 1 0}\left(\mathrm{CDCl}_{3}, 600 \mathrm{MHz}, 298 \mathrm{~K}\right)$
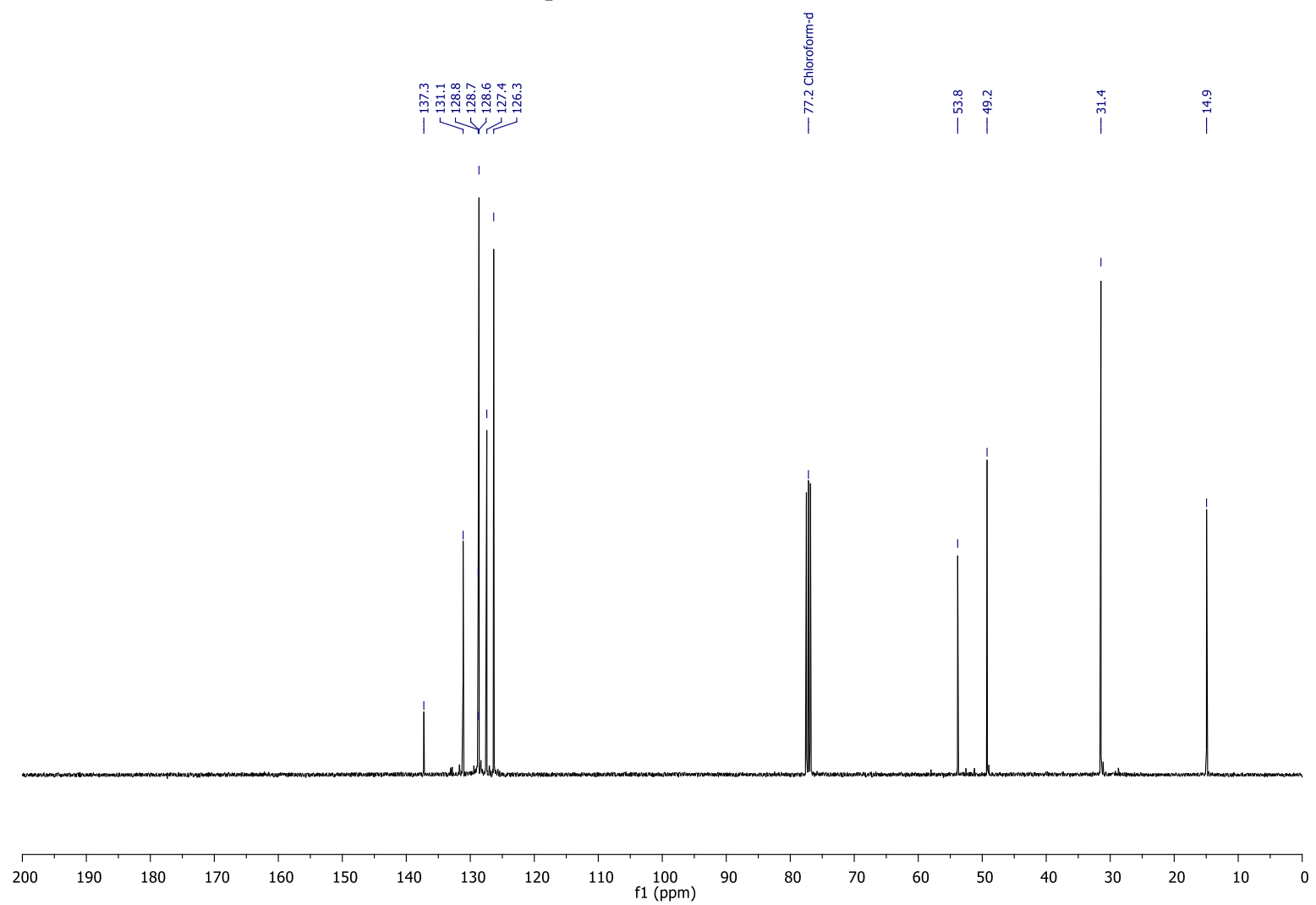

Figure $\mathbf{S}$-14. ${ }^{13} \mathrm{C}$ NMR spectrum of $\mathbf{S}-\mathbf{1 0}\left(\mathrm{CDCl}_{3}, 151 \mathrm{MHz}, 298 \mathrm{~K}\right)$ 


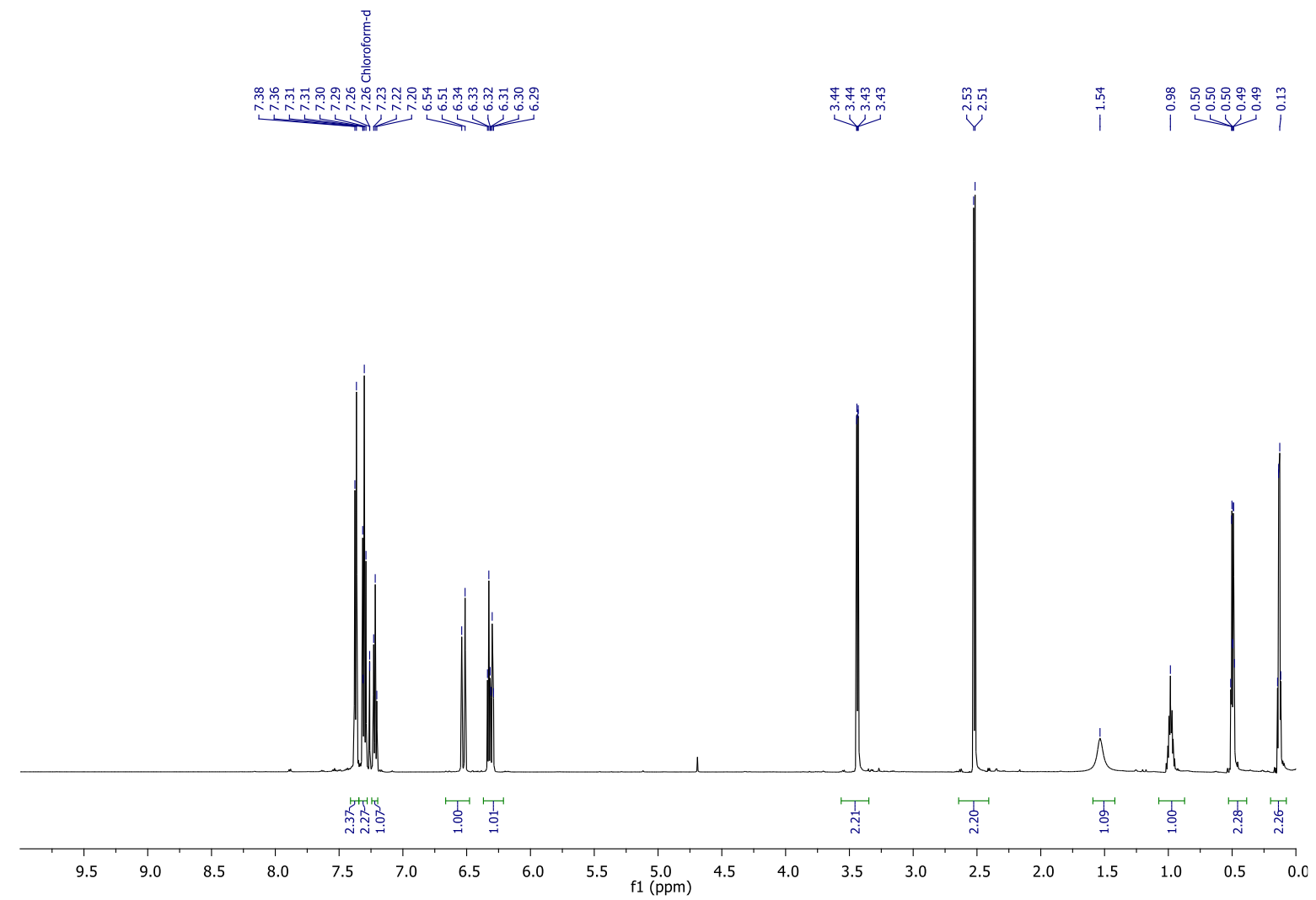

Figure $\mathbf{S}$-15. ${ }^{1} \mathrm{H}$ NMR spectrum of $\mathbf{S - 1 3}\left(\mathrm{CDCl}_{3}, 600 \mathrm{MHz}, 298 \mathrm{~K}\right)$
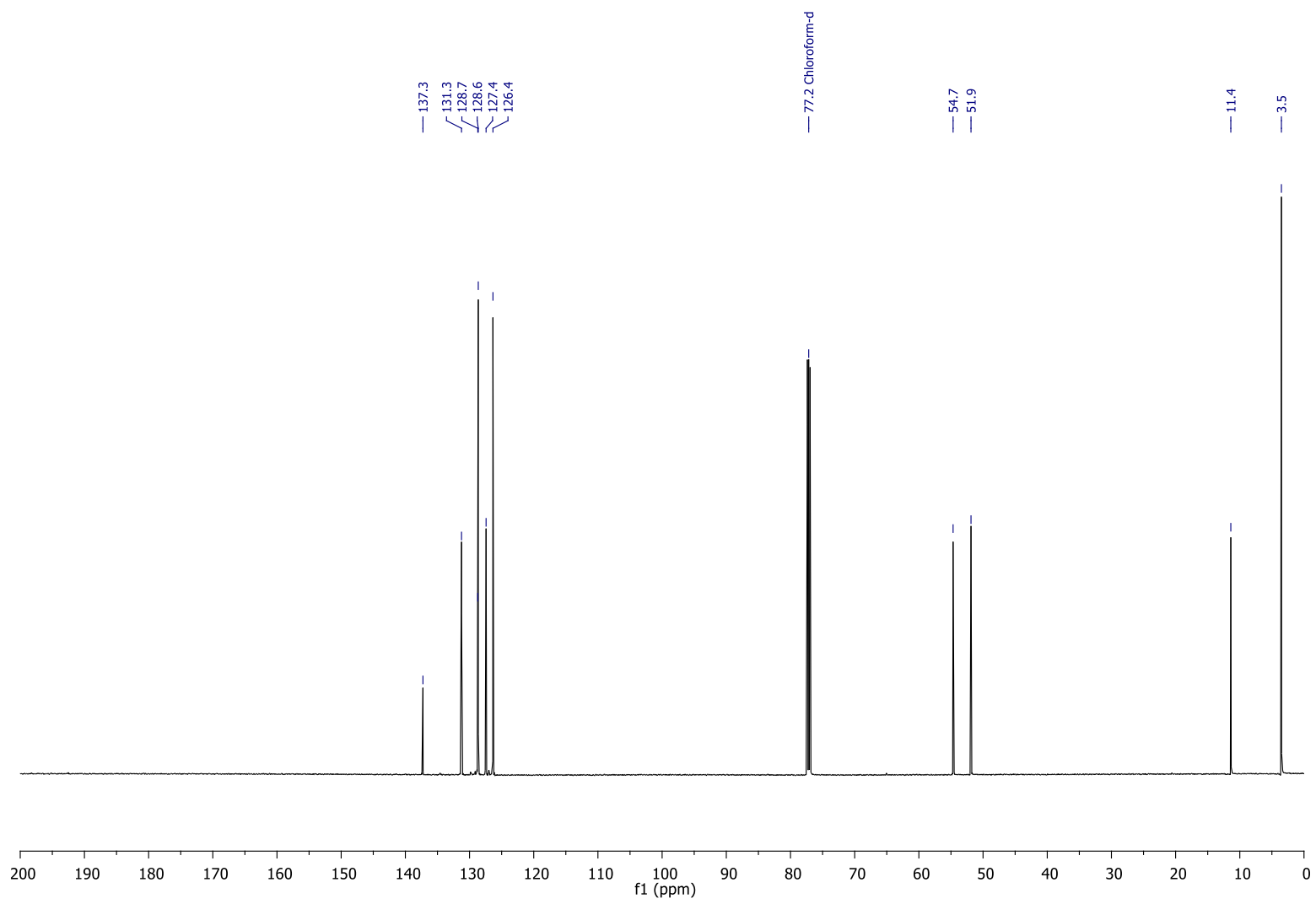

Figure $\mathbf{S}$-16. ${ }^{13} \mathrm{C}$ NMR spectrum of $\mathbf{S}-\mathbf{1 3}\left(\mathrm{CDCl}_{3}, 151 \mathrm{MHz}, 298 \mathrm{~K}\right)$ 


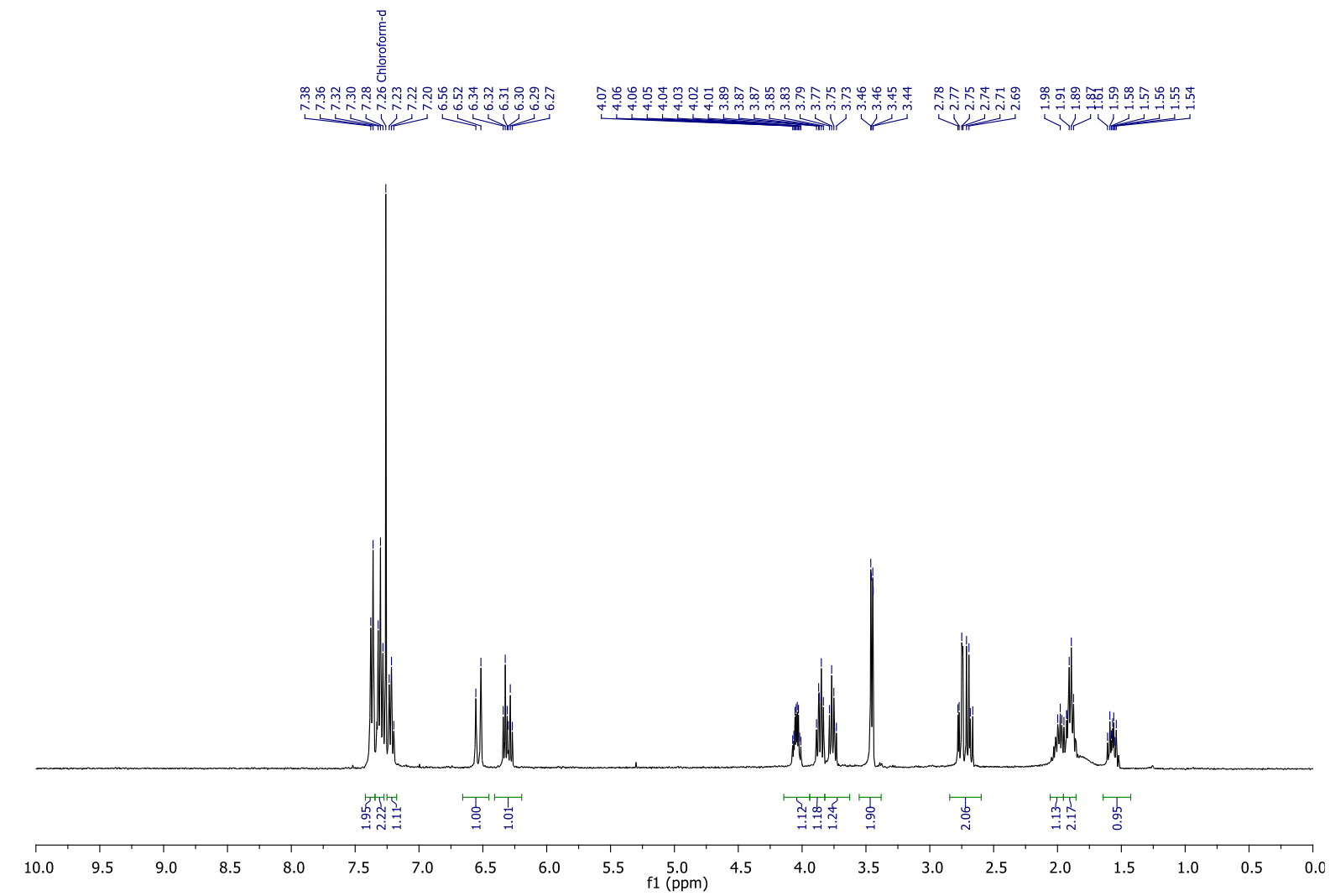

Figure S-17. ${ }^{1} \mathrm{H}$ NMR spectrum of $\mathbf{S - 1 4}\left(\mathrm{CDCl}_{3}, 600 \mathrm{MHz}, 298 \mathrm{~K}\right)$

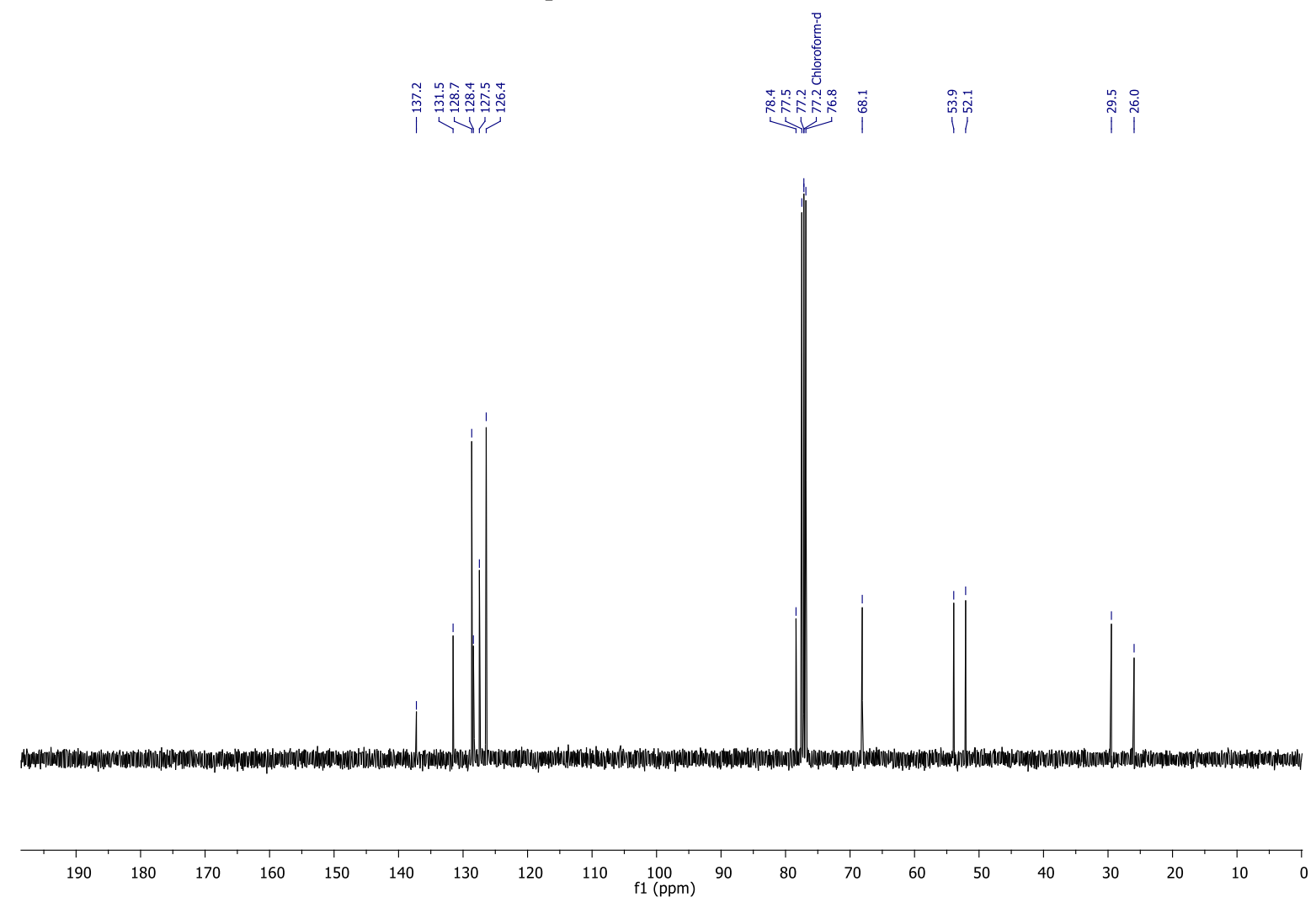

Figure $\mathbf{S}-18 .{ }^{13} \mathrm{C}$ NMR spectrum of $\mathbf{S - 1 4}\left(\mathrm{CDCl}_{3}, 151 \mathrm{MHz}, 298 \mathrm{~K}\right)$ 


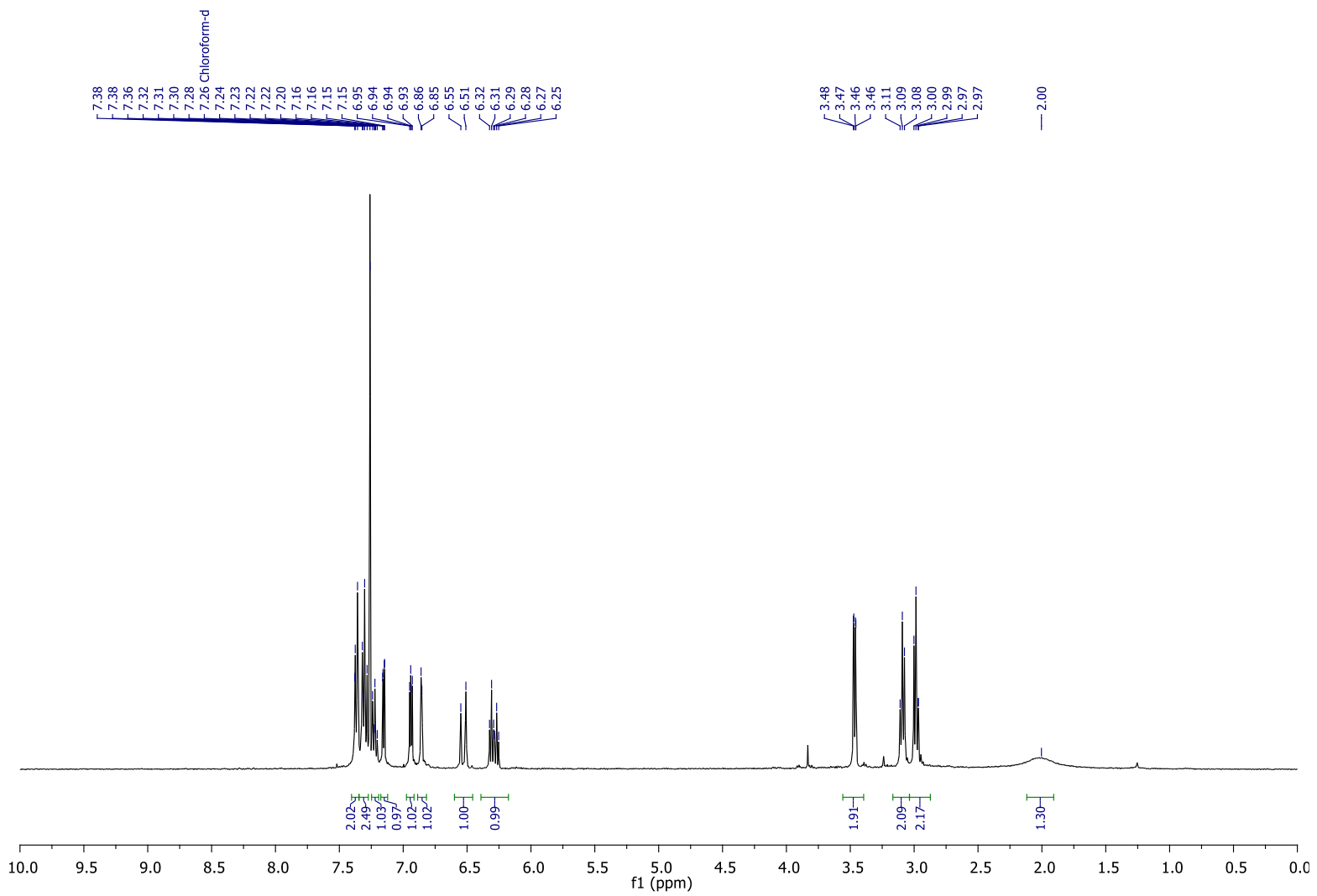

Figure $\mathbf{S}$-19. ${ }^{1} \mathrm{H}$ NMR spectrum of $\mathbf{S}-15\left(\mathrm{CDCl}_{3}, 600 \mathrm{MHz}, 298 \mathrm{~K}\right)$

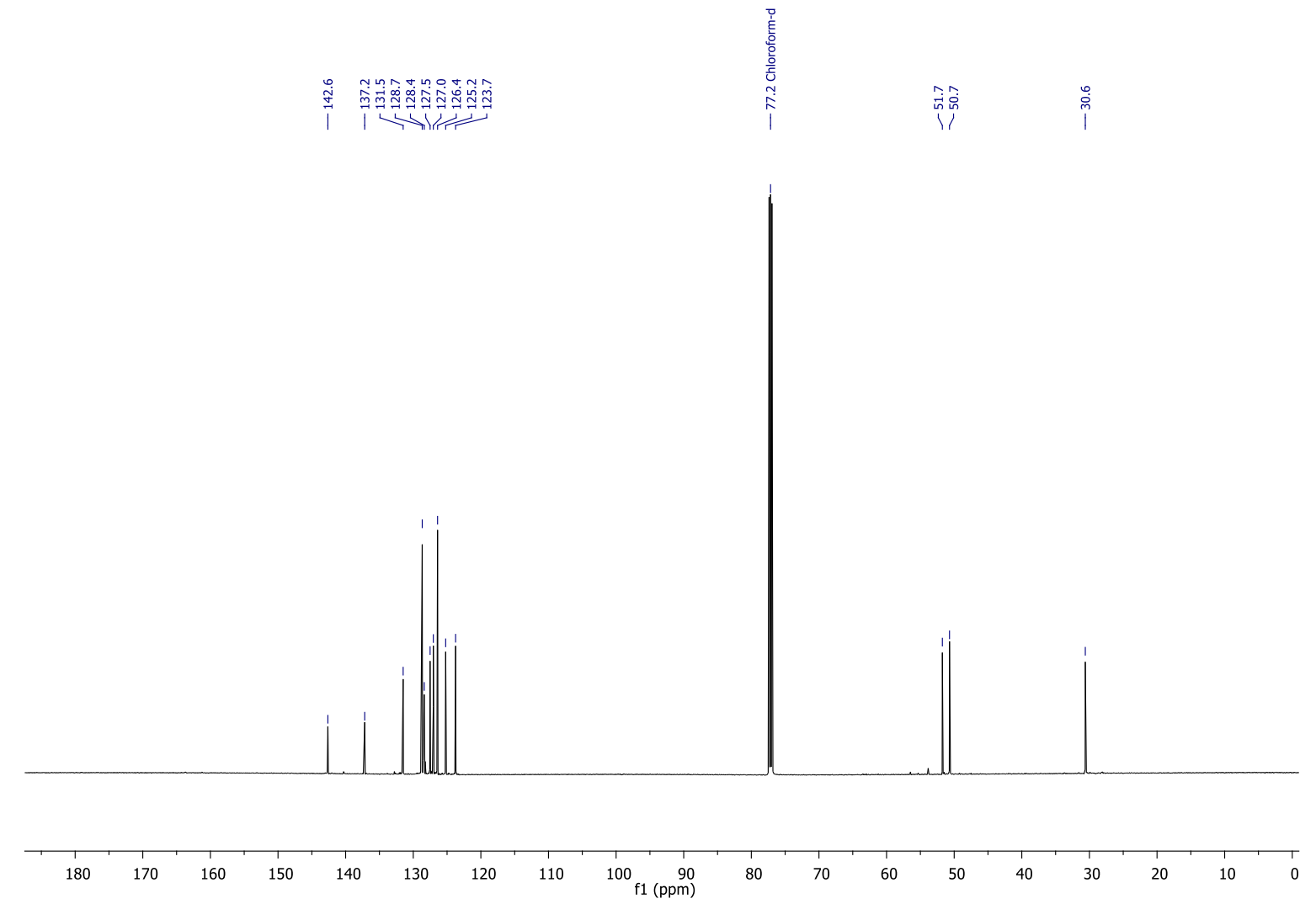

Figure S-20. ${ }^{13} \mathrm{C}$ NMR spectrum of $\mathbf{S}-15\left(\mathrm{CDCl}_{3}, 151 \mathrm{MHz}, 298 \mathrm{~K}\right)$ 

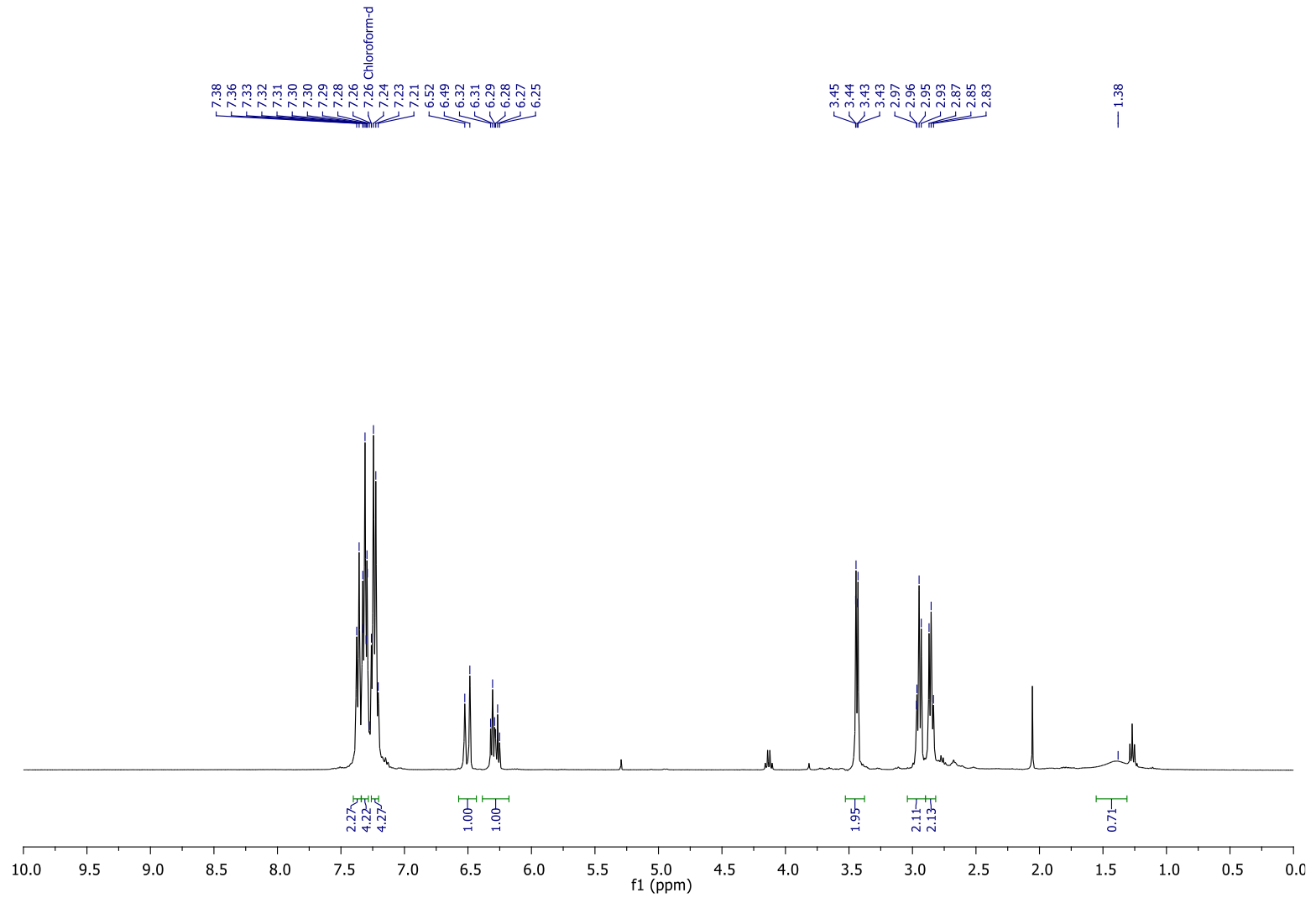

Figure S-21. ${ }^{1} \mathrm{H}$ NMR spectrum of $\mathbf{S - 1 8}\left(\mathrm{CDCl}_{3}, 400 \mathrm{MHz}, 298 \mathrm{~K}\right)$

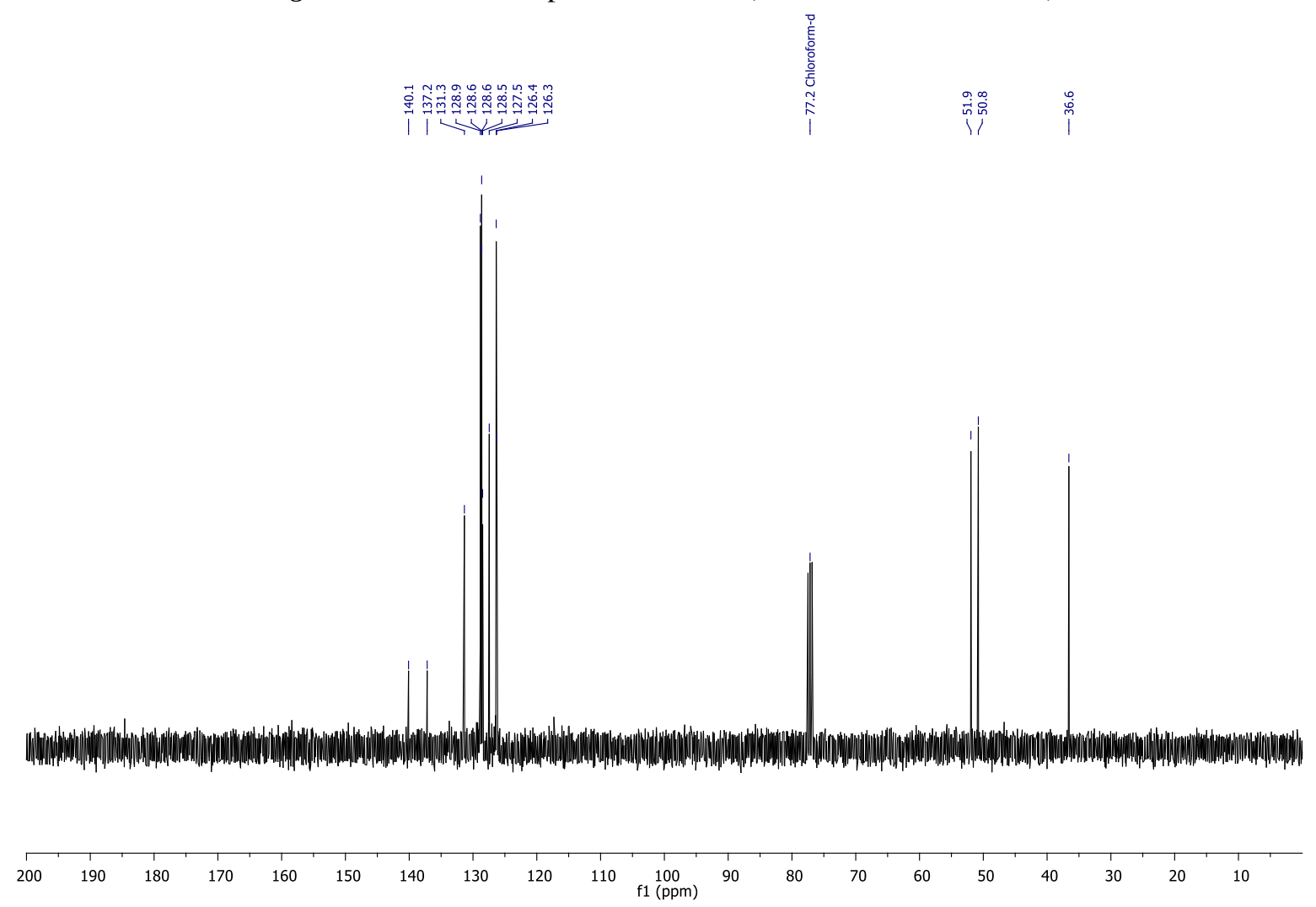

Figure $\mathbf{S - 2 2} .{ }^{13} \mathrm{C}$ NMR spectrum of $\mathbf{S - 1 8}\left(\mathrm{CDCl}_{3}, 101 \mathrm{MHz}, 298 \mathrm{~K}\right)$ 


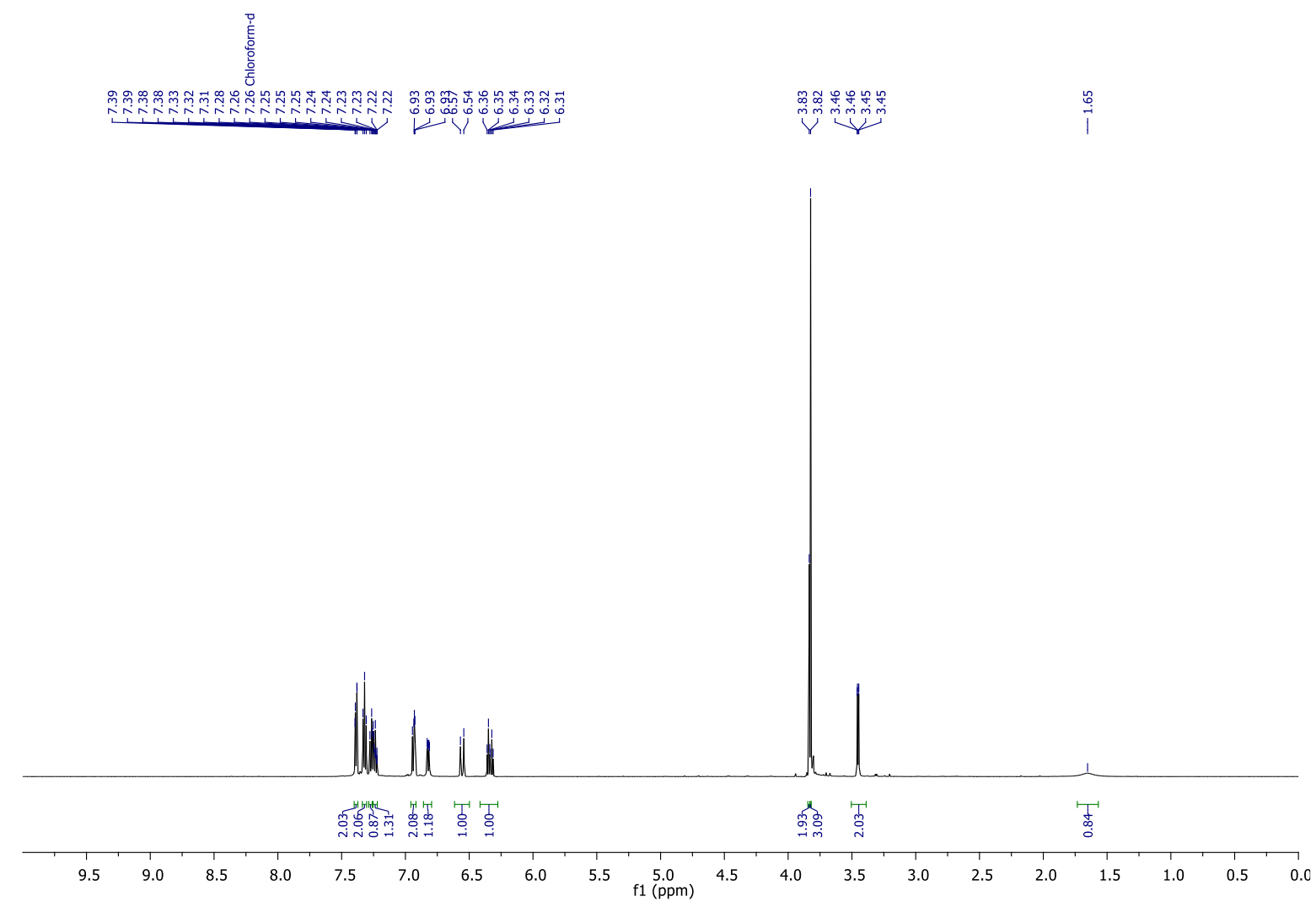

Figure S-23. ${ }^{1} \mathrm{H}$ NMR spectrum of $\mathbf{S - 1 9}\left(\mathrm{CDCl}_{3}, 600 \mathrm{MHz}, 298 \mathrm{~K}\right)$
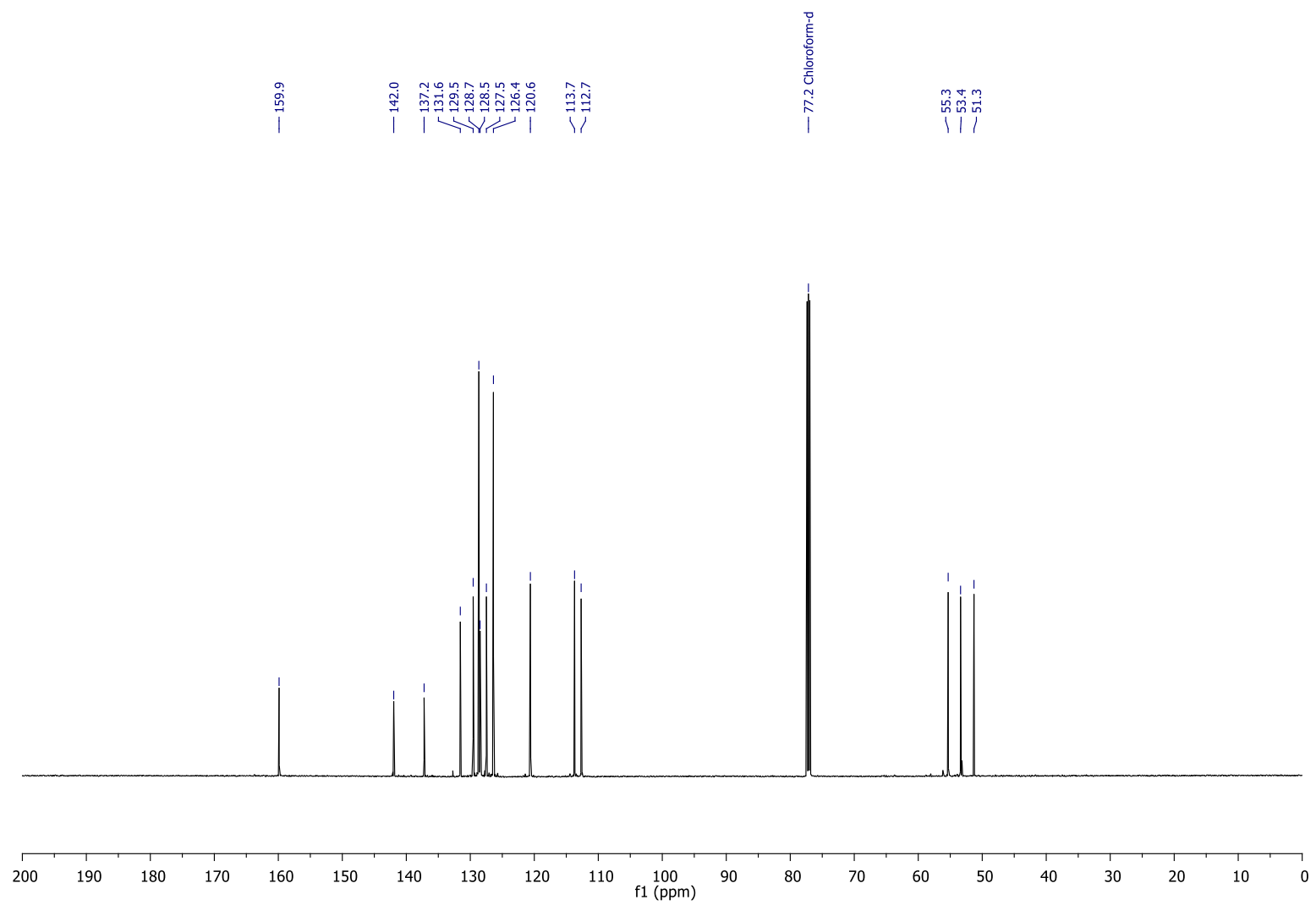

Figure $\mathbf{S}$-24. ${ }^{13} \mathrm{C}$ NMR spectrum of $\mathbf{S - 1 9}\left(\mathrm{CDCl}_{3}, 151 \mathrm{MHz}, 298 \mathrm{~K}\right)$ 


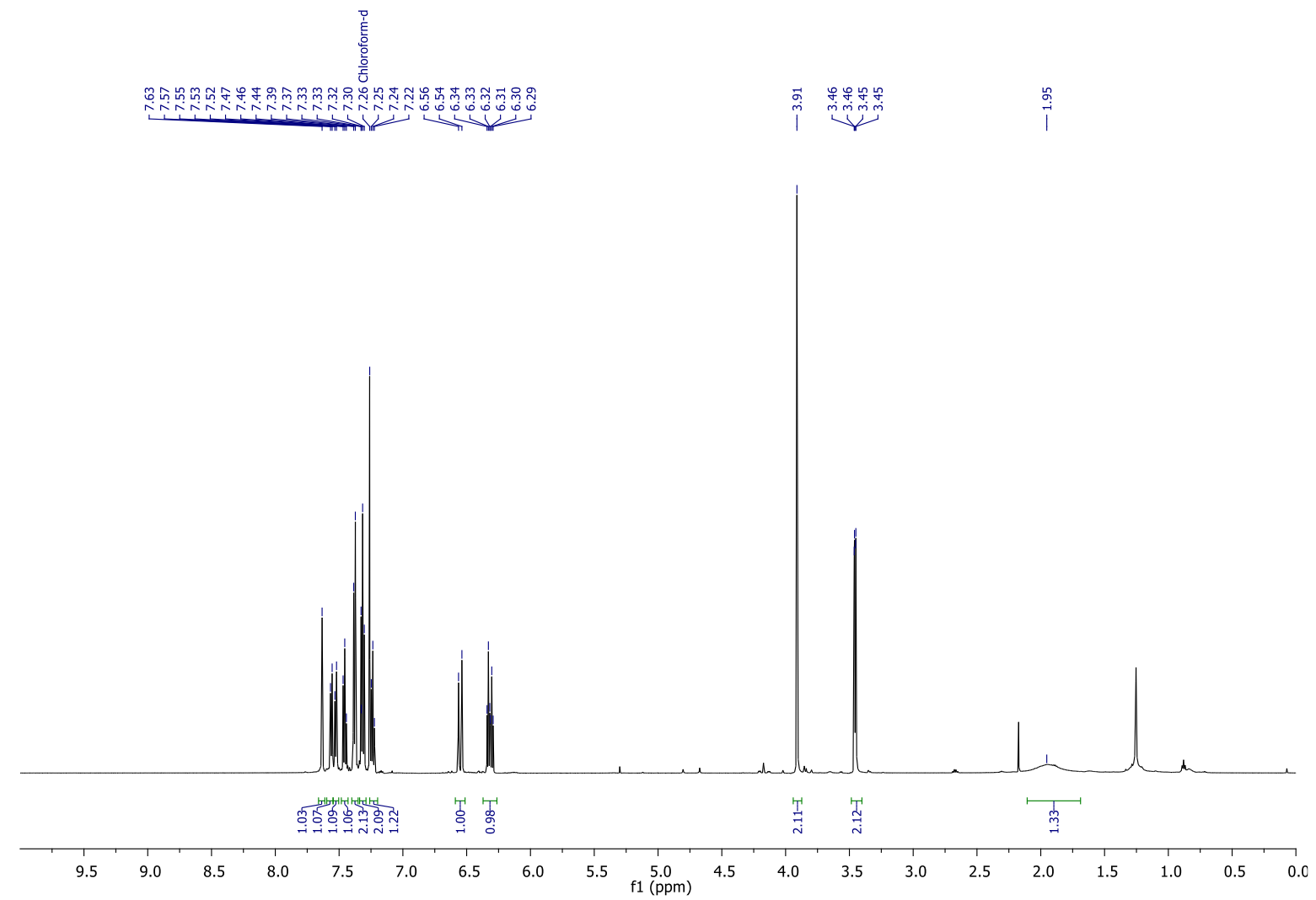

Figure S-25. ${ }^{1} \mathrm{H}$ NMR spectrum of $\mathbf{S - 2 0}\left(\mathrm{CDCl}_{3}, 600 \mathrm{MHz}, 298 \mathrm{~K}\right)$
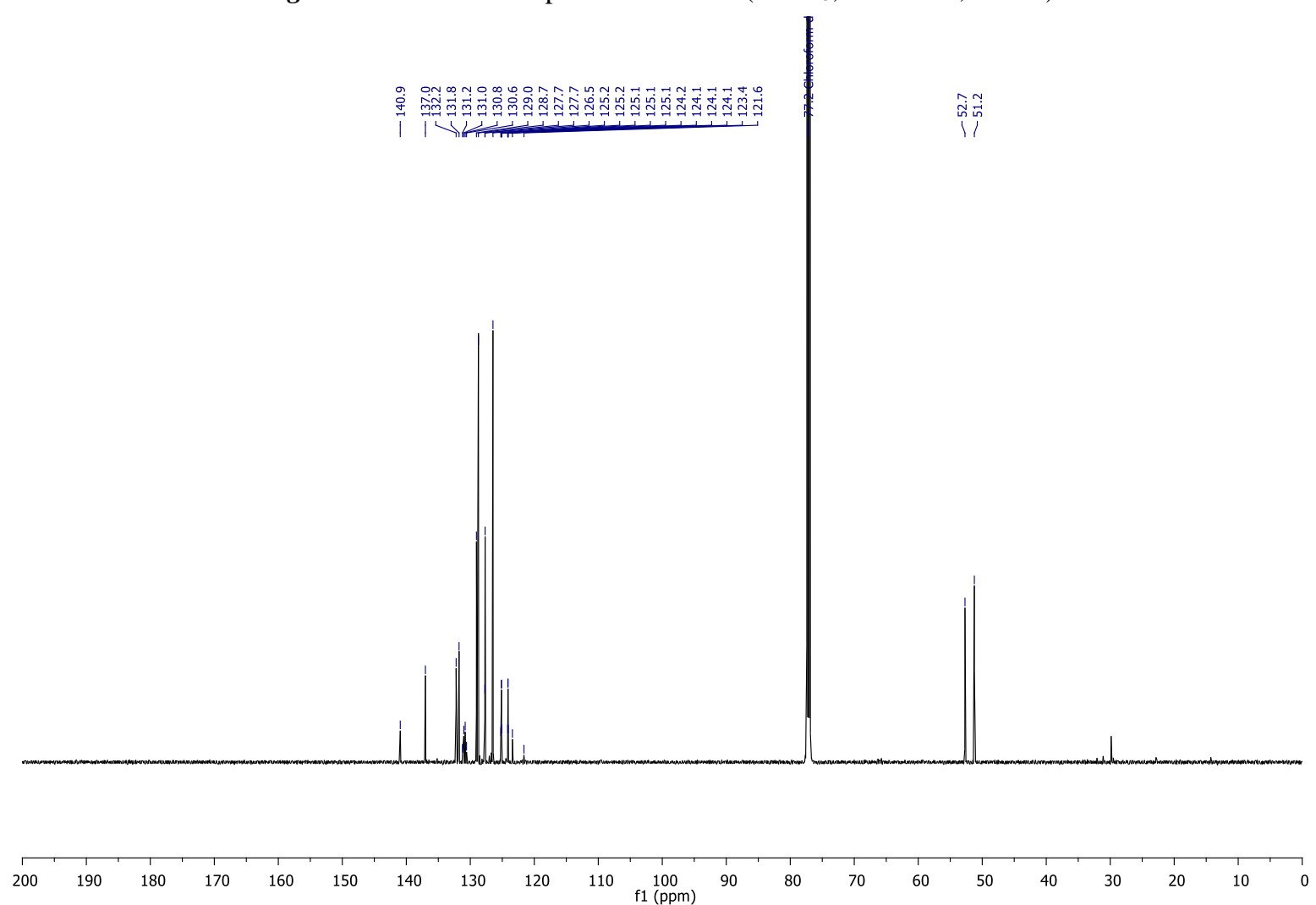

Figure $\mathbf{S}$-26. ${ }^{13} \mathrm{C}$ NMR spectrum of $\mathbf{S}-20\left(\mathrm{CDCl}_{3}, 151 \mathrm{MHz}, 298 \mathrm{~K}\right)$ 


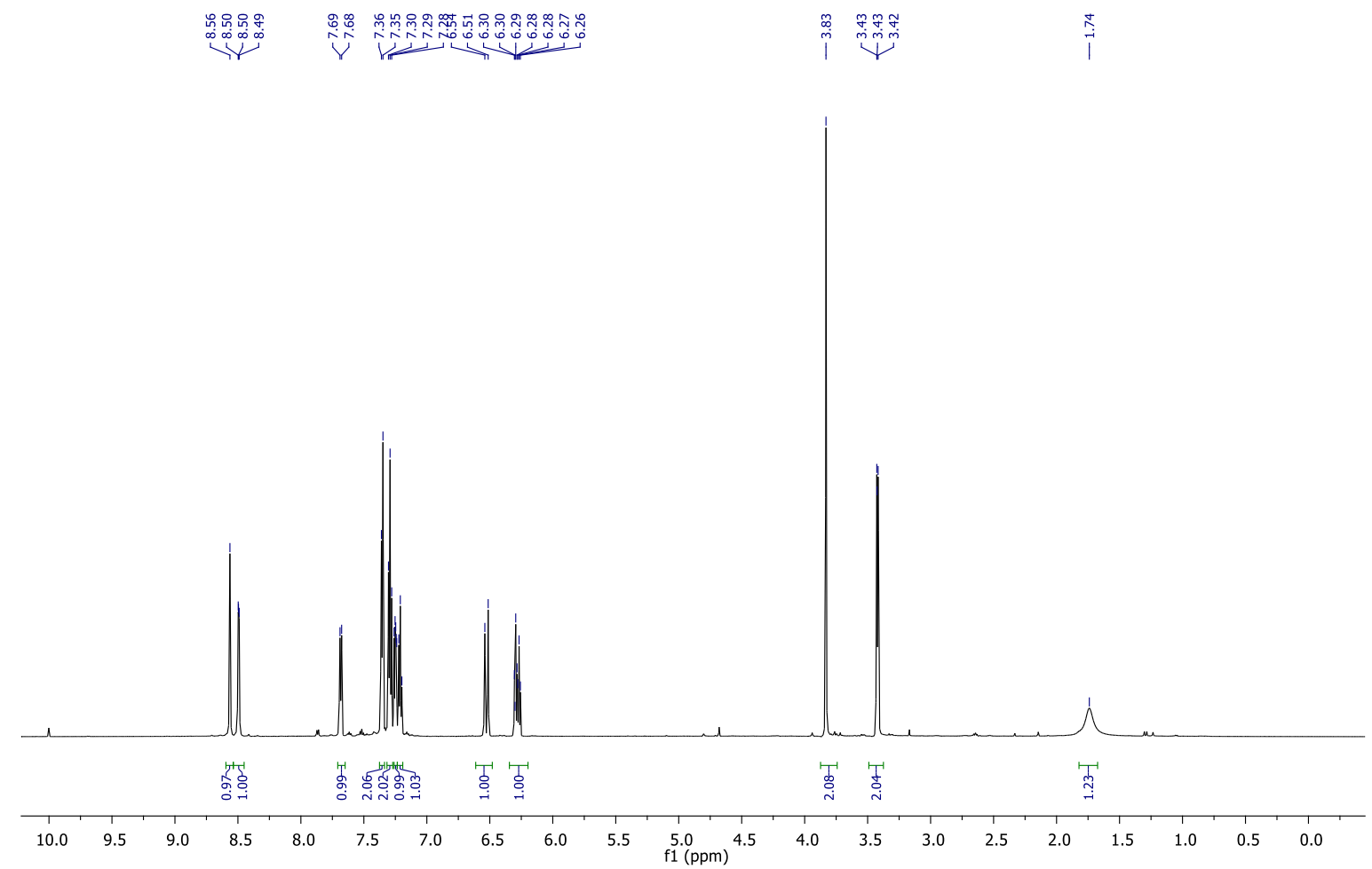

Figure S-27. ${ }^{1} \mathrm{H}$ NMR spectrum of $\mathbf{S - 2 1}\left(\mathrm{CDCl}_{3}, 600 \mathrm{MHz}, 298 \mathrm{~K}\right)$

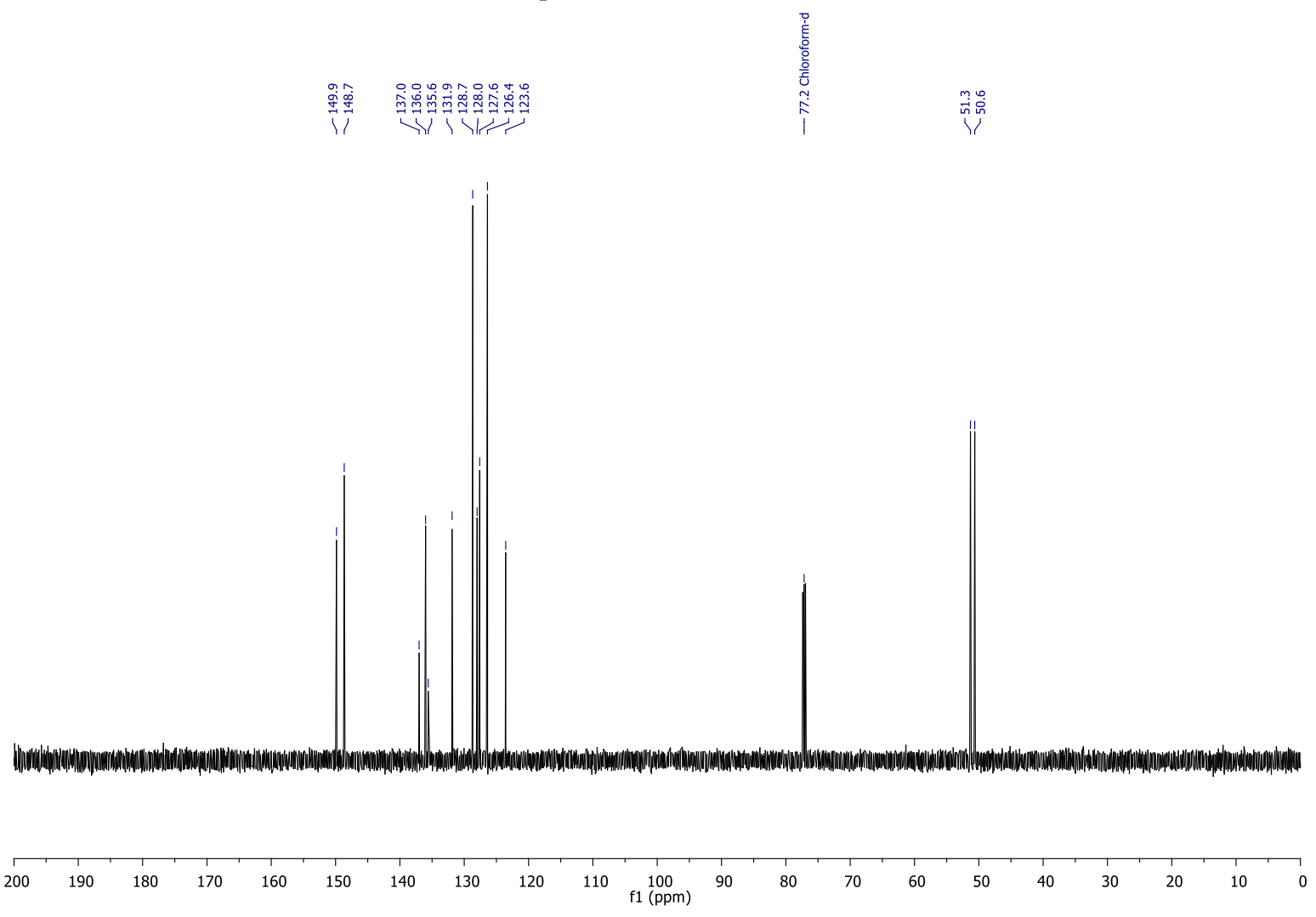

Figure $\mathbf{S}$-28. ${ }^{13} \mathrm{C}$ NMR spectrum of $\mathbf{S}-21\left(\mathrm{CDCl}_{3}, 151 \mathrm{MHz}, 298 \mathrm{~K}\right)$ 


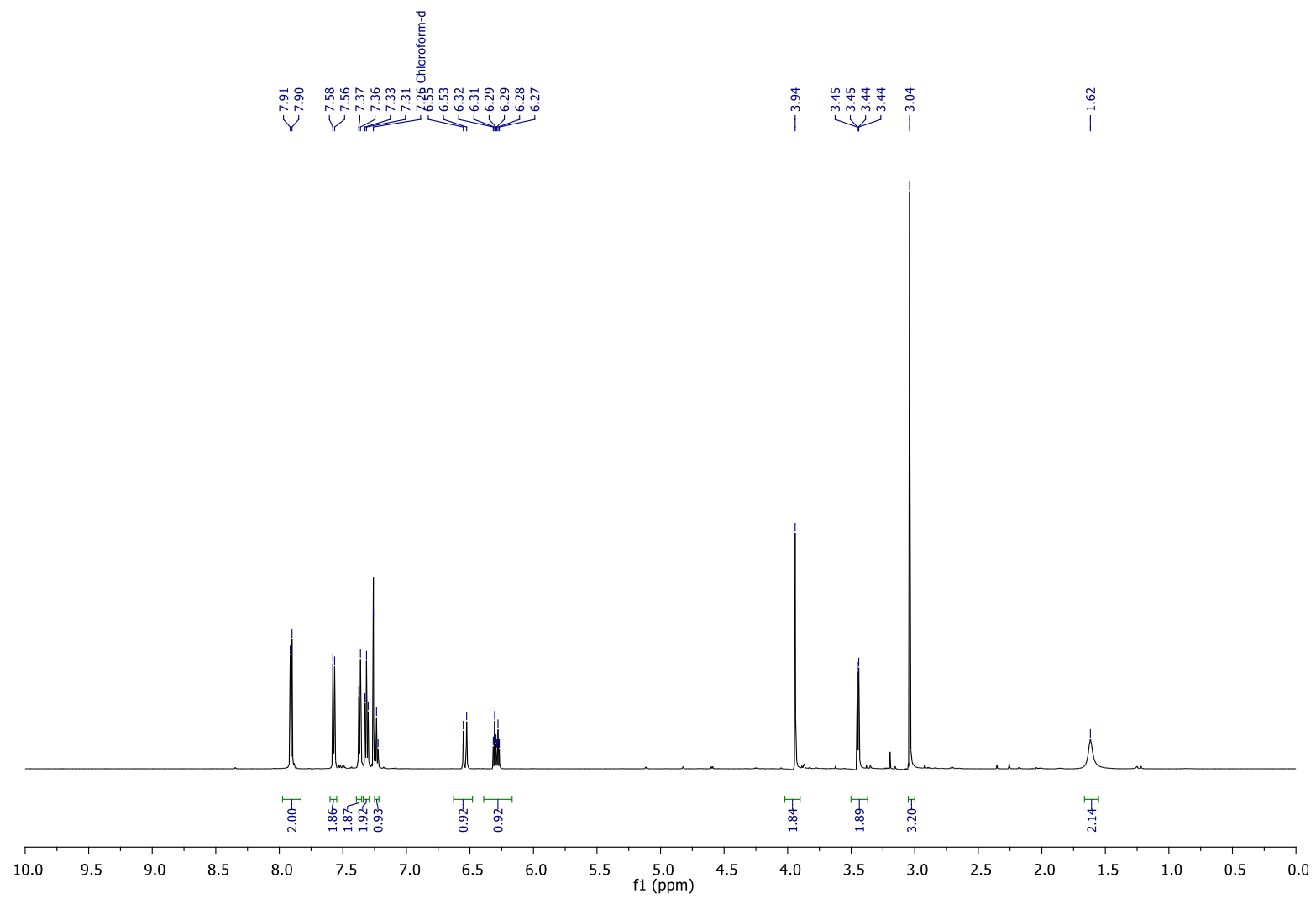

Figure S-29. ${ }^{1} \mathrm{H}$ NMR spectrum of $\mathbf{S - 2 2}\left(\mathrm{CDCl}_{3}, 600 \mathrm{MHz}, 298 \mathrm{~K}\right)$

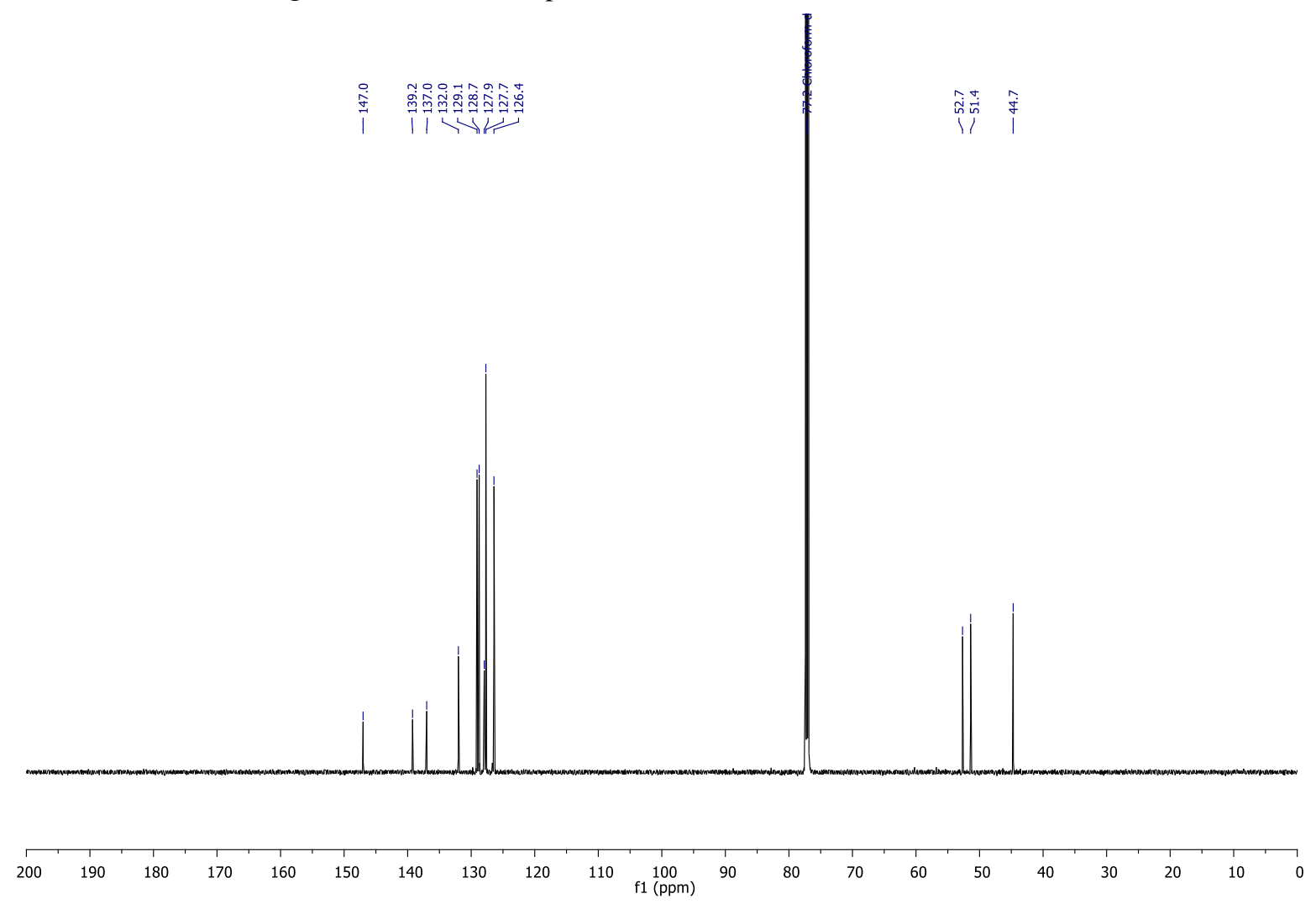

Figure S-30. ${ }^{13} \mathrm{C}$ NMR spectrum of $\mathbf{S - 2 2}\left(\mathrm{CDCl}_{3}, 151 \mathrm{MHz}, 298 \mathrm{~K}\right)$ 


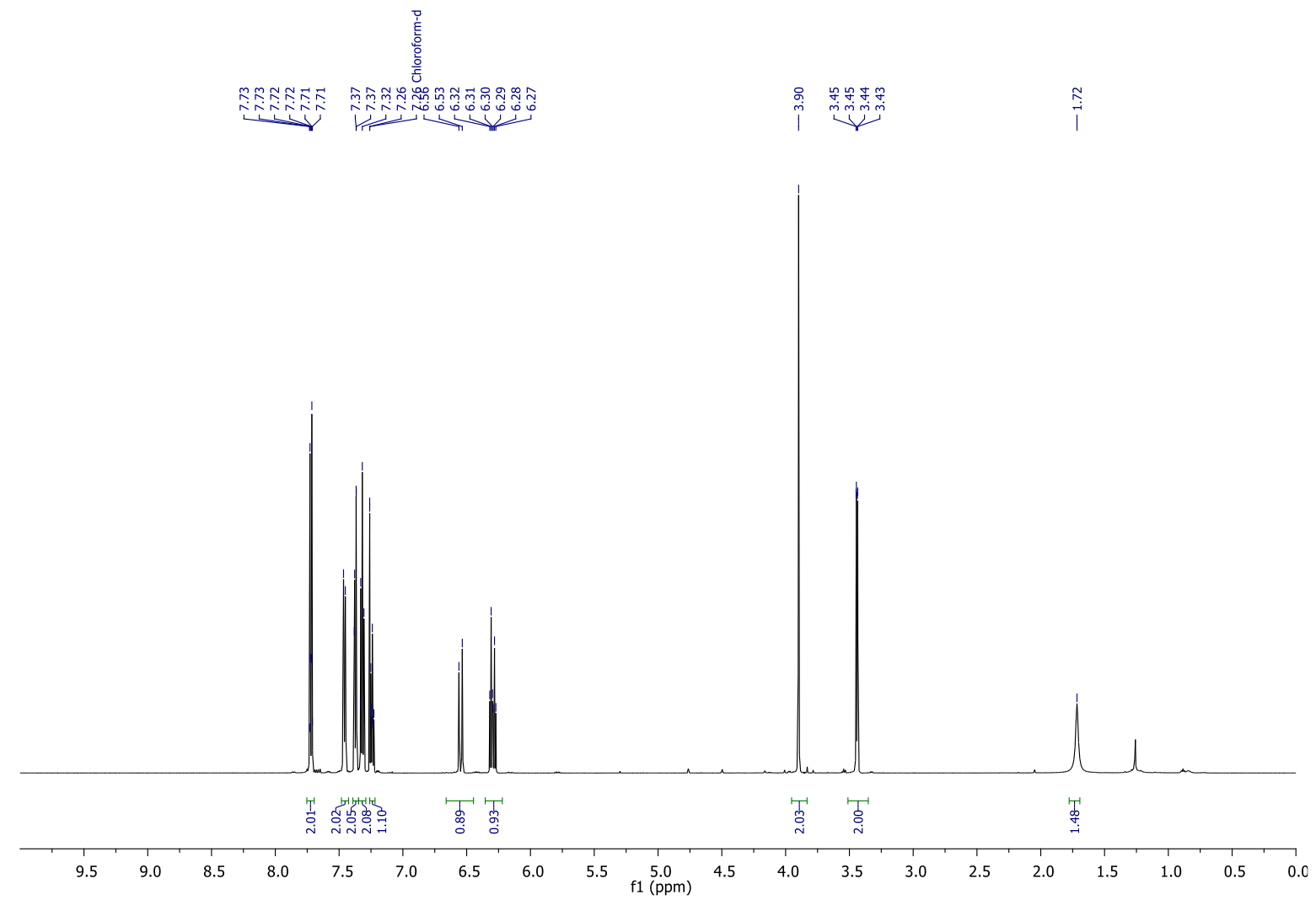

Figure S-31. ${ }^{1} \mathrm{H}$ NMR spectrum of $\mathbf{S - 2 3}\left(\mathrm{CDCl}_{3}, 600 \mathrm{MHz}, 298 \mathrm{~K}\right)$
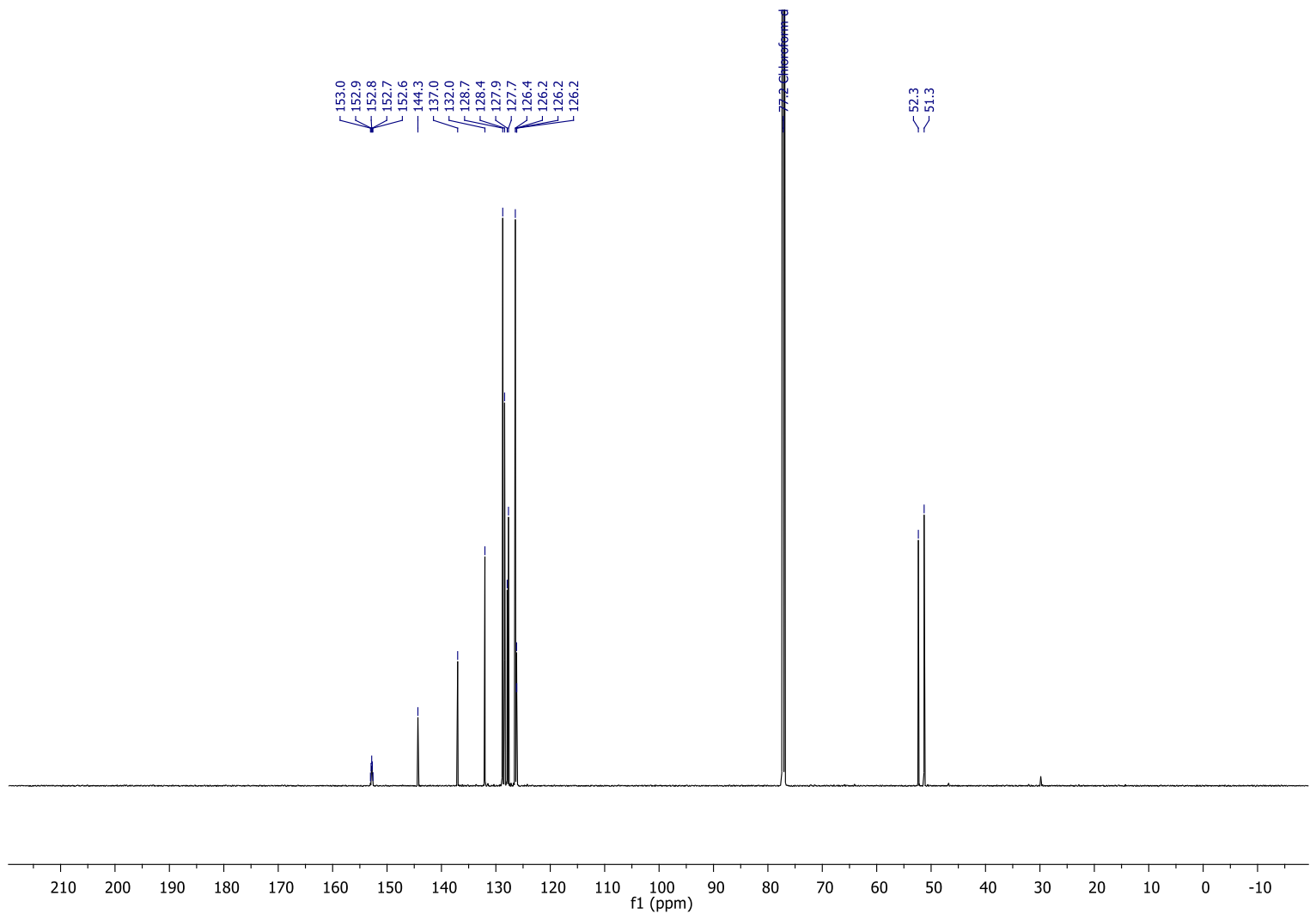

Figure $\mathbf{S - 3 2} .{ }^{13} \mathrm{C}$ NMR spectrum of $\mathbf{S - 2 3}\left(\mathrm{CDCl}_{3}, 151 \mathrm{MHz}, 298 \mathrm{~K}\right)$ 

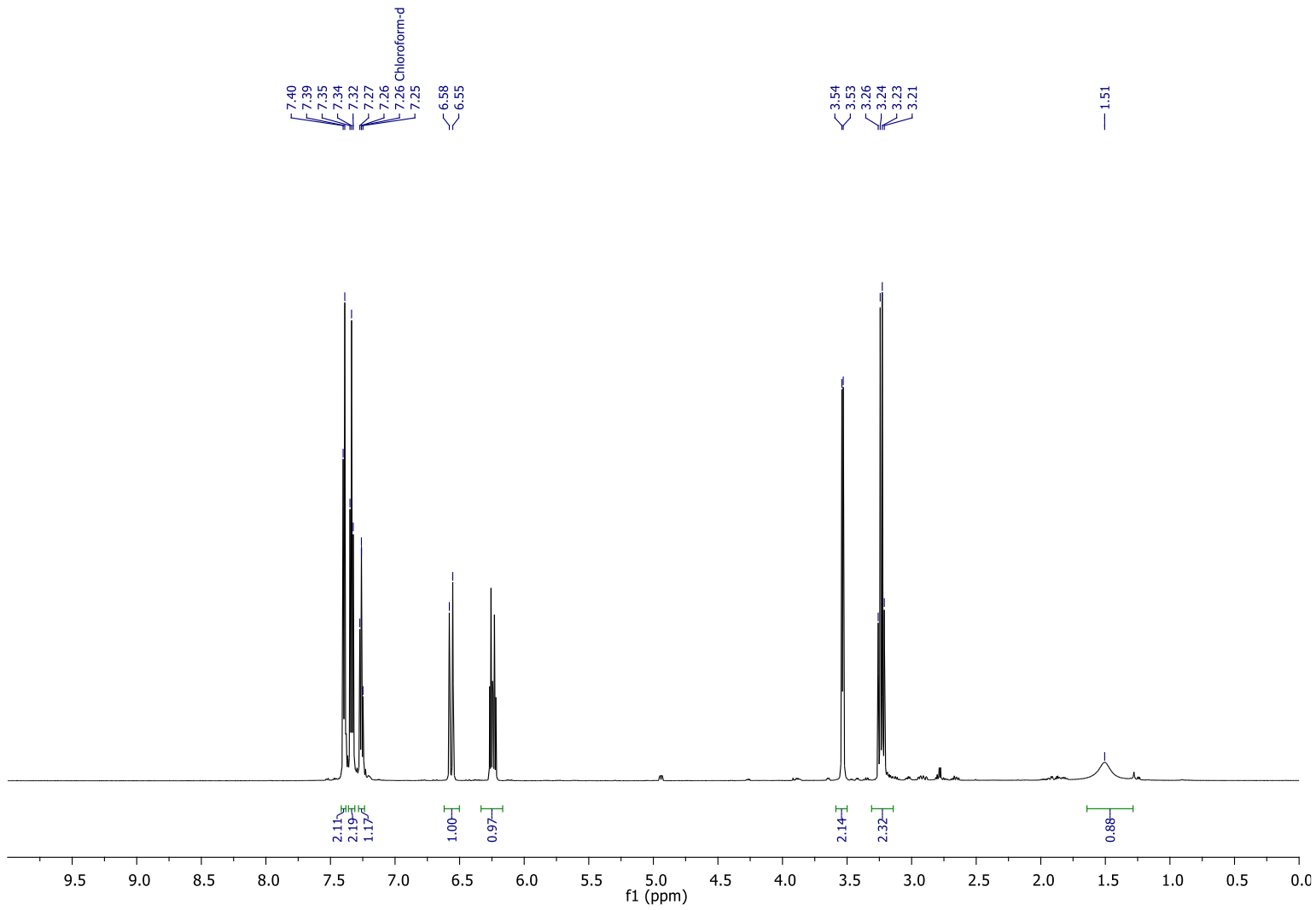

Figure S-33. ${ }^{1} \mathrm{H}$ NMR spectrum of S-24 $\left(\mathrm{CDCl}_{3}, 600 \mathrm{MHz}, 298 \mathrm{~K}\right)$
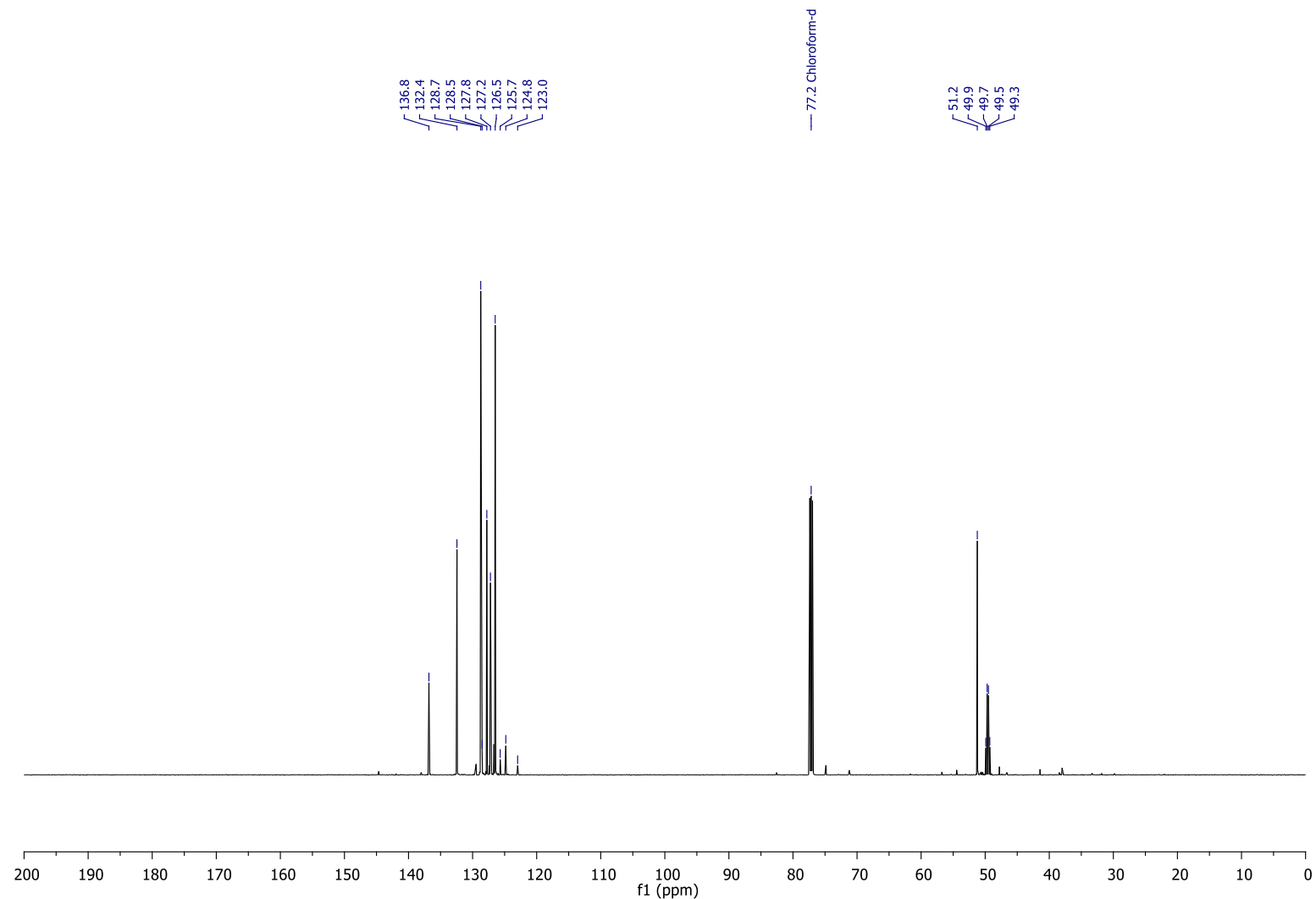

Figure $\mathbf{S - 3 4} .{ }^{13} \mathrm{C}$ NMR spectrum of $\mathbf{S}-24\left(\mathrm{CDCl}_{3}, 151 \mathrm{MHz}, 298 \mathrm{~K}\right)$ 


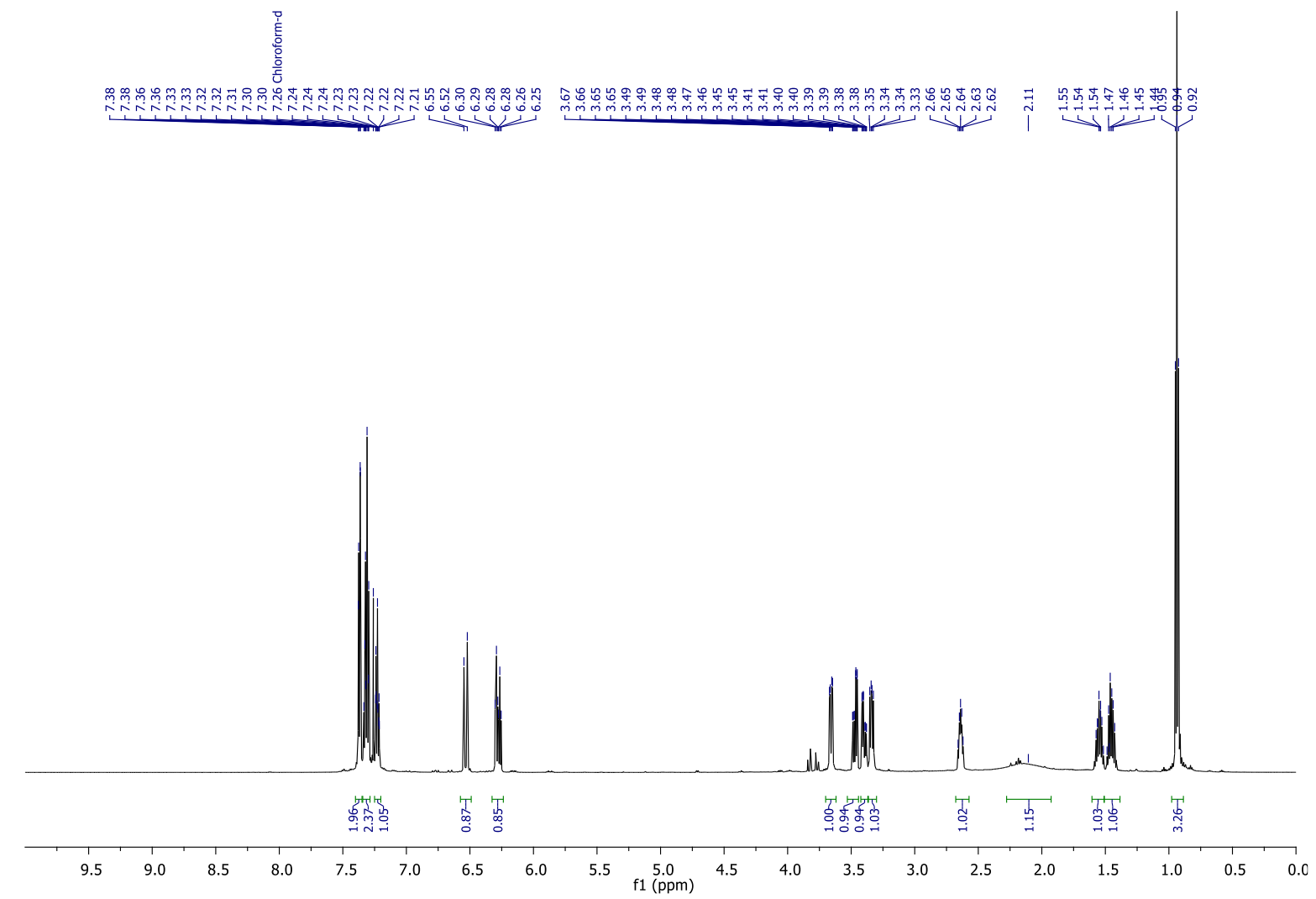

Figure S-35. ${ }^{1} \mathrm{H}$ NMR spectrum of $\mathbf{S - 2 5}\left(\mathrm{CDCl}_{3}, 600 \mathrm{MHz}, 298 \mathrm{~K}\right)$
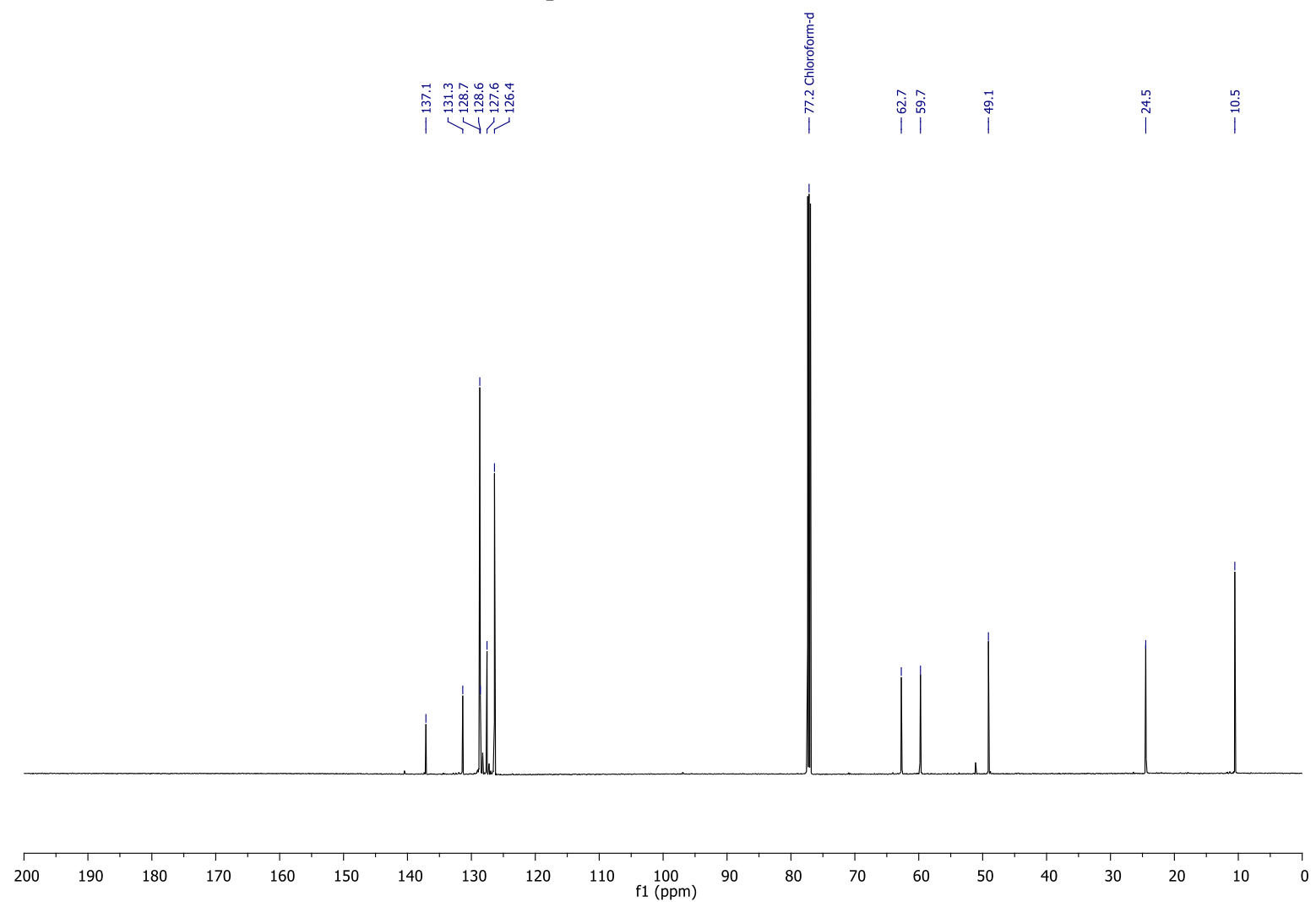

Figure $\mathbf{S}$-36. ${ }^{13} \mathrm{C}$ NMR spectrum of $\mathbf{S}-25\left(\mathrm{CDCl}_{3}, 151 \mathrm{MHz}, 298 \mathrm{~K}\right)$ 


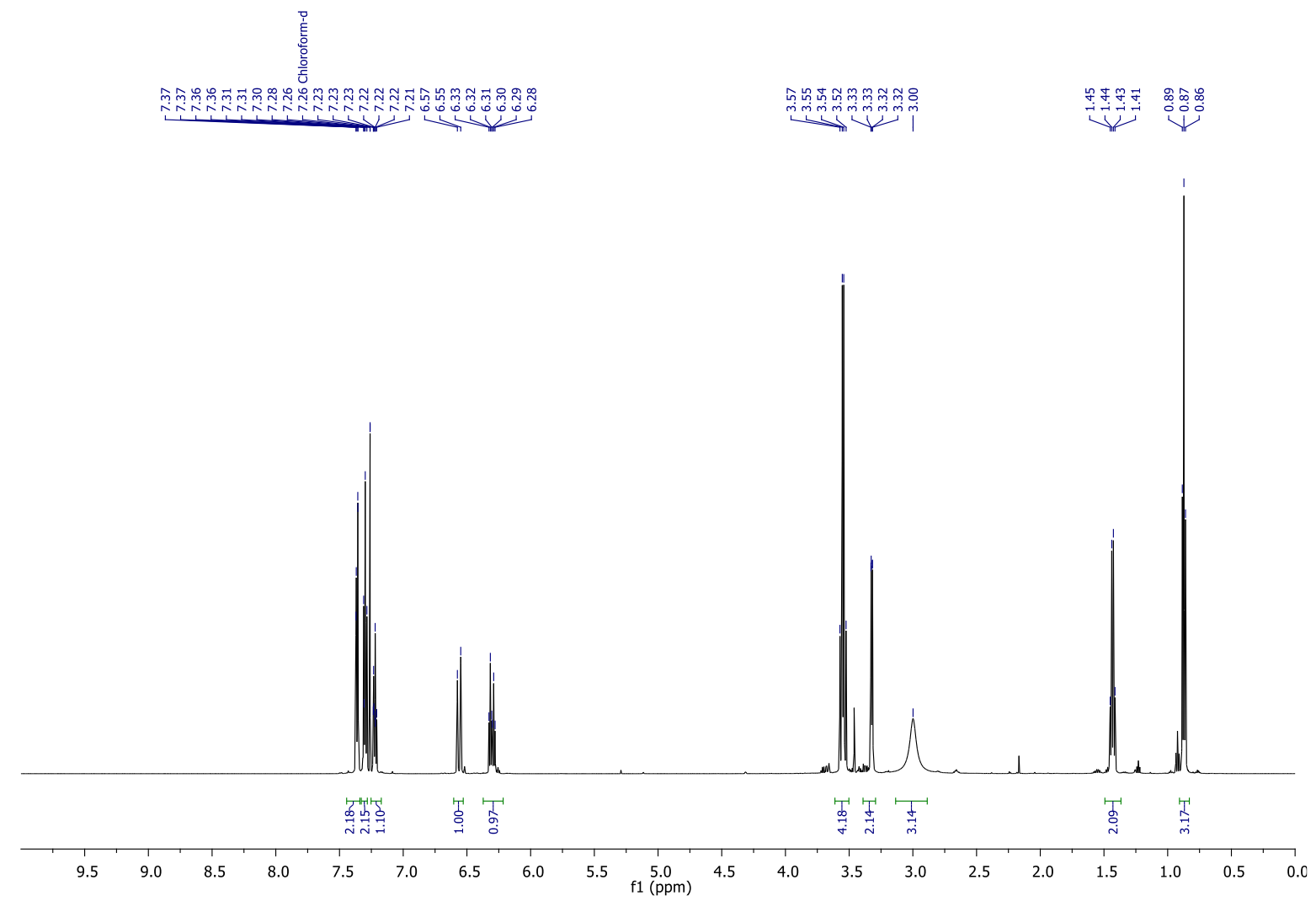

Figure S-37. ${ }^{1} \mathrm{H}$ NMR spectrum of $\mathbf{S - 2 6}\left(\mathrm{CDCl}_{3}, 600 \mathrm{MHz}, 298 \mathrm{~K}\right)$
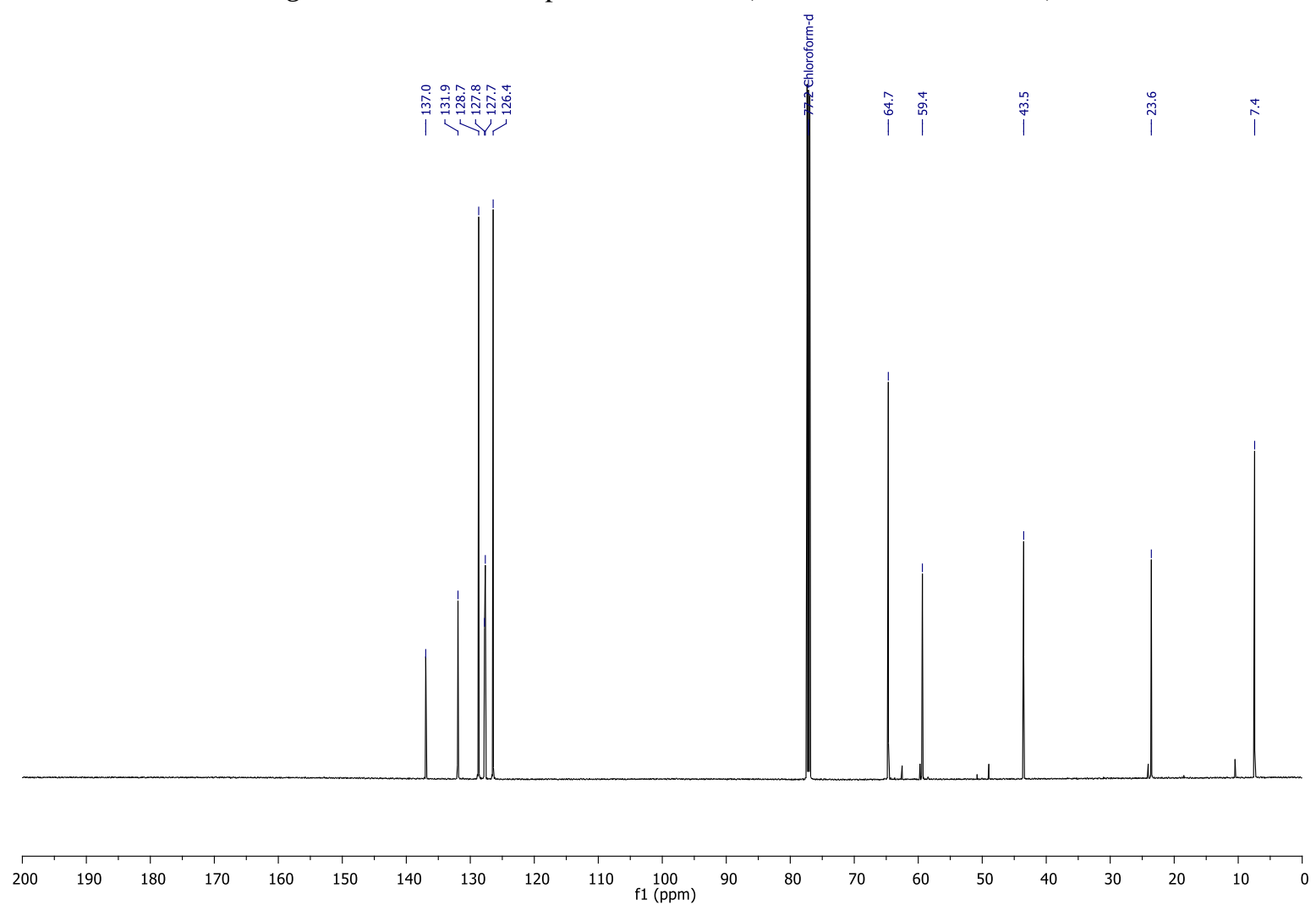

Figure $\mathbf{S}$-38. ${ }^{13} \mathrm{C}$ NMR spectrum of $\mathbf{S}-26\left(\mathrm{CDCl}_{3}, 151 \mathrm{MHz}, 298 \mathrm{~K}\right)$ 


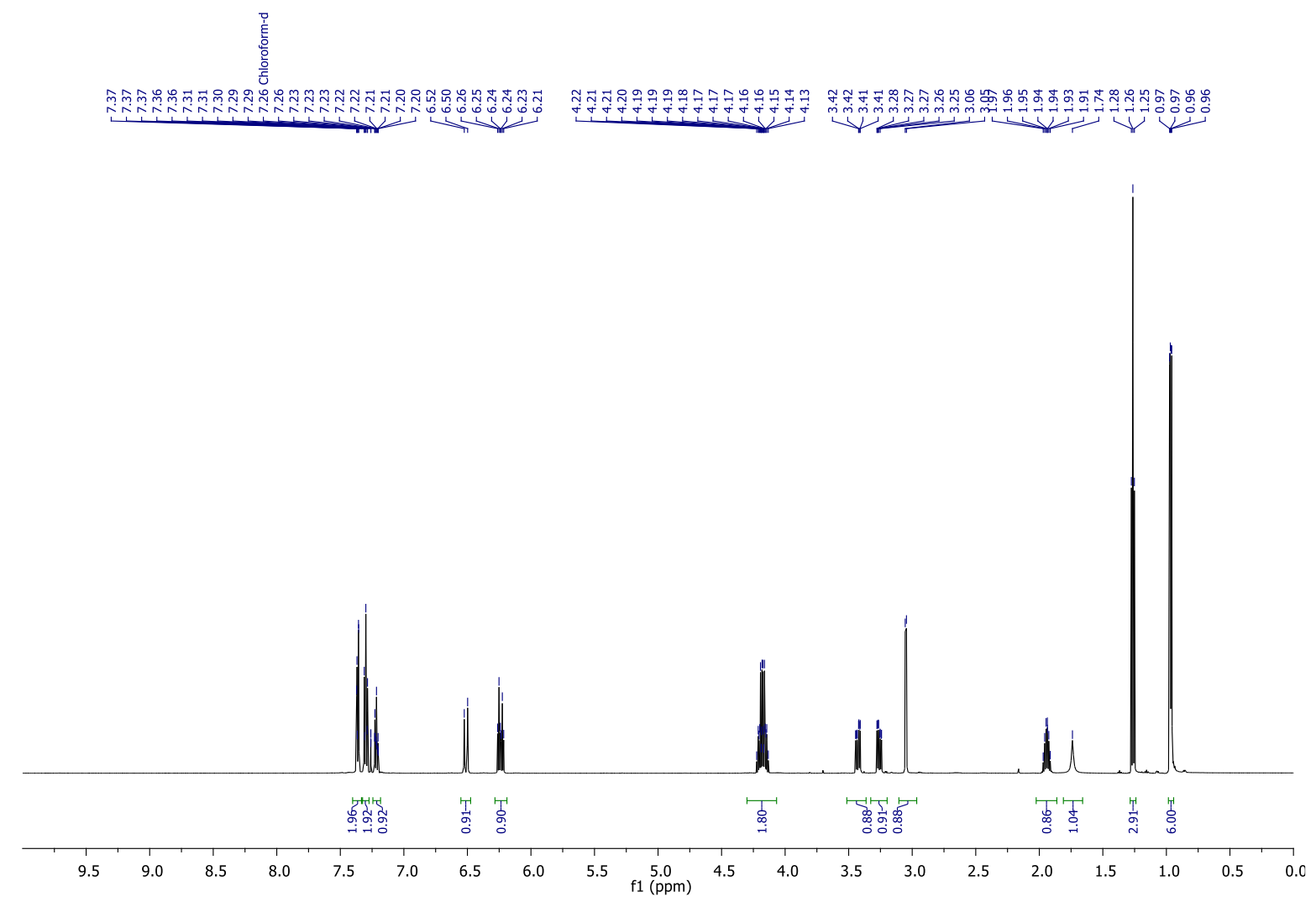

Figure S-39. ${ }^{1} \mathrm{H}$ NMR spectrum of $\mathbf{S - 2 7}\left(\mathrm{CDCl}_{3}, 600 \mathrm{MHz}, 298 \mathrm{~K}\right)$

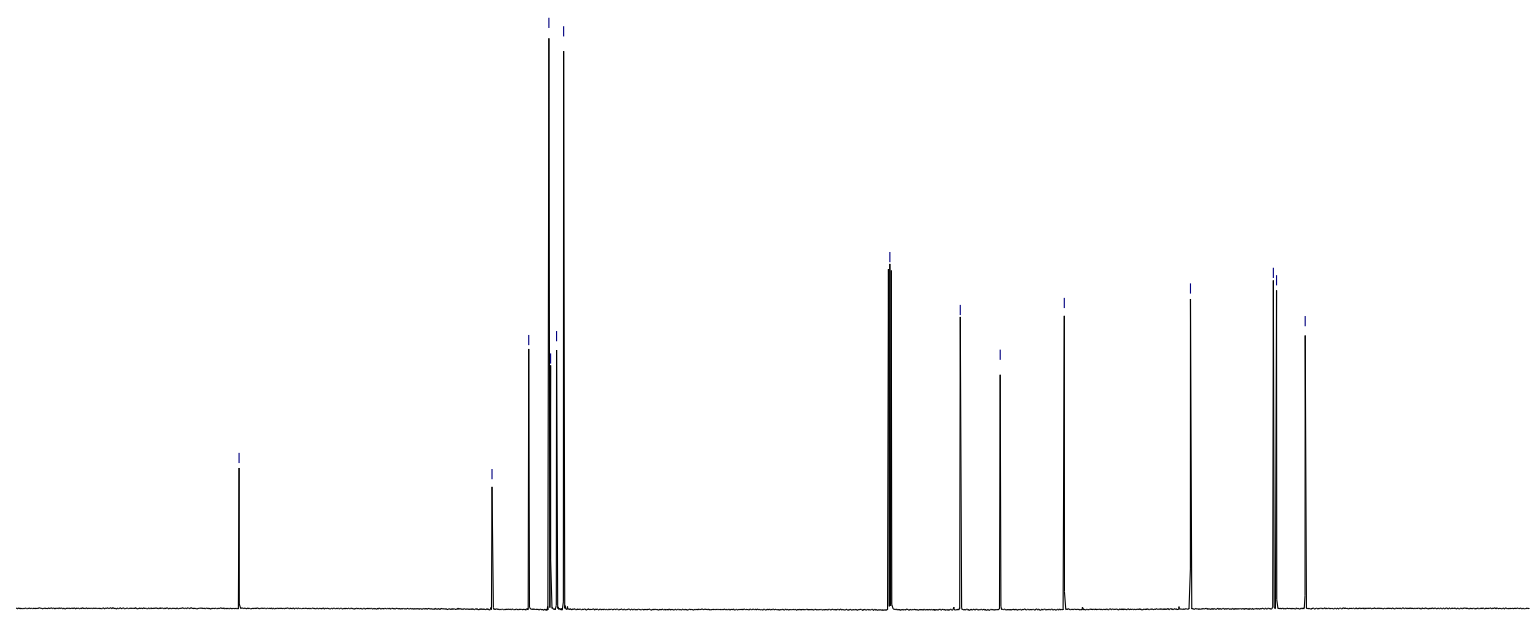

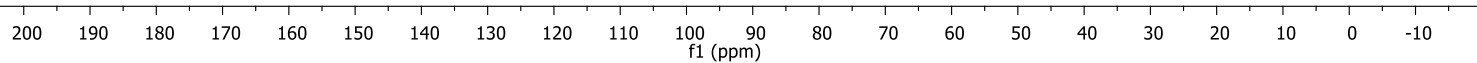

Figure $\mathbf{S}-40 .{ }^{13} \mathrm{C}$ NMR spectrum of $\mathbf{S}-27\left(\mathrm{CDCl}_{3}, 151 \mathrm{MHz}, 298 \mathrm{~K}\right)$ 


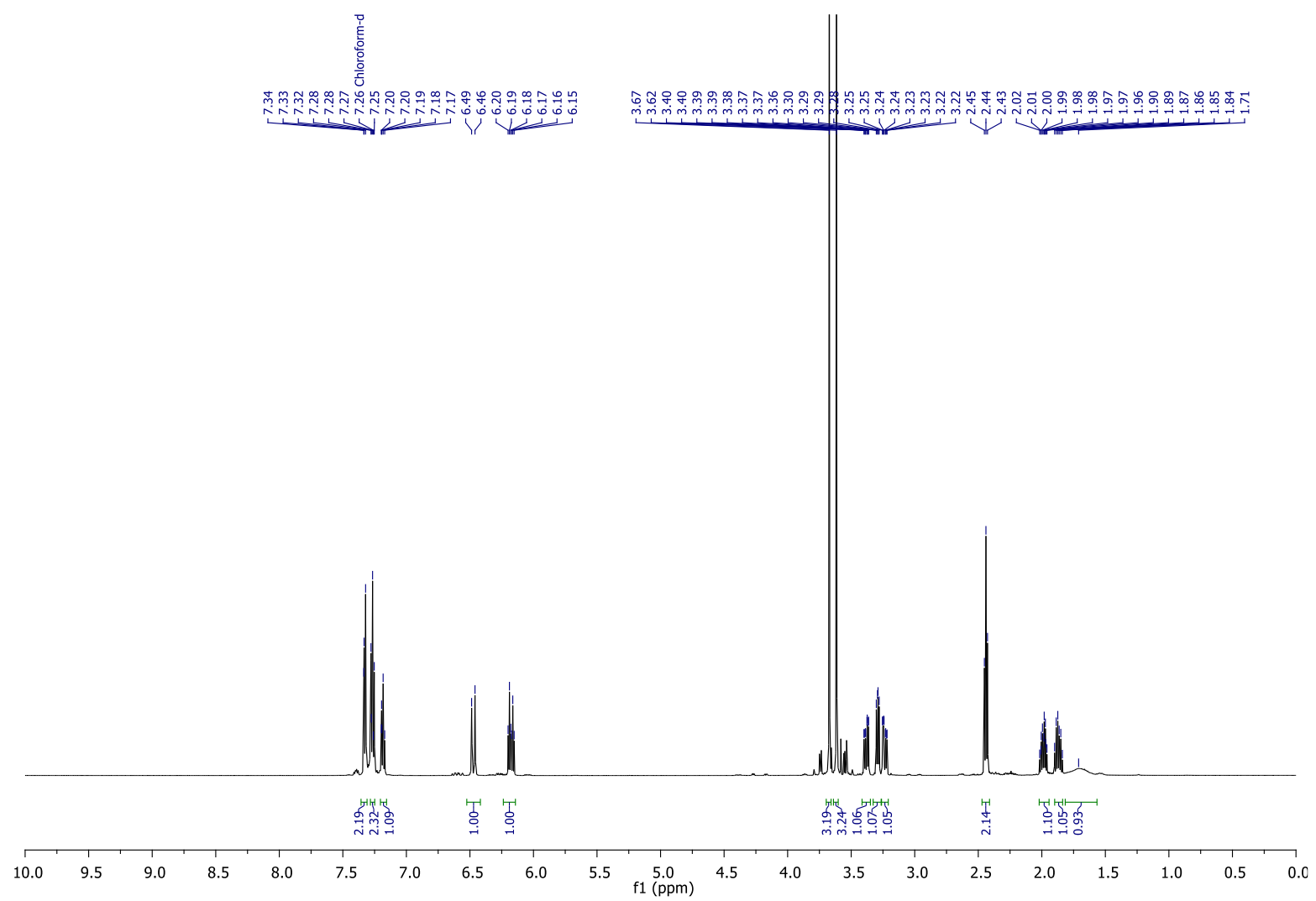

Figure S-41. ${ }^{1} \mathrm{H}$ NMR spectrum of $\mathbf{S - 2 8}\left(\mathrm{CDCl}_{3}, 600 \mathrm{MHz}, 298 \mathrm{~K}\right)$
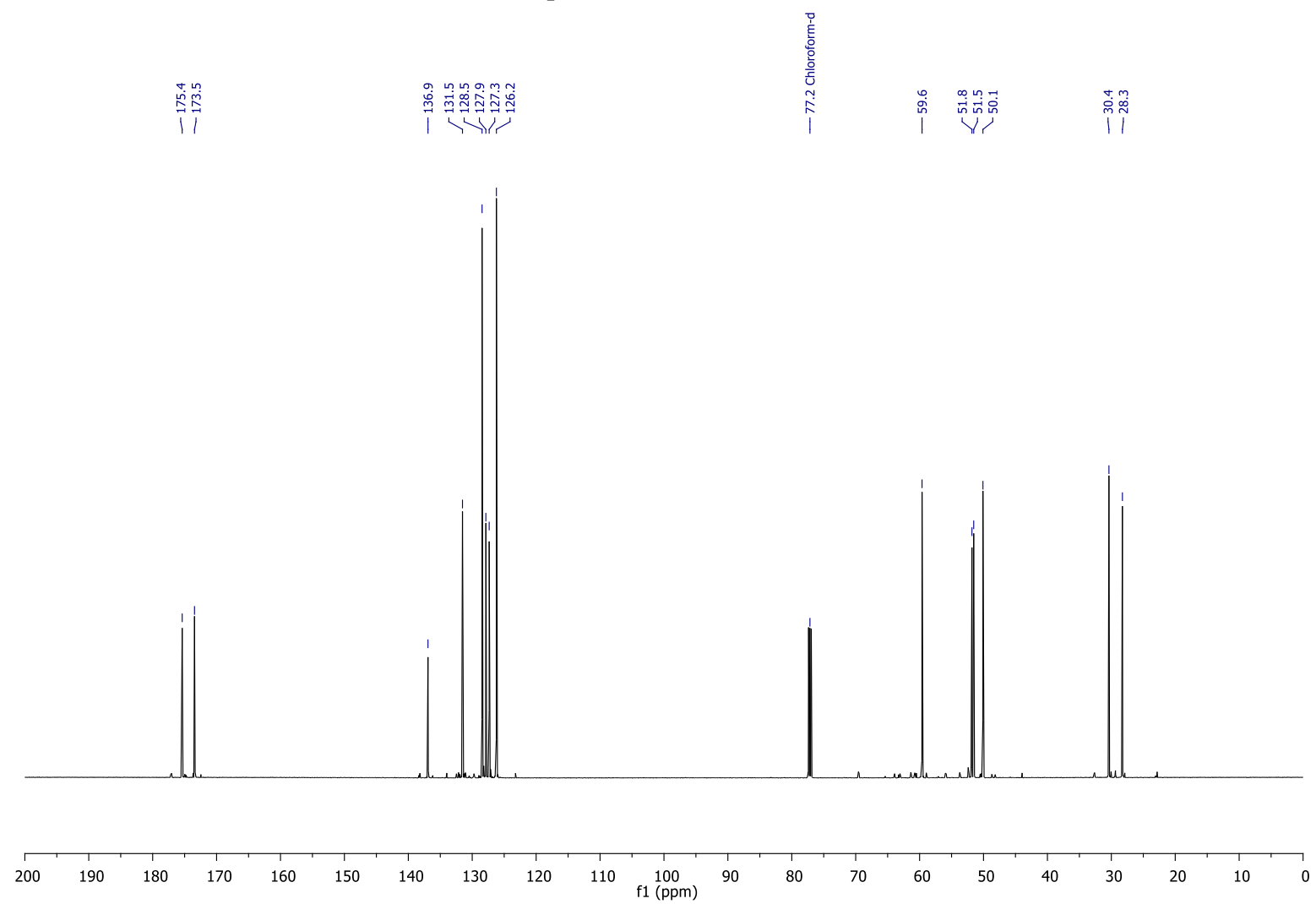

Figure $\mathbf{S - 4 2 .}{ }^{13} \mathrm{C}$ NMR spectrum of $\mathbf{S}-28\left(\mathrm{CDCl}_{3}, 151 \mathrm{MHz}, 298 \mathrm{~K}\right)$ 


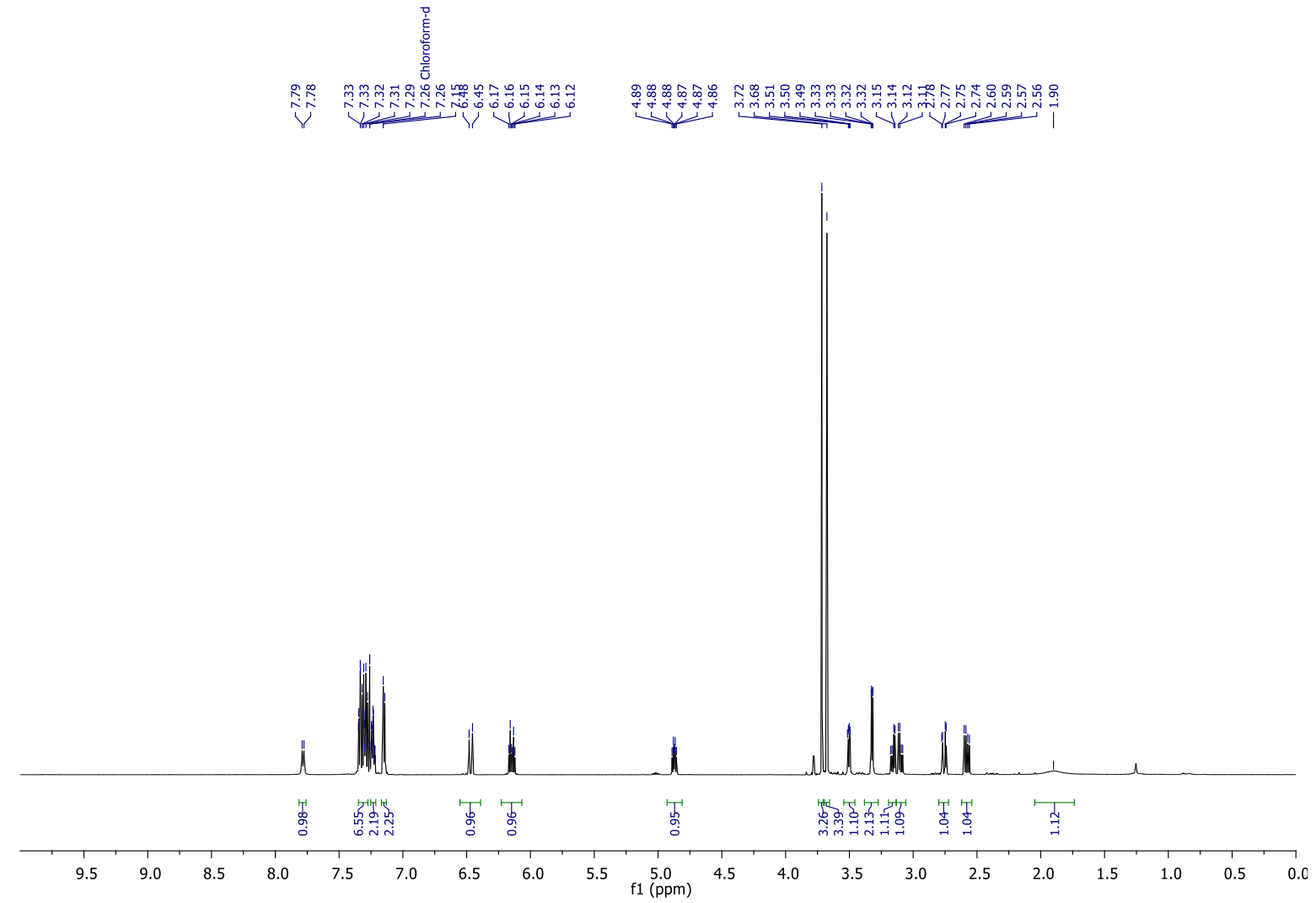

Figure S-43. ${ }^{1} \mathrm{H}$ NMR spectrum of $\mathbf{S - 2 9}\left(\mathrm{CDCl}_{3}, 600 \mathrm{MHz}, 298 \mathrm{~K}\right)$

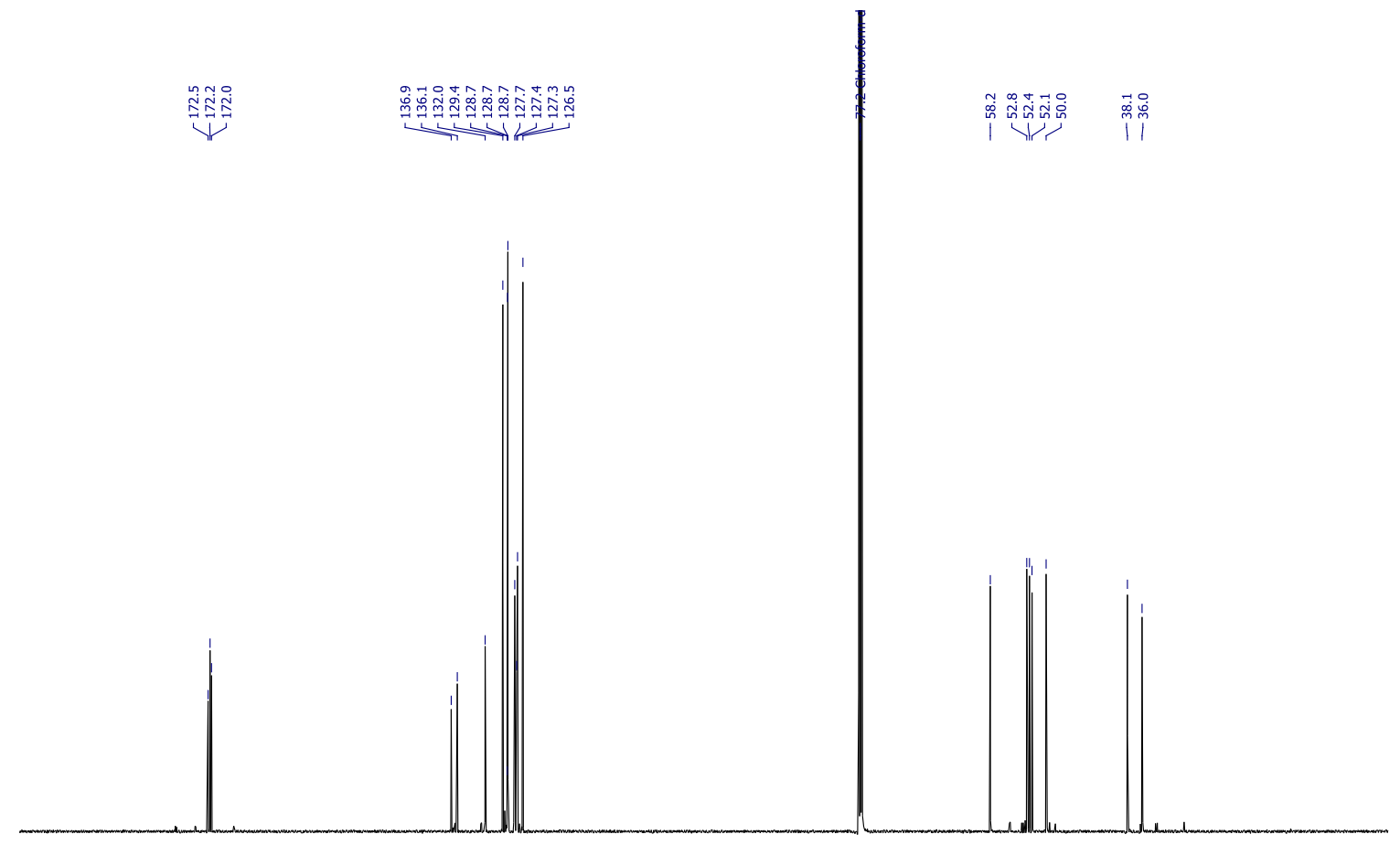

Figure $\mathbf{S}$-44. ${ }^{13} \mathrm{C}$ NMR spectrum of $\mathbf{S - 2 9}\left(\mathrm{CDCl}_{3}, 151 \mathrm{MHz}, 298 \mathrm{~K}\right)$ 


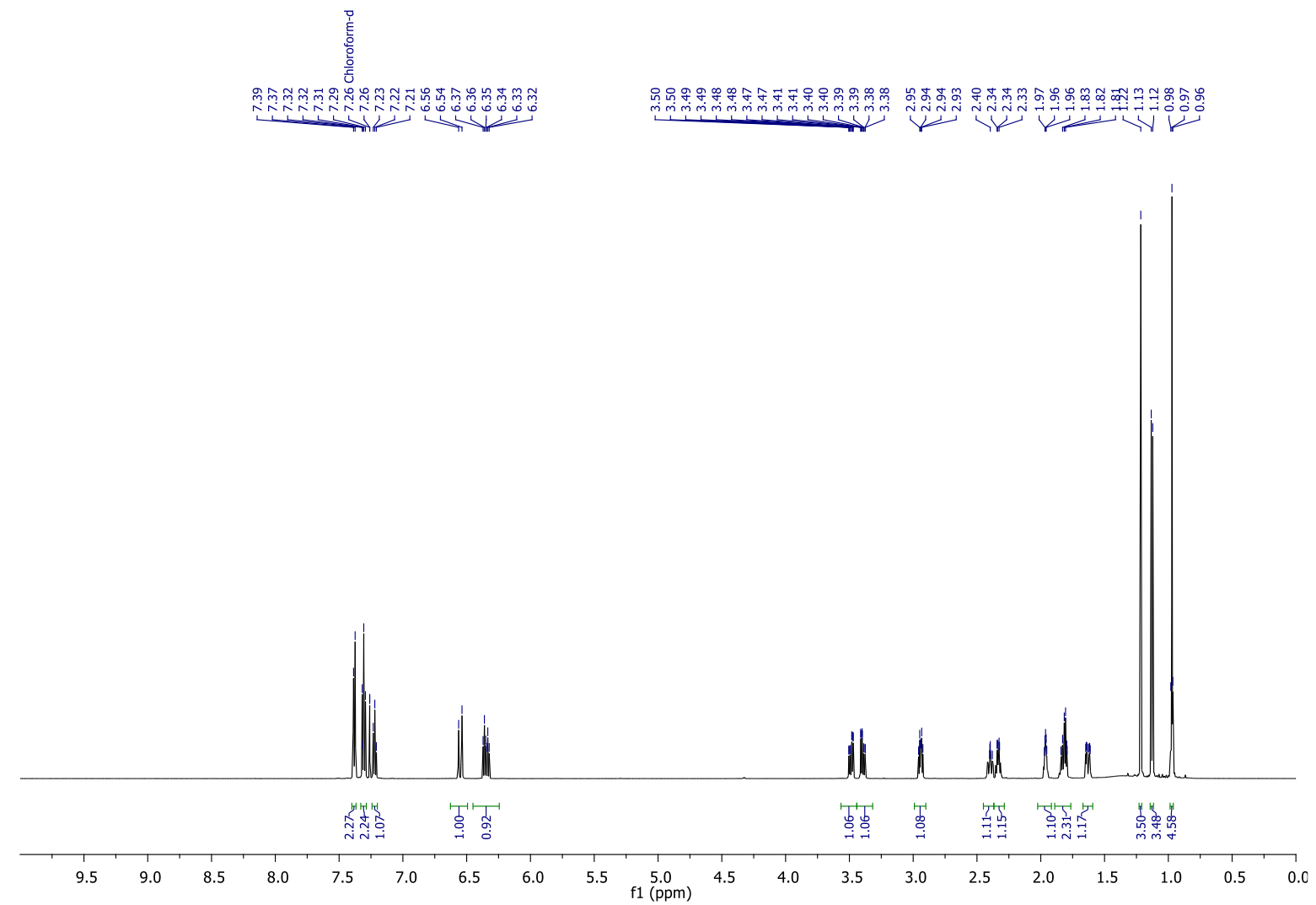

Figure S-45. ${ }^{1} \mathrm{H}$ NMR spectrum of $\mathbf{S - 3 0}\left(\mathrm{CDCl}_{3}, 600 \mathrm{MHz}, 298 \mathrm{~K}\right)$
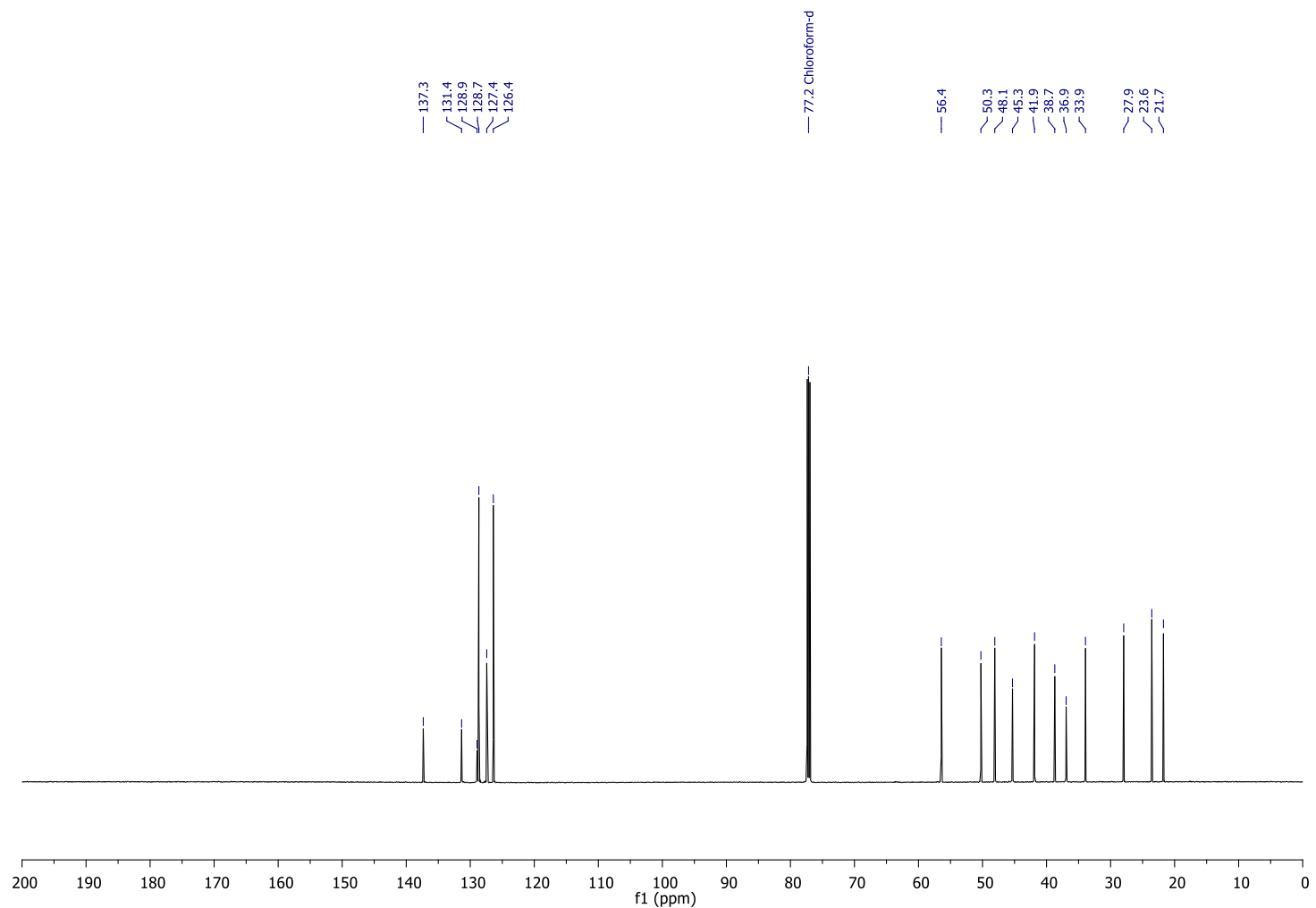

Figure S-46. ${ }^{13} \mathrm{C}$ NMR spectrum of $\mathbf{S - 3 0}\left(\mathrm{CDCl}_{3}, 151 \mathrm{MHz}, 298 \mathrm{~K}\right)$ 


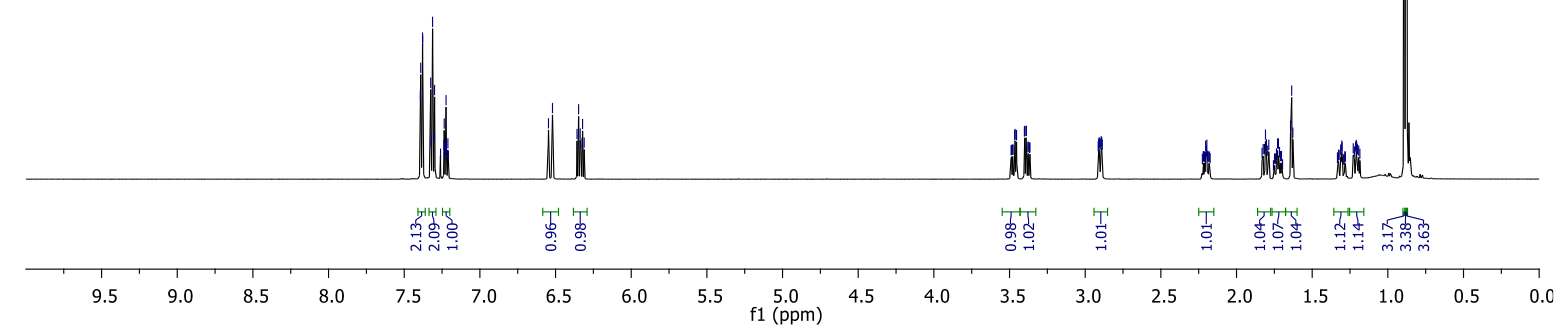

Figure S-47. ${ }^{1} \mathrm{H}$ NMR spectrum of $\mathbf{S - 3 1}\left(\mathrm{CDCl}_{3}, 600 \mathrm{MHz}, 298 \mathrm{~K}\right)$

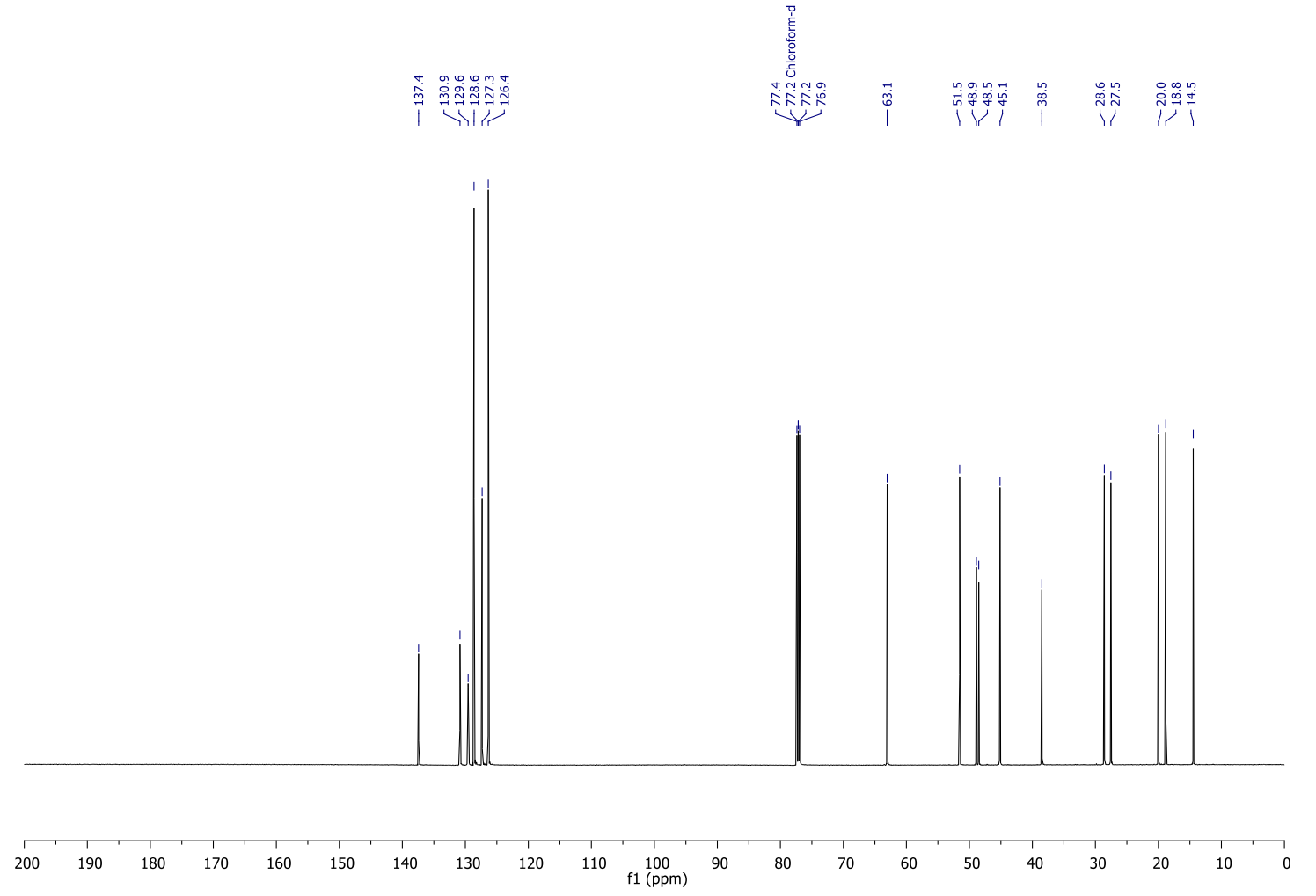

Figure S-48. ${ }^{13} \mathrm{C}$ NMR spectrum of $\mathbf{S - 3 1}\left(\mathrm{CDCl}_{3}, 151 \mathrm{MHz}, 298 \mathrm{~K}\right)$ 


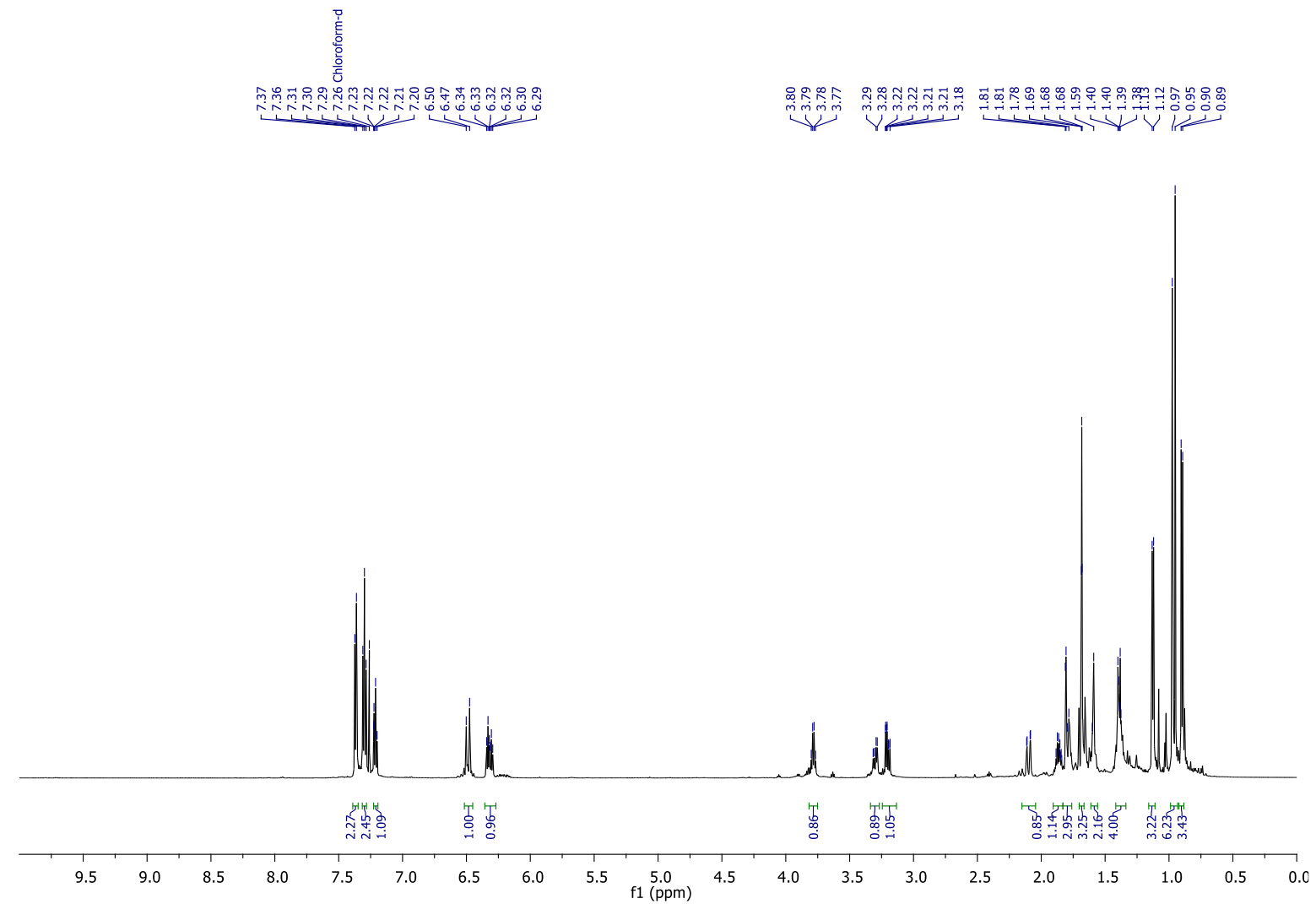

Figure S-49. ${ }^{1} \mathrm{H}$ NMR spectrum of $\mathbf{S - 3 2}\left(\mathrm{CDCl}_{3}, 600 \mathrm{MHz}, 298 \mathrm{~K}\right)$
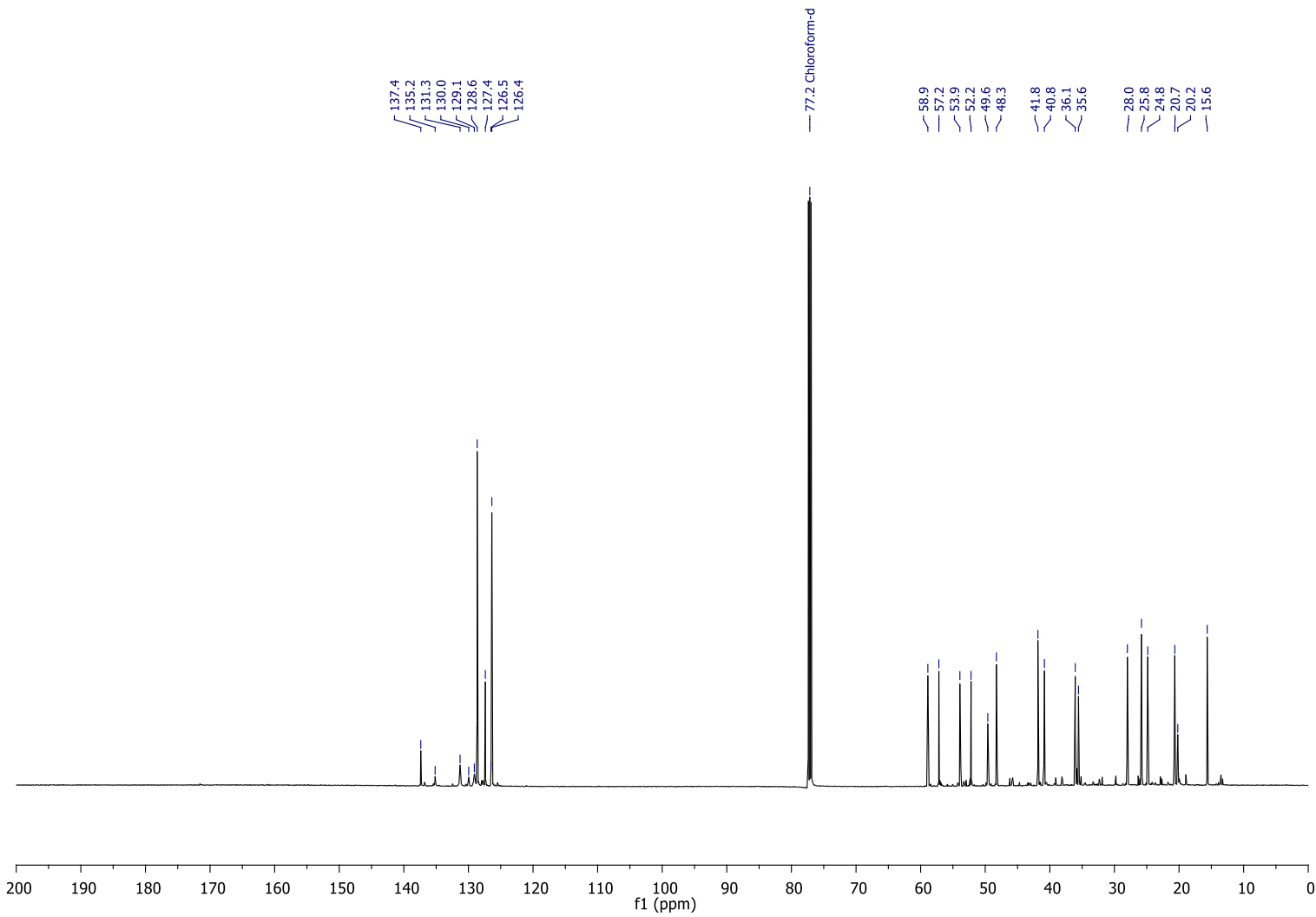

Figure S-50. ${ }^{13} \mathrm{C}$ NMR spectrum of $\mathbf{S - 3 2}\left(\mathrm{CDCl}_{3}, 151 \mathrm{MHz}, 298 \mathrm{~K}\right)$ 


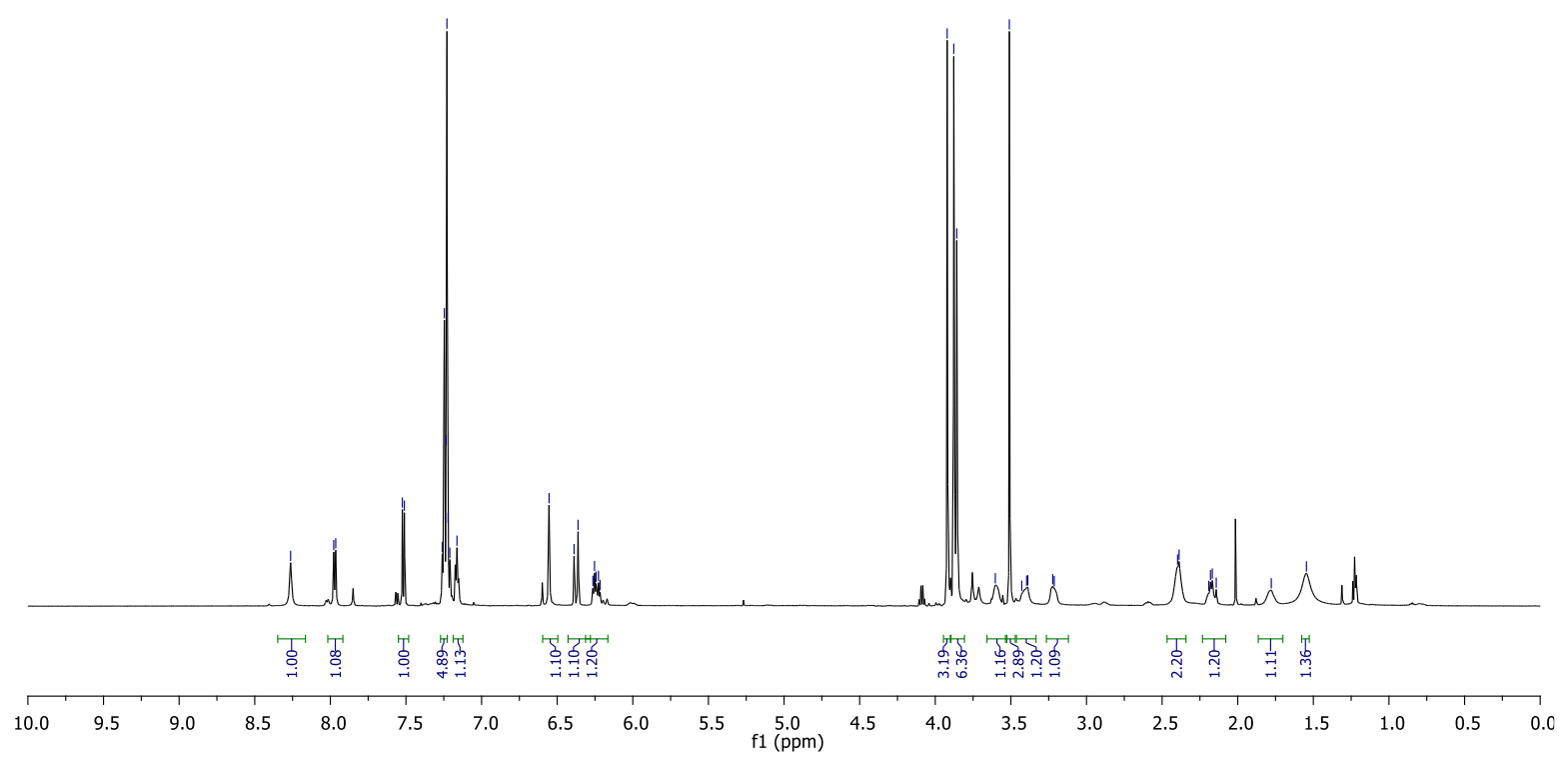

Figure S-51. ${ }^{1} \mathrm{H}$ NMR spectrum of $\mathbf{S - 3 3}\left(\mathrm{CDCl}_{3}, 600 \mathrm{MHz}, 298 \mathrm{~K}\right)$
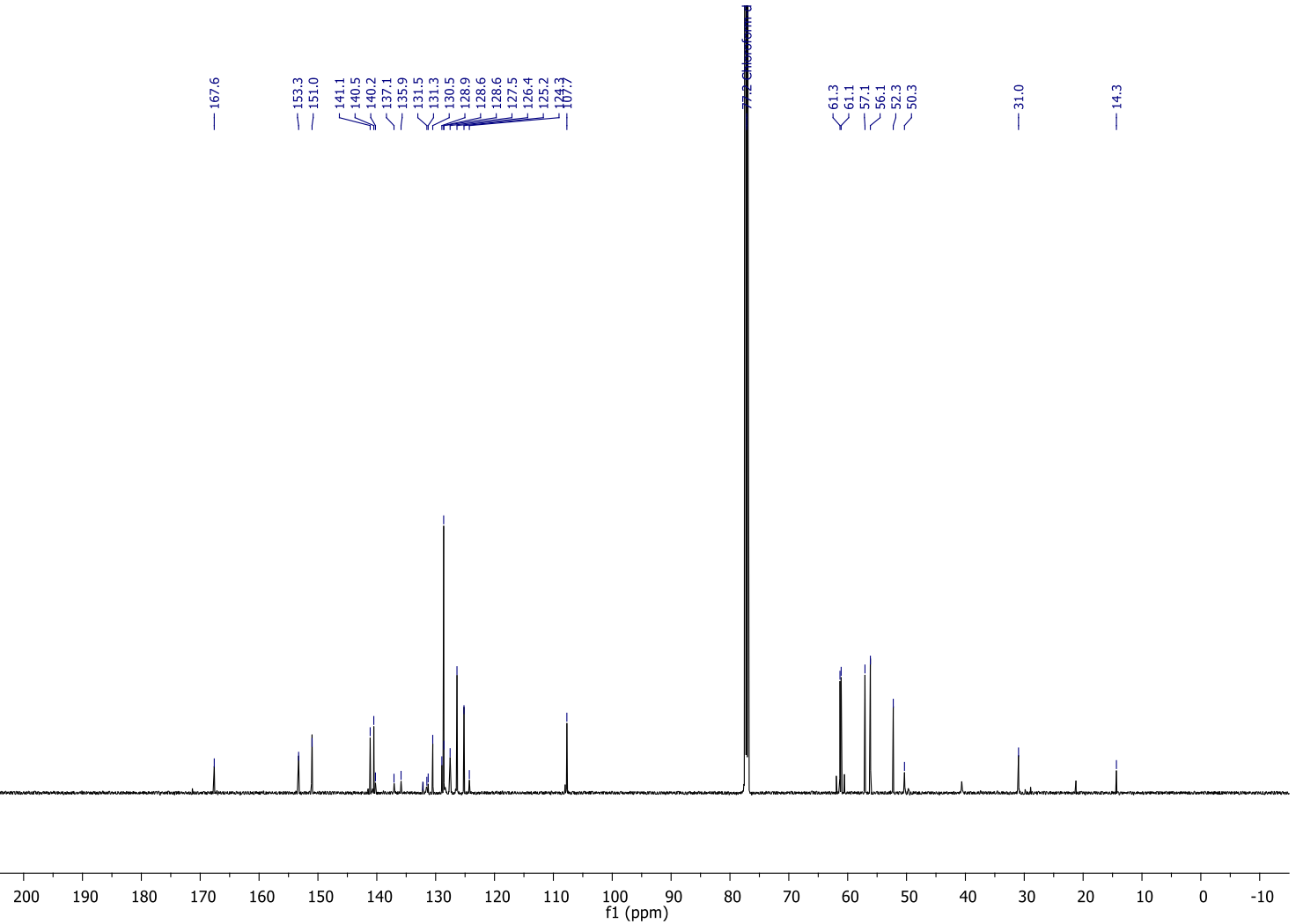

Figure $\mathbf{S - 5 2 .}{ }^{13} \mathrm{C}$ NMR spectrum of $\mathbf{S - 3 3}\left(\mathrm{CDCl}_{3}, 151 \mathrm{MHz}, 298 \mathrm{~K}\right)$ 


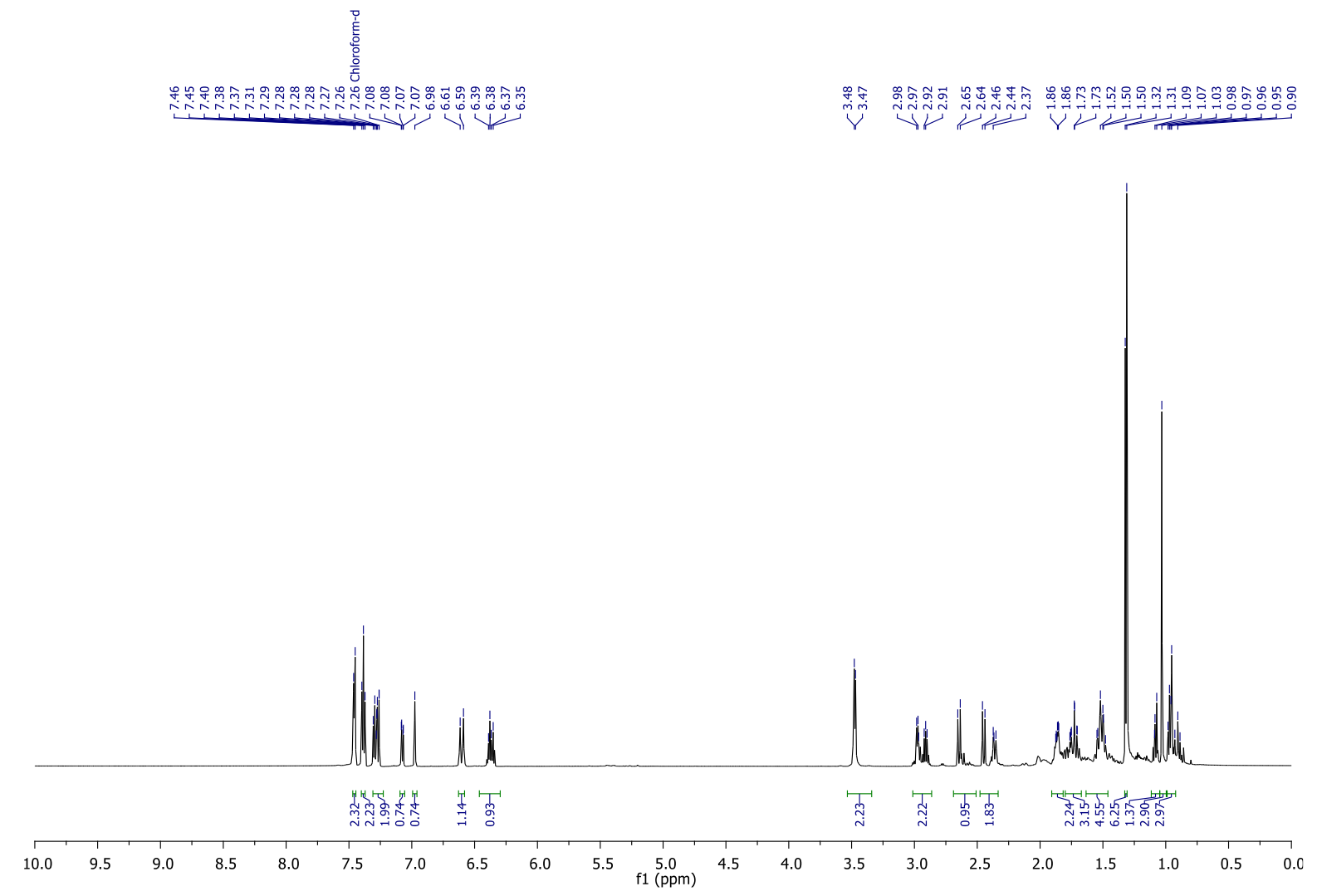

Figure S-53. ${ }^{1} \mathrm{H}$ NMR spectrum of $\mathbf{S - 3 4}\left(\mathrm{CDCl}_{3}, 600 \mathrm{MHz}, 298 \mathrm{~K}\right)$
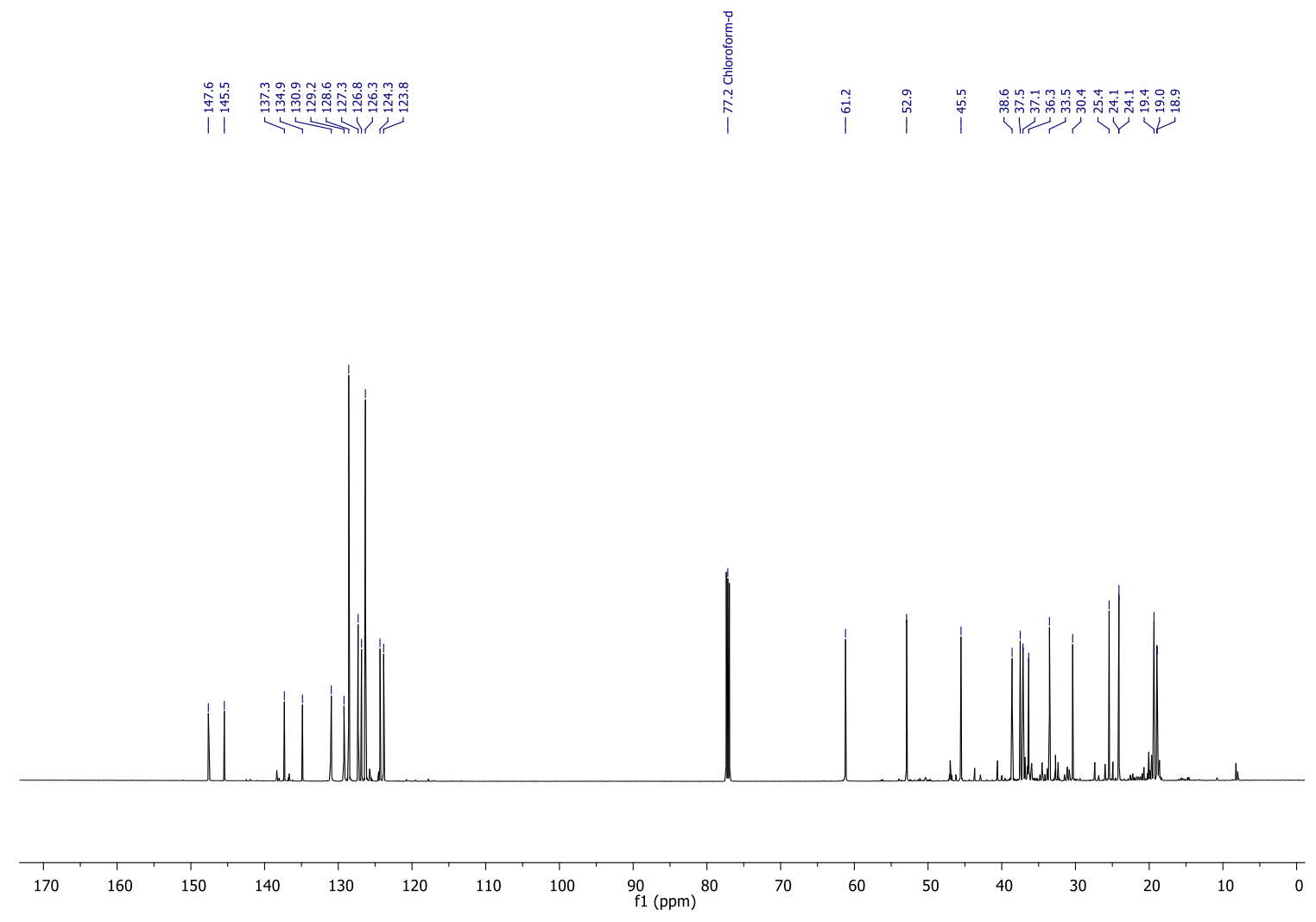

Figure S-54. ${ }^{13} \mathrm{C}$ NMR spectrum of $\mathbf{S - 3 4}\left(\mathrm{CDCl}_{3}, 151 \mathrm{MHz}, 298 \mathrm{~K}\right)$ 


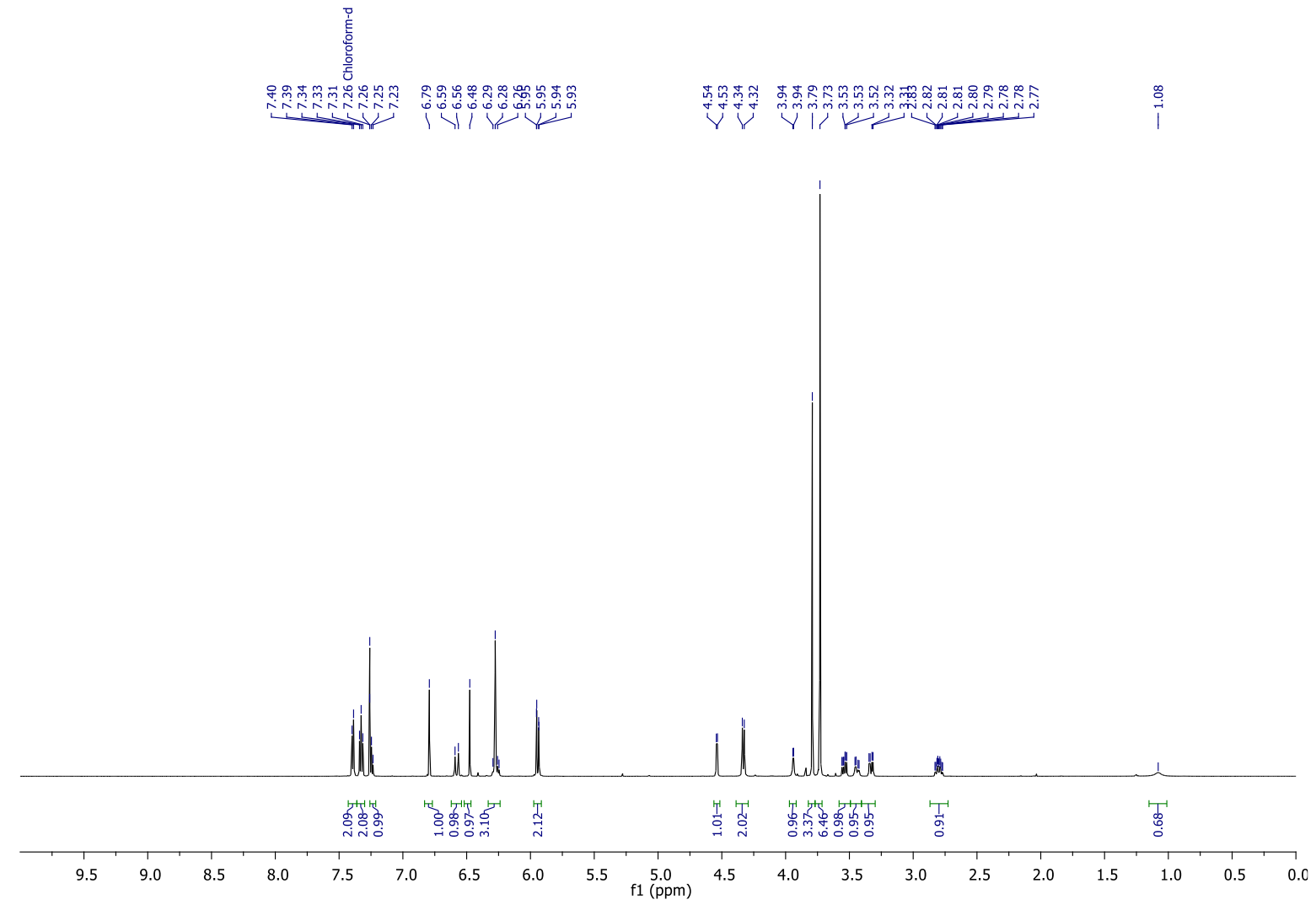

Figure S-55. ${ }^{1} \mathrm{H}$ NMR spectrum of $\mathbf{S - 3 5}\left(\mathrm{CDCl}_{3}, 600 \mathrm{MHz}, 298 \mathrm{~K}\right)$
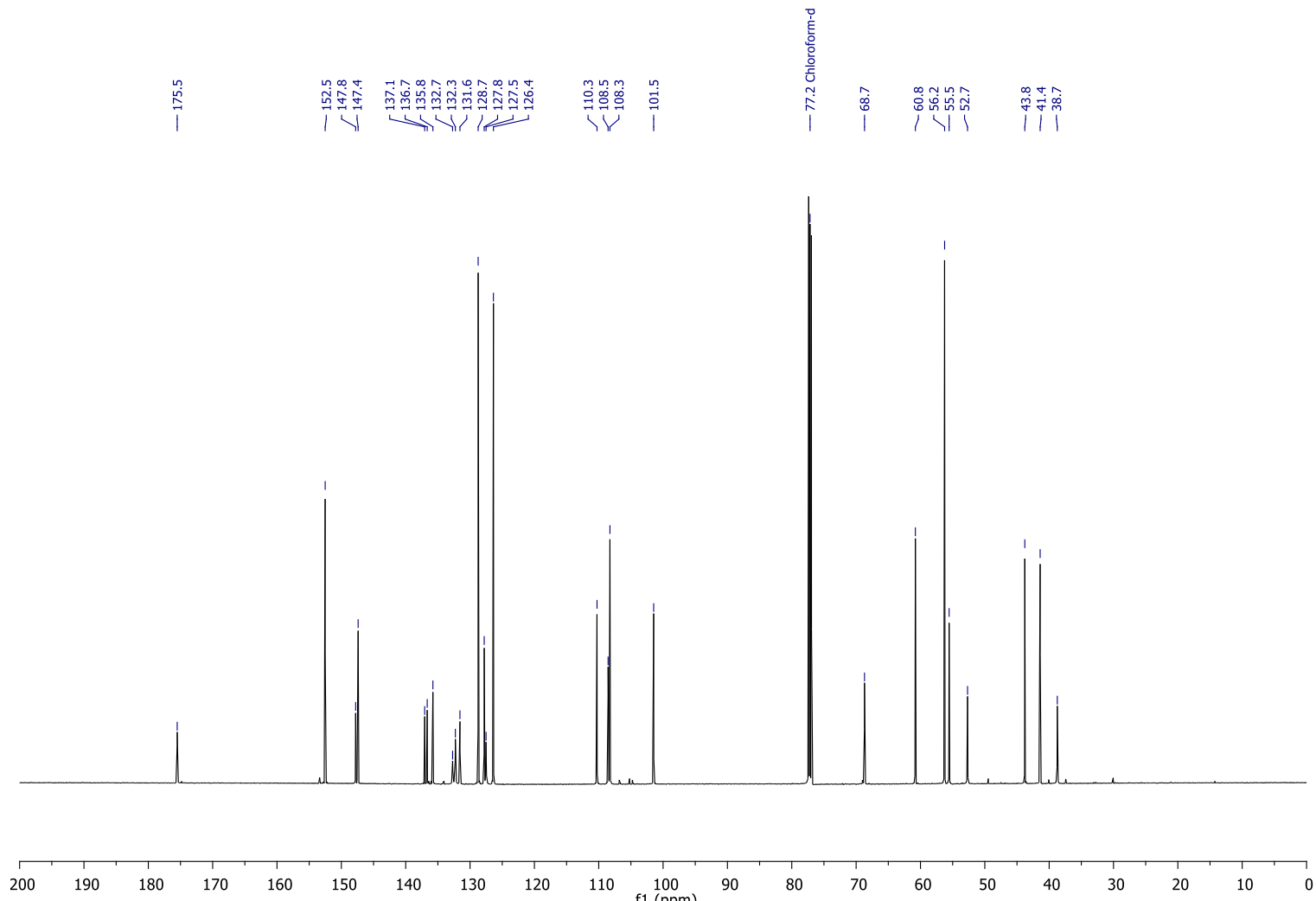

Figure S-56. ${ }^{13} \mathrm{C}$ NMR spectrum of $\mathbf{S - 3 5}\left(\mathrm{CDCl}_{3}, 151 \mathrm{MHz}, 298 \mathrm{~K}\right)$ 


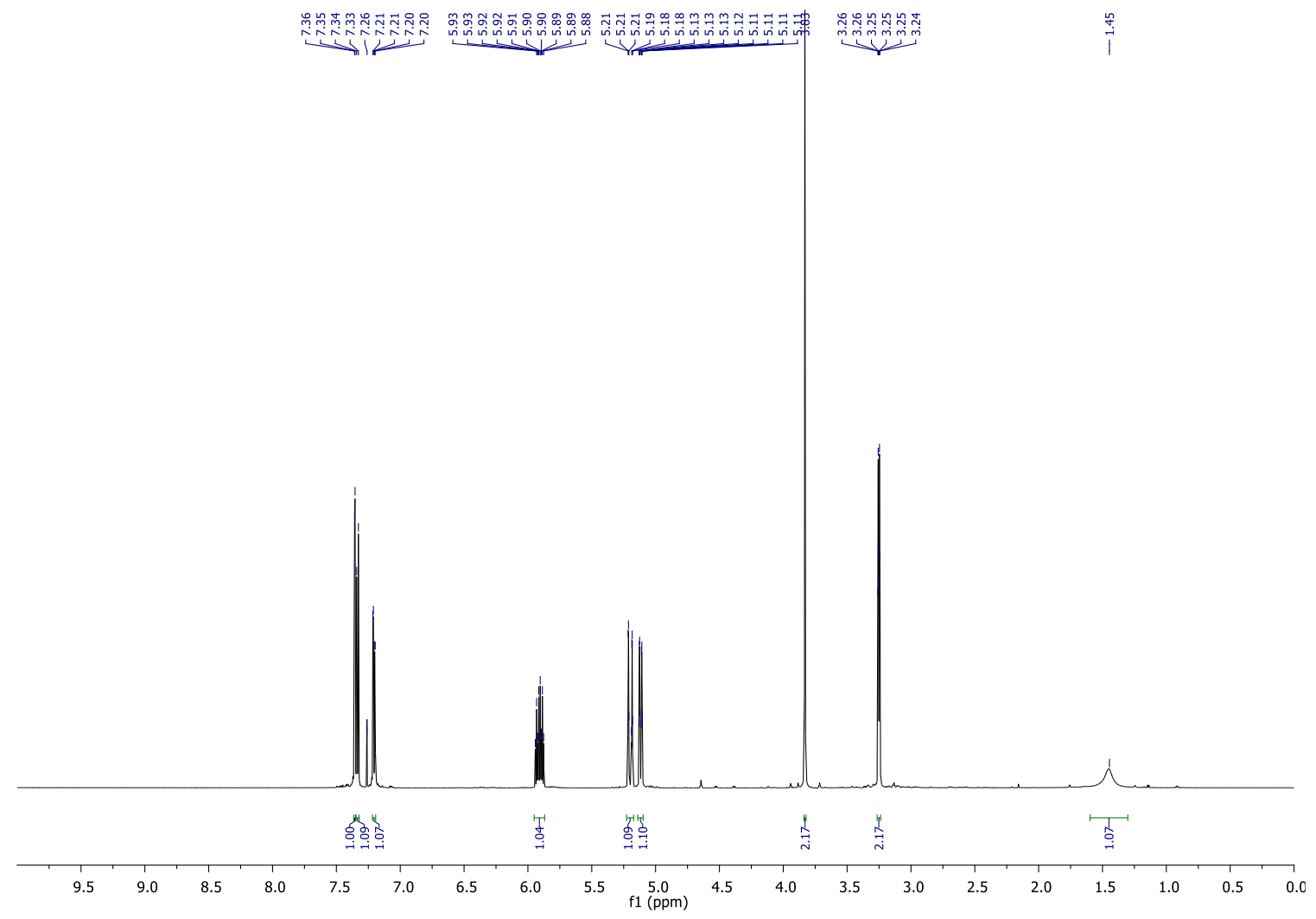

Figure S-57. ${ }^{1} \mathrm{H}$ NMR spectrum of $\mathbf{S - 3 6}\left(\mathrm{CDCl}_{3}, 600 \mathrm{MHz}, 298 \mathrm{~K}\right)$
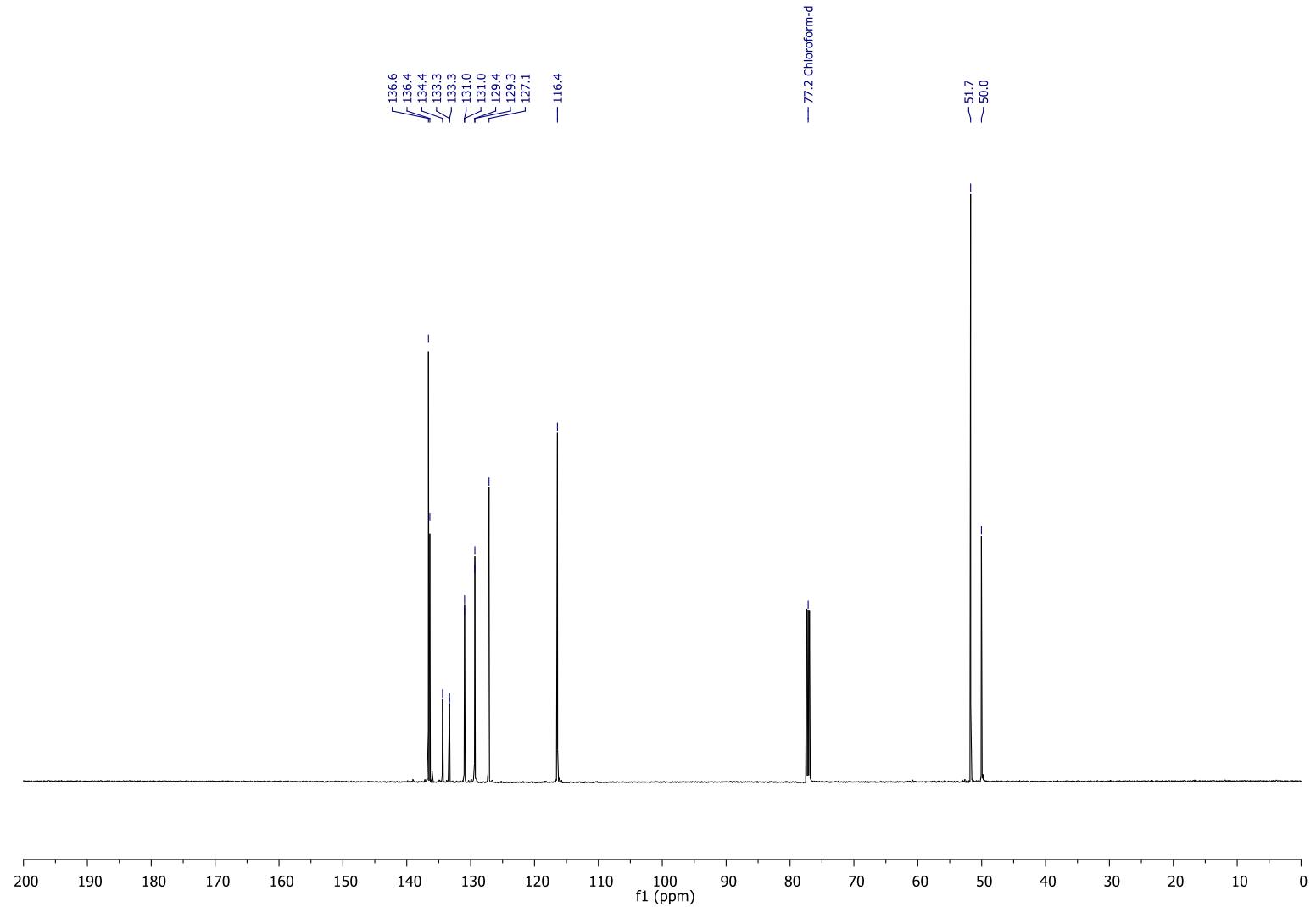

Figure $\mathbf{S - 5 8 .}{ }^{13} \mathrm{C}$ NMR spectrum of $\mathbf{S - 3 6}\left(\mathrm{CDCl}_{3}, 151 \mathrm{MHz}, 298 \mathrm{~K}\right)$ 

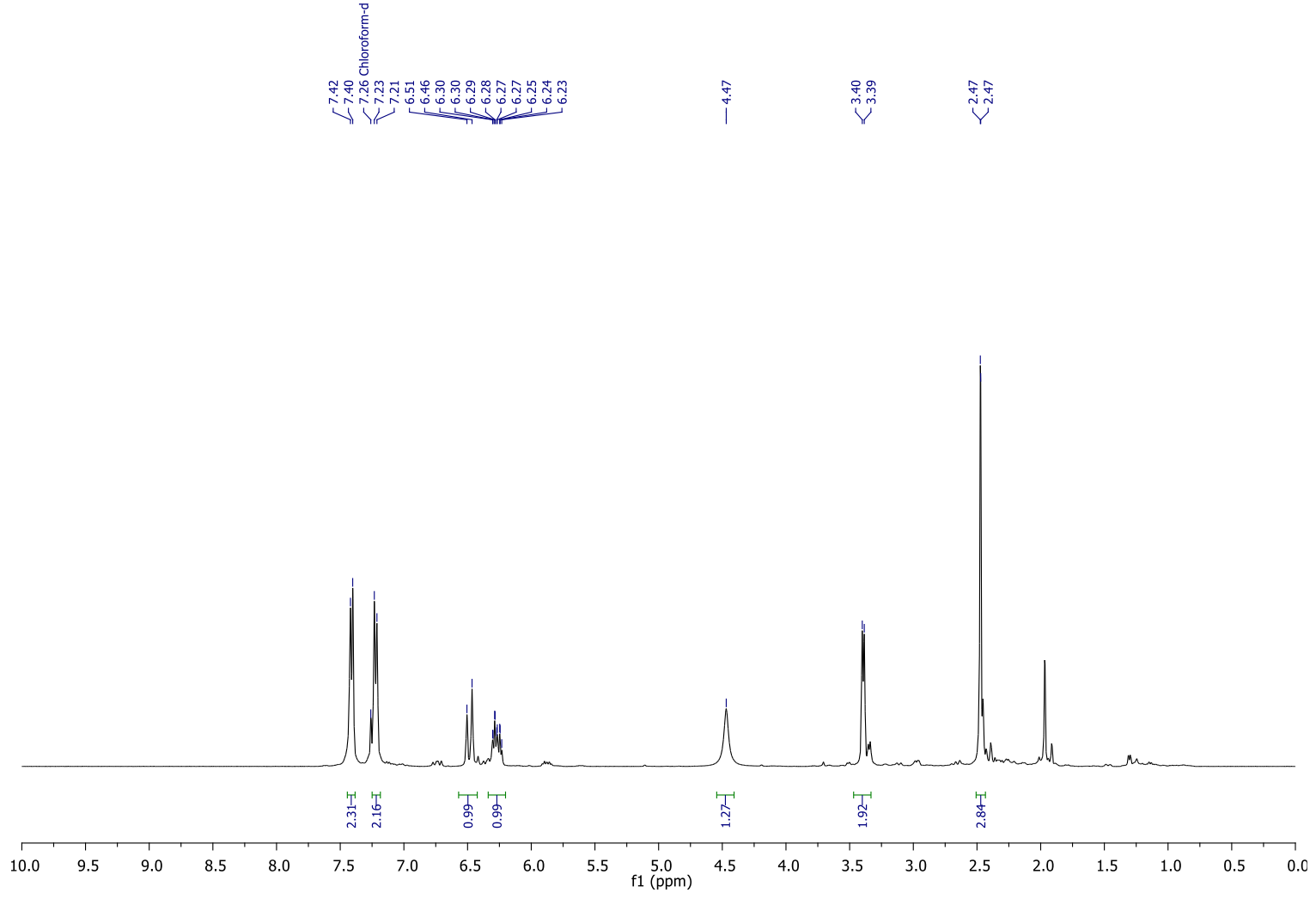

Figure S-59. ${ }^{1} \mathrm{H}$ NMR spectrum of $\mathbf{S - 3 8}\left(\mathrm{CDCl}_{3}, 600 \mathrm{MHz}, 298 \mathrm{~K}\right)$
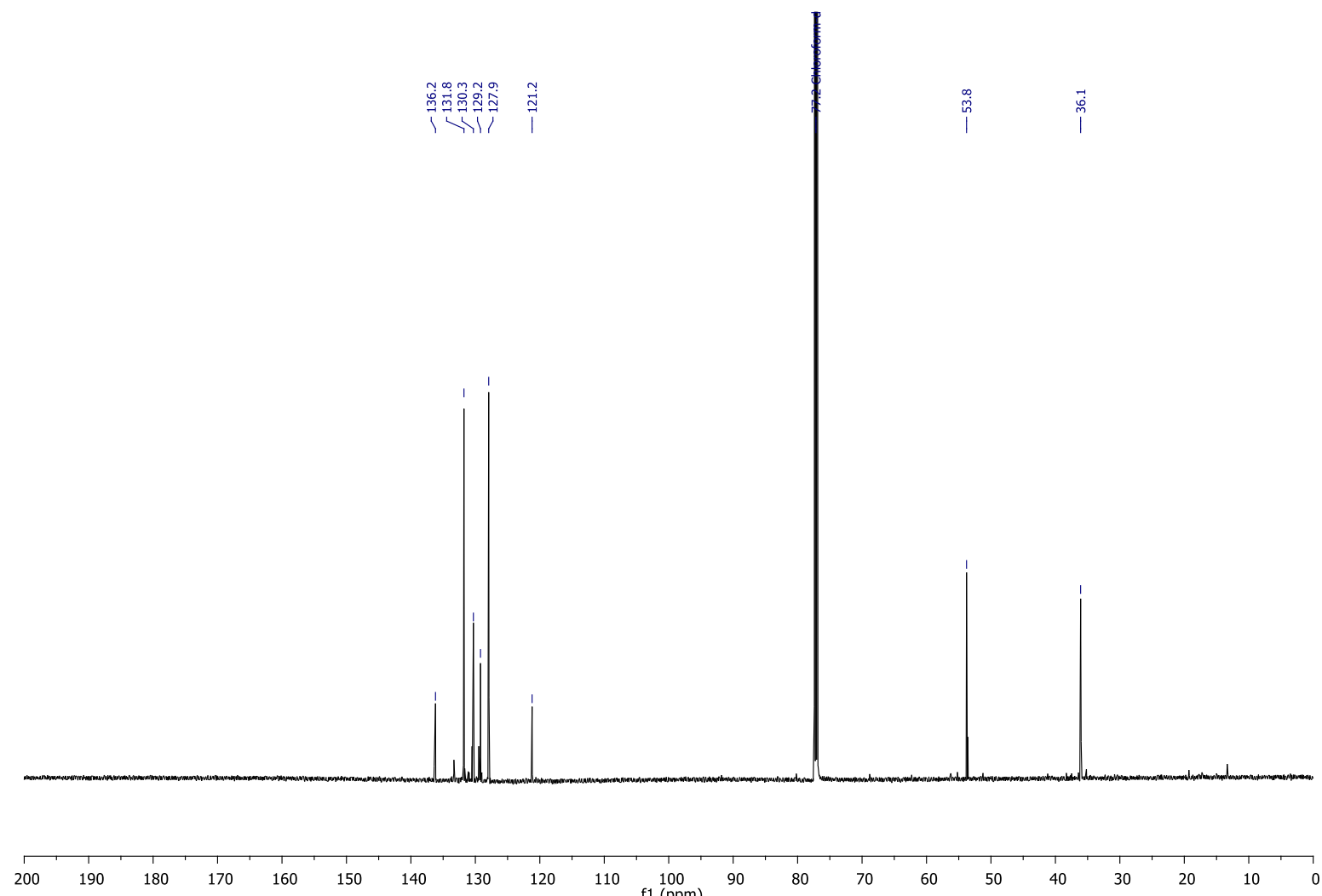

Figure $\mathbf{S}$-60. ${ }^{13} \mathrm{C}$ NMR spectrum of $\mathbf{S - 3 8}\left(\mathrm{CDCl}_{3}, 151 \mathrm{MHz}, 298 \mathrm{~K}\right)$ 


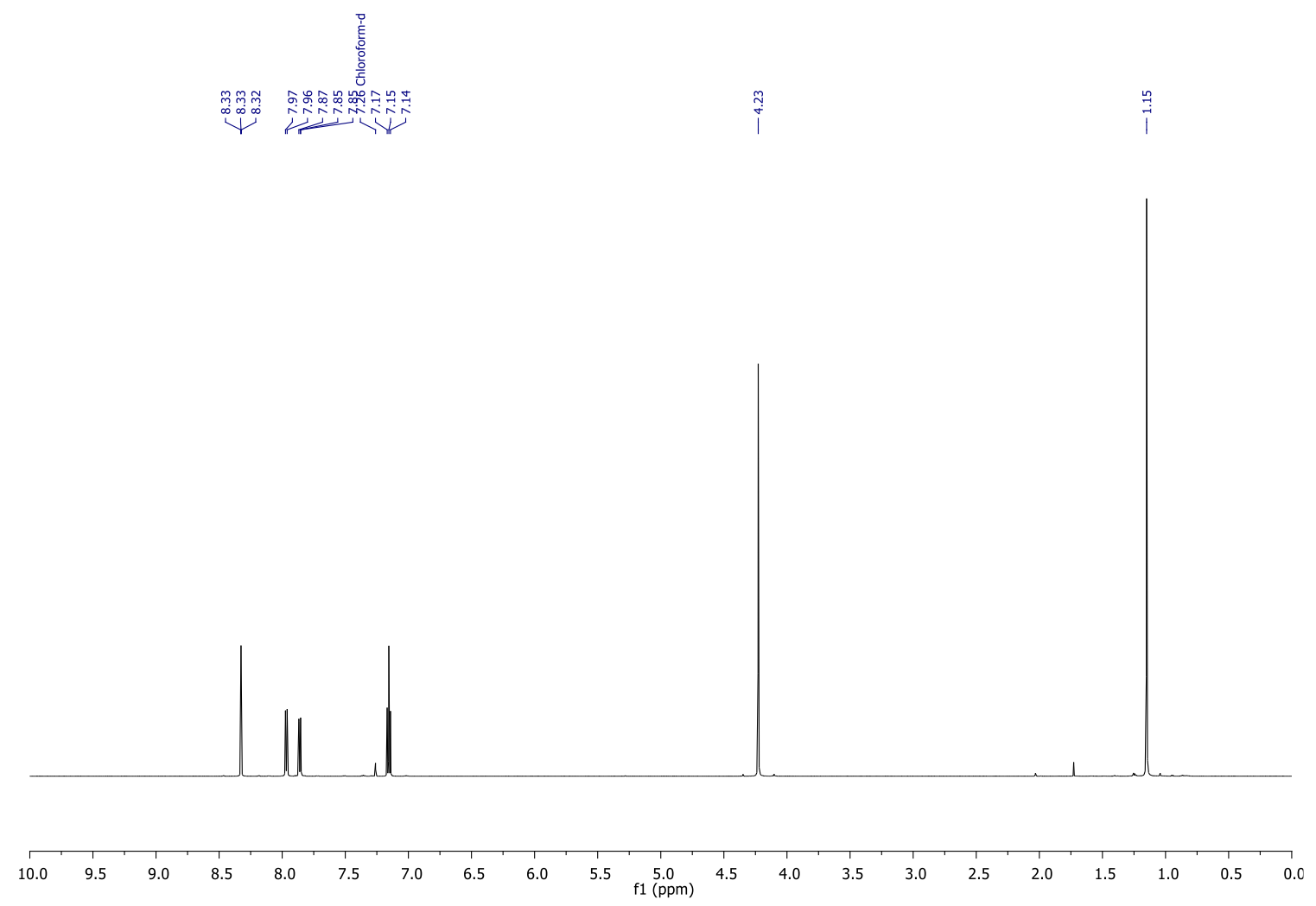

Figure S-61. ${ }^{1} \mathrm{H}$ NMR spectrum of $\mathbf{S}-41\left(\mathrm{CDCl}_{3}, 600 \mathrm{MHz}, 298 \mathrm{~K}\right)$

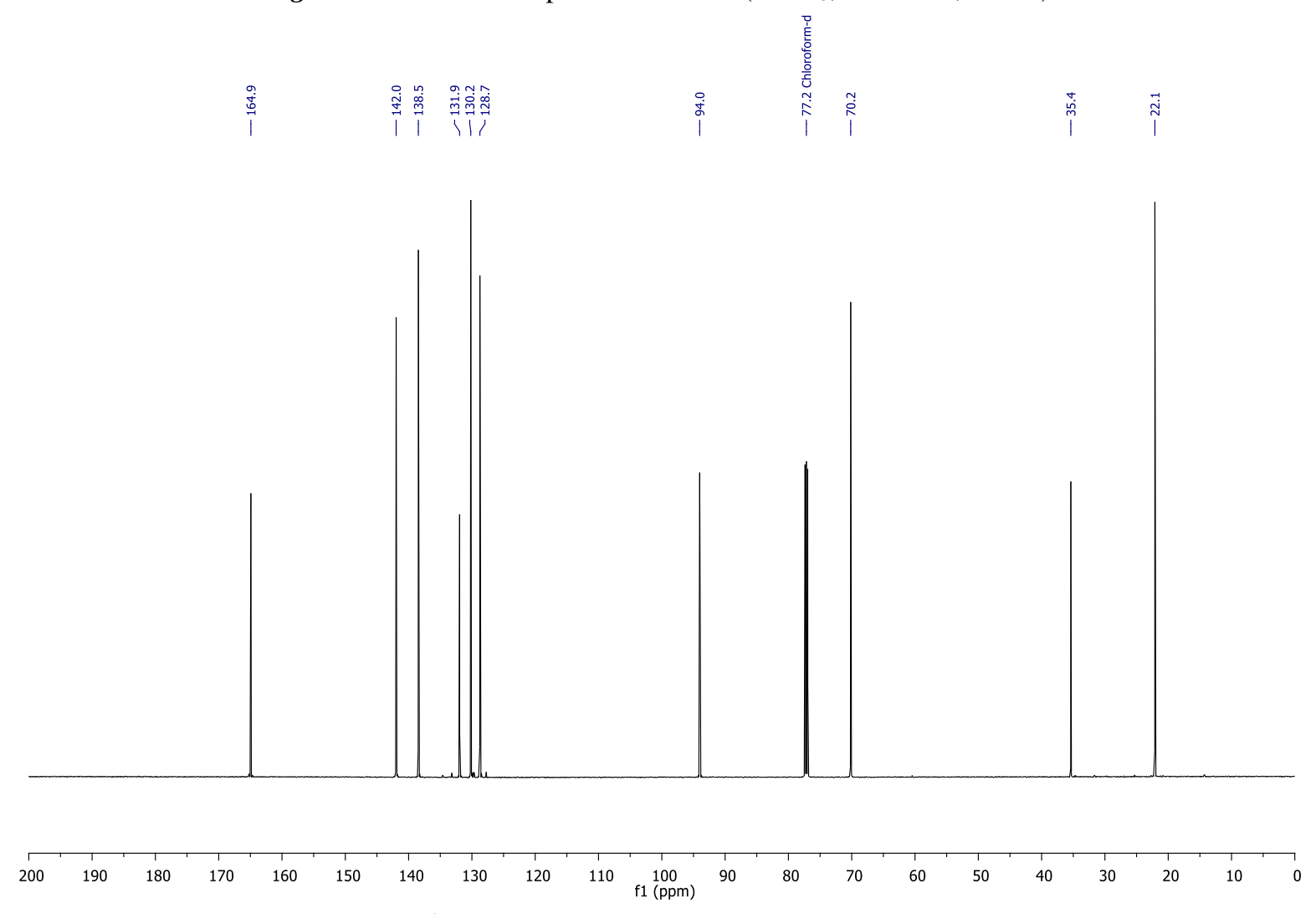

Figure S-62. ${ }^{13} \mathrm{C}$ NMR spectrum of $\mathbf{S}-41\left(\mathrm{CDCl}_{3}, 151 \mathrm{MHz}, 298 \mathrm{~K}\right)$ 


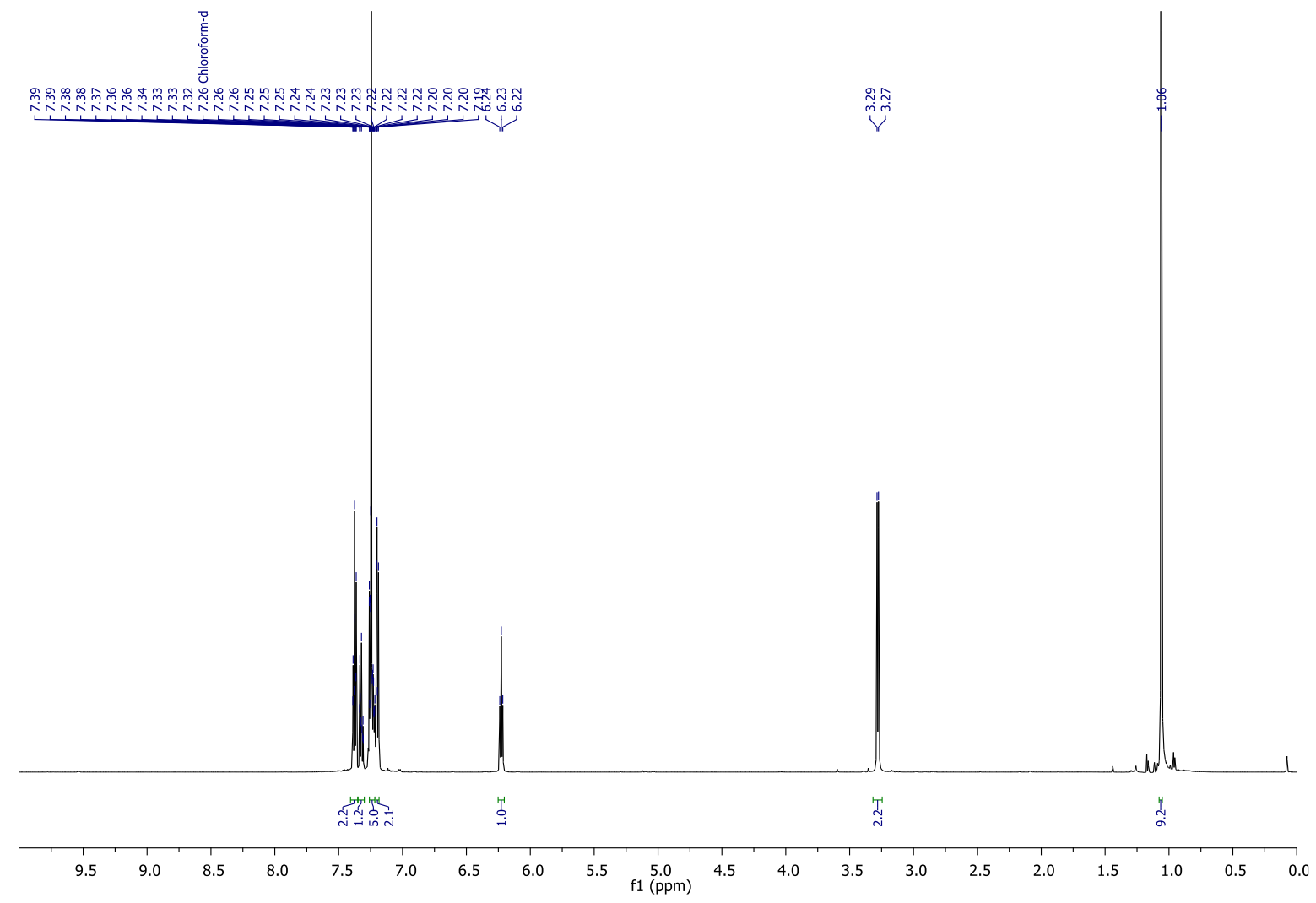

Figure S-63. ${ }^{1} \mathrm{H}$ NMR spectrum of $1 \mathrm{a}\left(\mathrm{CDCl}_{3}, 600 \mathrm{MHz}, 298 \mathrm{~K}\right)$
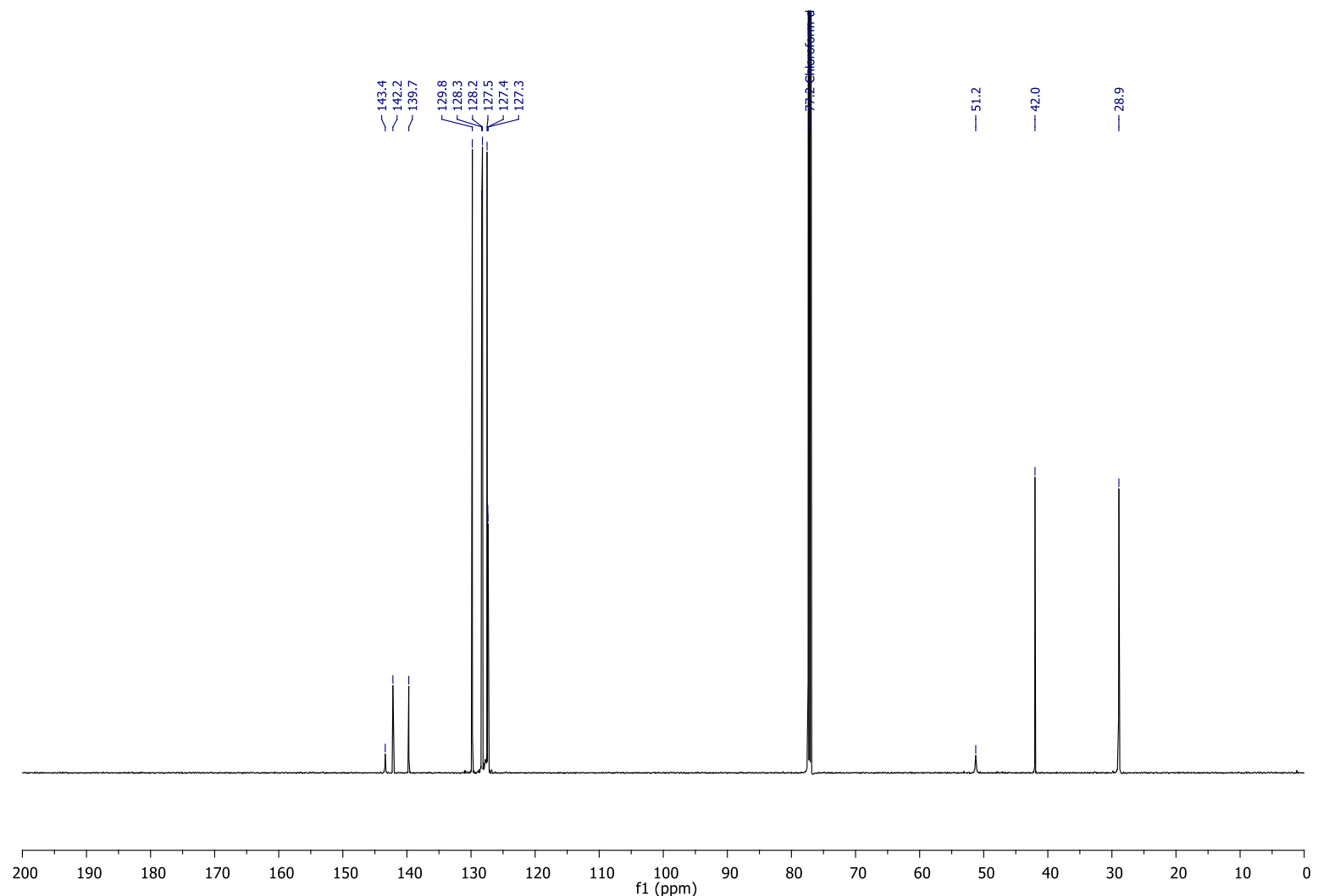

Figure S-64. ${ }^{13} \mathrm{C}$ NMR spectrum of $1 a\left(\mathrm{CDCl}_{3}, 151 \mathrm{MHz}, 298 \mathrm{~K}\right)$ 


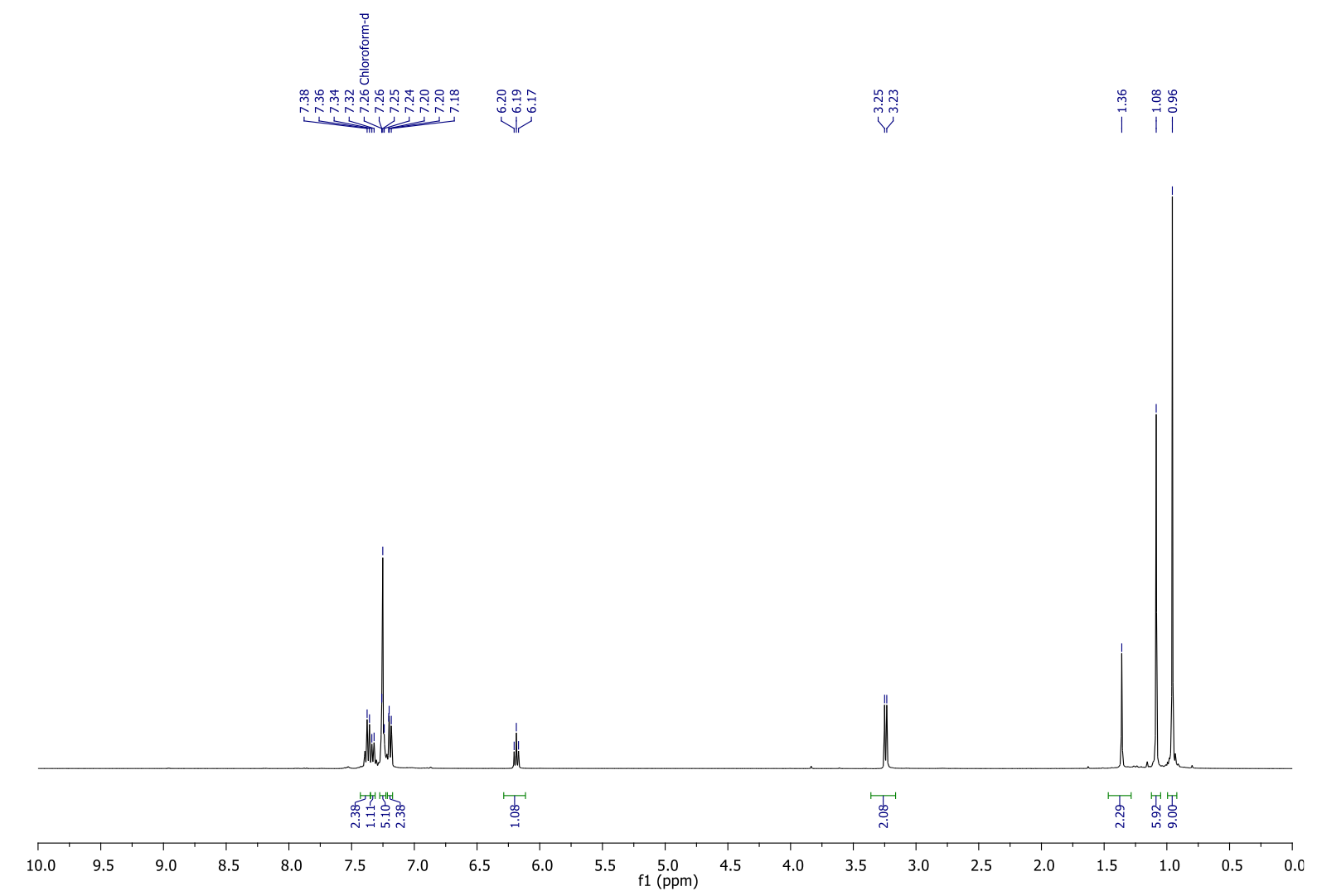

Figure $\mathbf{S - 6 5} .{ }^{1} \mathrm{H}$ NMR spectrum of $\mathbf{1 b}\left(\mathrm{CDCl}_{3}, 600 \mathrm{MHz}, 298 \mathrm{~K}\right)$
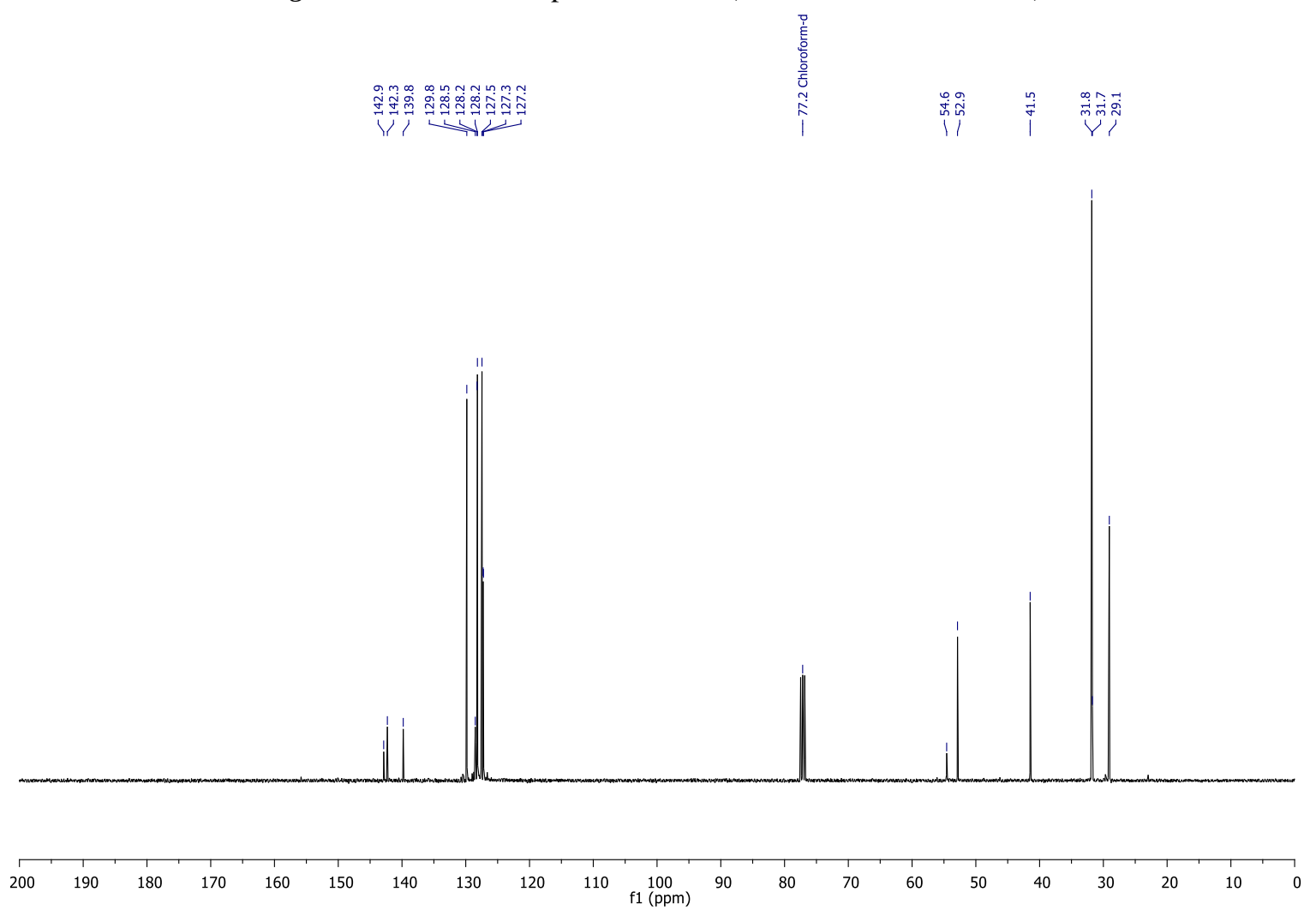

Figure S-66. ${ }^{13} \mathrm{C}$ NMR spectrum of $\mathbf{1 b}\left(\mathrm{CDCl}_{3}, 151 \mathrm{MHz}, 298 \mathrm{~K}\right)$ 


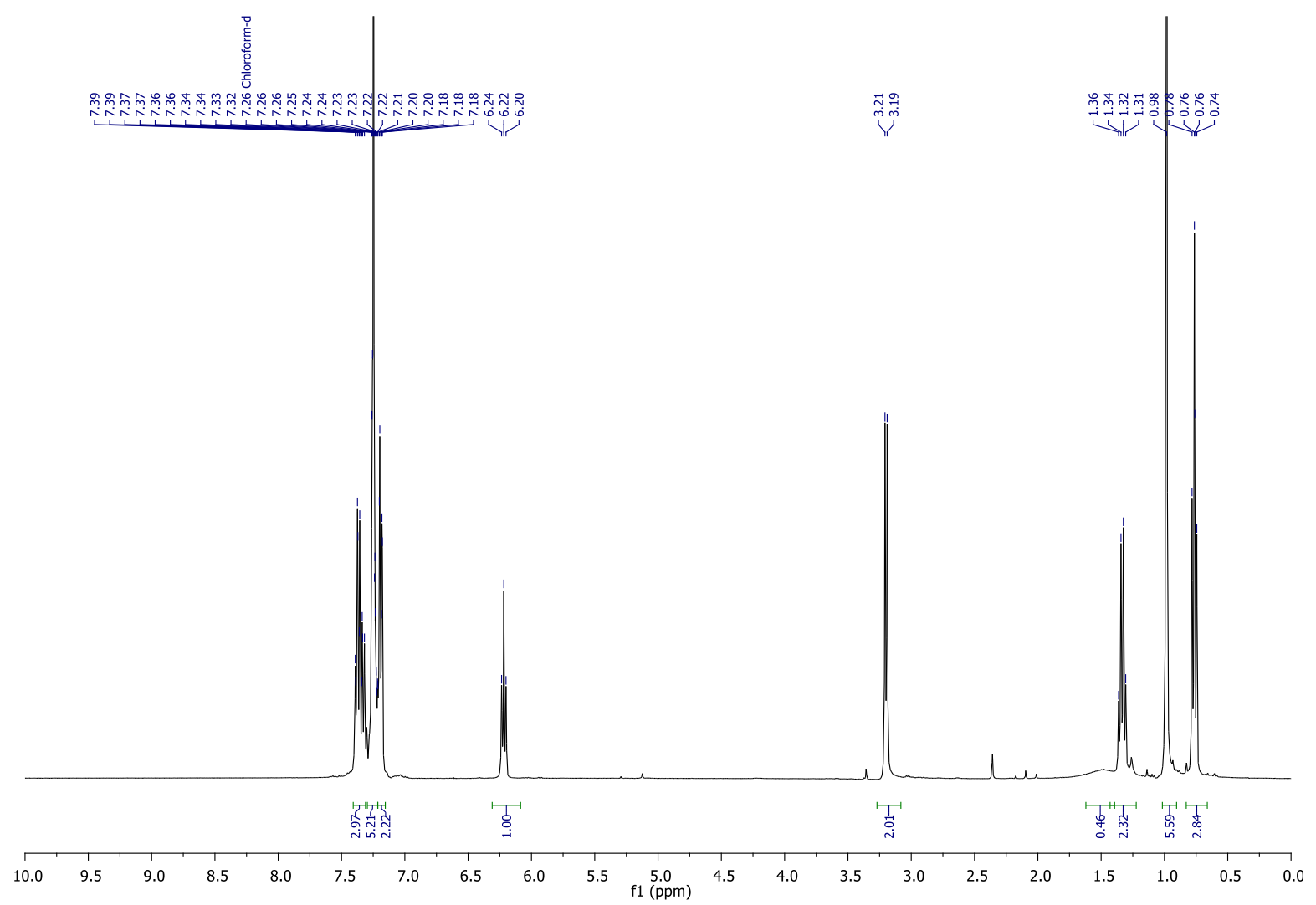

Figure S-67. ${ }^{1} \mathrm{H}$ NMR spectrum of $1 \mathrm{c}\left(\mathrm{CDCl}_{3}, 600 \mathrm{MHz}, 298 \mathrm{~K}\right)$
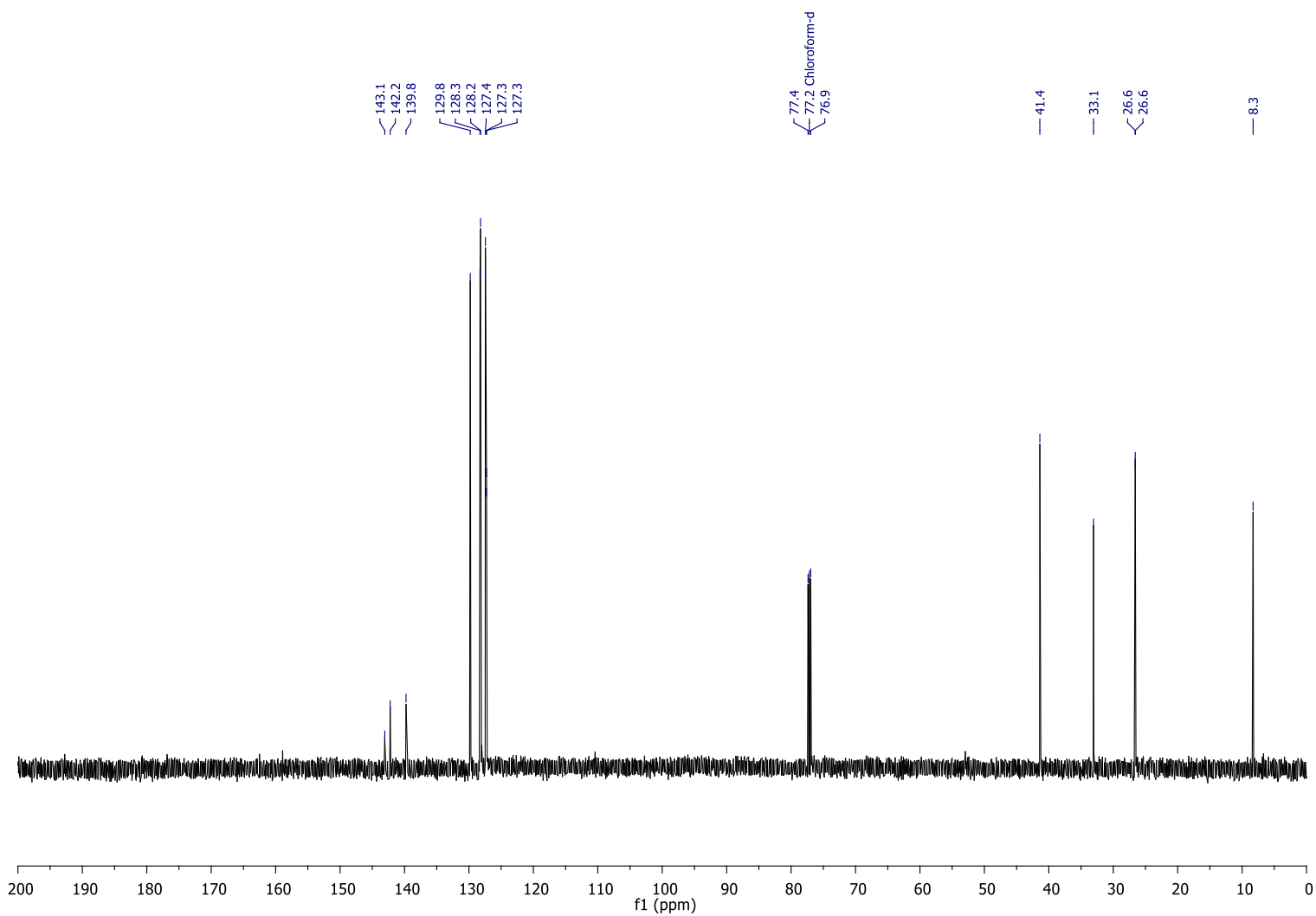

Figure S-68. ${ }^{13} \mathrm{C}$ NMR spectrum of $1 c\left(\mathrm{CDCl}_{3}, 151 \mathrm{MHz}, 298 \mathrm{~K}\right)$ 

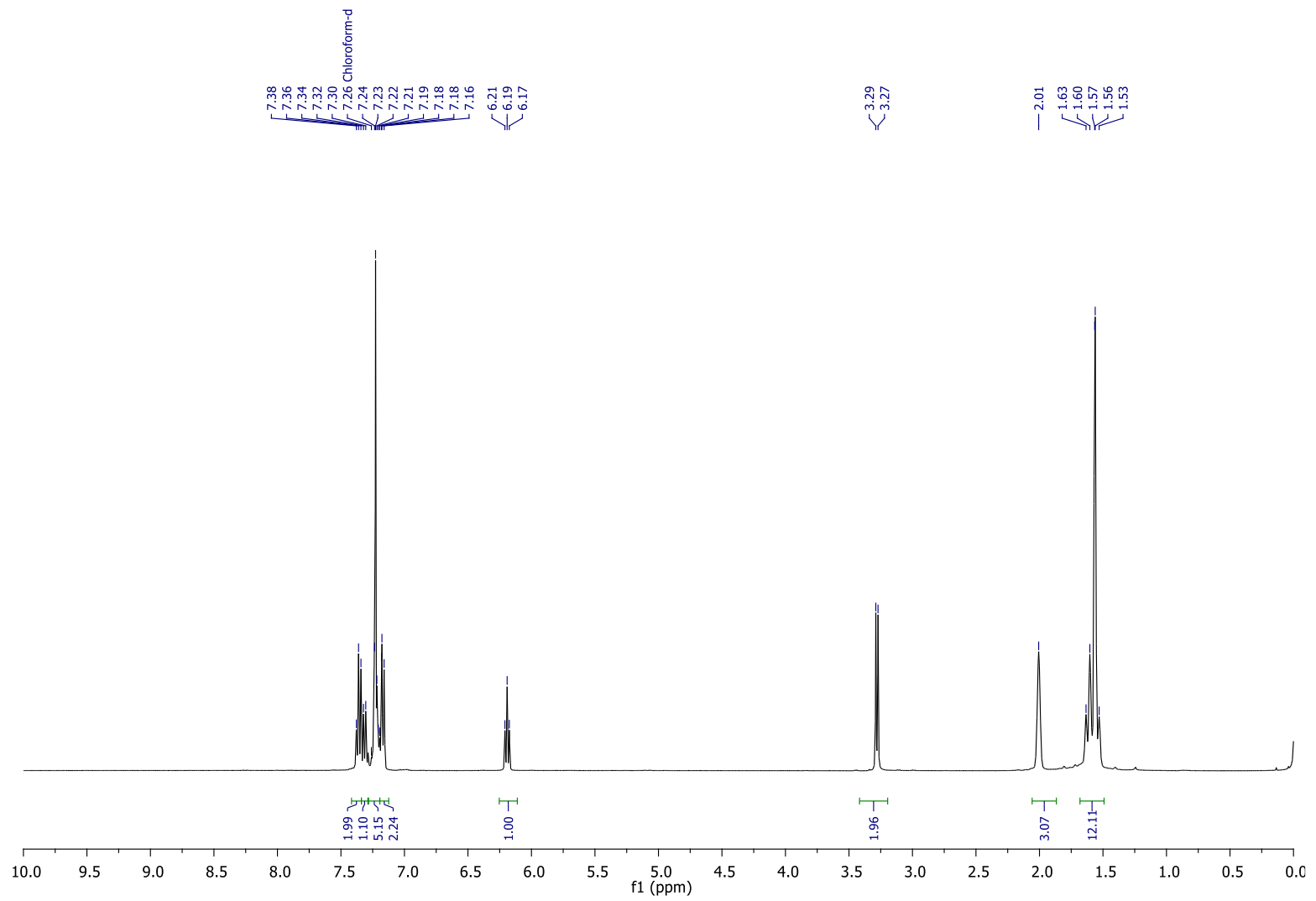

Figure S-69. ${ }^{1} \mathrm{H}$ NMR spectrum of $\mathbf{1 d}\left(\mathrm{CDCl}_{3}, 600 \mathrm{MHz}, 298 \mathrm{~K}\right)$
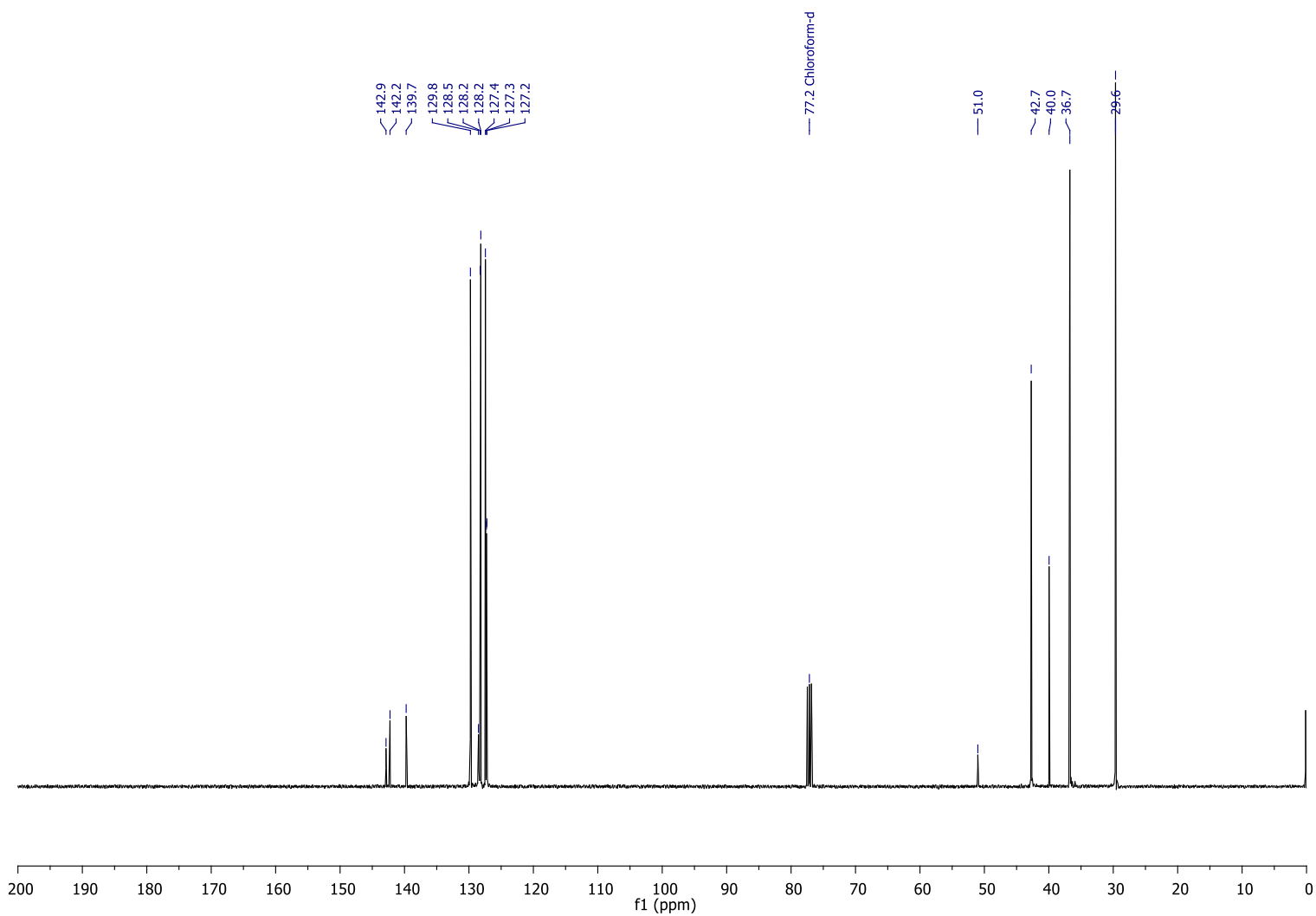

Figure S-70. ${ }^{13} \mathrm{C}$ NMR spectrum of $\mathbf{1 d}\left(\mathrm{CDCl}_{3}, 151 \mathrm{MHz}, 298 \mathrm{~K}\right)$ 

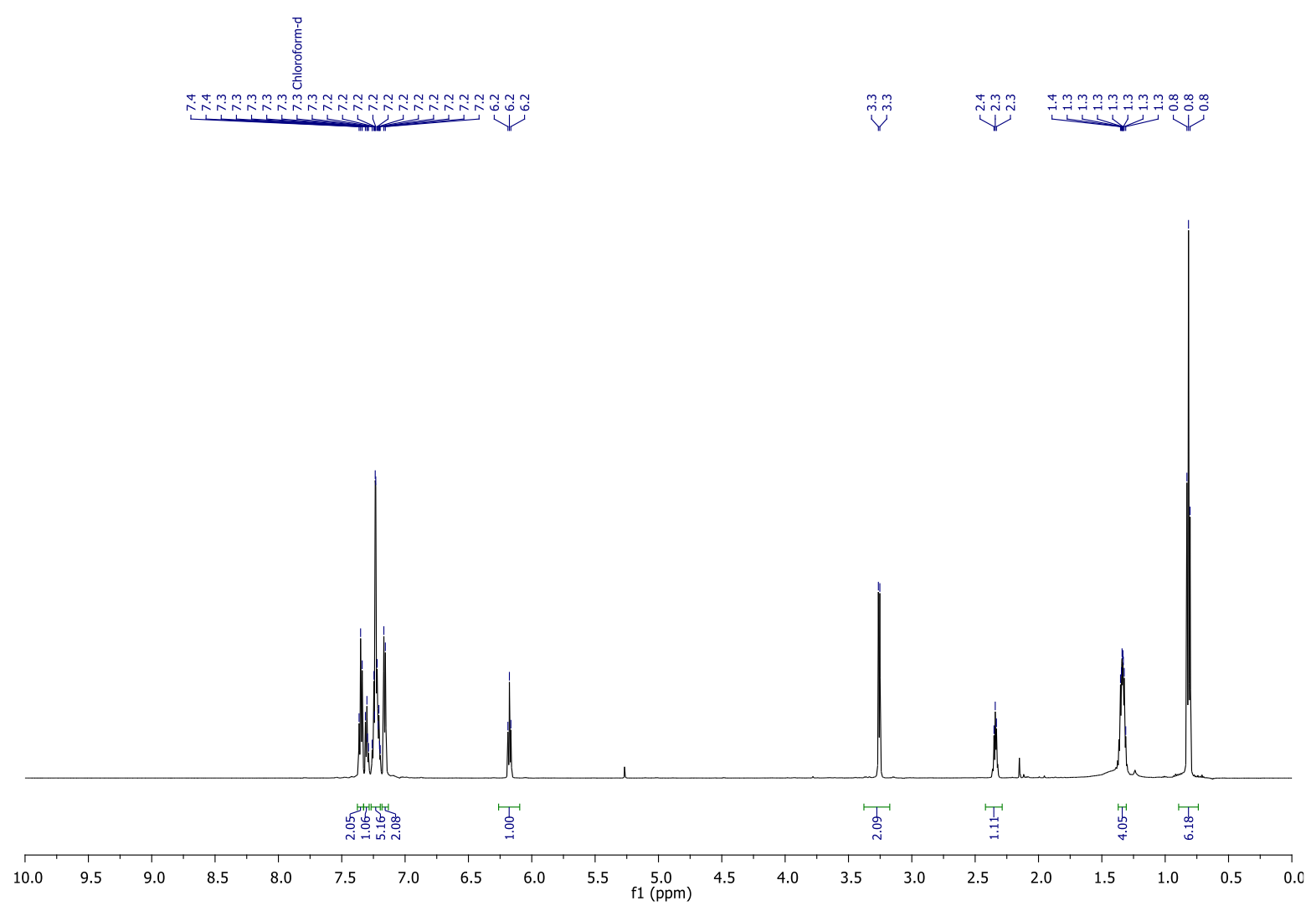

Figure S-71. ${ }^{1} \mathrm{H}$ NMR spectrum of $1 \mathrm{e}\left(\mathrm{CDCl}_{3}, 600 \mathrm{MHz}, 298 \mathrm{~K}\right)$

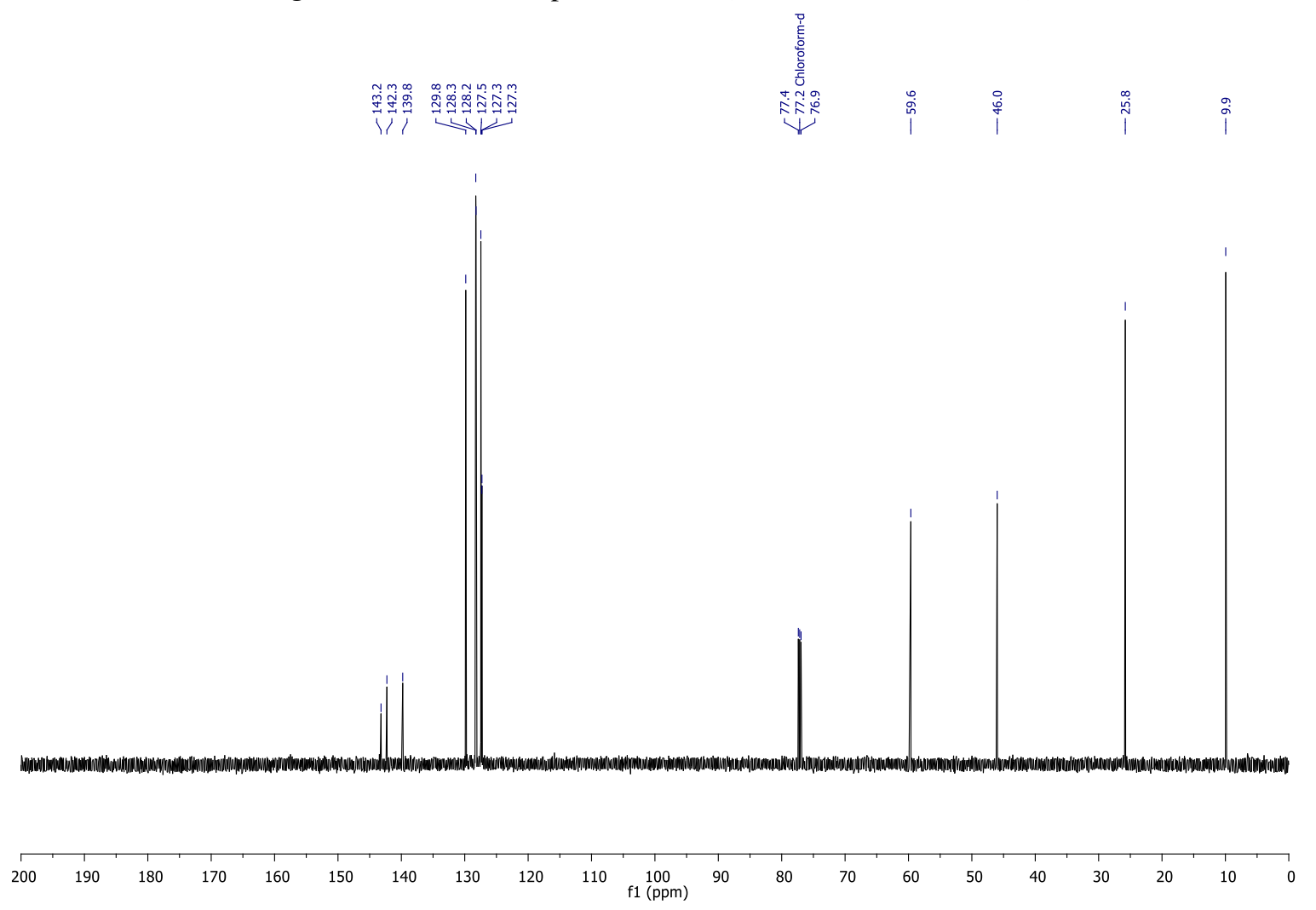

Figure S-72. ${ }^{13} \mathrm{C}$ NMR spectrum of $1 \mathbf{e}\left(\mathrm{CDCl}_{3}, 151 \mathrm{MHz}, 298 \mathrm{~K}\right)$ 

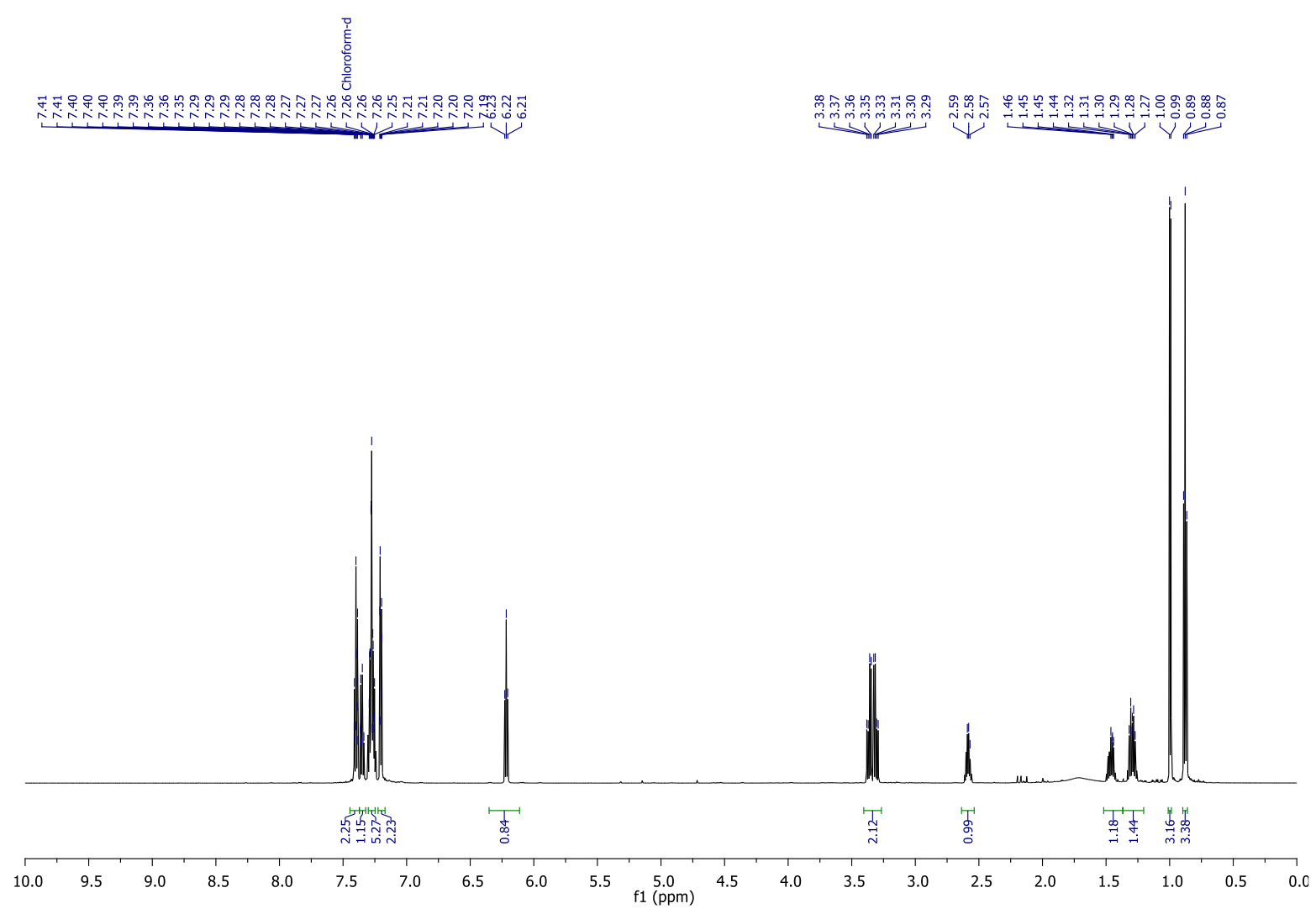

Figure S-73. ${ }^{1} \mathrm{H}$ NMR spectrum of $\mathbf{1 f}\left(\mathrm{CDCl}_{3}, 600 \mathrm{MHz}, 298 \mathrm{~K}\right)$
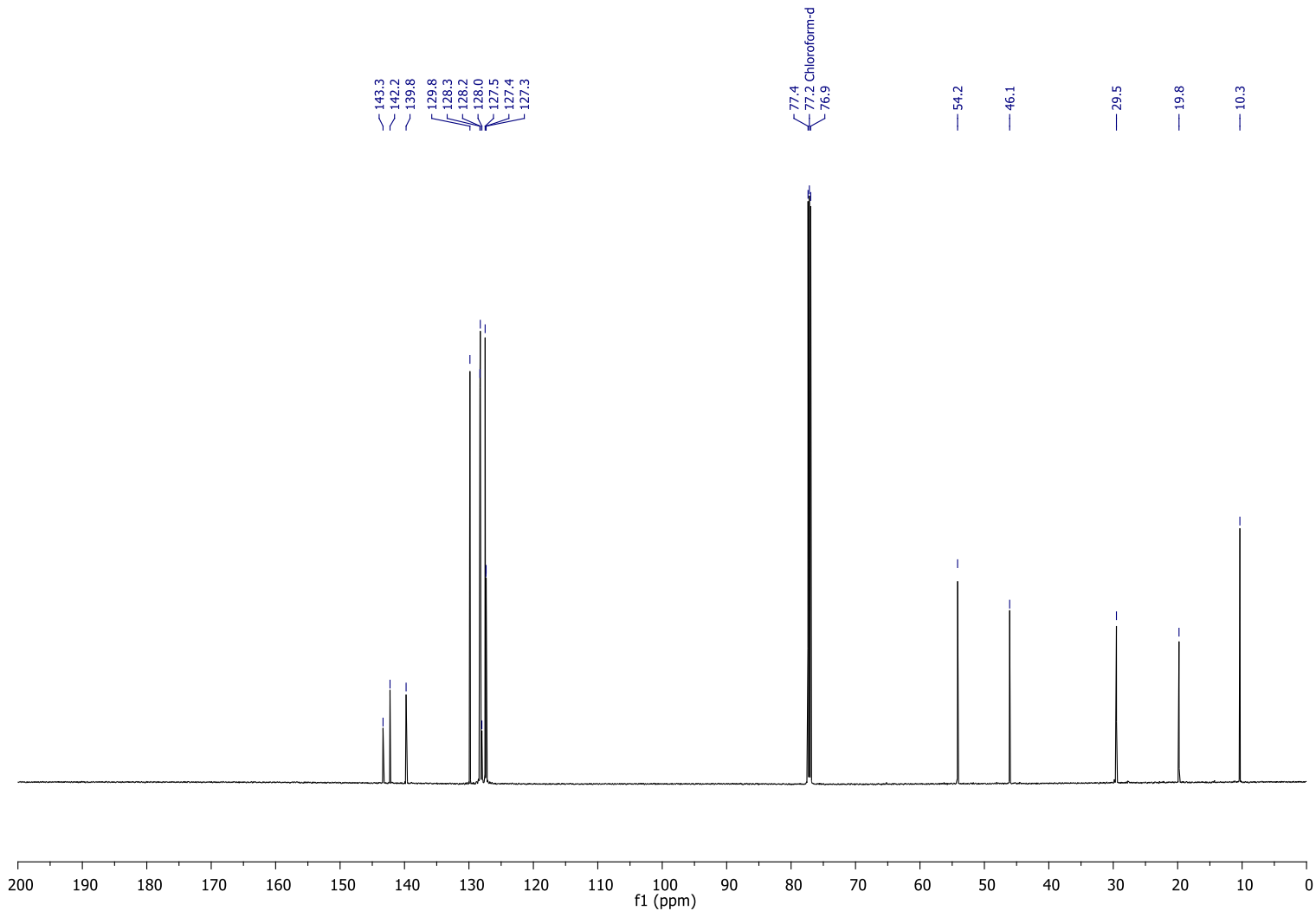

Figure S-74. ${ }^{13} \mathrm{C}$ NMR spectrum of $\mathbf{1 f}\left(\mathrm{CDCl}_{3}, 151 \mathrm{MHz}, 298 \mathrm{~K}\right)$ 

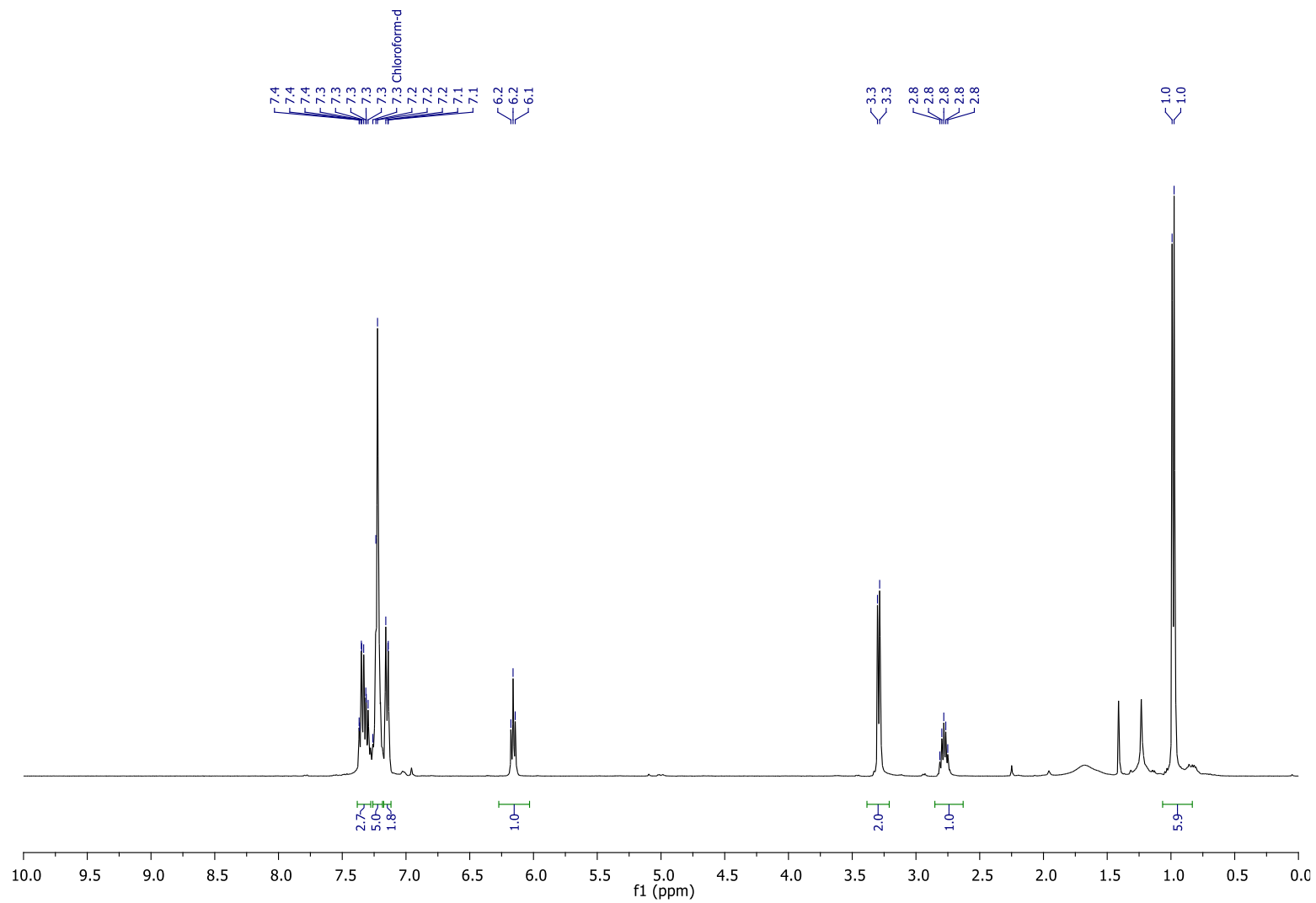

Figure S-75. ${ }^{1} \mathrm{H}$ NMR spectrum of $1 \mathrm{~g}\left(\mathrm{CDCl}_{3}, 600 \mathrm{MHz}, 298 \mathrm{~K}\right)$
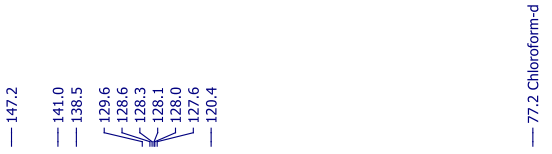

$\stackrel{\substack{\infty \\ \infty}}{\substack{j \\ j}}$
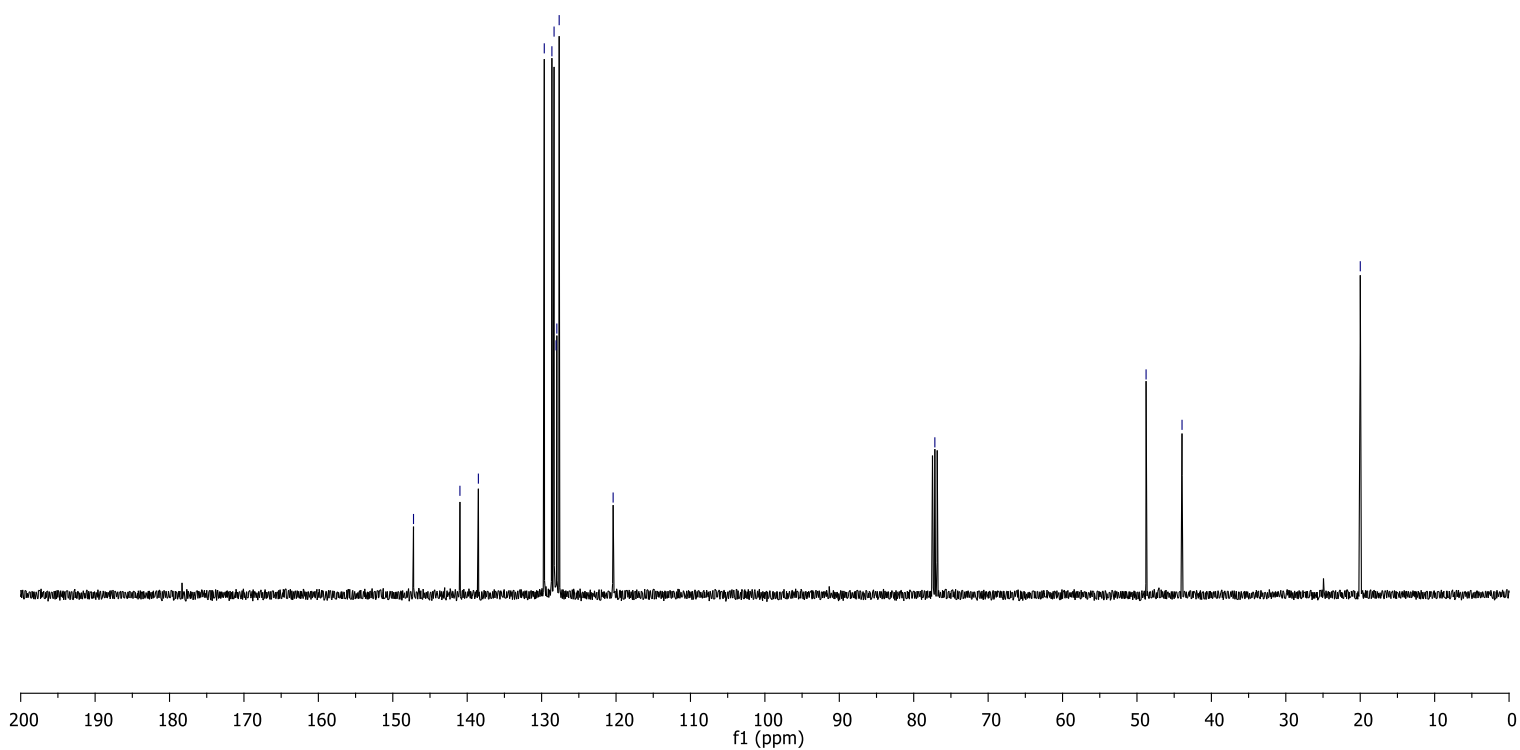

Figure S-76. ${ }^{13} \mathrm{C}$ NMR spectrum of $\mathbf{1 g}\left(\mathrm{CDCl}_{3}, 151 \mathrm{MHz}, 298 \mathrm{~K}\right)$ 

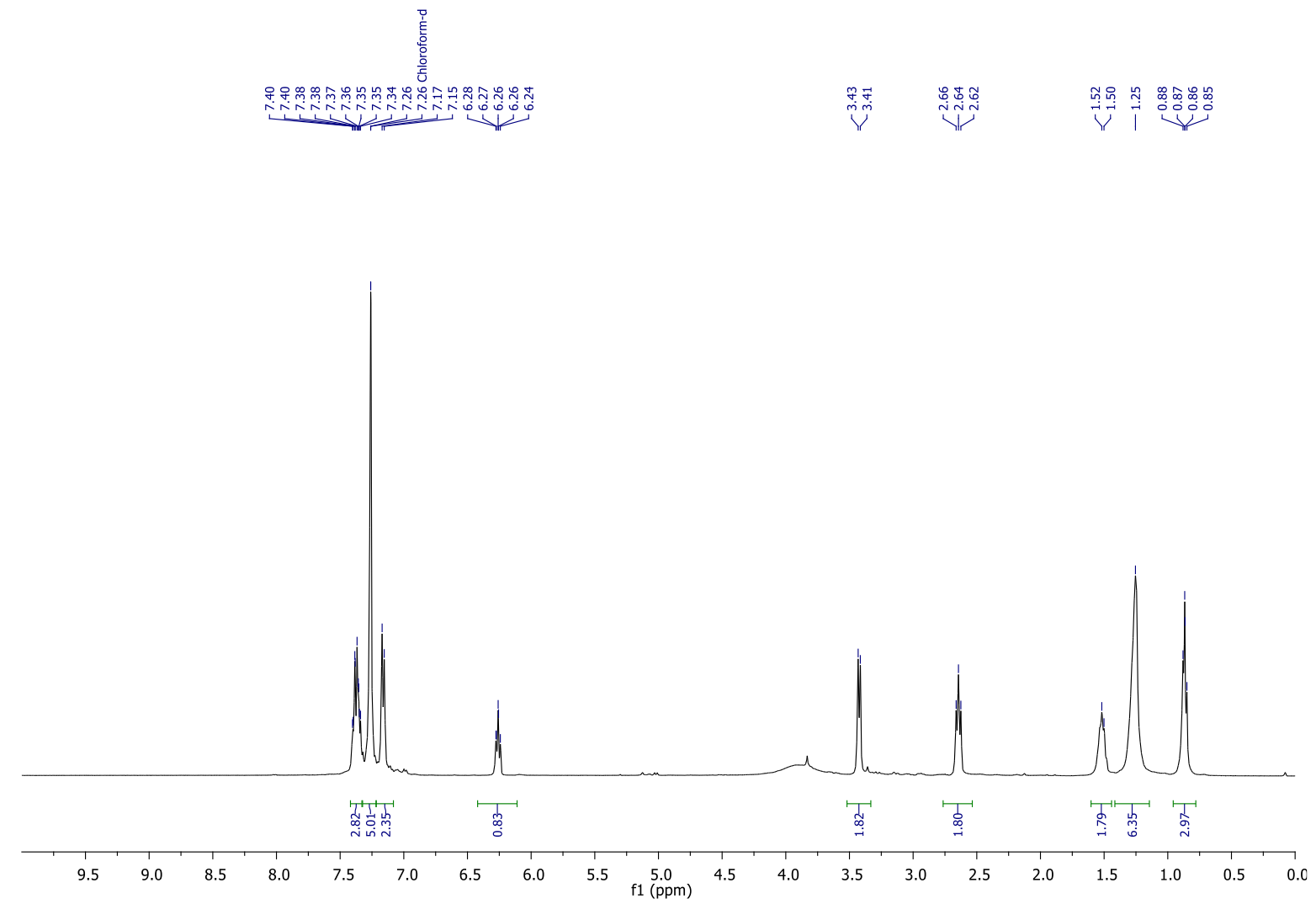

Figure S-77. ${ }^{1} \mathrm{H}$ NMR spectrum of $\mathbf{1 h}\left(\mathrm{CDCl}_{3}, 600 \mathrm{MHz}, 298 \mathrm{~K}\right)$
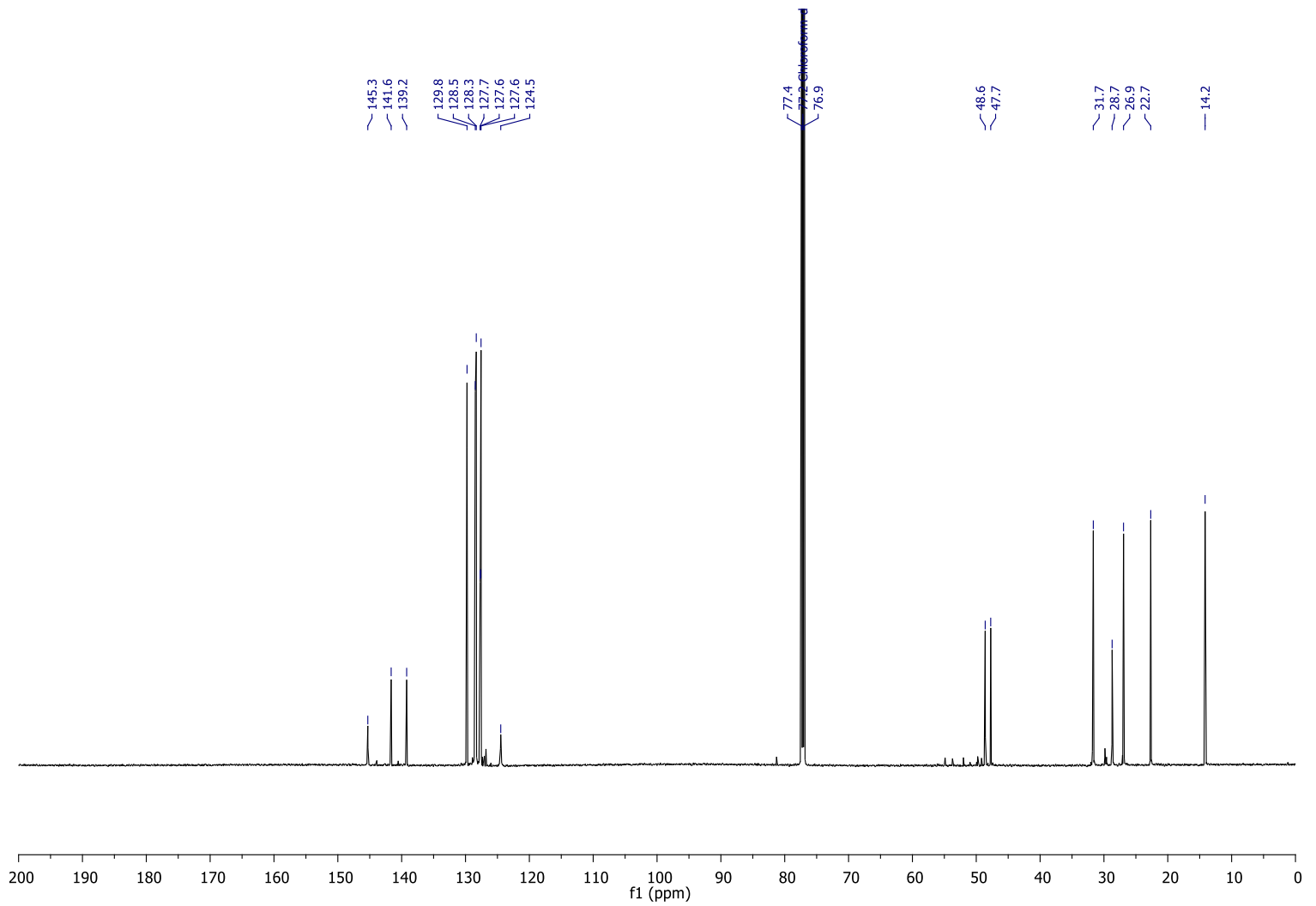

Figure S-78. ${ }^{13} \mathrm{C}$ NMR spectrum of $\mathbf{1 h}\left(\mathrm{CDCl}_{3}, 151 \mathrm{MHz}, 298 \mathrm{~K}\right)$ 

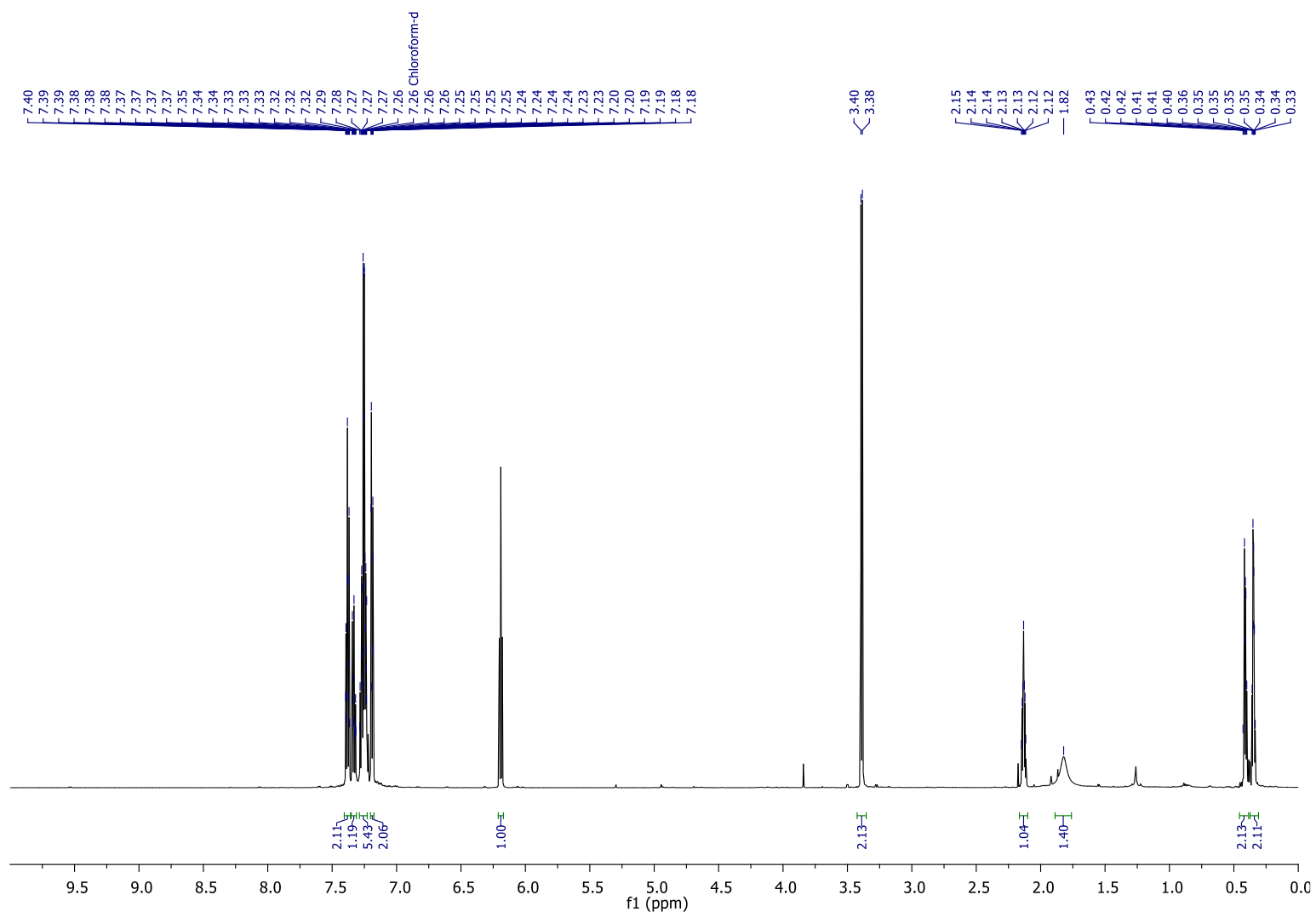

Figure S-79. ${ }^{1} \mathrm{H}$ NMR spectrum of $\mathbf{1 i}\left(\mathrm{CDCl}_{3}, 600 \mathrm{MHz}, 298 \mathrm{~K}\right)$
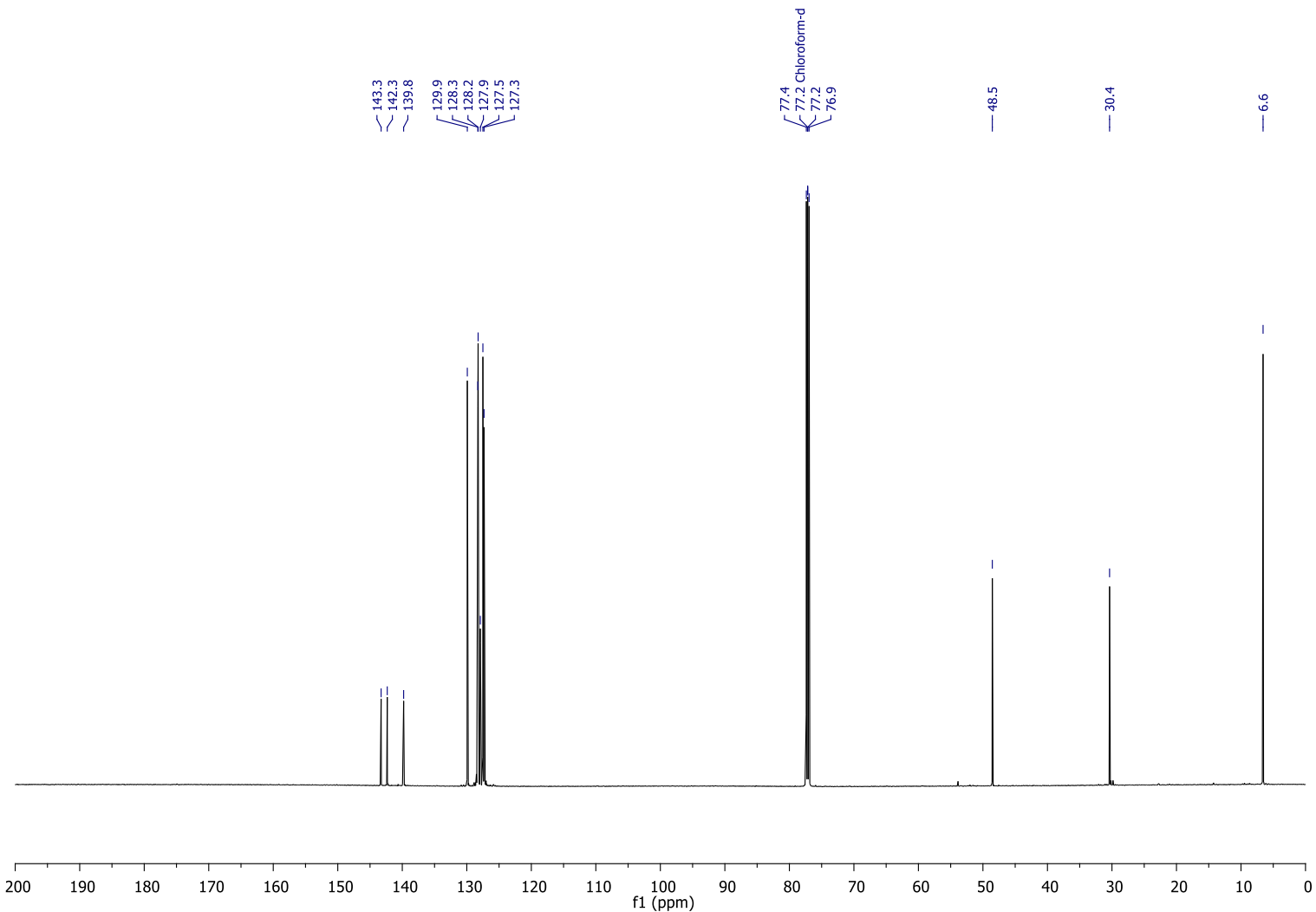

Figure S-80. ${ }^{13} \mathrm{C}$ NMR spectrum of $\mathbf{1 i}\left(\mathrm{CDCl}_{3}, 151 \mathrm{MHz}, 298 \mathrm{~K}\right)$ 


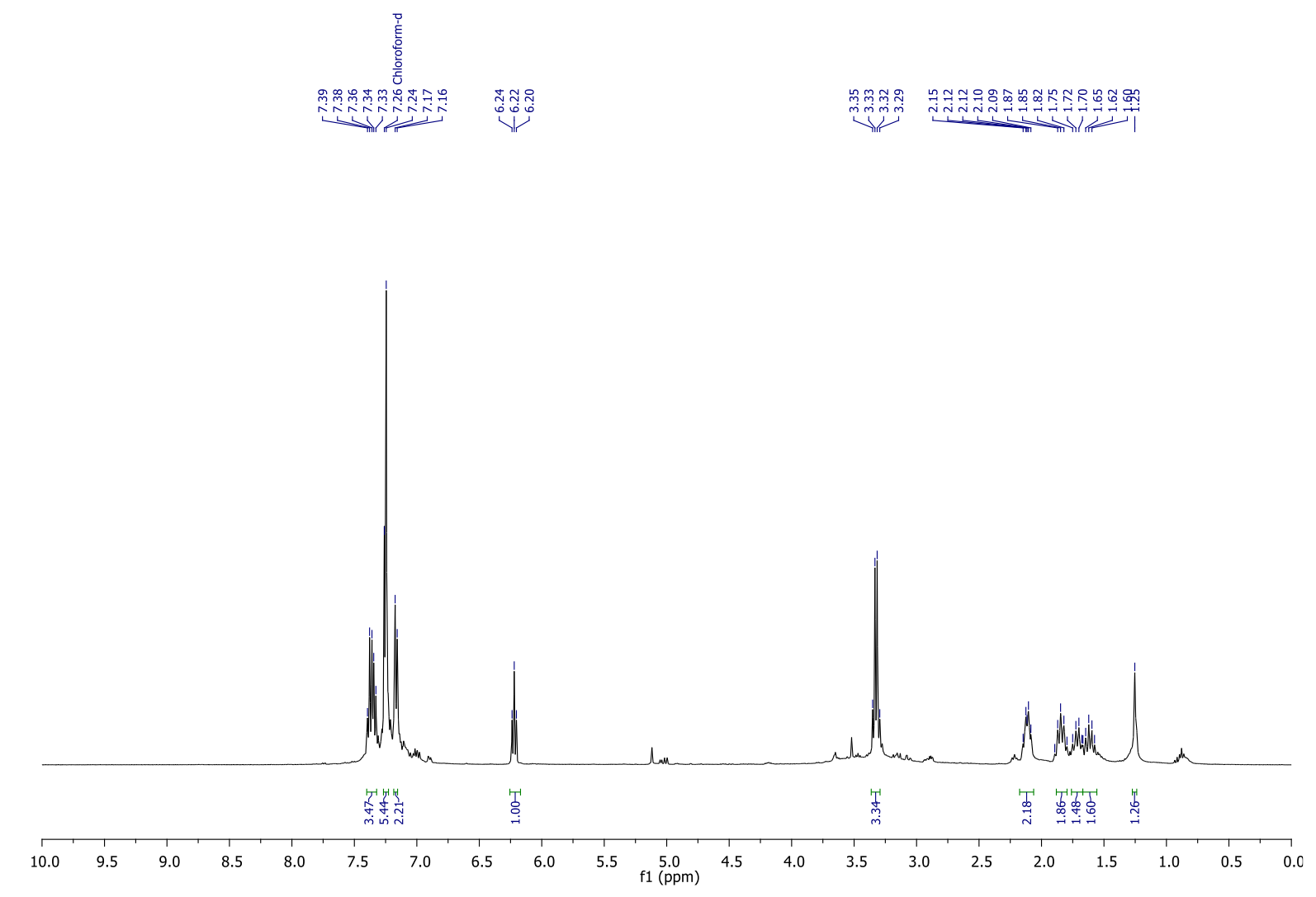

Figure S-81. ${ }^{1} \mathrm{H}$ NMR spectrum of $\mathbf{1 j}\left(\mathrm{CDCl}_{3}, 600 \mathrm{MHz}, 298 \mathrm{~K}\right)$
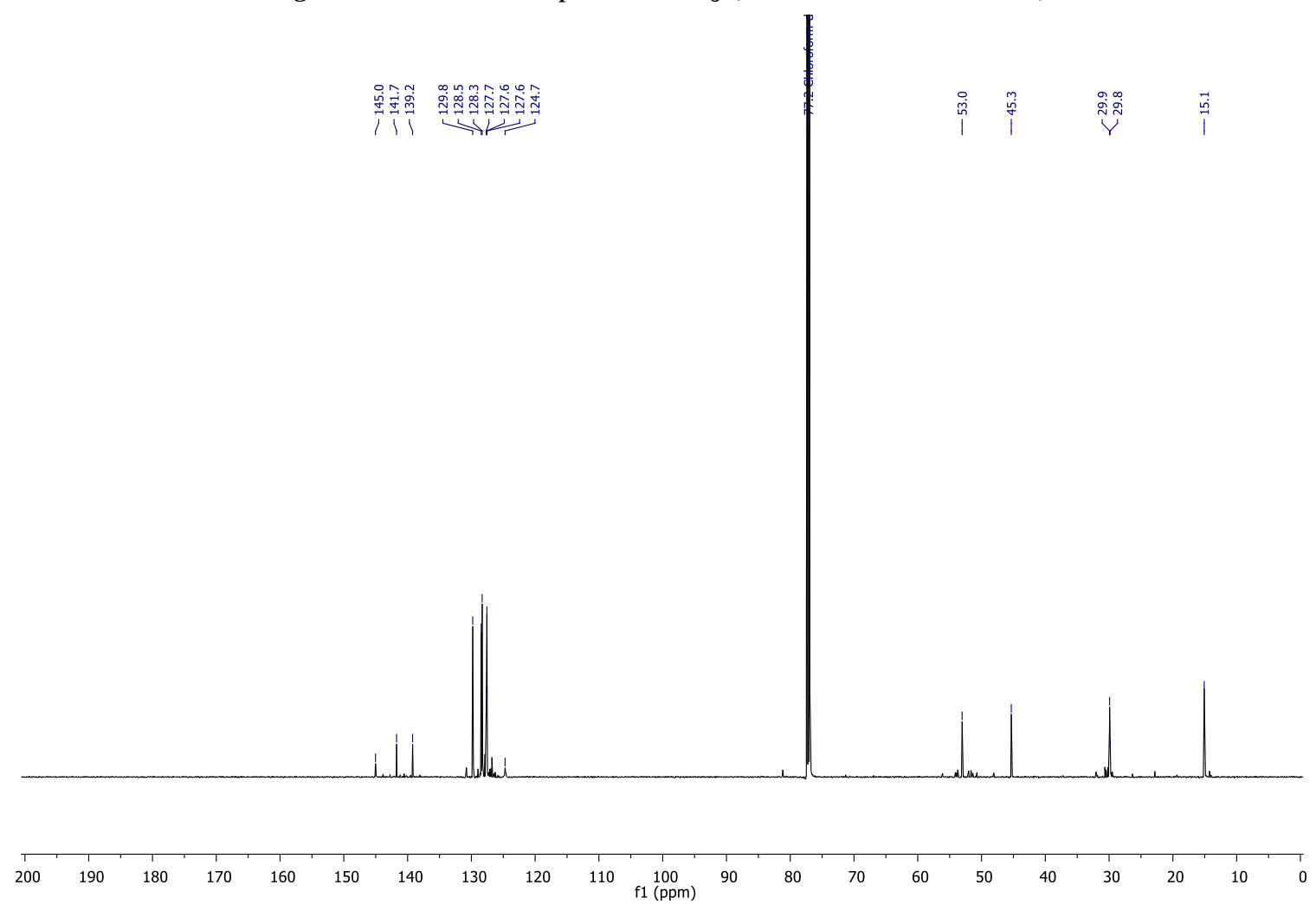

Figure S-82. ${ }^{13} \mathrm{C}$ NMR spectrum of $\mathbf{1 j}\left(\mathrm{CDCl}_{3}, 151 \mathrm{MHz}, 298 \mathrm{~K}\right)$ 


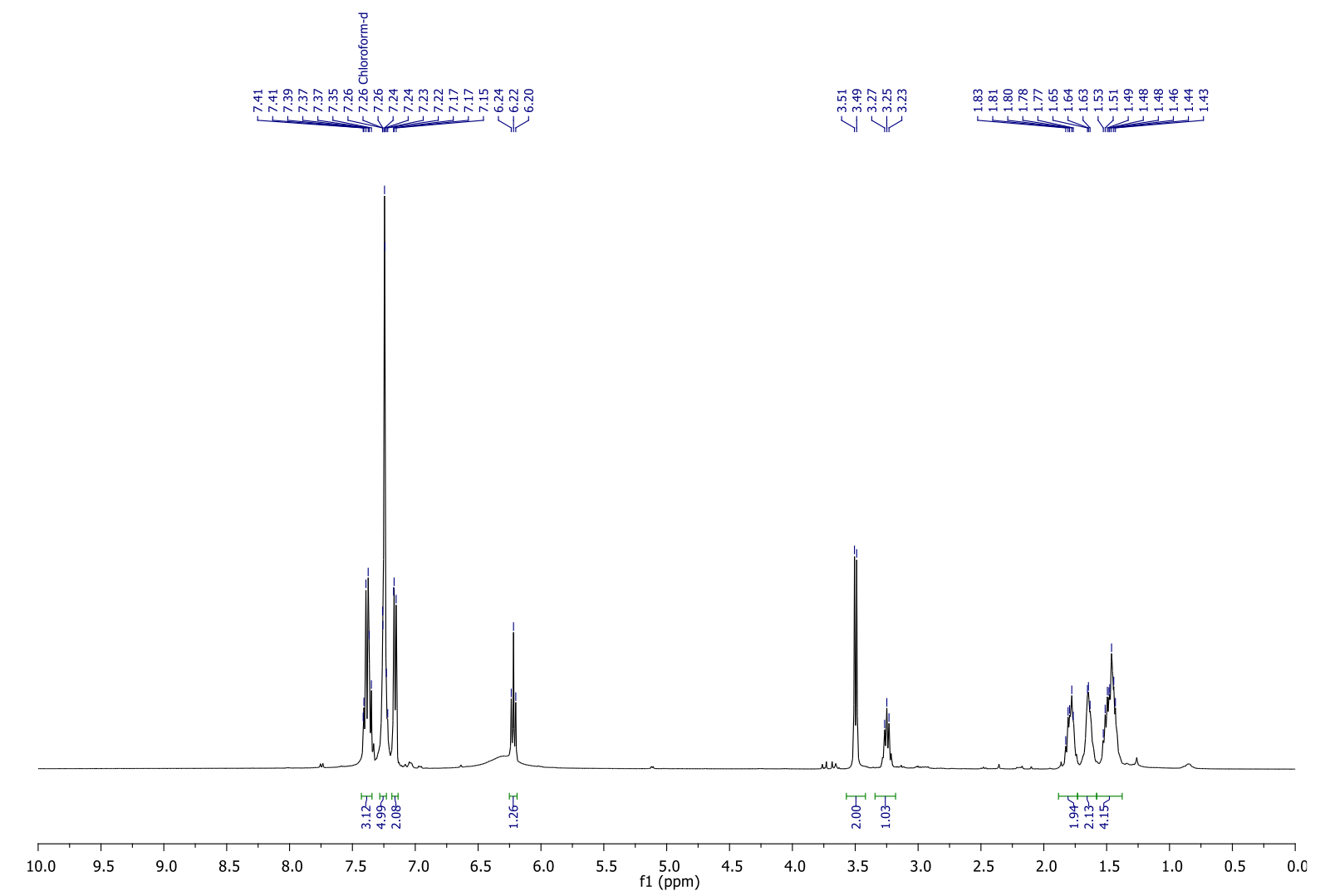

Figure $\mathbf{S - 8 3 .}{ }^{1} \mathrm{H}$ NMR spectrum of $1 \mathbf{k}\left(\mathrm{CDCl}_{3}, 600 \mathrm{MHz}, 298 \mathrm{~K}\right)$
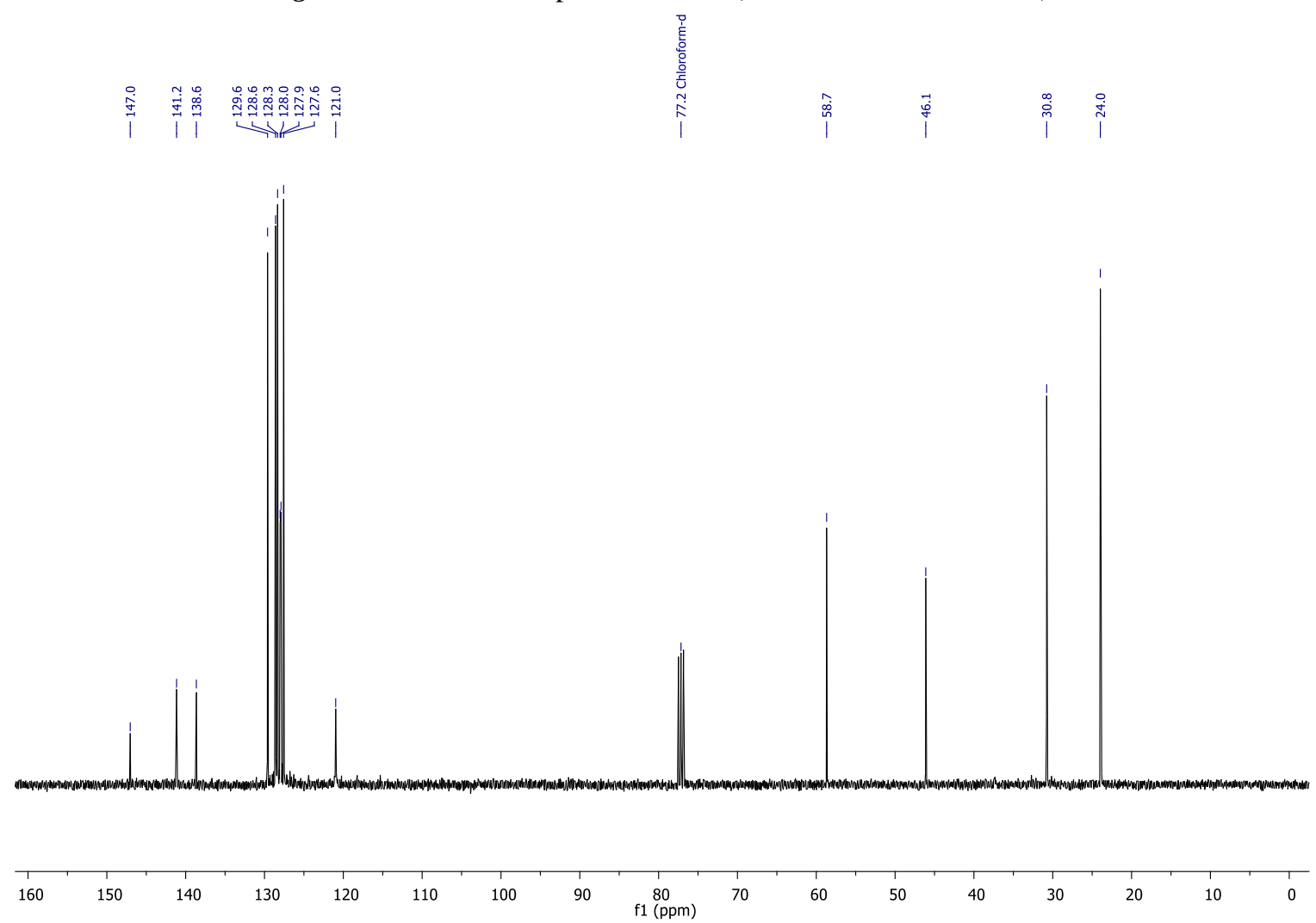

Figure S-84. ${ }^{13} \mathrm{C}$ NMR spectrum of $\mathbf{1 k}\left(\mathrm{CDCl}_{3}, 151 \mathrm{MHz}, 298 \mathrm{~K}\right)$ 


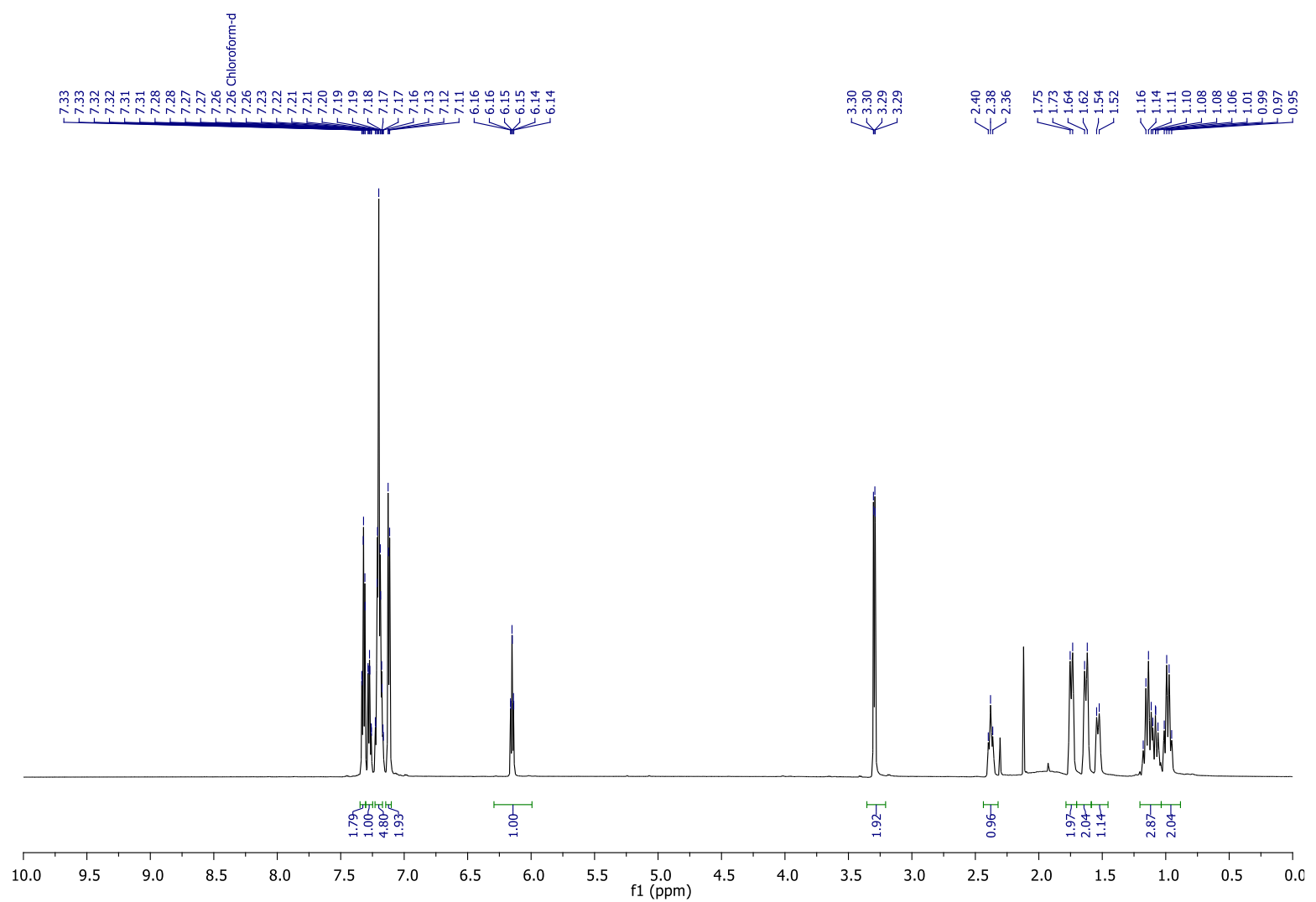

Figure S-85. ${ }^{1} \mathrm{H}$ NMR spectrum of $11\left(\mathrm{CDCl}_{3}, 600 \mathrm{MHz}, 298 \mathrm{~K}\right)$
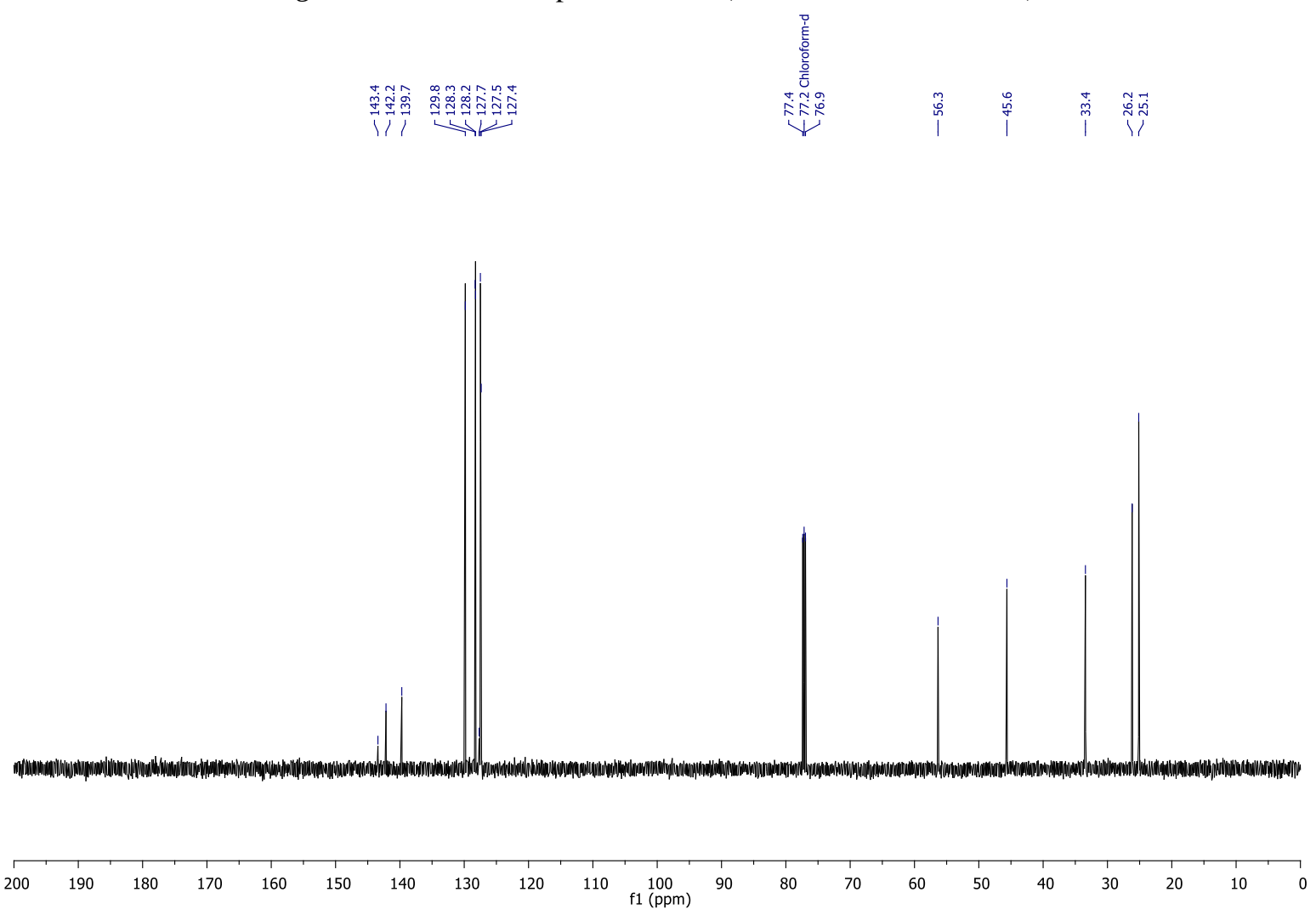

Figure S-86. ${ }^{13} \mathrm{C}$ NMR spectrum of $11\left(\mathrm{CDCl}_{3}, 151 \mathrm{MHz}, 298 \mathrm{~K}\right)$ 

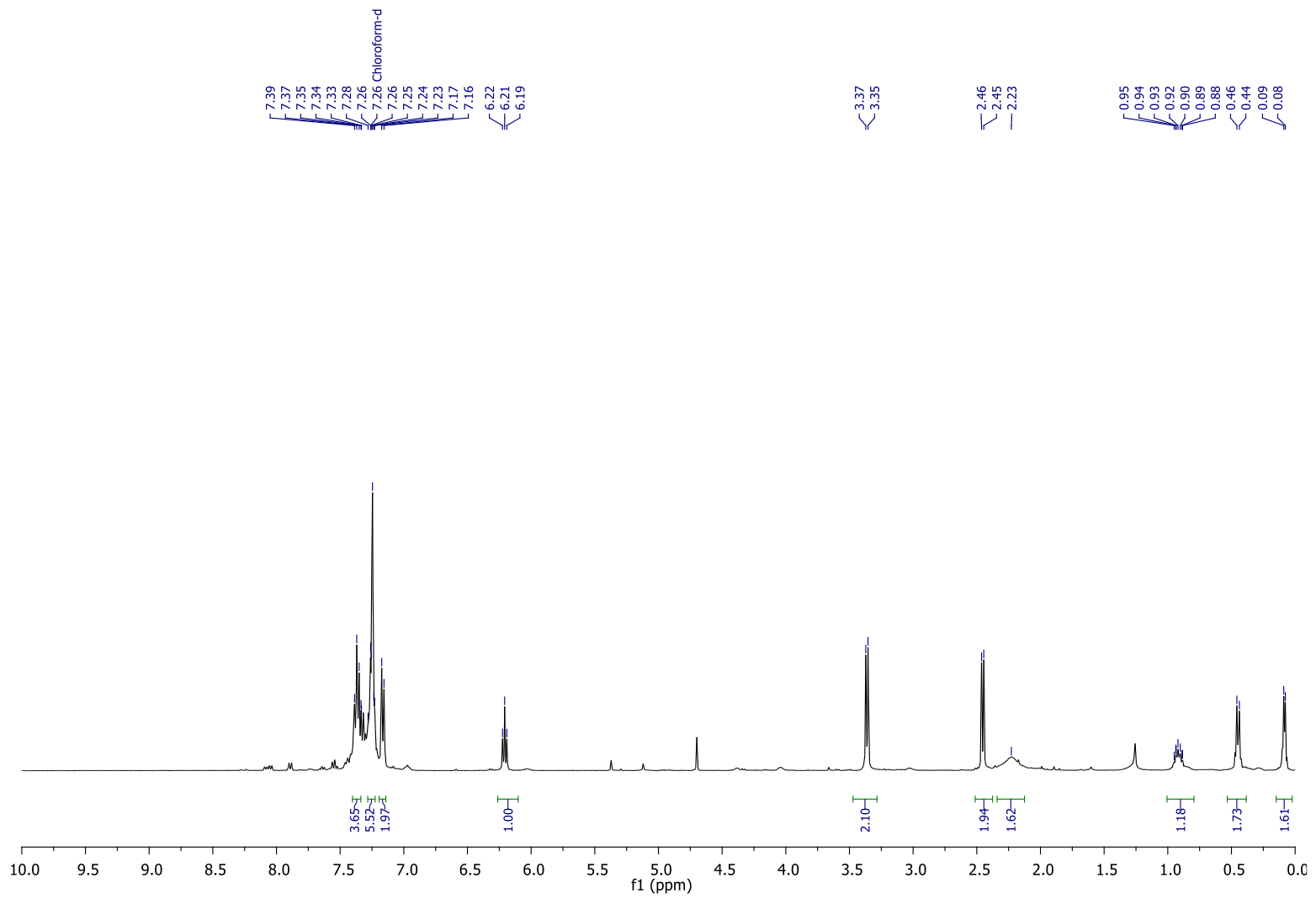

Figure S-87. ${ }^{1} \mathrm{H}$ NMR spectrum of $1 \mathrm{~m}\left(\mathrm{CDCl}_{3}, 600 \mathrm{MHz}, 298 \mathrm{~K}\right)$

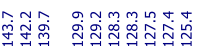

ओ门

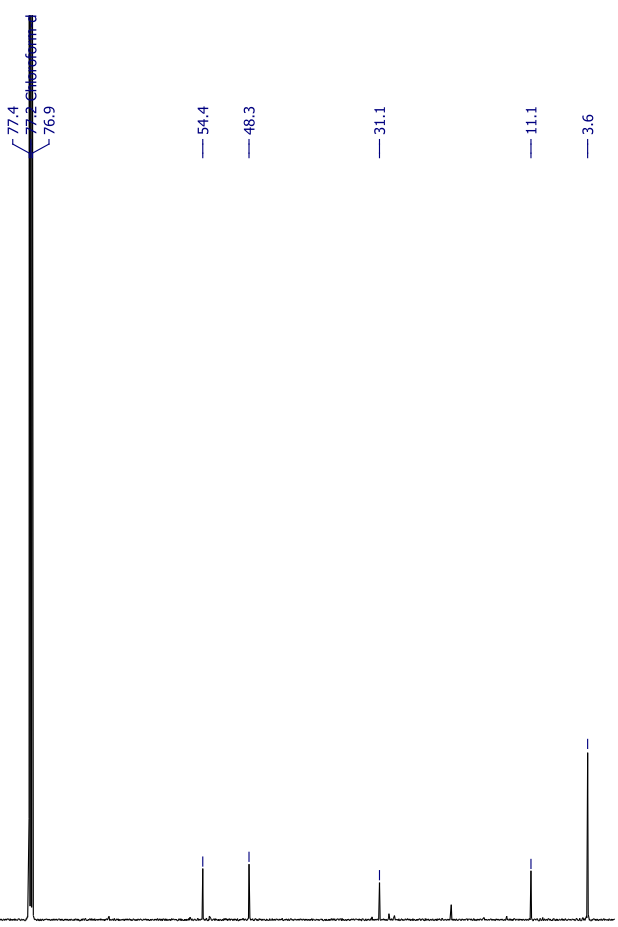

200

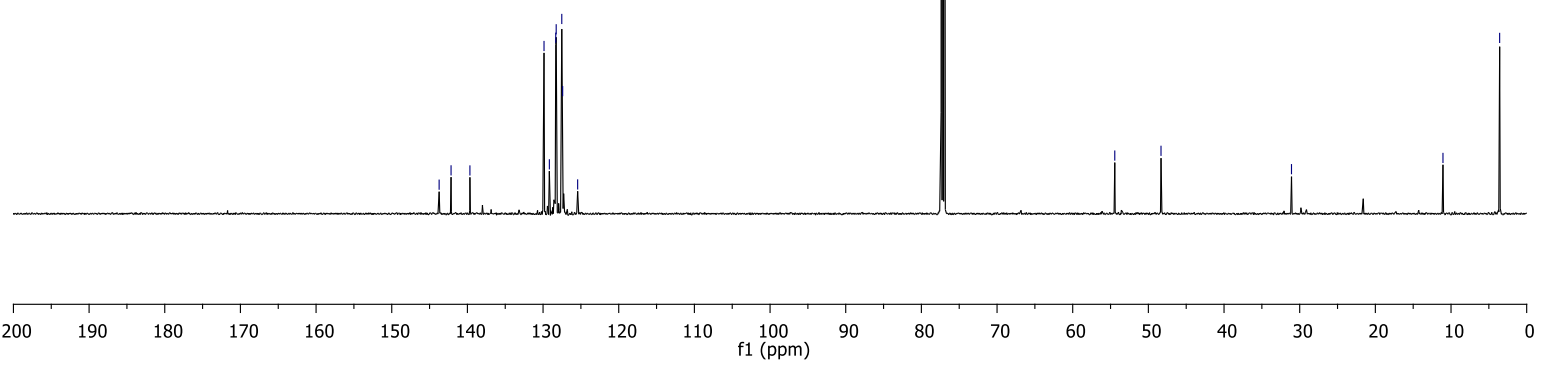

Figure S-88. ${ }^{13} \mathrm{C}$ NMR spectrum of $1 \mathbf{m}\left(\mathrm{CDCl}_{3}, 151 \mathrm{MHz}, 298 \mathrm{~K}\right)$ 

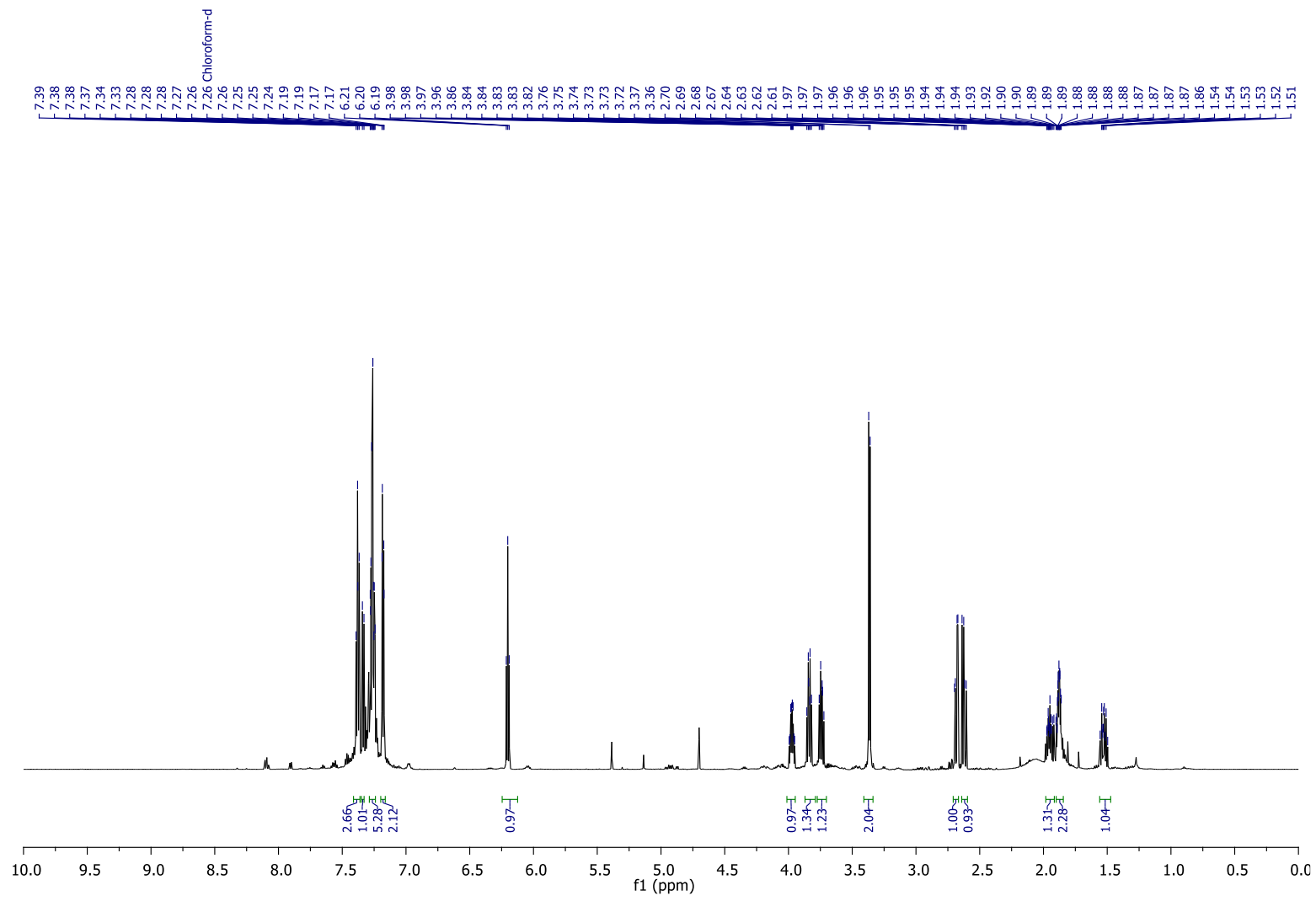

Figure S-89. ${ }^{1} \mathrm{H}$ NMR spectrum of $\mathbf{1 n}\left(\mathrm{CDCl}_{3}, 600 \mathrm{MHz}, 298 \mathrm{~K}\right)$

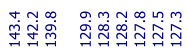

1)
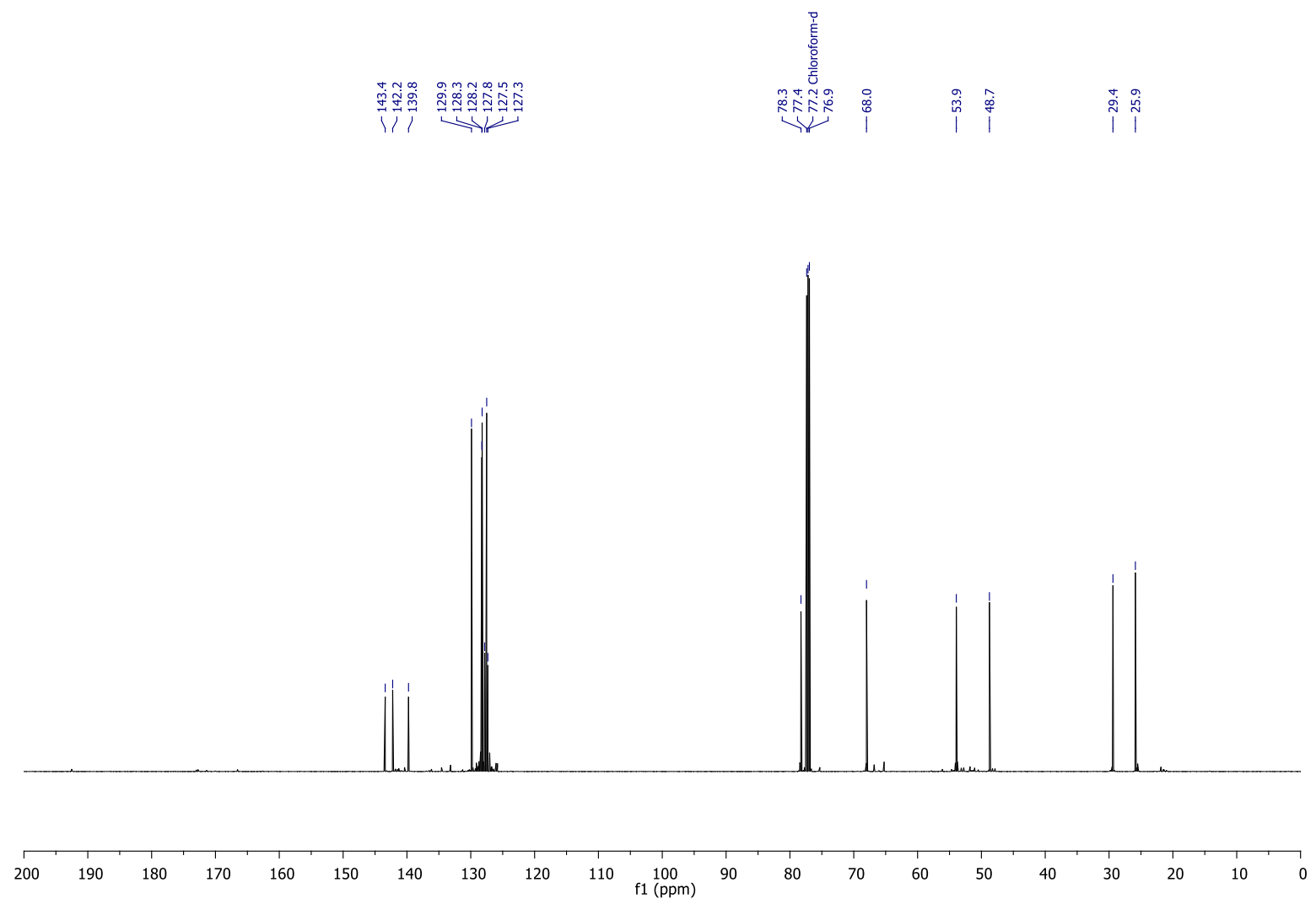

Figure S-90. ${ }^{13} \mathrm{C}$ NMR spectrum of $\mathbf{1 n}\left(\mathrm{CDCl}_{3}, 151 \mathrm{MHz}, 298 \mathrm{~K}\right)$ 

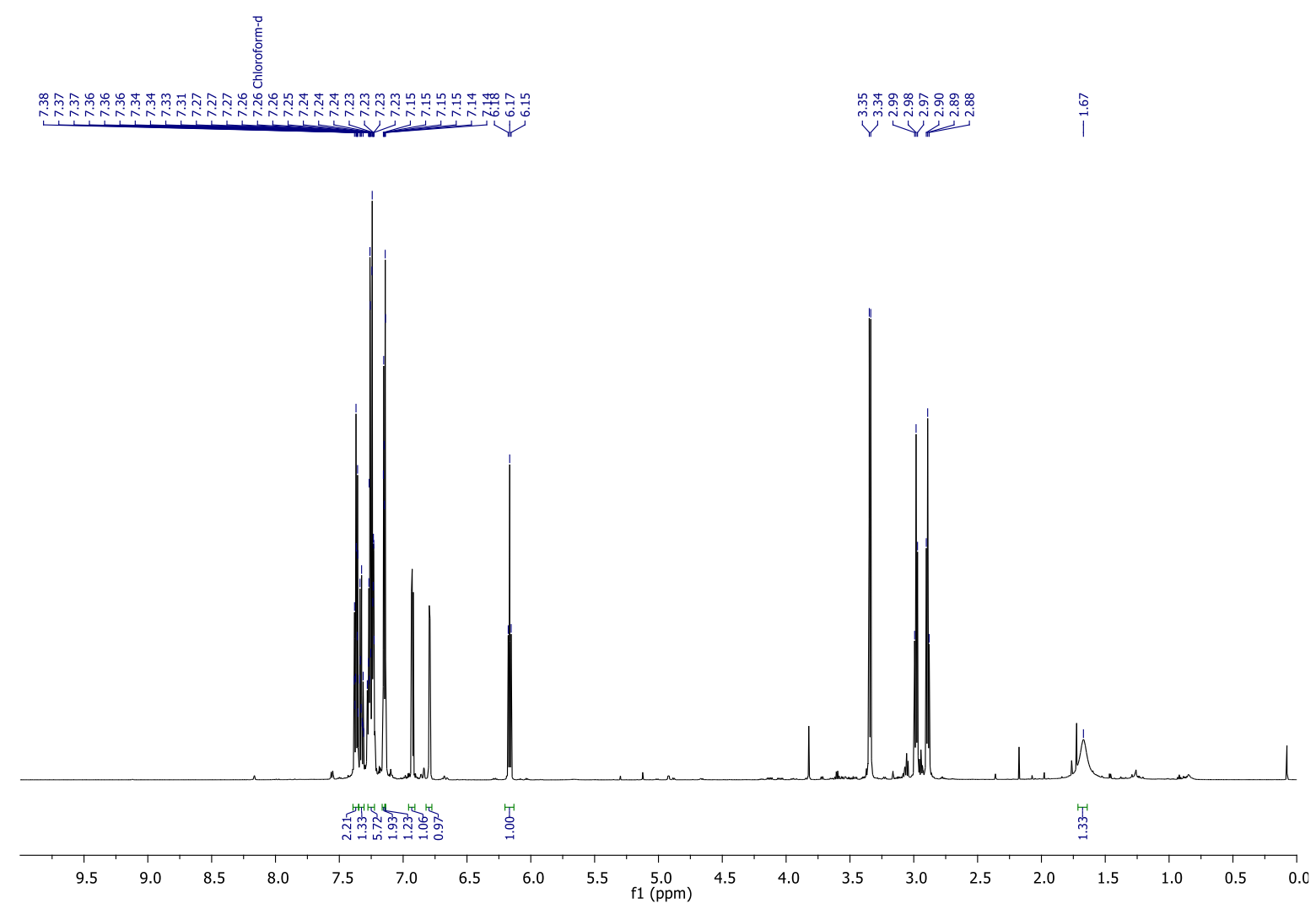

Figure S-91. ${ }^{1} \mathrm{H}$ NMR spectrum of $10\left(\mathrm{CDCl}_{3}, 600 \mathrm{MHz}, 298 \mathrm{~K}\right)$
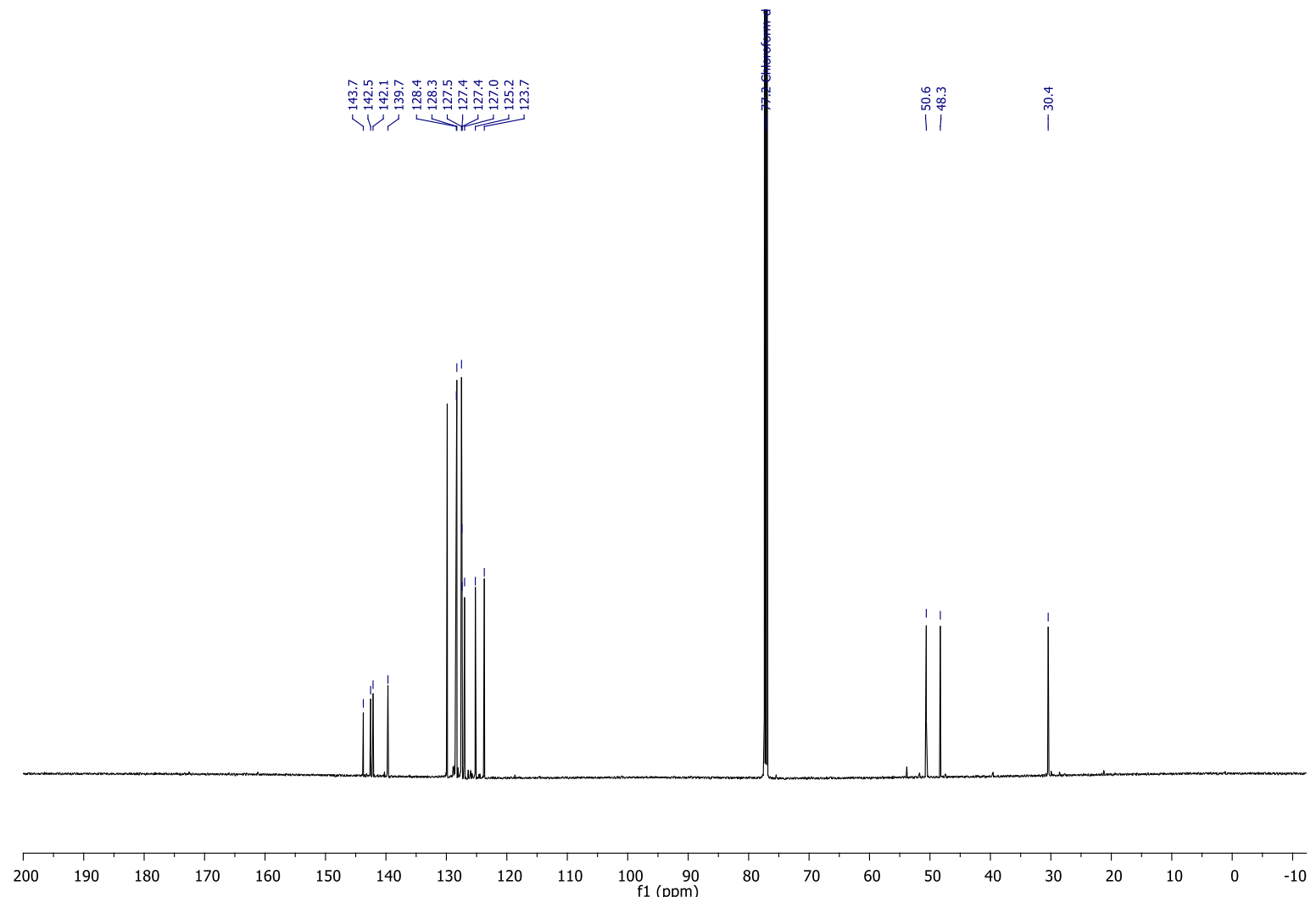

Figure S-92. ${ }^{13} \mathrm{C}$ NMR spectrum of $10\left(\mathrm{CDCl}_{3}, 151 \mathrm{MHz}, 298 \mathrm{~K}\right)$ 


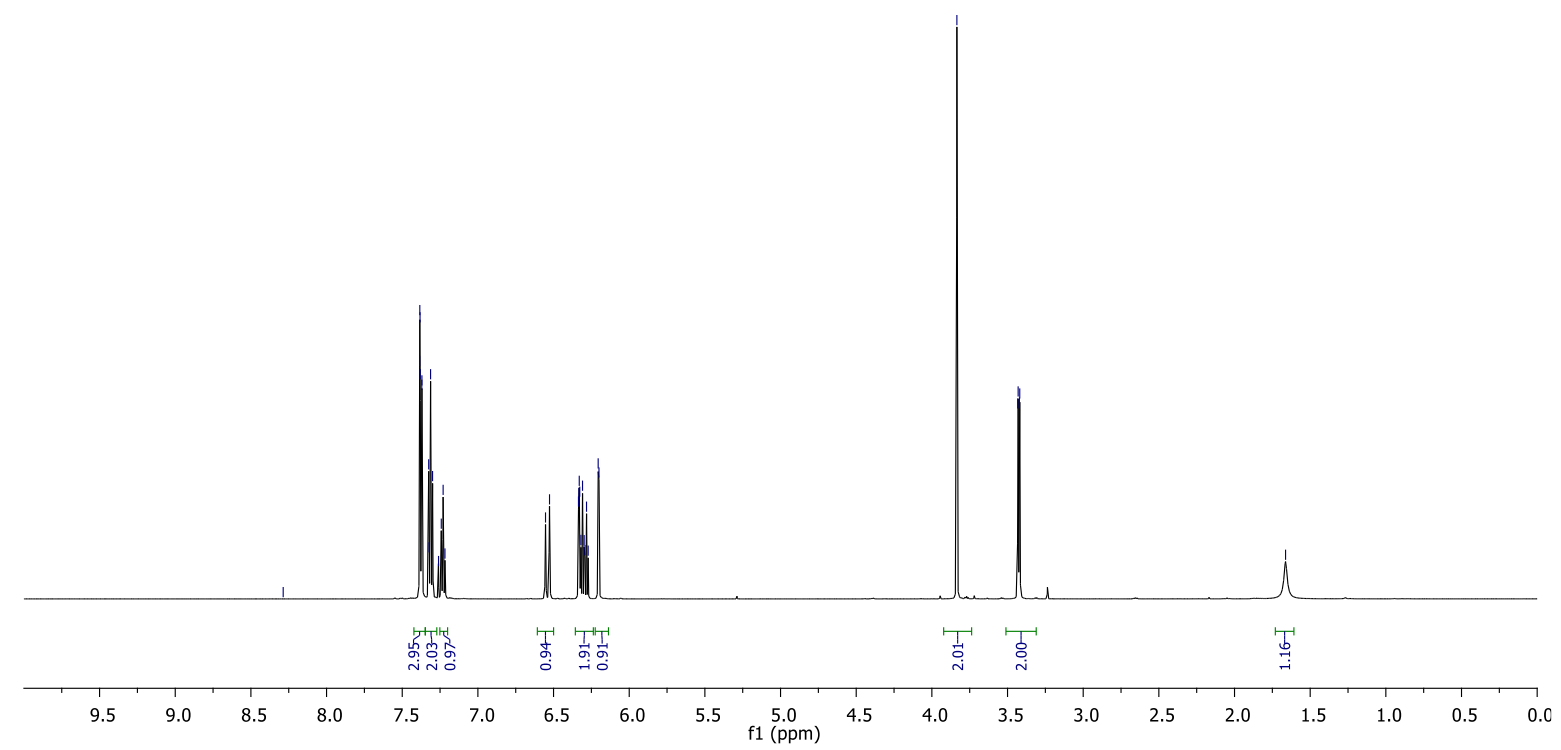

Figure S-93. ${ }^{1} \mathrm{H}$ NMR spectrum of $1 p\left(\mathrm{CDCl}_{3}, 600 \mathrm{MHz}, 298 \mathrm{~K}\right)$
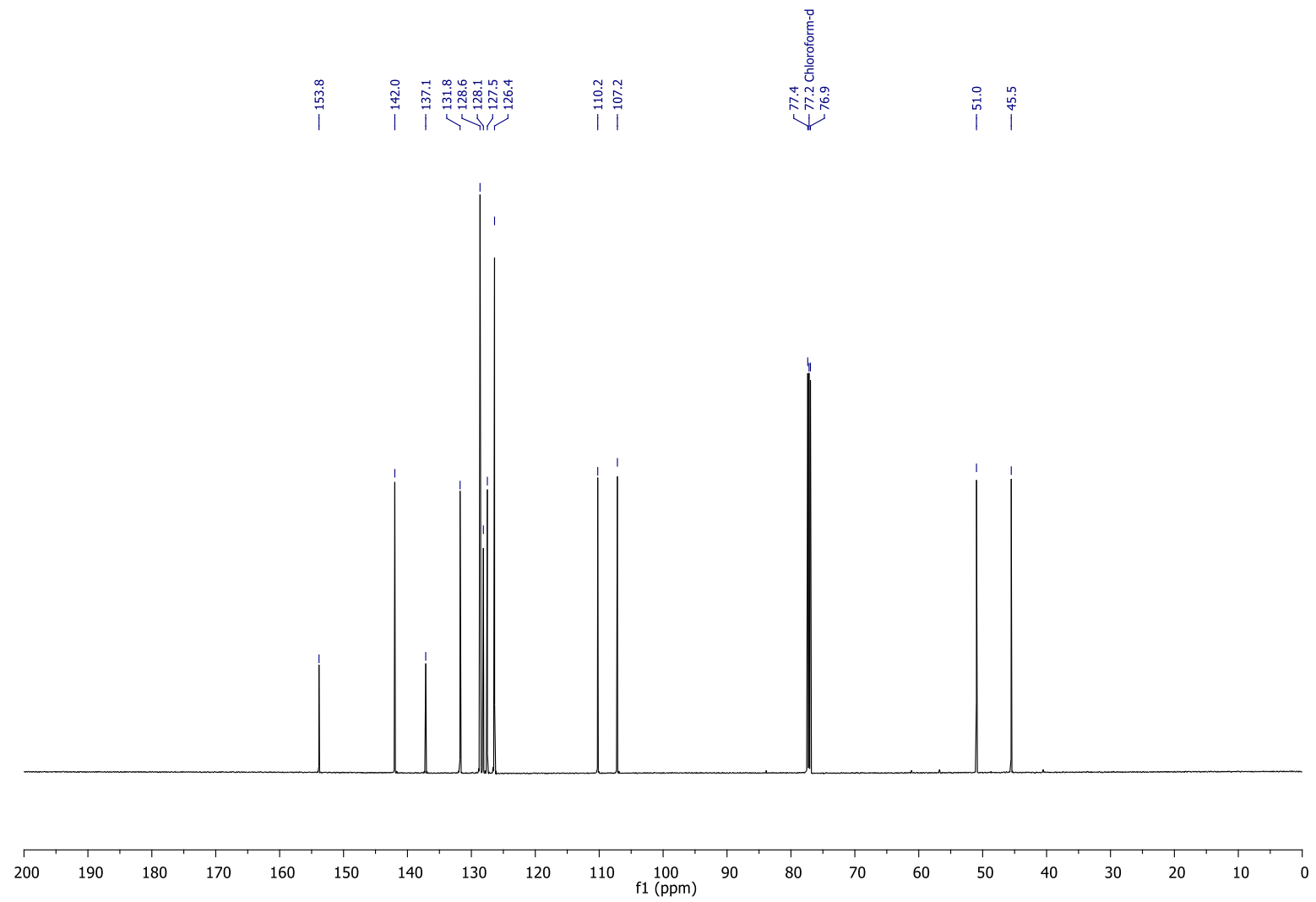

Figure S-94. ${ }^{13} \mathrm{C}$ NMR spectrum of $1 \mathbf{p}\left(\mathrm{CDCl}_{3}, 151 \mathrm{MHz}, 298 \mathrm{~K}\right)$ 

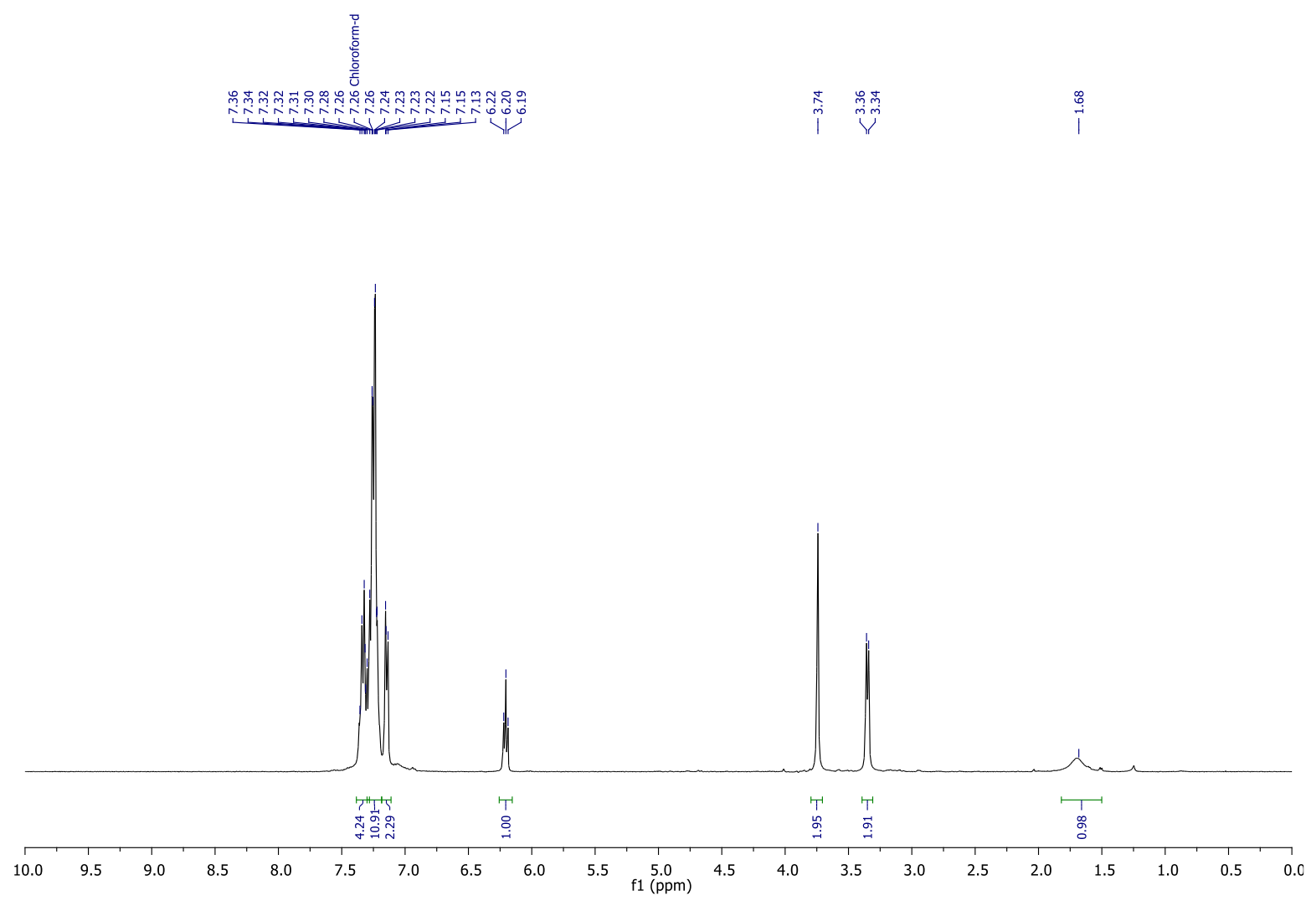

Figure S-95. ${ }^{1} \mathrm{H}$ NMR spectrum of $\mathbf{1 q}\left(\mathrm{CDCl}_{3}, 600 \mathrm{MHz}, 298 \mathrm{~K}\right)$
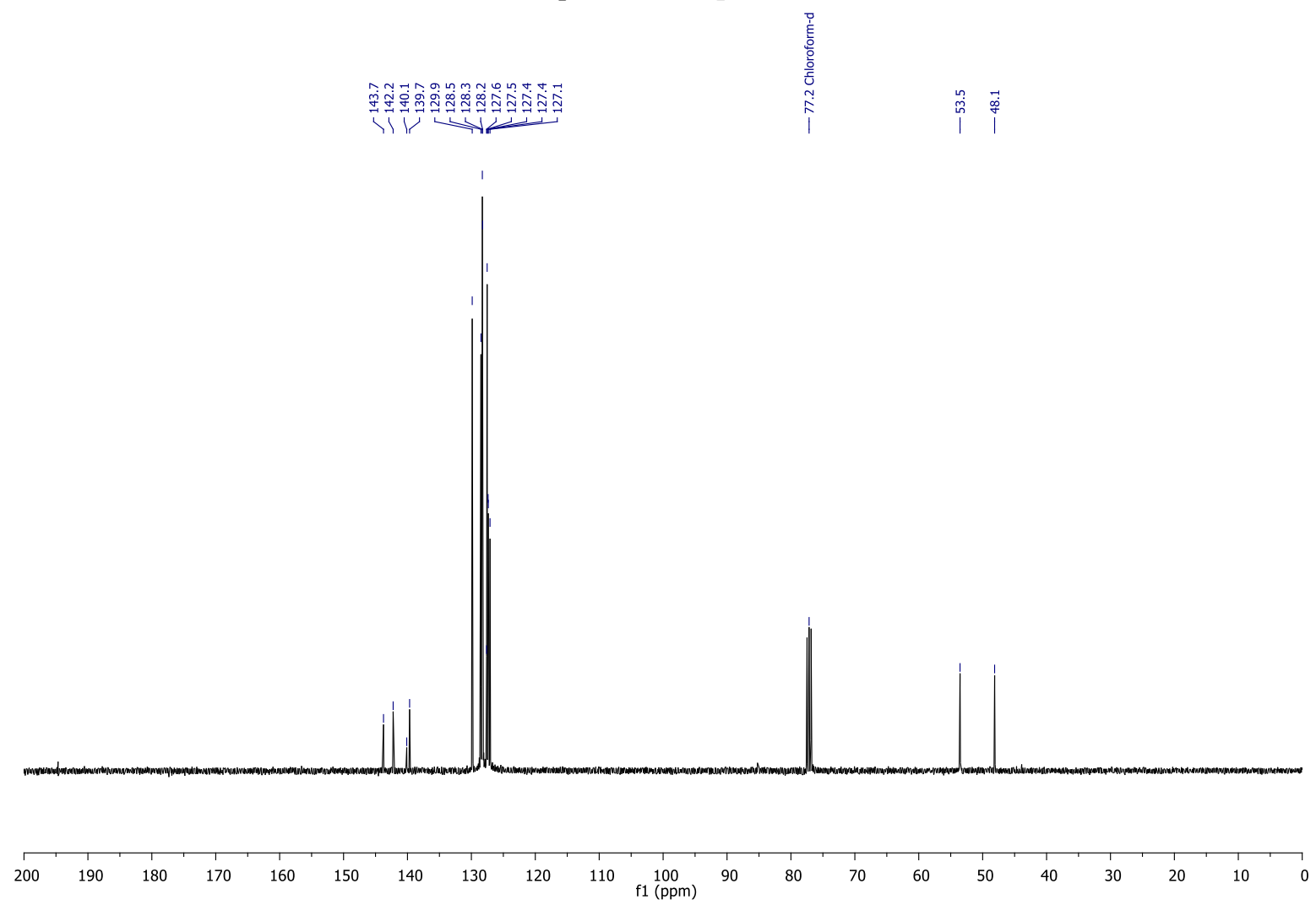

Figure S-96. ${ }^{13} \mathrm{C}$ NMR spectrum of $\mathbf{1 q}\left(\mathrm{CDCl}_{3}, 151 \mathrm{MHz}, 298 \mathrm{~K}\right)$ 


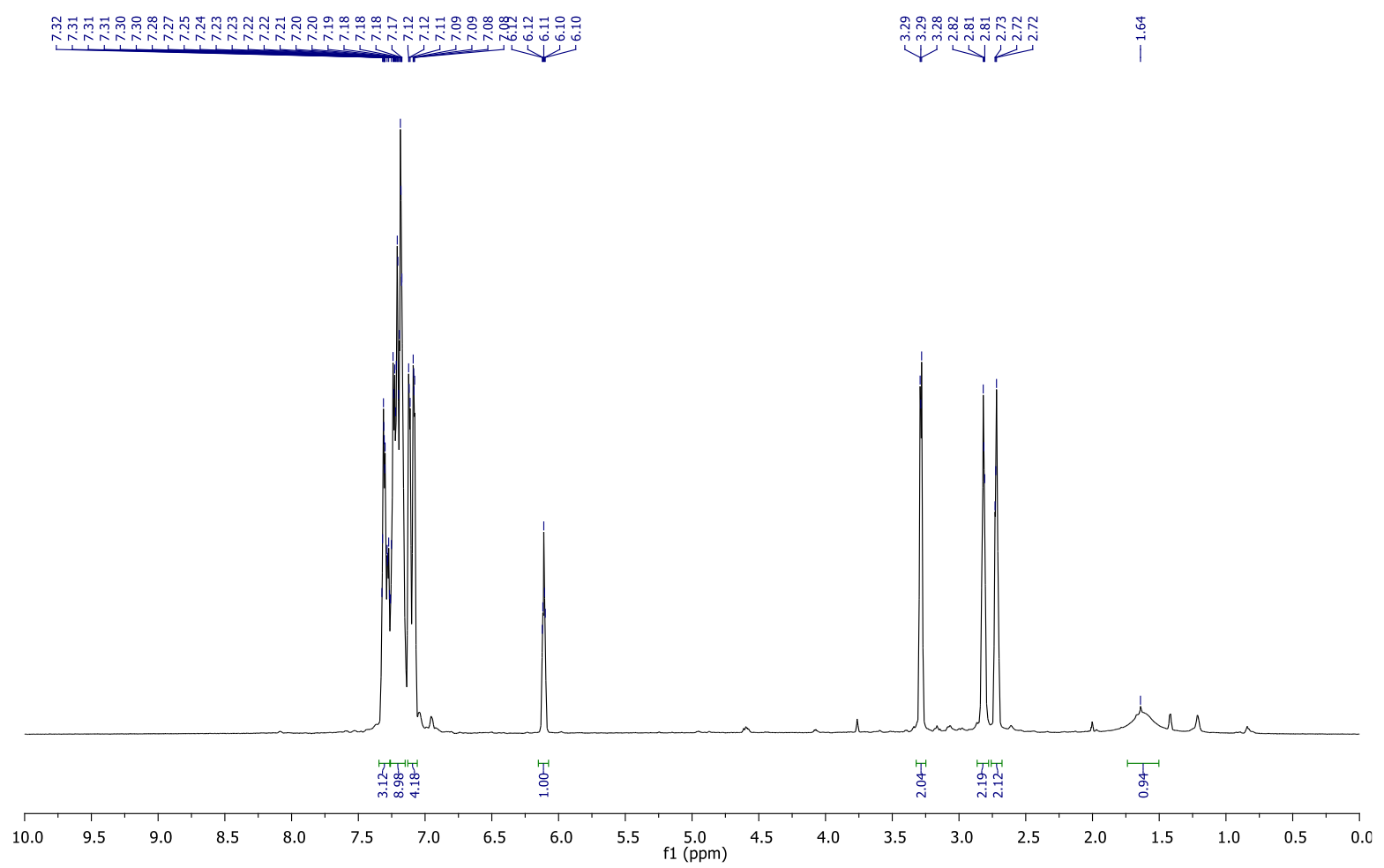

Figure S-97. ${ }^{1} \mathrm{H}$ NMR spectrum of $1 \mathbf{r}\left(\mathrm{CDCl}_{3}, 600 \mathrm{MHz}, 298 \mathrm{~K}\right)$

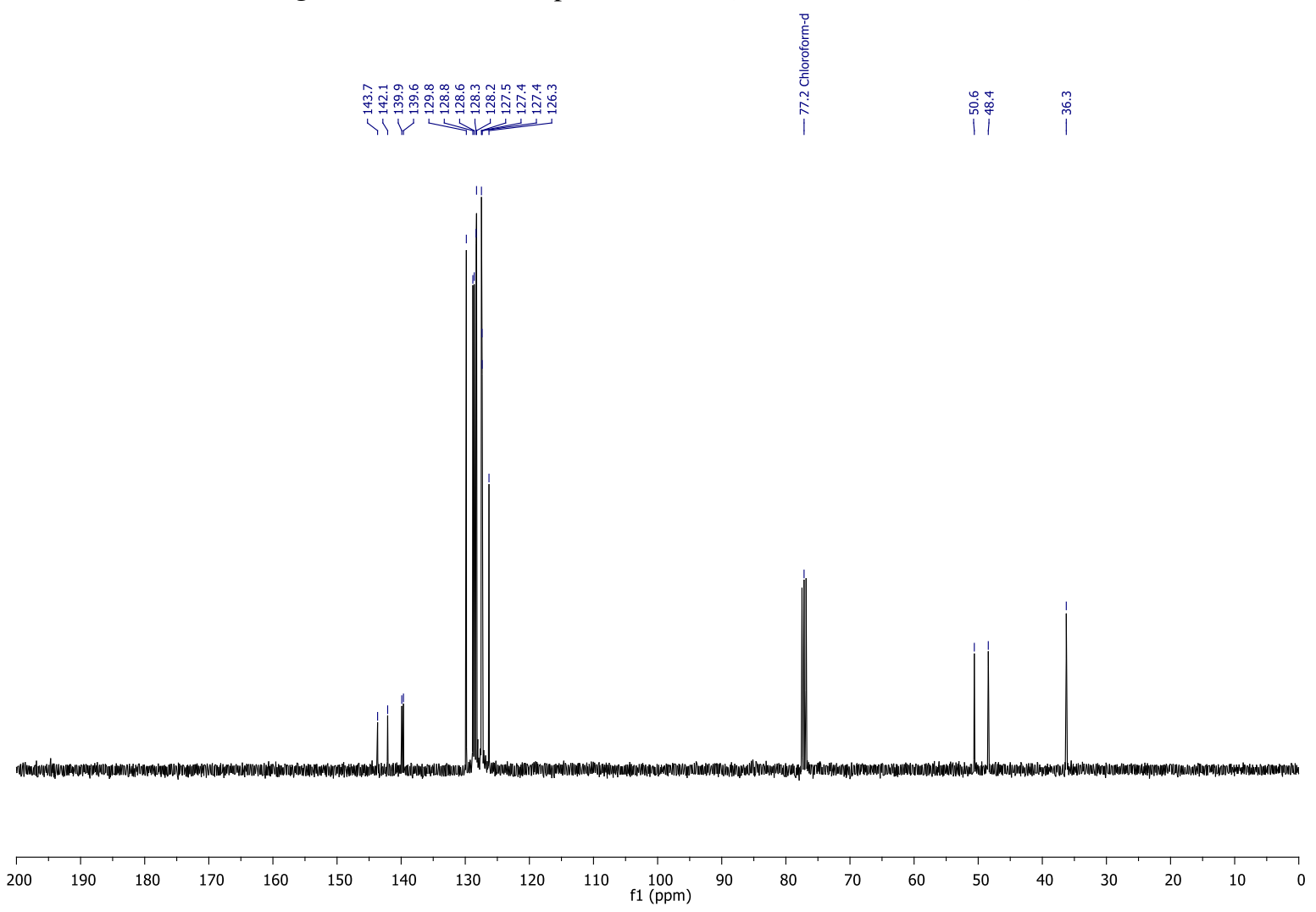

Figure S-98. ${ }^{13} \mathrm{C}$ NMR spectrum of $1 \mathbf{r}\left(\mathrm{CDCl}_{3}, 151 \mathrm{MHz}, 298 \mathrm{~K}\right)$ 


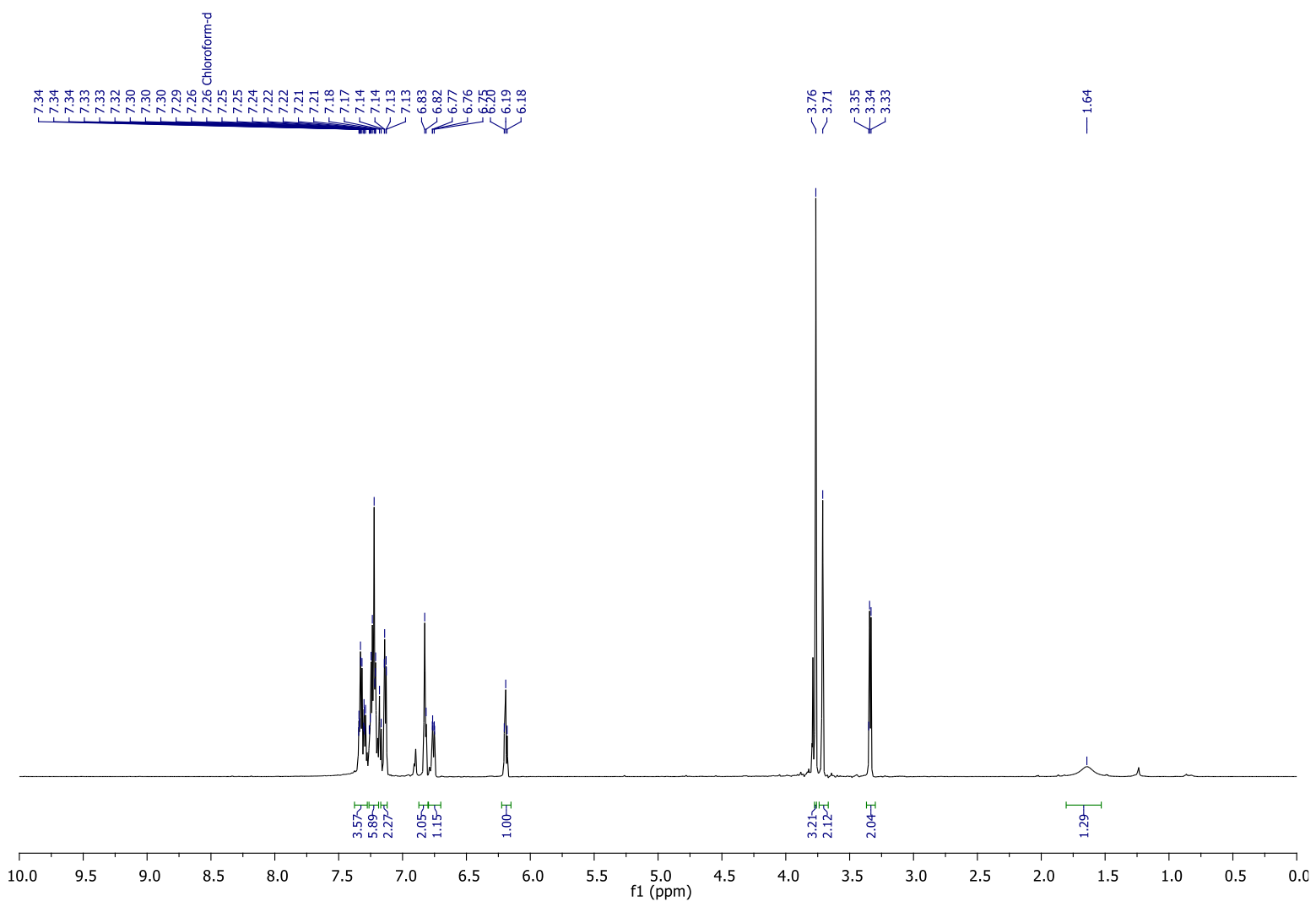

Figure S-99. ${ }^{1} \mathrm{H}$ NMR spectrum of $1 \mathrm{~s}\left(\mathrm{CDCl}_{3}, 600 \mathrm{MHz}, 298 \mathrm{~K}\right)$

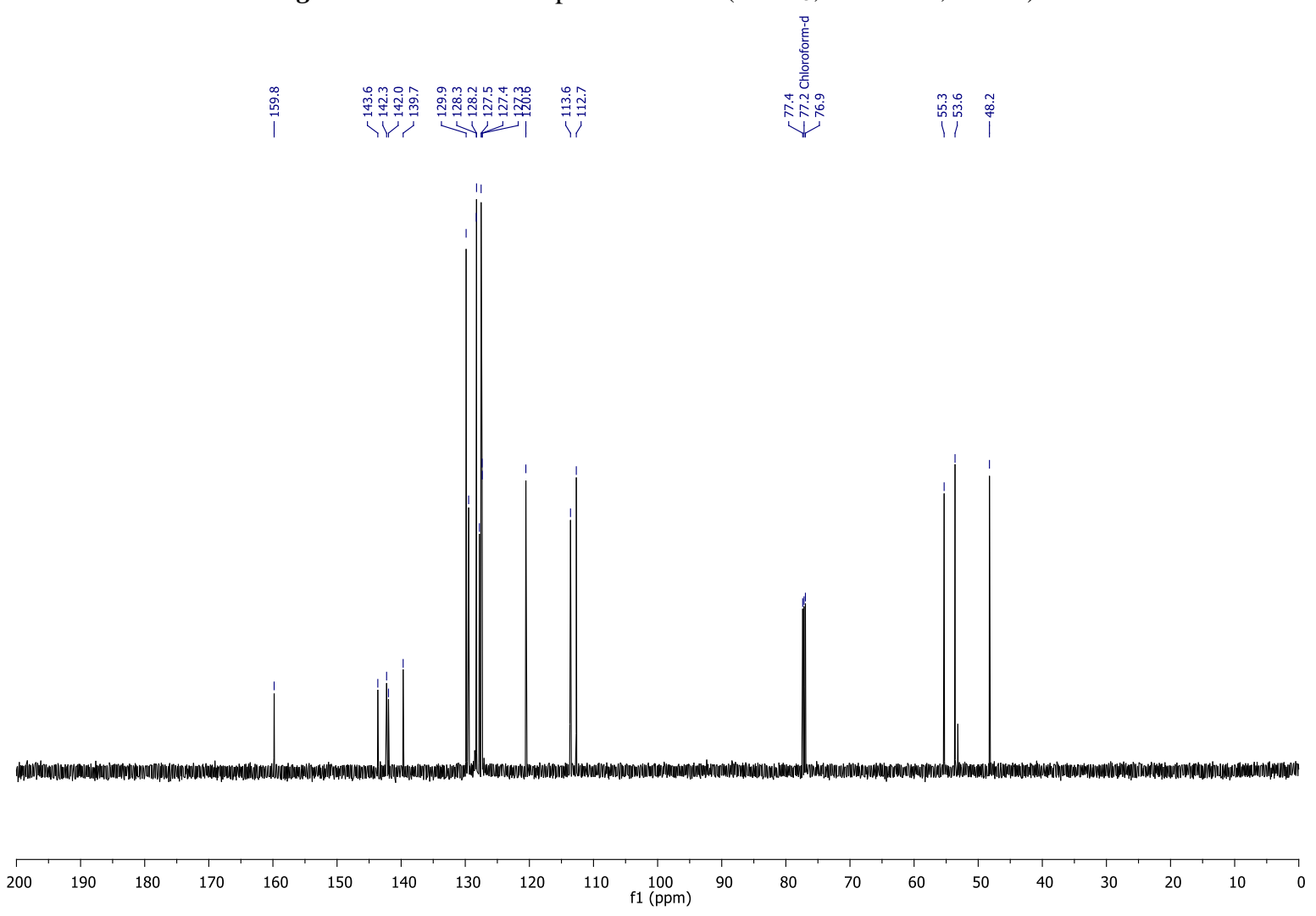

Figure S-100. ${ }^{13} \mathrm{C}$ NMR spectrum of $1 \mathrm{~s}\left(\mathrm{CDCl}_{3}, 151 \mathrm{MHz}, 298 \mathrm{~K}\right)$ 


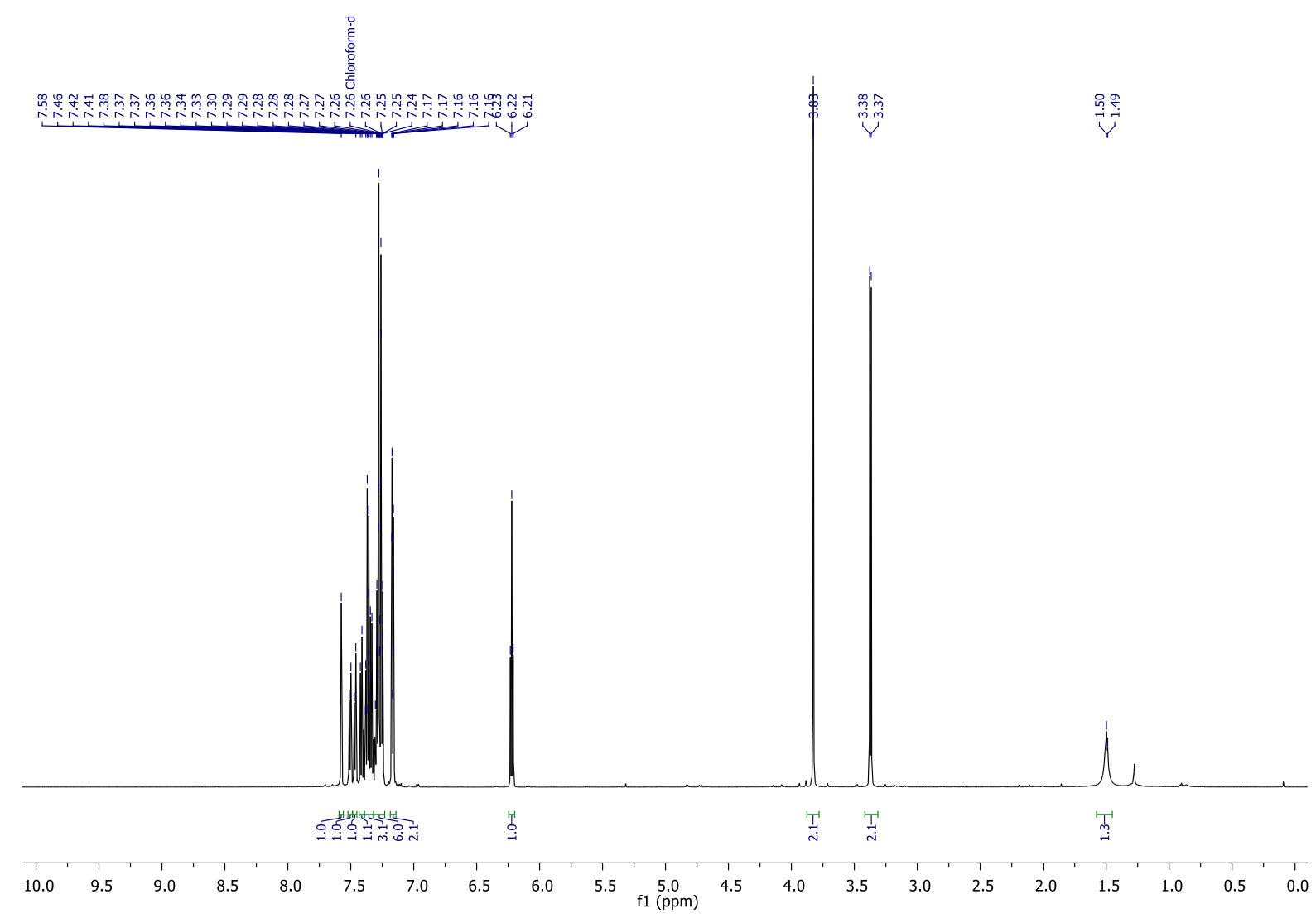

Figure S-101. ${ }^{1} \mathrm{H}$ NMR spectrum of $\mathbf{1 t}\left(\mathrm{CDCl}_{3}, 600 \mathrm{MHz}, 298 \mathrm{~K}\right)$
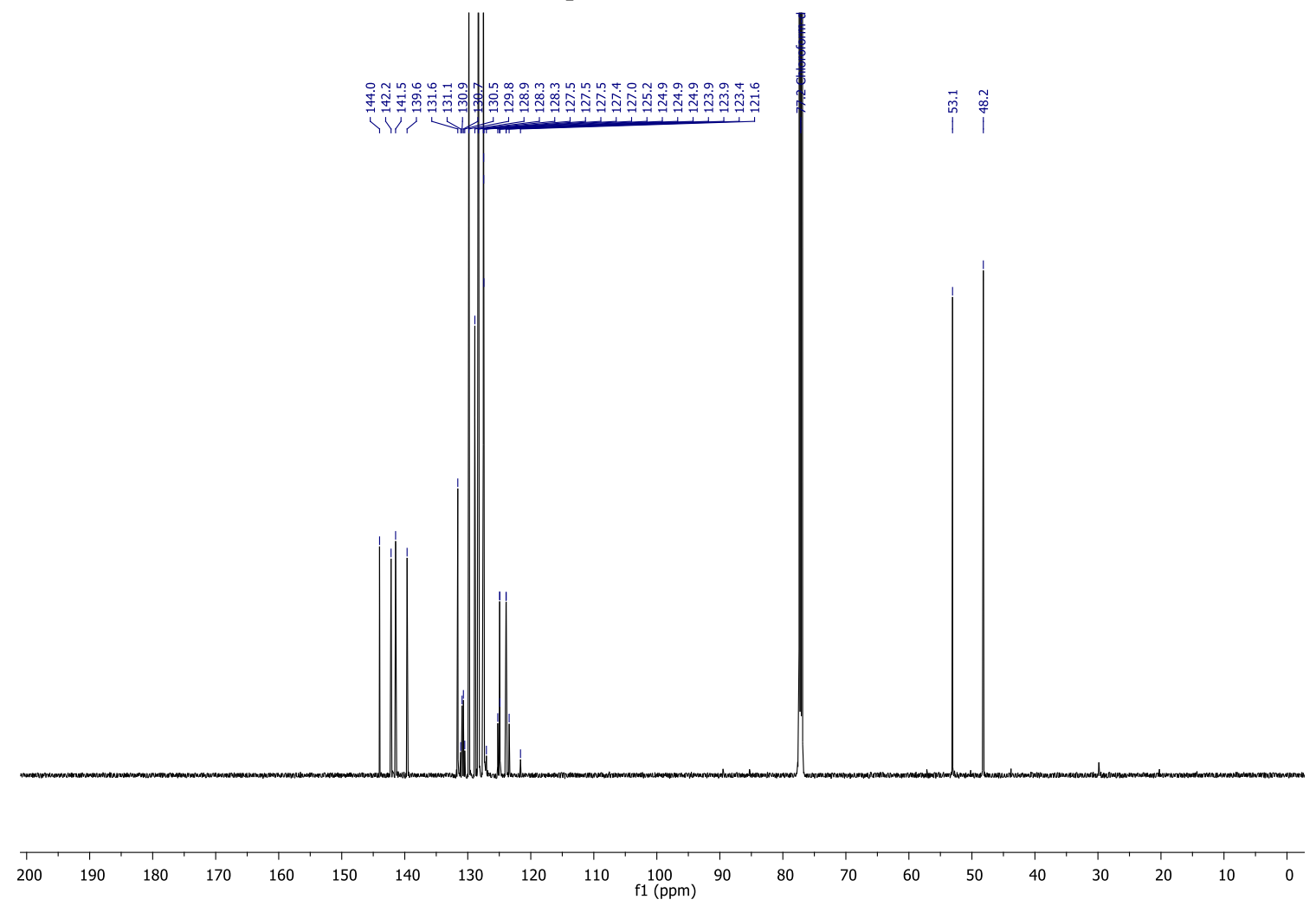

Figure S-102. ${ }^{13} \mathrm{C}$ NMR spectrum of $1 \mathbf{t}\left(\mathrm{CDCl}_{3}, 151 \mathrm{MHz}, 298 \mathrm{~K}\right)$ 


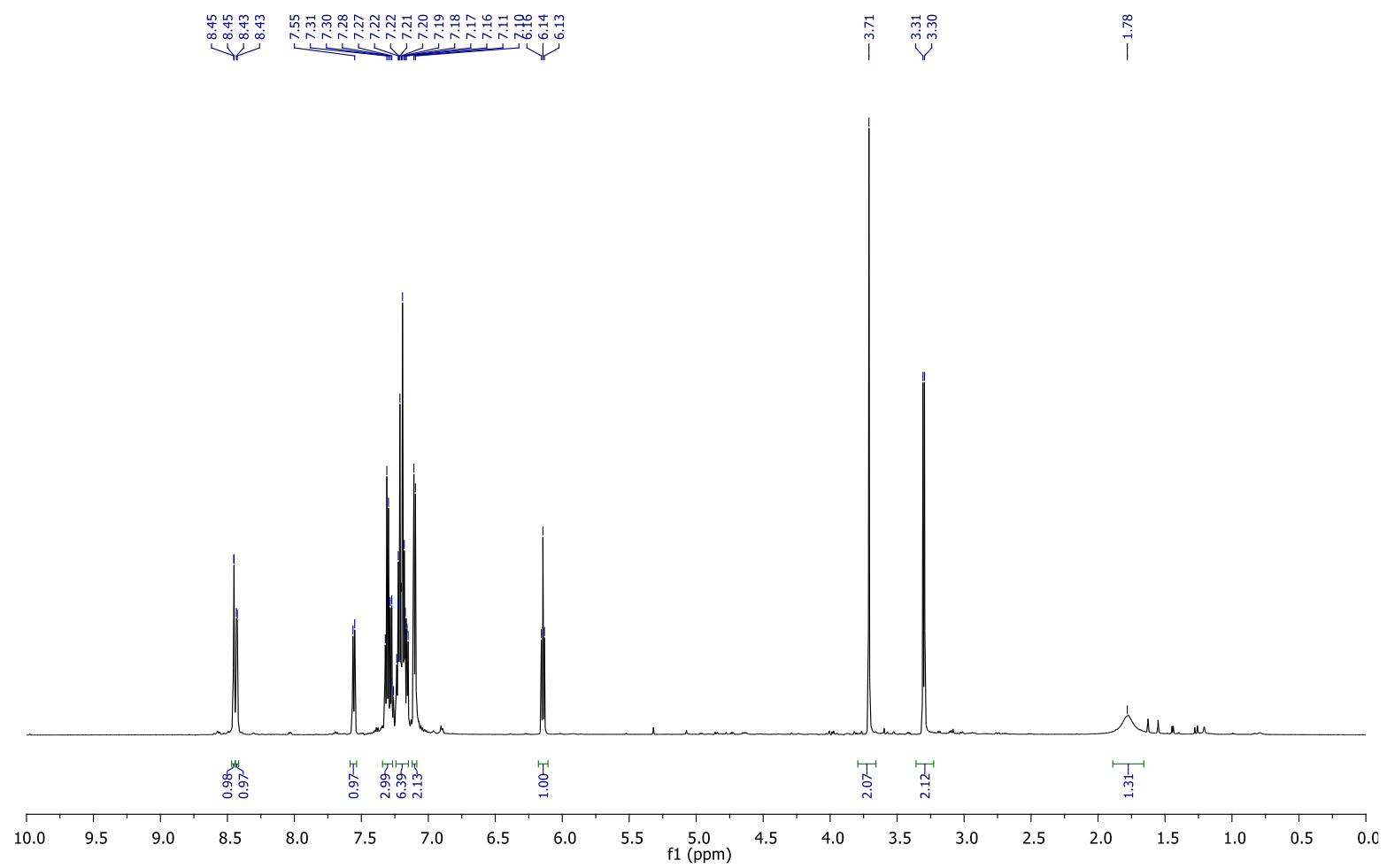

Figure $S$-103. ${ }^{1} \mathrm{H}$ NMR spectrum of $1 \mathbf{u}\left(\mathrm{CDCl}_{3}, 600 \mathrm{MHz}, 298 \mathrm{~K}\right)$

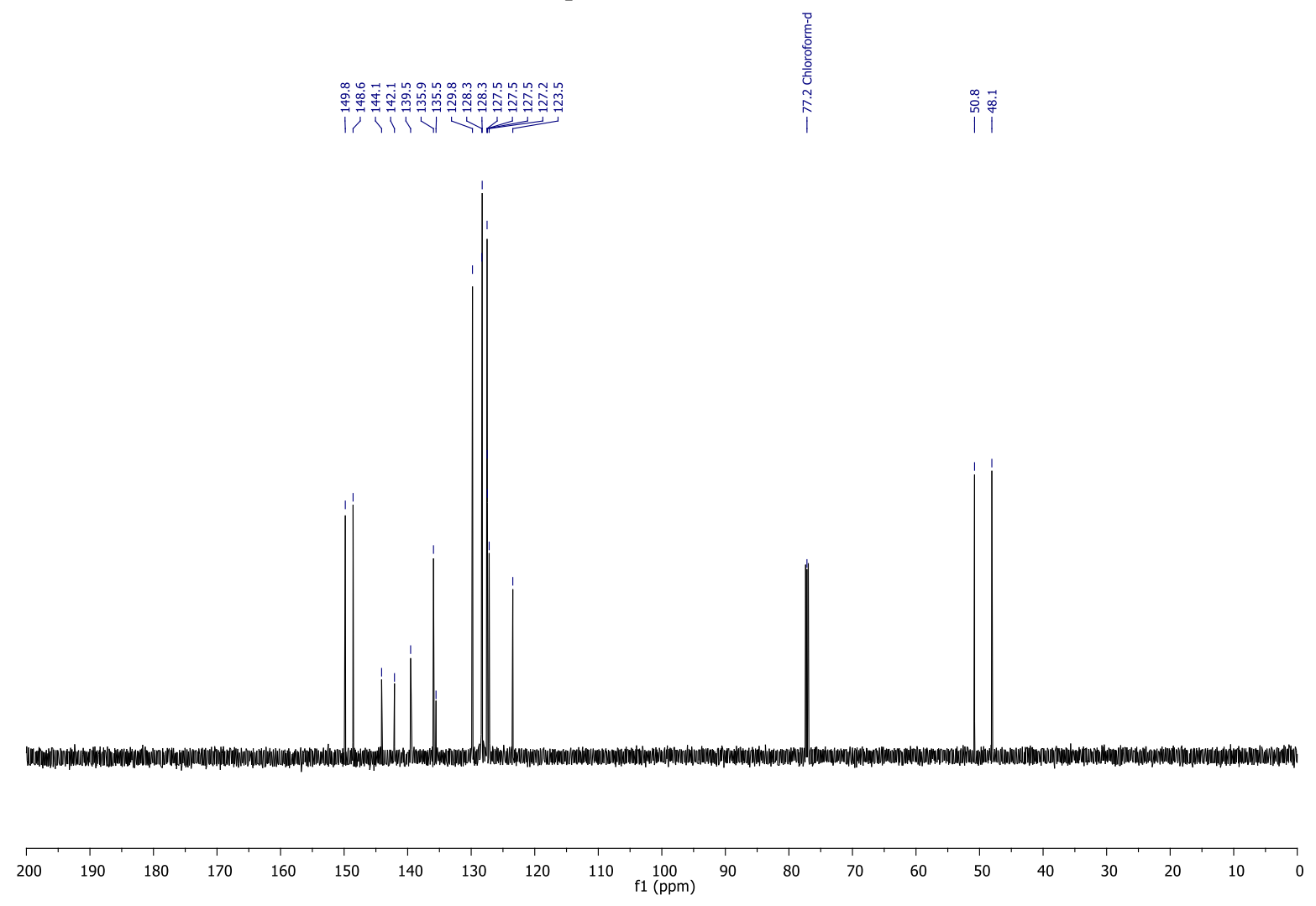

Figure S-104. ${ }^{13} \mathrm{C}$ NMR spectrum of $1 \mathbf{u}\left(\mathrm{CDCl}_{3}, 151 \mathrm{MHz}, 298 \mathrm{~K}\right)$ 


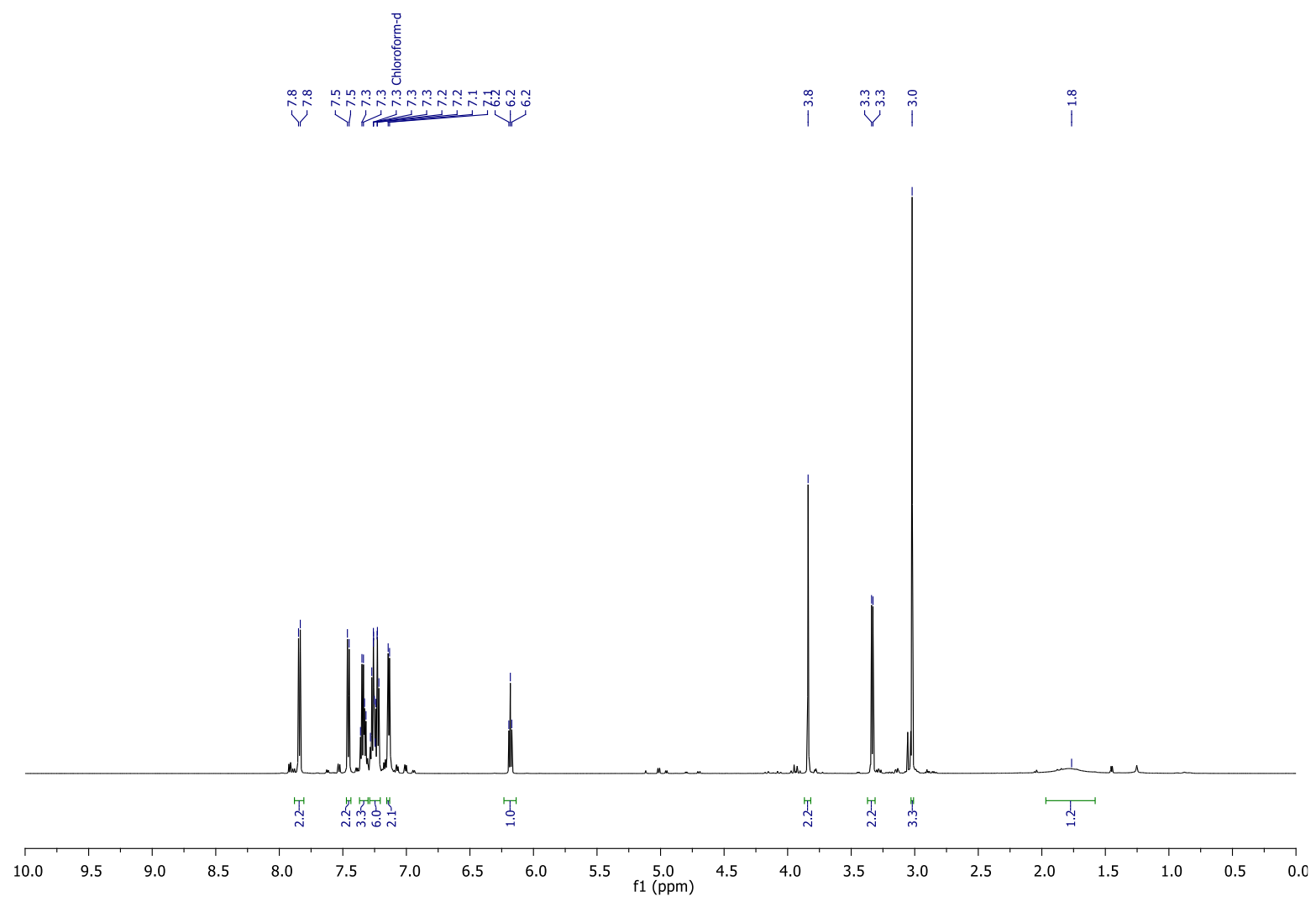

Figure $\boldsymbol{S - 1 0 5 . ~}{ }^{1} \mathrm{H}$ NMR spectrum of $\mathbf{1 v}\left(\mathrm{CDCl}_{3}, 600 \mathrm{MHz}, 298 \mathrm{~K}\right)$
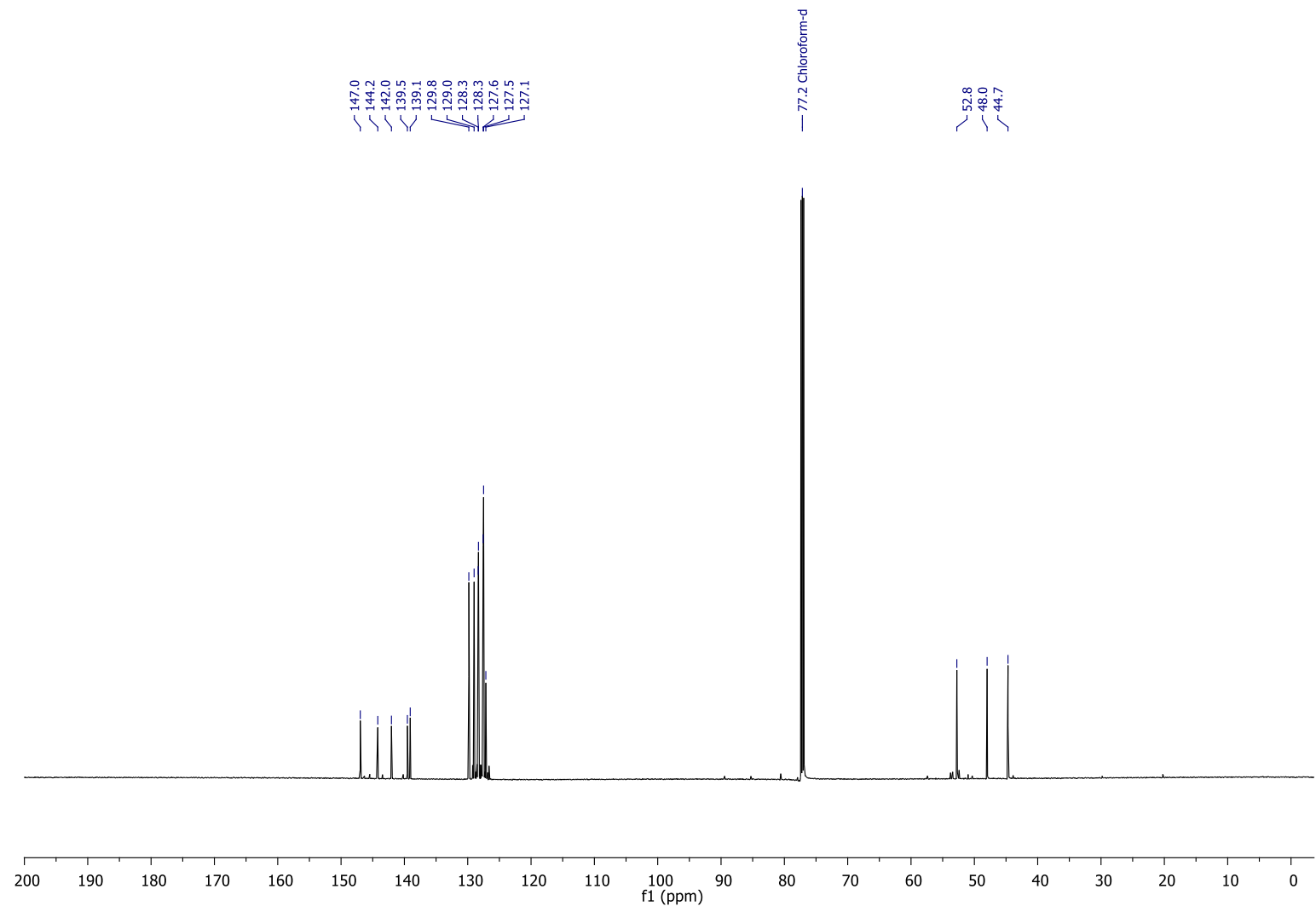

Figure S-106. ${ }^{13} \mathrm{C}$ NMR spectrum of $1 \mathrm{v}\left(\mathrm{CDCl}_{3}, 151 \mathrm{MHz}, 298 \mathrm{~K}\right)$ 


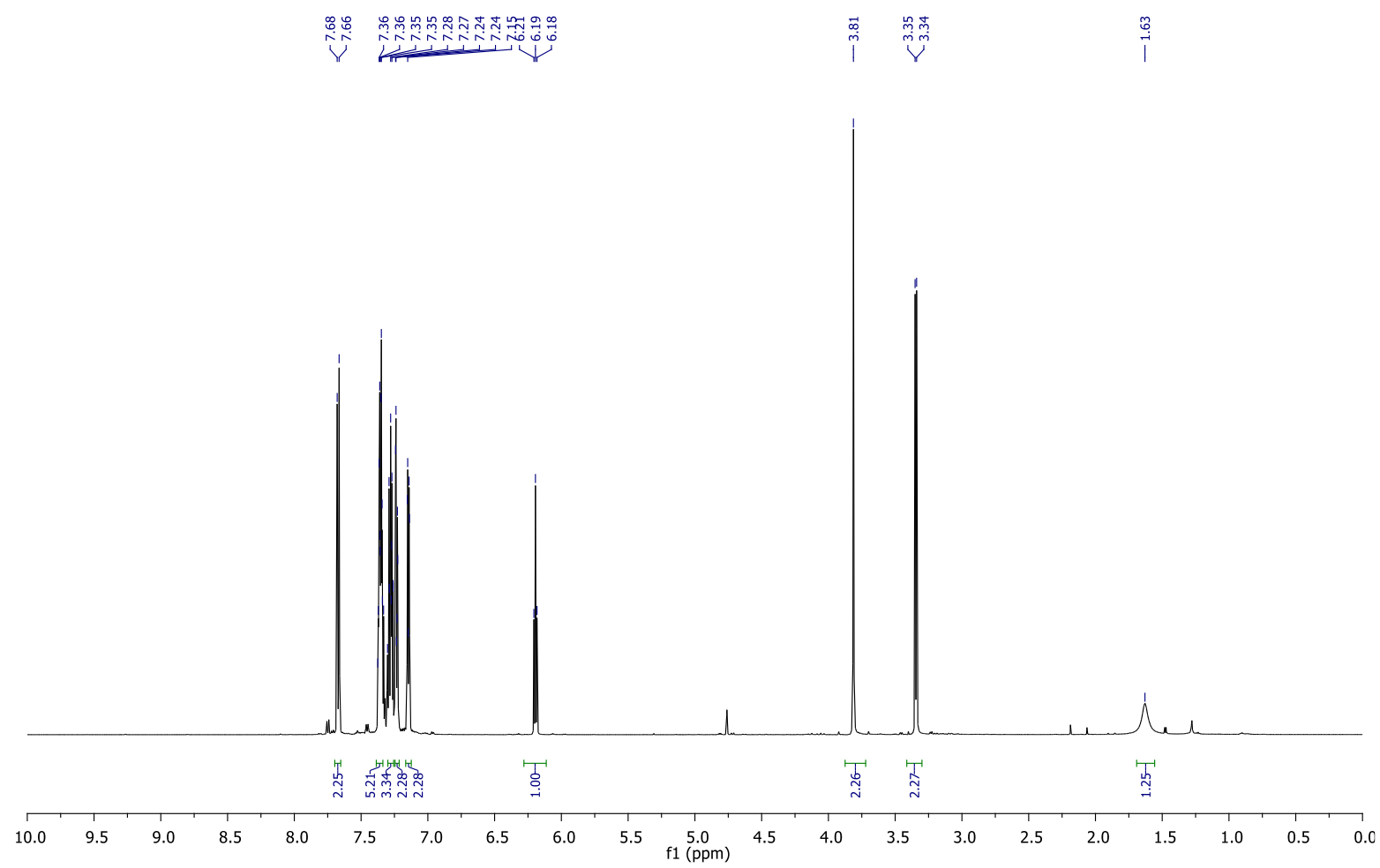

Figure S-107. ${ }^{1} \mathrm{H}$ NMR spectrum of $1 \mathbf{w}\left(\mathrm{CDCl}_{3}, 600 \mathrm{MHz}, 298 \mathrm{~K}\right)$
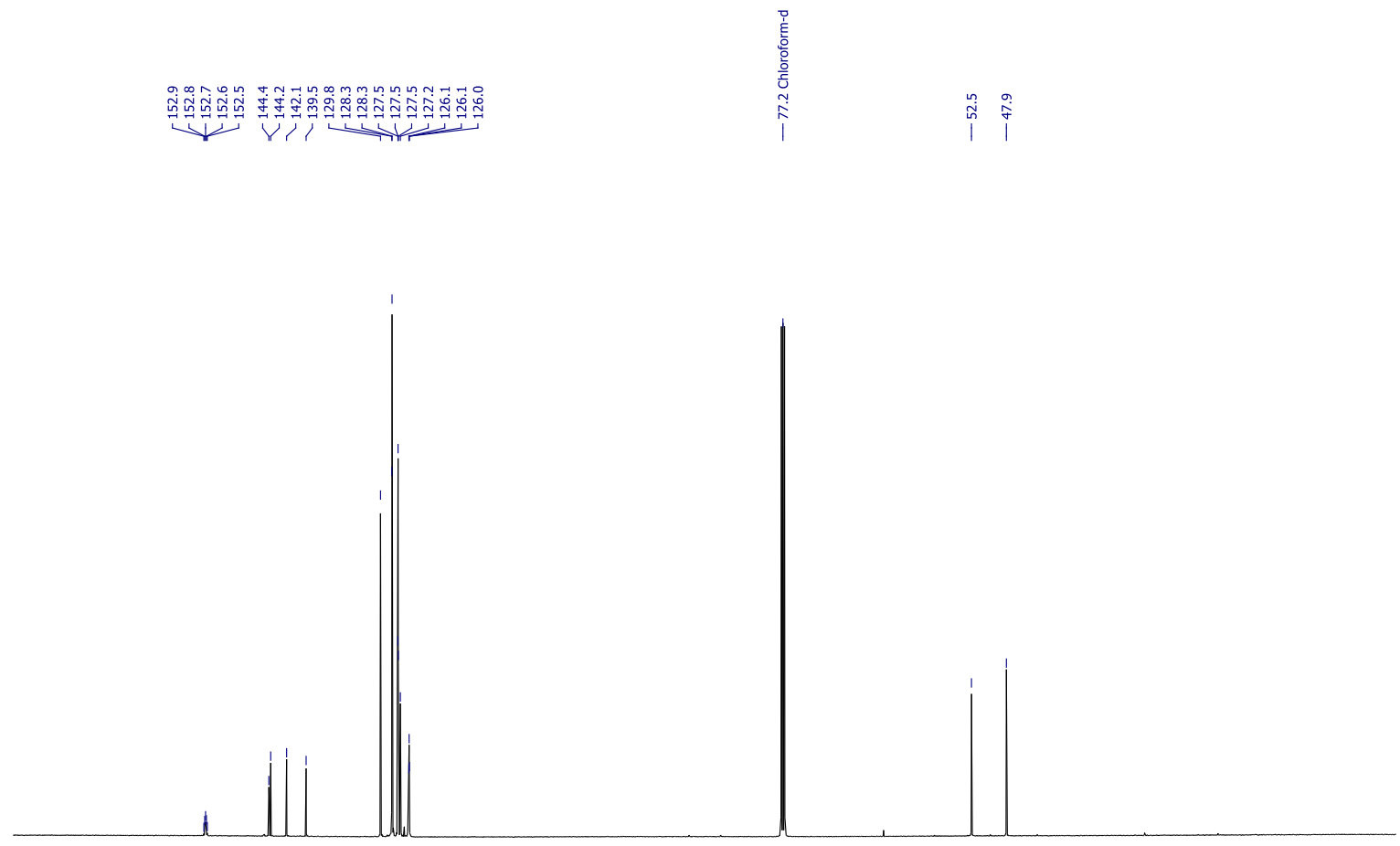

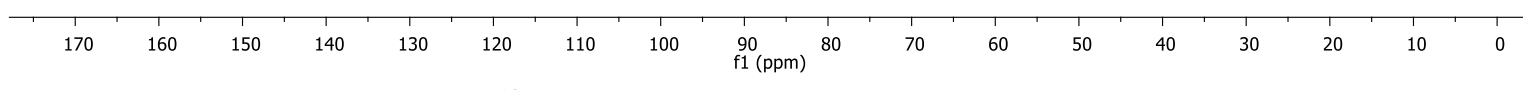

Figure $S$-108. ${ }^{13} \mathrm{C}$ NMR spectrum of $1 \mathbf{w}\left(\mathrm{CDCl}_{3}, 151 \mathrm{MHz}, 298 \mathrm{~K}\right)$ 

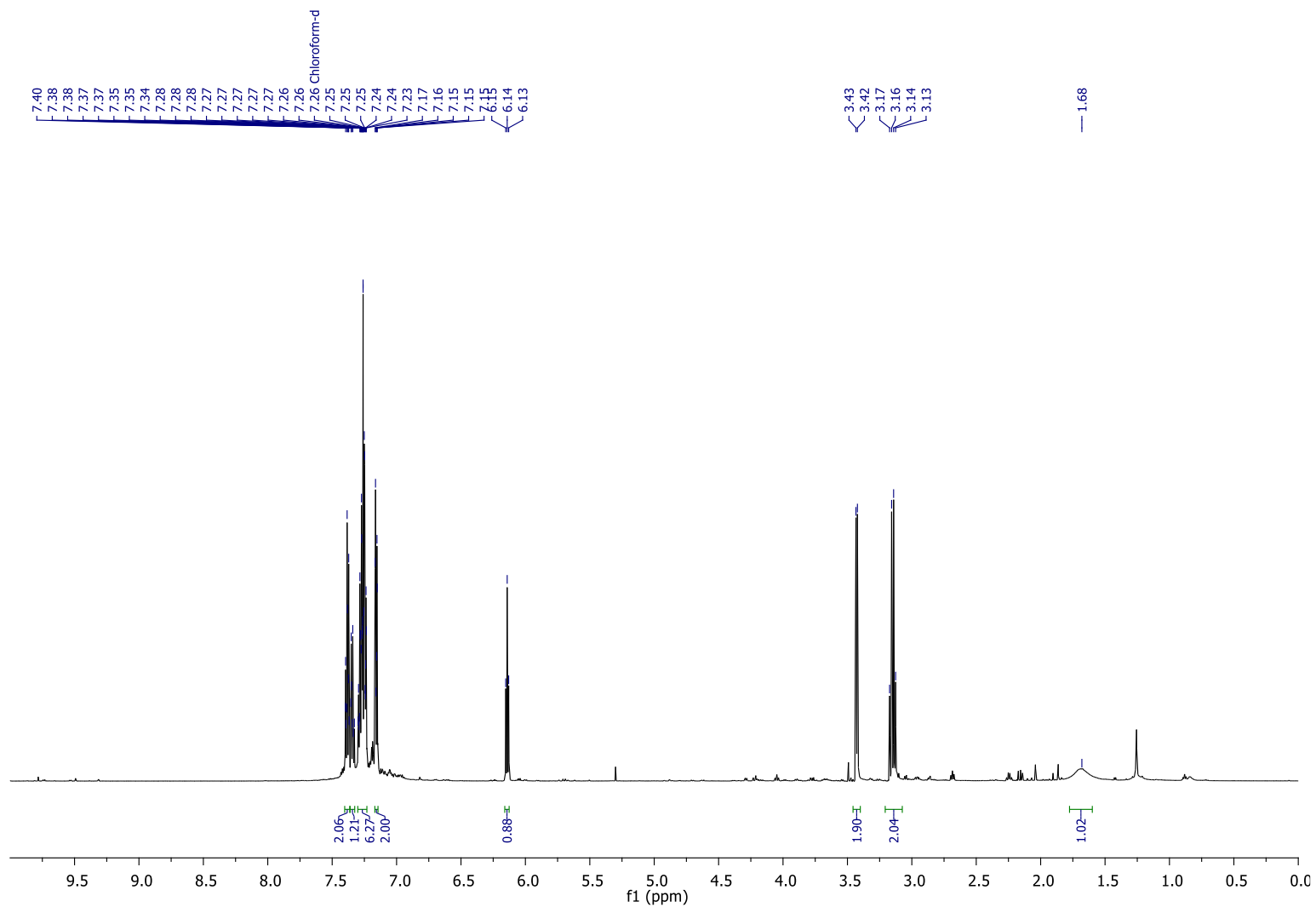

Figure S-109. ${ }^{1} \mathrm{H}$ NMR spectrum of $\mathbf{1 x}\left(\mathrm{CDCl}_{3}, 600 \mathrm{MHz}, 298 \mathrm{~K}\right)$

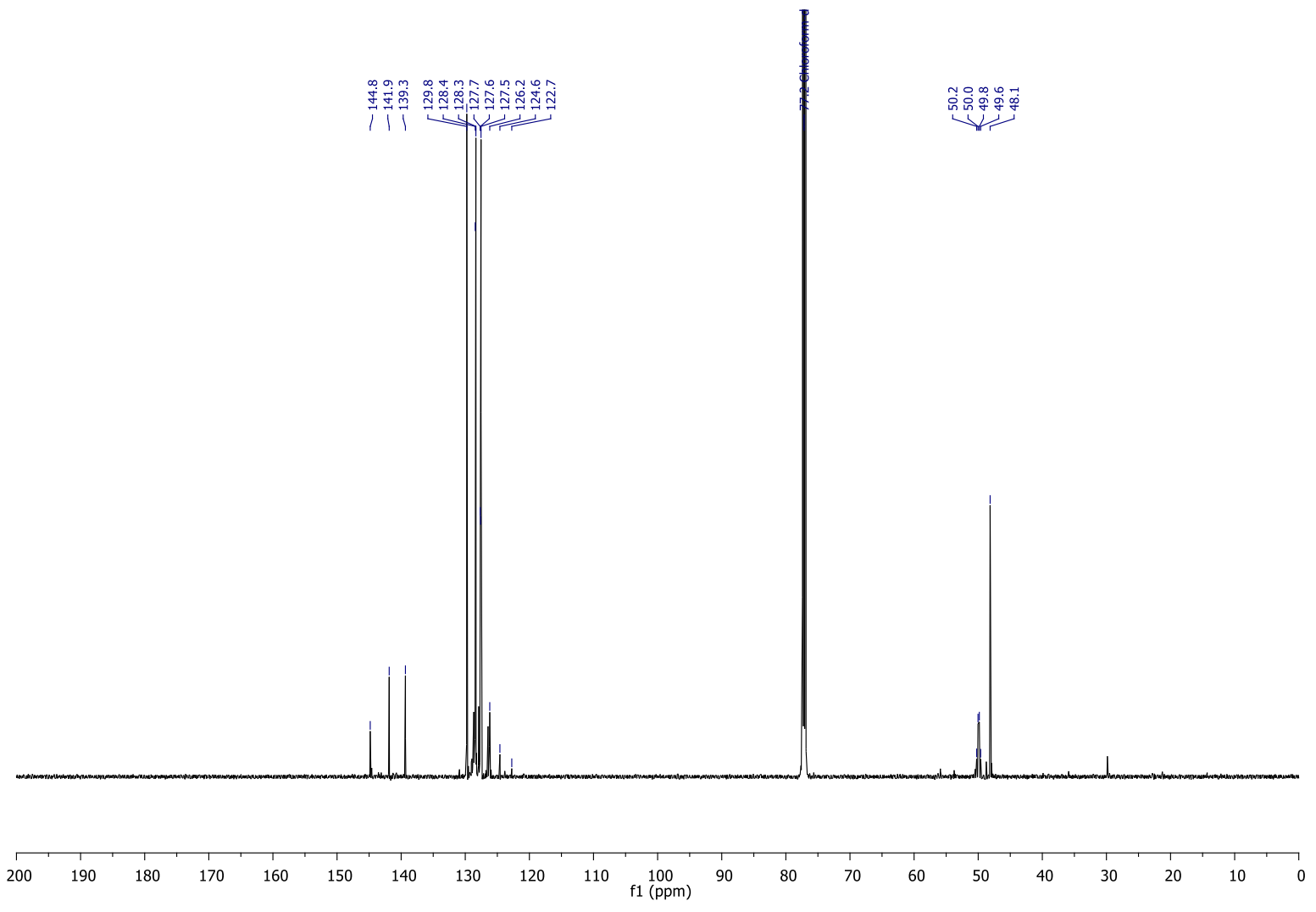

Figure $S$-110. ${ }^{13} \mathrm{C}$ NMR spectrum of $\mathbf{1 x}\left(\mathrm{CDCl}_{3}, 151 \mathrm{MHz}, 298 \mathrm{~K}\right)$ 


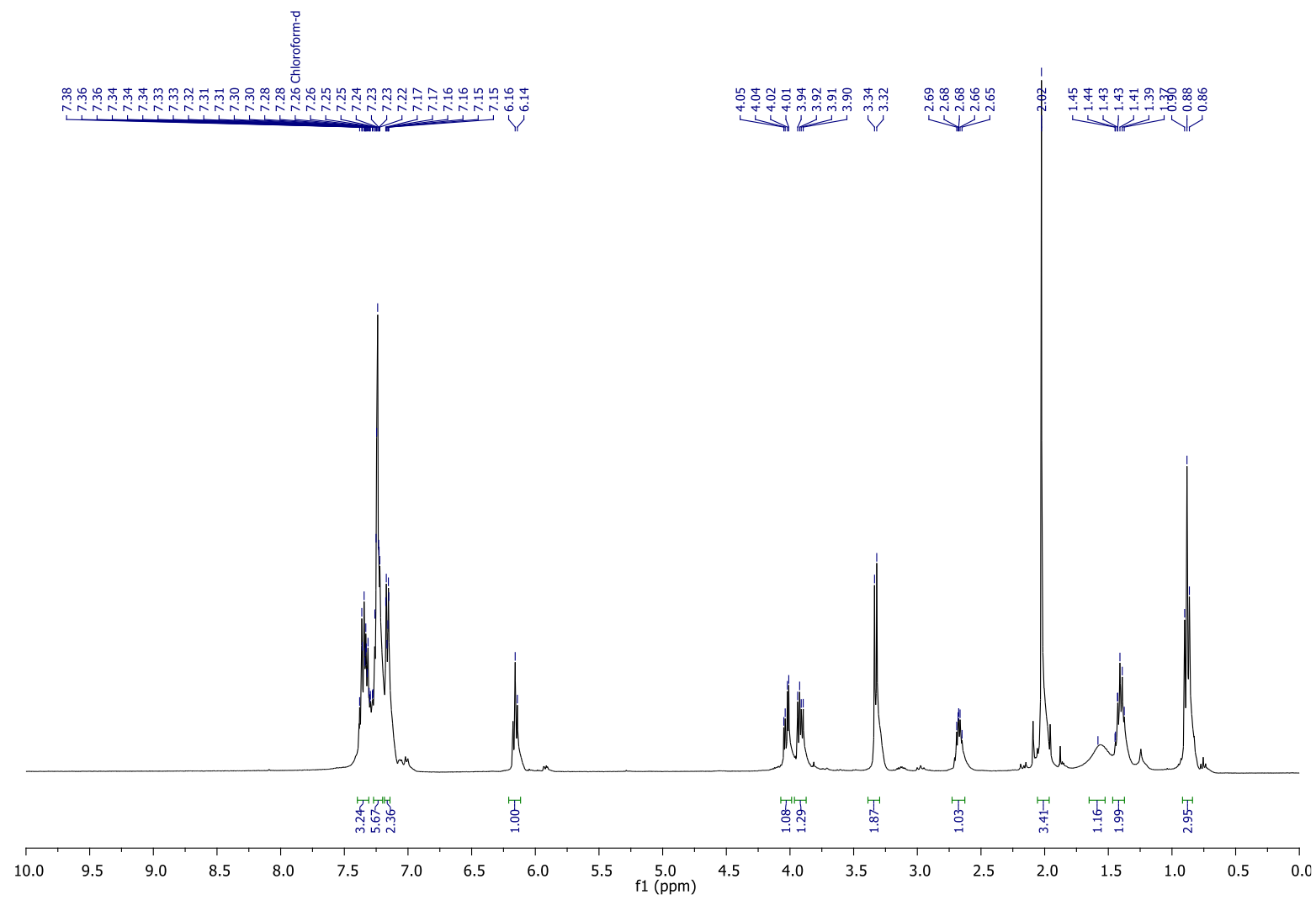

Figure S-111. ${ }^{1} \mathrm{H}$ NMR spectrum of $1 \mathbf{y}\left(\mathrm{CDCl}_{3}, 600 \mathrm{MHz}, 298 \mathrm{~K}\right)$

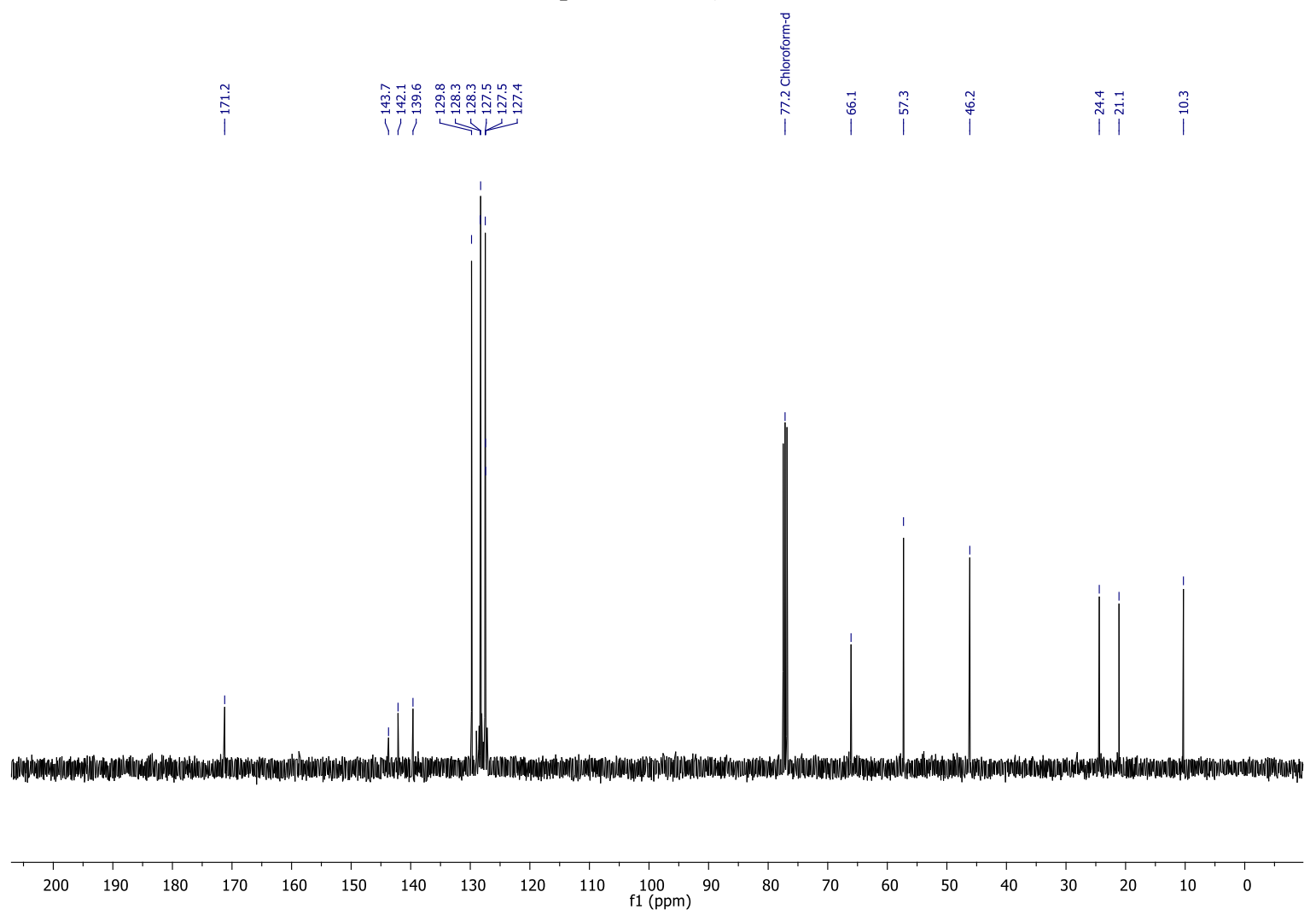

Figure $S$-112. ${ }^{13} \mathrm{C}$ NMR spectrum of $1 \mathbf{y}\left(\mathrm{CDCl}_{3}, 151 \mathrm{MHz}, 298 \mathrm{~K}\right)$ 


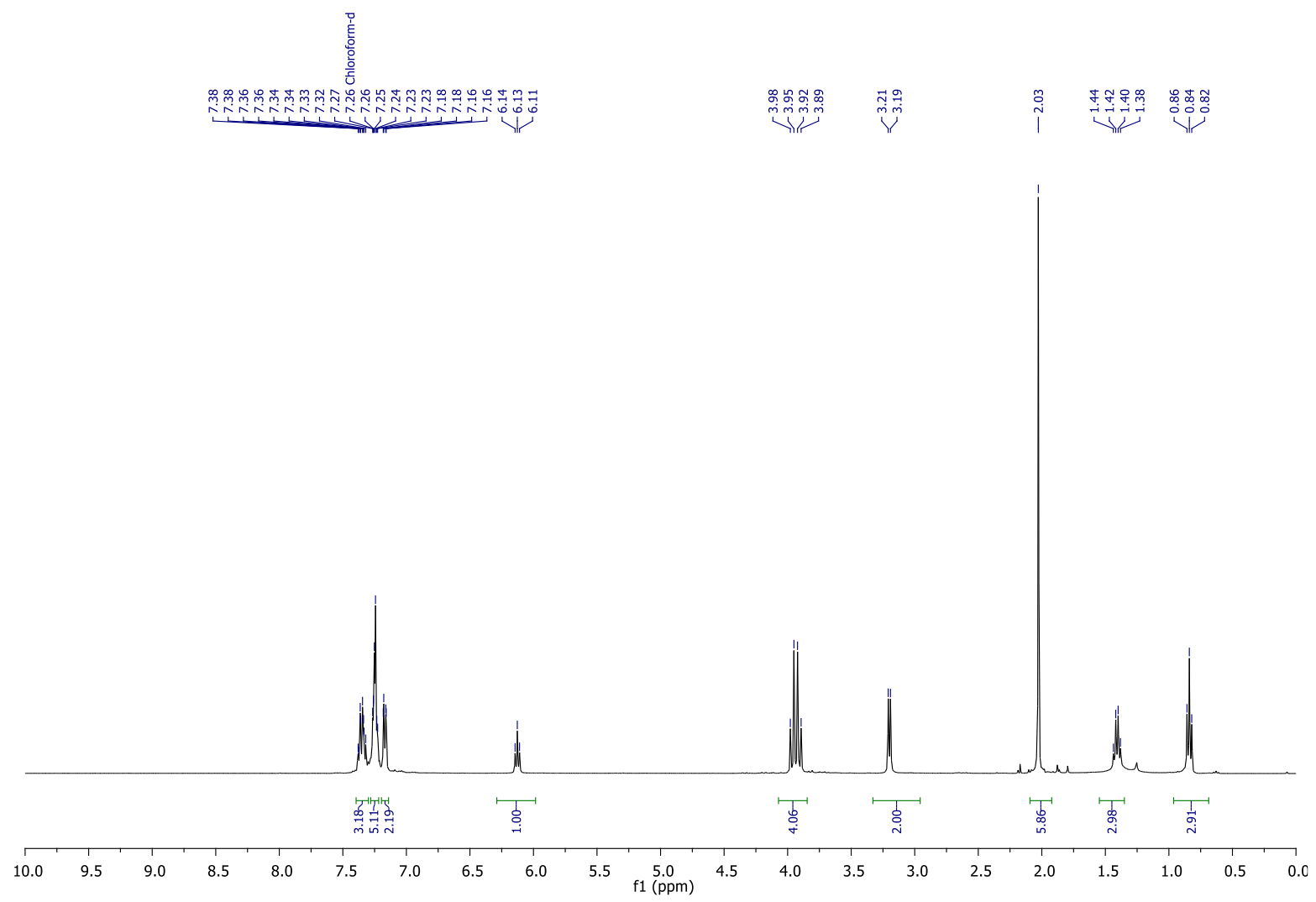

Figure $\boldsymbol{S - 1 1 3 .}{ }^{1} \mathrm{H}$ NMR spectrum of $\mathbf{1 z}\left(\mathrm{CDCl}_{3}, 600 \mathrm{MHz}, 298 \mathrm{~K}\right)$
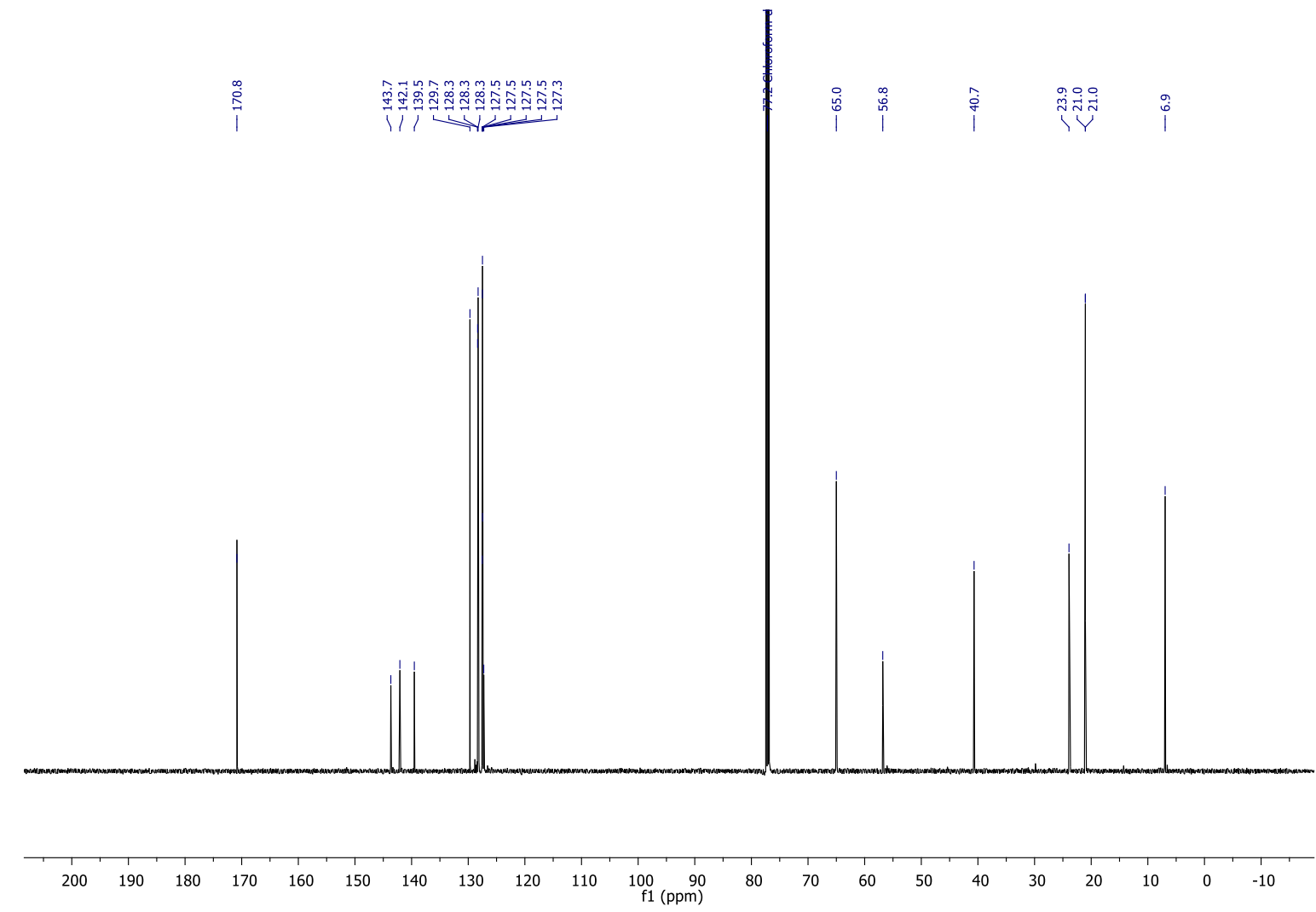

Figure $S$-114. ${ }^{13} \mathrm{C}$ NMR spectrum of $\mathbf{1 z}\left(\mathrm{CDCl}_{3}, 151 \mathrm{MHz}, 298 \mathrm{~K}\right)$ 


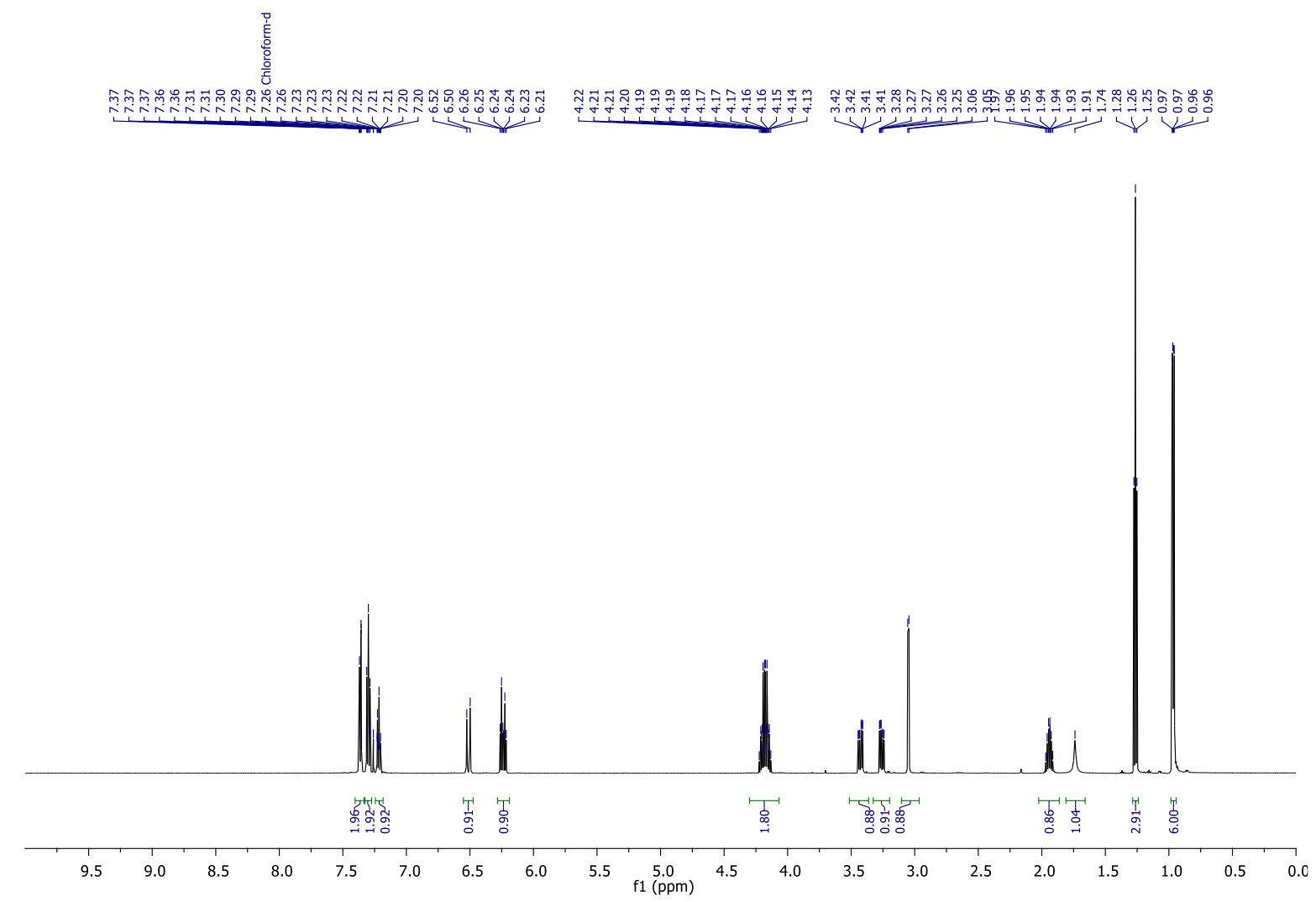

Figure S-115. ${ }^{1} \mathrm{H}$ NMR spectrum of $1 \mathrm{aa}\left(\mathrm{CDCl}_{3}, 600 \mathrm{MHz}, 298 \mathrm{~K}\right)$
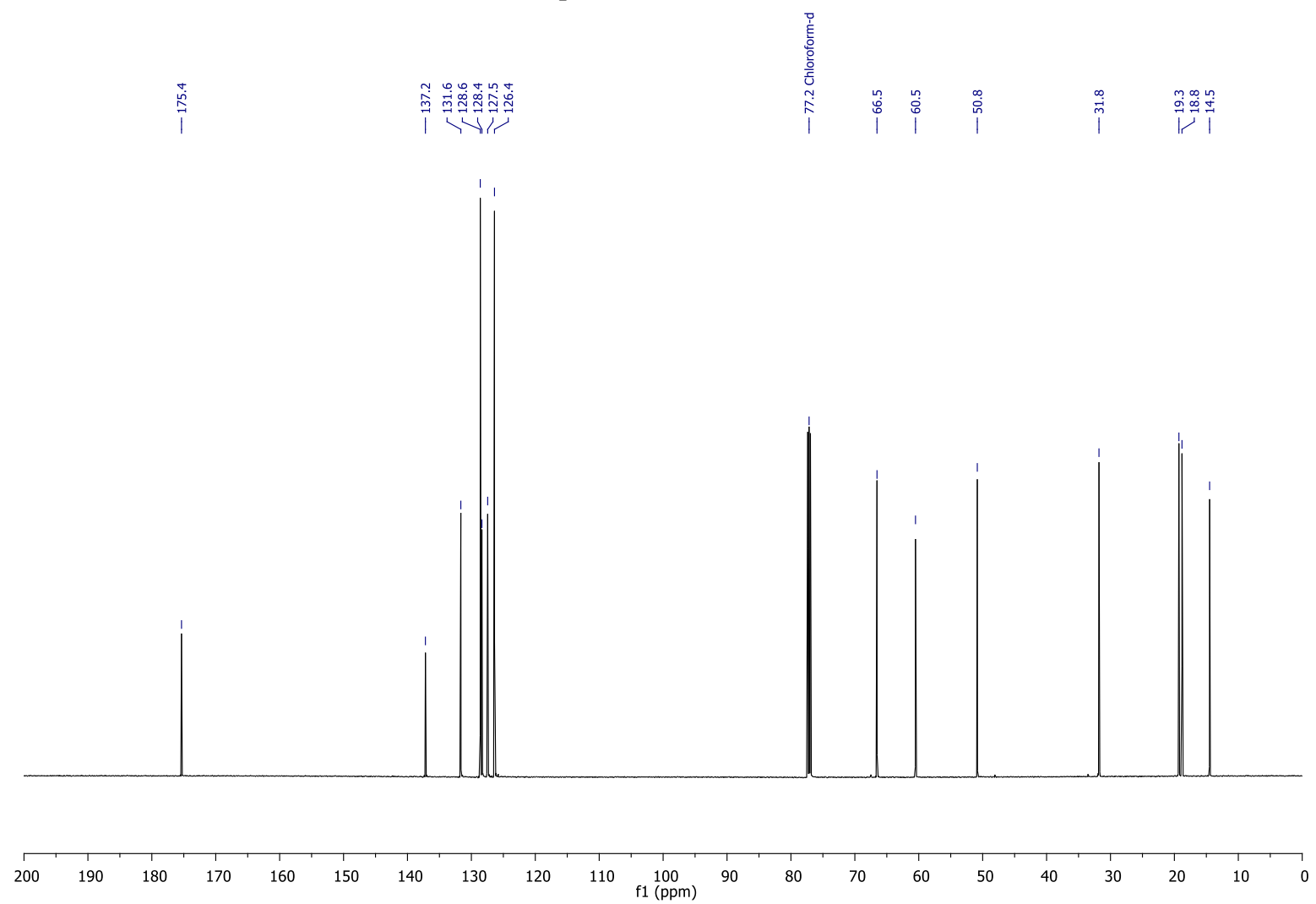

Figure $S$-116. ${ }^{13} \mathrm{C}$ NMR spectrum of 1 aa $\left(\mathrm{CDCl}_{3}, 151 \mathrm{MHz}, 298 \mathrm{~K}\right)$ 


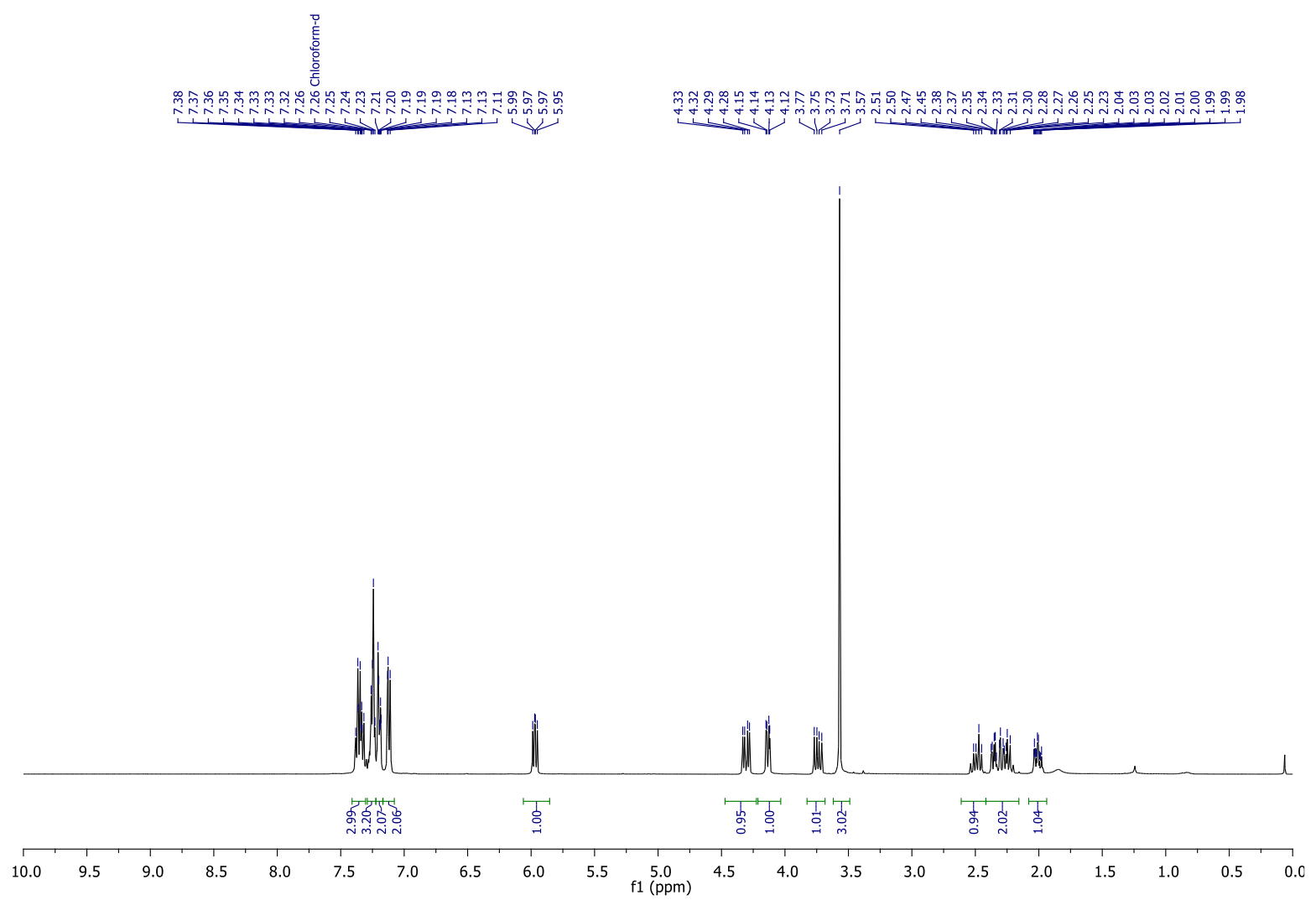

Figure S-117. ${ }^{1} \mathrm{H}$ NMR spectrum of $1 \mathbf{a b}\left(\mathrm{CDCl}_{3}, 600 \mathrm{MHz}, 298 \mathrm{~K}\right)$

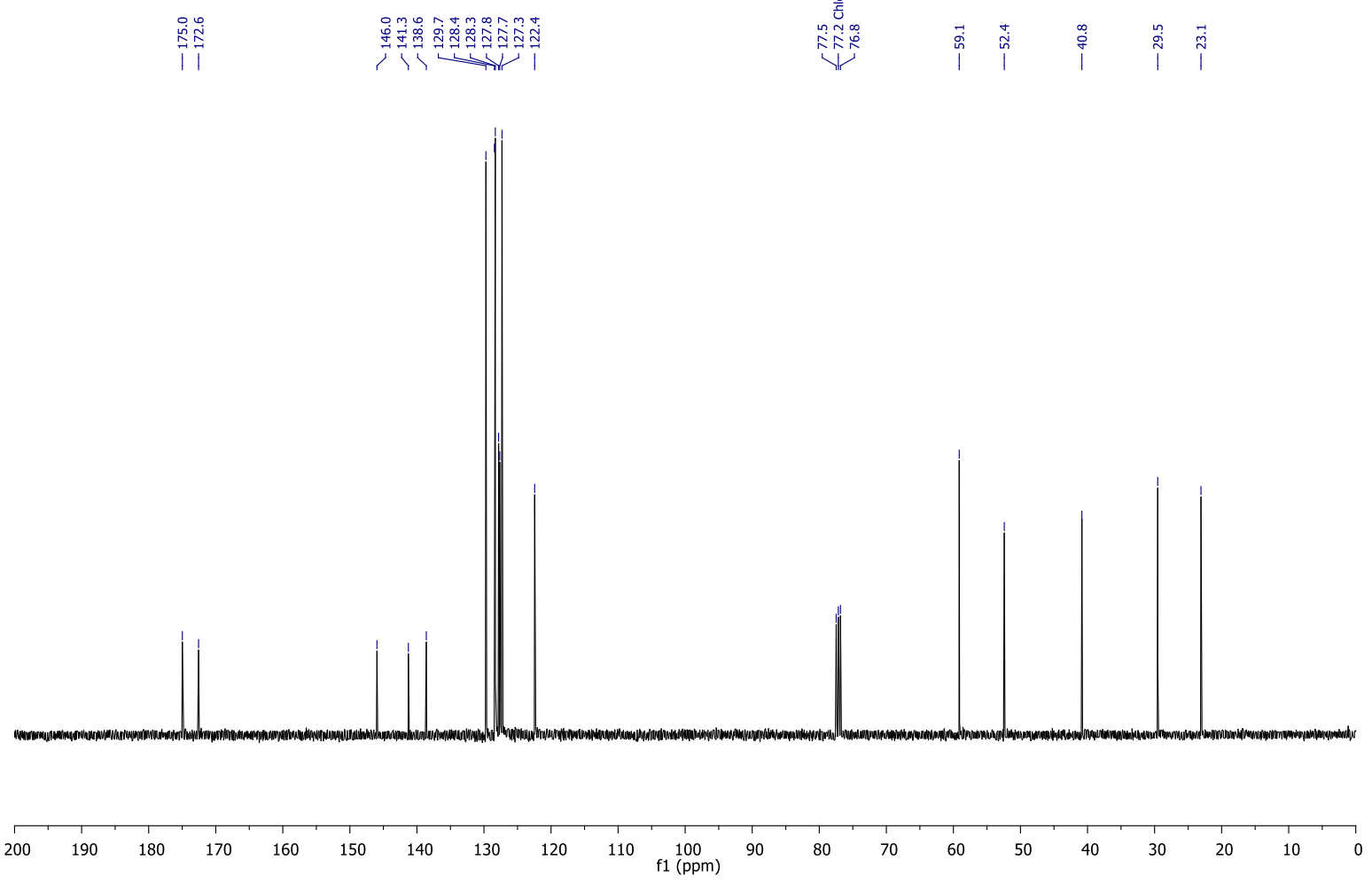

Figure S-118. ${ }^{13} \mathrm{C}$ NMR spectrum of $1 \mathbf{a b}\left(\mathrm{CDCl}_{3}, 151 \mathrm{MHz}, 298 \mathrm{~K}\right)$ 

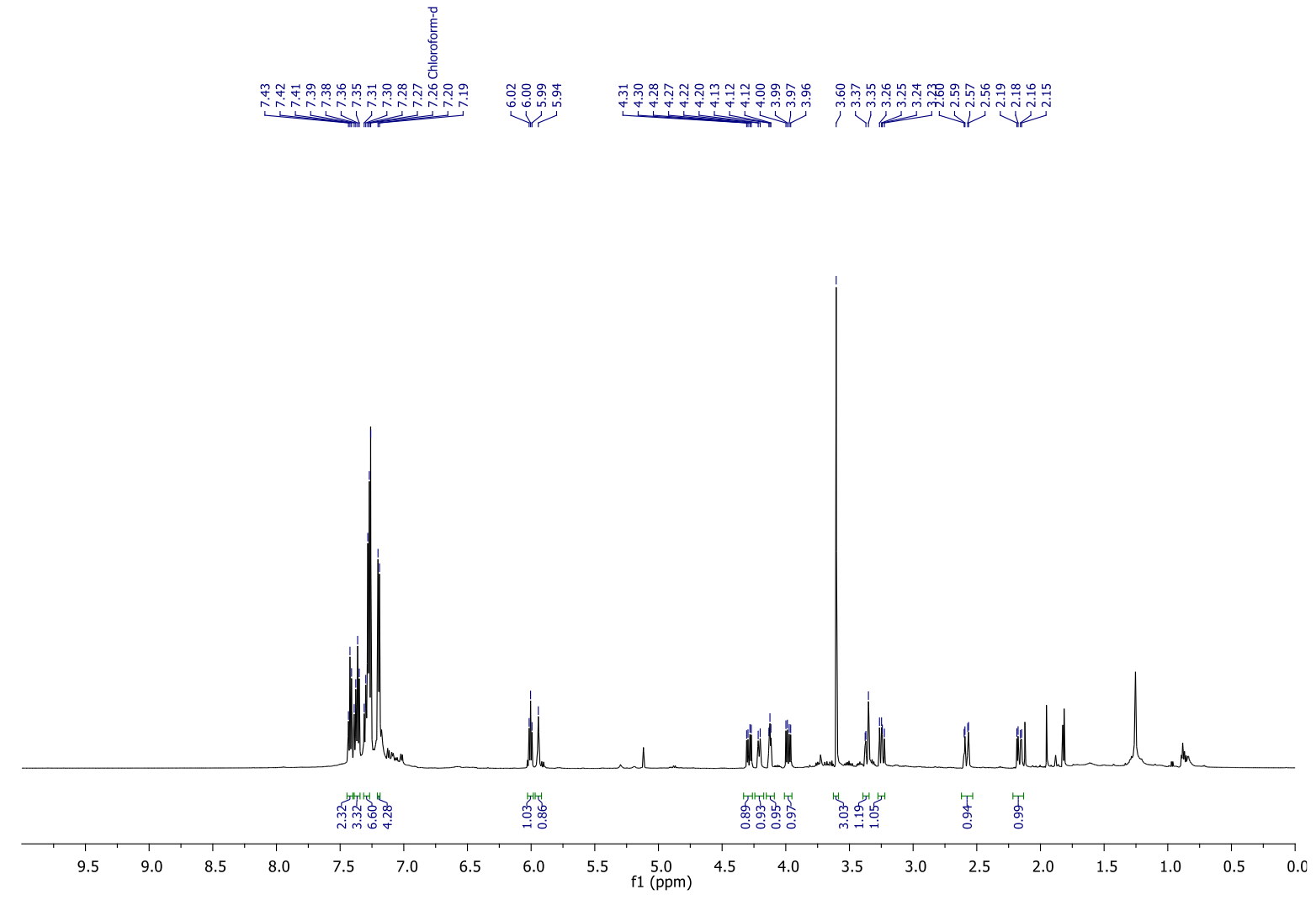

Figure S-119. ${ }^{1} \mathrm{H}$ NMR spectrum of $1 \mathrm{ac}\left(\mathrm{CDCl}_{3}, 600 \mathrm{MHz}, 298 \mathrm{~K}\right)$
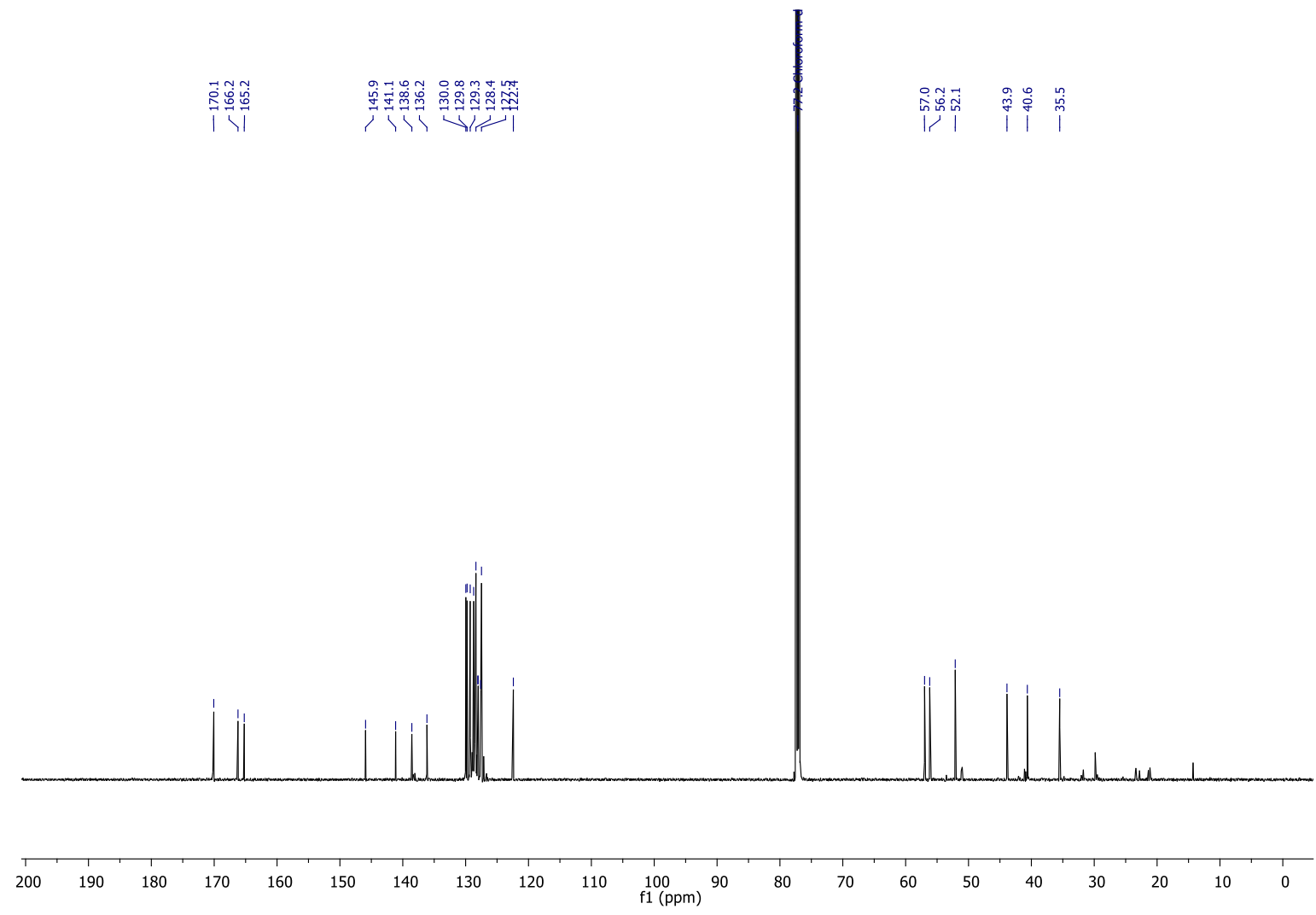

Figure $S$-120. ${ }^{13} \mathrm{C}$ NMR spectrum of $1 \mathbf{a c}\left(\mathrm{CDCl}_{3}, 151 \mathrm{MHz}, 298 \mathrm{~K}\right)$ 


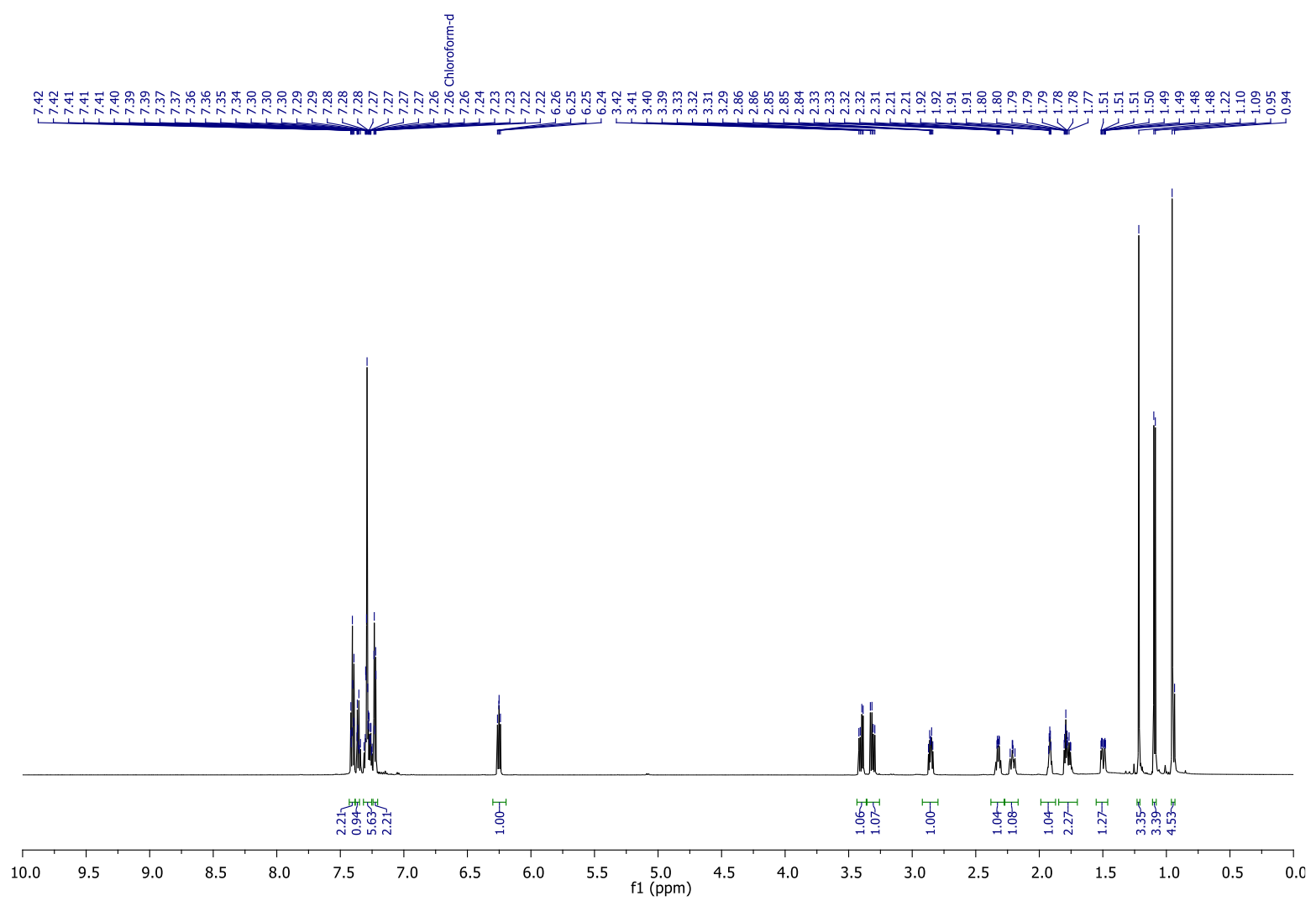

Figure $\mathbf{S - 1 2 1} .{ }^{1} \mathrm{H}$ NMR spectrum of $\mathbf{1 a d}\left(\mathrm{CDCl}_{3}, 600 \mathrm{MHz}, 298 \mathrm{~K}\right)$

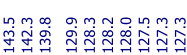

औi
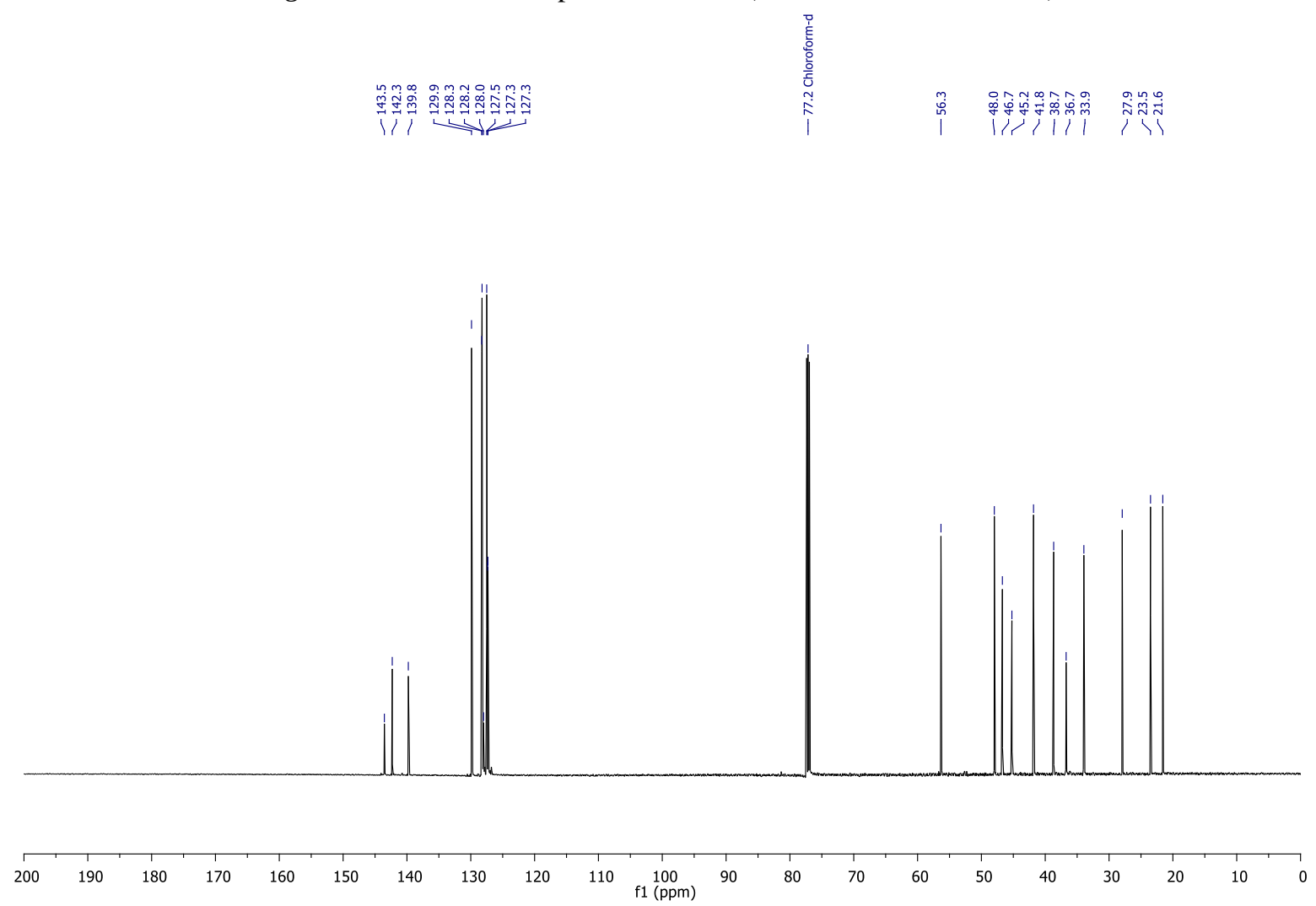

Figure $\mathbf{S}$-122. ${ }^{13} \mathrm{C}$ NMR spectrum of 1 ad $\left(\mathrm{CDCl}_{3}, 151 \mathrm{MHz}, 298 \mathrm{~K}\right)$ 

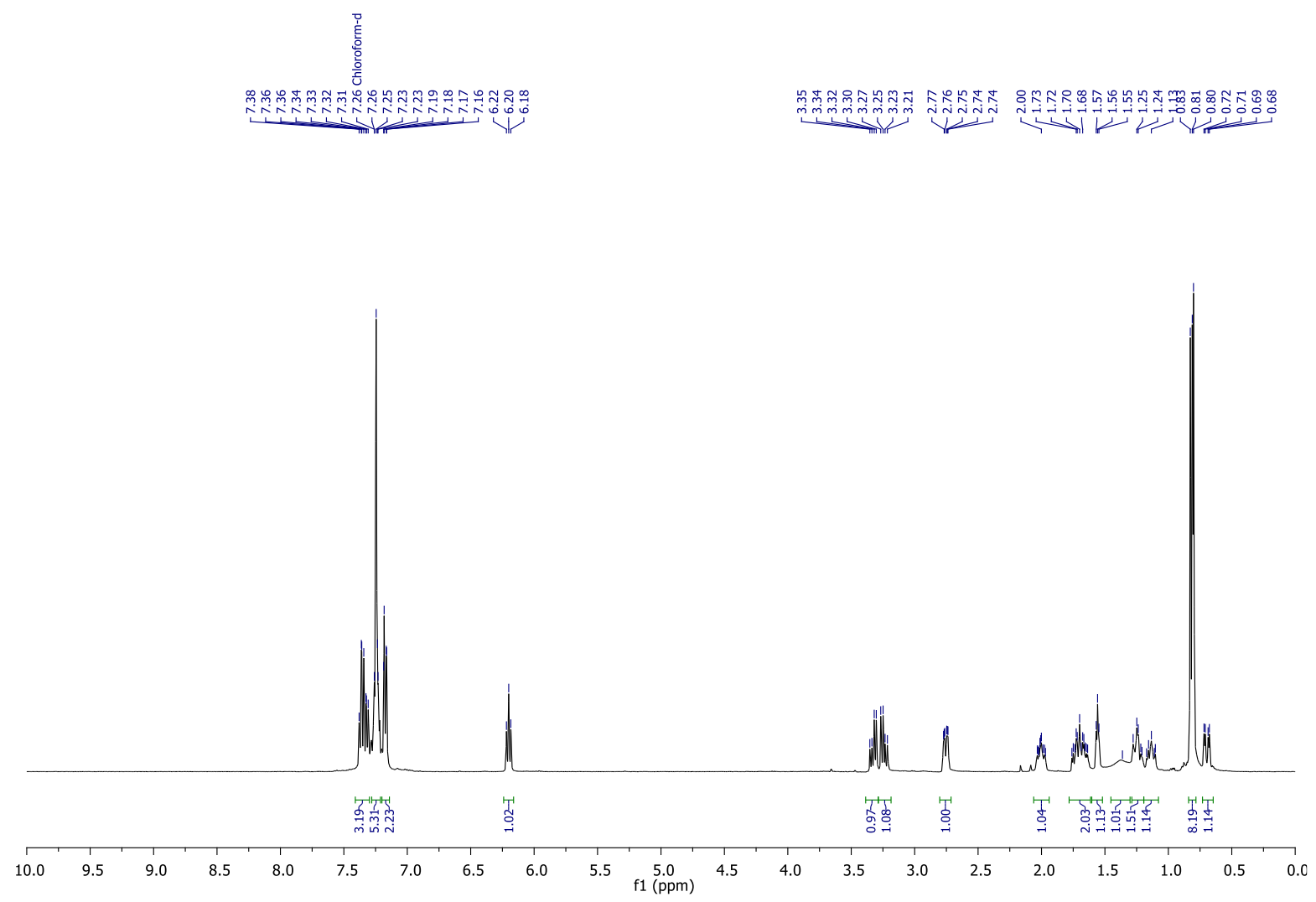

Figure $\boldsymbol{S - 1 2 3 .}{ }^{1} \mathrm{H}$ NMR spectrum of $\mathbf{1 a e}\left(\mathrm{CDCl}_{3}, 600 \mathrm{MHz}, 298 \mathrm{~K}\right)$

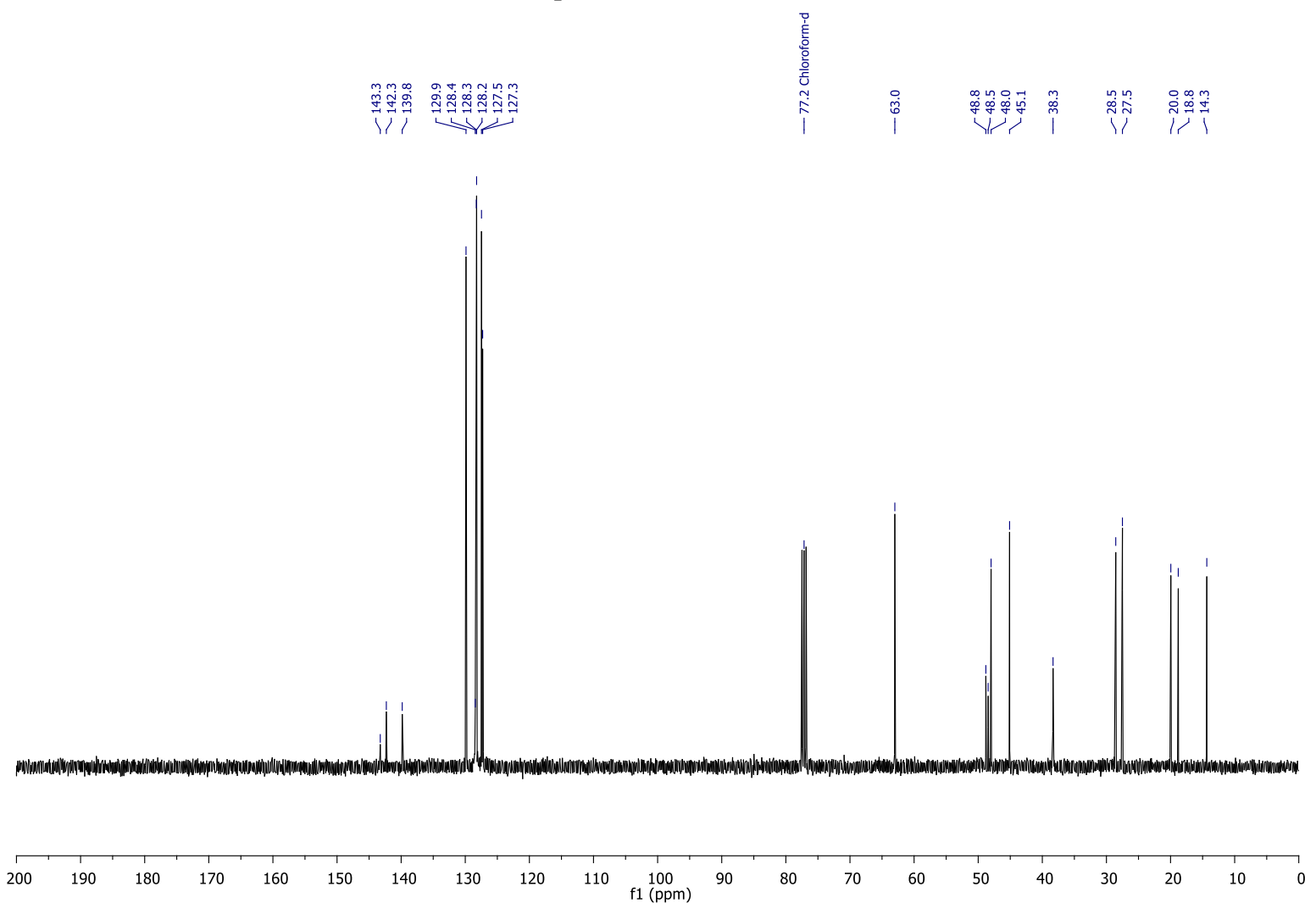

Figure $\boldsymbol{S}$-124. ${ }^{13} \mathrm{C}$ NMR spectrum of $1 \mathrm{ae}\left(\mathrm{CDCl}_{3}, 151 \mathrm{MHz}, 298 \mathrm{~K}\right)$ 


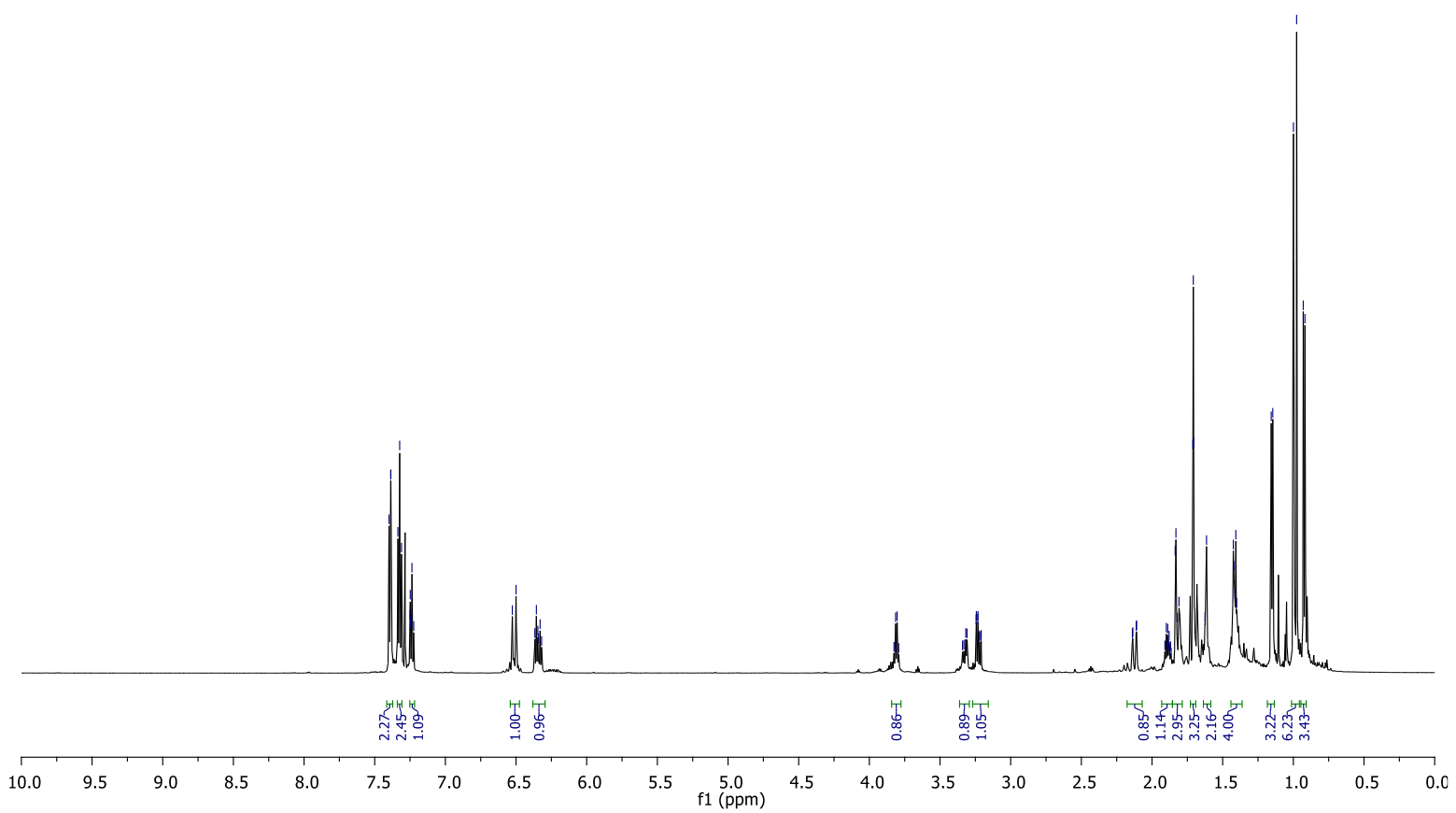

Figure S-125. ${ }^{1} \mathrm{H}$ NMR spectrum of $\mathbf{1 a f}\left(\mathrm{CDCl}_{3}, 600 \mathrm{MHz}, 298 \mathrm{~K}\right)$

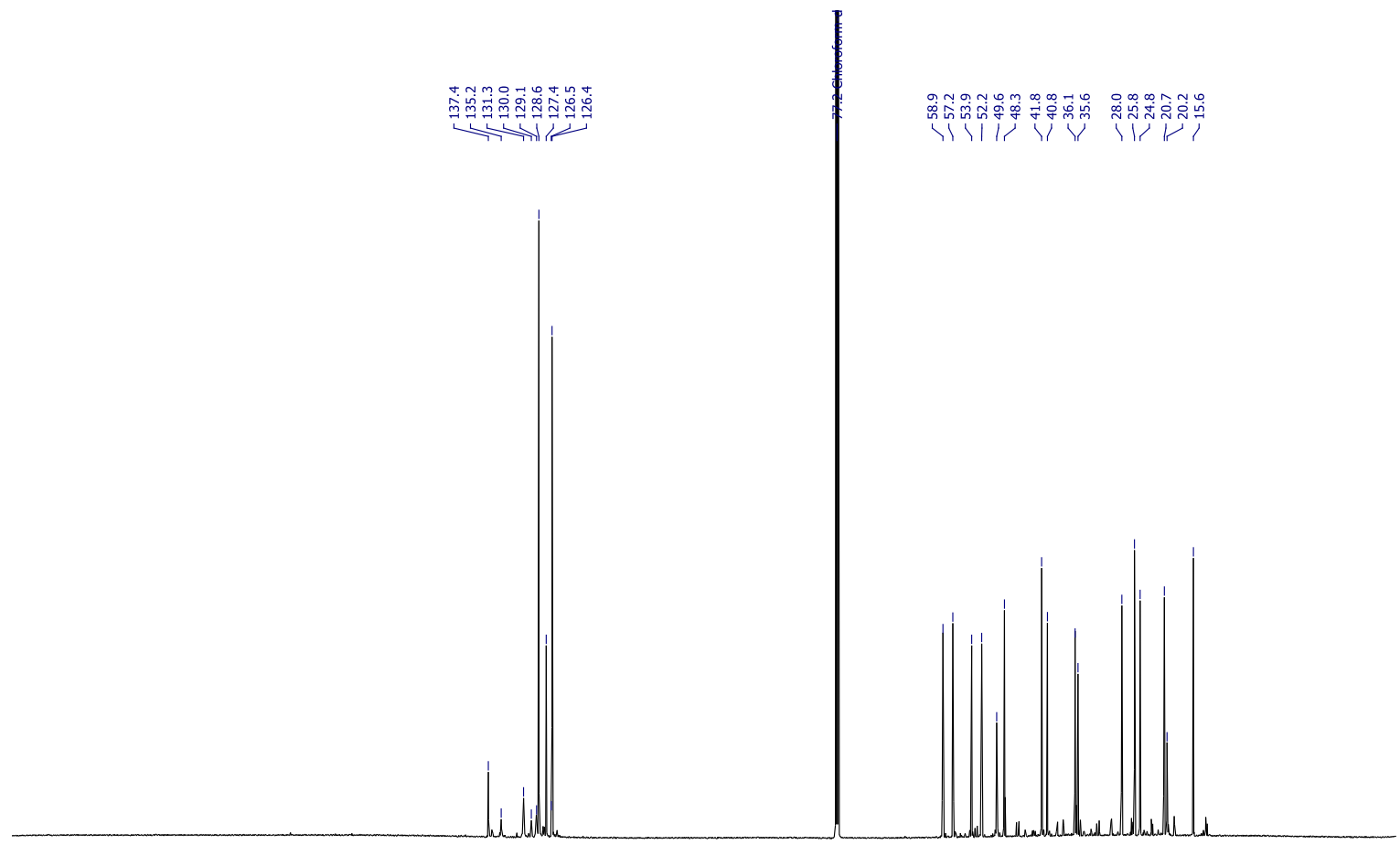

$\begin{array}{lllllllllllllllllllllllllllllll}210 & 200 & 190 & 180 & 170 & 160 & 150 & 140 & 130 & 120 & 110 & 100 & 90 & 80 & 70 & 60 & 50 & 40 & 30 & 20 & 10 & 0 & -10\end{array}$

Figure $S$-126. ${ }^{13} \mathrm{C}$ NMR spectrum of $1 \mathrm{af}\left(\mathrm{CDCl}_{3}, 151 \mathrm{MHz}, 298 \mathrm{~K}\right)$ 

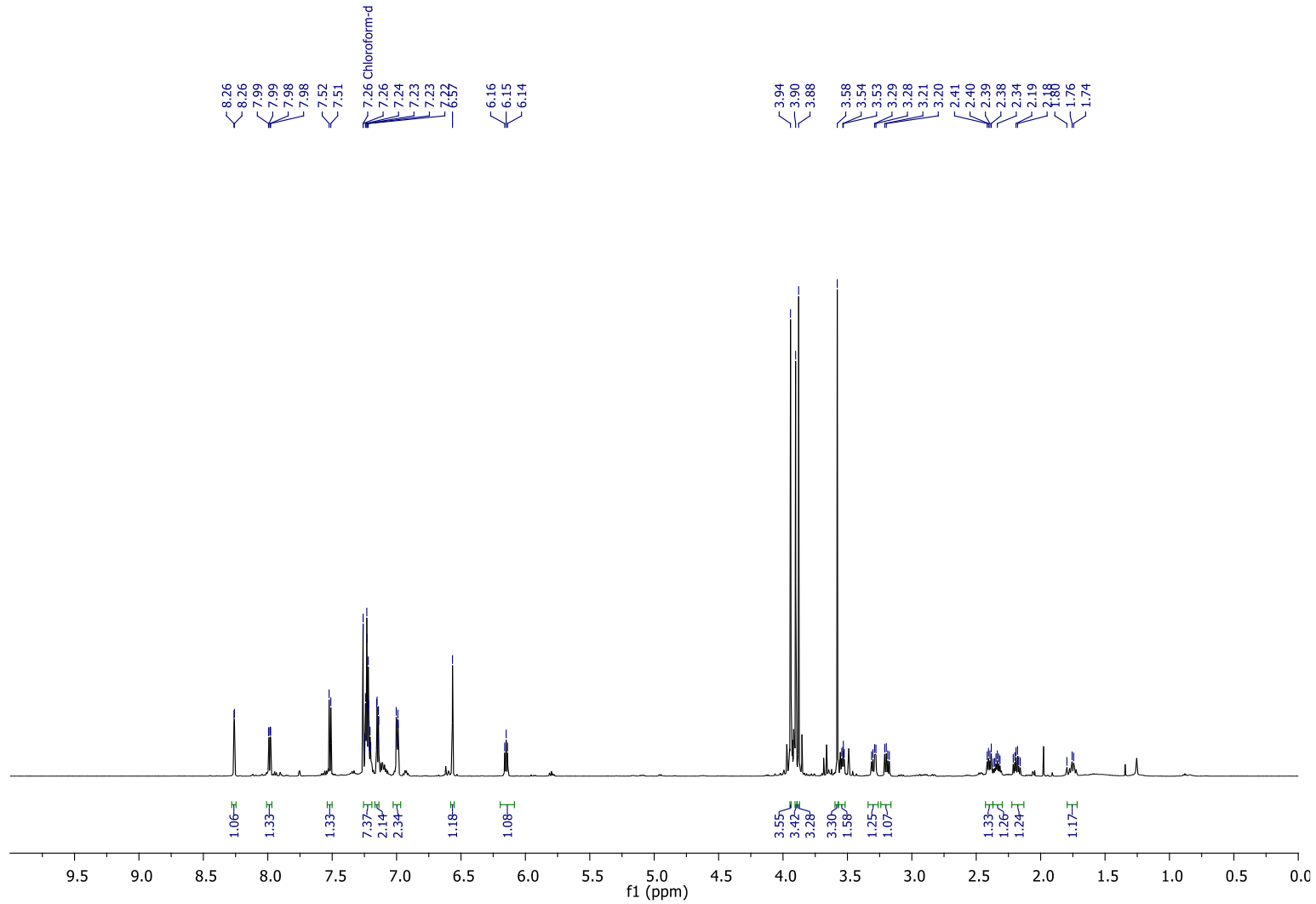

Figure S-127. ${ }^{1} \mathrm{H}$ NMR spectrum of $1 \mathrm{ag}\left(\mathrm{CDCl}_{3}, 600 \mathrm{MHz}, 298 \mathrm{~K}\right)$
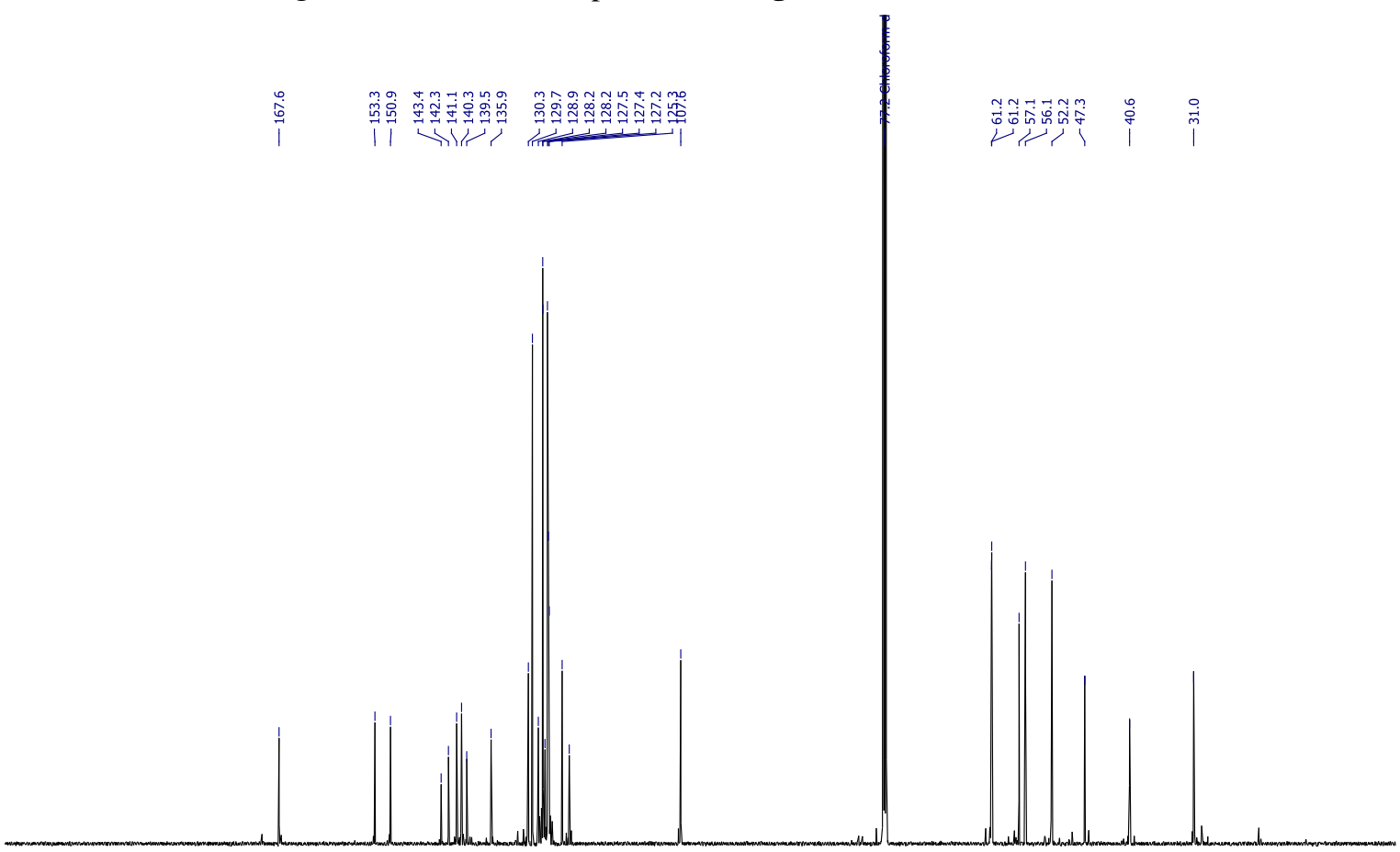

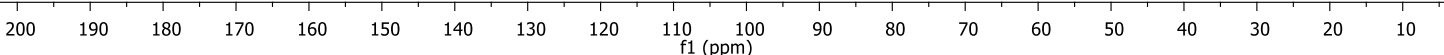

Figure $S$-128. ${ }^{13} \mathrm{C}$ NMR spectrum of $1 \mathrm{ag}\left(\mathrm{CDCl}_{3}, 151 \mathrm{MHz}, 298 \mathrm{~K}\right)$ 


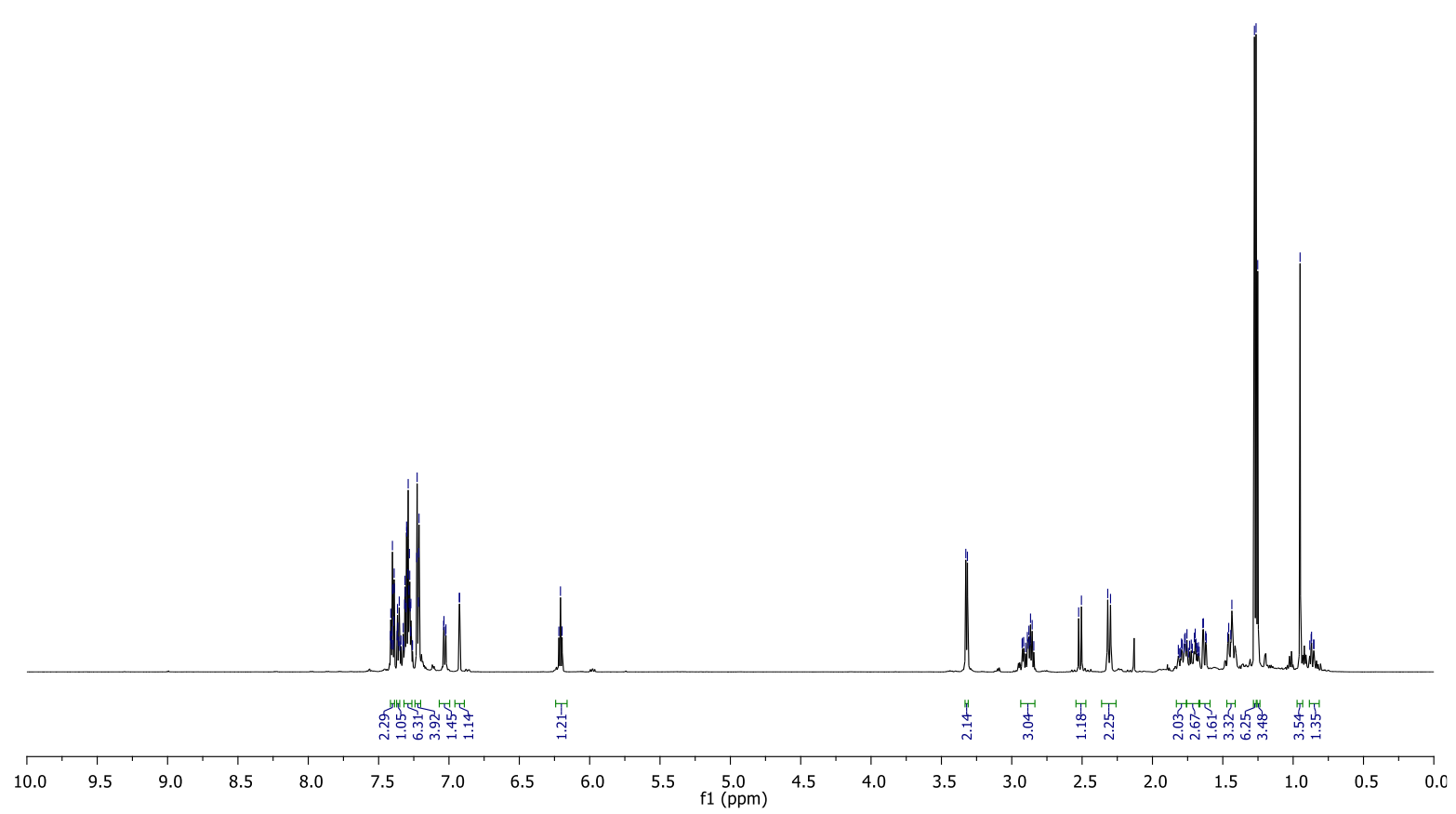

Figure $S$-129. ${ }^{1} \mathrm{H}$ NMR spectrum of 1 ah $\left(\mathrm{CDCl}_{3}, 600 \mathrm{MHz}, 298 \mathrm{~K}\right)$

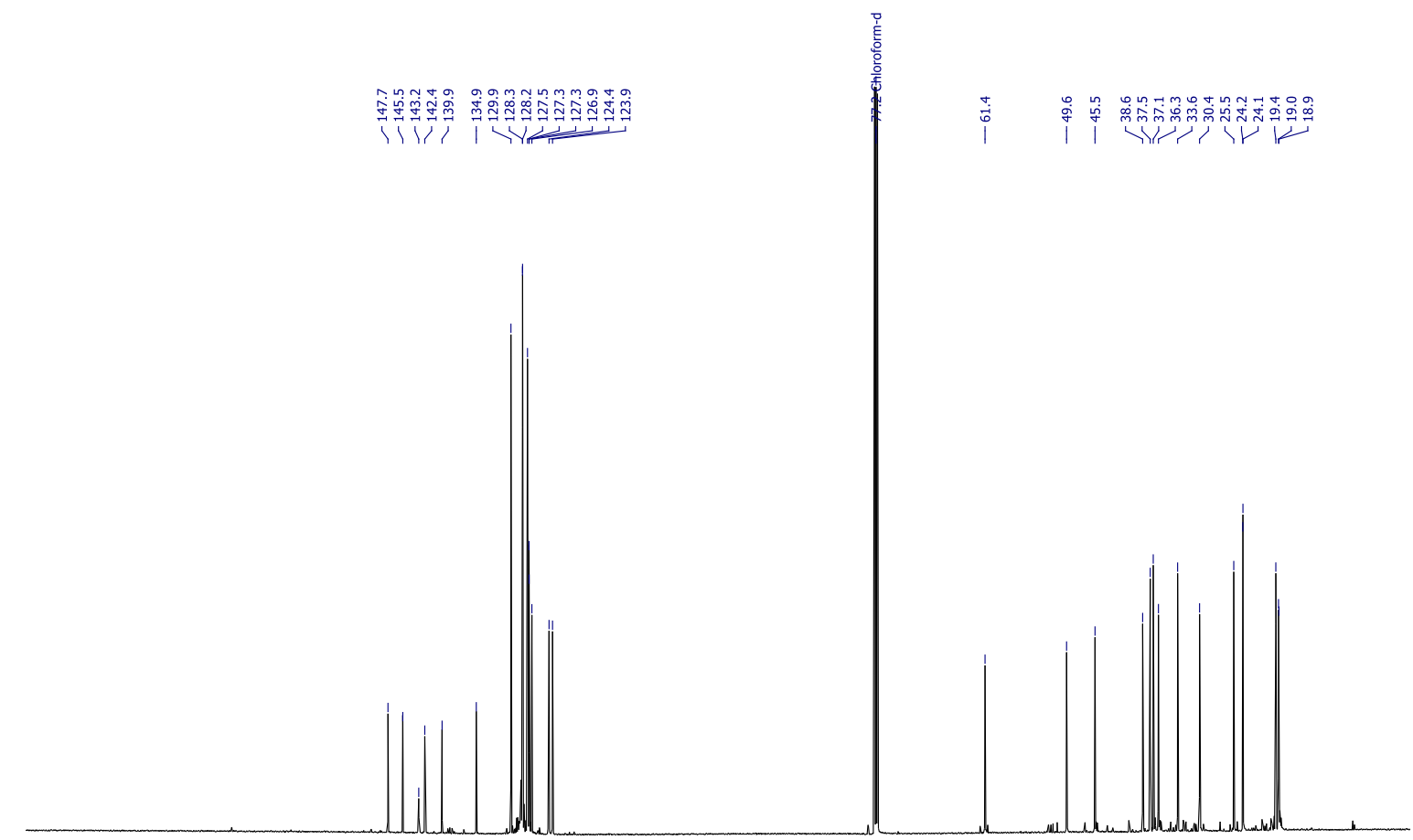

$200 \quad 190 \quad 180 \quad 170$

160150

$150 \quad 140$

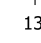

$\begin{array}{llc}120 & 110 & 100 \\ & & \mathrm{f} 1(\mathrm{ppm})\end{array}$
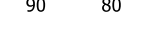

Figure S-130. ${ }^{13} \mathrm{C}$ NMR spectrum of 1 ah $\left(\mathrm{CDCl}_{3}, 151 \mathrm{MHz}, 298 \mathrm{~K}\right)$ 


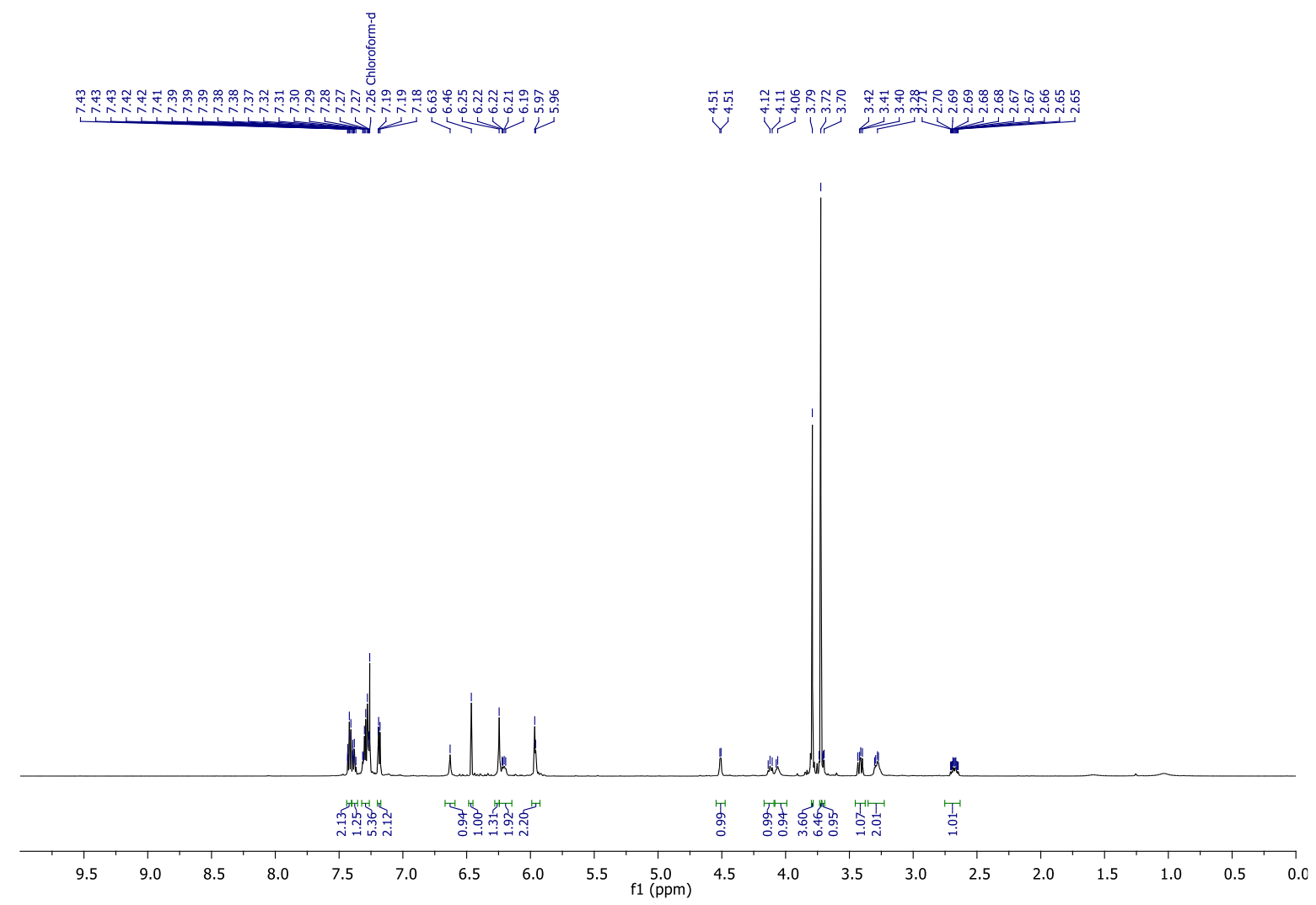

Figure S-131. ${ }^{1} \mathrm{H}$ NMR spectrum of $\mathbf{1 a i}\left(\mathrm{CDCl}_{3}, 600 \mathrm{MHz}, 298 \mathrm{~K}\right)$
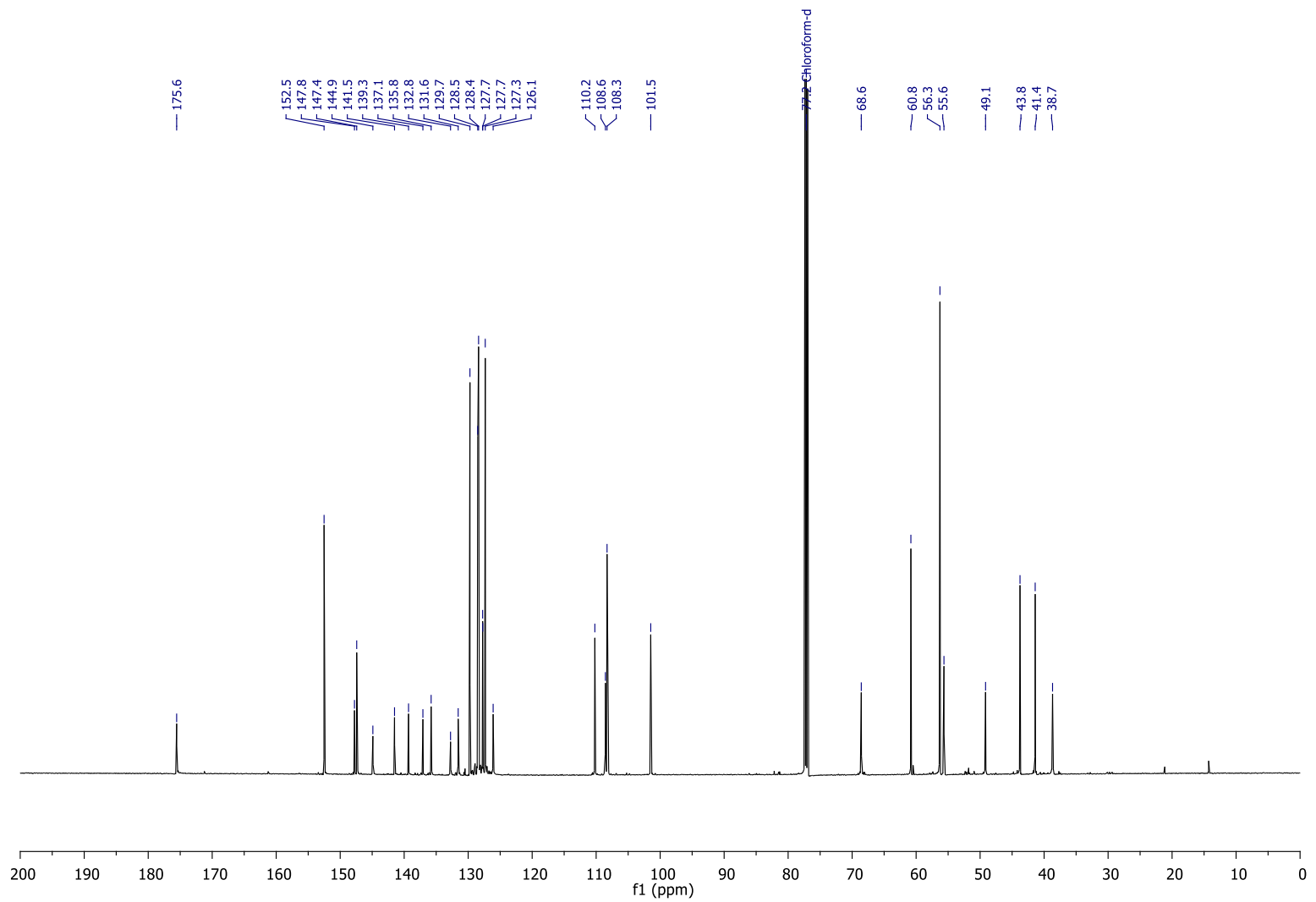

Figure S-132. ${ }^{13} \mathrm{C}$ NMR spectrum of 1 ai $\left(\mathrm{CDCl}_{3}, 151 \mathrm{MHz}, 298 \mathrm{~K}\right)$ 


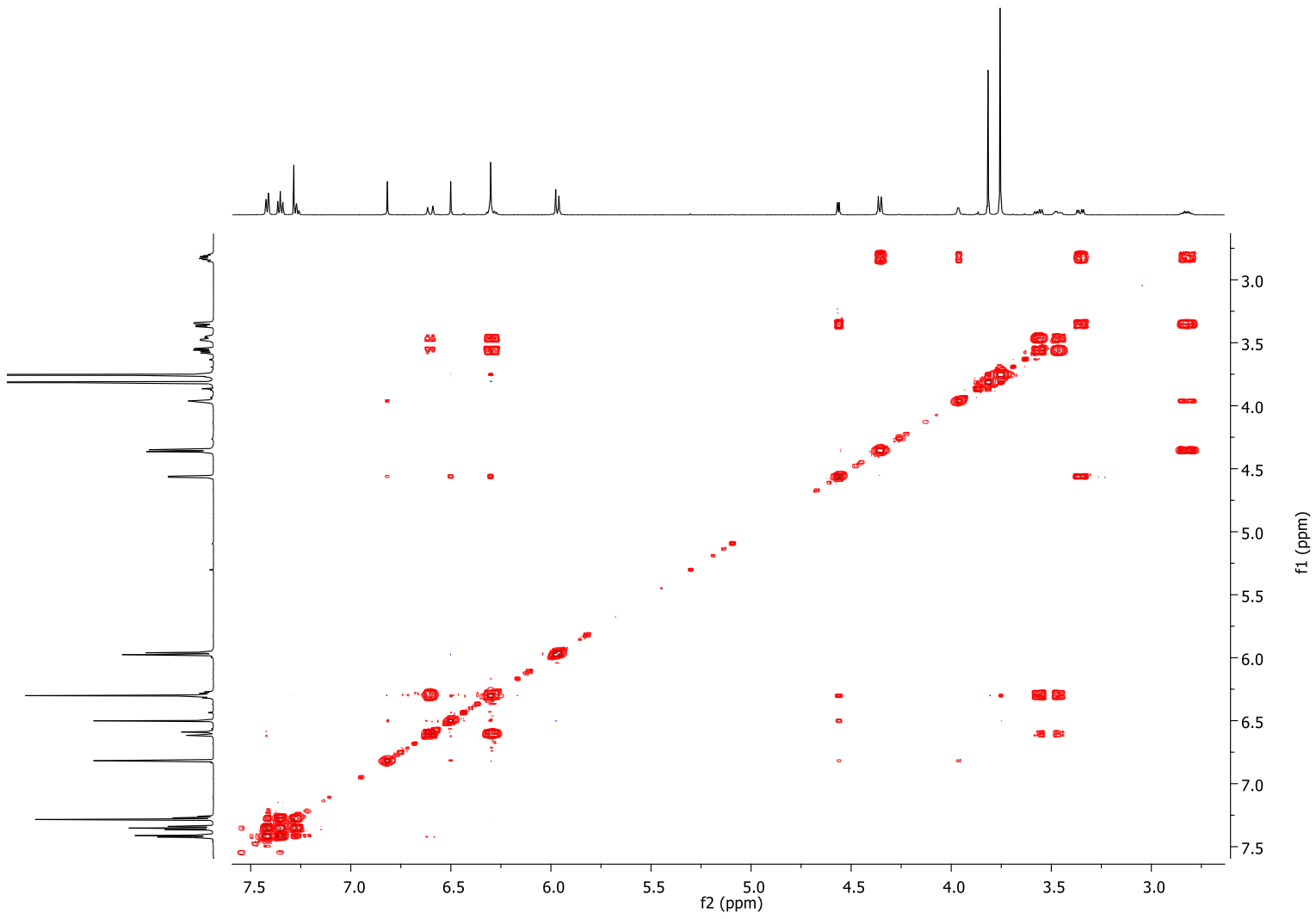

Figure $\boldsymbol{S - 1 3 3}$. Full COSY spectrum of $\boldsymbol{S - 3 5}\left(\mathrm{CDCl}_{3}, 600 \mathrm{MHz}, 298 \mathrm{~K}\right)$

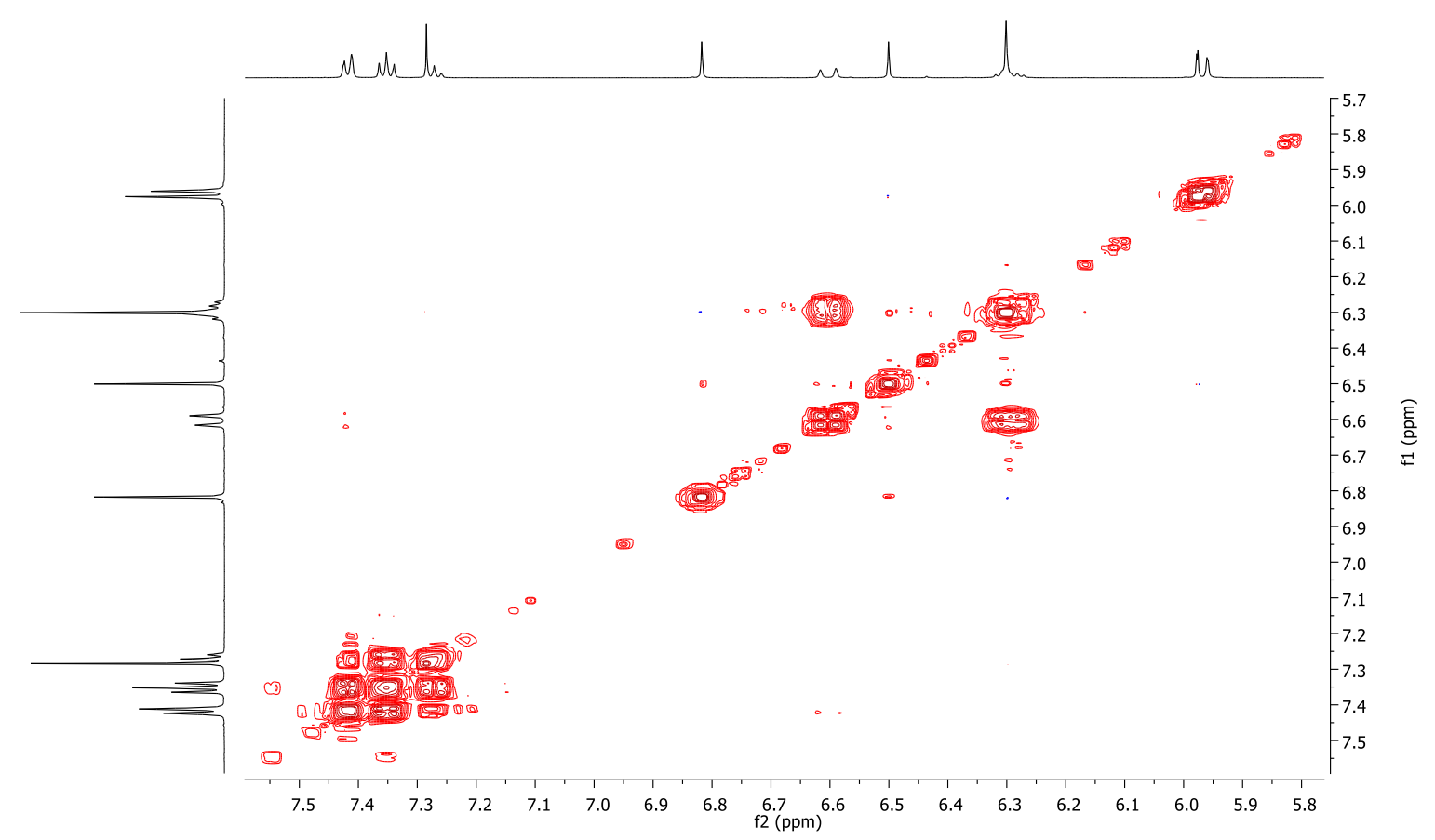

Figure $\boldsymbol{S}$-134. Zoomed-in Downfield Region of COSY spectrum of $\boldsymbol{S}-\mathbf{3 5}\left(\mathrm{CDCl}_{3}, 600 \mathrm{MHz}, 298 \mathrm{~K}\right)$ 


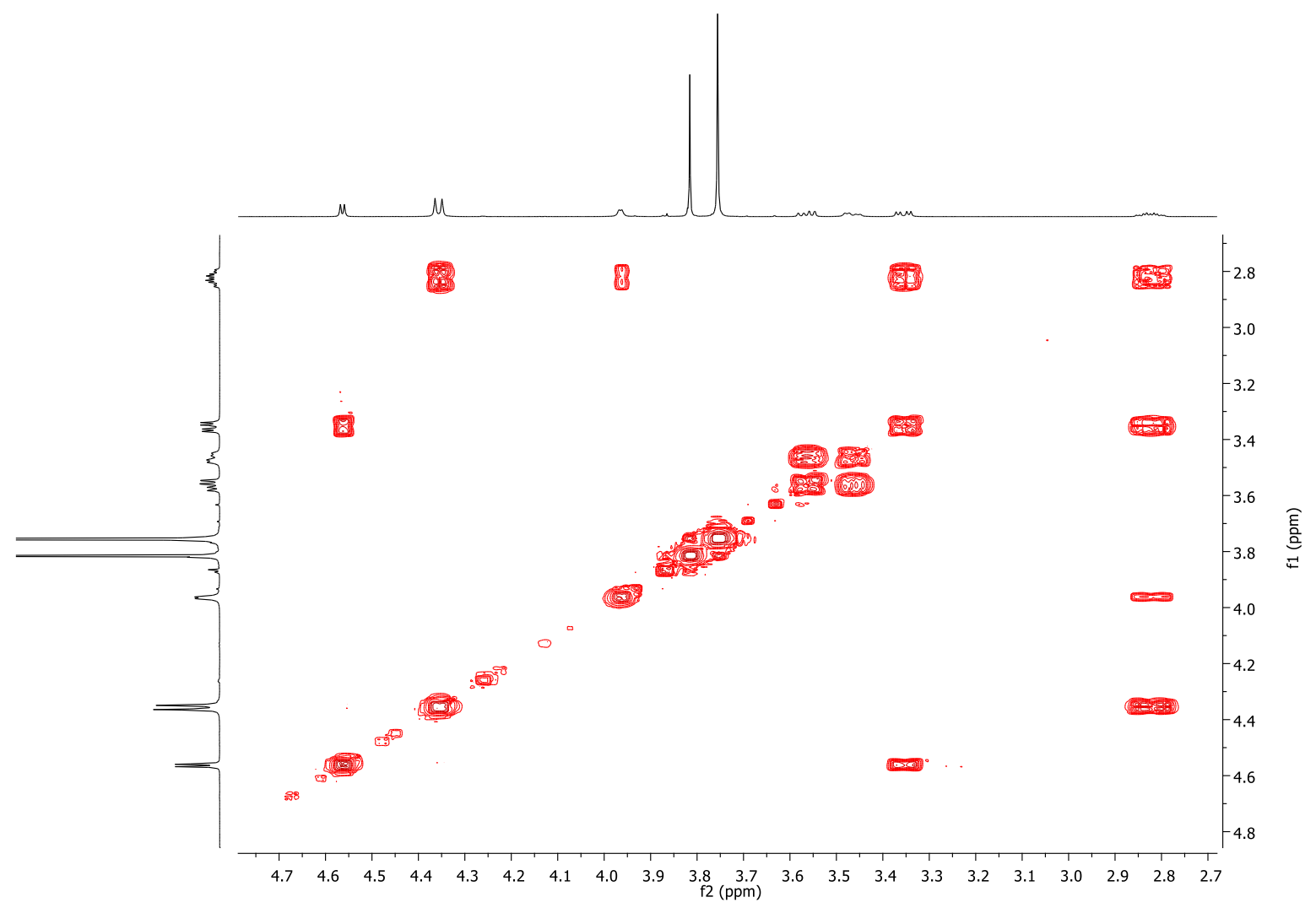

Figure $\boldsymbol{S - 1 3 5}$. Zoomed-in Upfield Region of COSY spectrum of $\boldsymbol{S - 3 5}\left(\mathrm{CDCl}_{3}, 600 \mathrm{MHz}, 298 \mathrm{~K}\right)$

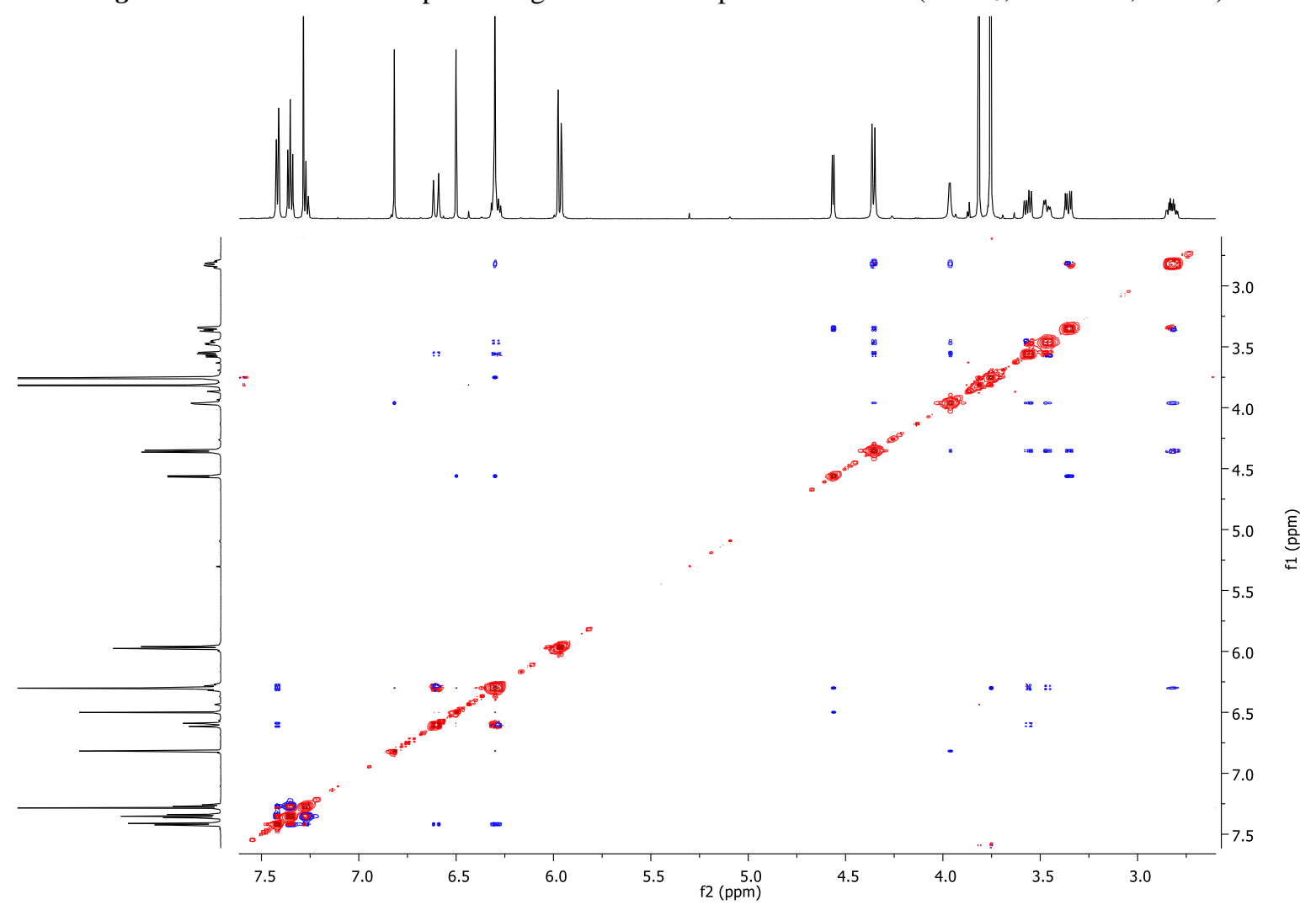

Figure $\boldsymbol{S}$-136. Full NOESY spectrum of $\boldsymbol{S}-\mathbf{3 5}\left(\mathrm{CDCl}_{3}, 600 \mathrm{MHz}, 298 \mathrm{~K}\right)$ 


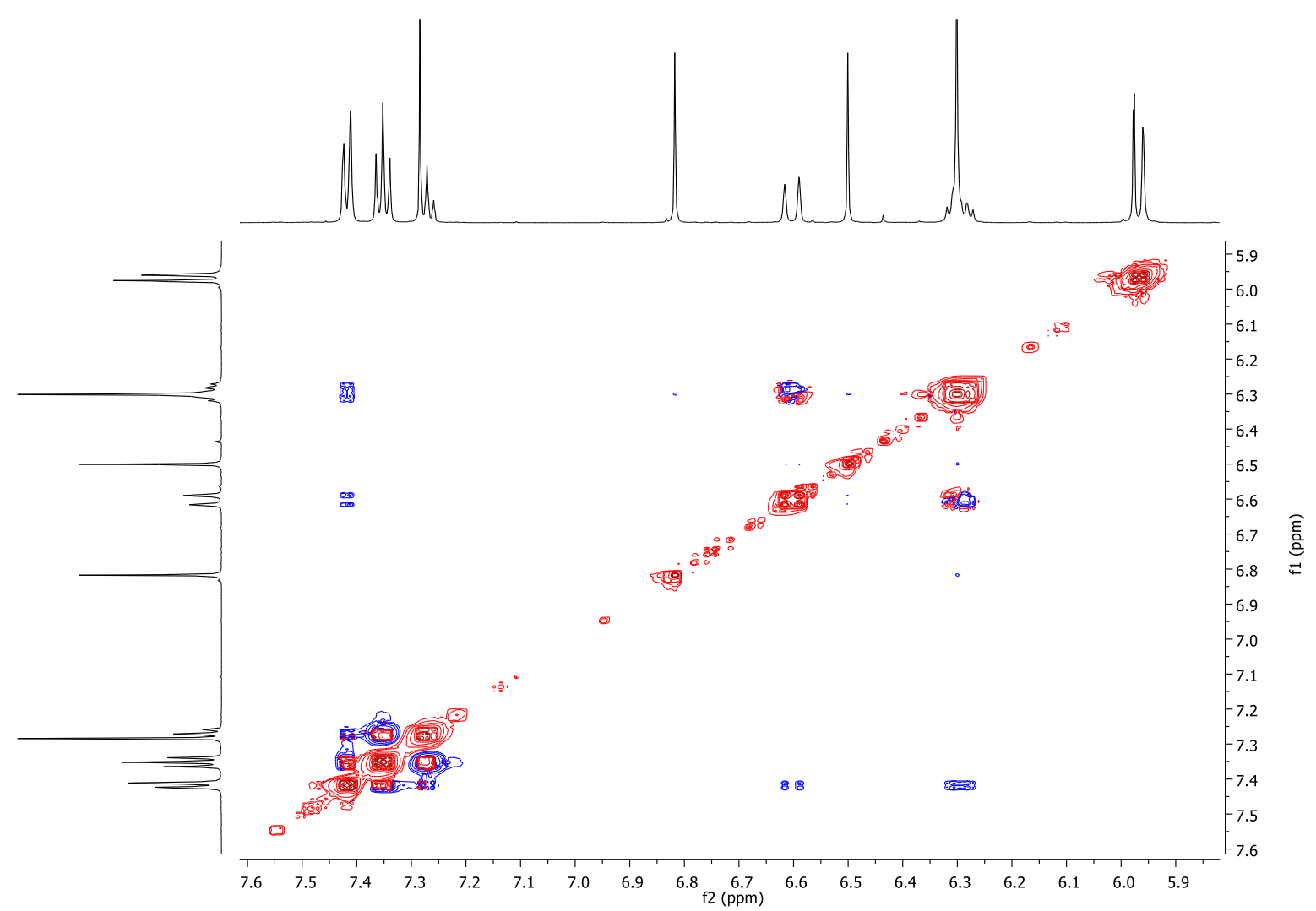

Figure S-137. Zoomed-in Downfield Region of NOESY spectrum of $\boldsymbol{S - 3 5}\left(\mathrm{CDCl}_{3}, 600 \mathrm{MHz}, 298 \mathrm{~K}\right)$

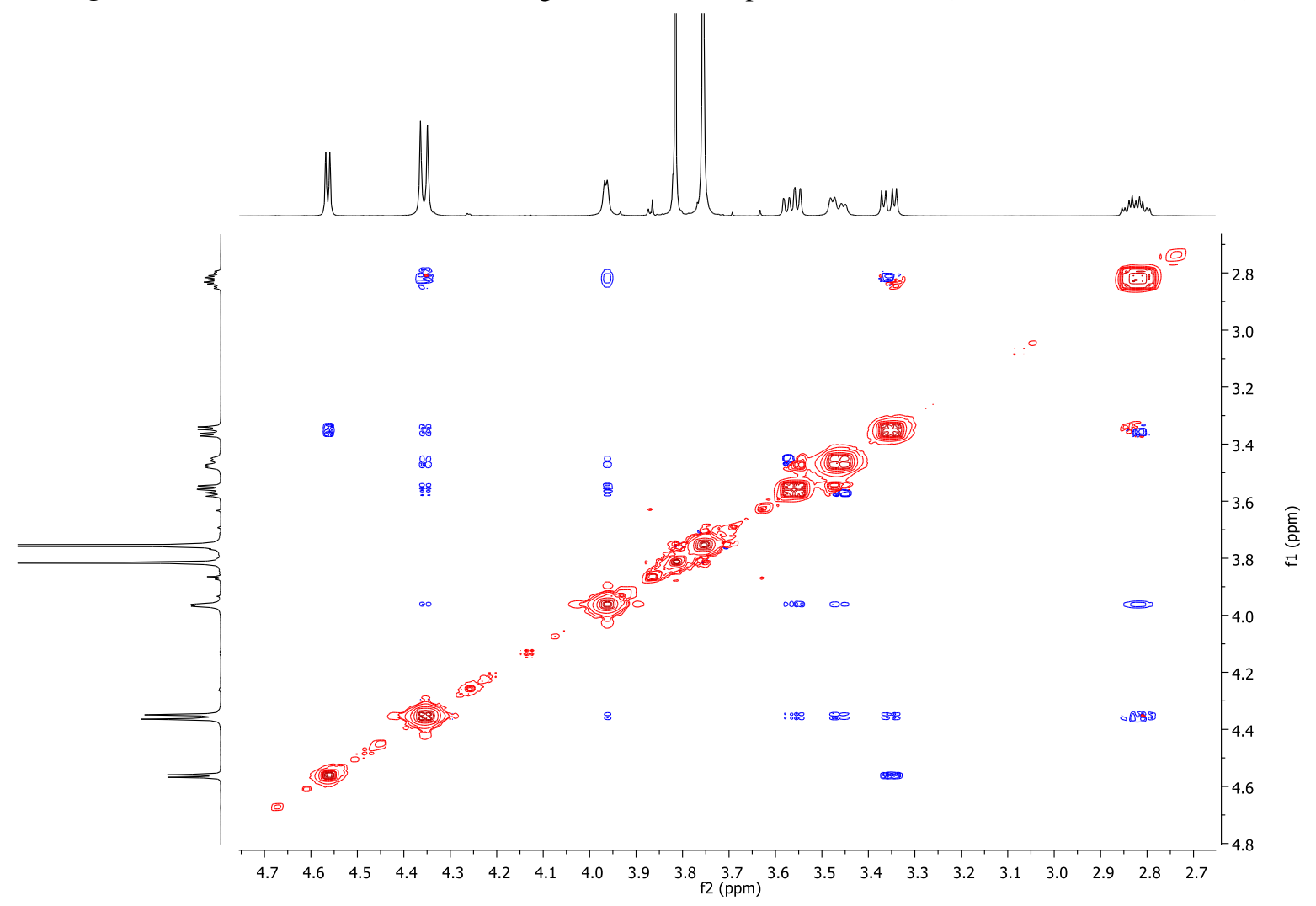

Figure $\boldsymbol{S - 1 3 8}$. Zoomed-in Upfield Region of NOESY spectrum of $\boldsymbol{S - 3 5}\left(\mathrm{CDCl}_{3}, 600 \mathrm{MHz}, 298 \mathrm{~K}\right)$ 

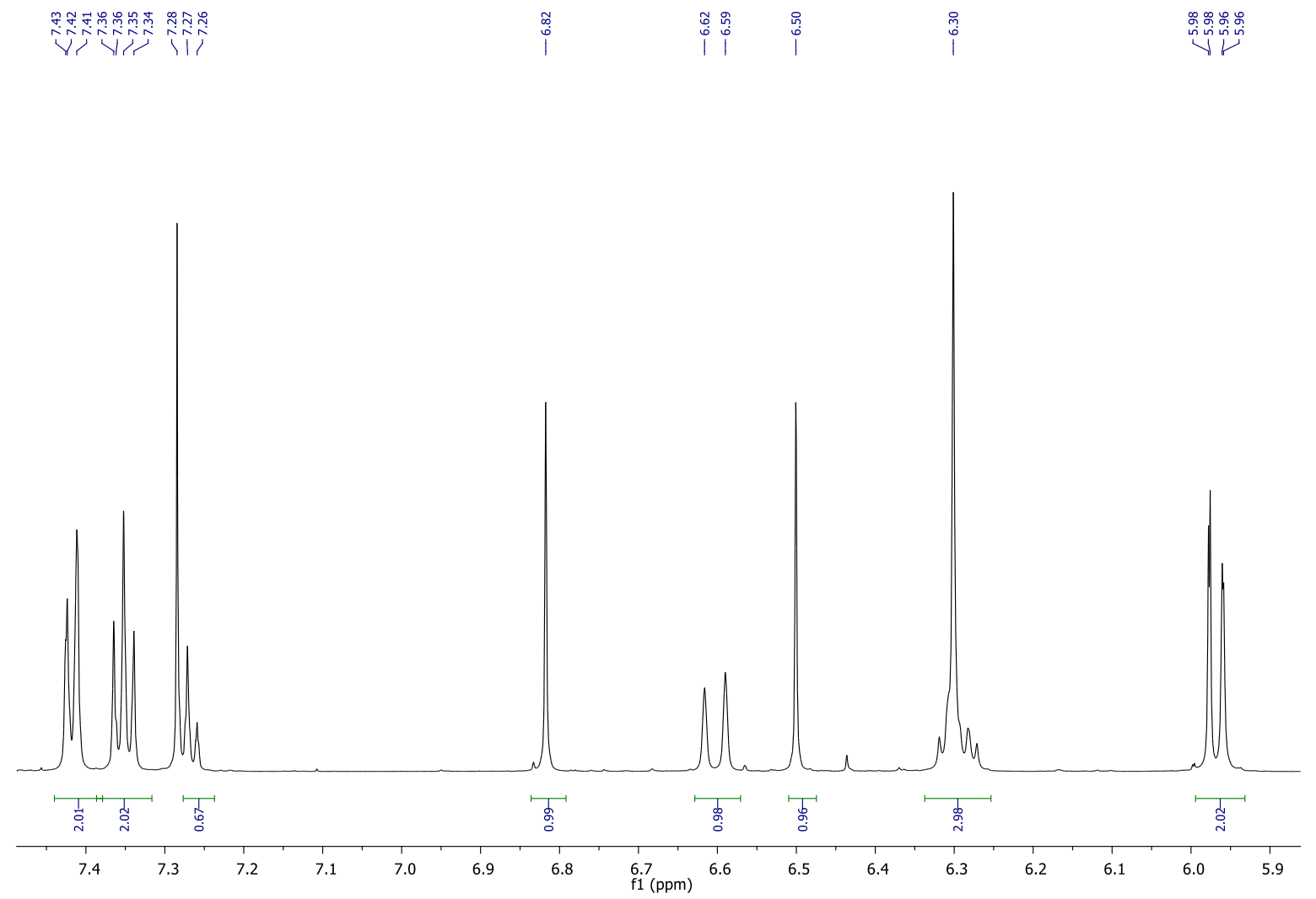

Figure $\boldsymbol{S}$-139. Zoomed-in ${ }^{1} \mathrm{H}$ spectrum of $\boldsymbol{S}$-35 showing key coupling constants $\left(\mathrm{CDCl}_{3}, 600 \mathrm{MHz}, 298 \mathrm{~K}\right)$

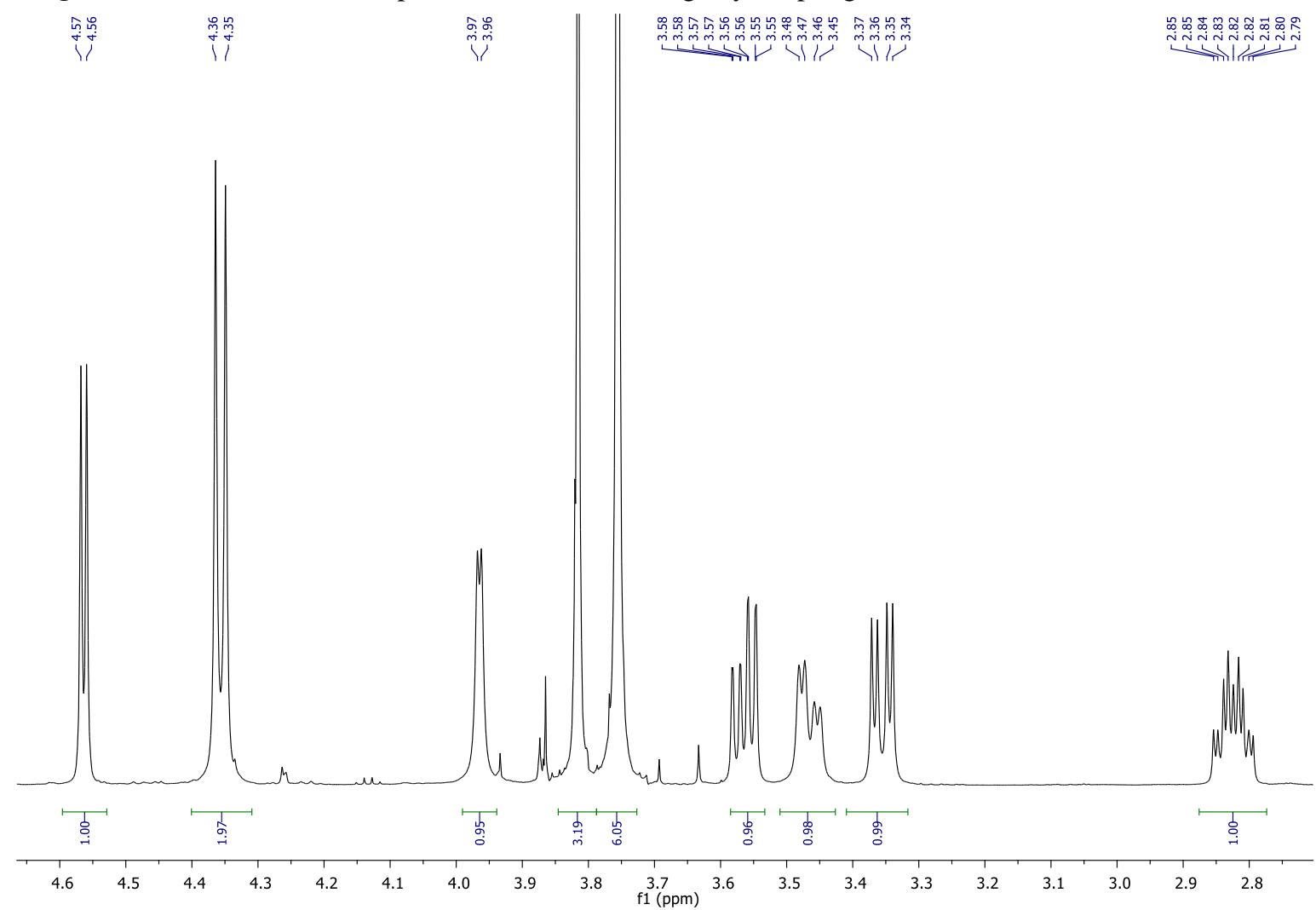

Figure $S$-140. Zoomed-in ${ }^{1} \mathrm{H}$ spectrum of $\boldsymbol{S}$-35 showing key coupling constants $\left(\mathrm{CDCl}_{3}, 600 \mathrm{MHz}, 298 \mathrm{~K}\right)$ 


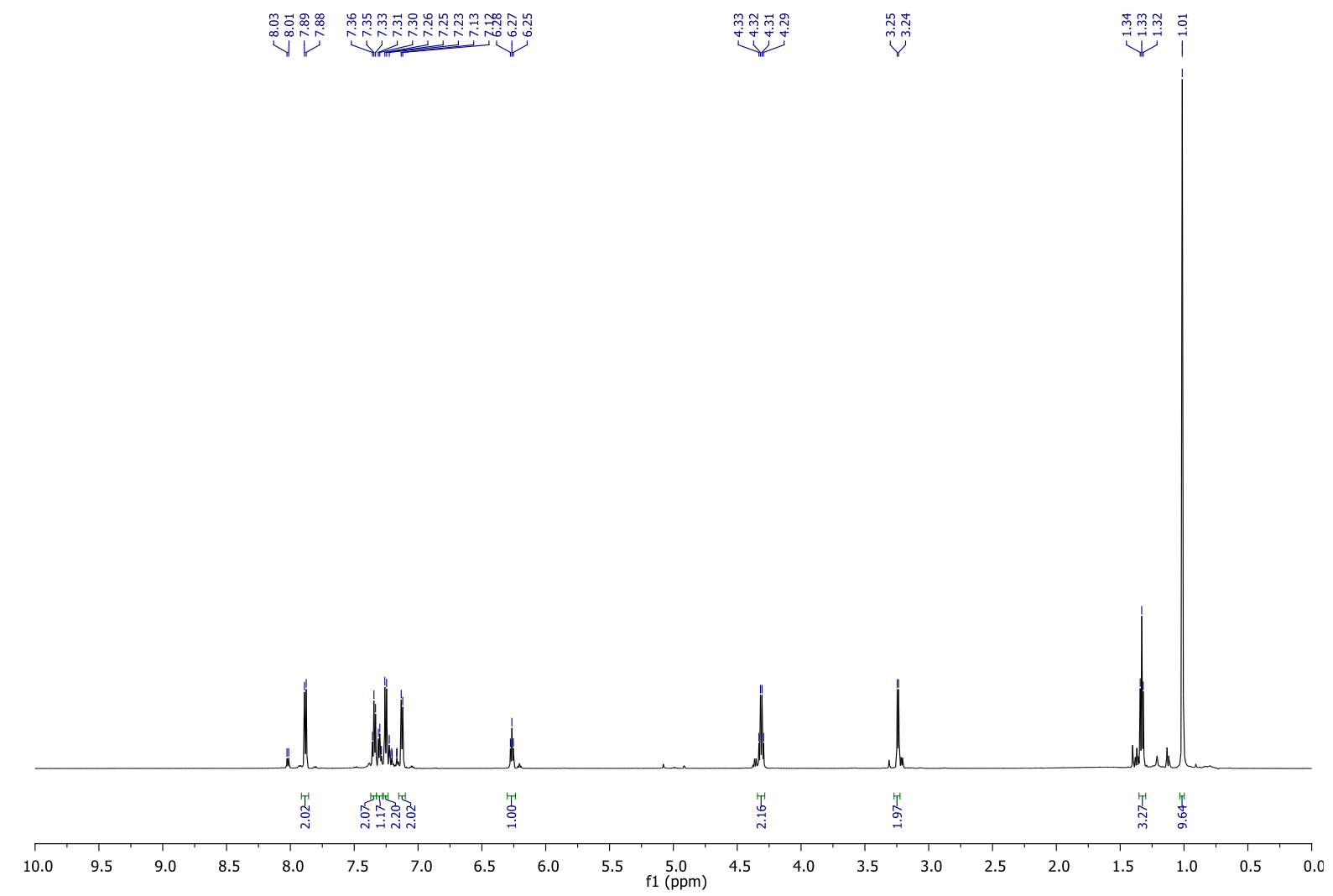

Figure $S$-141. ${ }^{1} \mathrm{H}$ NMR spectrum of $\mathbf{2 a}\left(\mathrm{CDCl}_{3}, 600 \mathrm{MHz}, 298 \mathrm{~K}\right)$

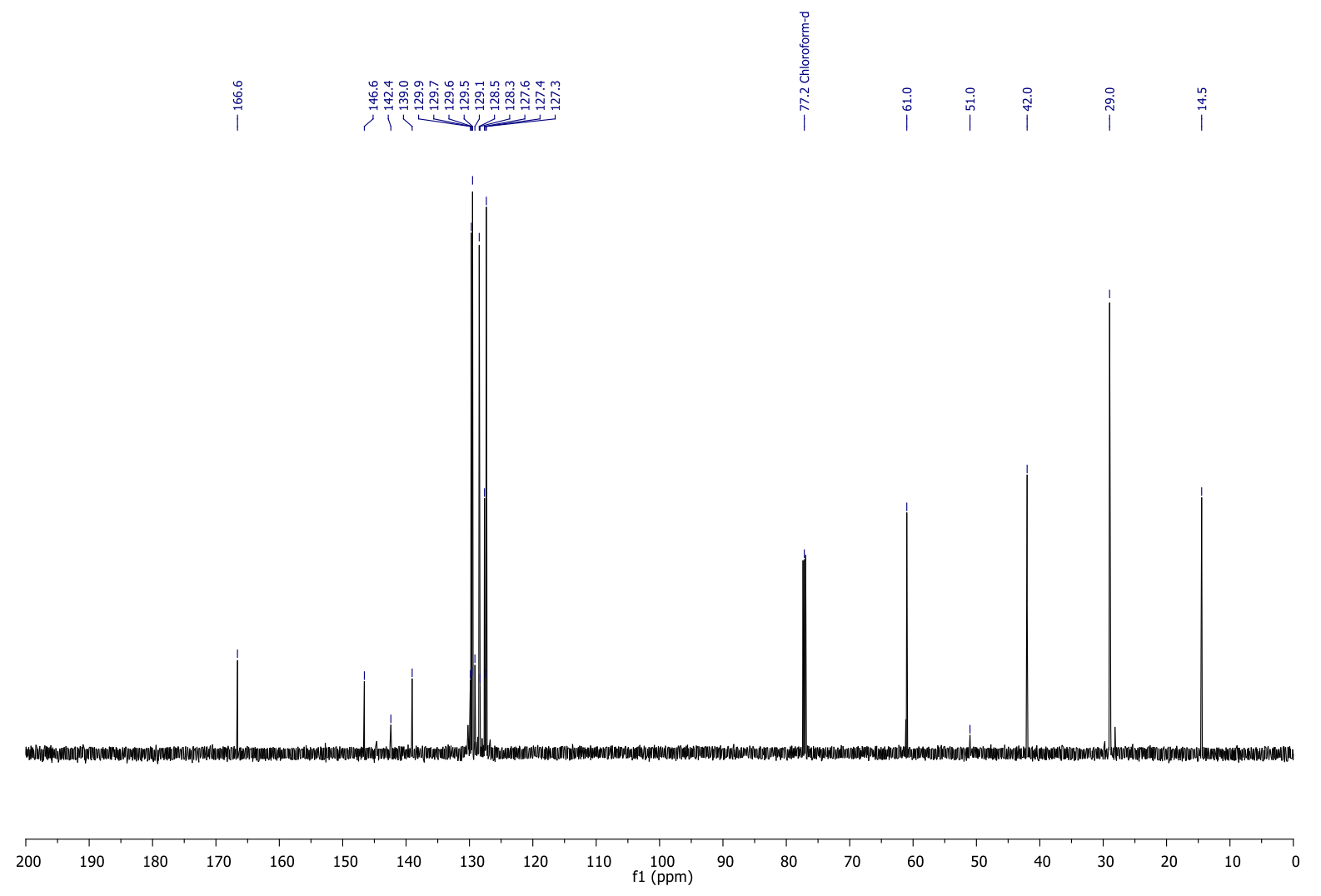

Figure S-142. ${ }^{13} \mathrm{C}$ NMR spectrum of $\mathbf{2 a}\left(\mathrm{CDCl}_{3}, 151 \mathrm{MHz}, 298 \mathrm{~K}\right)$ 


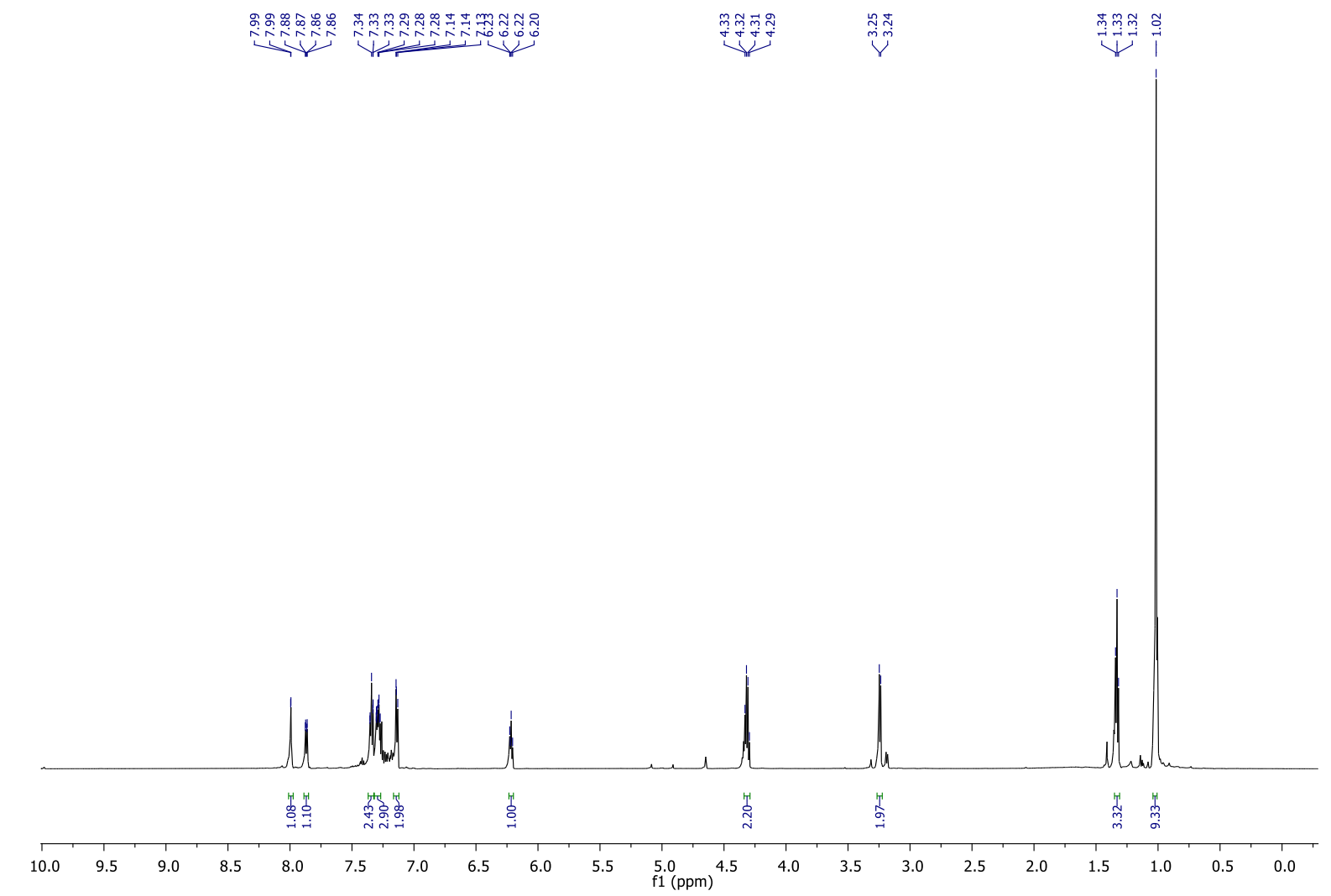

Figure S-143. ${ }^{1} \mathrm{H}$ NMR spectrum of $\mathbf{2 b}\left(\mathrm{CDCl}_{3}, 600 \mathrm{MHz}, 298 \mathrm{~K}\right)$

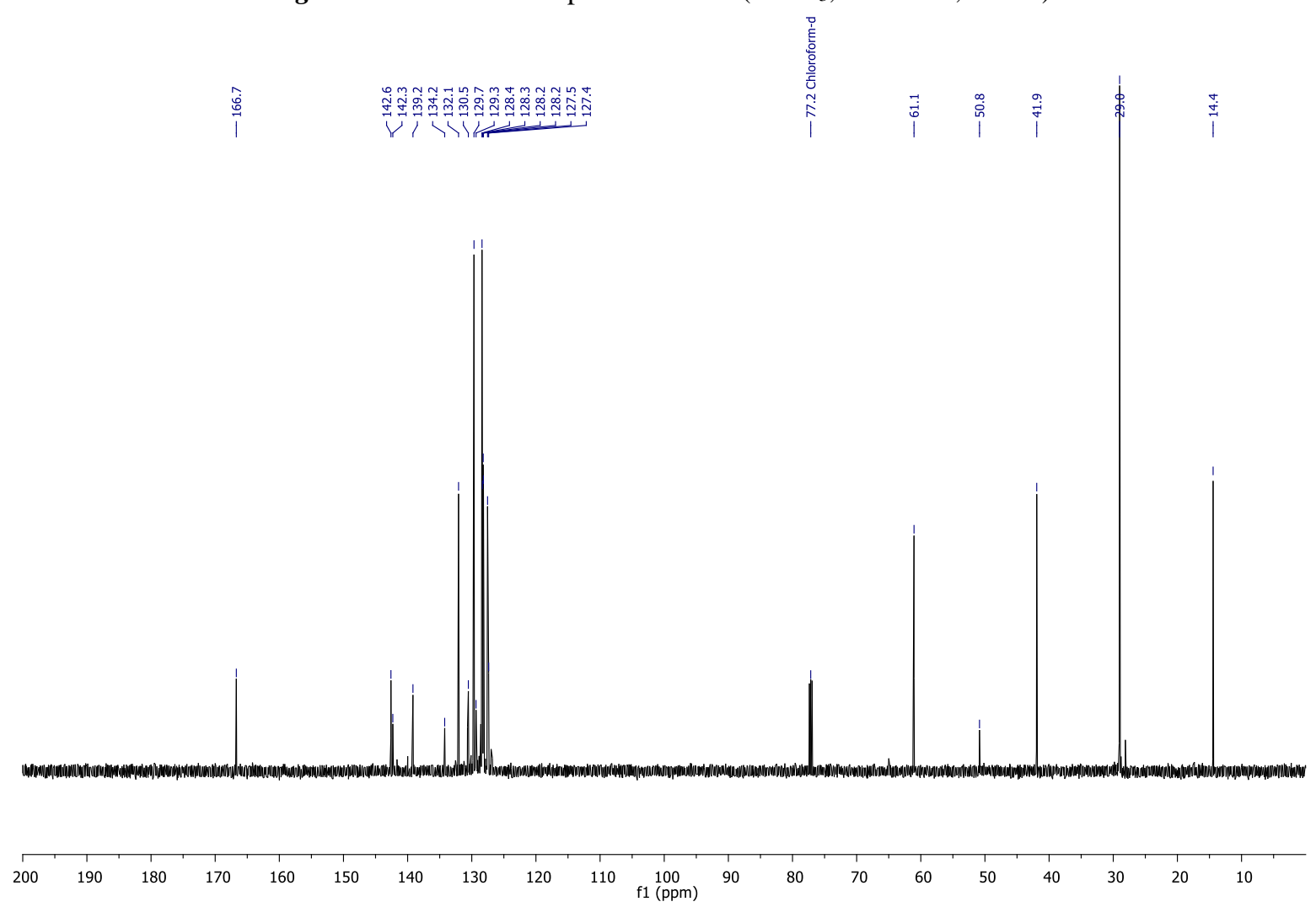

Figure S-144. ${ }^{13} \mathrm{C}$ NMR spectrum of $\mathbf{2 b}\left(\mathrm{CDCl}_{3}, 151 \mathrm{MHz}, 298 \mathrm{~K}\right)$ 


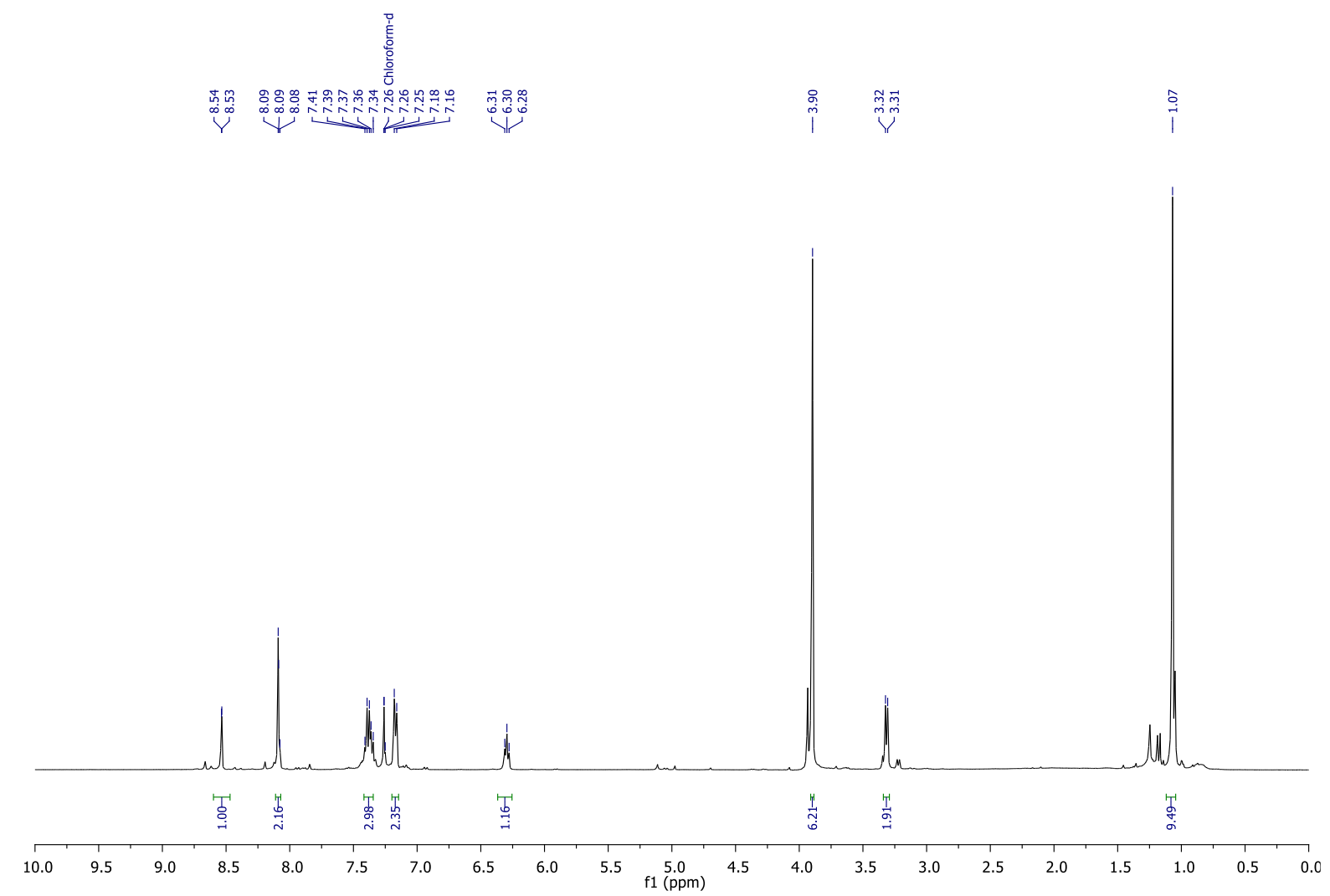

Figure S-145. ${ }^{1} \mathrm{H}$ NMR spectrum of $2 c\left(\mathrm{CDCl}_{3}, 600 \mathrm{MHz}, 298 \mathrm{~K}\right)$
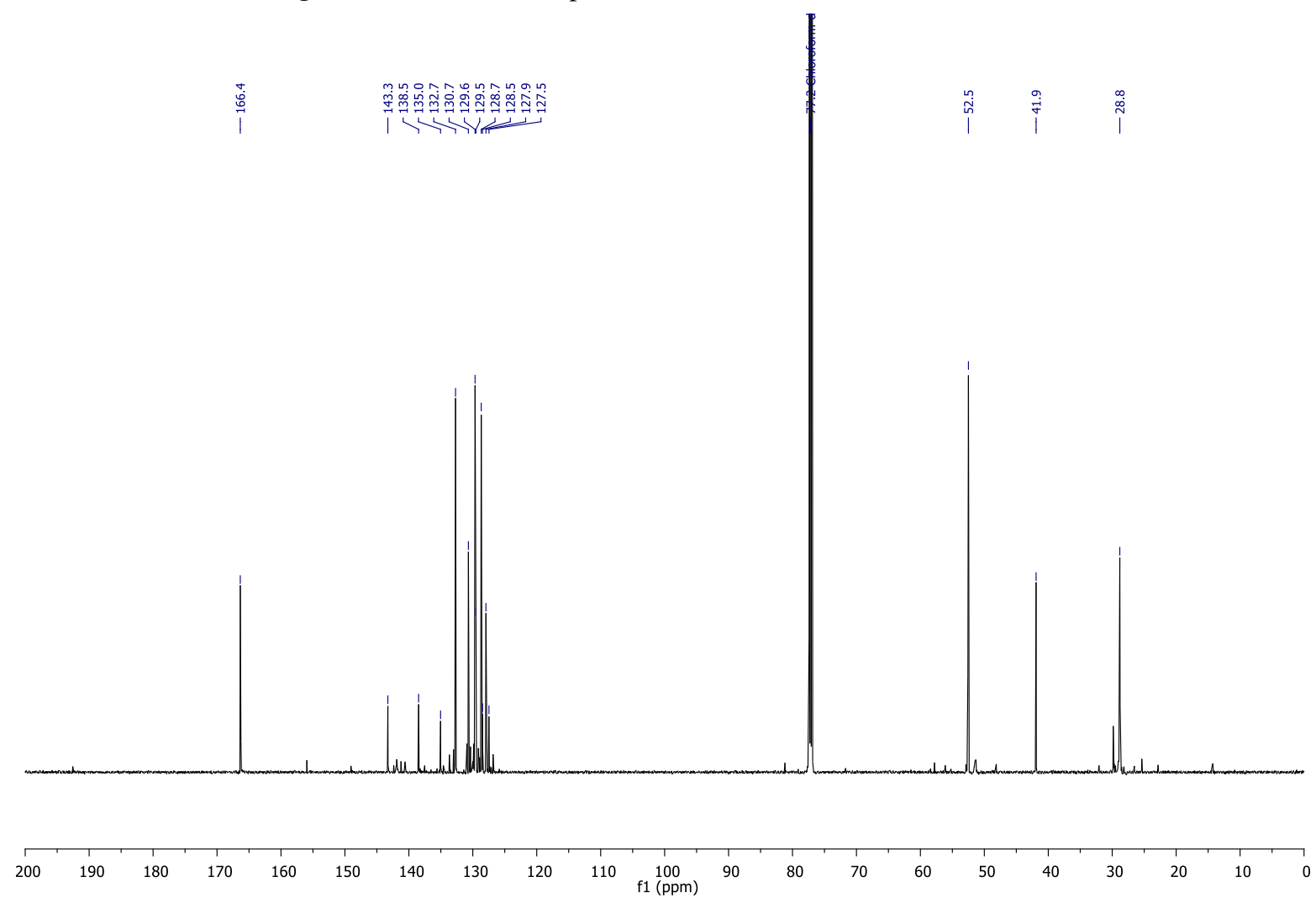

Figure $\mathbf{S}$-146. ${ }^{13} \mathrm{C}$ NMR spectrum of $2 \mathrm{c}\left(\mathrm{CDCl}_{3}, 151 \mathrm{MHz}, 298 \mathrm{~K}\right)$ 


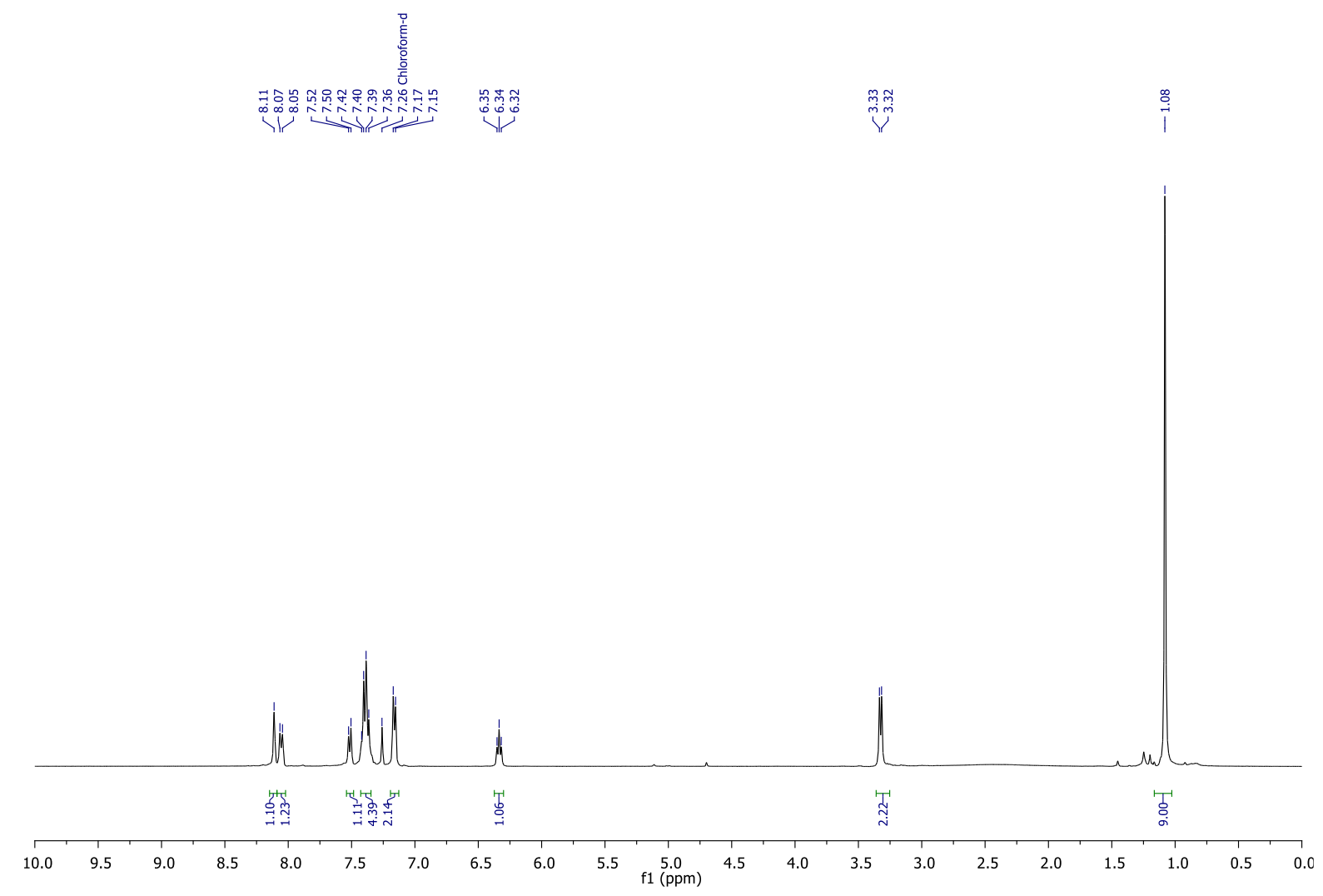

Figure S-147. ${ }^{1} \mathrm{H}$ NMR spectrum of $\mathbf{2 d}\left(\mathrm{CDCl}_{3}, 600 \mathrm{MHz}, 298 \mathrm{~K}\right)$
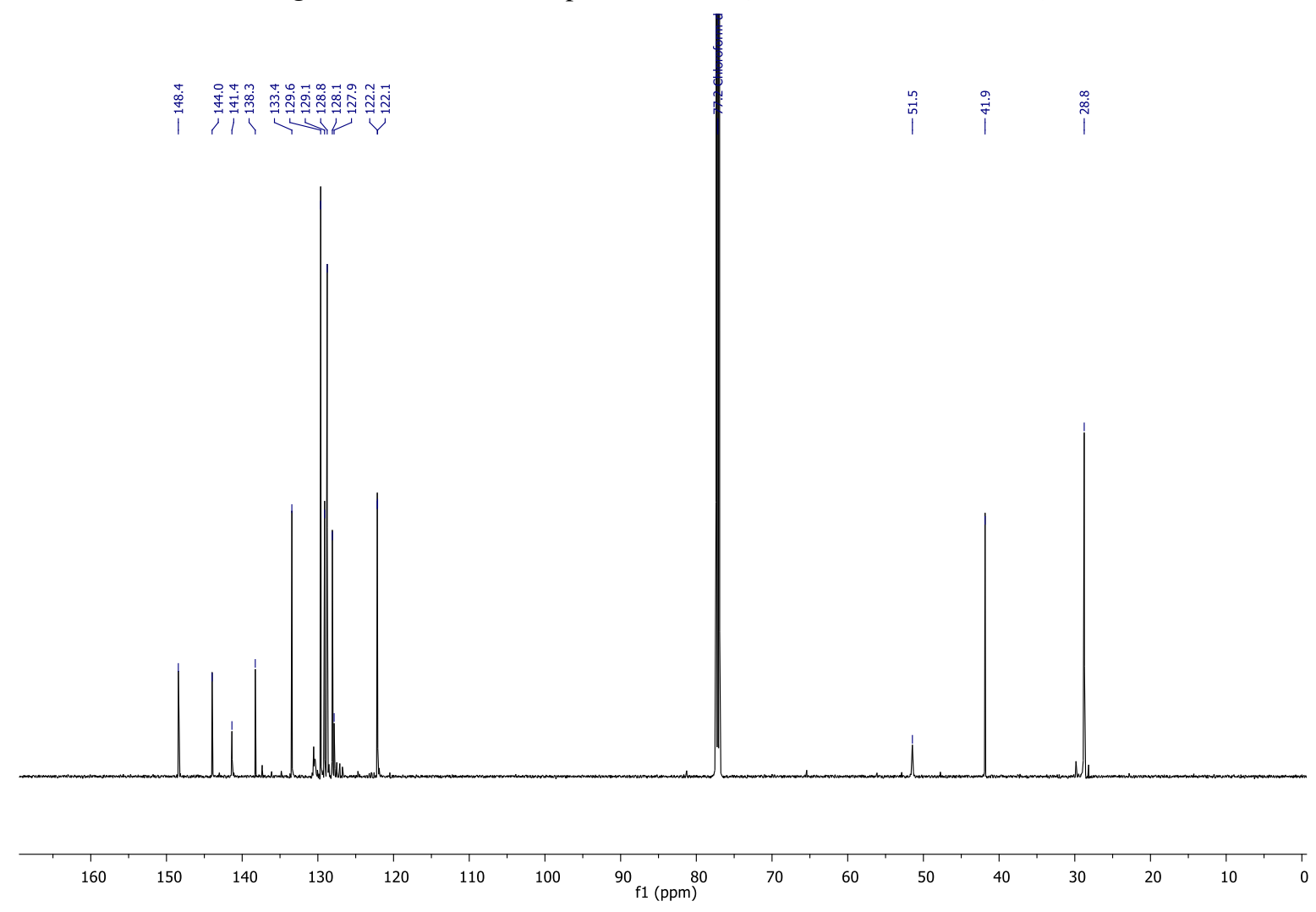

Figure $\mathbf{S}$-148. ${ }^{13} \mathrm{C}$ NMR spectrum of $\mathbf{2 d}\left(\mathrm{CDCl}_{3}, 151 \mathrm{MHz}, 298 \mathrm{~K}\right)$ 

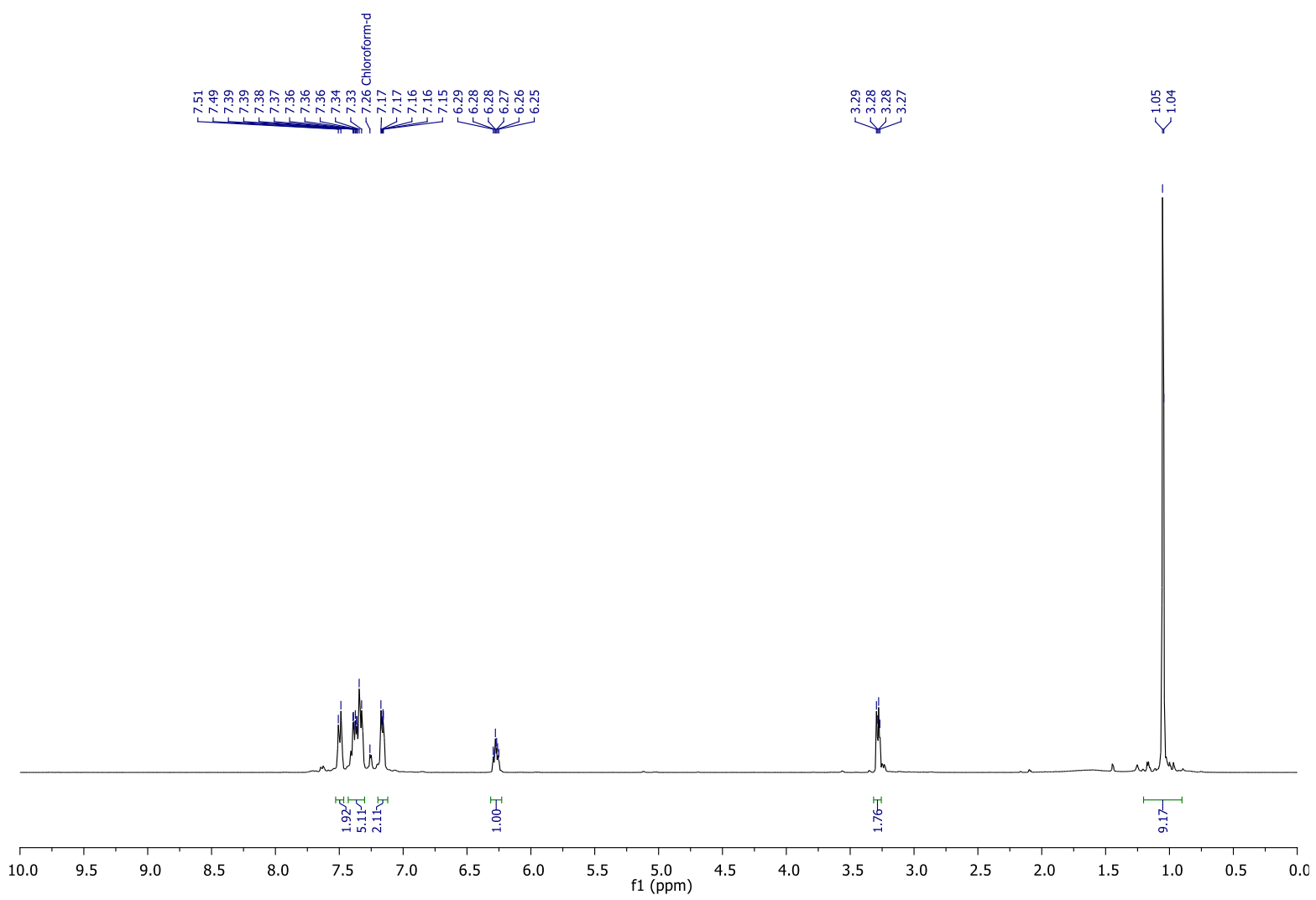

Figure S-149. ${ }^{1} \mathrm{H}$ NMR spectrum of $2 \mathrm{e}\left(\mathrm{CDCl}_{3}, 600 \mathrm{MHz}, 298 \mathrm{~K}\right)$
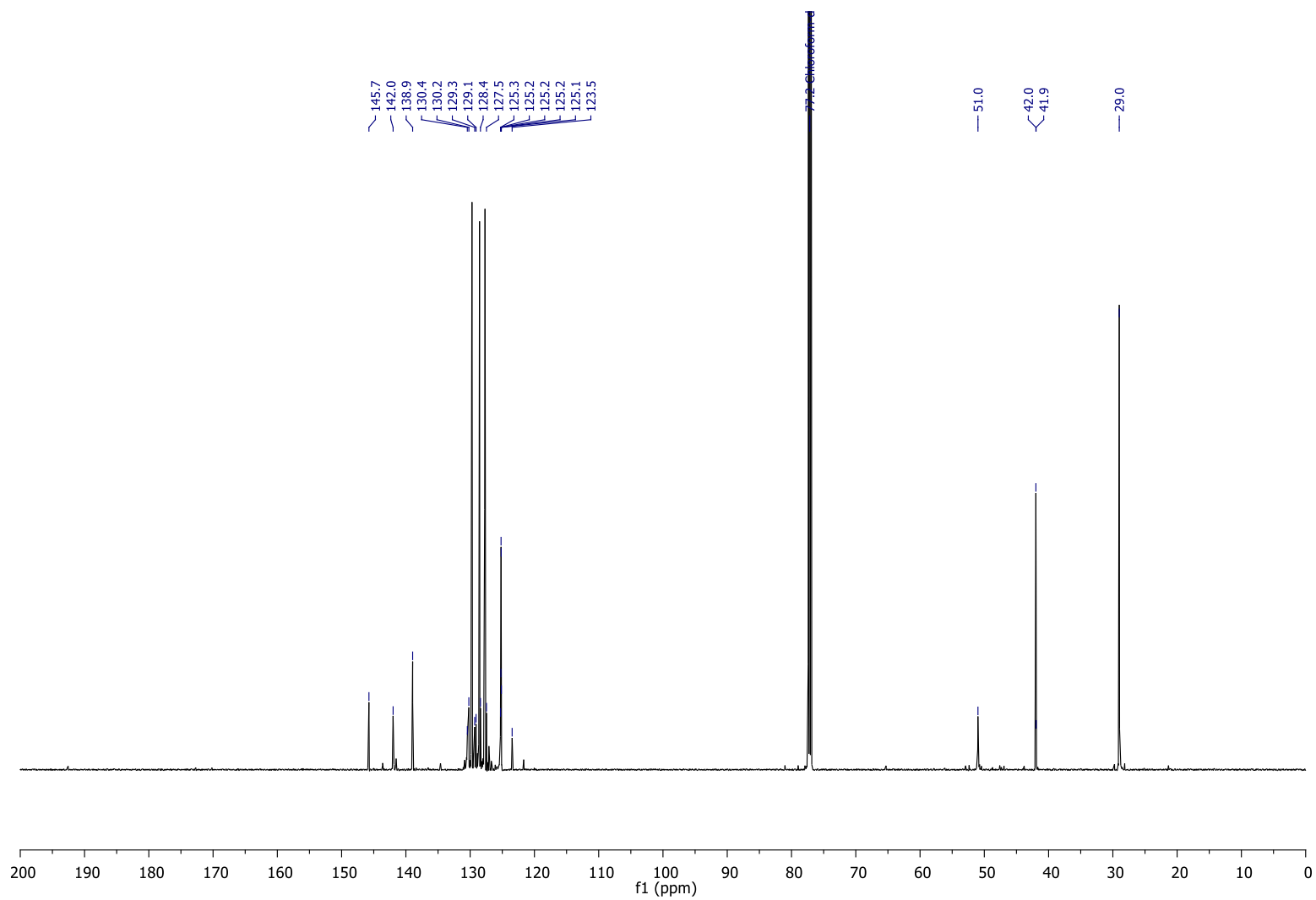

Figure S-150. ${ }^{13} \mathrm{C}$ NMR spectrum of $2 \mathrm{e}\left(\mathrm{CDCl}_{3}, 151 \mathrm{MHz}, 298 \mathrm{~K}\right)$ 


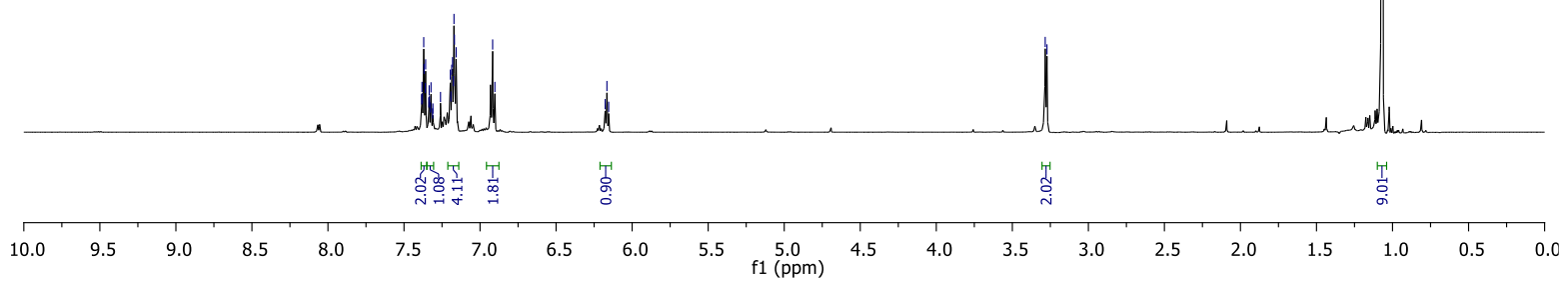

Figure S-151. ${ }^{1} \mathrm{H}$ NMR spectrum of $2 \mathbf{f}\left(\mathrm{CDCl}_{3}, 600 \mathrm{MHz}, 298 \mathrm{~K}\right)$
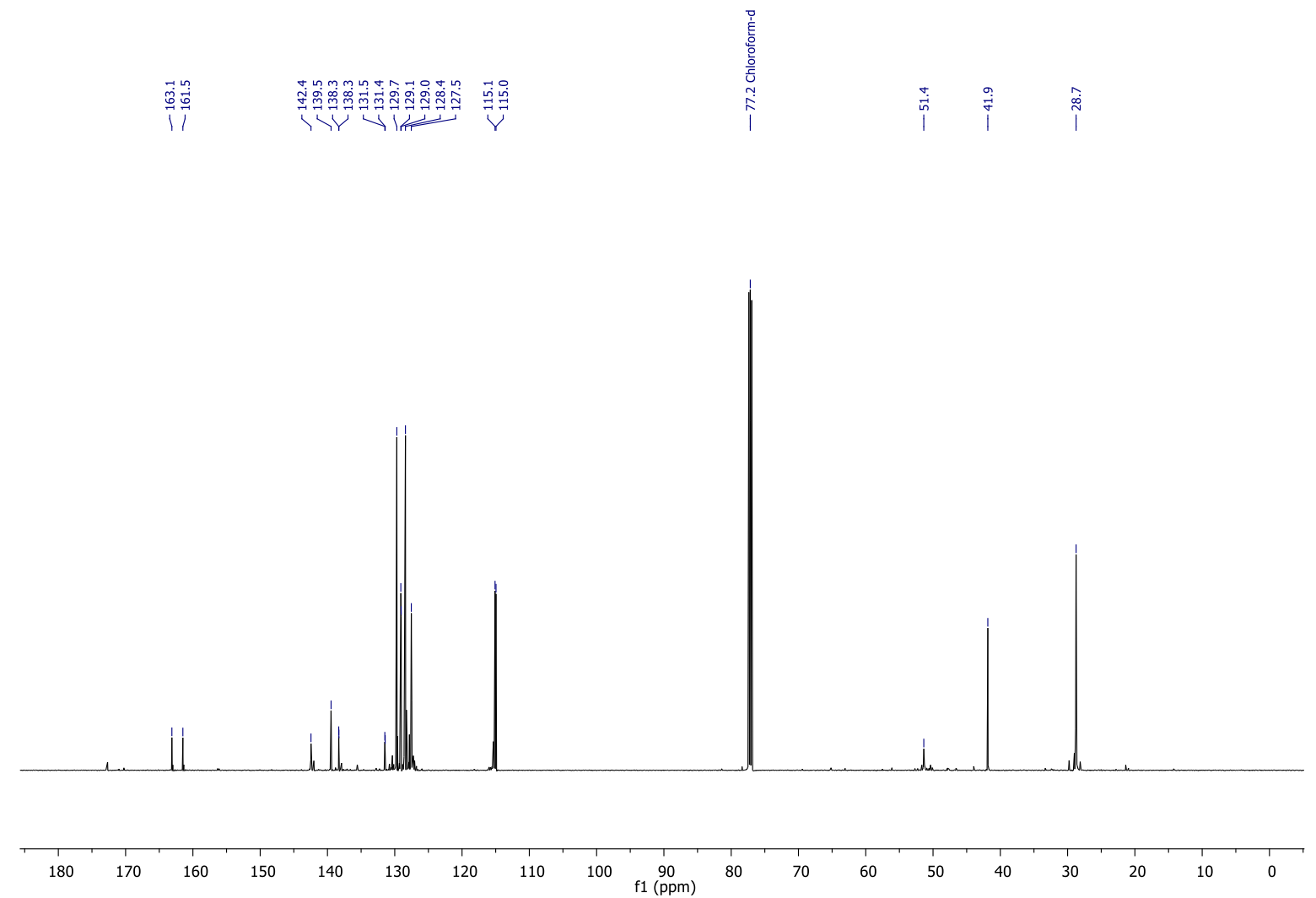

Figure S-152. ${ }^{13} \mathrm{C}$ NMR spectrum of $2 \mathbf{f}\left(\mathrm{CDCl}_{3}, 151 \mathrm{MHz}, 298 \mathrm{~K}\right)$ 


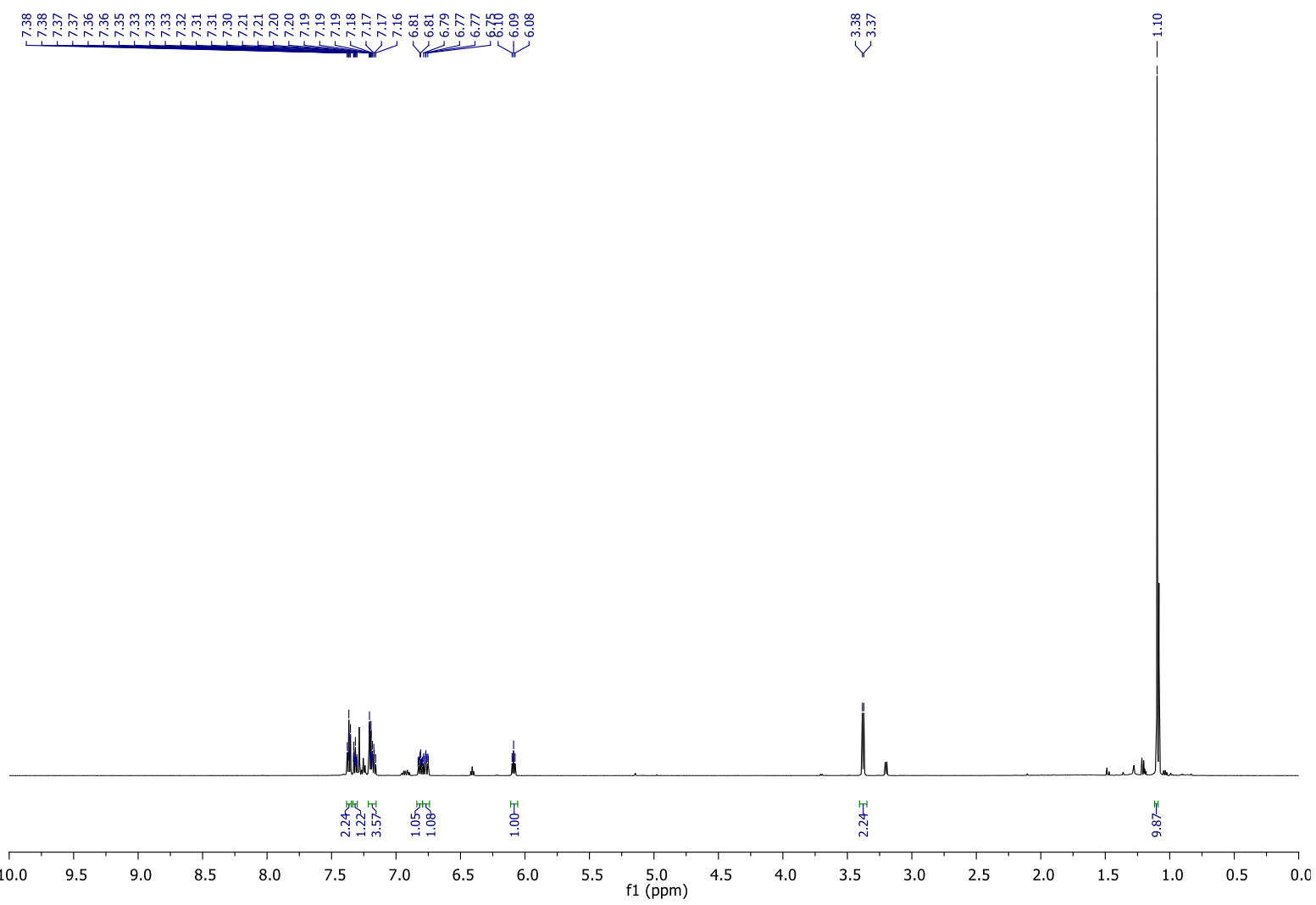

Figure S-153. ${ }^{1} \mathrm{H}$ NMR spectrum of $\mathbf{2 g}\left(\mathrm{CDCl}_{3}, 600 \mathrm{MHz}, 298 \mathrm{~K}\right)$
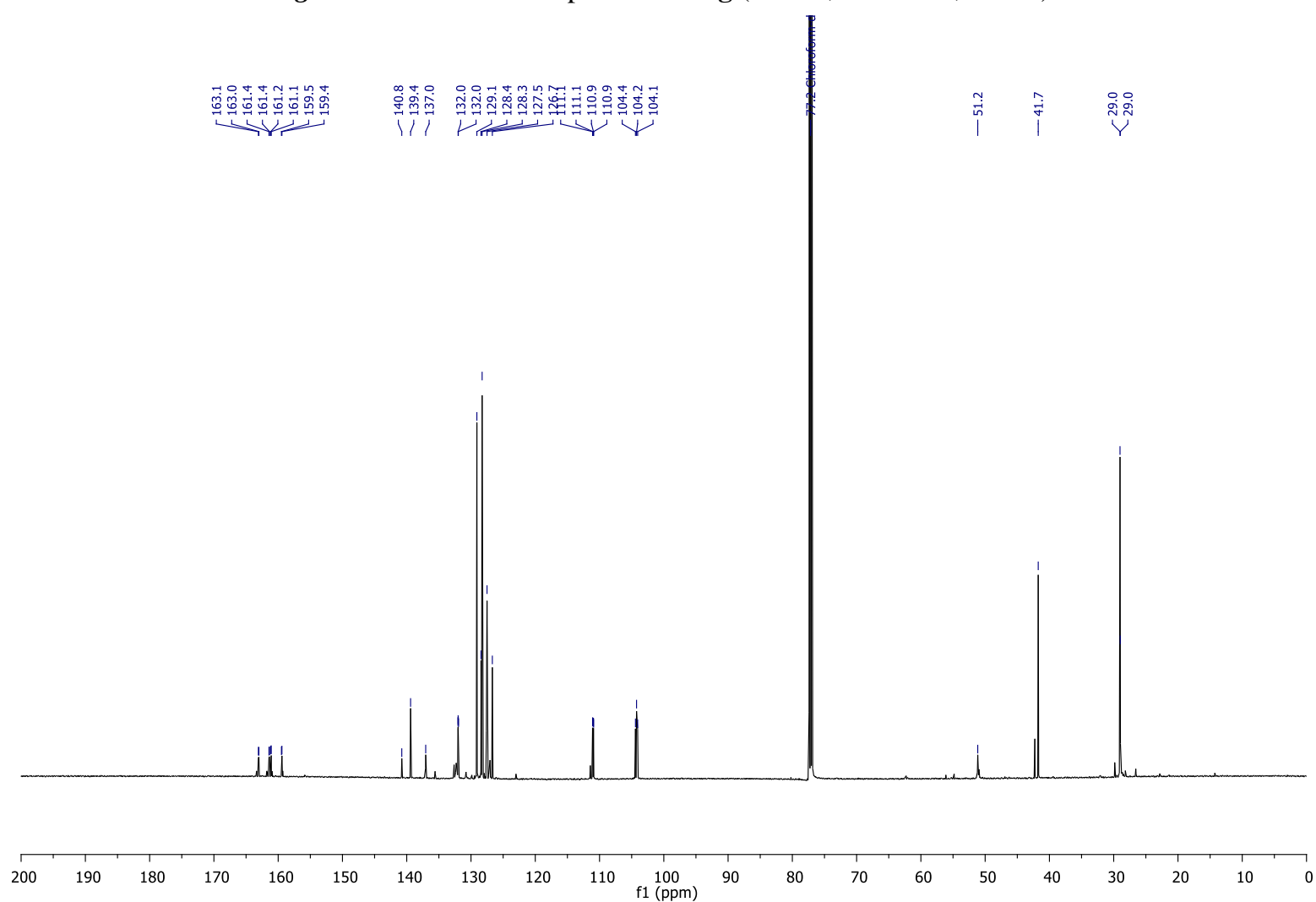

Figure $S$-154. ${ }^{13} \mathrm{C}$ NMR spectrum of $2 \mathrm{~g}\left(\mathrm{CDCl}_{3}, 151 \mathrm{MHz}, 298 \mathrm{~K}\right)$ 

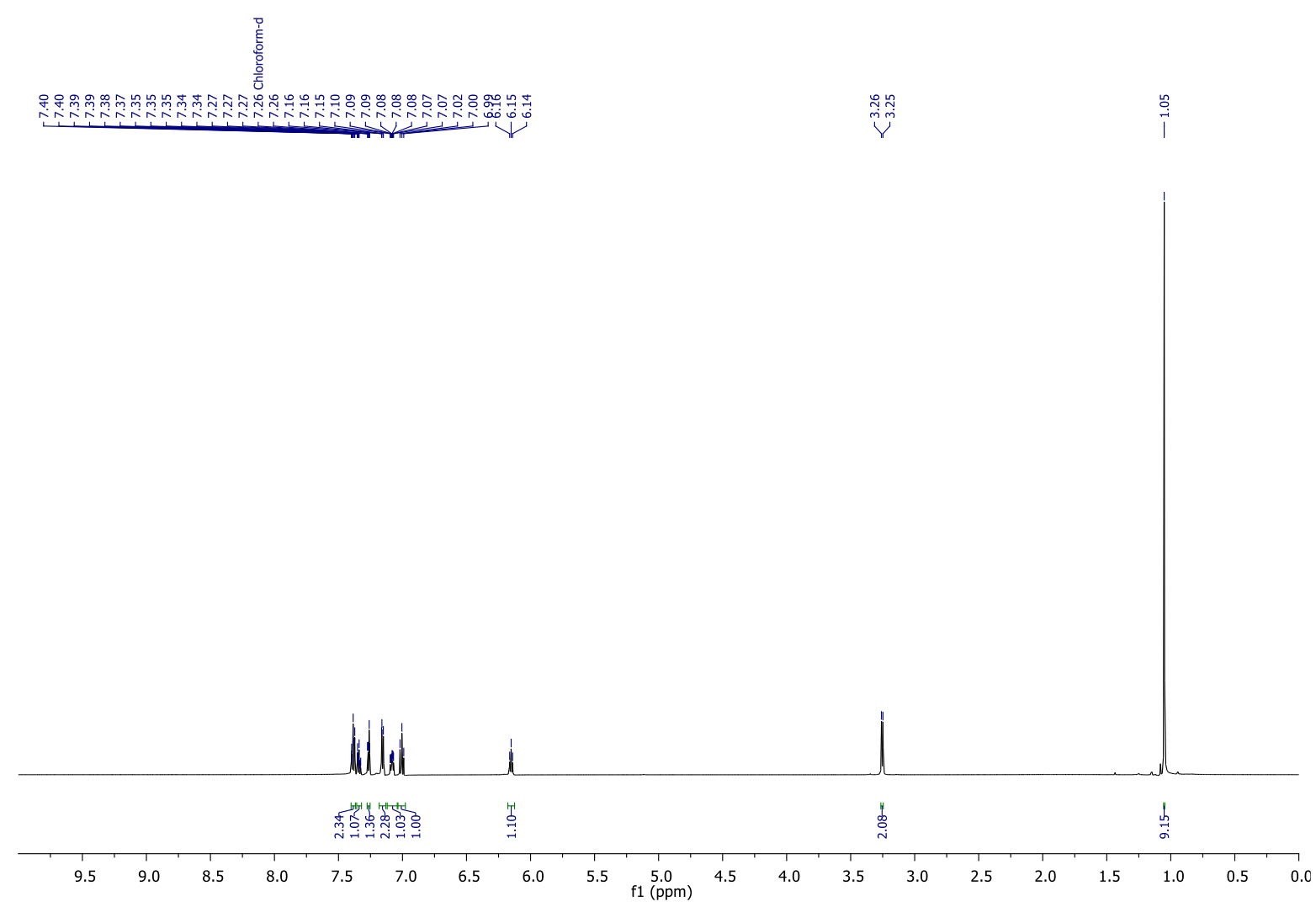

Figure S-155. ${ }^{1} \mathrm{H}$ NMR spectrum of $\mathbf{2 h}\left(\mathrm{CDCl}_{3}, 600 \mathrm{MHz}, 298 \mathrm{~K}\right)$
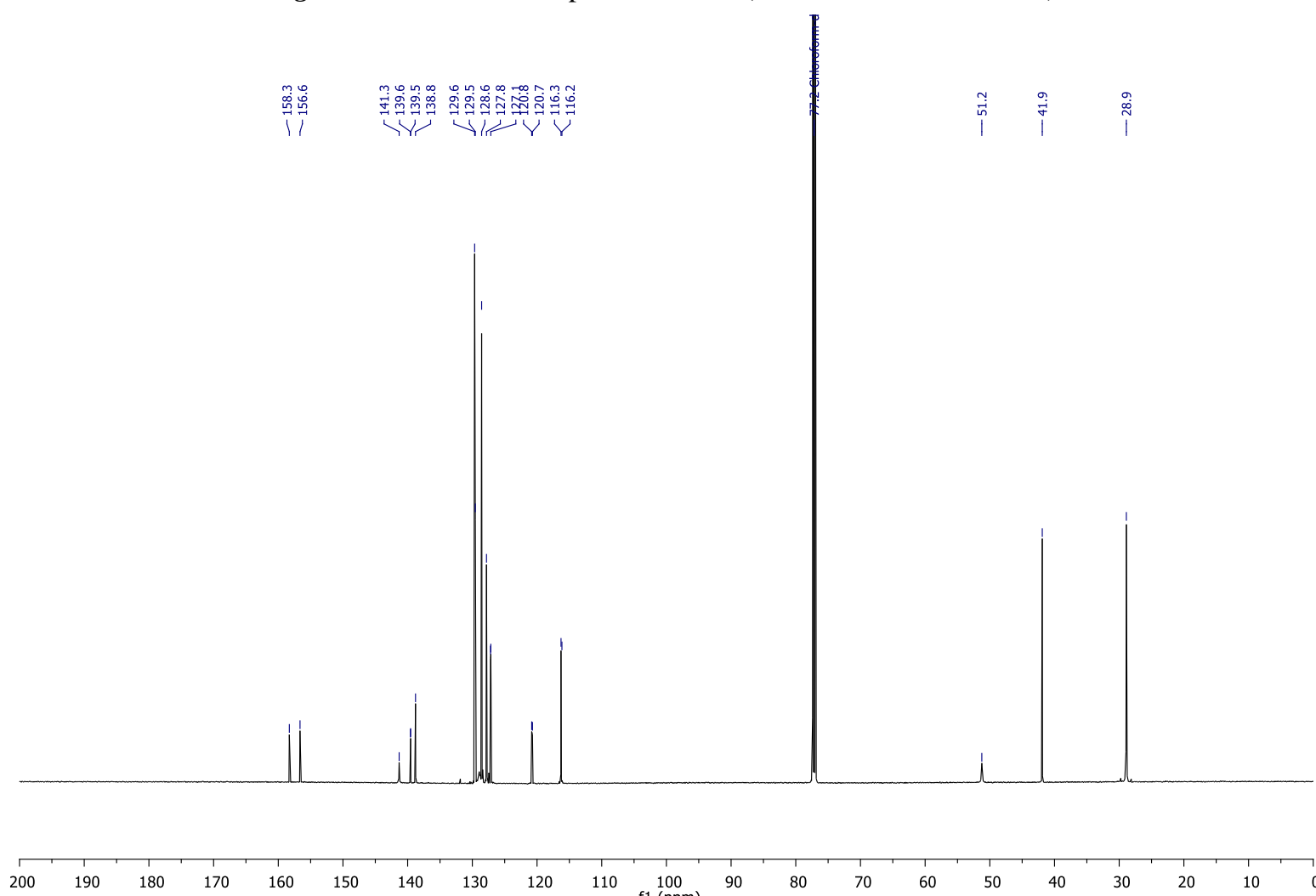

Figure $\boldsymbol{S}$-156. ${ }^{13} \mathrm{C}$ NMR spectrum of $\mathbf{2 h}\left(\mathrm{CDCl}_{3}, 151 \mathrm{MHz}, 298 \mathrm{~K}\right)$ 


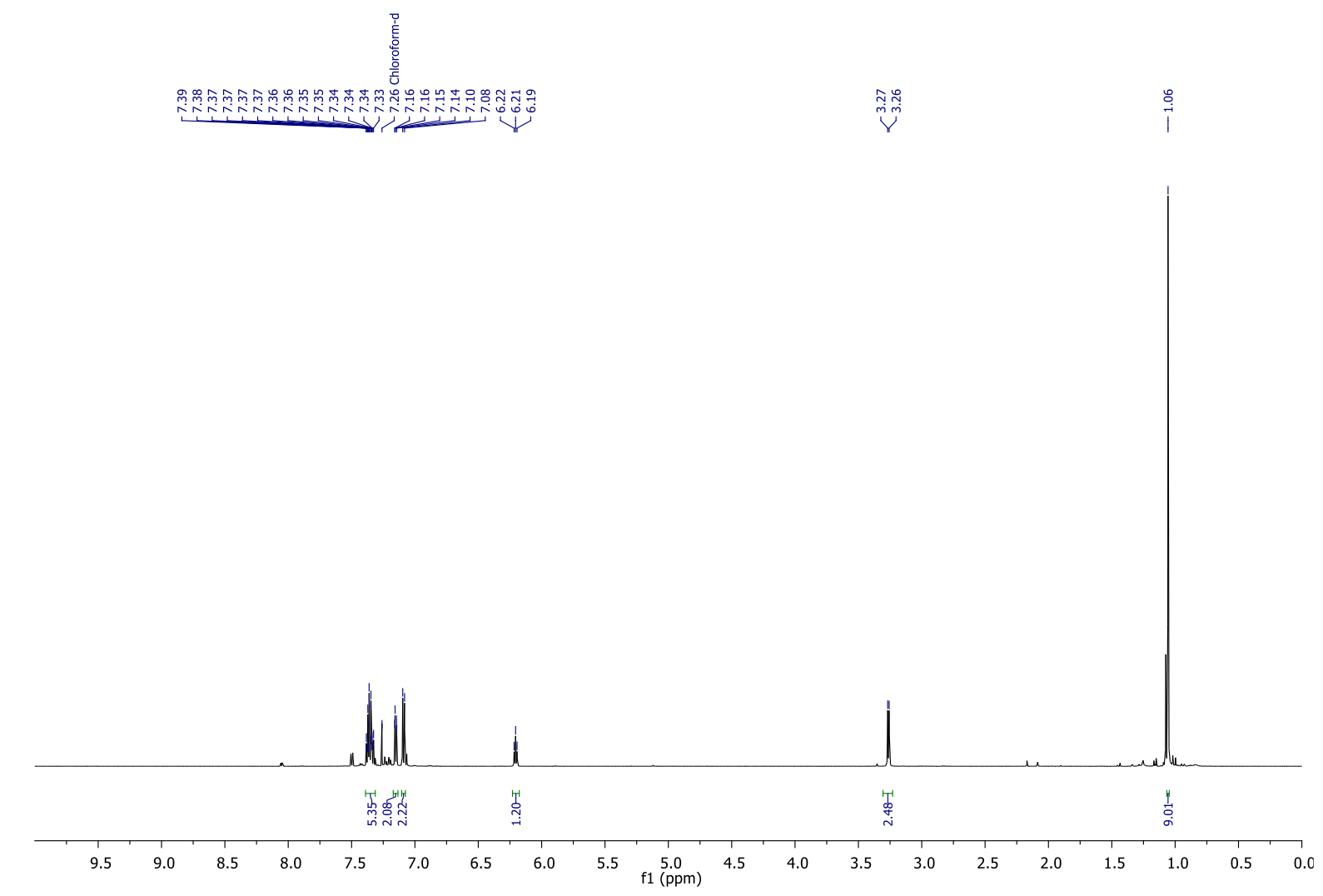

Figure S-157. ${ }^{1} \mathrm{H}$ NMR spectrum of $2 \mathbf{i}\left(\mathrm{CDCl}_{3}, 600 \mathrm{MHz}, 298 \mathrm{~K}\right)$
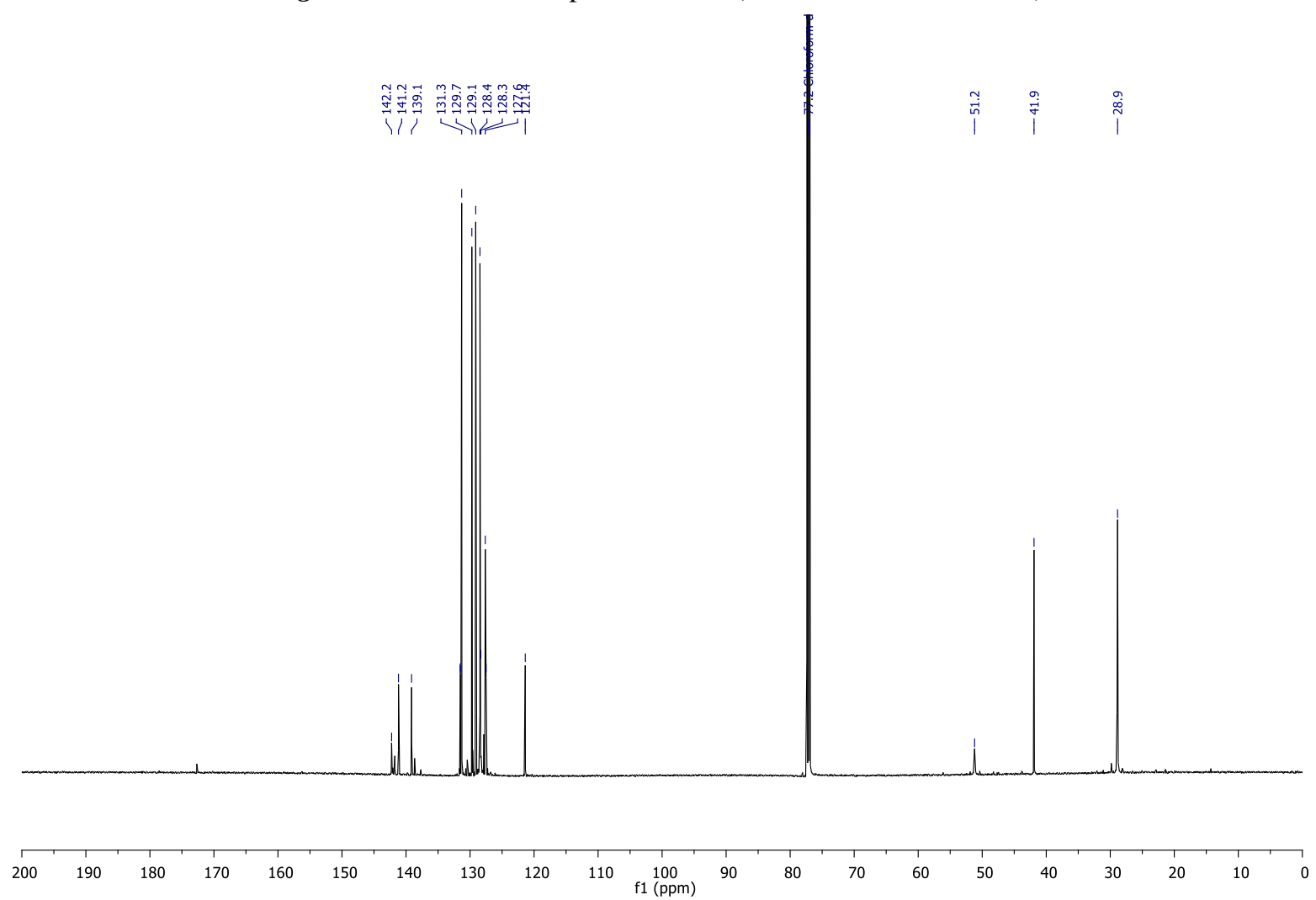

Figure S-158. ${ }^{13} \mathrm{C}$ NMR spectrum of $\mathbf{2 i}\left(\mathrm{CDCl}_{3}, 151 \mathrm{MHz}, 298 \mathrm{~K}\right)$ 


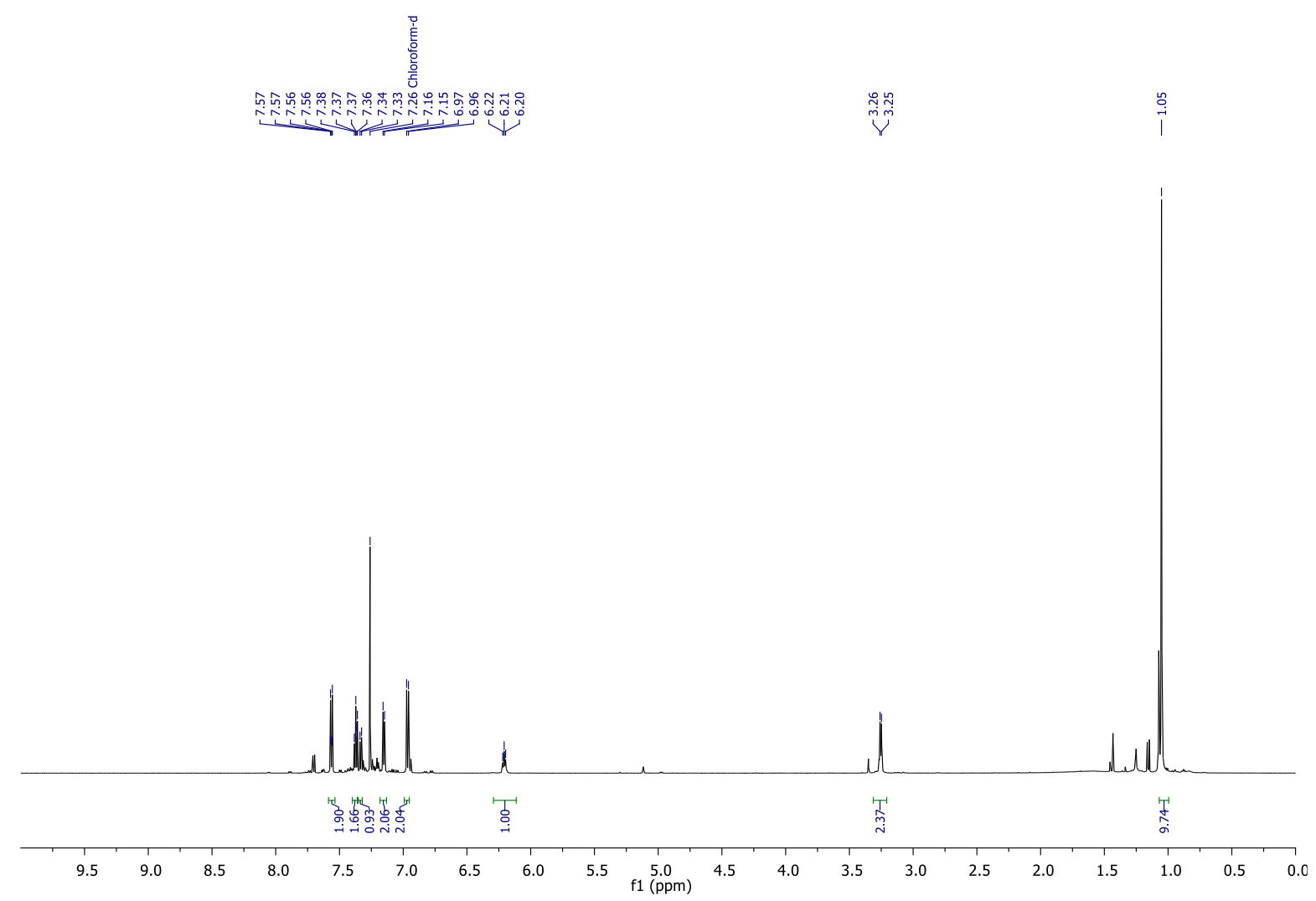

Figure S-159. ${ }^{1} \mathrm{H}$ NMR spectrum of $\mathbf{2 j}\left(\mathrm{CDCl}_{3}, 600 \mathrm{MHz}, 298 \mathrm{~K}\right)$
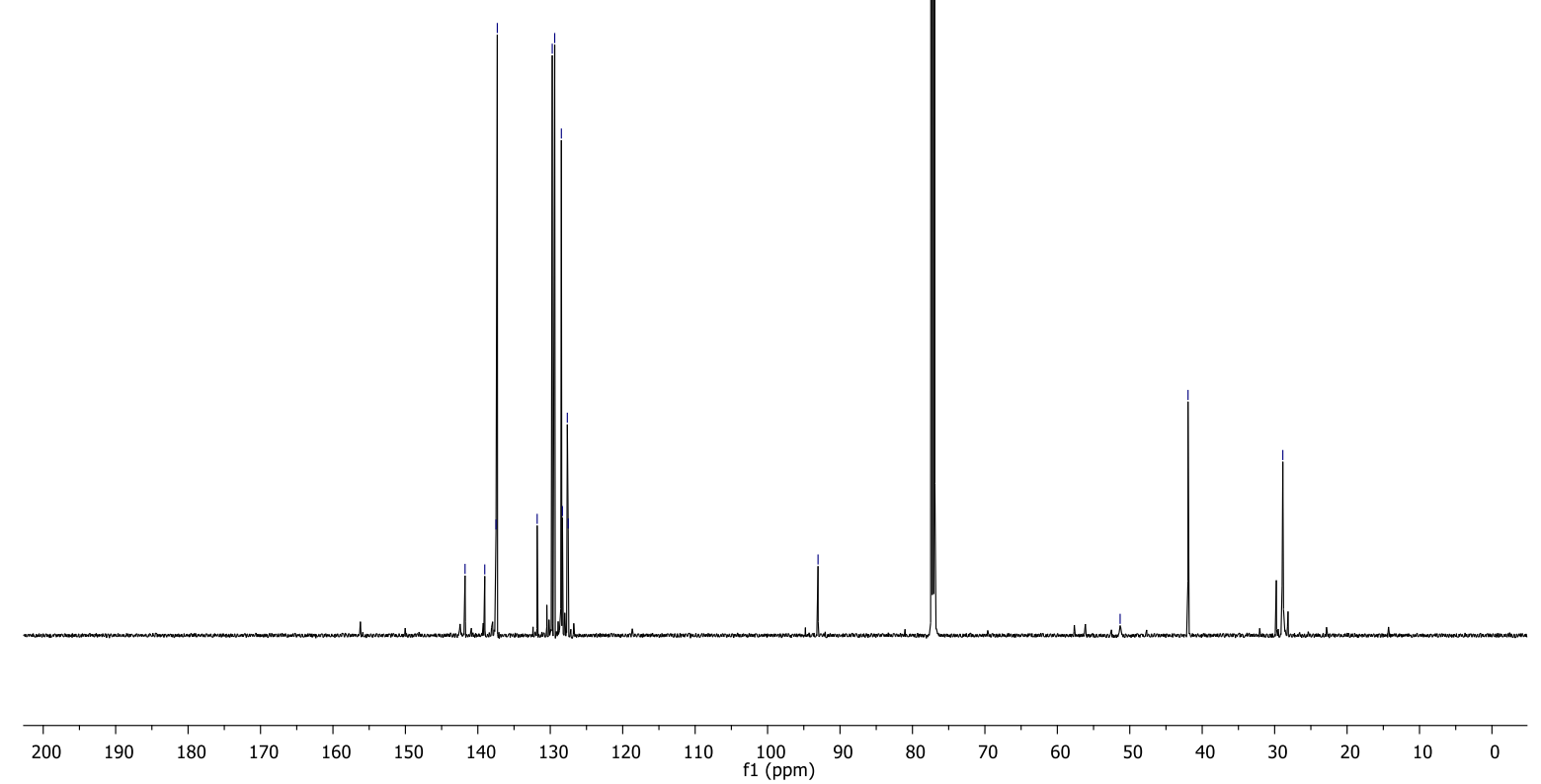

Figure $\mathbf{S}$-160. ${ }^{13} \mathrm{C}$ NMR spectrum of $\mathbf{2 j}\left(\mathrm{CDCl}_{3}, 151 \mathrm{MHz}, 298 \mathrm{~K}\right)$ 


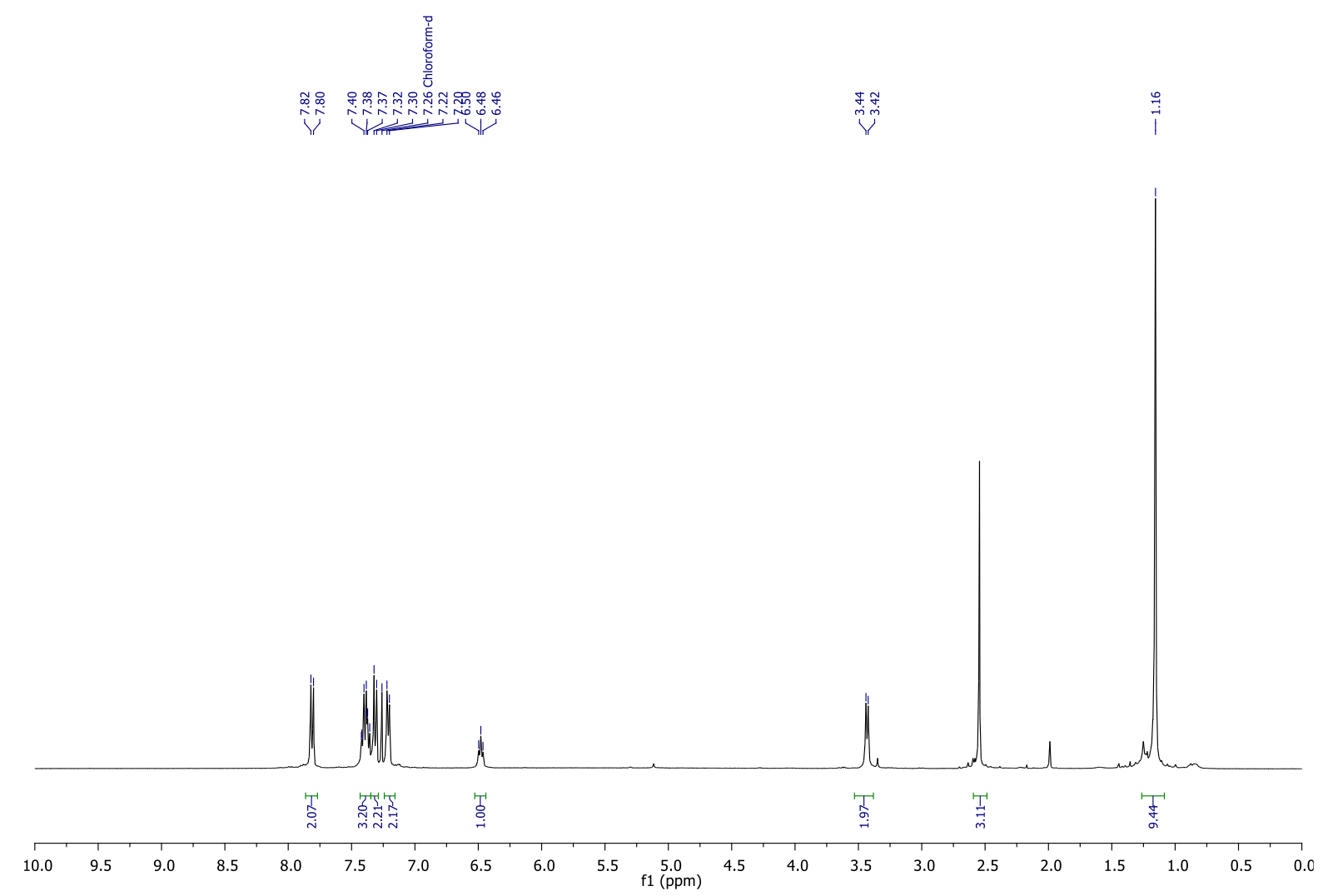

Figure S-161. ${ }^{1} \mathrm{H}$ NMR spectrum of $\mathbf{2 k}\left(\mathrm{CDCl}_{3}, 600 \mathrm{MHz}, 298 \mathrm{~K}\right)$
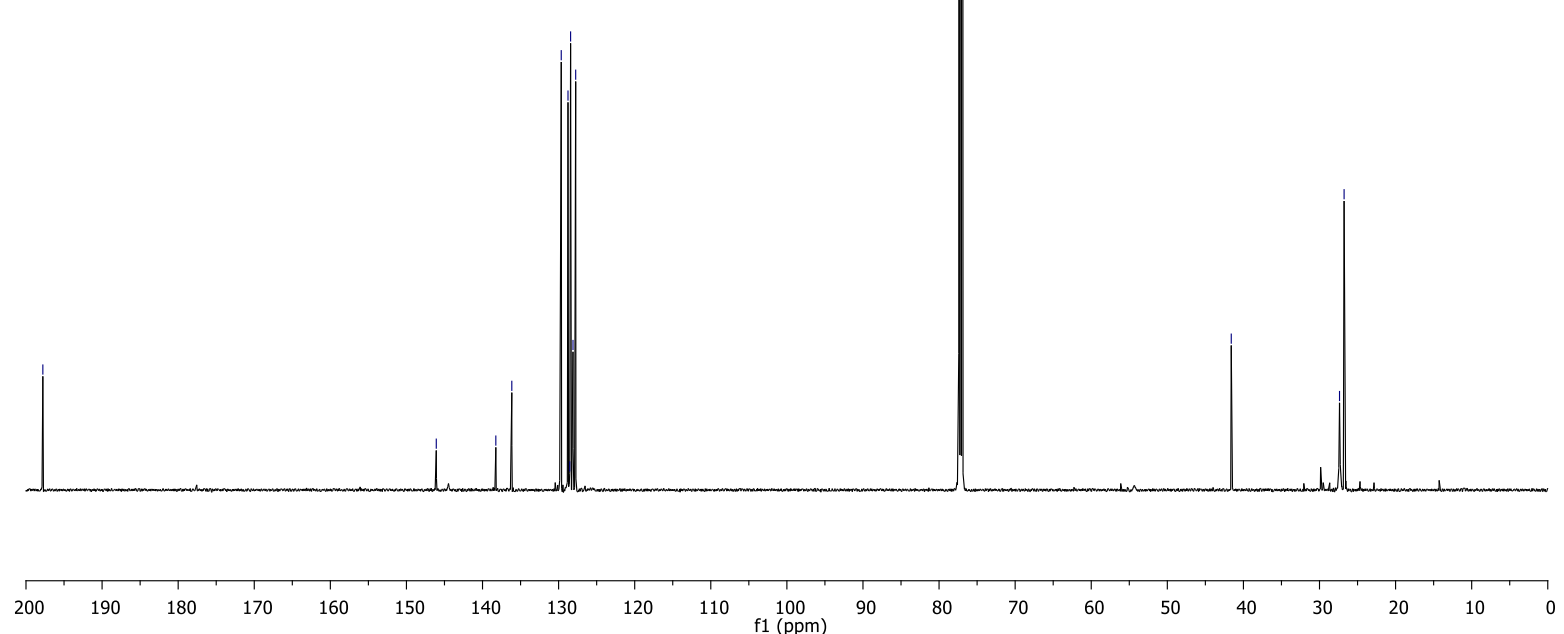

Figure $\mathbf{S - 1 6 2} .{ }^{13} \mathrm{C}$ NMR spectrum of $\mathbf{2 k}\left(\mathrm{CDCl}_{3}, 151 \mathrm{MHz}, 298 \mathrm{~K}\right)$ 


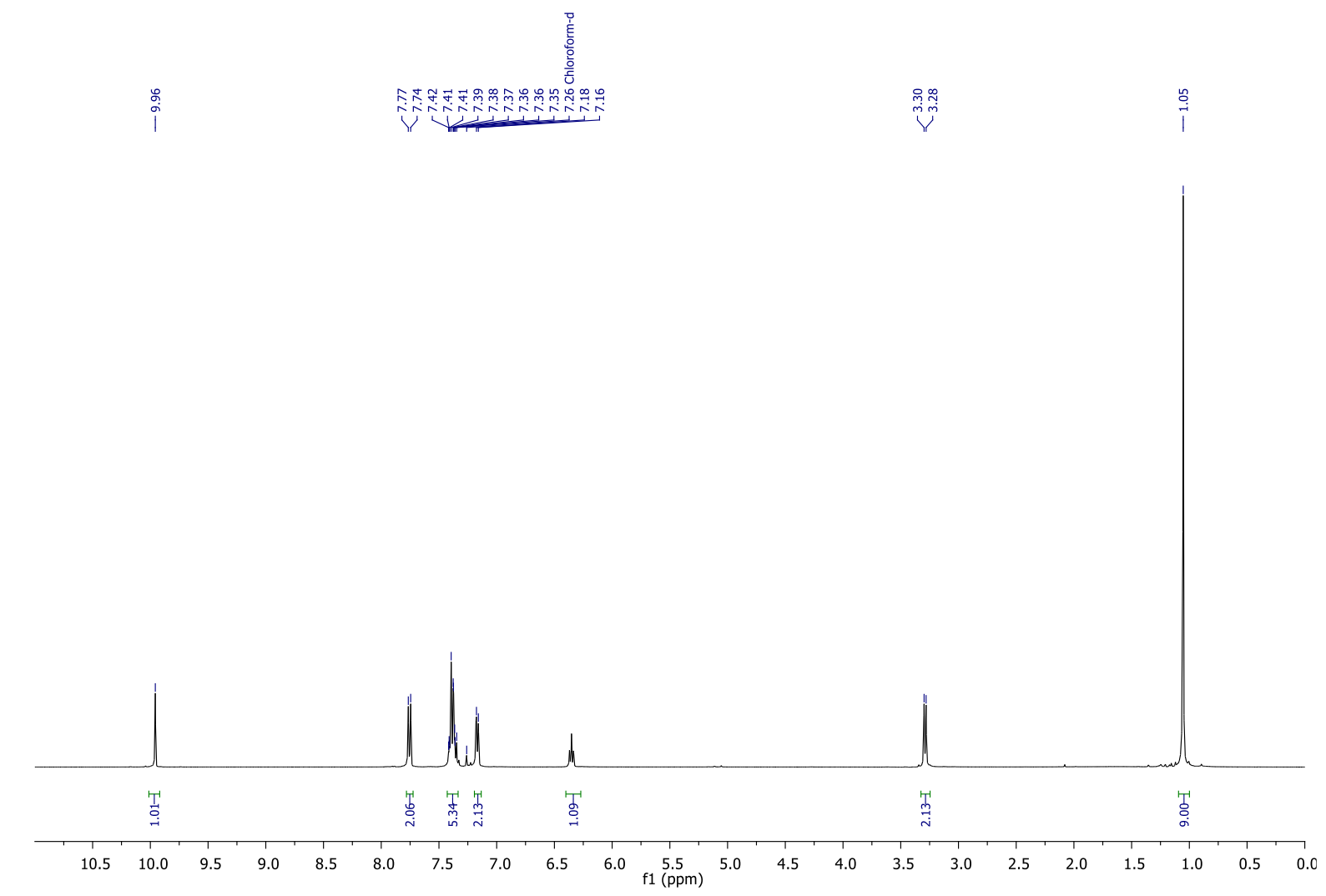

Figure S-163. ${ }^{1} \mathrm{H}$ NMR spectrum of $21\left(\mathrm{CDCl}_{3}, 600 \mathrm{MHz}, 298 \mathrm{~K}\right)$
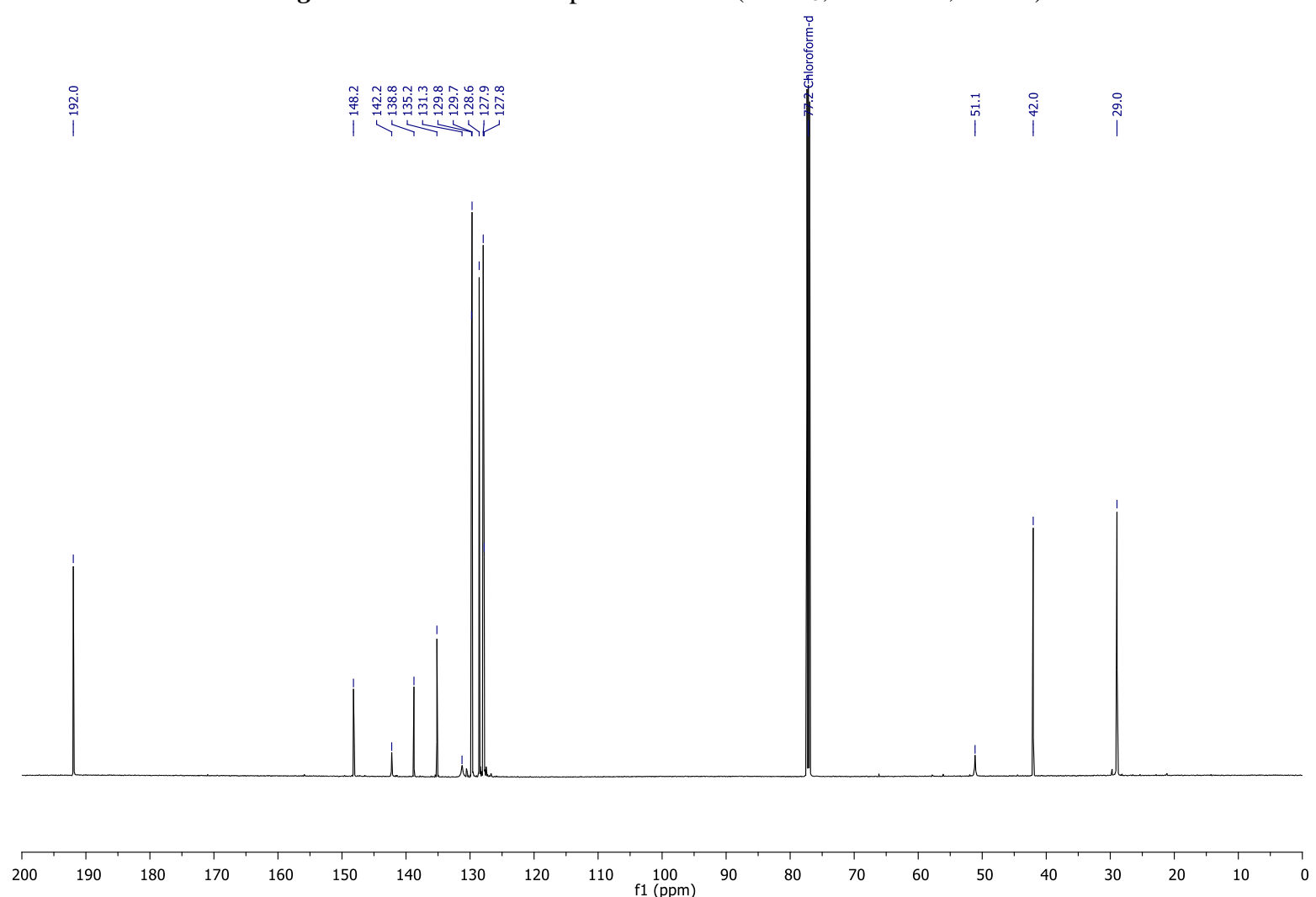

Figure S-164. ${ }^{13} \mathrm{C}$ NMR spectrum of $21\left(\mathrm{CDCl}_{3}, 151 \mathrm{MHz}, 298 \mathrm{~K}\right)$ 


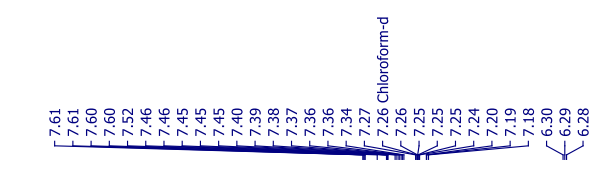

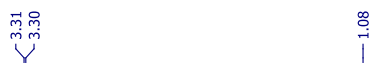

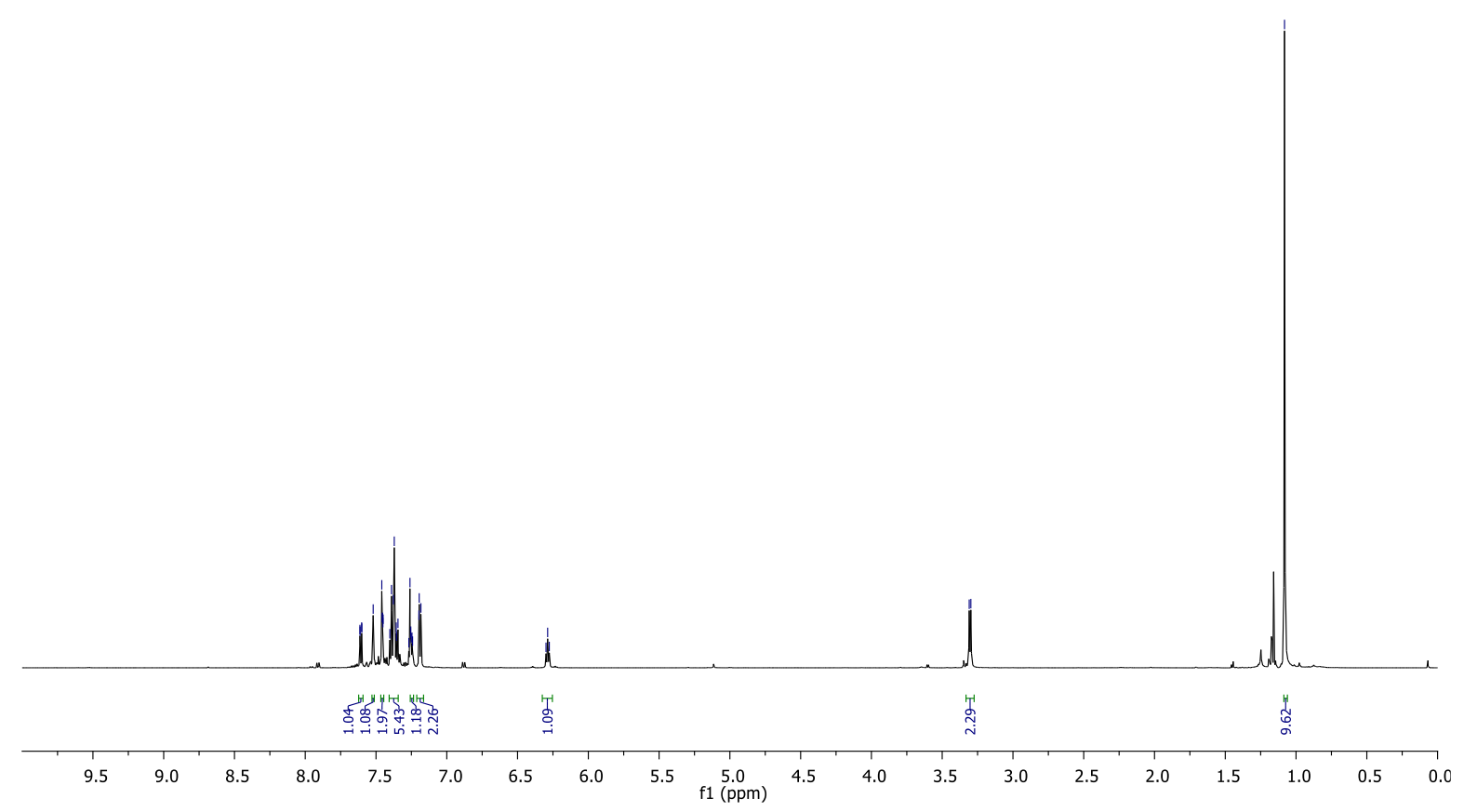

Figure S-165. ${ }^{1} \mathrm{H}$ NMR spectrum of $\mathbf{2 m}\left(\mathrm{CDCl}_{3}, 600 \mathrm{MHz}, 298 \mathrm{~K}\right)$
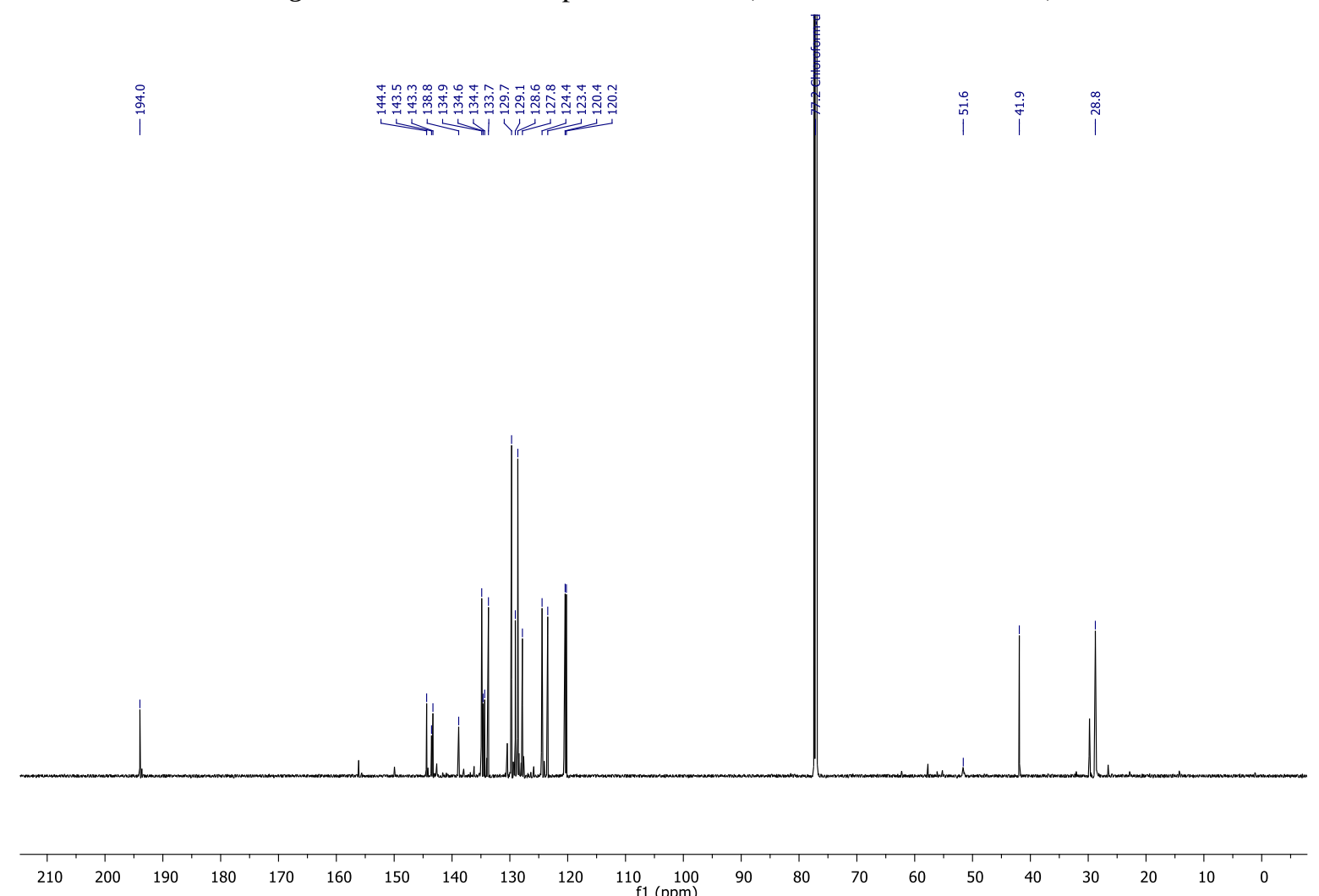

Figure $\mathbf{S - 1 6 6 .}{ }^{13} \mathrm{C}$ NMR spectrum of $\mathbf{2 m}\left(\mathrm{CDCl}_{3}, 151 \mathrm{MHz}, 298 \mathrm{~K}\right)$ 


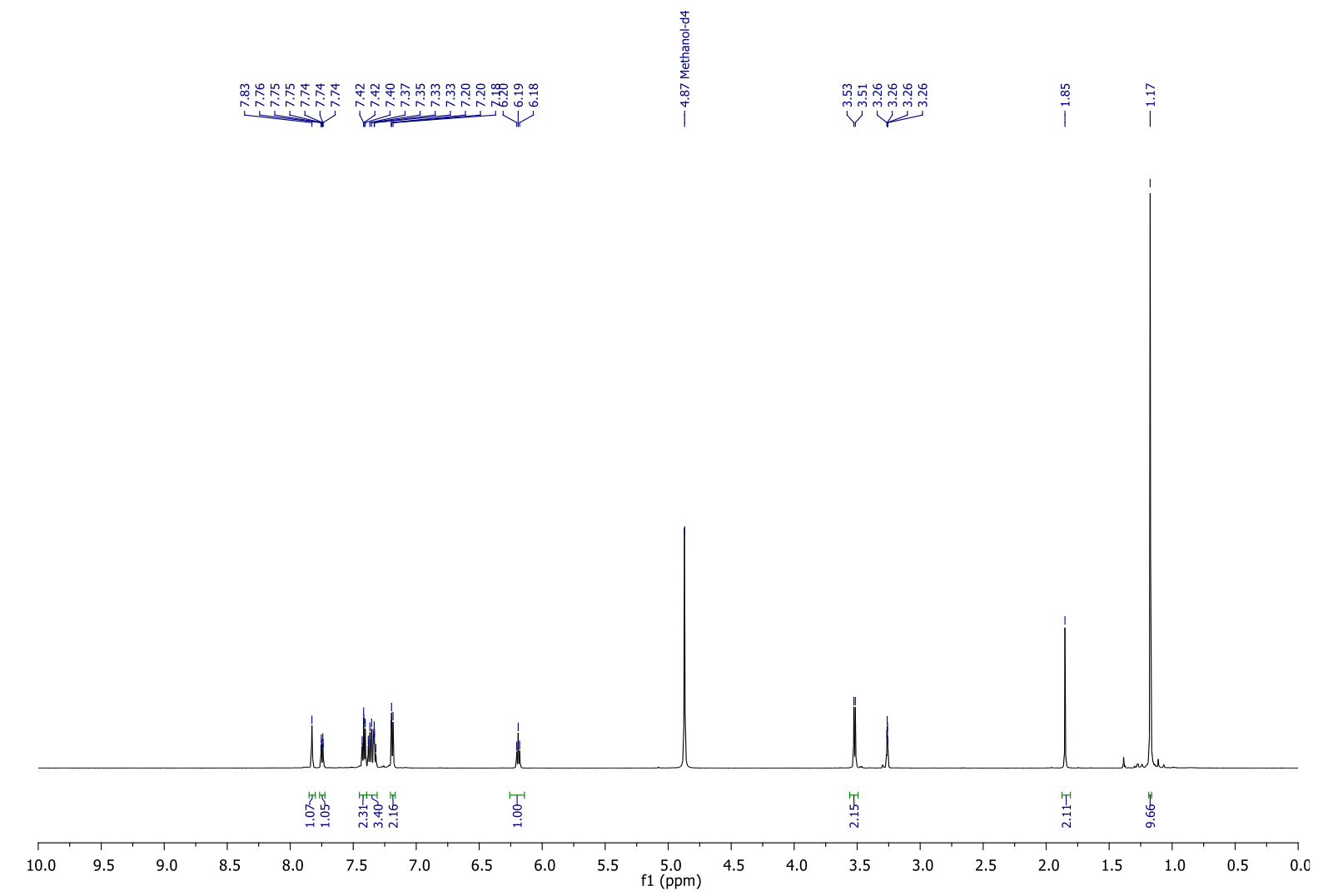

Figure S-167. ${ }^{1} \mathrm{H}$ NMR spectrum of $\mathbf{2 n}\left(\mathrm{CD}_{3} \mathrm{OD}, 600 \mathrm{MHz}, 298 \mathrm{~K}\right)$

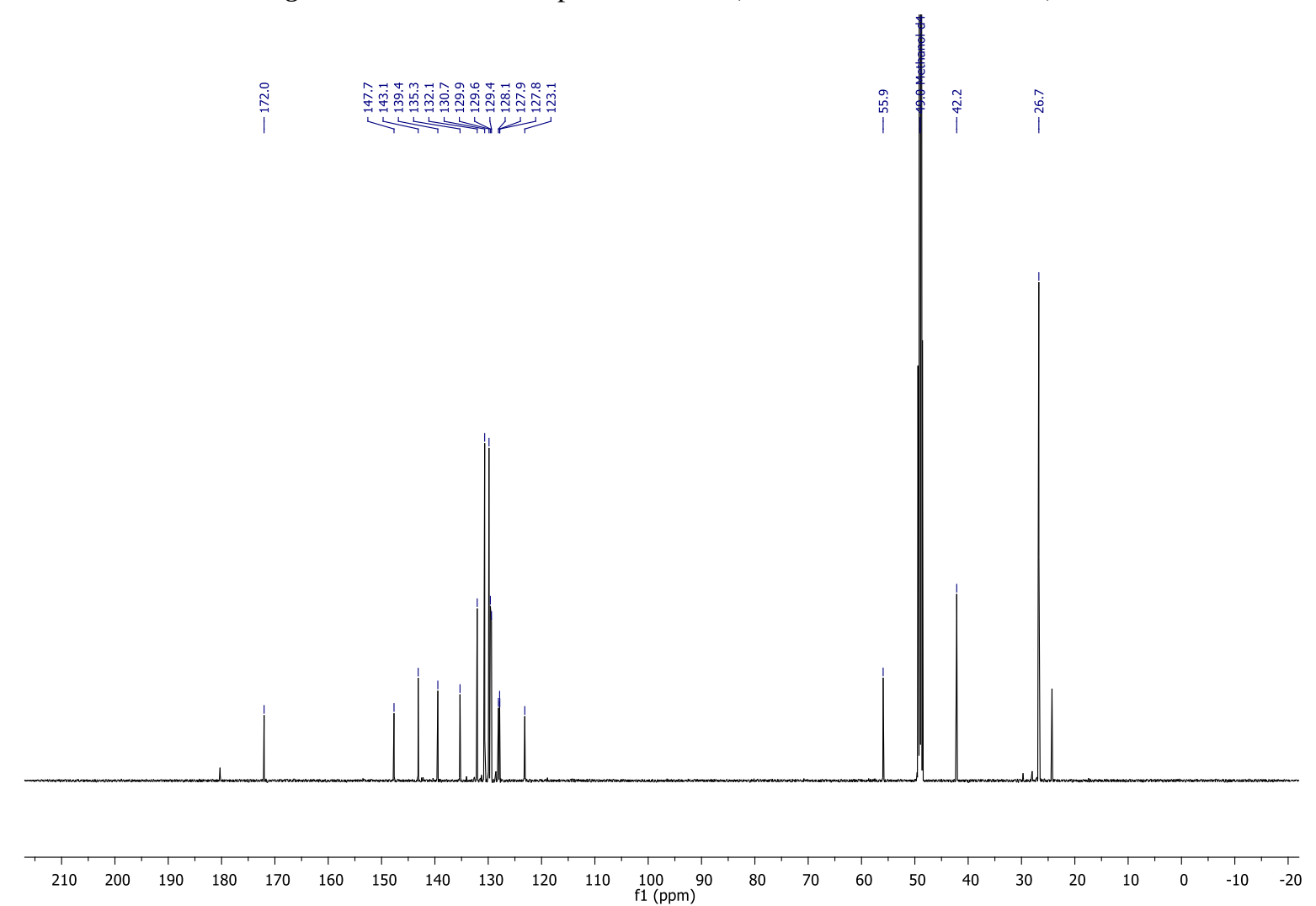

Figure $S$-168. ${ }^{13} \mathrm{C}$ NMR spectrum of $2 \mathrm{n}\left(\mathrm{CD}_{3} \mathrm{OD}, 151 \mathrm{MHz}, 298 \mathrm{~K}\right)$ 


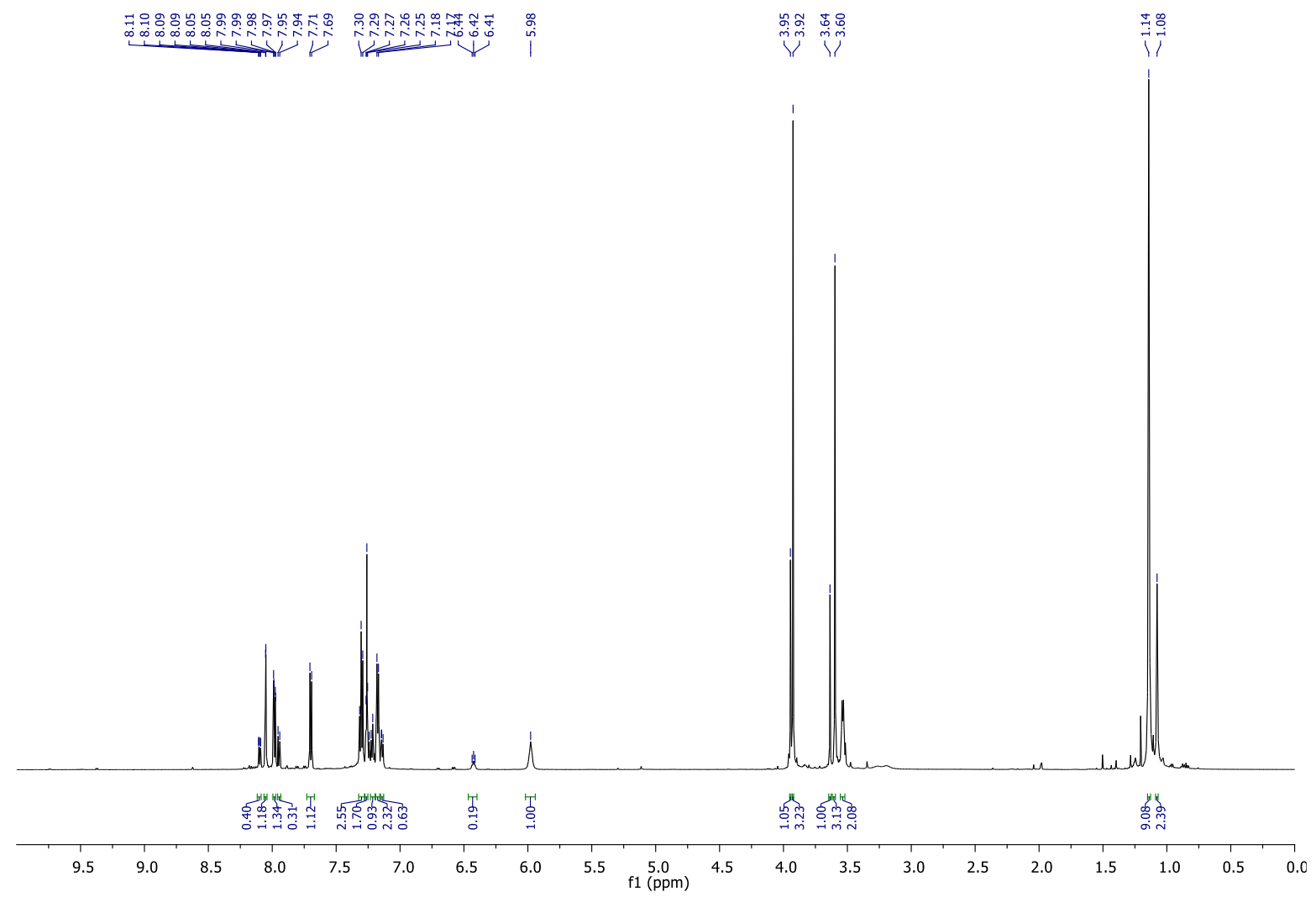

Figure S-169. ${ }^{1} \mathrm{H}$ NMR spectrum of $20\left(\mathrm{CDCl}_{3}, 600 \mathrm{MHz}, 298 \mathrm{~K}\right)$
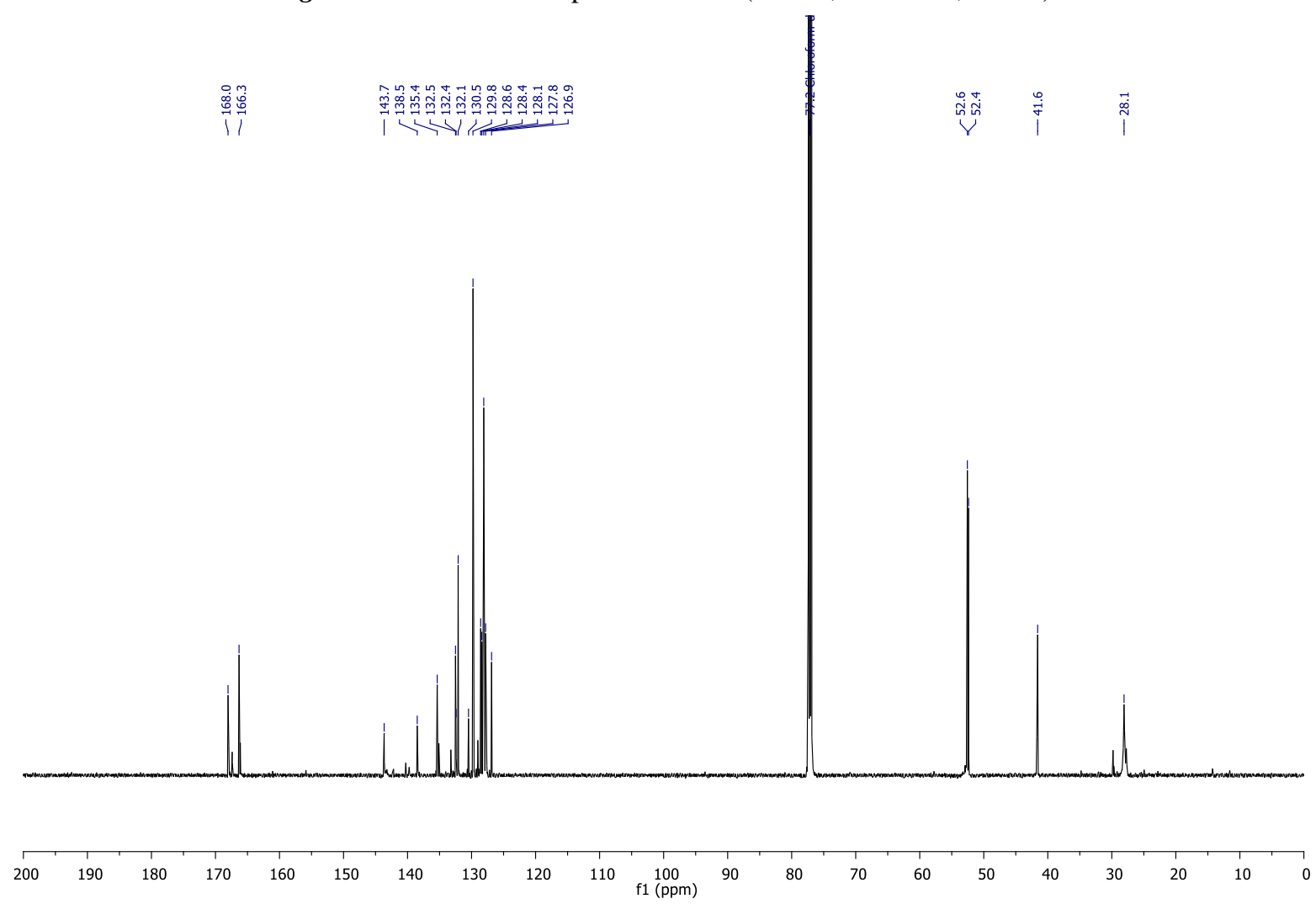

Figure S-170. ${ }^{13} \mathrm{C}$ NMR spectrum of $20\left(\mathrm{CDCl}_{3}, 151 \mathrm{MHz}, 298 \mathrm{~K}\right)$ 


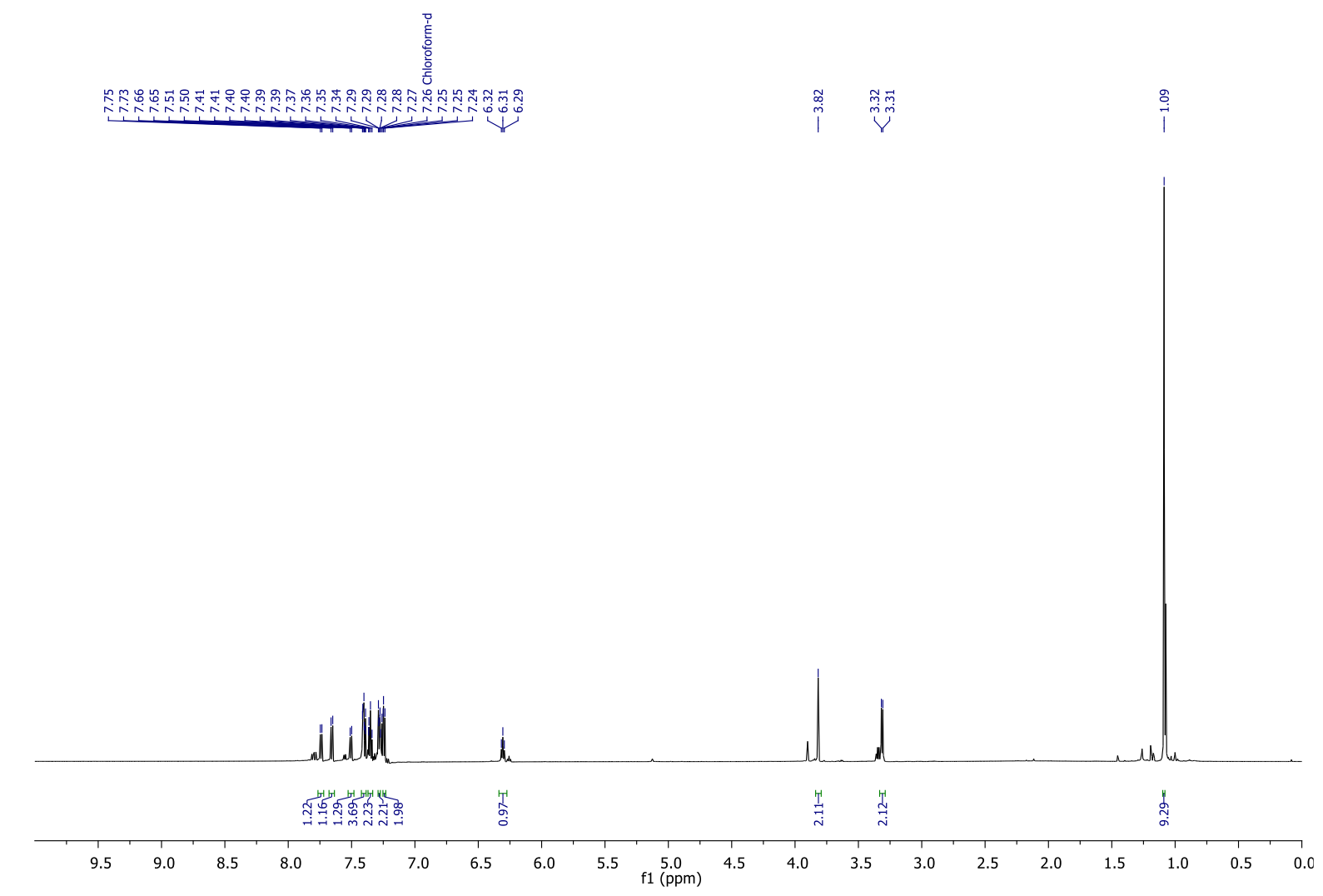

Figure S-171. ${ }^{1} \mathrm{H}$ NMR spectrum of $2 \mathbf{p}\left(\mathrm{CDCl}_{3}, 600 \mathrm{MHz}, 298 \mathrm{~K}\right)$
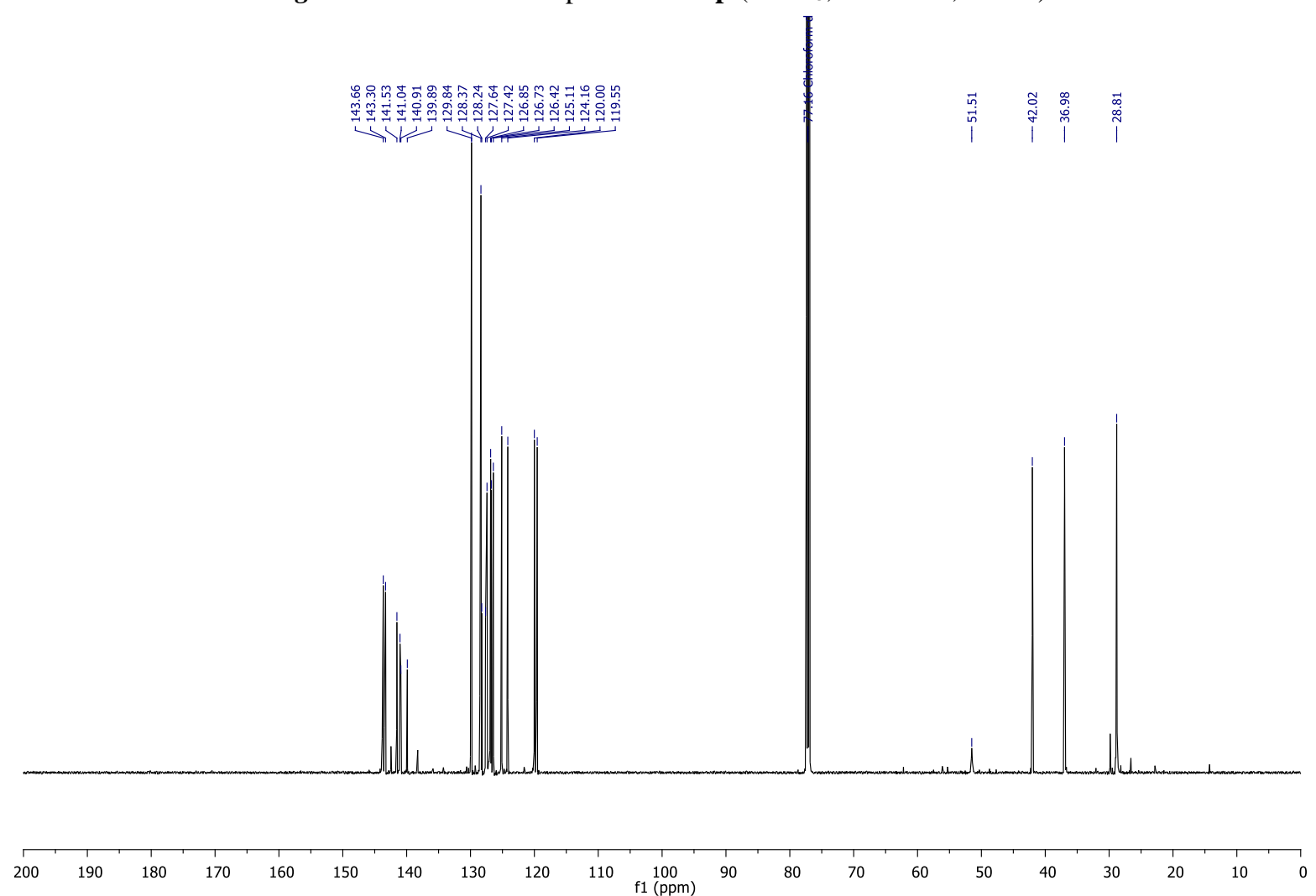

Figure $\mathbf{S - 1 7 2} .{ }^{13} \mathrm{C}$ NMR spectrum of $\mathbf{2 p}\left(\mathrm{CDCl}_{3}, 151 \mathrm{MHz}, 298 \mathrm{~K}\right)$ 

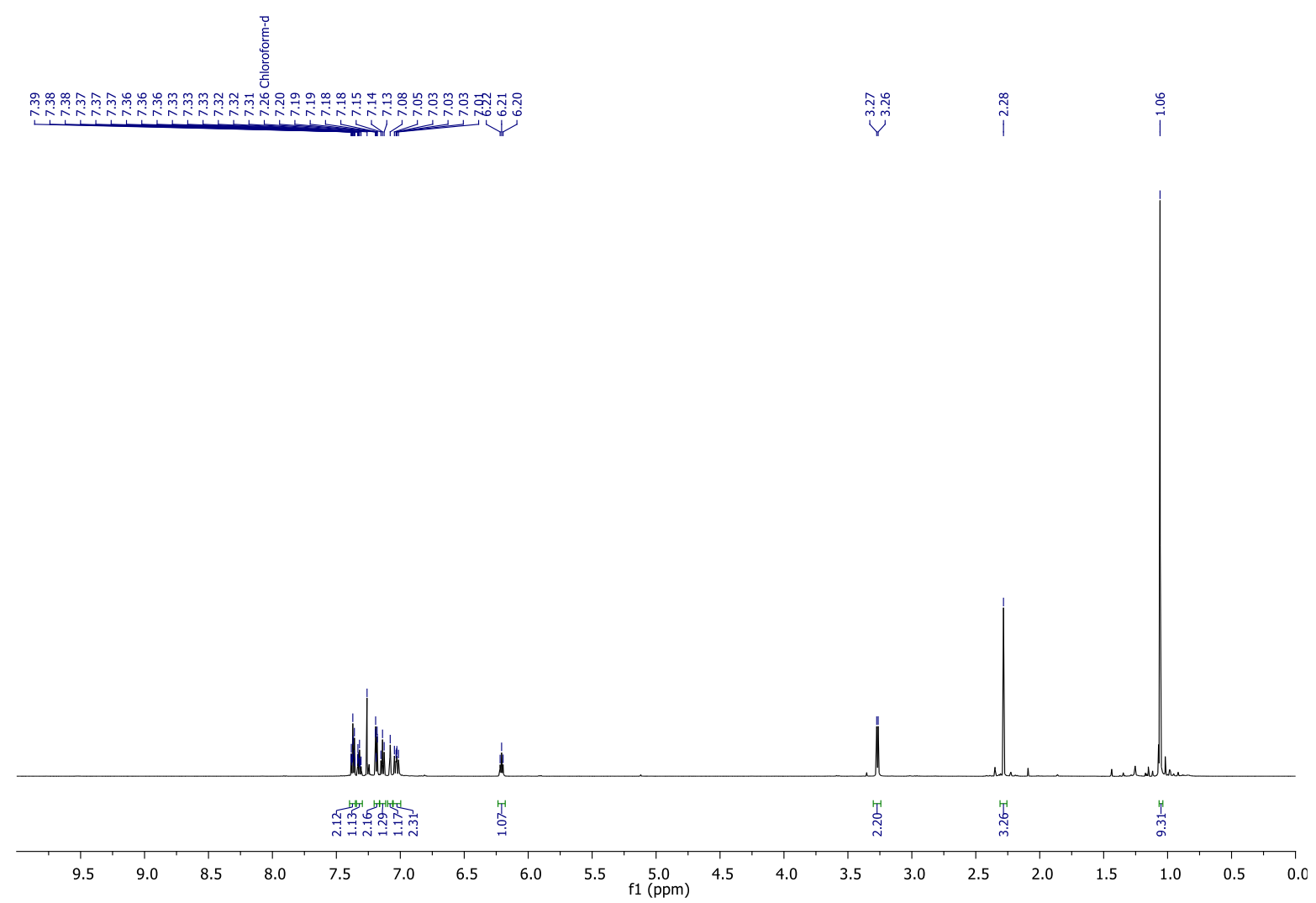

Figure S-173. ${ }^{1} \mathrm{H}$ NMR spectrum of $2 \mathbf{2 q}\left(\mathrm{CDCl}_{3}, 600 \mathrm{MHz}, 298 \mathrm{~K}\right)$
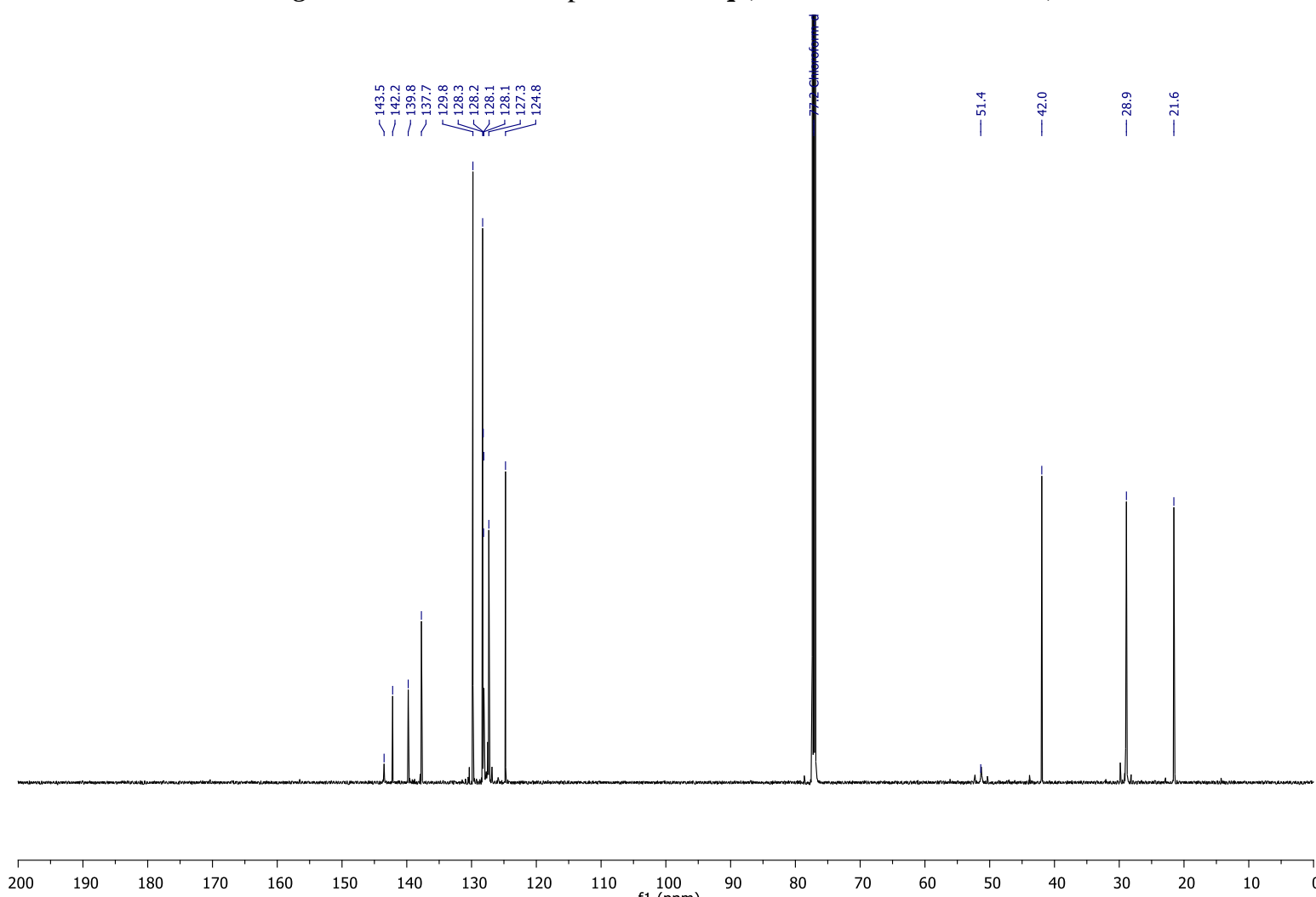

Figure $\mathbf{S - 1 7 4 .}{ }^{13} \mathrm{C}$ NMR spectrum of $\mathbf{2 q}\left(\mathrm{CDCl}_{3}, 151 \mathrm{MHz}, 298 \mathrm{~K}\right)$ 


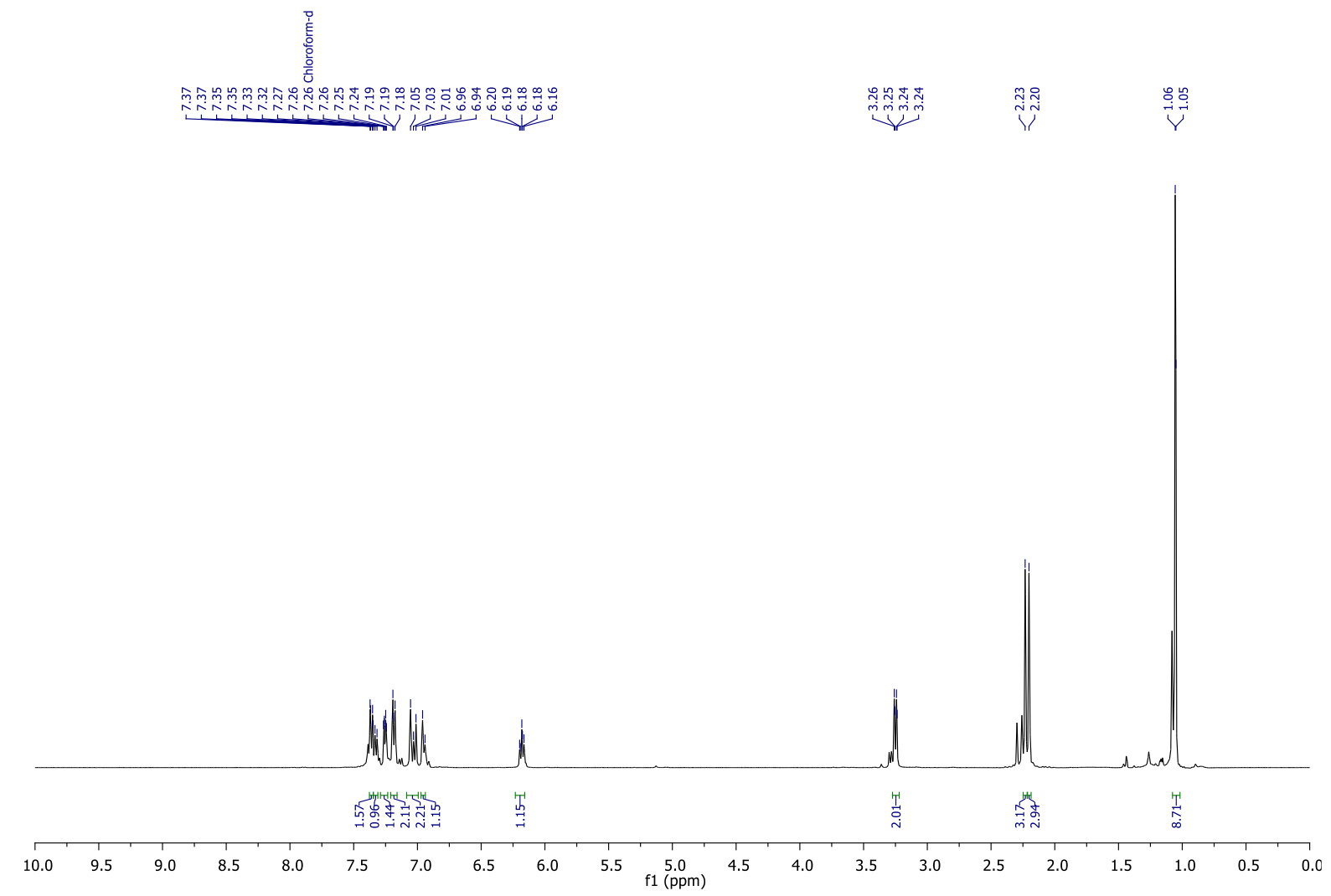

Figure S-175. ${ }^{1} \mathrm{H}$ NMR spectrum of $2 \mathbf{r}\left(\mathrm{CDCl}_{3}, 600 \mathrm{MHz}, 298 \mathrm{~K}\right)$
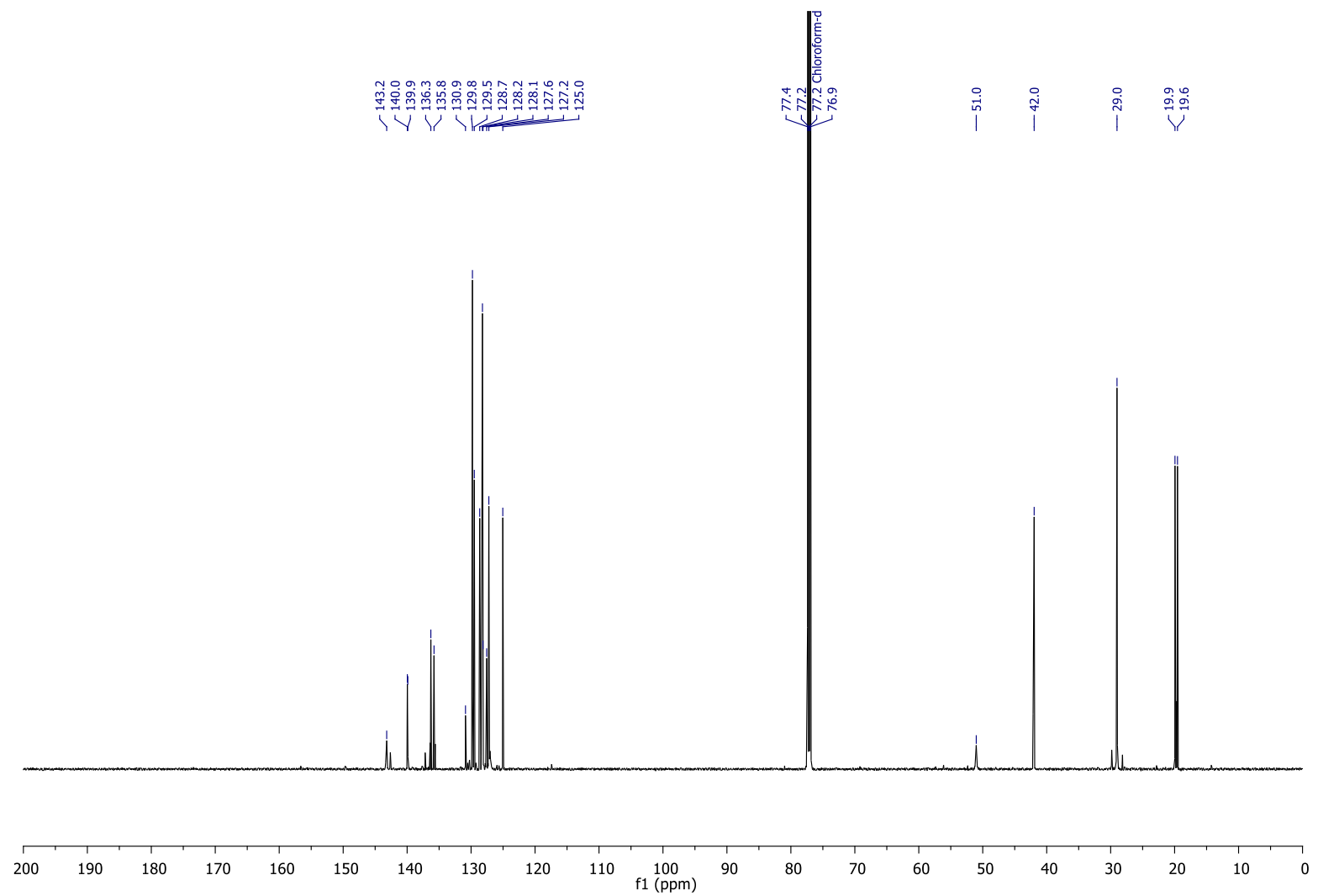

Figure $\mathbf{S}$-176. ${ }^{13} \mathrm{C}$ NMR spectrum of $2 \mathbf{r}\left(\mathrm{CDCl}_{3}, 151 \mathrm{MHz}, 298 \mathrm{~K}\right)$ 


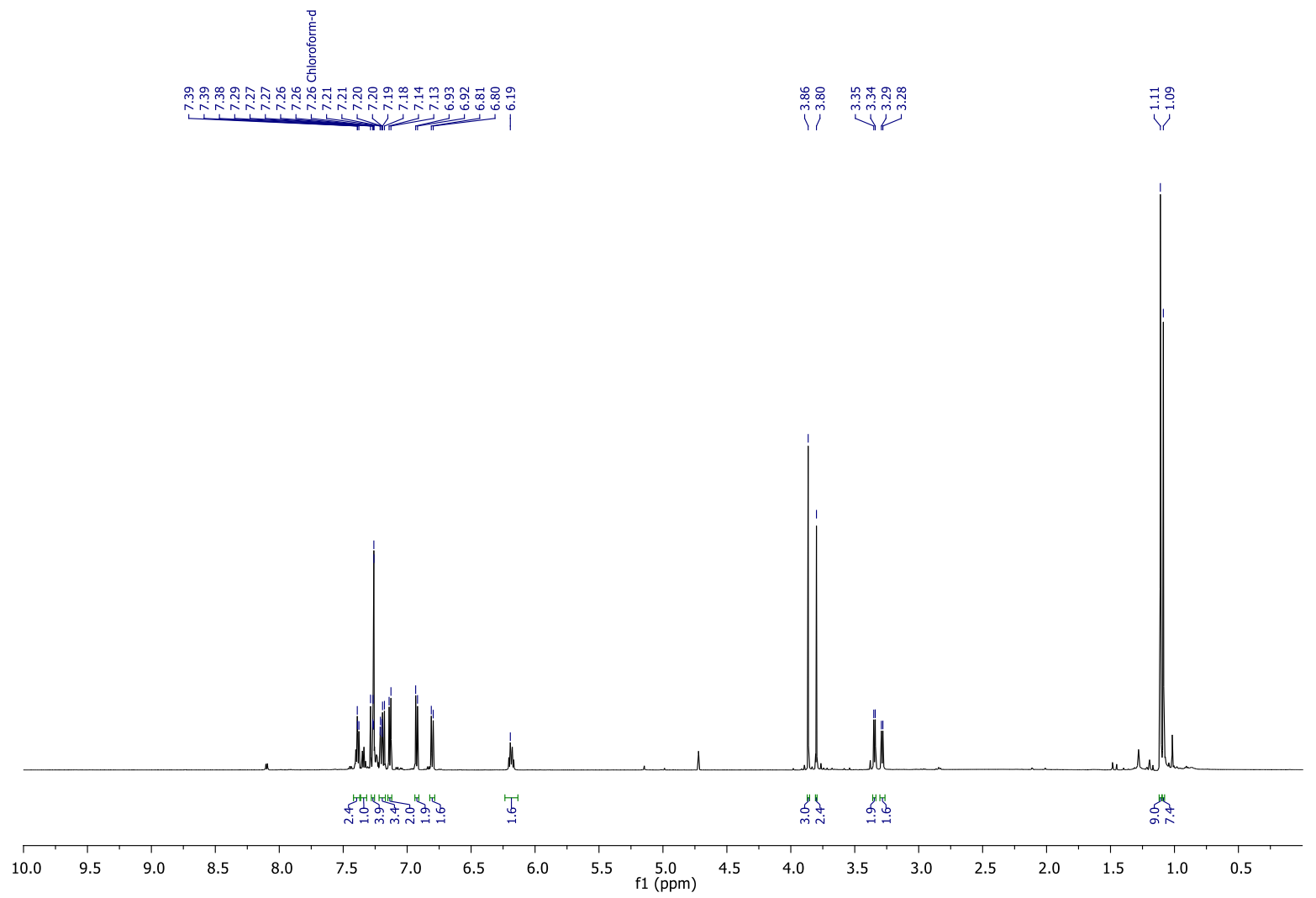

Figure S-177. ${ }^{1} \mathrm{H}$ NMR spectrum of $2 \mathbf{s}\left(\mathrm{CDCl}_{3}, 600 \mathrm{MHz}, 298 \mathrm{~K}\right)$
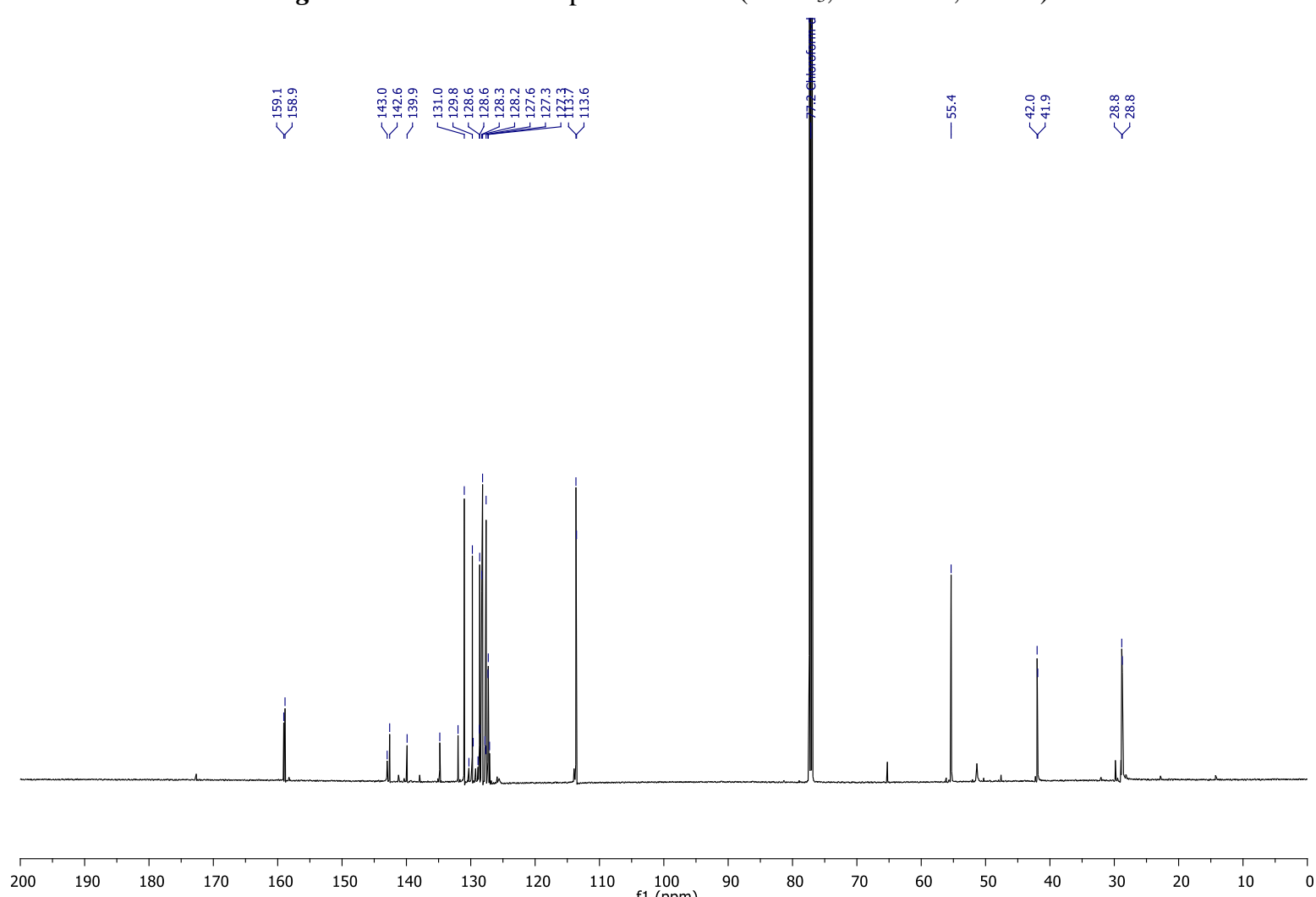

Figure S-178. ${ }^{13} \mathrm{C}$ NMR spectrum of $2 \mathbf{s}\left(\mathrm{CDCl}_{3}, 151 \mathrm{MHz}, 298 \mathrm{~K}\right)$ 


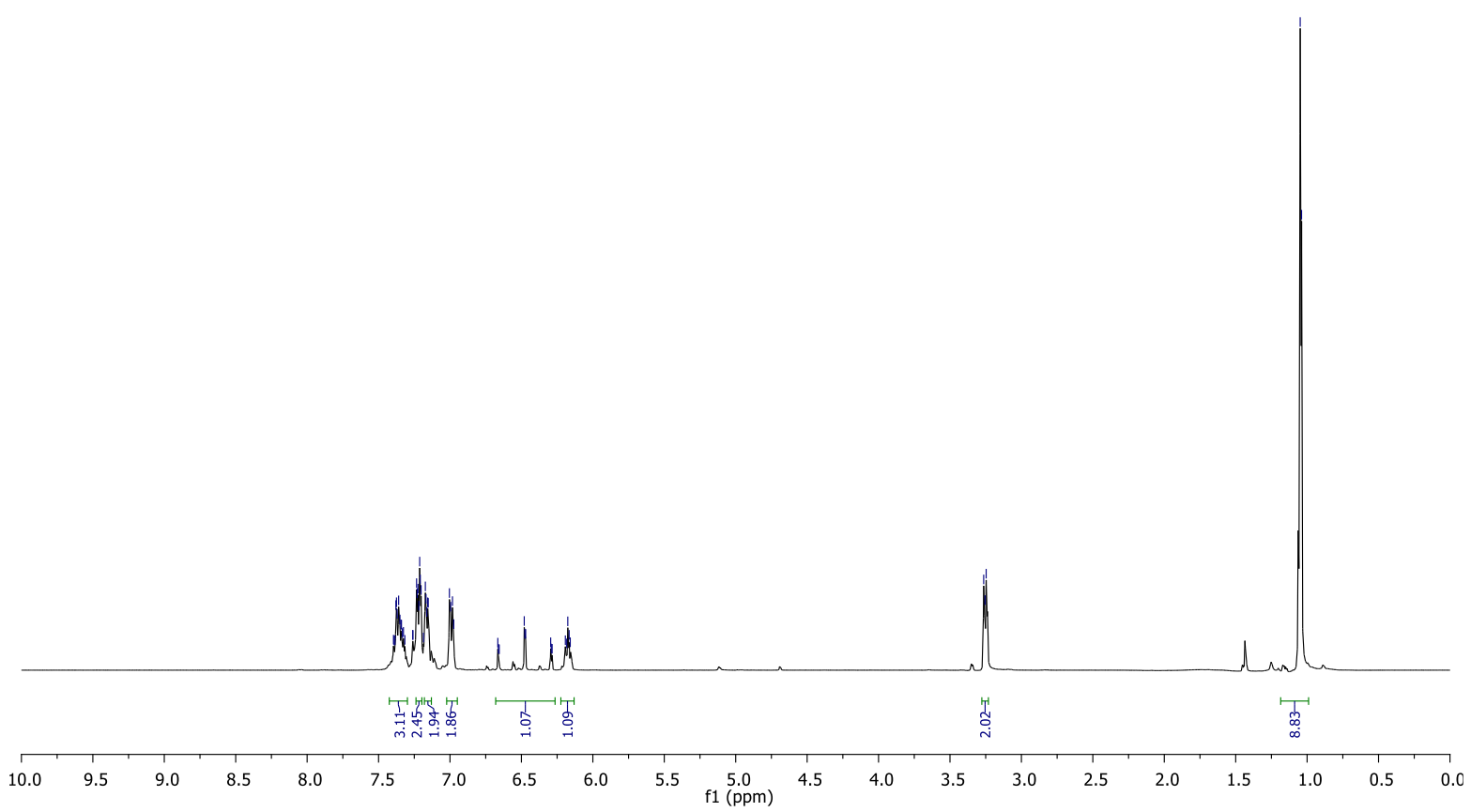

Figure S-179. ${ }^{1} \mathrm{H}$ NMR spectrum of $2 \mathbf{t}\left(\mathrm{CDCl}_{3}, 600 \mathrm{MHz}, 298 \mathrm{~K}\right)$
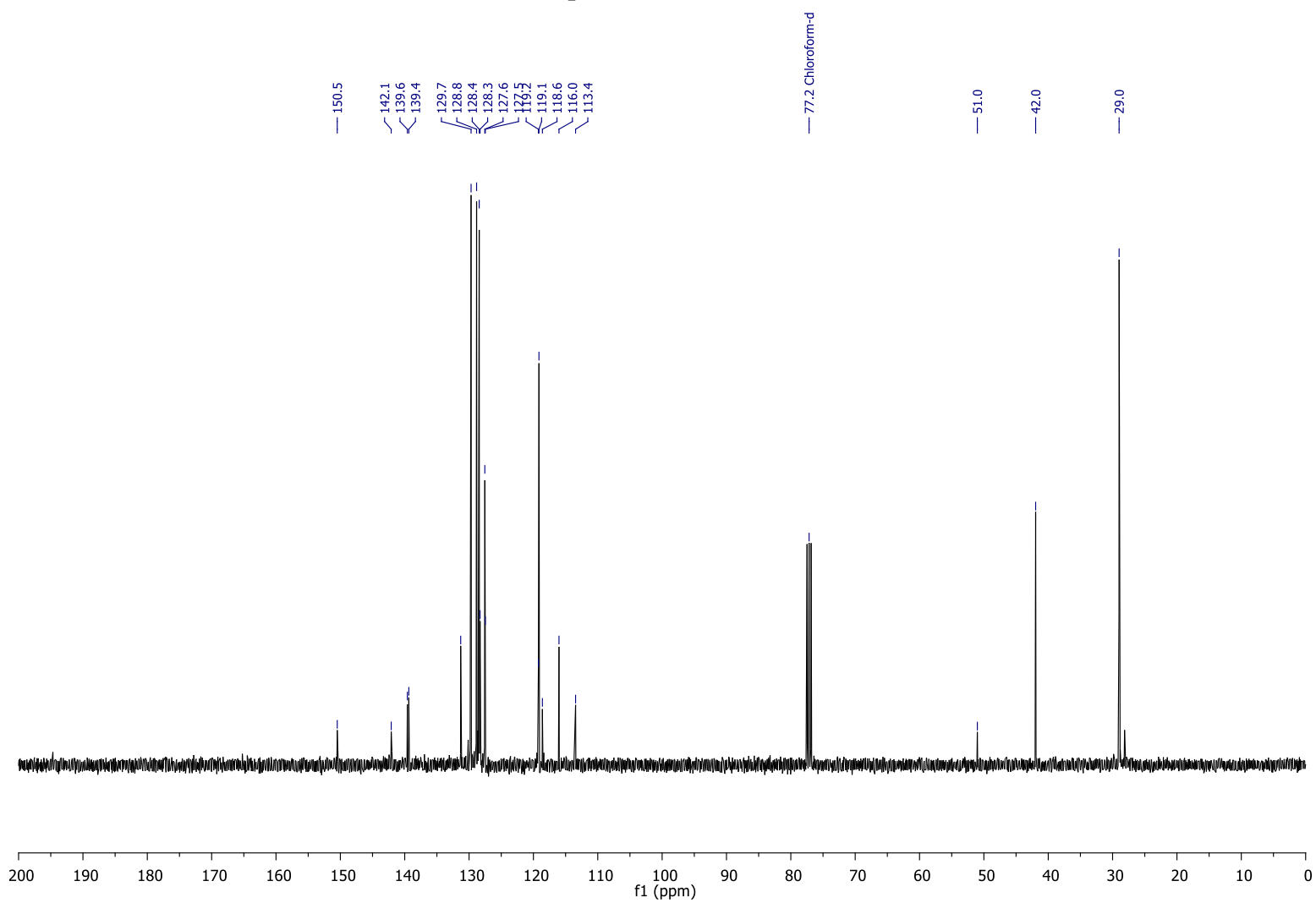

Figure $S$-180. ${ }^{13} \mathrm{C}$ NMR spectrum of $2 \mathbf{t}\left(\mathrm{CDCl}_{3}, 151 \mathrm{MHz}, 298 \mathrm{~K}\right)$ 


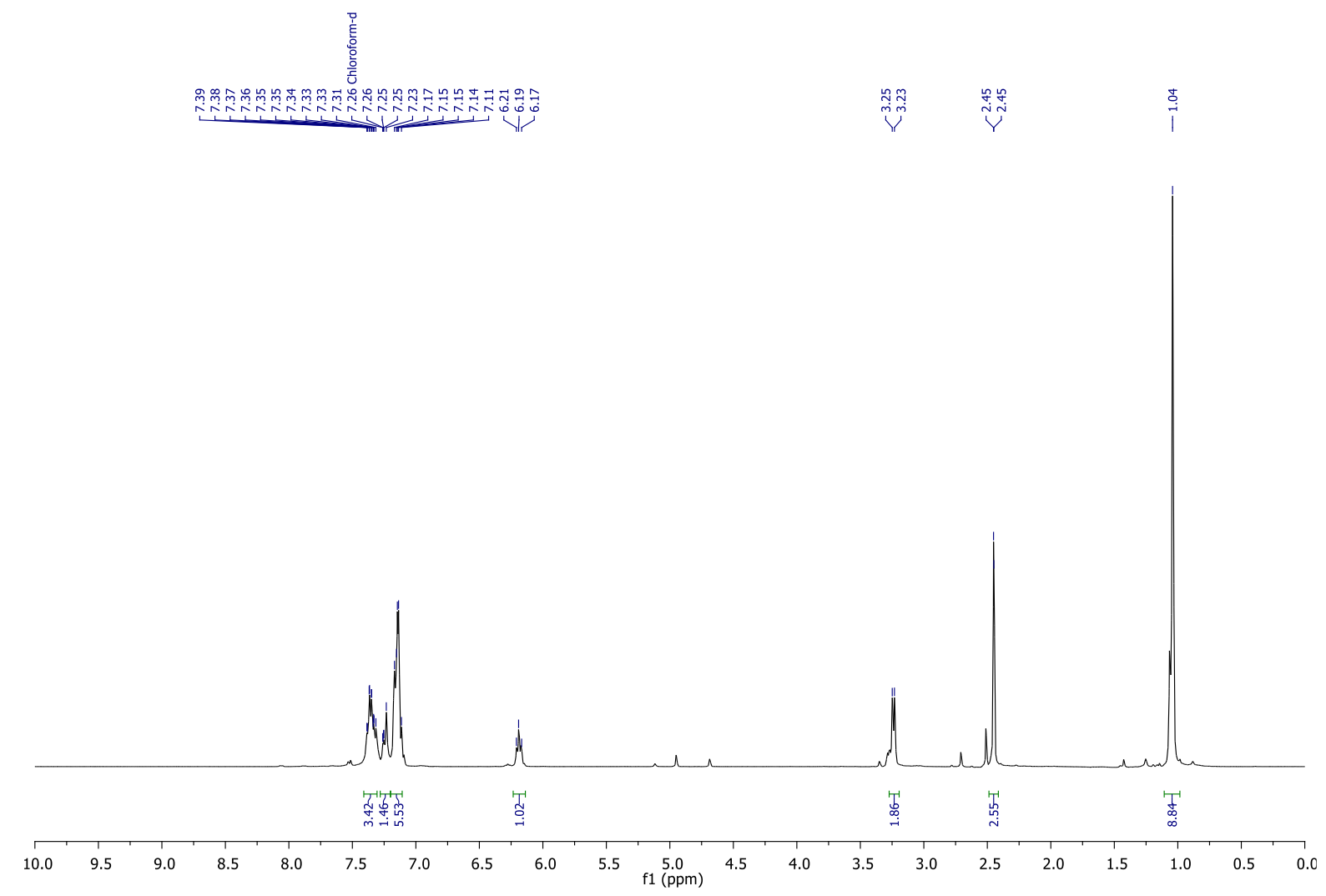

Figure S-181. ${ }^{1} \mathrm{H}$ NMR spectrum of $\mathbf{2 u}\left(\mathrm{CDCl}_{3}, 600 \mathrm{MHz}, 298 \mathrm{~K}\right)$

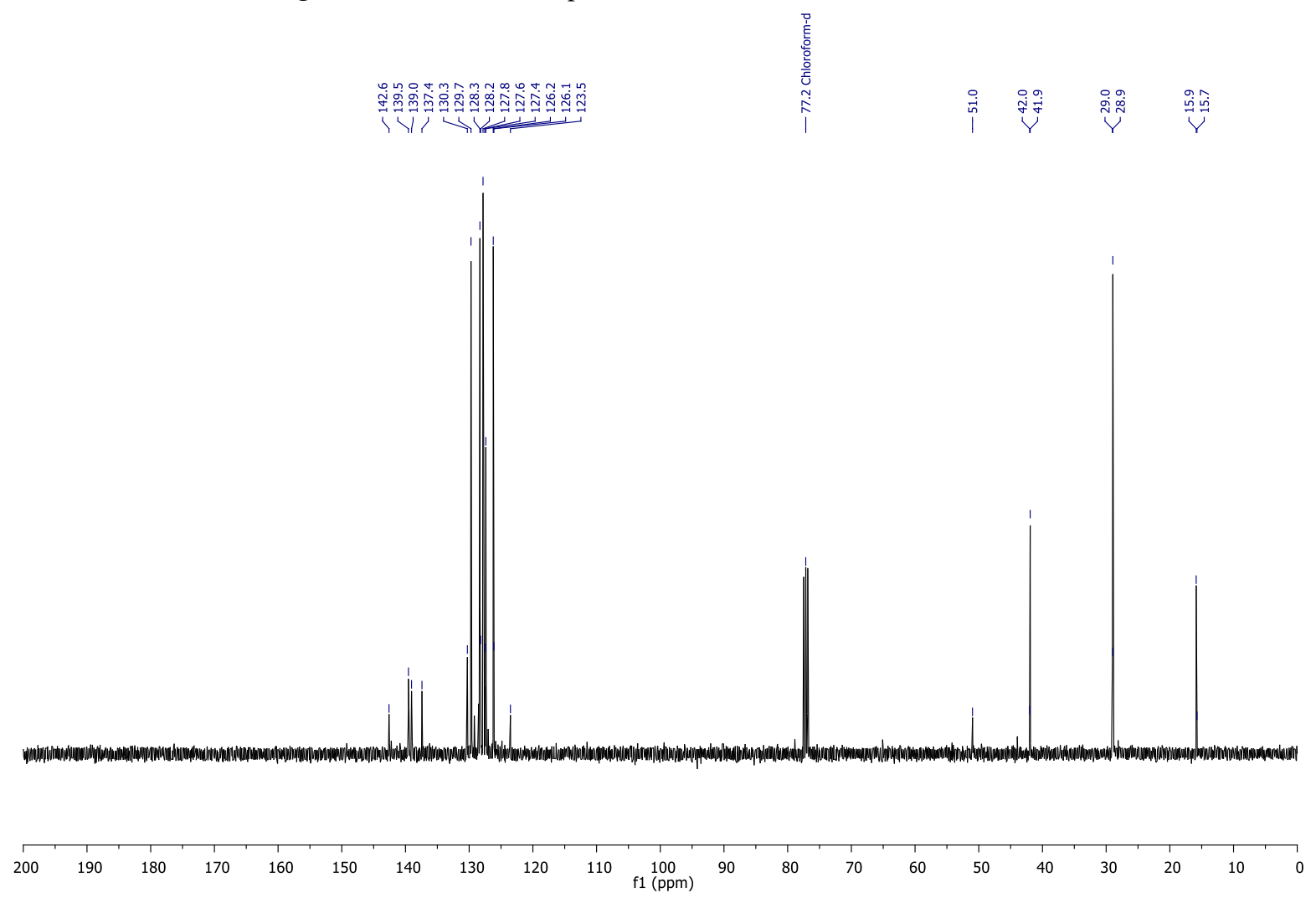

Figure S-182. ${ }^{13} \mathrm{C}$ NMR spectrum of $\mathbf{2 u}\left(\mathrm{CDCl}_{3}, 151 \mathrm{MHz}, 298 \mathrm{~K}\right)$ 


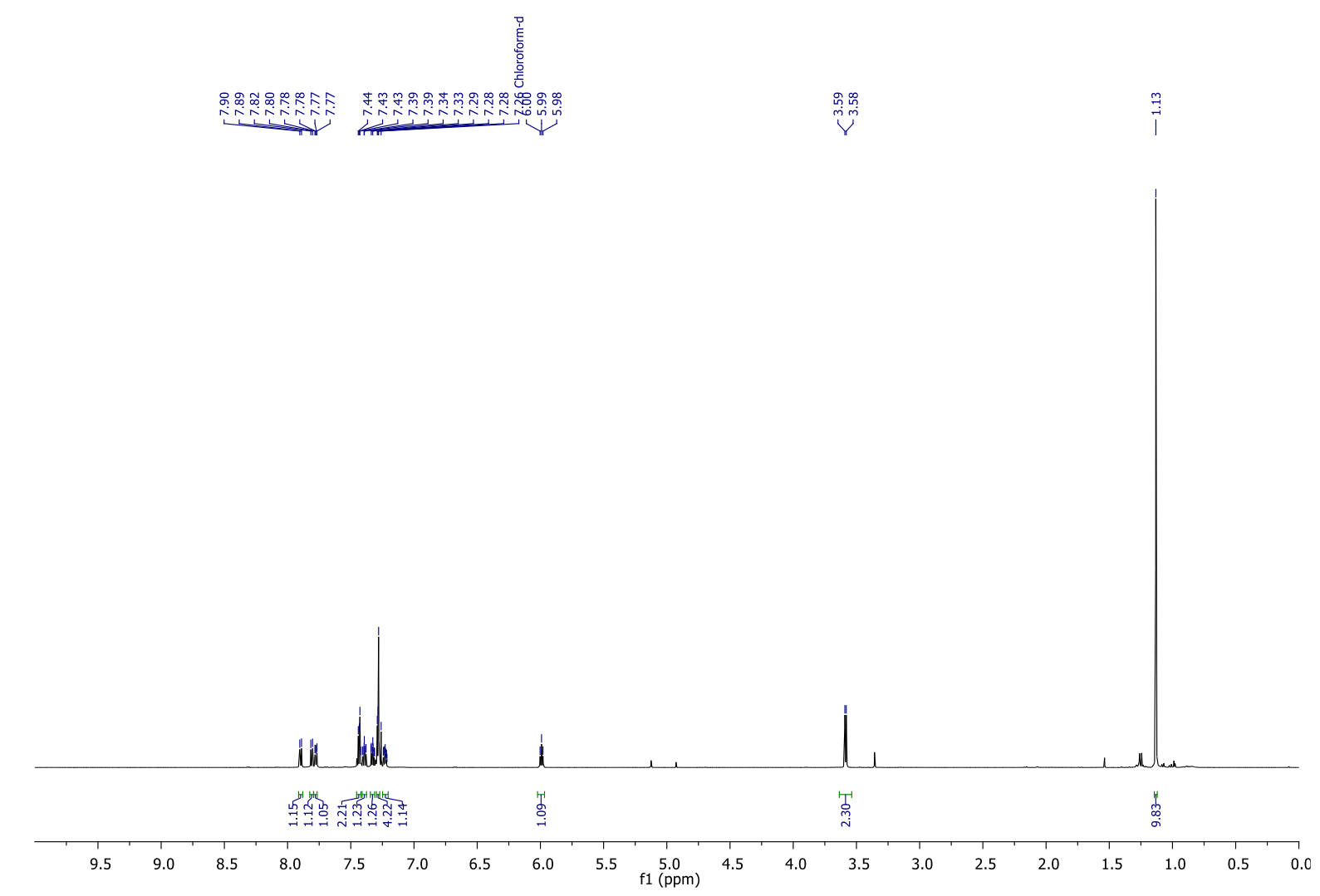

Figure S-183. ${ }^{1} \mathrm{H}$ NMR spectrum of $\mathbf{2 v}\left(\mathrm{CDCl}_{3}, 600 \mathrm{MHz}, 298 \mathrm{~K}\right)$
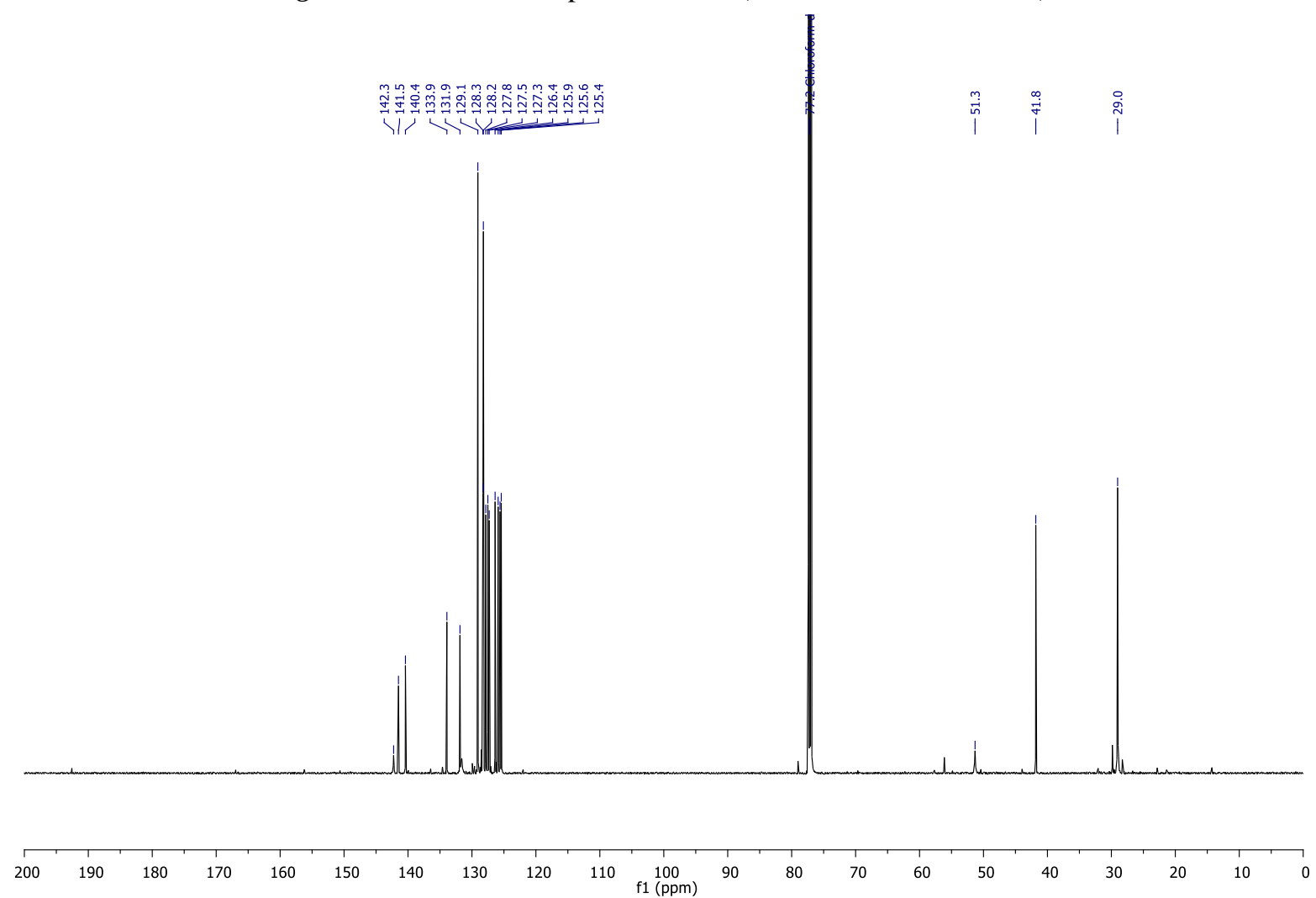

Figure $S$-184. ${ }^{13} \mathrm{C}$ NMR spectrum of $2 \mathbf{v}\left(\mathrm{CDCl}_{3}, 151 \mathrm{MHz}, 298 \mathrm{~K}\right)$ 


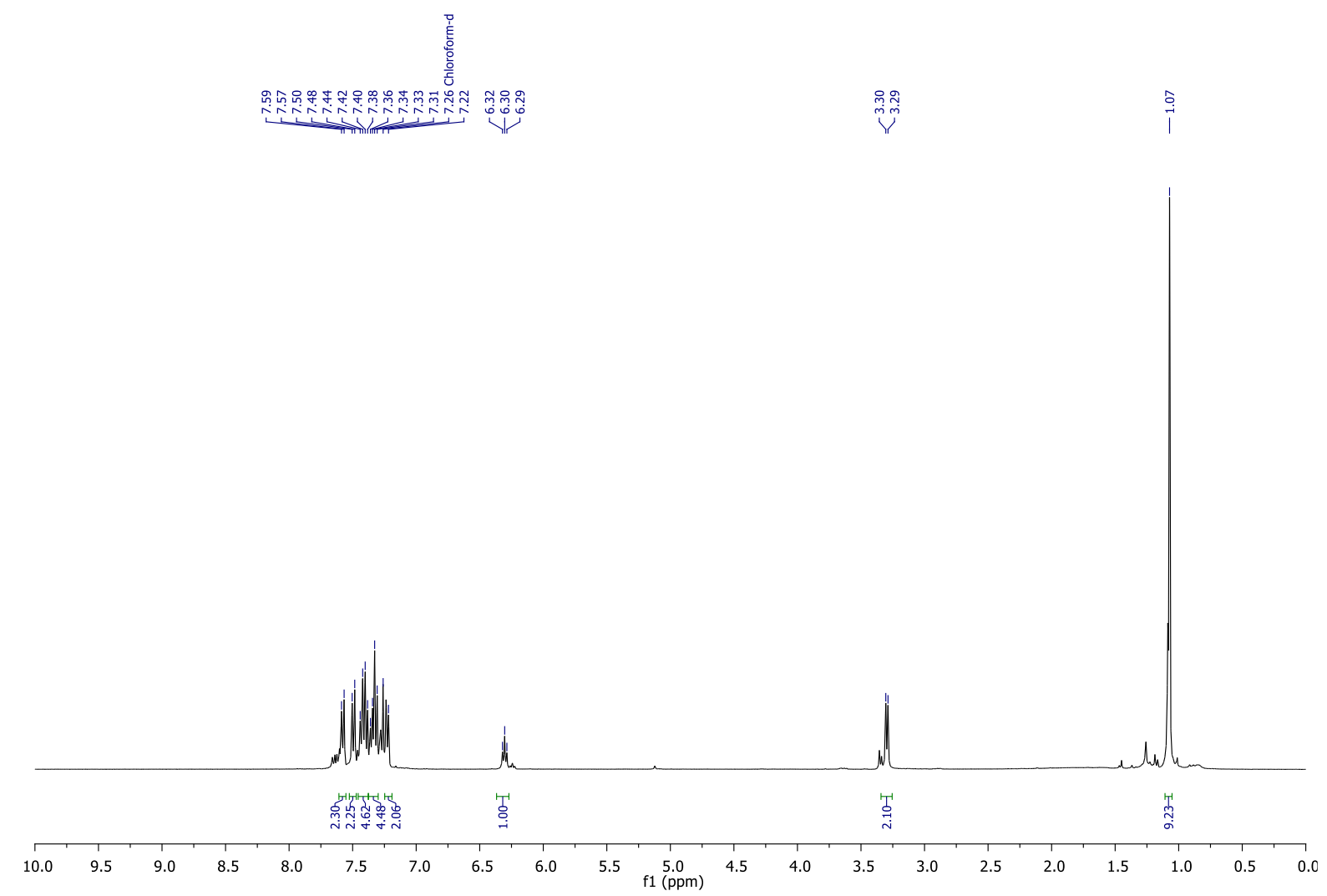

Figure S-185. ${ }^{1} \mathrm{H}$ NMR spectrum of $\mathbf{2} \mathbf{w}\left(\mathrm{CDCl}_{3}, 600 \mathrm{MHz}, 298 \mathrm{~K}\right)$
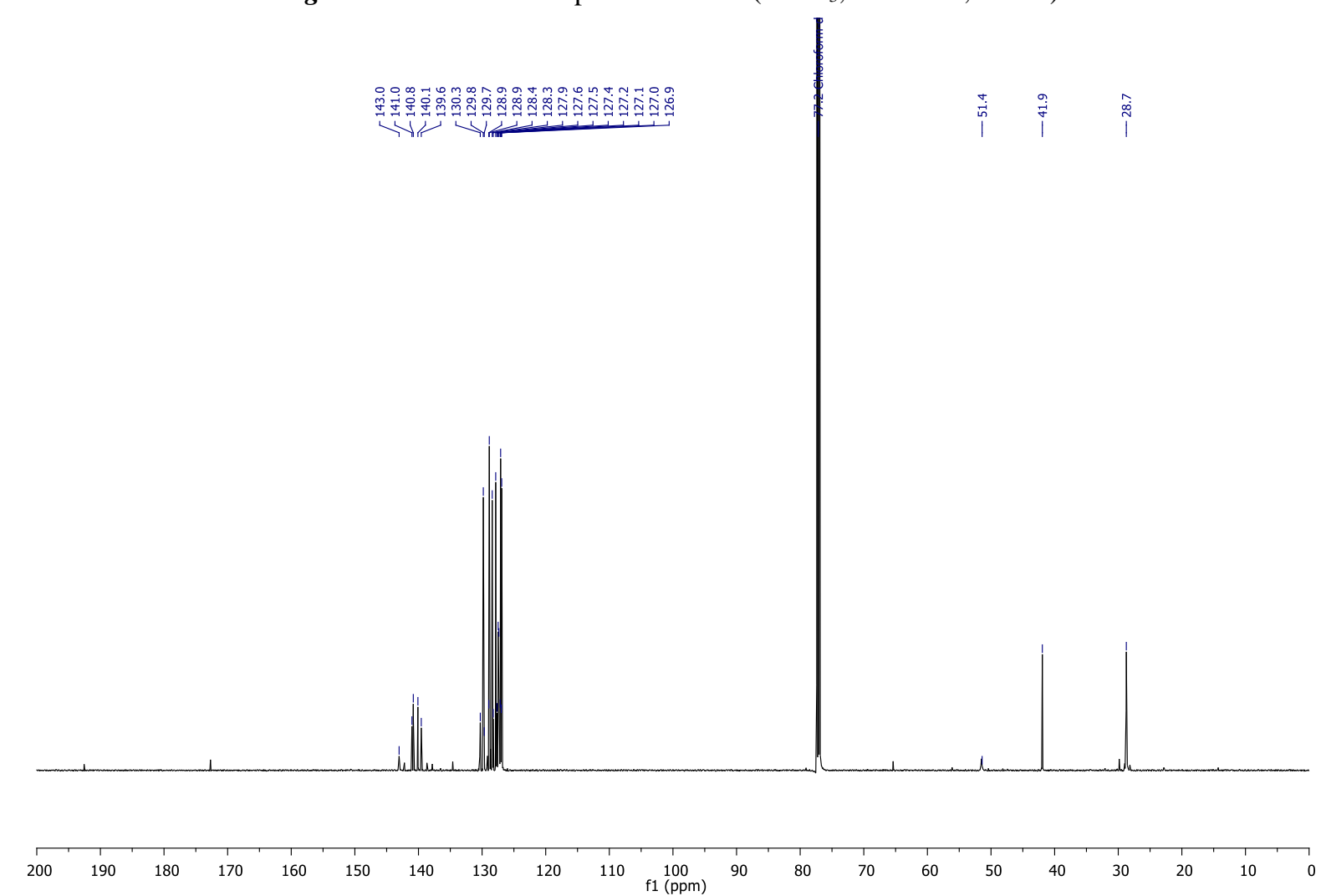

Figure $\mathbf{S}$-186. ${ }^{13} \mathrm{C}$ NMR spectrum of $\mathbf{2} \mathbf{w}\left(\mathrm{CDCl}_{3}, 151 \mathrm{MHz}, 298 \mathrm{~K}\right)$ 


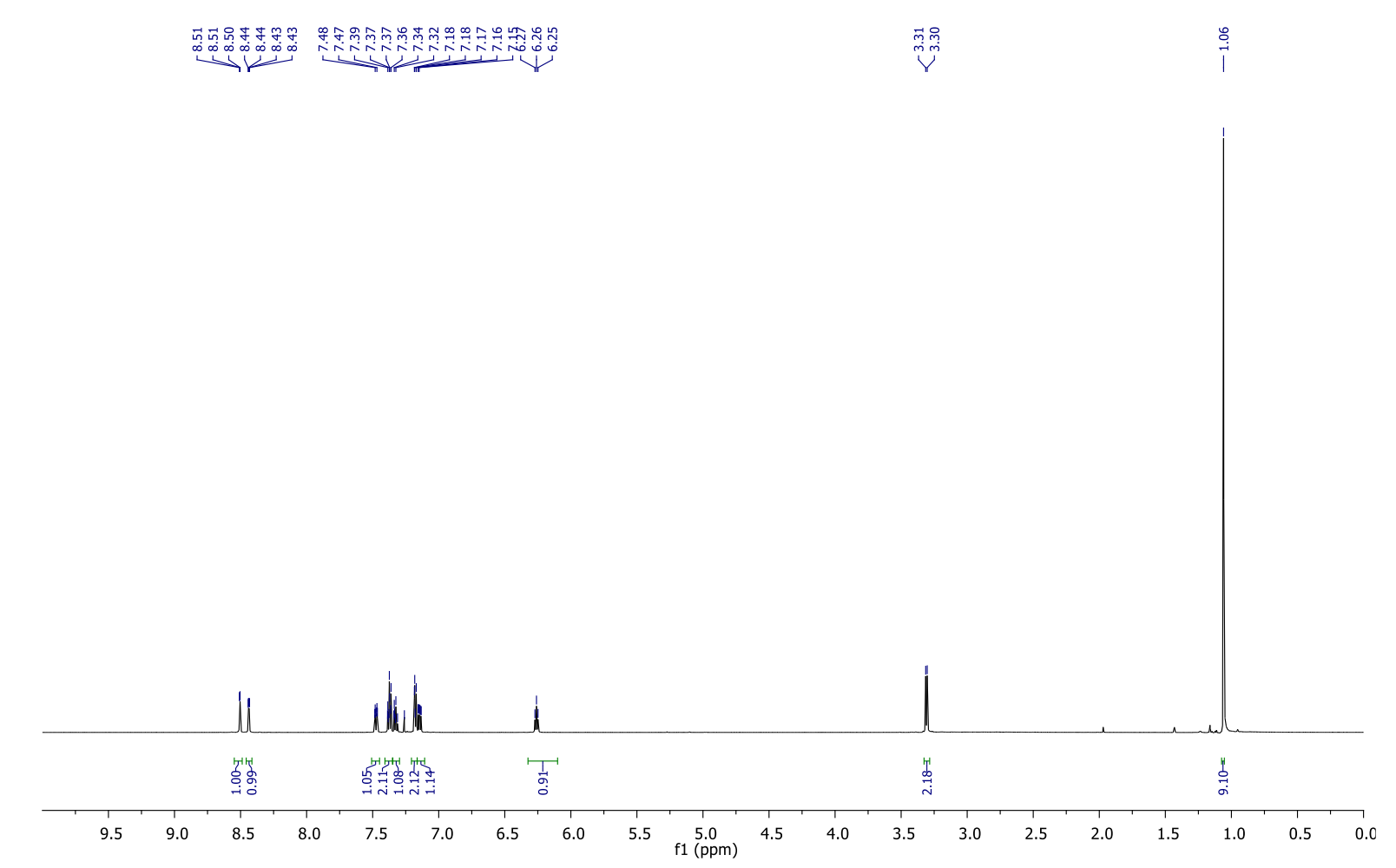

Figure S-187. ${ }^{1} \mathrm{H}$ NMR spectrum of $\mathbf{2 x}\left(\mathrm{CDCl}_{3}, 600 \mathrm{MHz}, 298 \mathrm{~K}\right)$
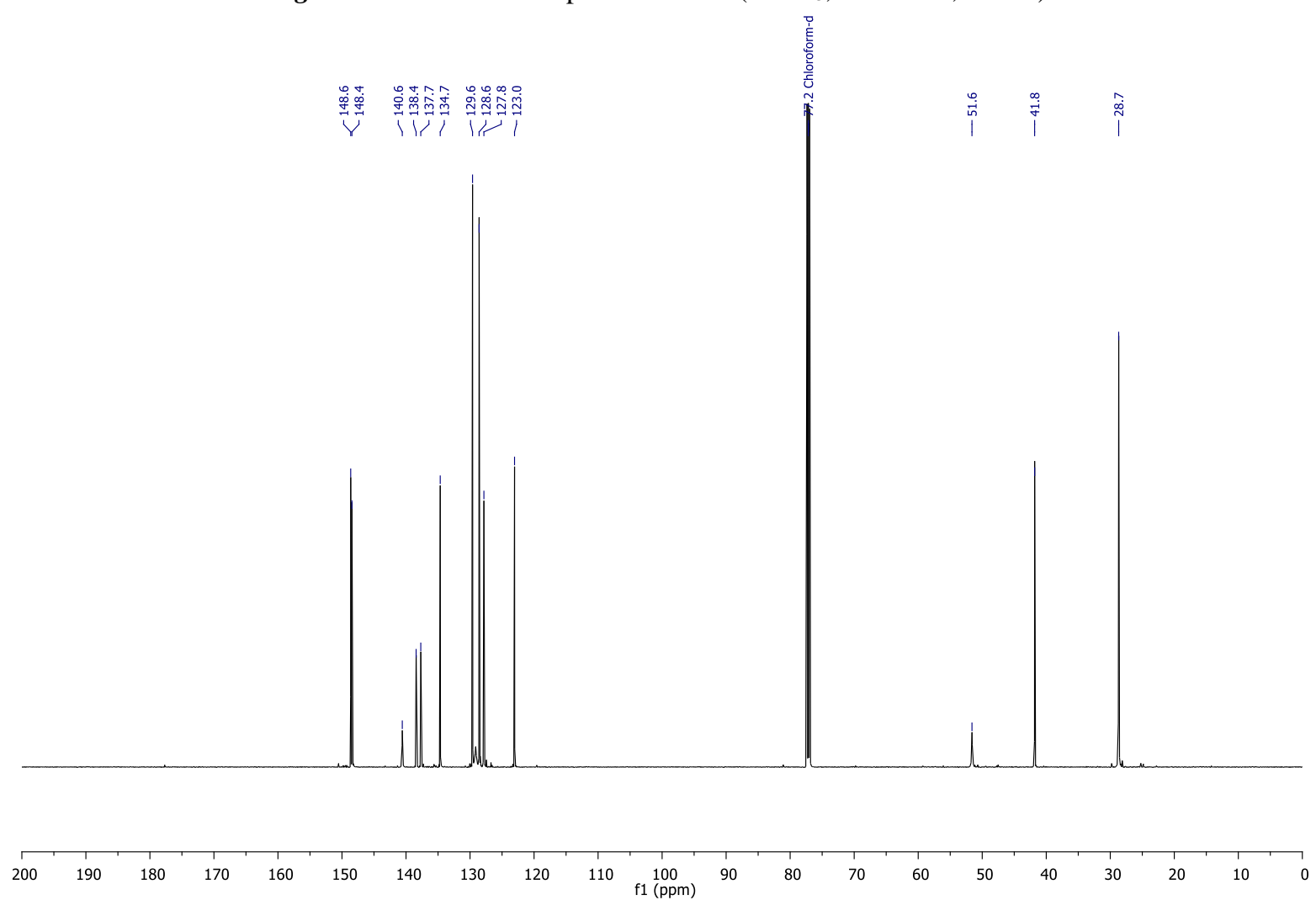

Figure $\mathbf{S}$-188. ${ }^{13} \mathrm{C}$ NMR spectrum of $\mathbf{2 x}\left(\mathrm{CDCl}_{3}, 151 \mathrm{MHz}, 298 \mathrm{~K}\right)$ 


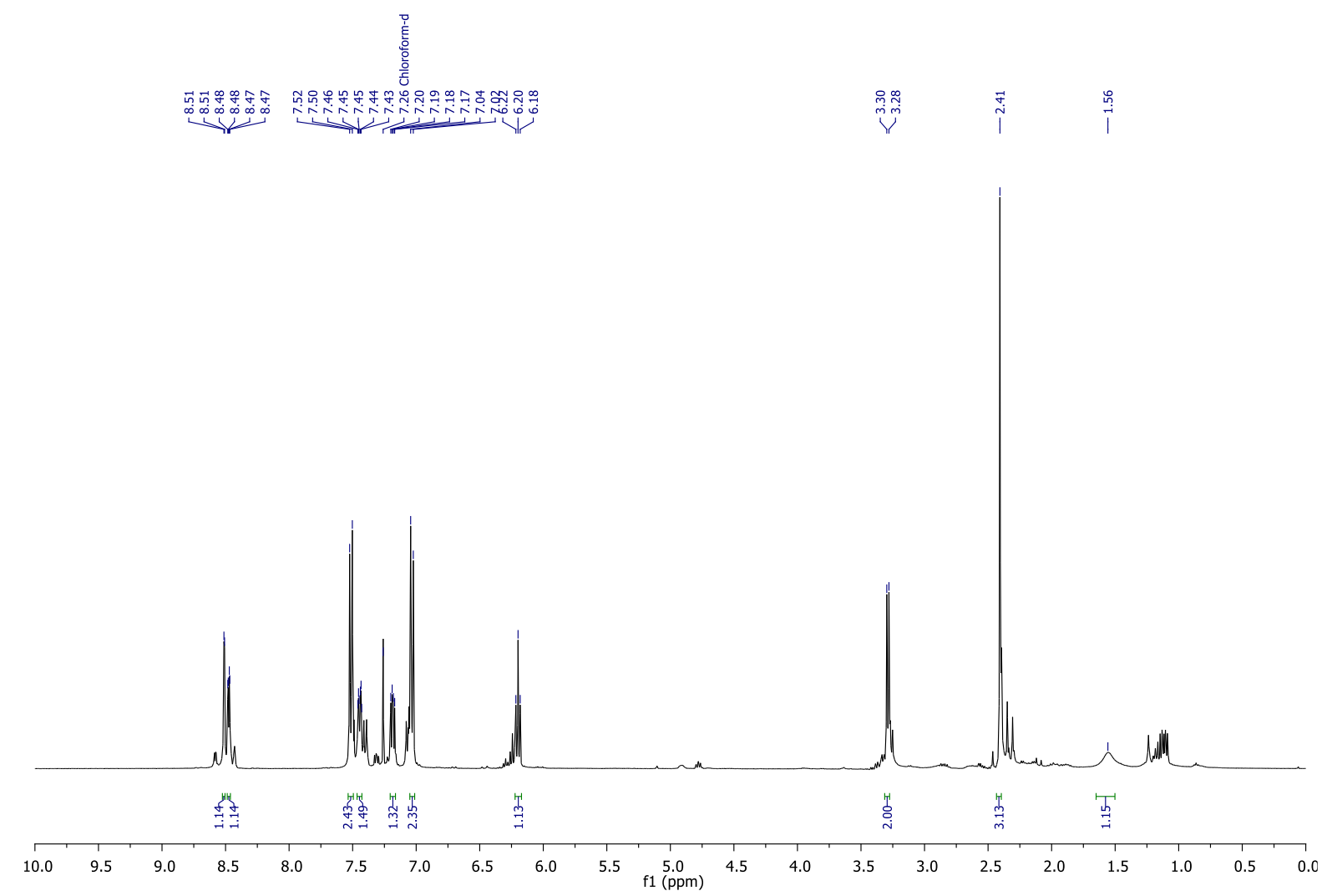

Figure S-189. ${ }^{1} \mathrm{H}$ NMR spectrum of $\mathbf{2 y}\left(\mathrm{CDCl}_{3}, 600 \mathrm{MHz}, 298 \mathrm{~K}\right)$
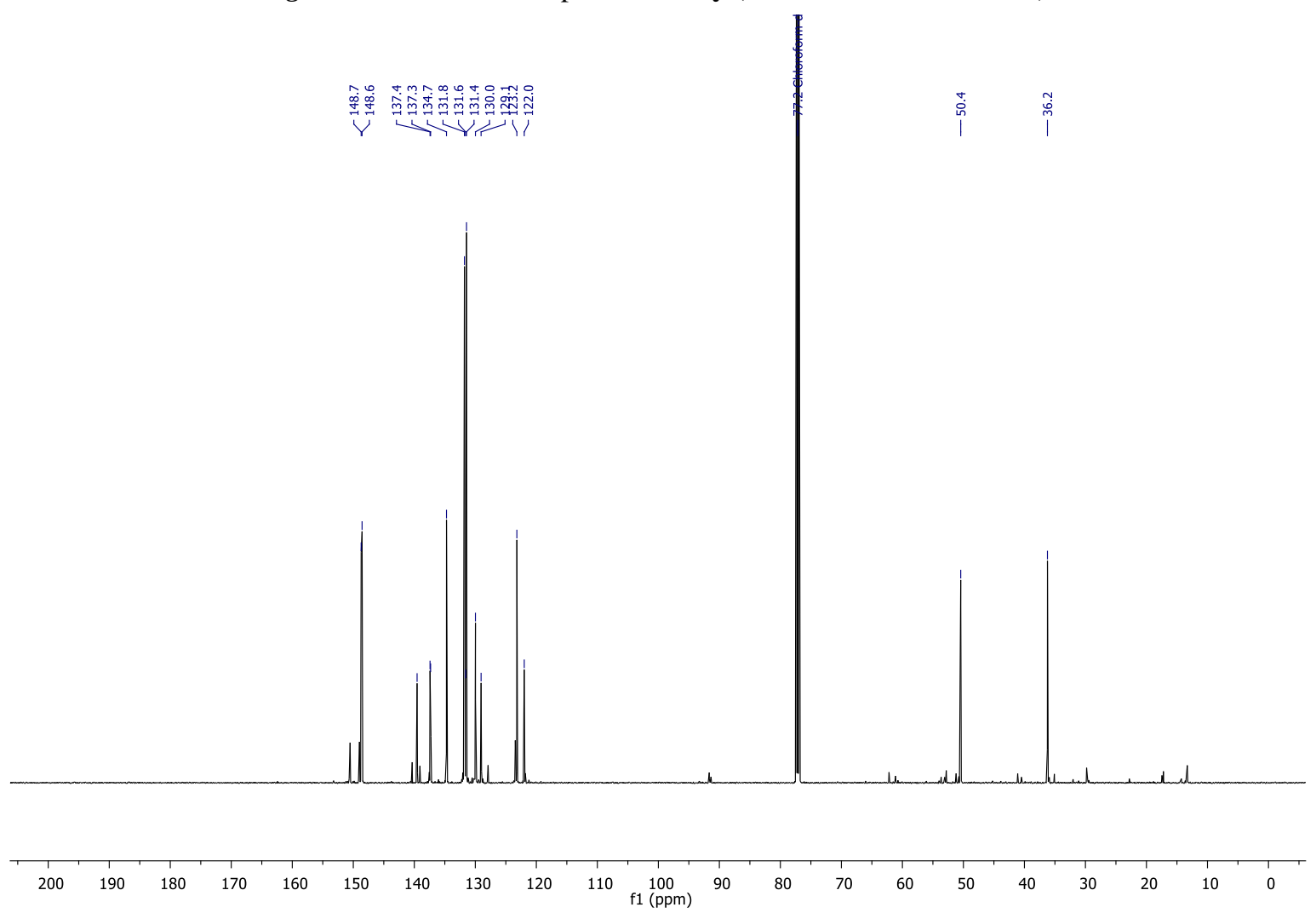

Figure $S$-190. ${ }^{13} \mathrm{C}$ NMR spectrum of $2 \mathbf{y}\left(\mathrm{CDCl}_{3}, 151 \mathrm{MHz}, 298 \mathrm{~K}\right)$ 


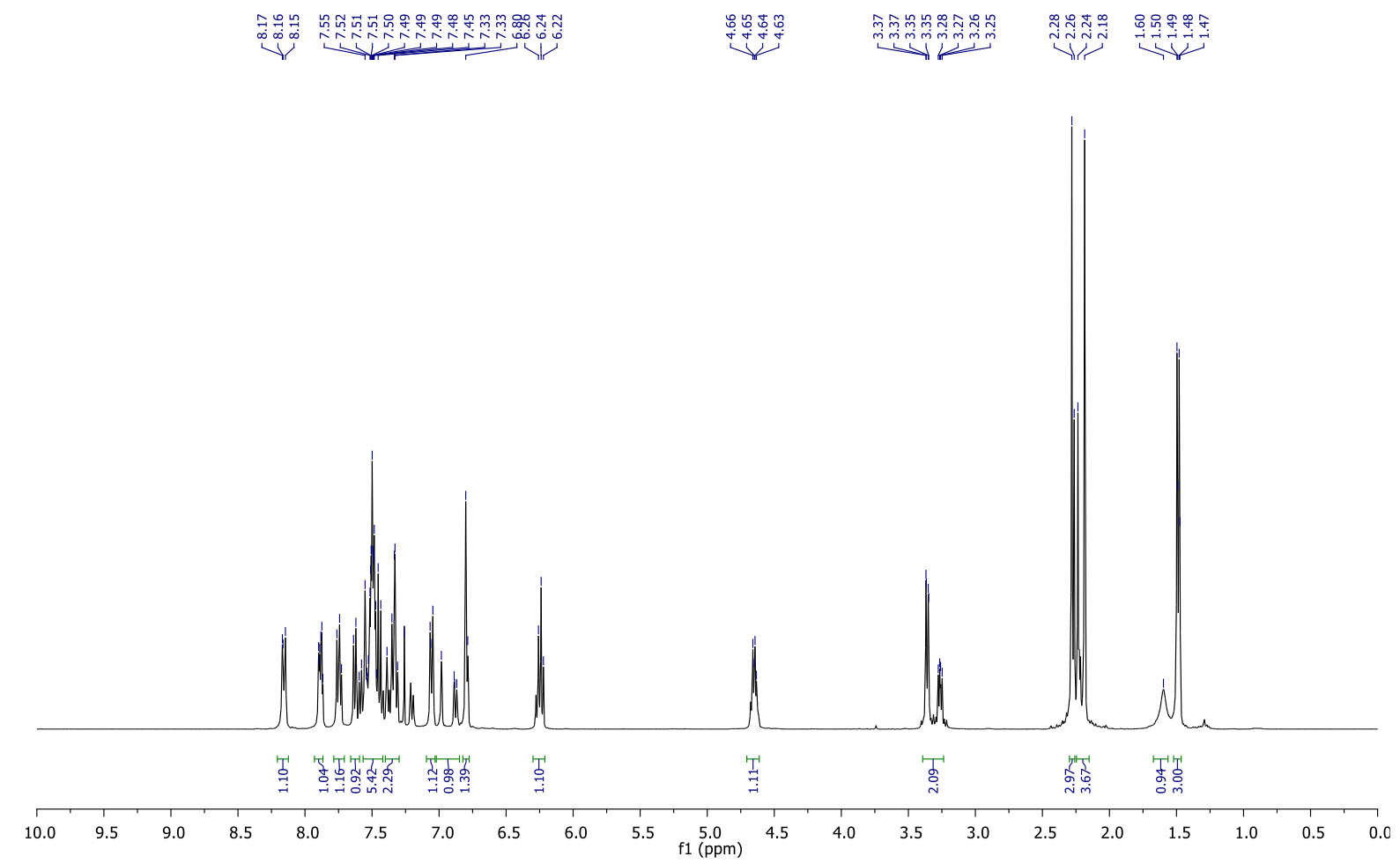

Figure S-191. ${ }^{1} \mathrm{H}$ NMR spectrum of $\mathbf{2 z}\left(\mathrm{CDCl}_{3}, 600 \mathrm{MHz}, 298 \mathrm{~K}\right)$
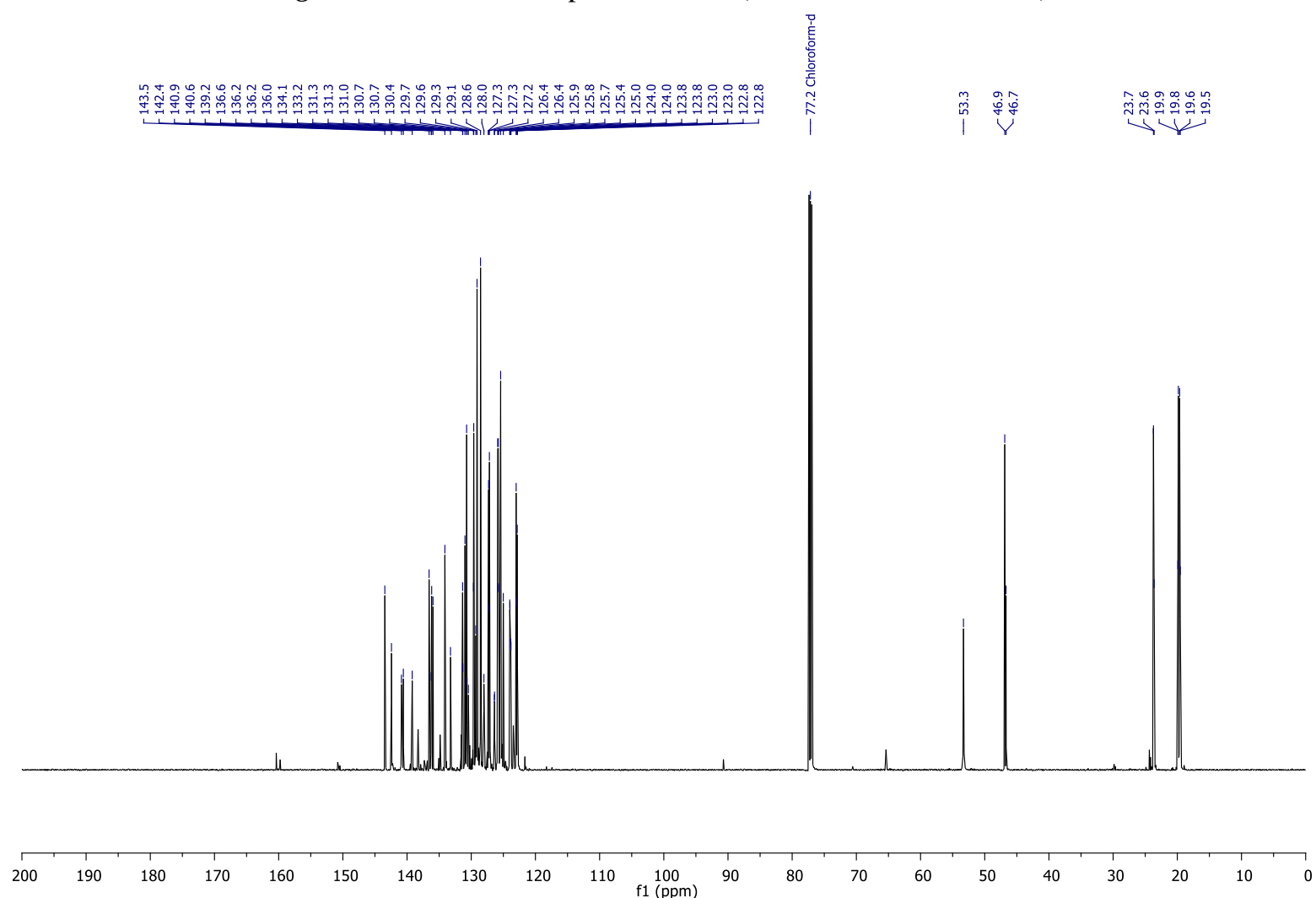

Figure $\mathbf{S}$-192. ${ }^{13} \mathrm{C}$ NMR spectrum of $\mathbf{2 z}\left(\mathrm{CDCl}_{3}, 151 \mathrm{MHz}, 298 \mathrm{~K}\right)$ 


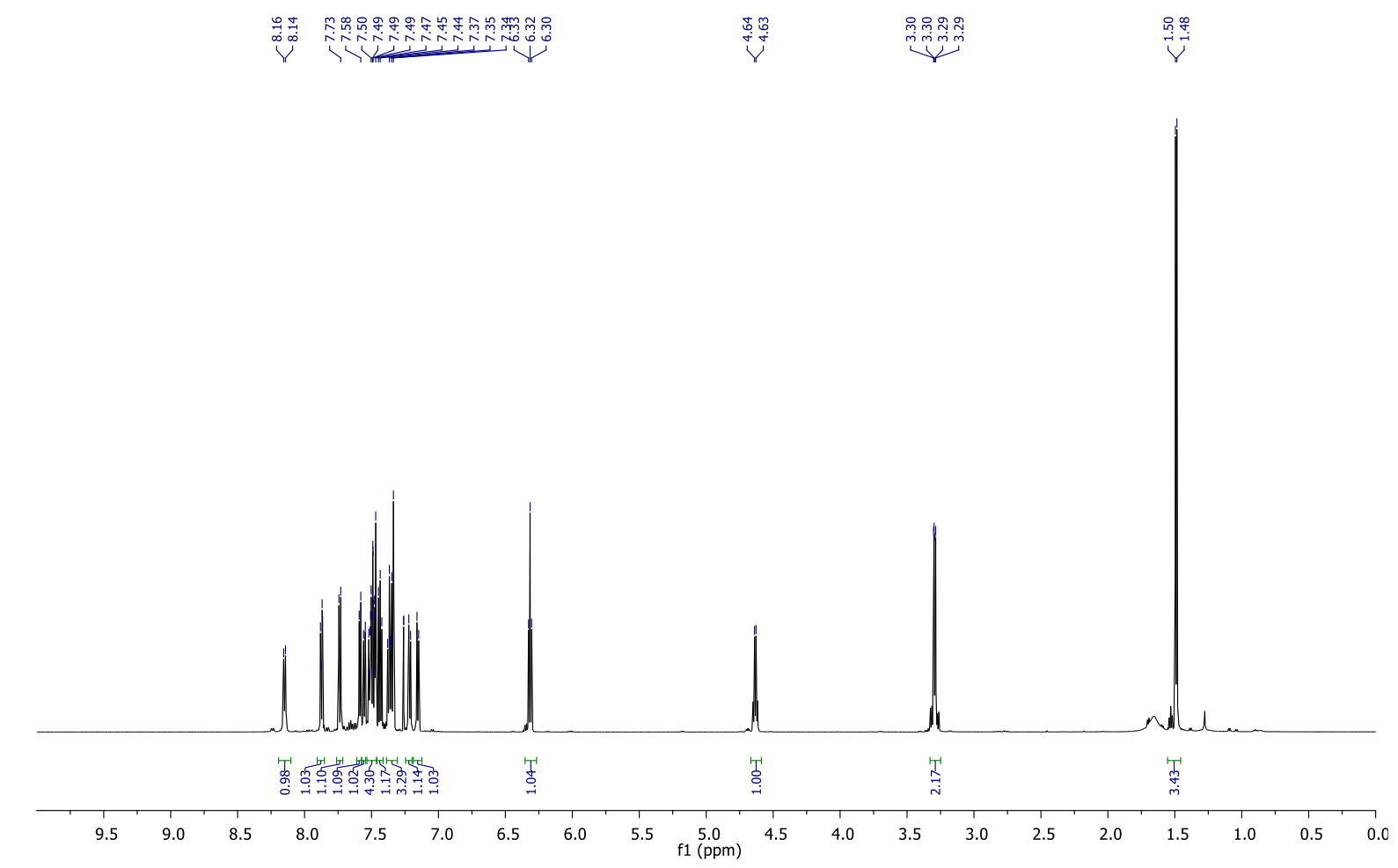

Figure S-193. ${ }^{1} \mathrm{H}$ NMR spectrum of $\mathbf{2 a a}\left(\mathrm{CDCl}_{3}, 600 \mathrm{MHz}, 298 \mathrm{~K}\right)$
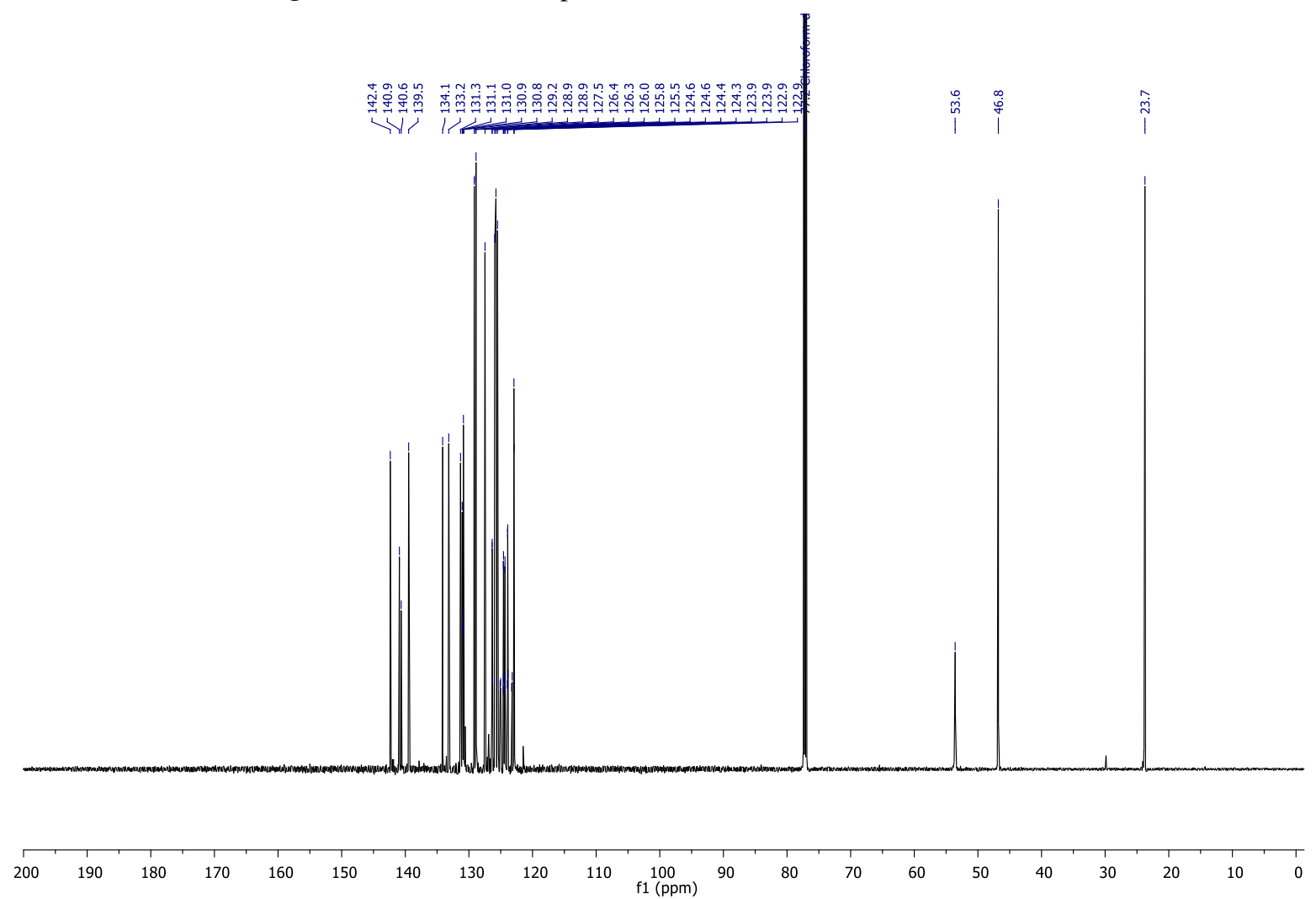

Figure $\mathbf{S - 1 9 4 .}{ }^{13} \mathrm{C} \mathrm{NMR}$ spectrum of $\mathbf{2} \mathbf{a a}\left(\mathrm{CDCl}_{3}, 151 \mathrm{MHz}, 298 \mathrm{~K}\right)$ 


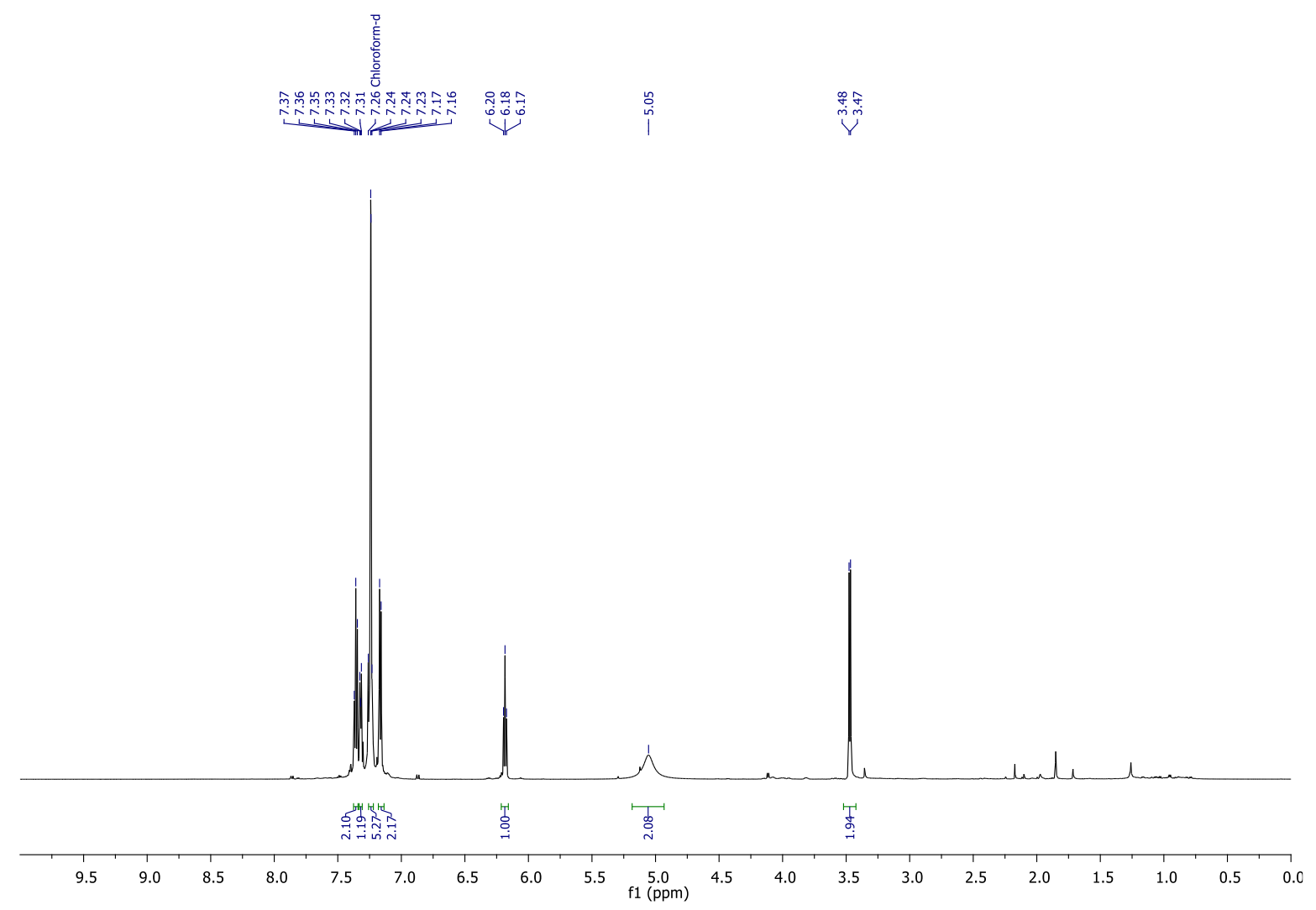

Figure S-195. ${ }^{1} \mathrm{H}$ NMR spectrum of $\mathbf{3 a}\left(\mathrm{CDCl}_{3}, 600 \mathrm{MHz}, 298 \mathrm{~K}\right)$

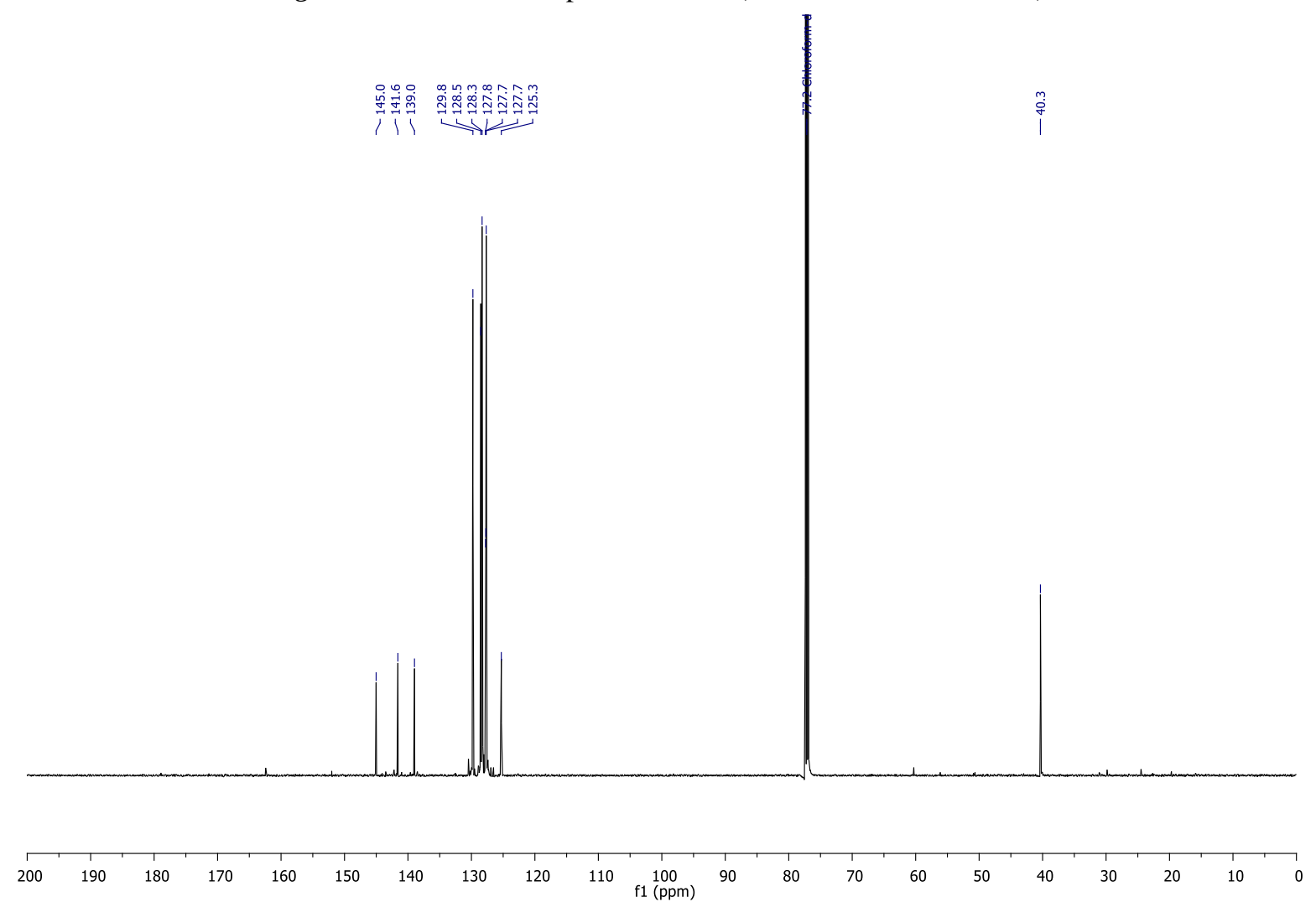

Figure $\mathbf{S - 1 9 6 .}{ }^{13} \mathrm{C}$ NMR spectrum of $\mathbf{3 a}\left(\mathrm{CDCl}_{3}, 151 \mathrm{MHz}, 298 \mathrm{~K}\right)$ 


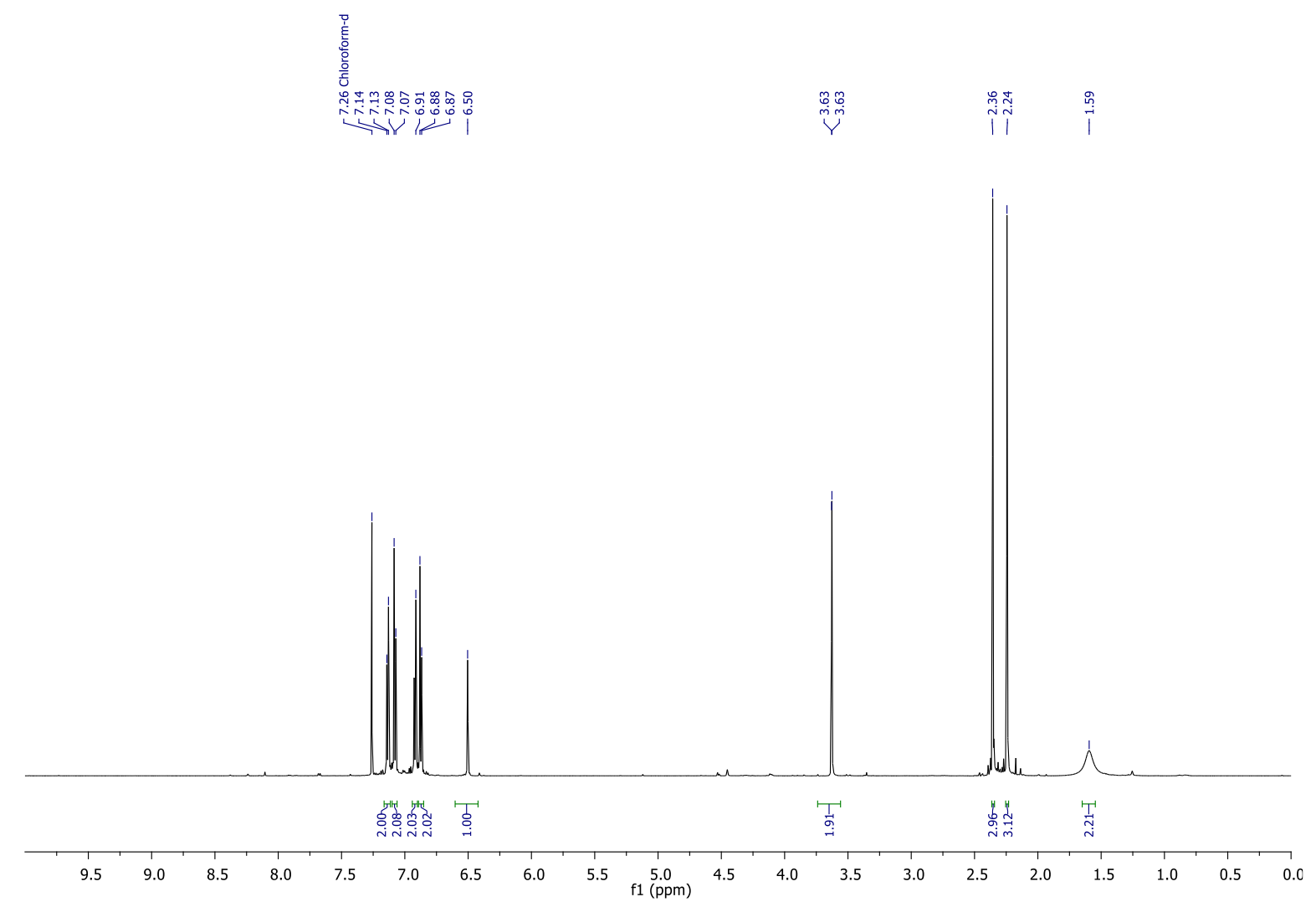

Figure S-197. ${ }^{1} \mathrm{H}$ NMR spectrum of $\mathbf{3 b}\left(\mathrm{CDCl}_{3}, 600 \mathrm{MHz}, 298 \mathrm{~K}\right)$

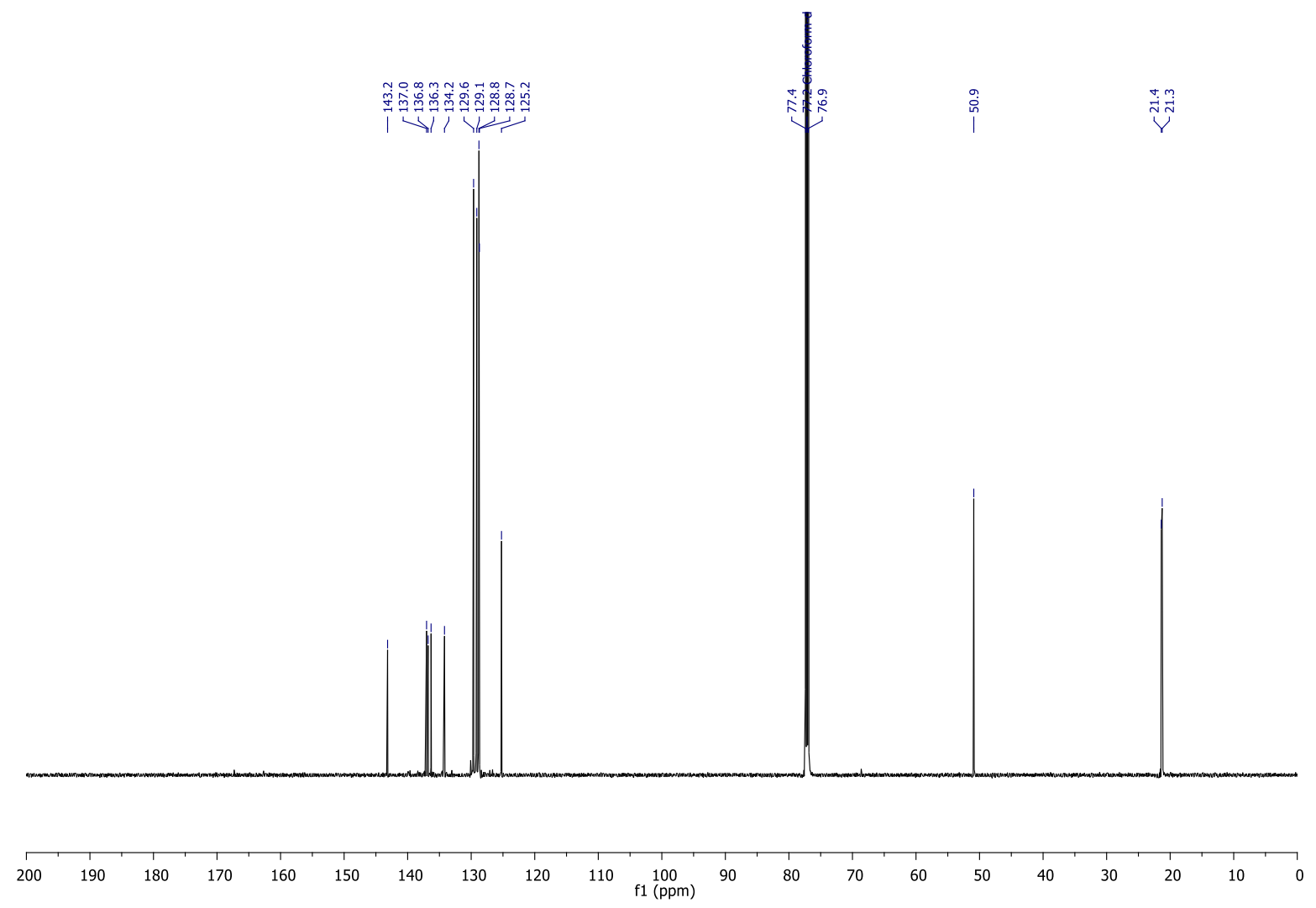

Figure $\mathbf{S}$-198. ${ }^{13} \mathrm{C}$ NMR spectrum of $\mathbf{3 b}\left(\mathrm{CDCl}_{3}, 151 \mathrm{MHz}, 298 \mathrm{~K}\right)$ 

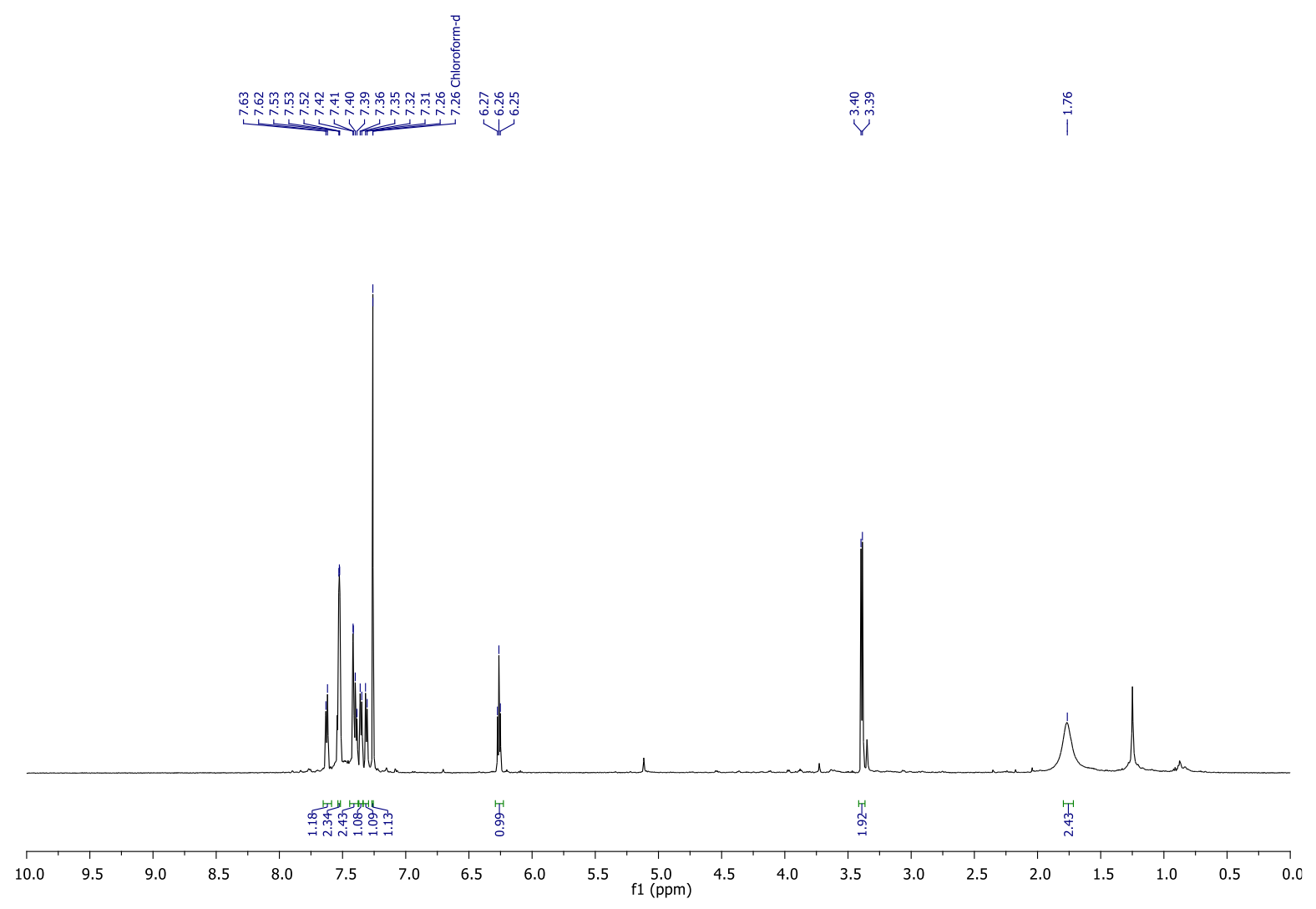

Figure S-199. ${ }^{1} \mathrm{H}$ NMR spectrum of $3 \mathbf{c}\left(\mathrm{CDCl}_{3}, 600 \mathrm{MHz}, 298 \mathrm{~K}\right)$

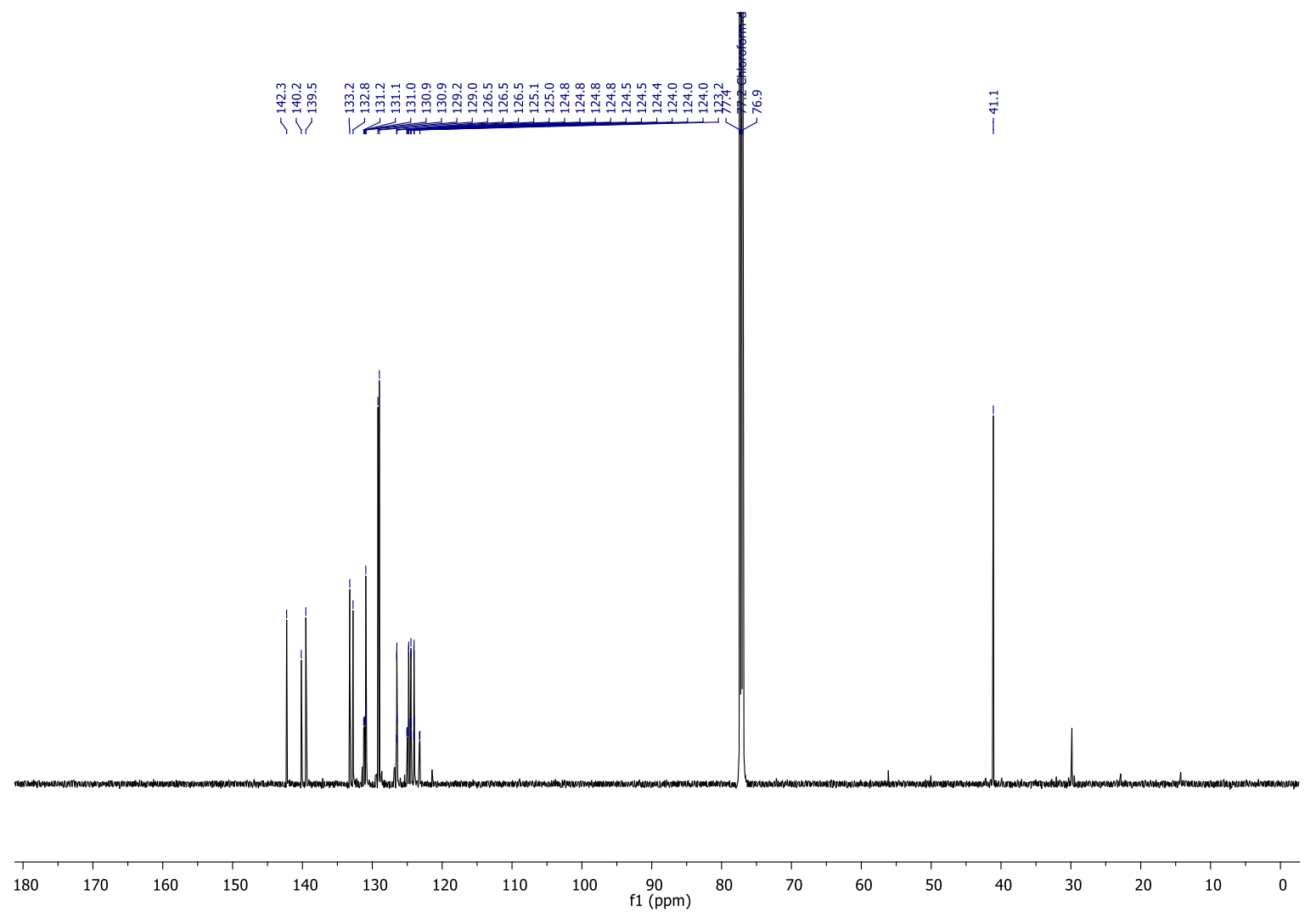

Figure S-200. ${ }^{13} \mathrm{C}$ NMR spectrum of $\mathbf{3 c}\left(\mathrm{CDCl}_{3}, 151 \mathrm{MHz}, 298 \mathrm{~K}\right)$ 

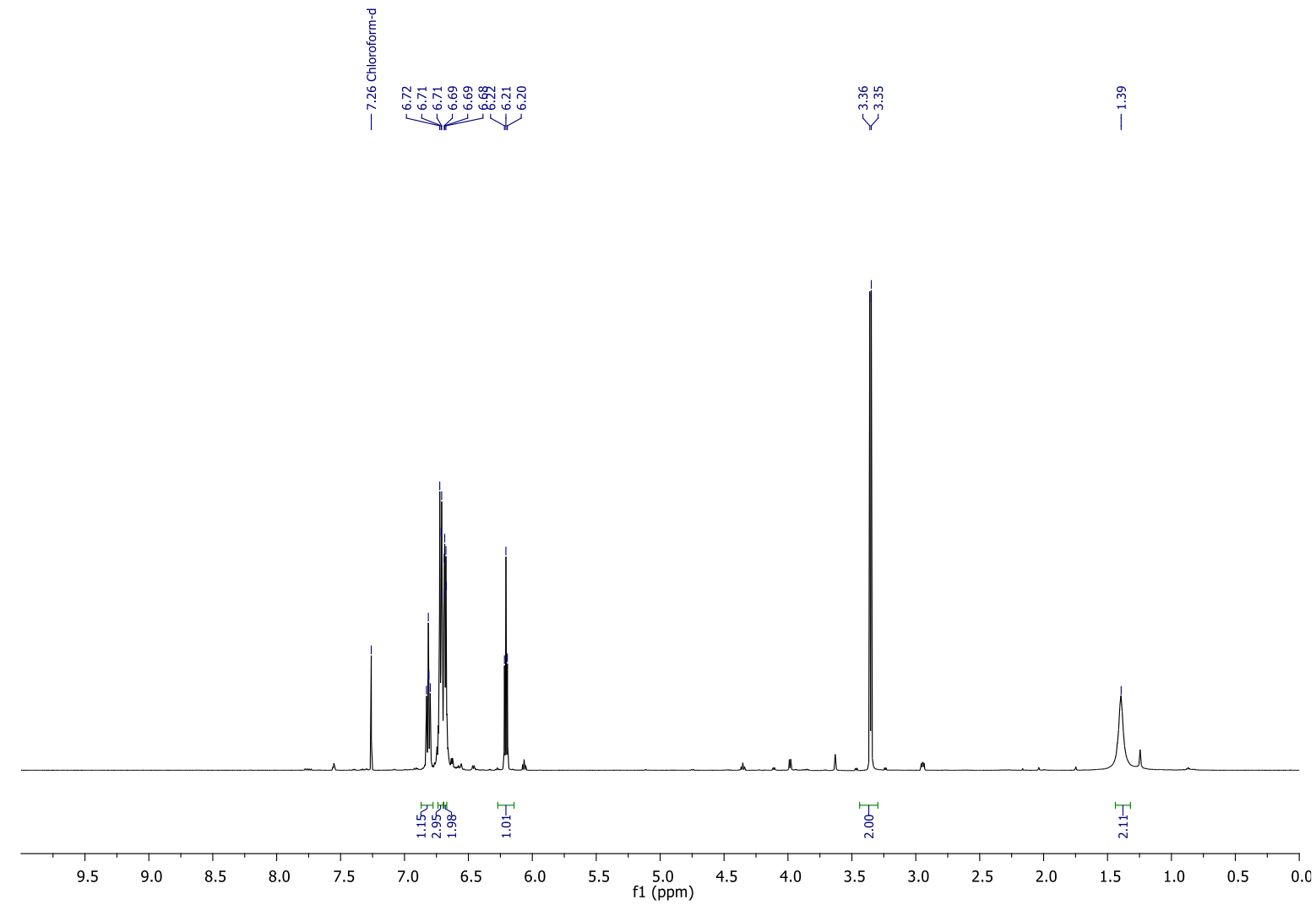

Figure S-201. ${ }^{1} \mathrm{H}$ NMR spectrum of $\mathbf{3 d}\left(\mathrm{CDCl}_{3}, 600 \mathrm{MHz}, 298 \mathrm{~K}\right)$

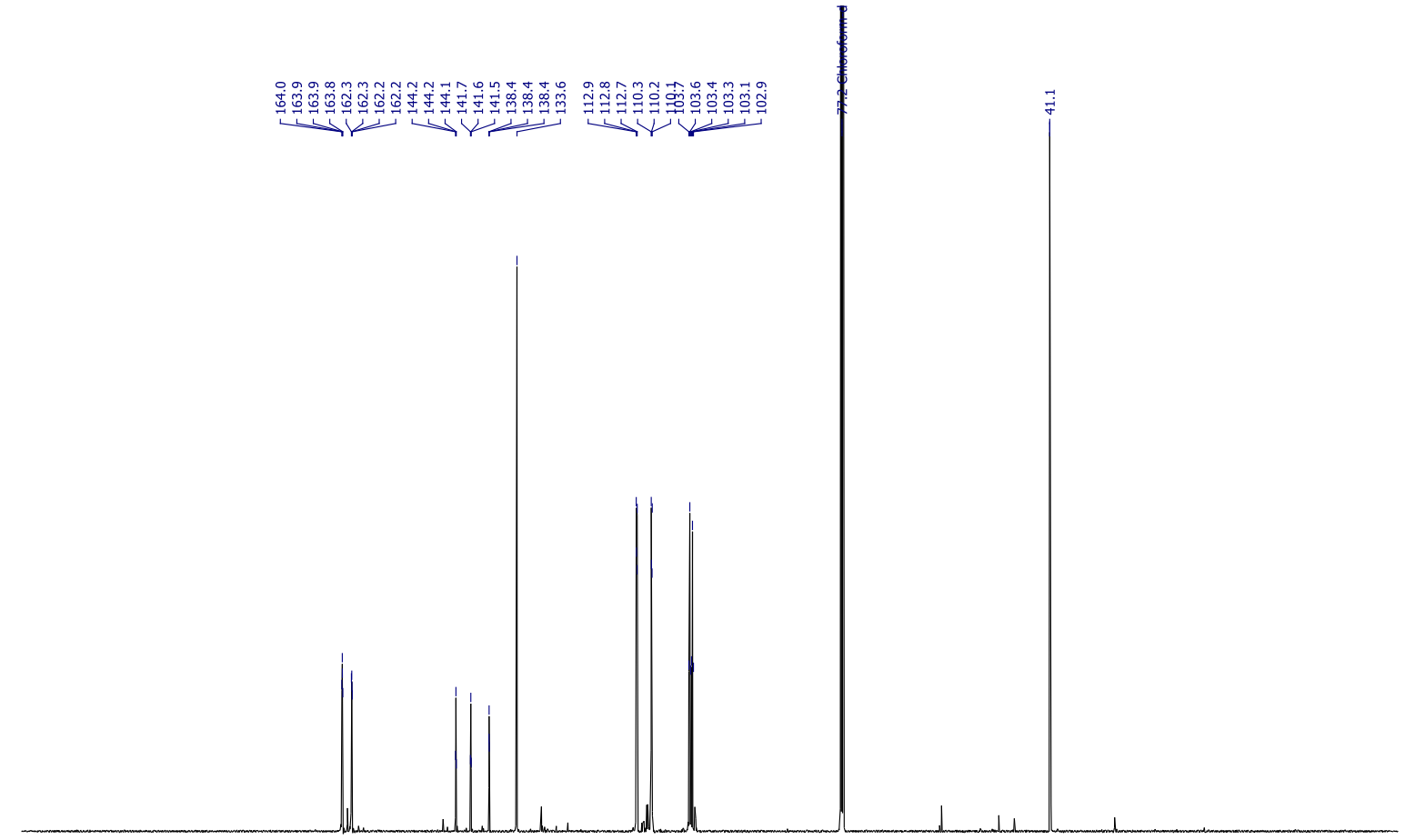

$\begin{array}{llllllllllllllllllllllll}210 & 200 & 190 & 180 & 170 & 160 & 150 & 140 & 130 & 120 & 110 & 100 & 90 & 80 & 70 & 60 & 50 & 40 & 30 & 20 & 10 & 0 & -10 & \end{array}$

Figure $\mathbf{S}$-202. ${ }^{13} \mathrm{C}$ NMR spectrum of $\mathbf{3 d}\left(\mathrm{CDCl}_{3}, 151 \mathrm{MHz}, 298 \mathrm{~K}\right)$ 


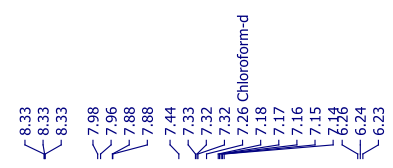

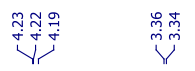

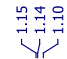

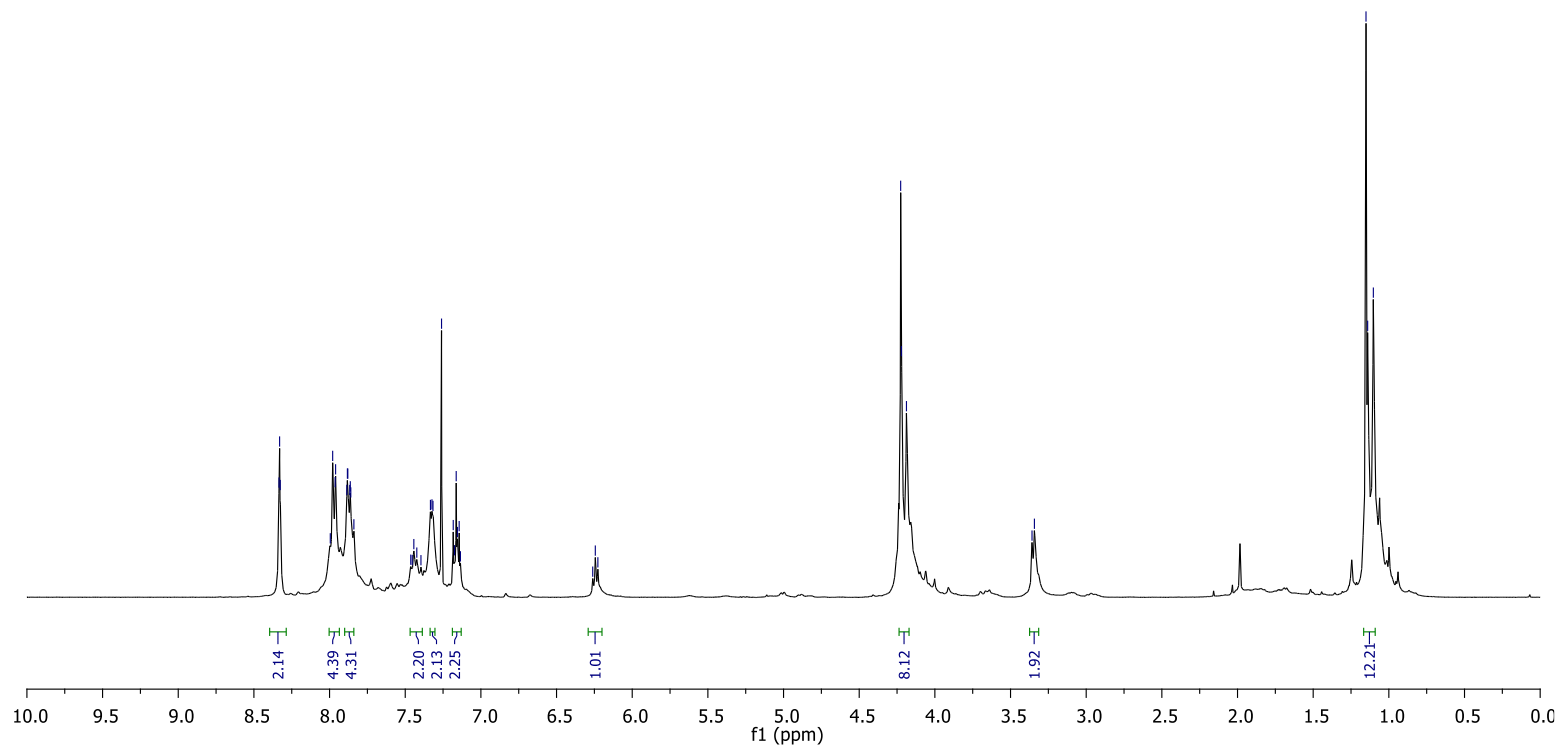

Figure S-203. ${ }^{1} \mathrm{H}$ NMR spectrum of $3 \mathbf{e}\left(\mathrm{CDCl}_{3}, 600 \mathrm{MHz}, 298 \mathrm{~K}\right)$
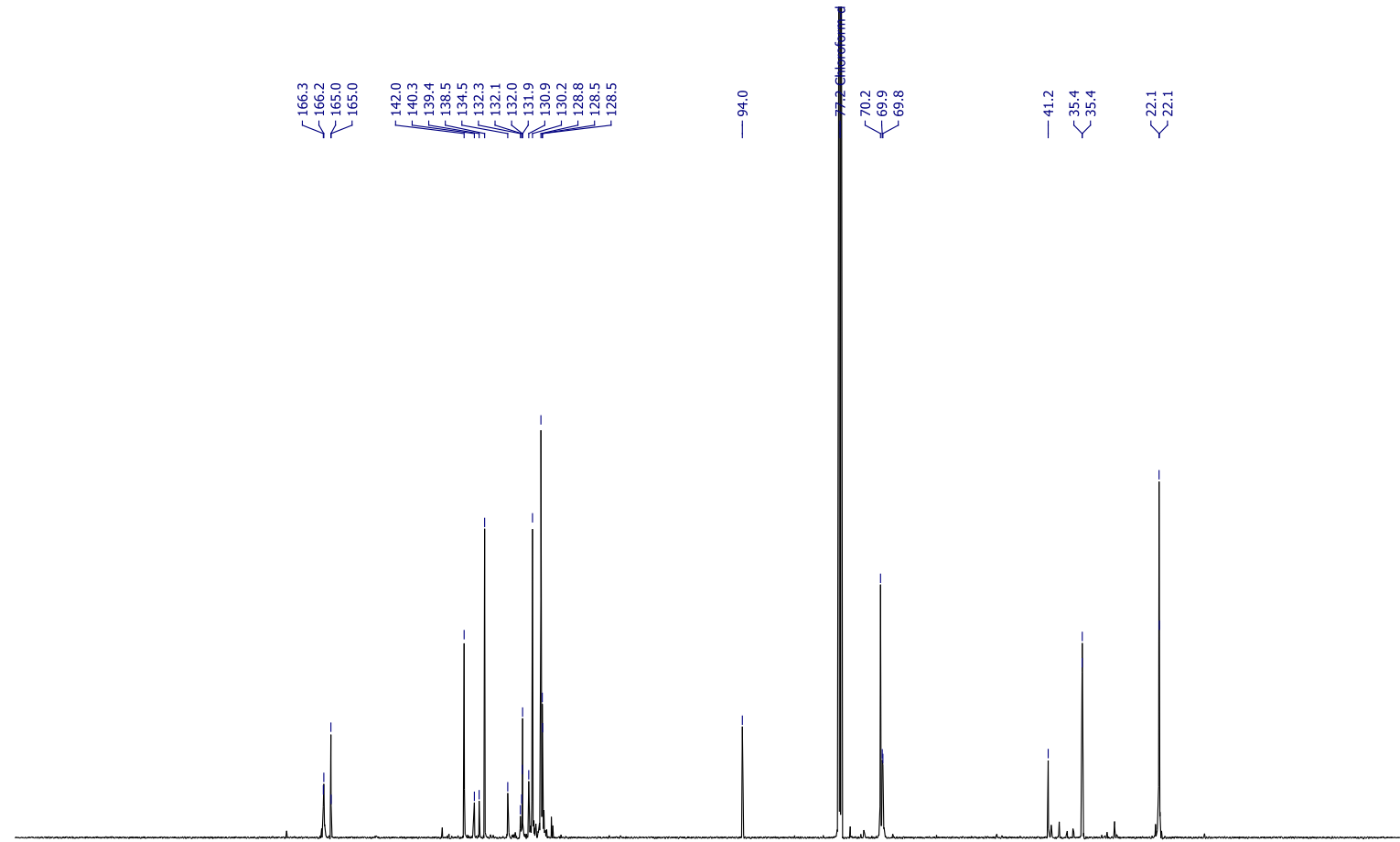

Figure $\mathbf{S}$-204. ${ }^{13} \mathrm{C}$ NMR spectrum of $3 \mathrm{e}\left(\mathrm{CDCl}_{3}, 151 \mathrm{MHz}, 298 \mathrm{~K}\right)$ 


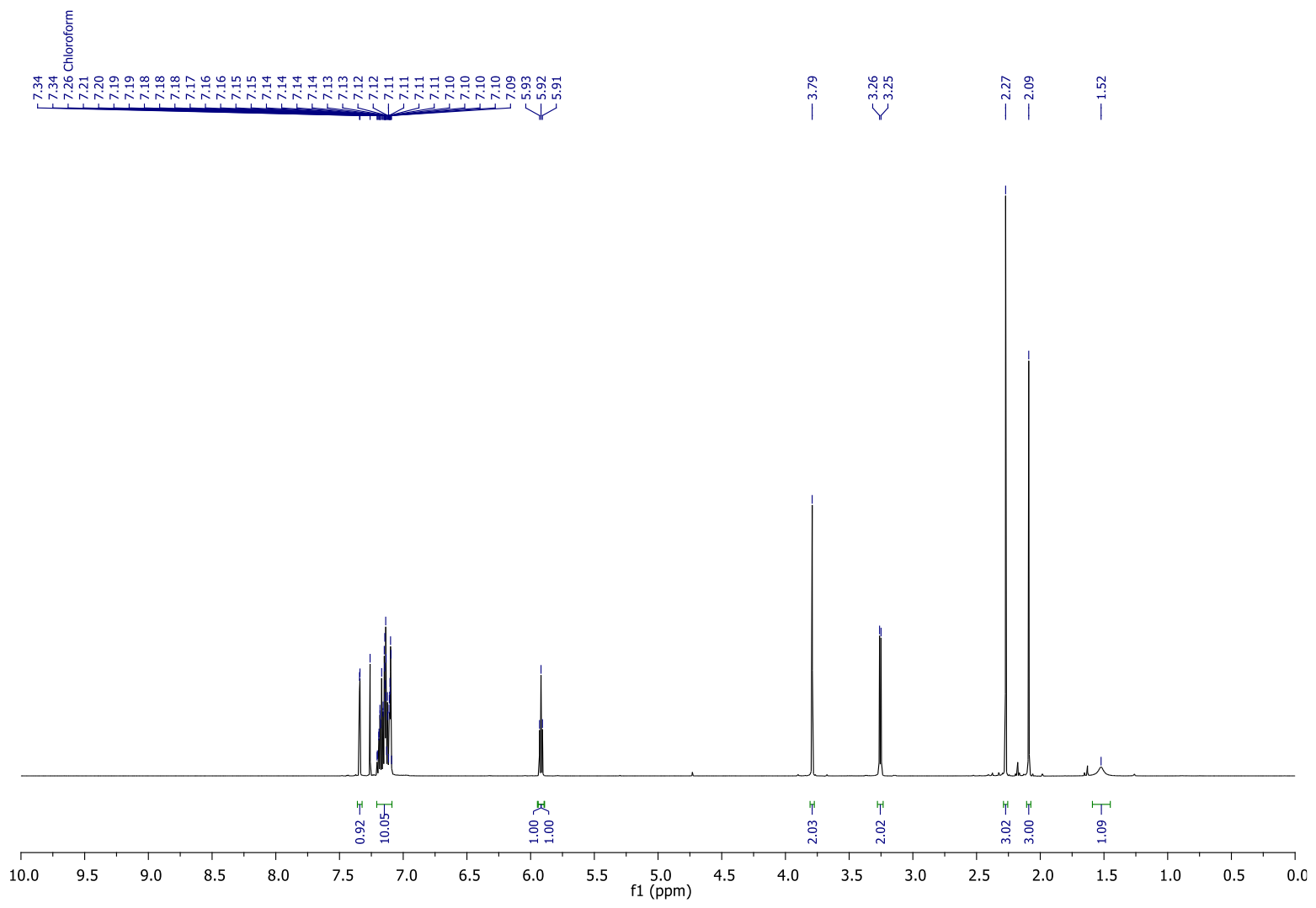

Figure S-205. ${ }^{1} \mathrm{H}$ NMR spectrum of $\mathbf{3 f}\left(\mathrm{CDCl}_{3}, 600 \mathrm{MHz}, 298 \mathrm{~K}\right)$
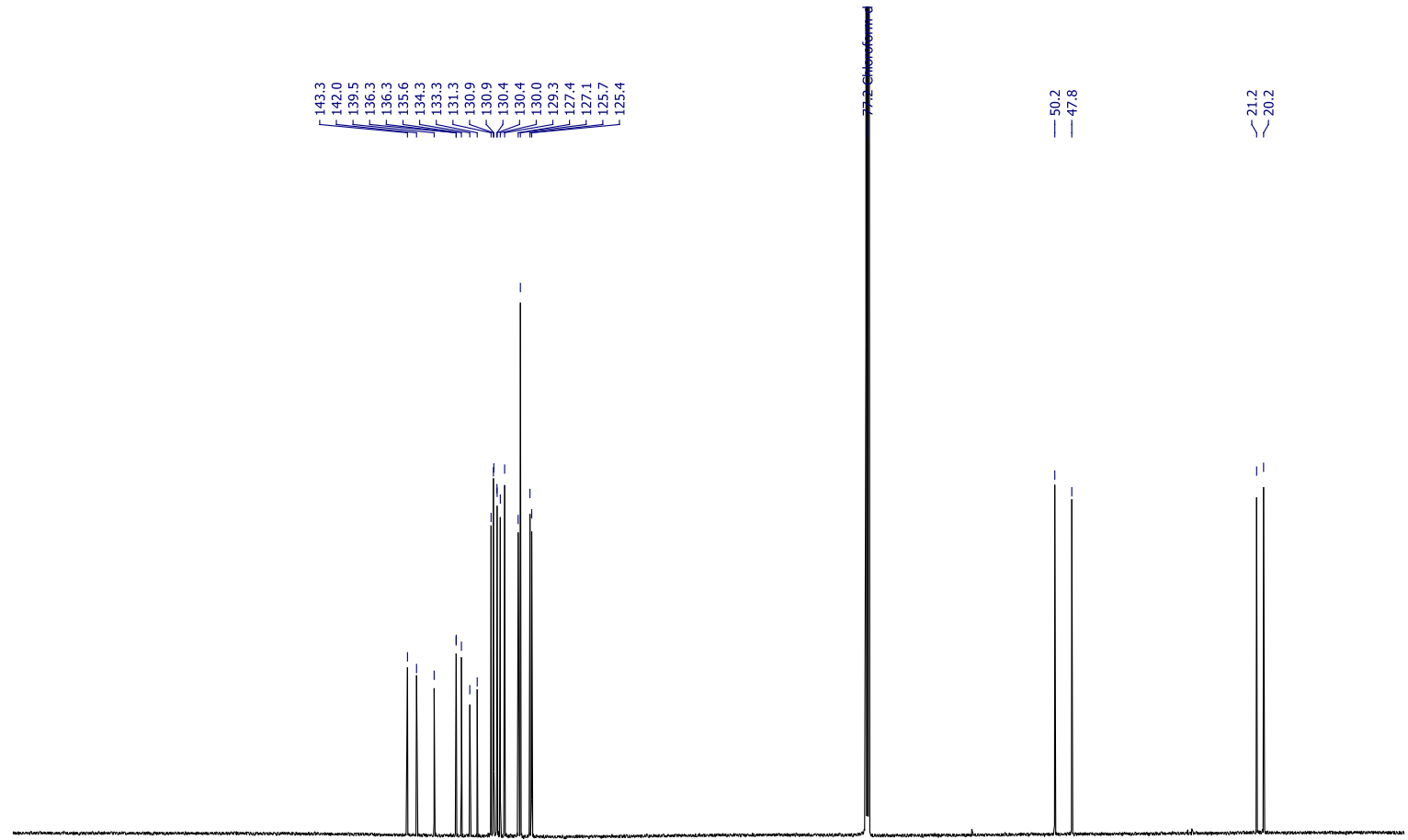

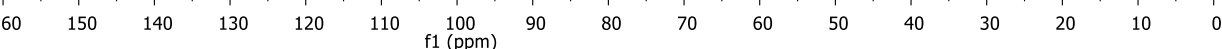

Figure S-206. ${ }^{13} \mathrm{C}$ NMR spectrum of $\mathbf{3 f}\left(\mathrm{CDCl}_{3}, 151 \mathrm{MHz}, 298 \mathrm{~K}\right)$ 


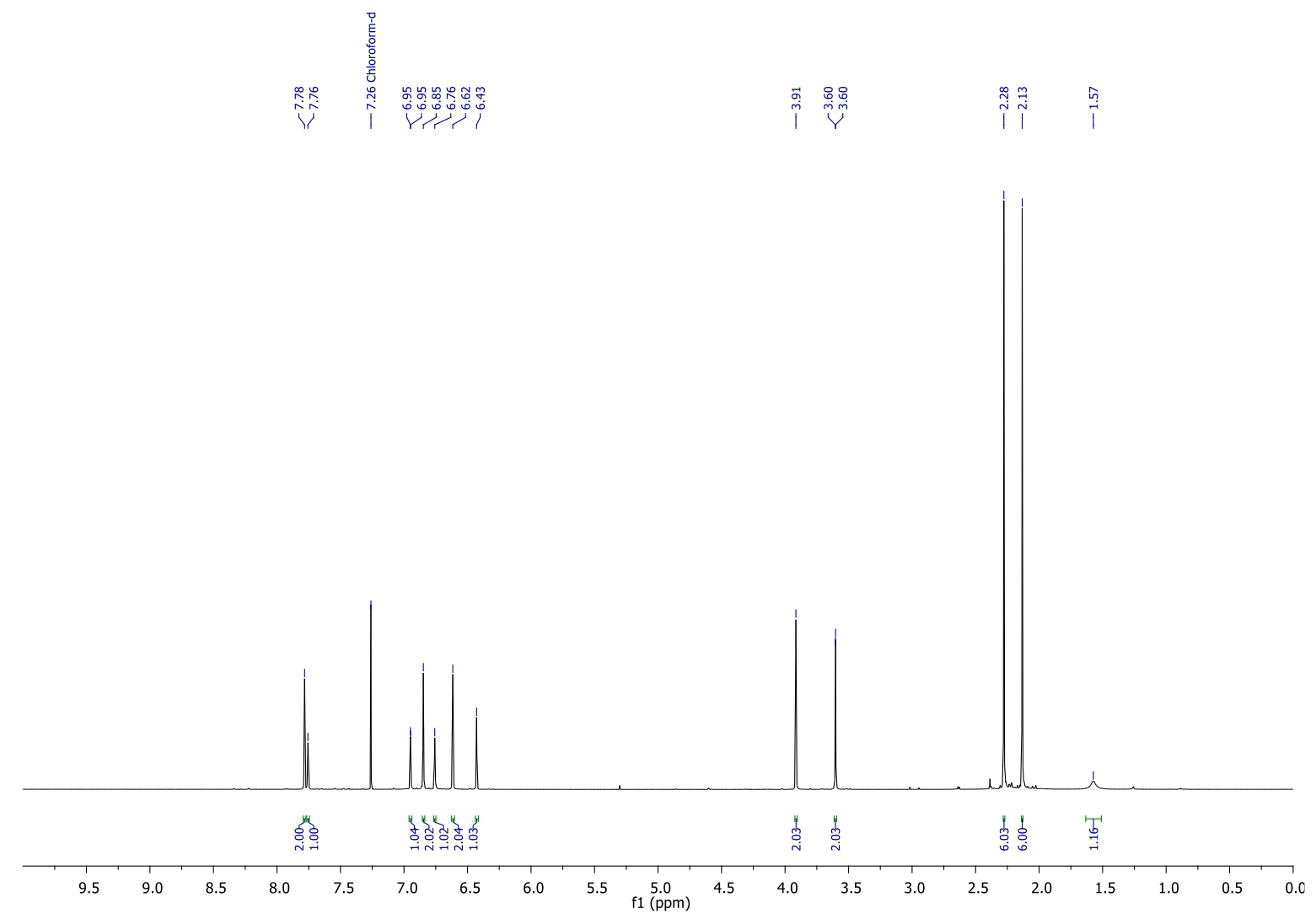

Figure S-207. ${ }^{1} \mathrm{H}$ NMR spectrum of $\mathbf{3 g}\left(\mathrm{CDCl}_{3}, 600 \mathrm{MHz}, 298 \mathrm{~K}\right)$

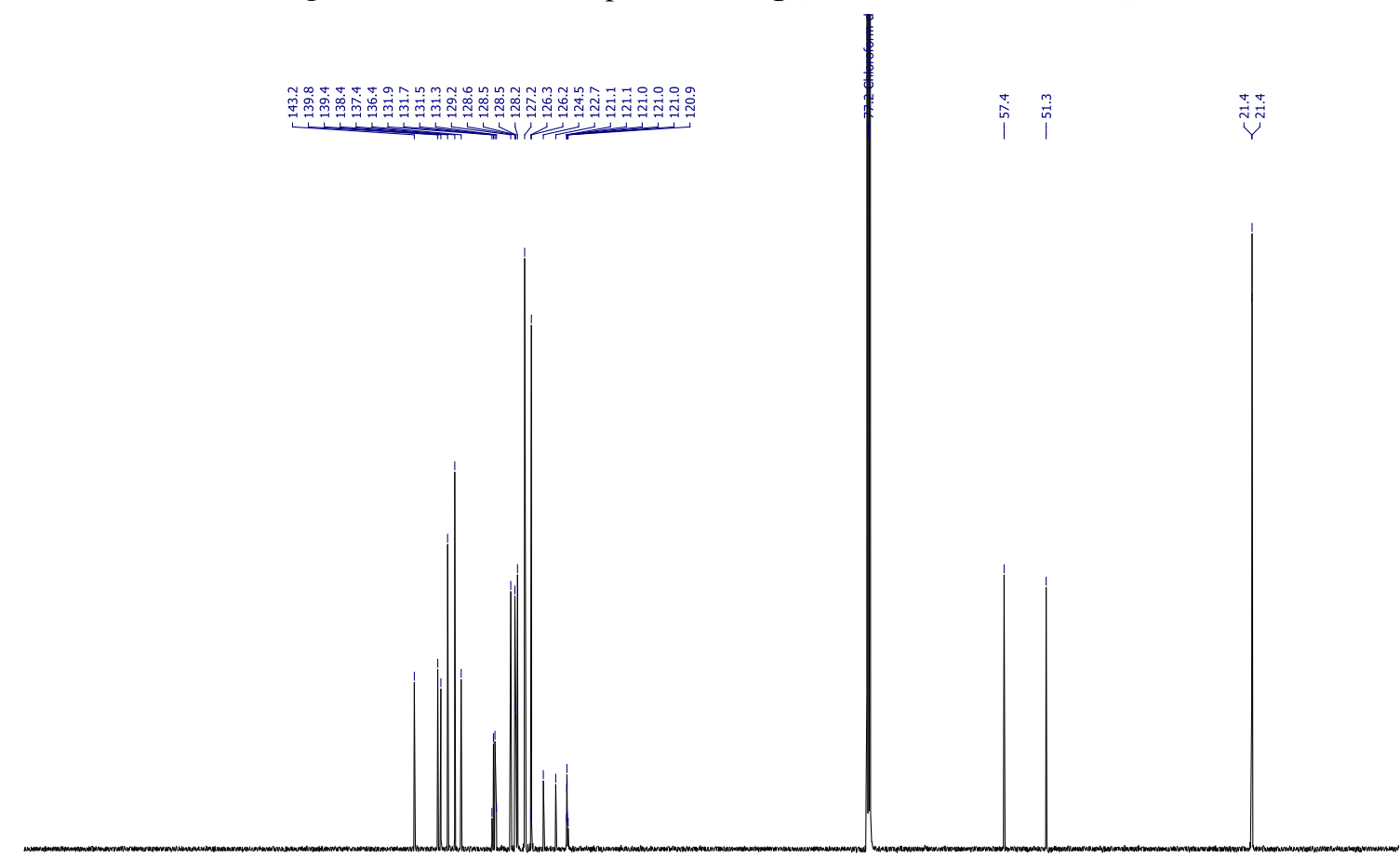

Figure $\mathbf{S - 2 0 8} .{ }^{13} \mathrm{C}$ NMR spectrum of $\mathbf{3 g}\left(\mathrm{CDCl}_{3}, 151 \mathrm{MHz}, 298 \mathrm{~K}\right)$ 

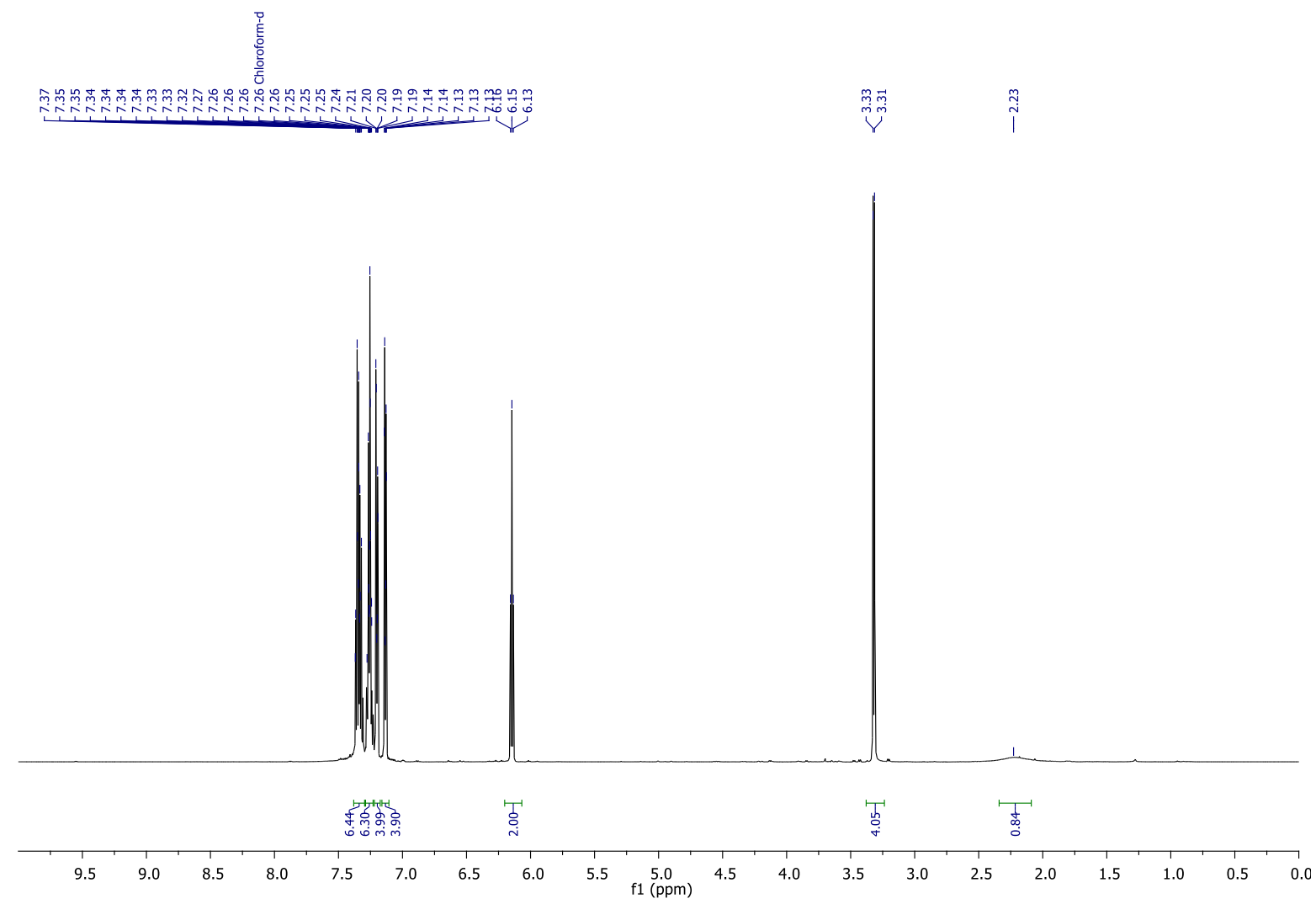

Figure S-209. ${ }^{1} \mathrm{H}$ NMR spectrum of $3 \mathbf{h}\left(\mathrm{CDCl}_{3}, 600 \mathrm{MHz}, 298 \mathrm{~K}\right)$
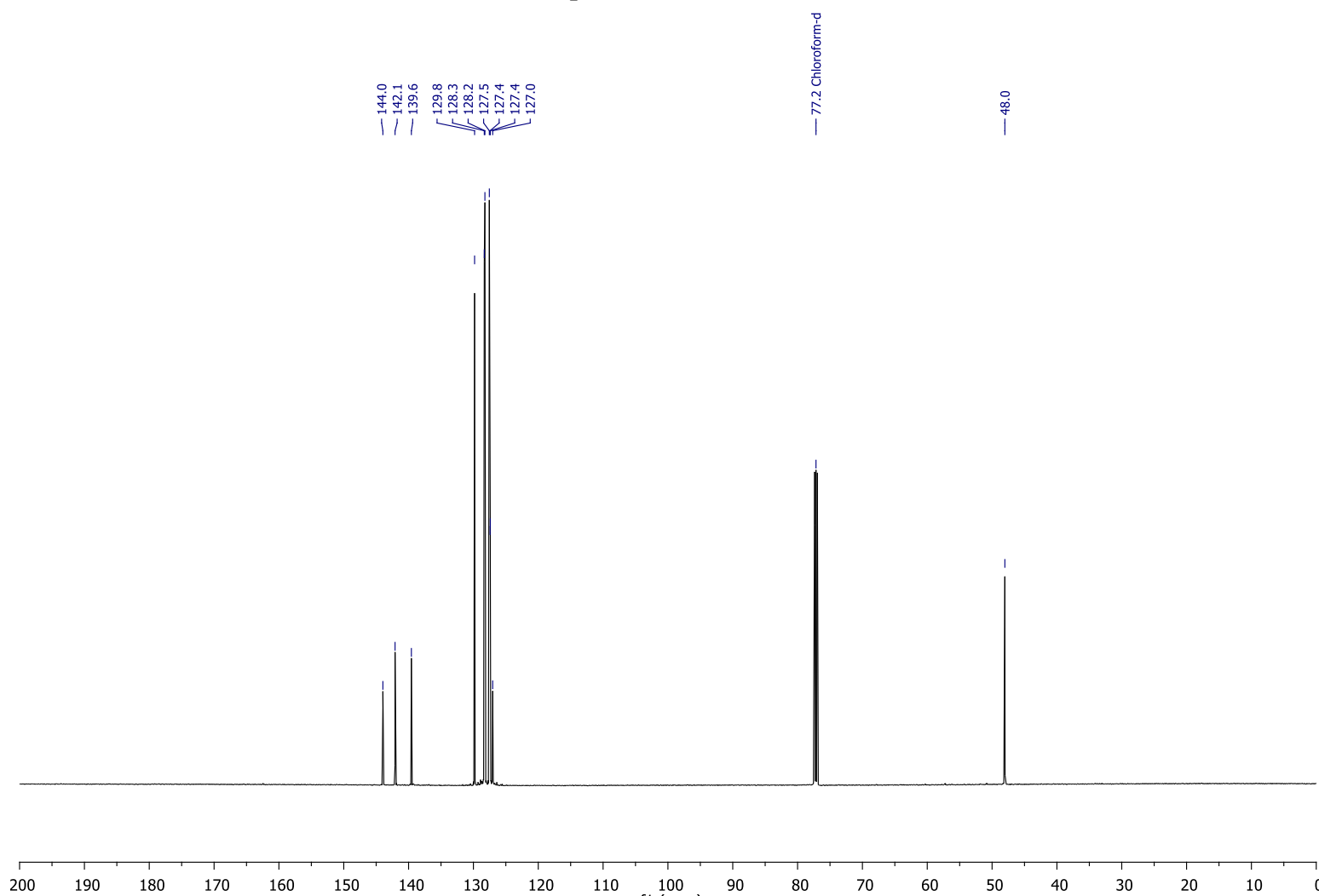

Figure S-210. ${ }^{13} \mathrm{C}$ NMR spectrum of $\mathbf{3 h}\left(\mathrm{CDCl}_{3}, 151 \mathrm{MHz}, 298 \mathrm{~K}\right)$ 


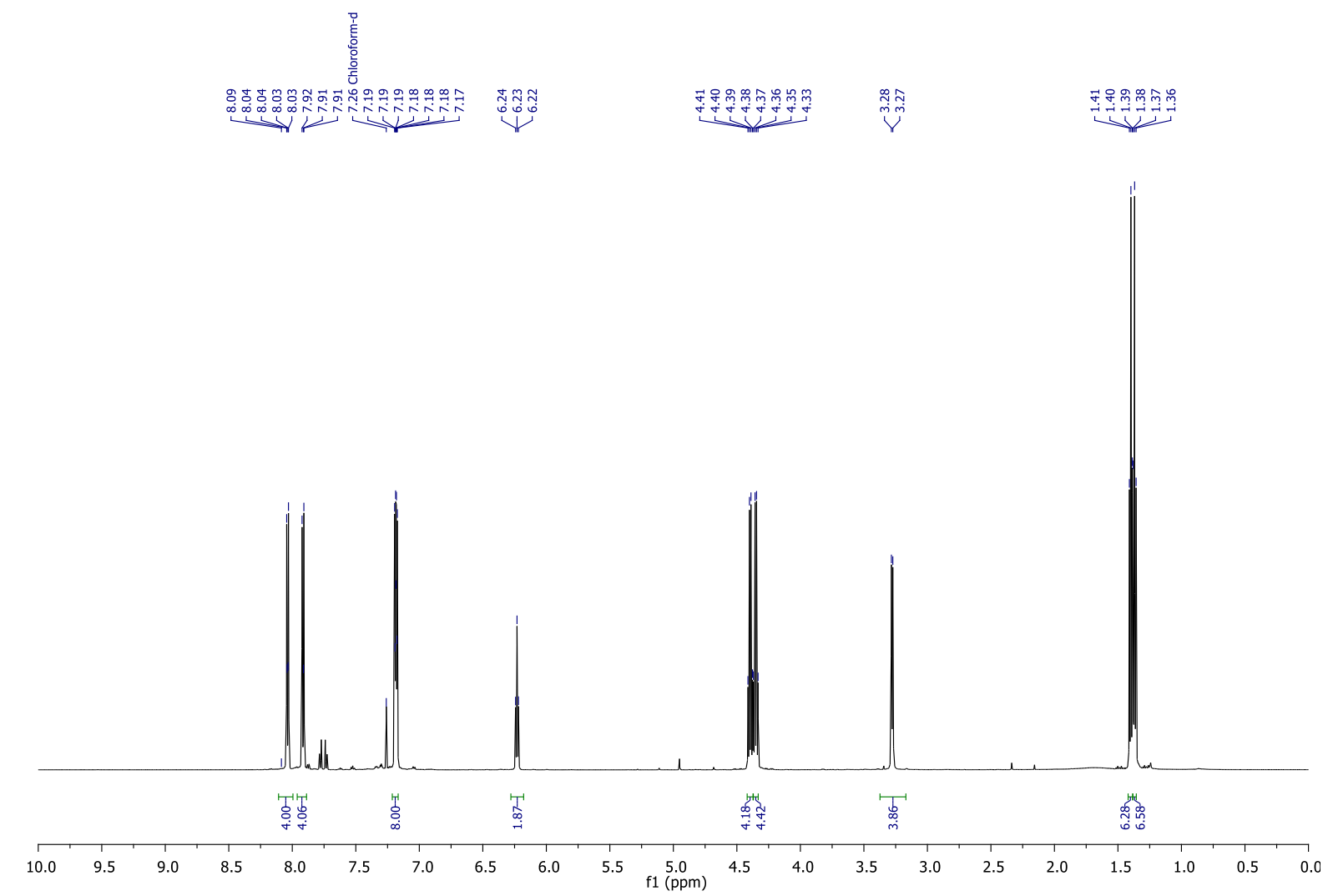

Figure S-211. ${ }^{1} \mathrm{H}$ NMR spectrum of $3 \mathbf{i}\left(\mathrm{CDCl}_{3}, 600 \mathrm{MHz}, 298 \mathrm{~K}\right)$

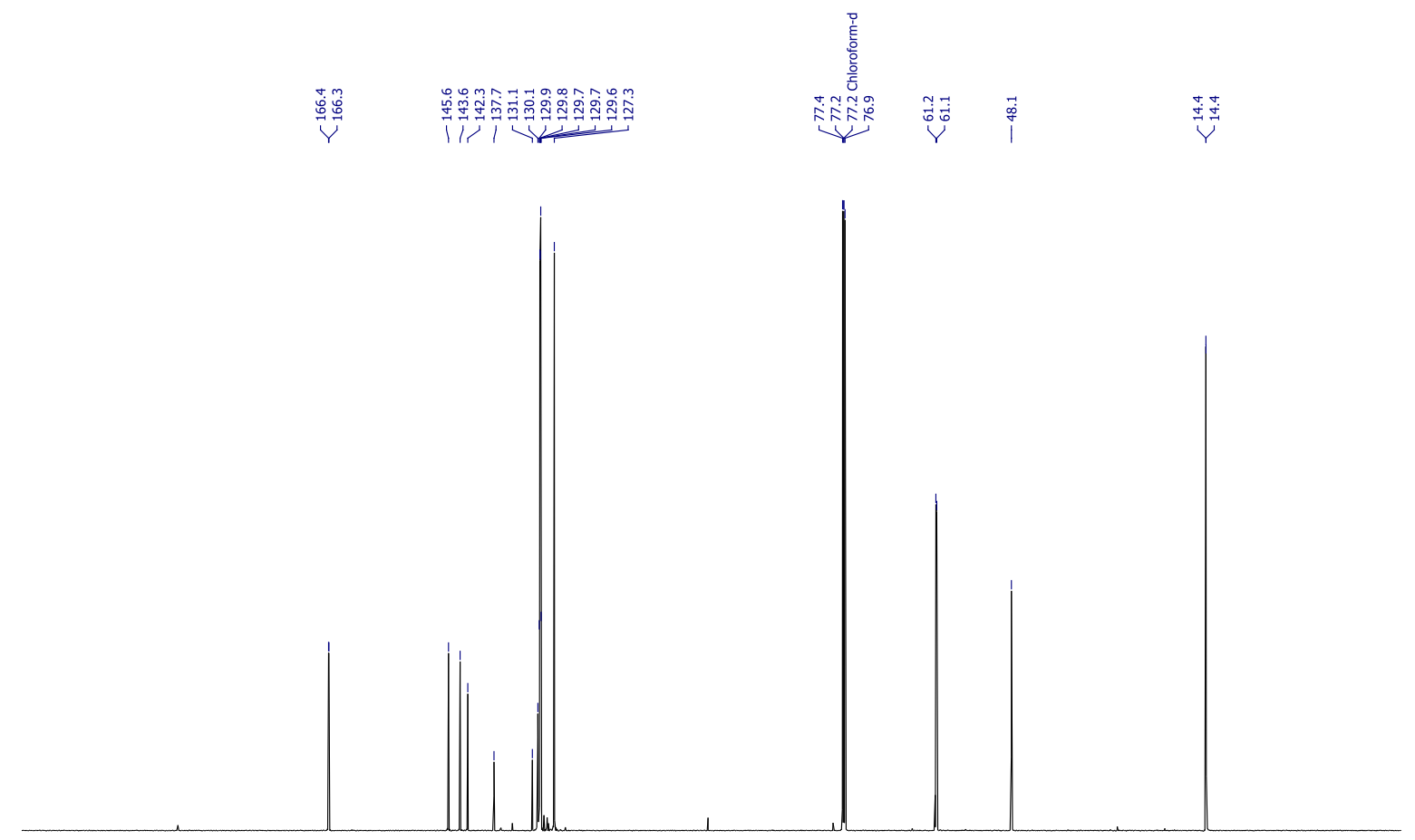

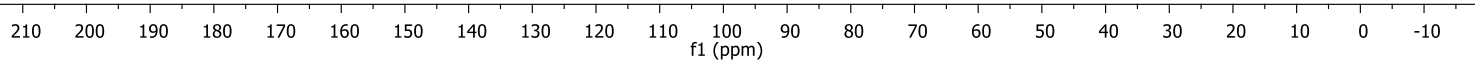

Figure S-212. ${ }^{13} \mathrm{C}$ NMR spectrum of $\mathbf{3 i}\left(\mathrm{CDCl}_{3}, 151 \mathrm{MHz}, 298 \mathrm{~K}\right)$ 


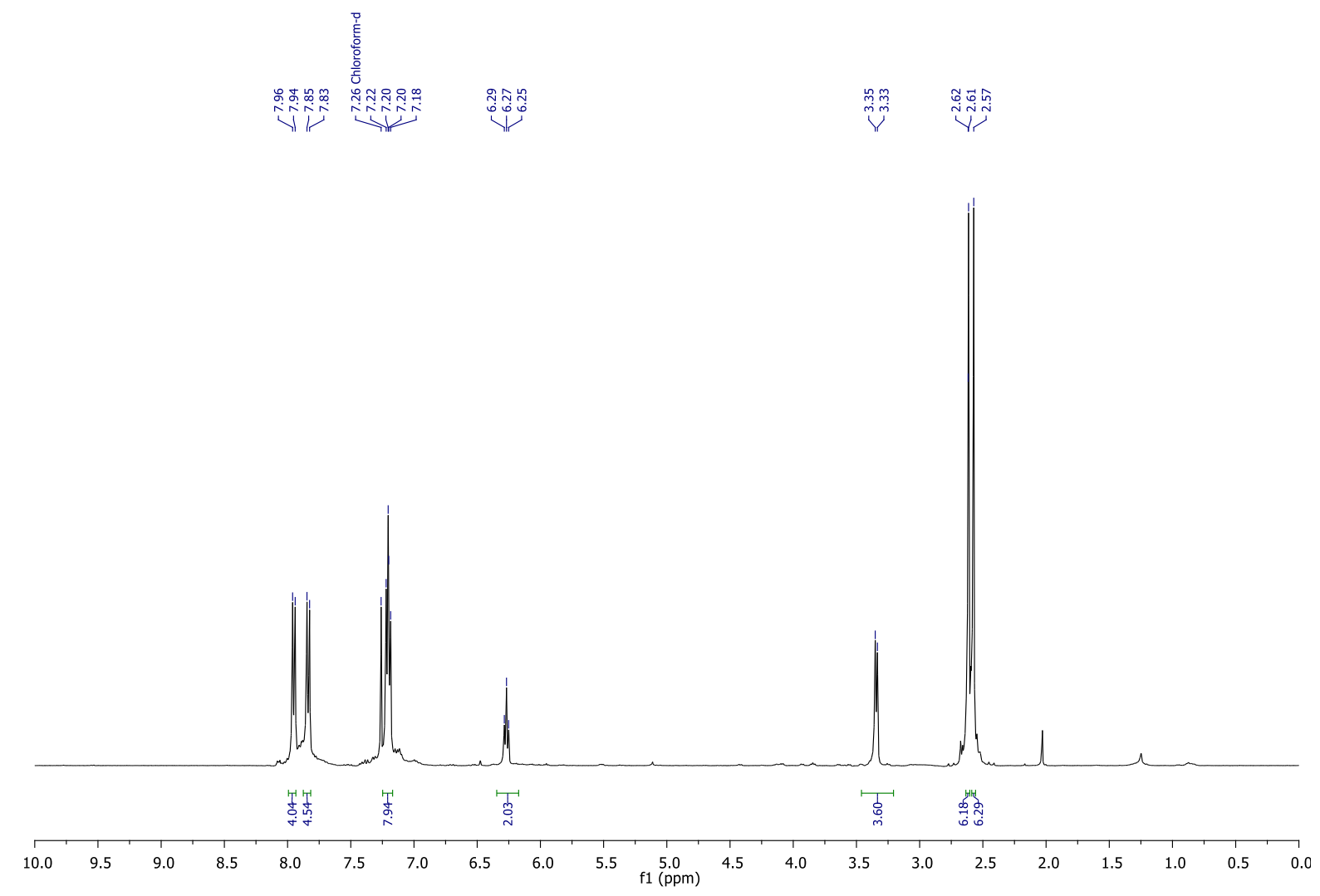

Figure S-213. ${ }^{1} \mathrm{H}$ NMR spectrum of $\mathbf{3 j}\left(\mathrm{CDCl}_{3}, 600 \mathrm{MHz}, 298 \mathrm{~K}\right)$

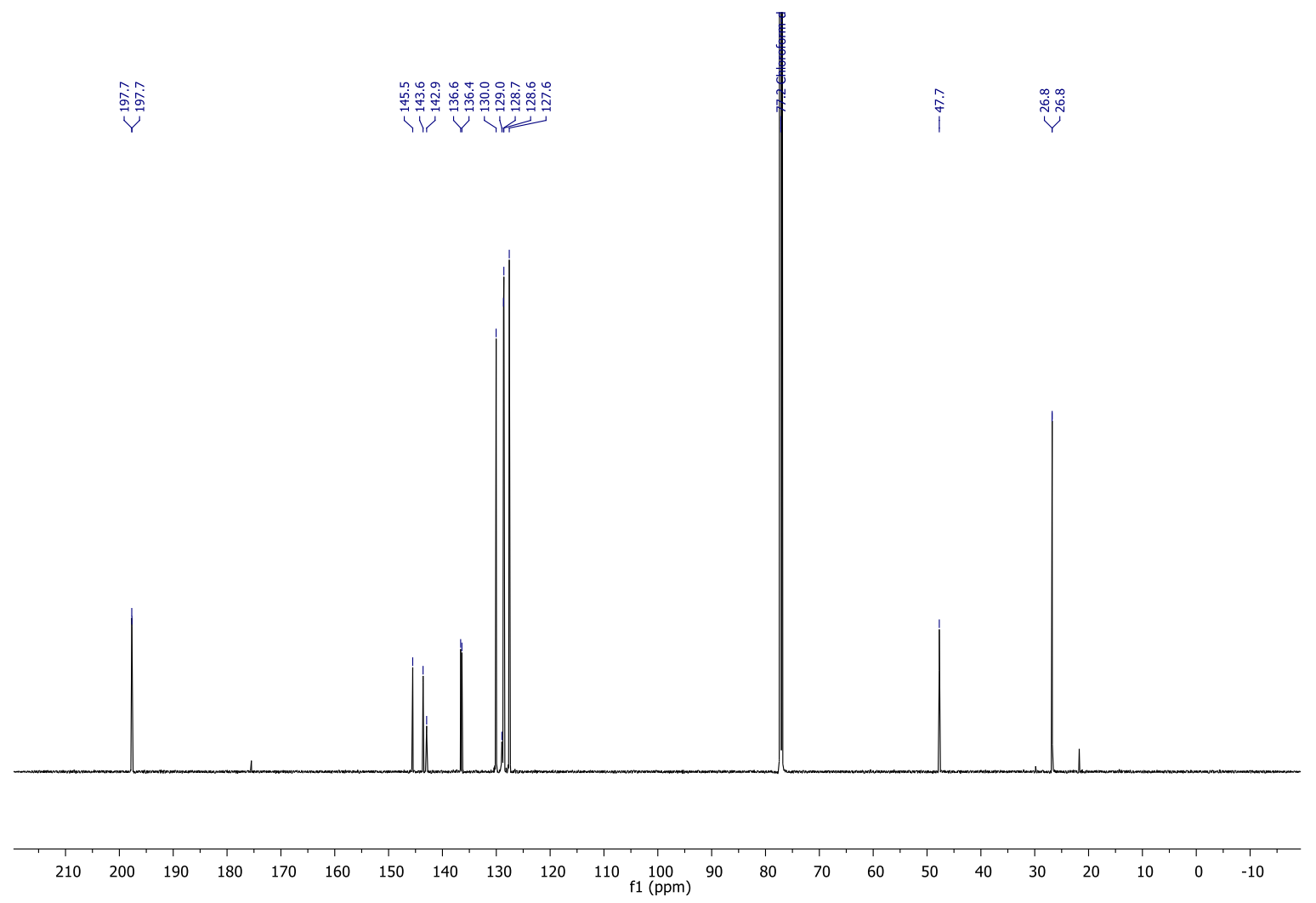

Figure S-214. ${ }^{13} \mathrm{C}$ NMR spectrum of $\mathbf{3 j}\left(\mathrm{CDCl}_{3}, 151 \mathrm{MHz}, 298 \mathrm{~K}\right)$ 


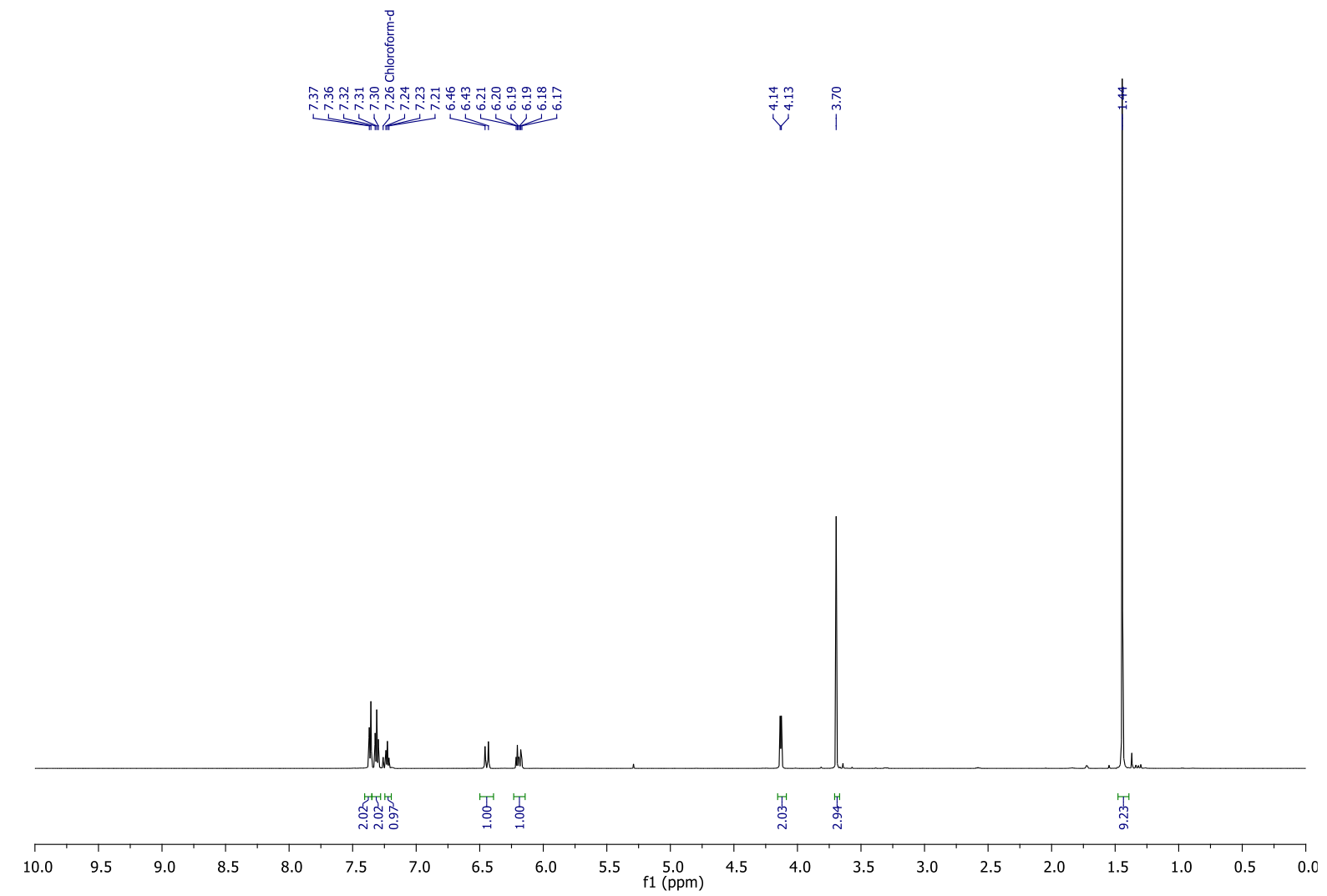

Figure S-215. ${ }^{1} \mathrm{H}$ NMR spectrum of $4\left(\mathrm{CDCl}_{3}, 600 \mathrm{MHz}, 298 \mathrm{~K}\right)$
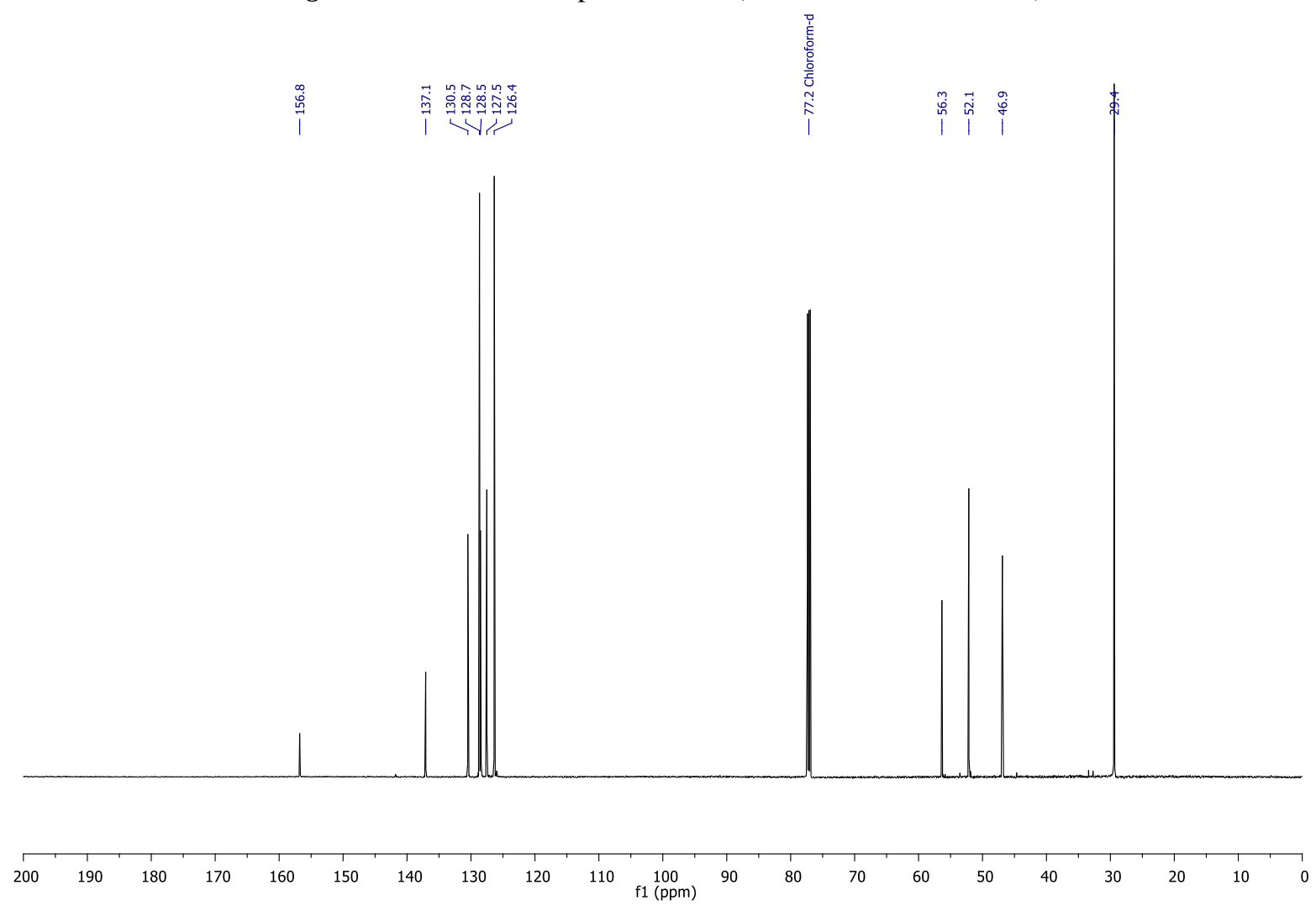

Figure S-216. ${ }^{13} \mathrm{C}$ NMR spectrum of $4\left(\mathrm{CDCl}_{3}, 151 \mathrm{MHz}, 298 \mathrm{~K}\right)$ 


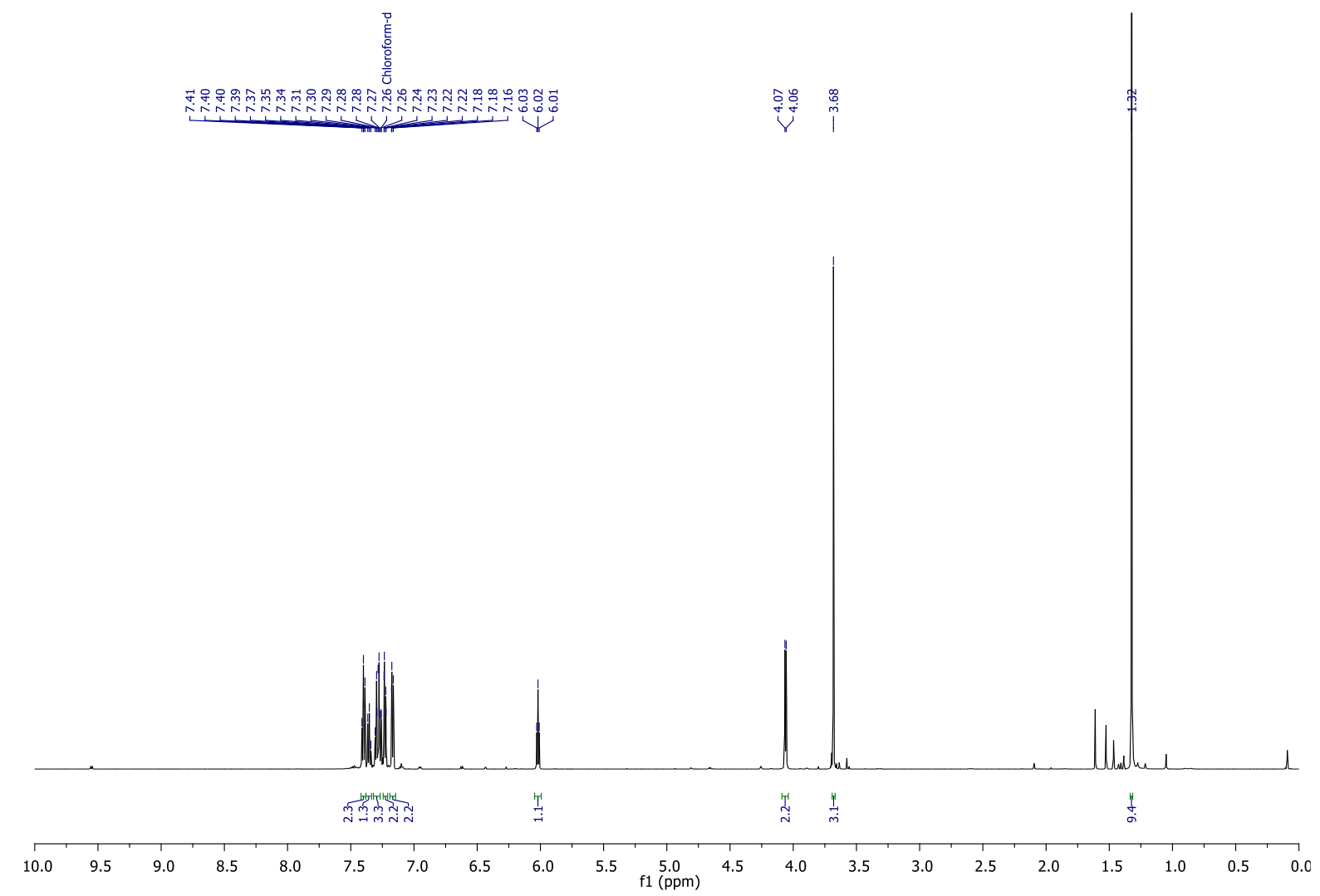

Figure S-217. ${ }^{1} \mathrm{H}$ NMR spectrum of $5\left(\mathrm{CDCl}_{3}, 600 \mathrm{MHz}, 298 \mathrm{~K}\right)$

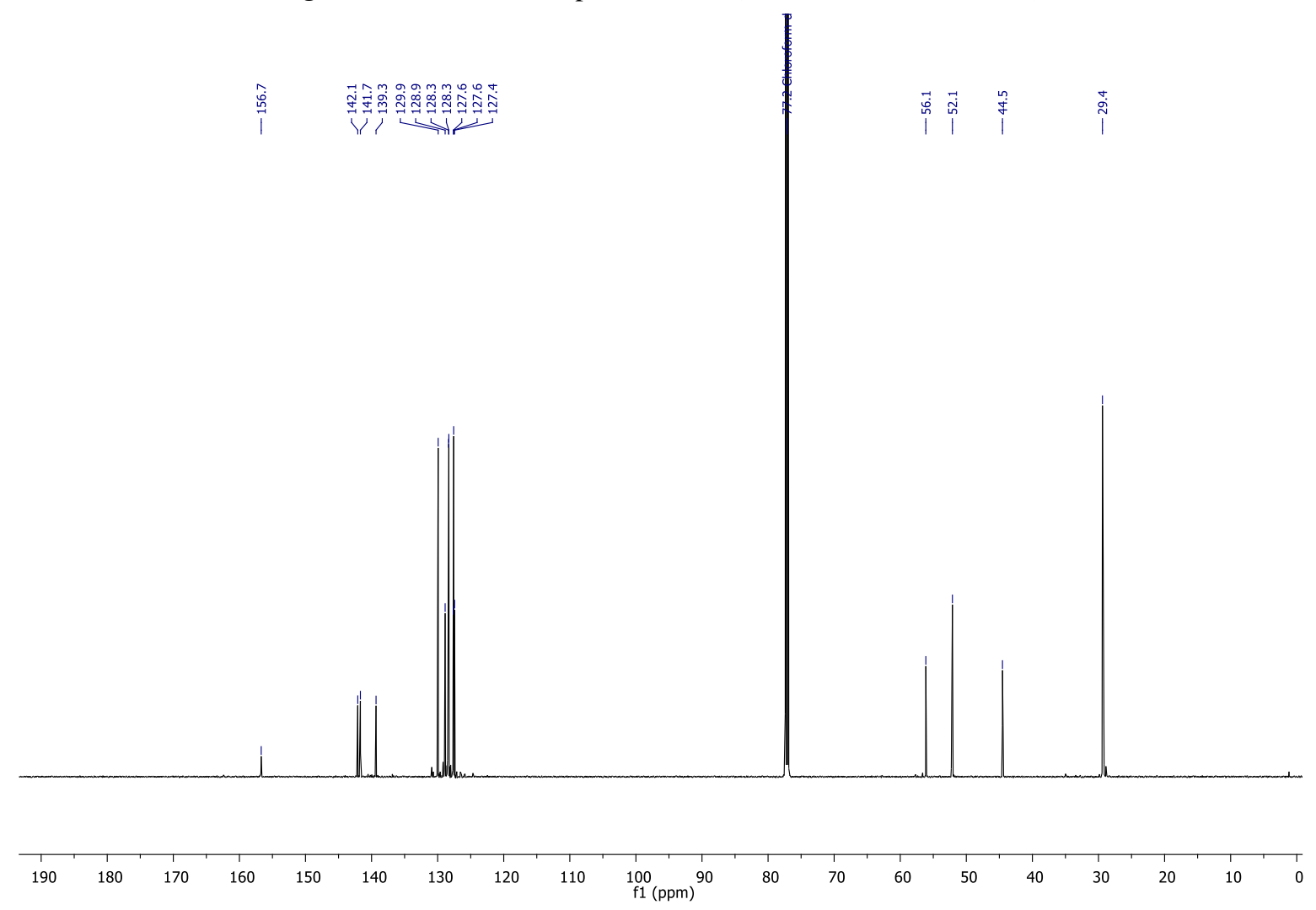

Figure S-218. ${ }^{13} \mathrm{C}$ NMR spectrum of $5\left(\mathrm{CDCl}_{3}, 151 \mathrm{MHz}, 298 \mathrm{~K}\right)$ 


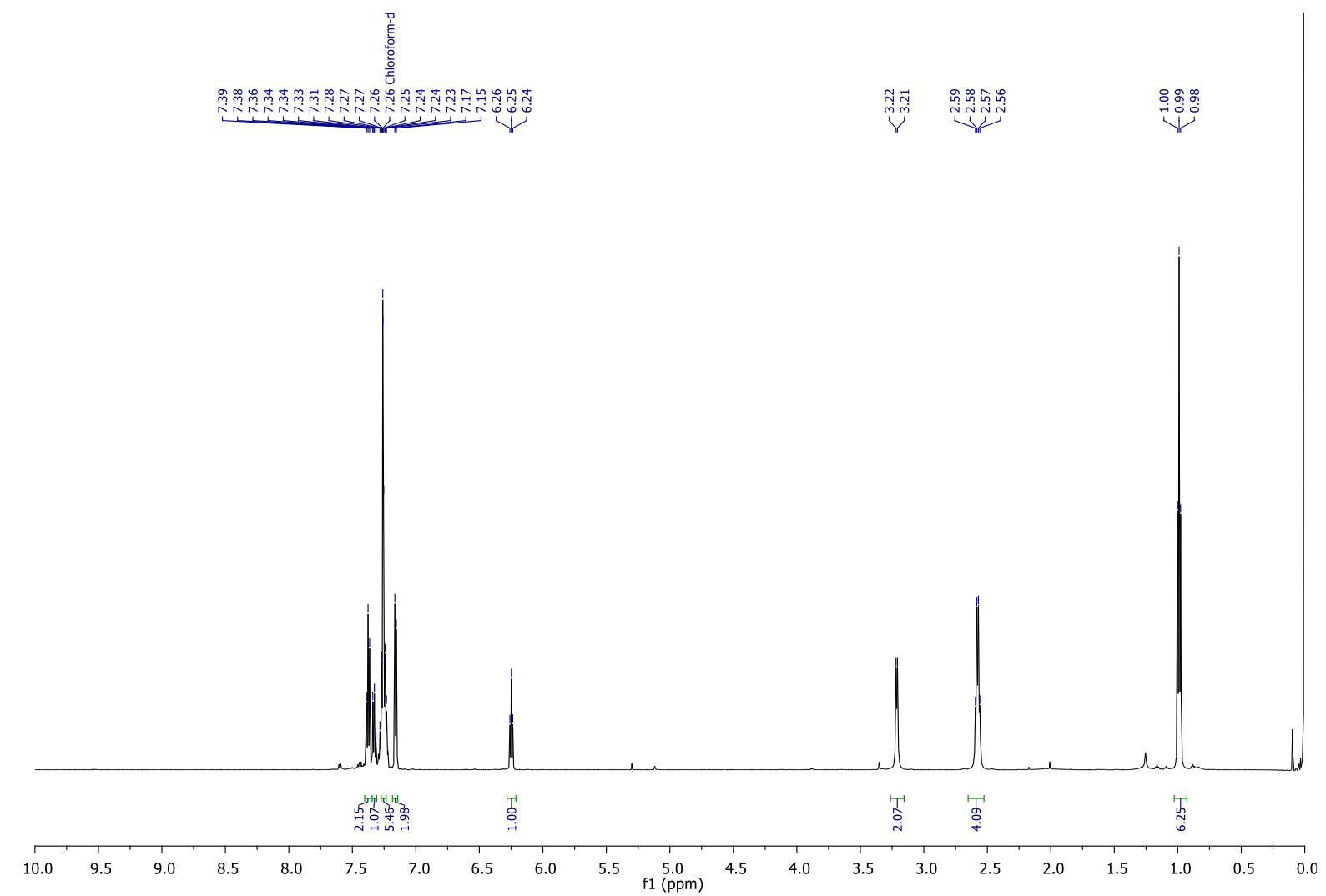

Figure $\boldsymbol{S}$-219. ${ }^{1} \mathrm{H} \mathrm{NMR}$ spectrum of $9\left(\mathrm{CDCl}_{3}, 600 \mathrm{MHz}, 298 \mathrm{~K}\right)$
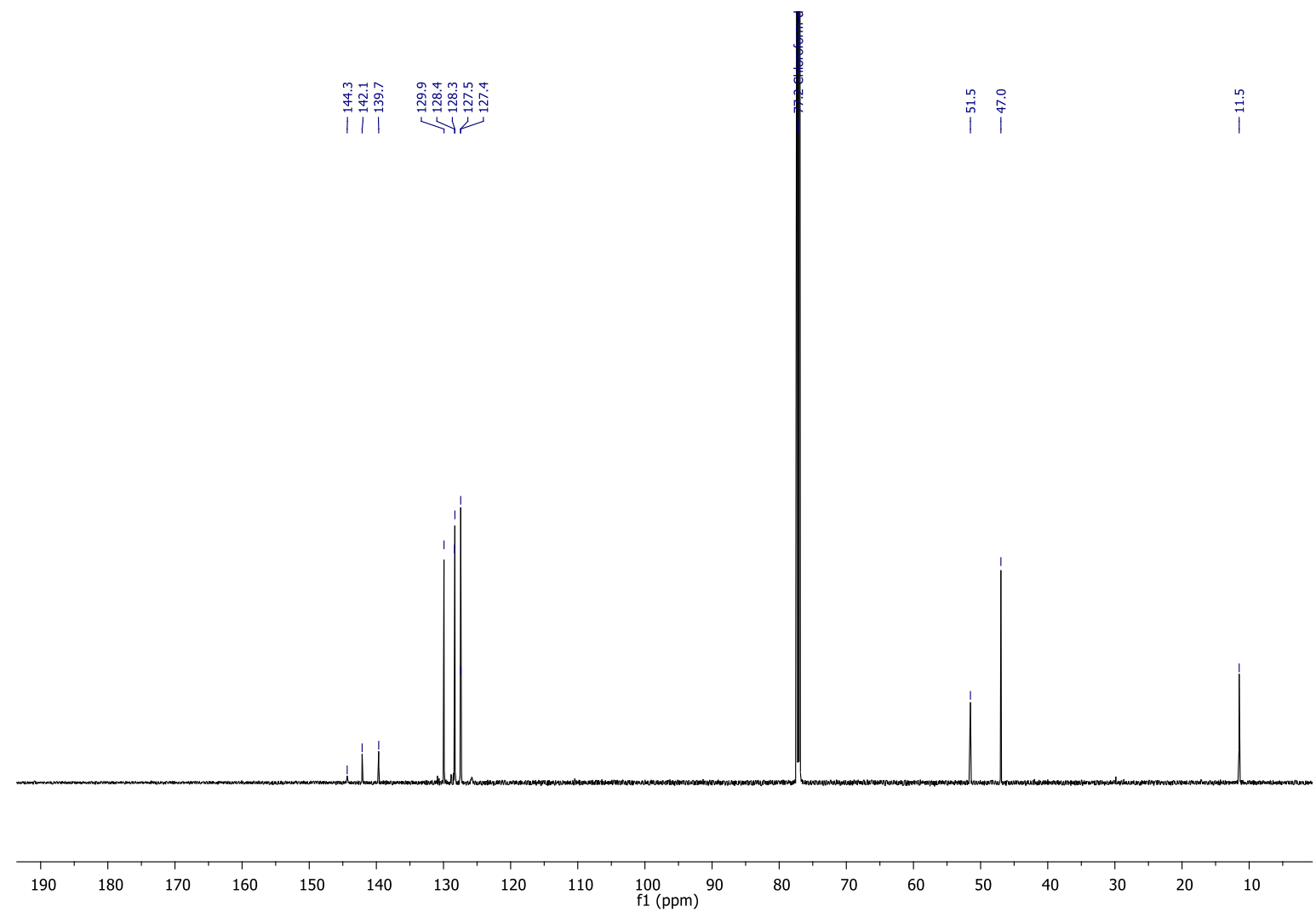

Figure S-220. ${ }^{13} \mathrm{C}$ NMR spectrum of $9\left(\mathrm{CDCl}_{3}, 151 \mathrm{MHz}, 298 \mathrm{~K}\right)$ 

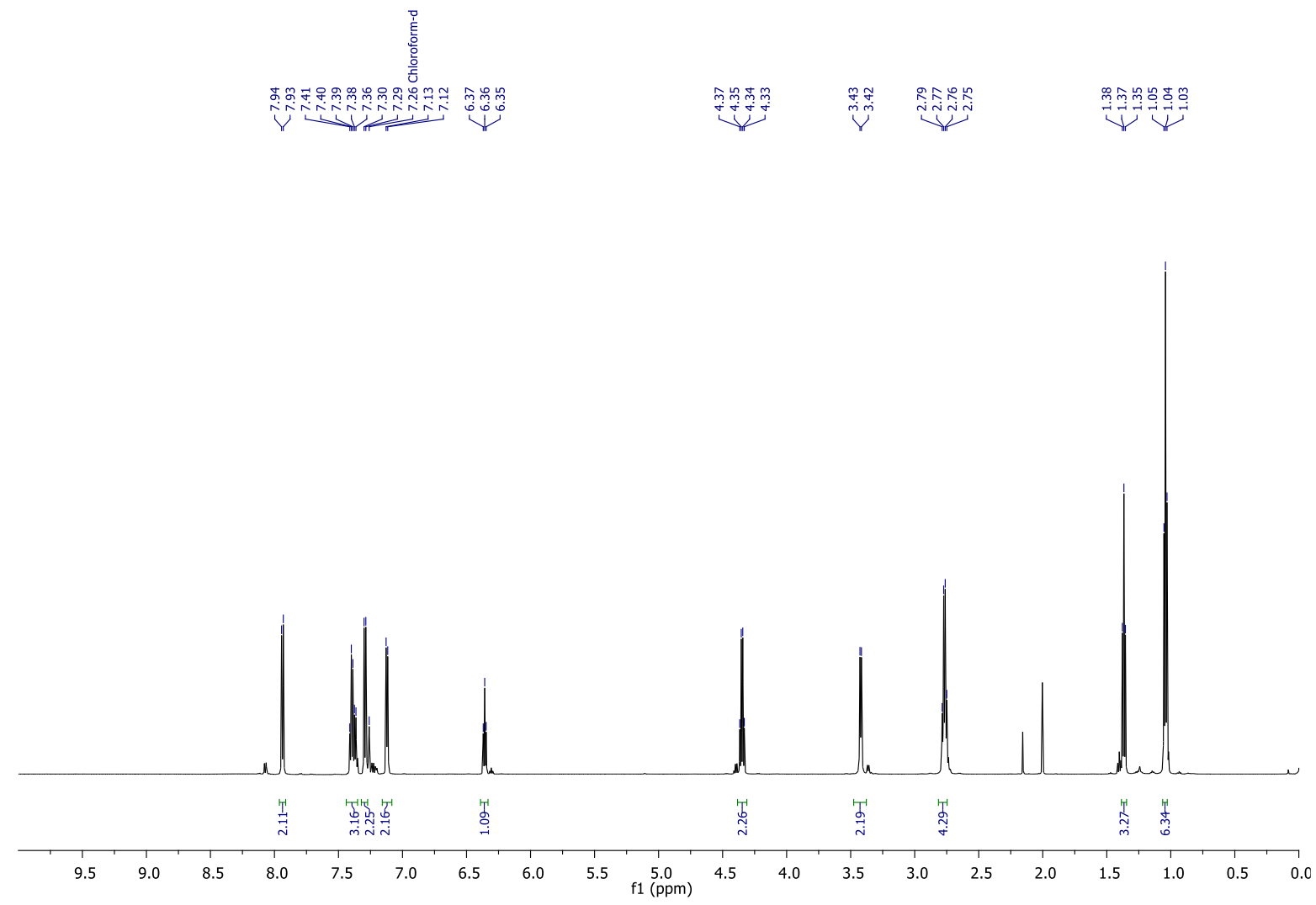

Figure $\boldsymbol{S}$-221. ${ }^{1} \mathrm{H}$ NMR spectrum of $\mathbf{1 0}\left(\mathrm{CDCl}_{3}, 600 \mathrm{MHz}, 298 \mathrm{~K}\right)$
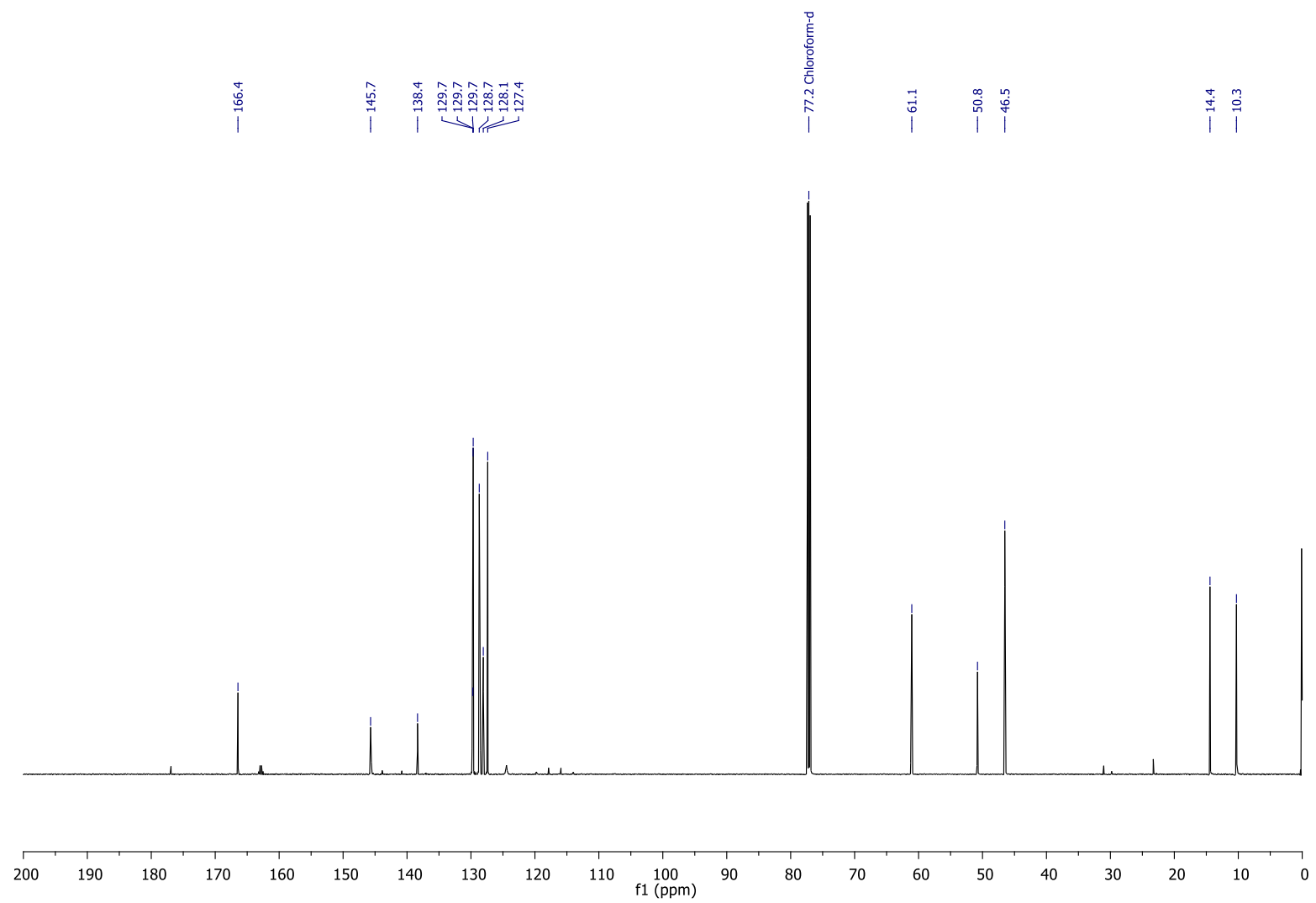

Figure S-222. ${ }^{13} \mathrm{C}$ NMR spectrum of $\mathbf{1 0}\left(\mathrm{CDCl}_{3}, 151 \mathrm{MHz}, 298 \mathrm{~K}\right)$ 


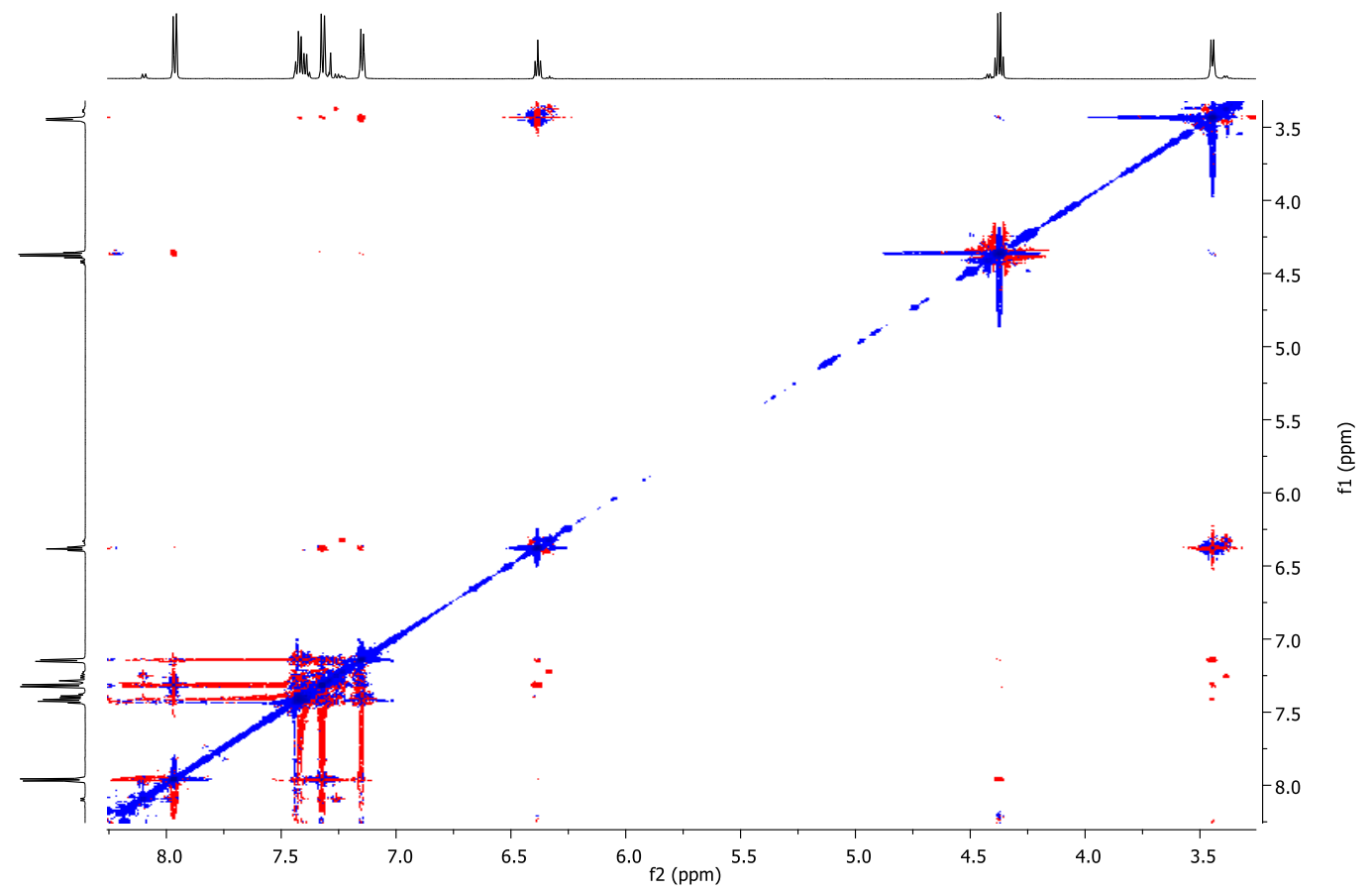

Figure S-223. Zoomed-in Upfield Region of COSY spectrum of $\mathbf{1 0}\left(\mathrm{CDCl}_{3}, 600 \mathrm{MHz}, 298 \mathrm{~K}\right)$

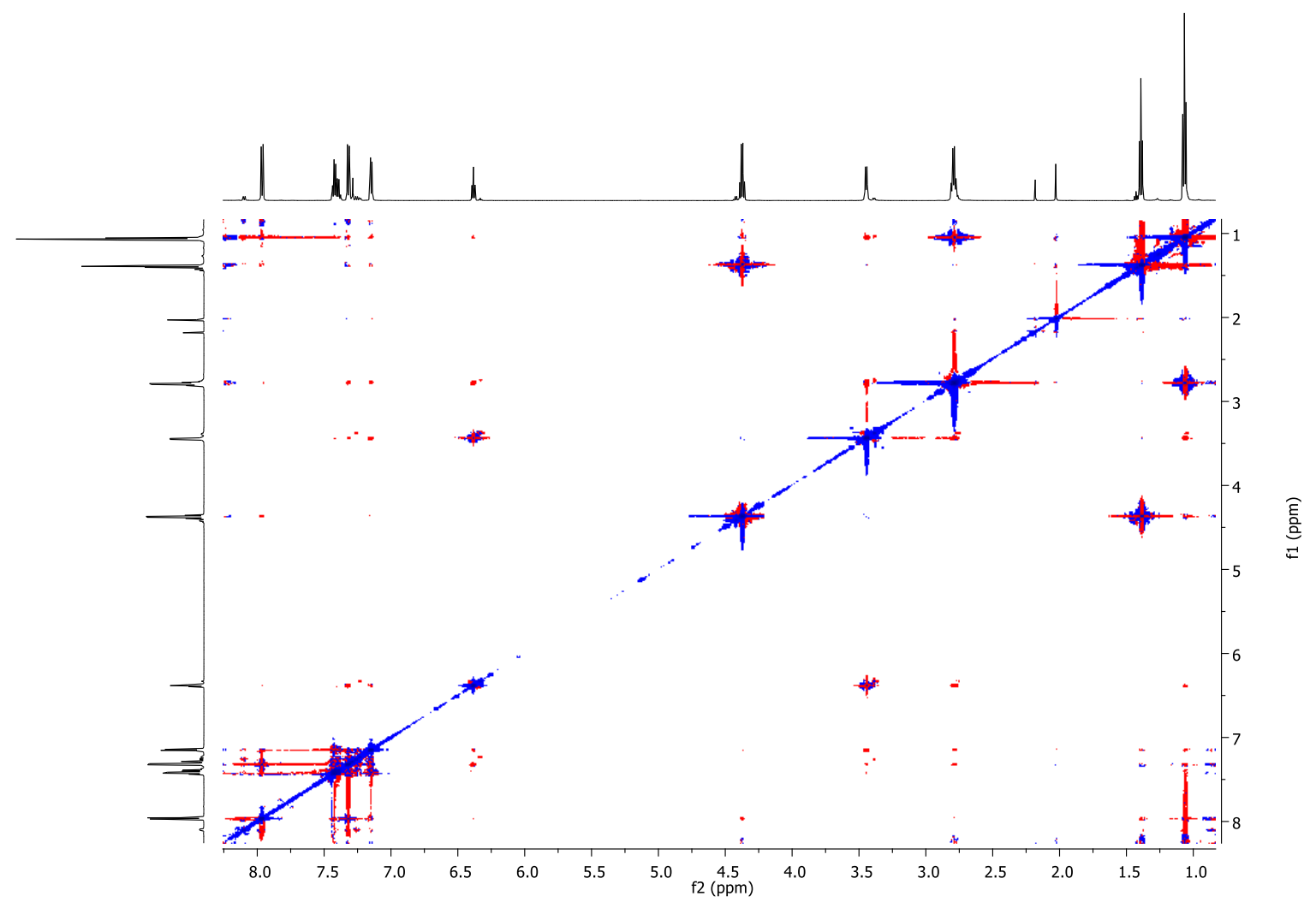

Figure S-224. Full NOESY spectrum of $10\left(\mathrm{CDCl}_{3}, 600 \mathrm{MHz}, 298 \mathrm{~K}\right)$ 


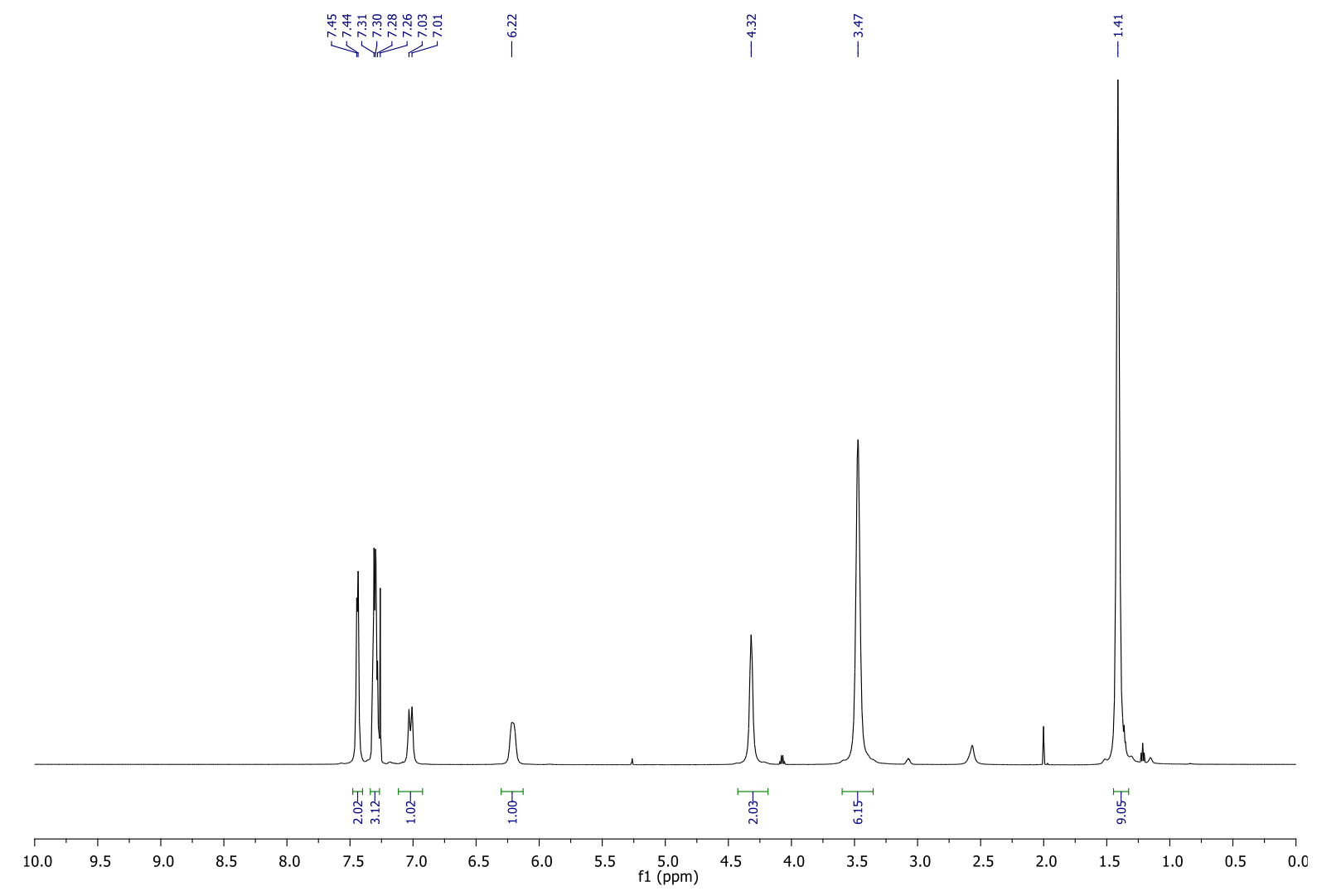

Figure S-225. ${ }^{1} \mathrm{H}$ NMR spectrum of $\mathbf{1 1}\left(\mathrm{CDCl}_{3}, 600 \mathrm{MHz}, 298 \mathrm{~K}\right)$
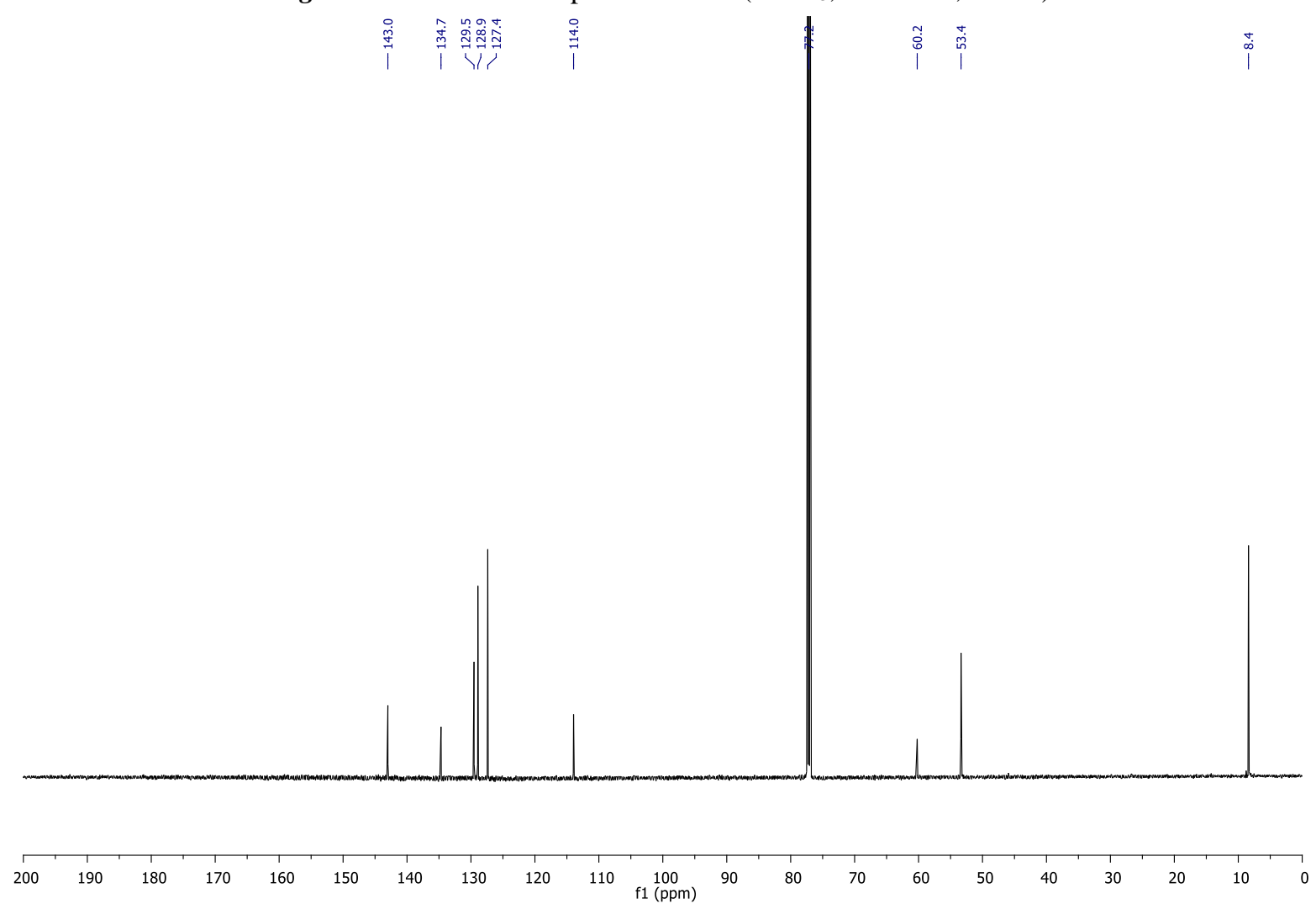

Figure S-226. ${ }^{13} \mathrm{C}$ NMR spectrum of $11\left(\mathrm{CDCl}_{3}, 151 \mathrm{MHz}, 298 \mathrm{~K}\right)$ 


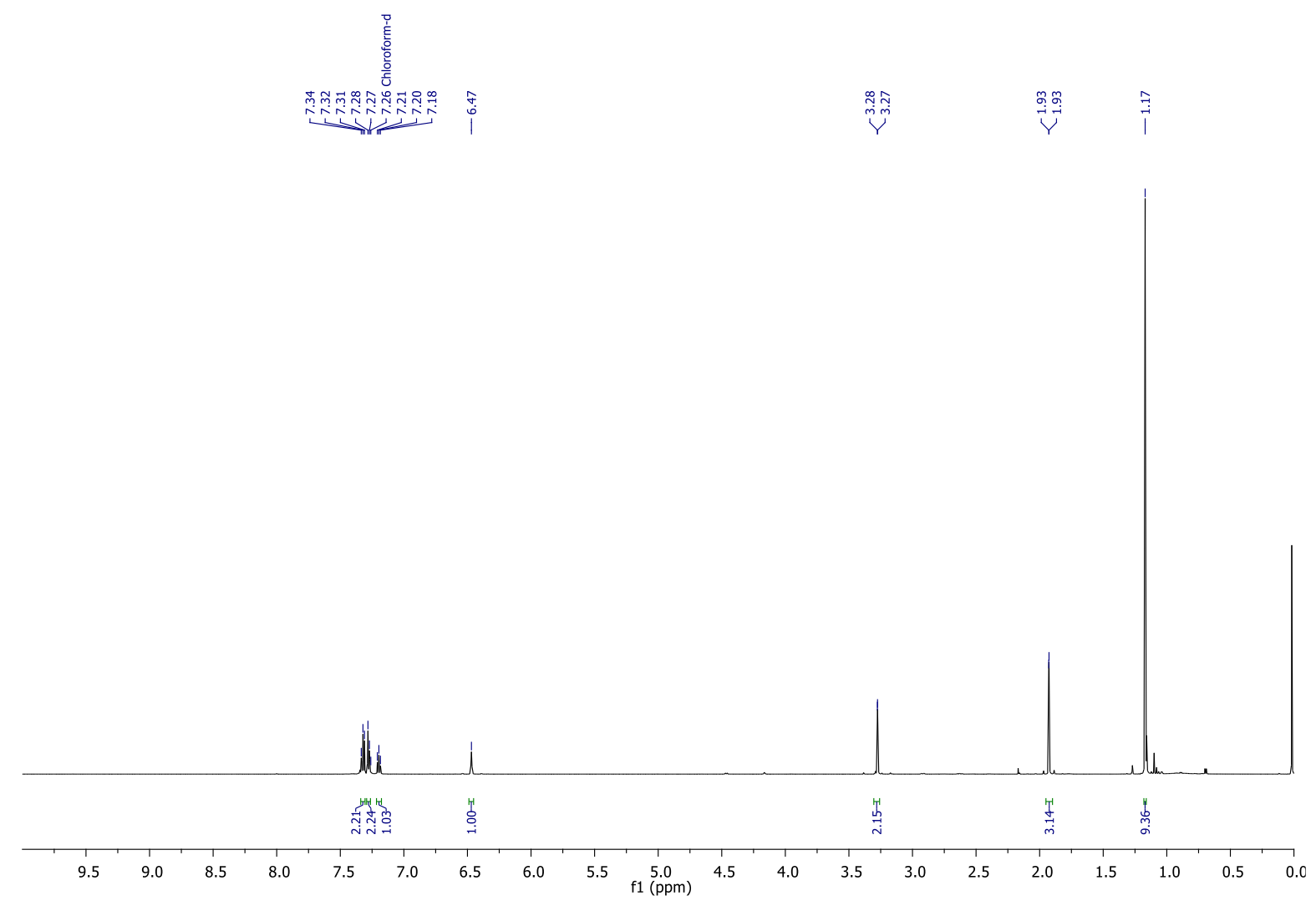

Figure $\boldsymbol{S - 2 2 7 .}{ }^{1} \mathrm{H}$ NMR spectrum of $12\left(\mathrm{CDCl}_{3}, 600 \mathrm{MHz}, 298 \mathrm{~K}\right)$

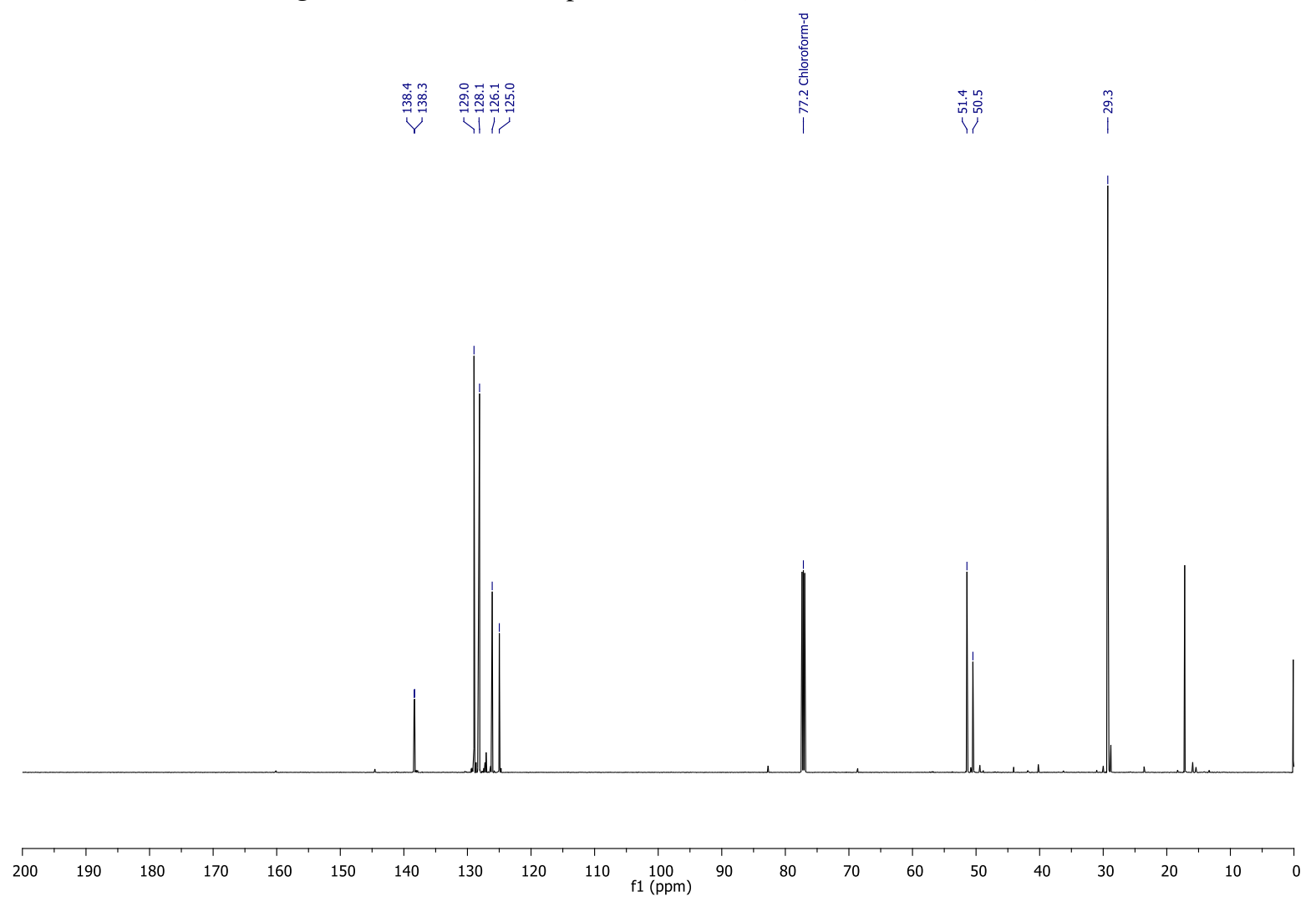

Figure S-228. ${ }^{13} \mathrm{C}$ NMR spectrum of $12\left(\mathrm{CDCl}_{3}, 151 \mathrm{MHz}, 298 \mathrm{~K}\right)$ 


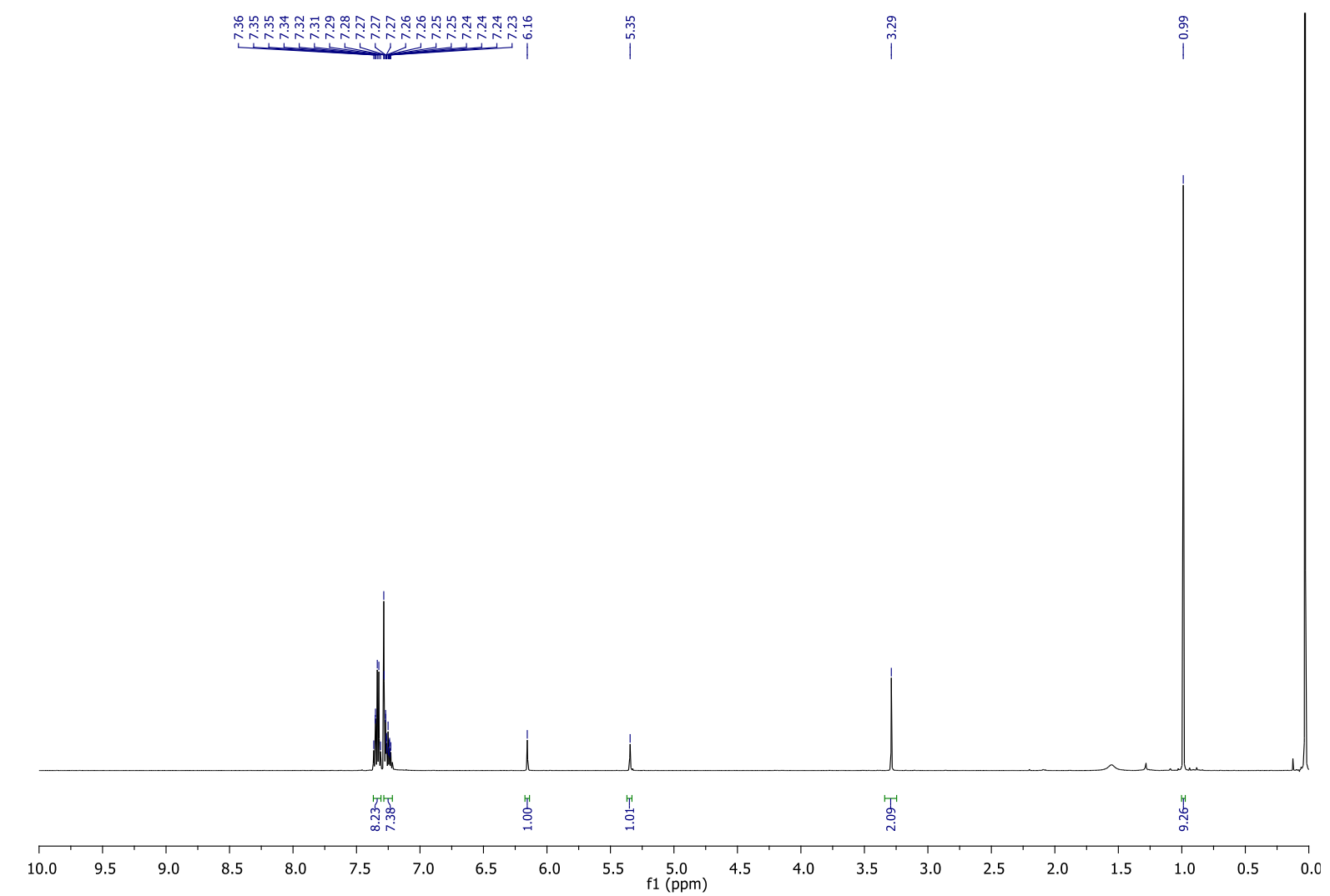

Figure S-229. ${ }^{1} \mathrm{H}$ NMR spectrum of $13\left(\mathrm{CDCl}_{3}, 600 \mathrm{MHz}, 298 \mathrm{~K}\right)$

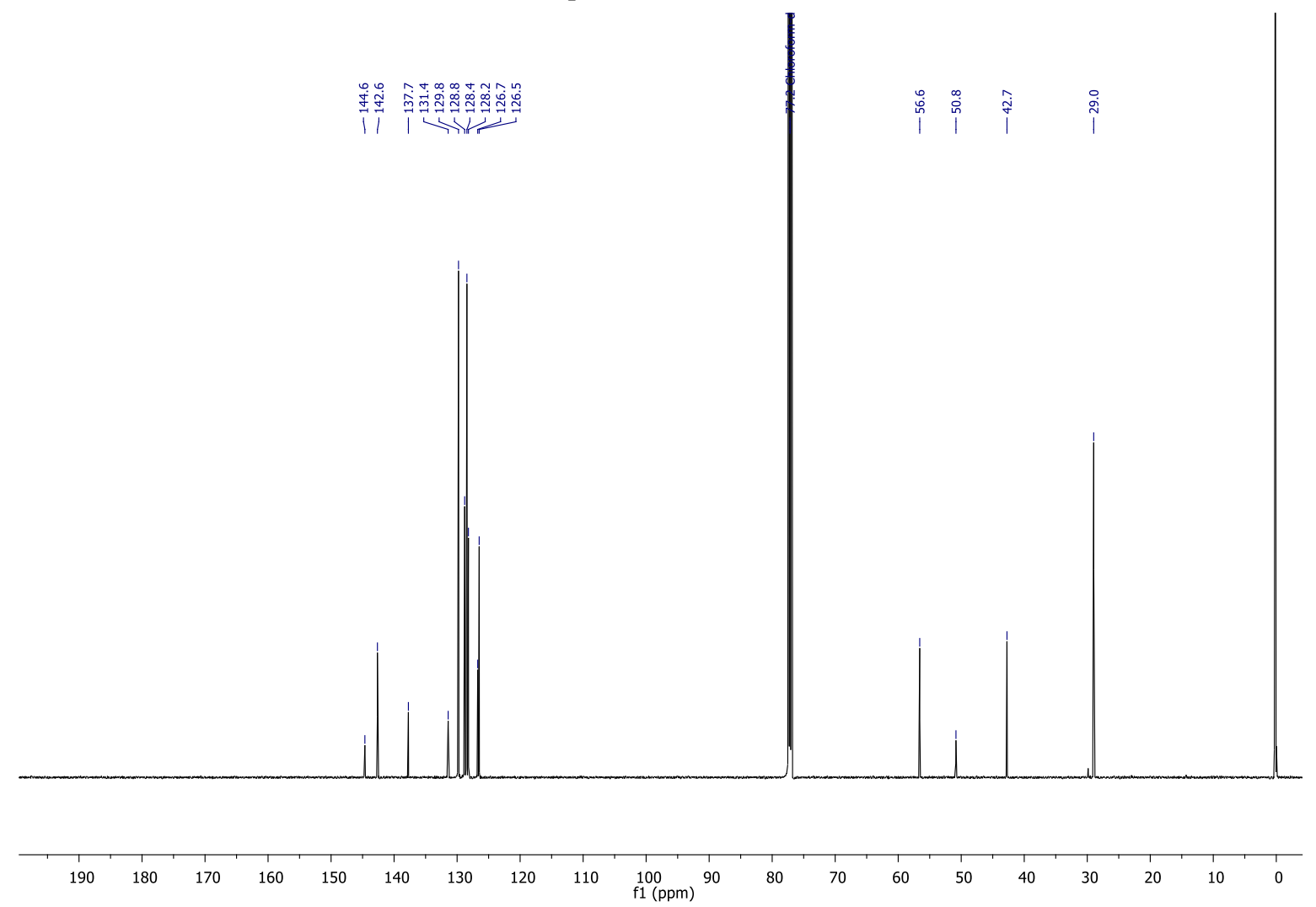

Figure S-230. ${ }^{13} \mathrm{C}$ NMR spectrum of $\mathbf{1 3}\left(\mathrm{CDCl}_{3}, 151 \mathrm{MHz}, 298 \mathrm{~K}\right)$ 

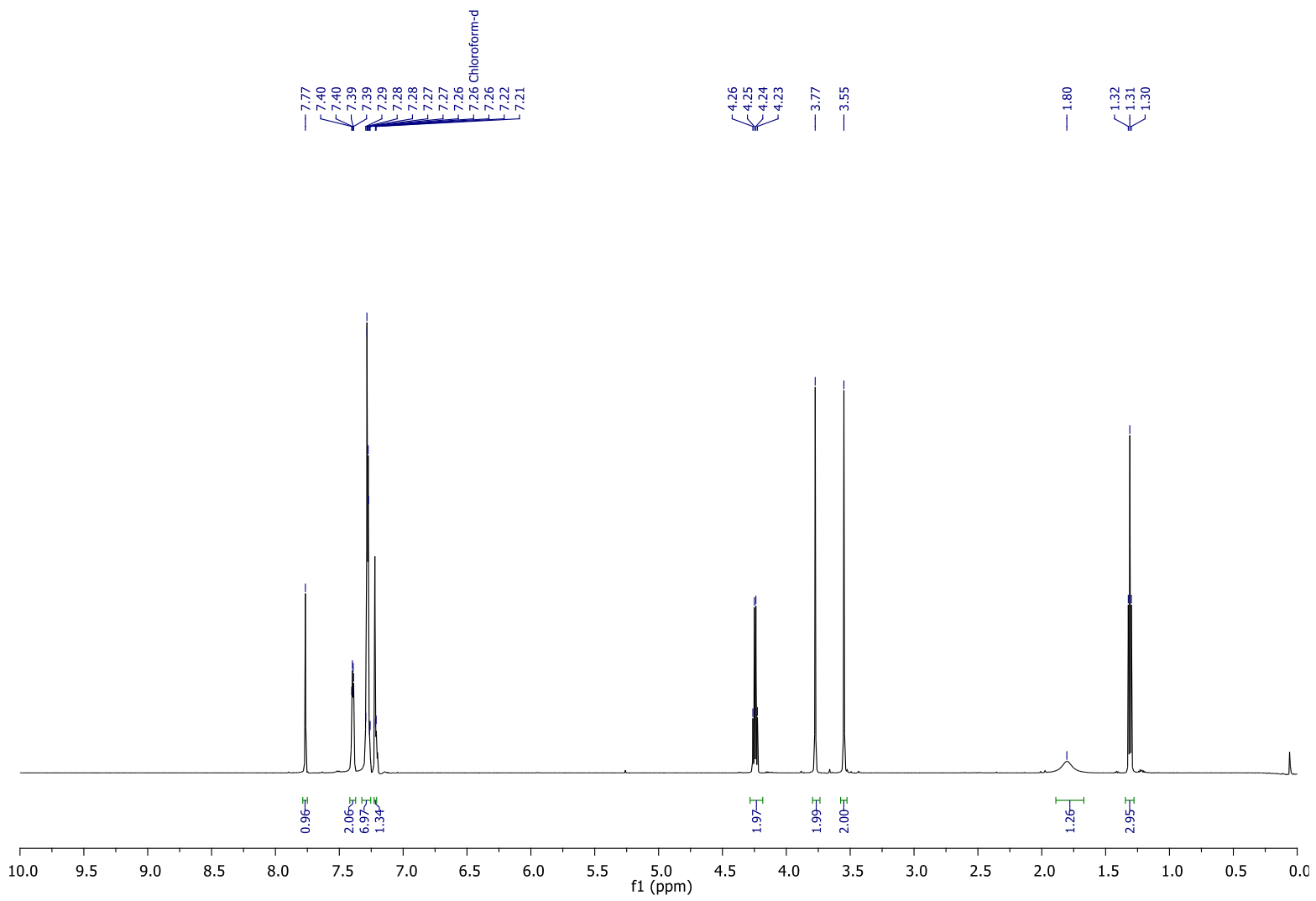

Figure $\boldsymbol{S}$-231. ${ }^{1} \mathrm{H}$ NMR spectrum of $14\left(\mathrm{CDCl}_{3}, 600 \mathrm{MHz}, 298 \mathrm{~K}\right)$

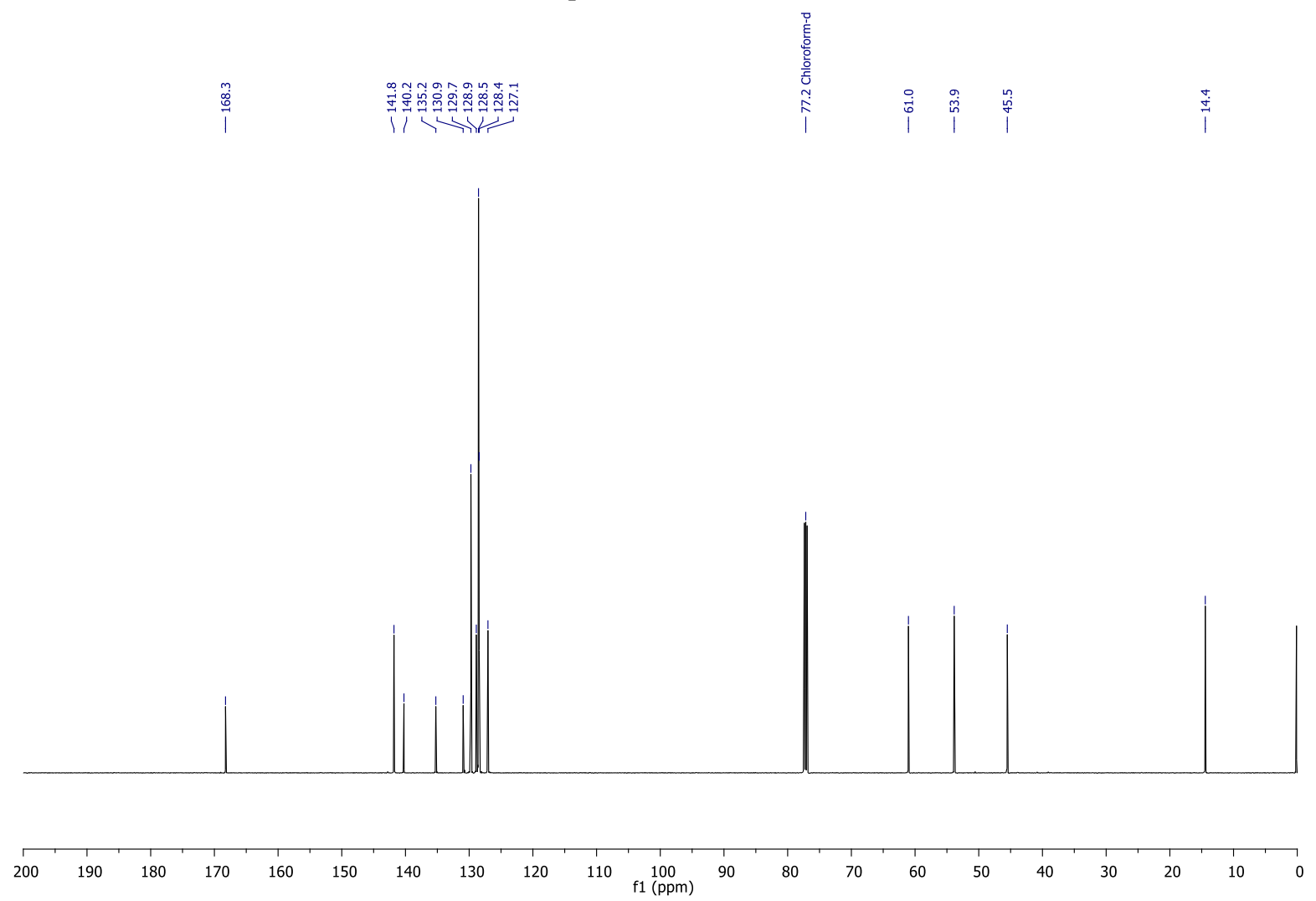

Figure S-232. ${ }^{13} \mathrm{C}$ NMR spectrum of $14\left(\mathrm{CDCl}_{3}, 151 \mathrm{MHz}, 298 \mathrm{~K}\right)$ 


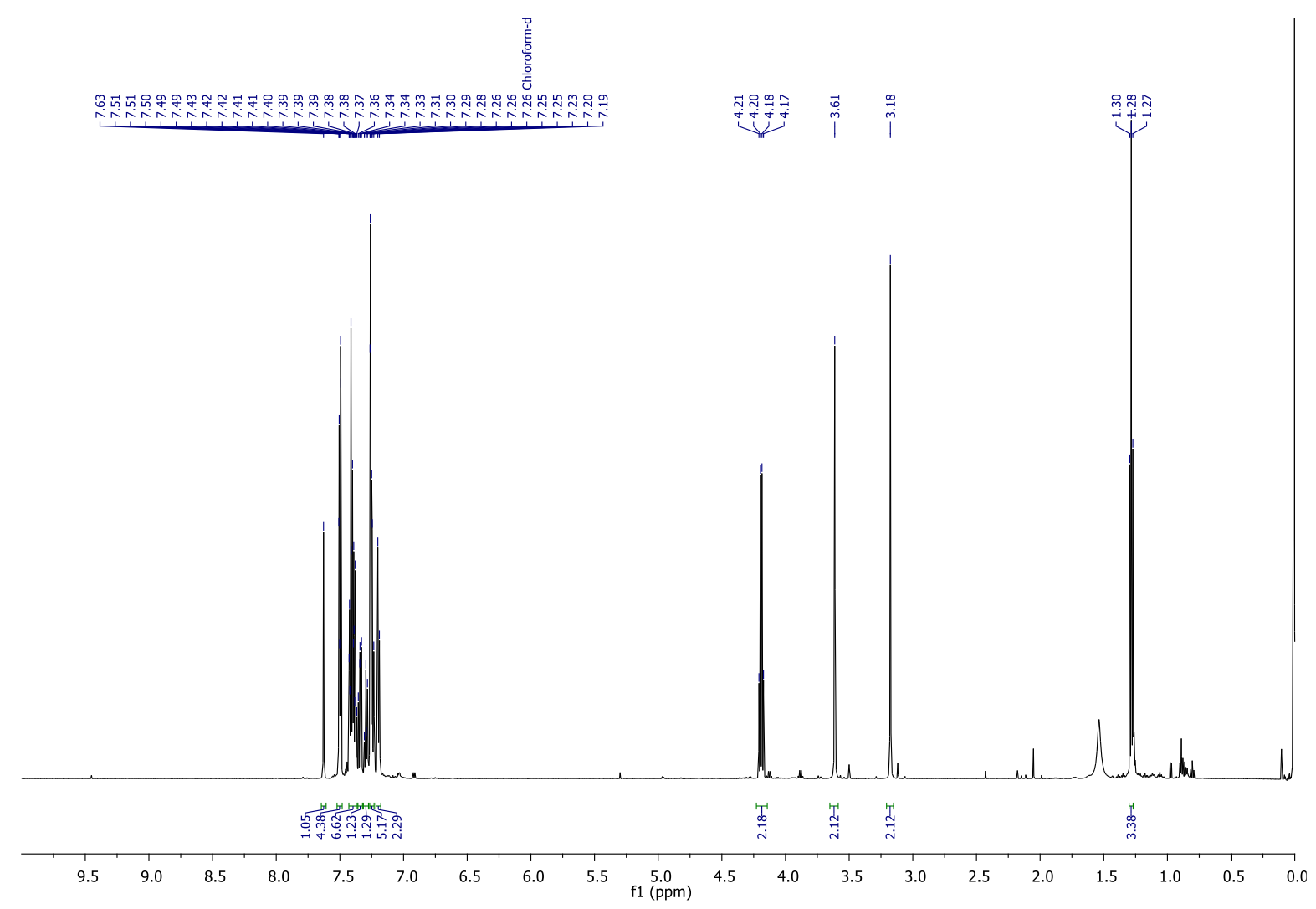

Figure $\boldsymbol{S - 2 3 3 .}{ }^{1} \mathrm{H}$ NMR spectrum of $15\left(\mathrm{CDCl}_{3}, 600 \mathrm{MHz}, 298 \mathrm{~K}\right)$
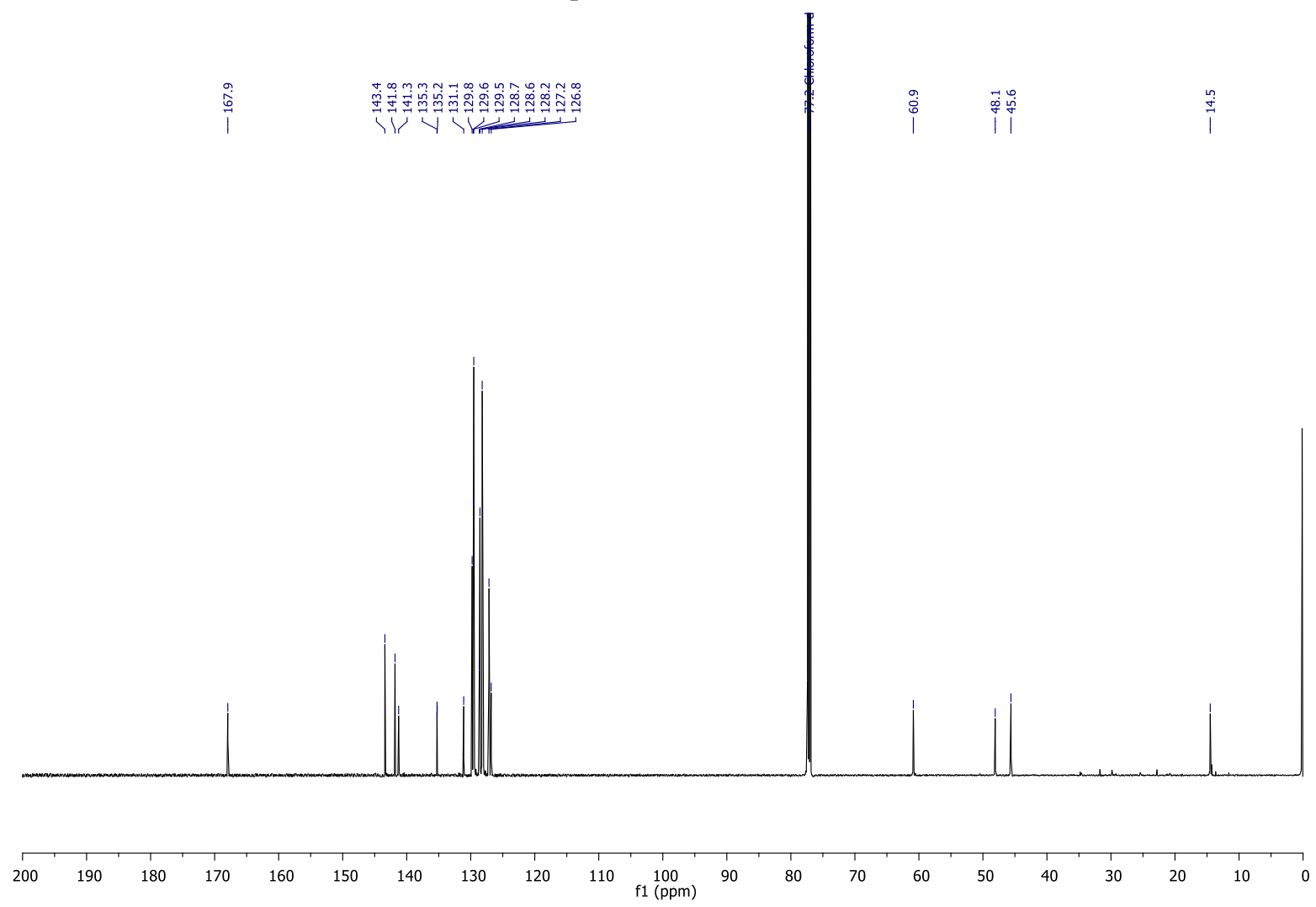

Figure S-234. ${ }^{13} \mathrm{C}$ NMR spectrum of $15\left(\mathrm{CDCl}_{3}, 151 \mathrm{MHz}, 298 \mathrm{~K}\right)$ 


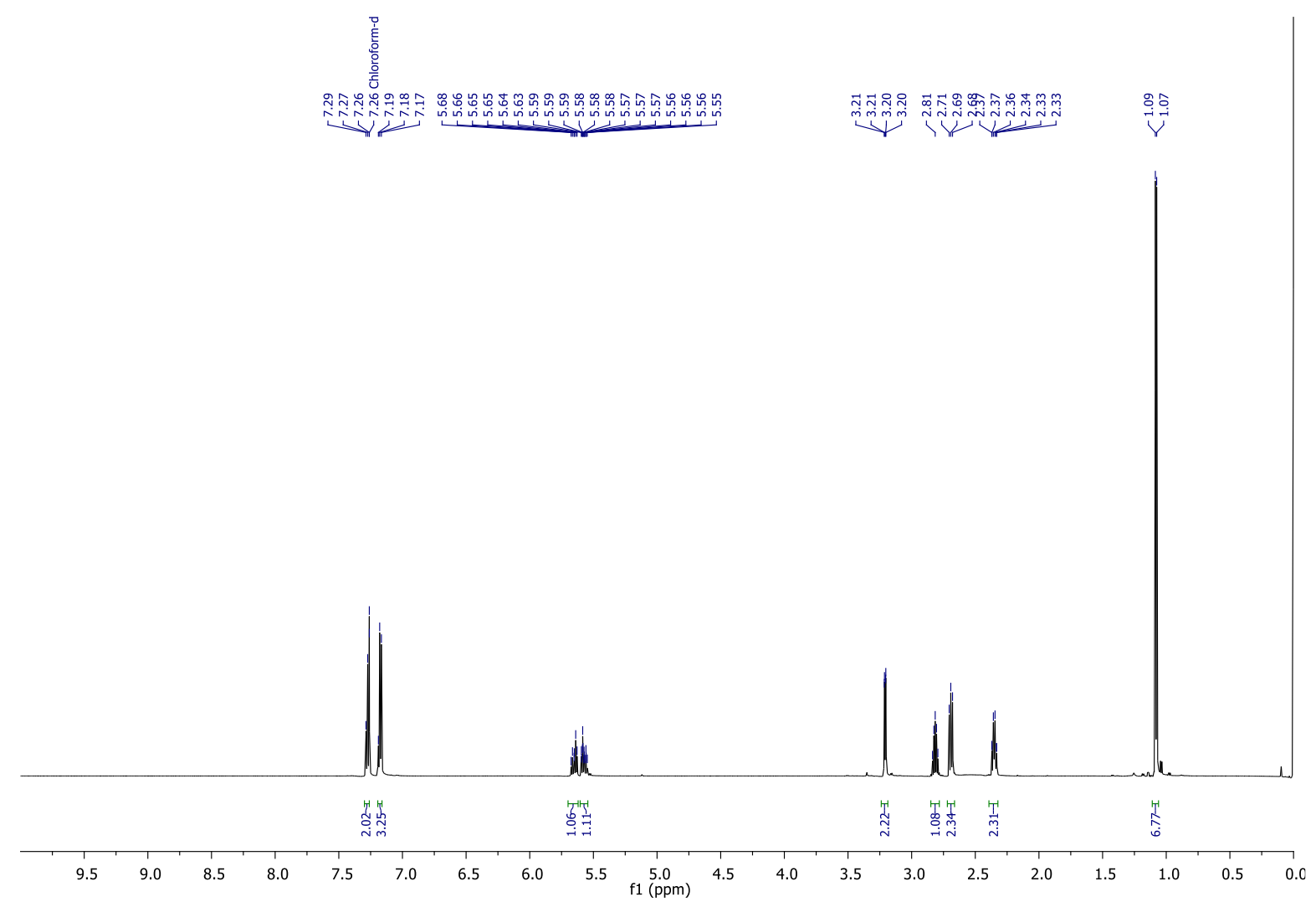

Figure S-235. ${ }^{1} \mathrm{H}$ NMR spectrum of $\mathbf{1 6}\left(\mathrm{CDCl}_{3}, 600 \mathrm{MHz}, 298 \mathrm{~K}\right)$
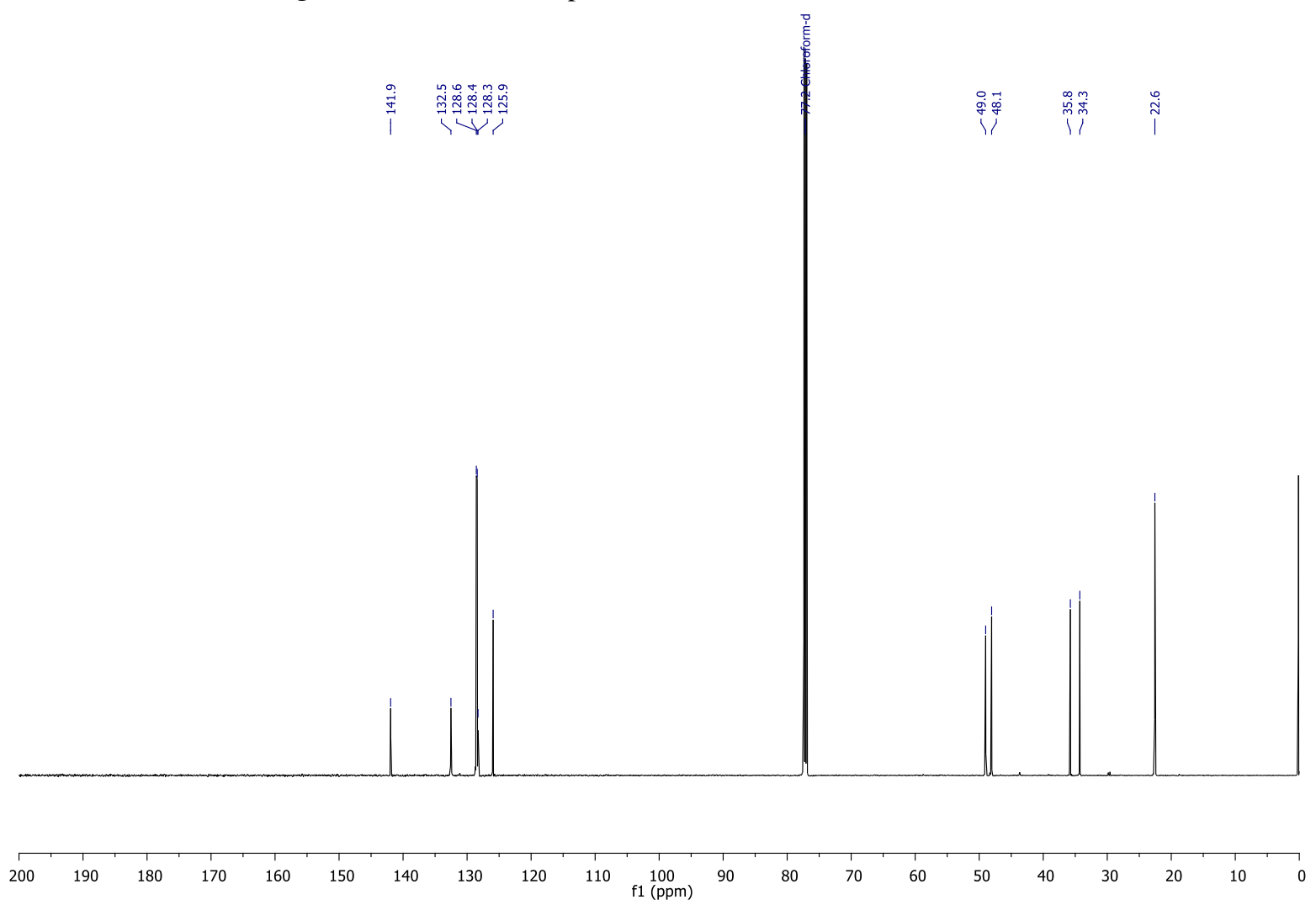

Figure S-236. ${ }^{13} \mathrm{C}$ NMR spectrum of $16\left(\mathrm{CDCl}_{3}, 151 \mathrm{MHz}, 298 \mathrm{~K}\right)$ 


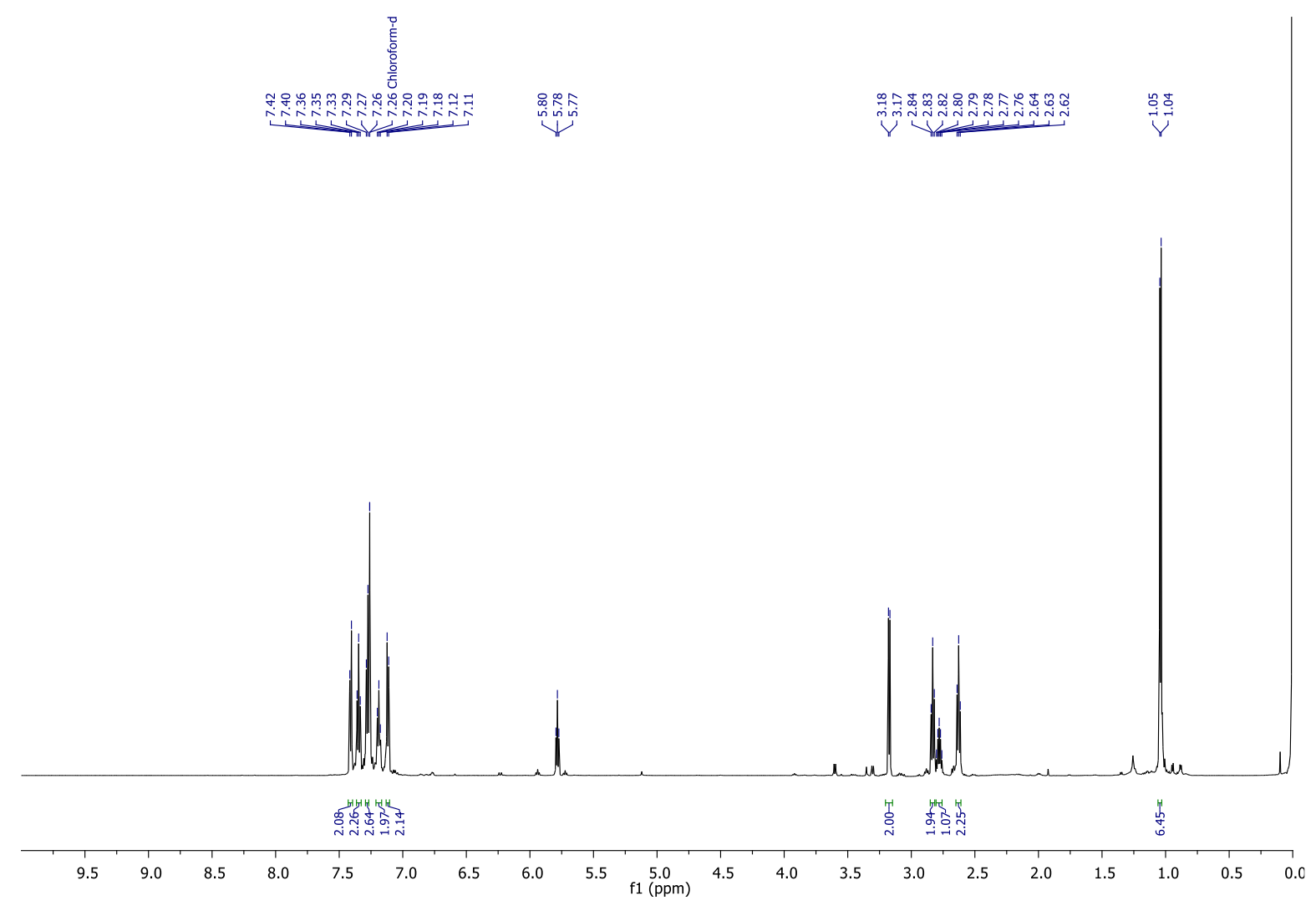

Figure S-237. ${ }^{1} \mathrm{H}$ NMR spectrum of $17\left(\mathrm{CDCl}_{3}, 600 \mathrm{MHz}, 298 \mathrm{~K}\right)$

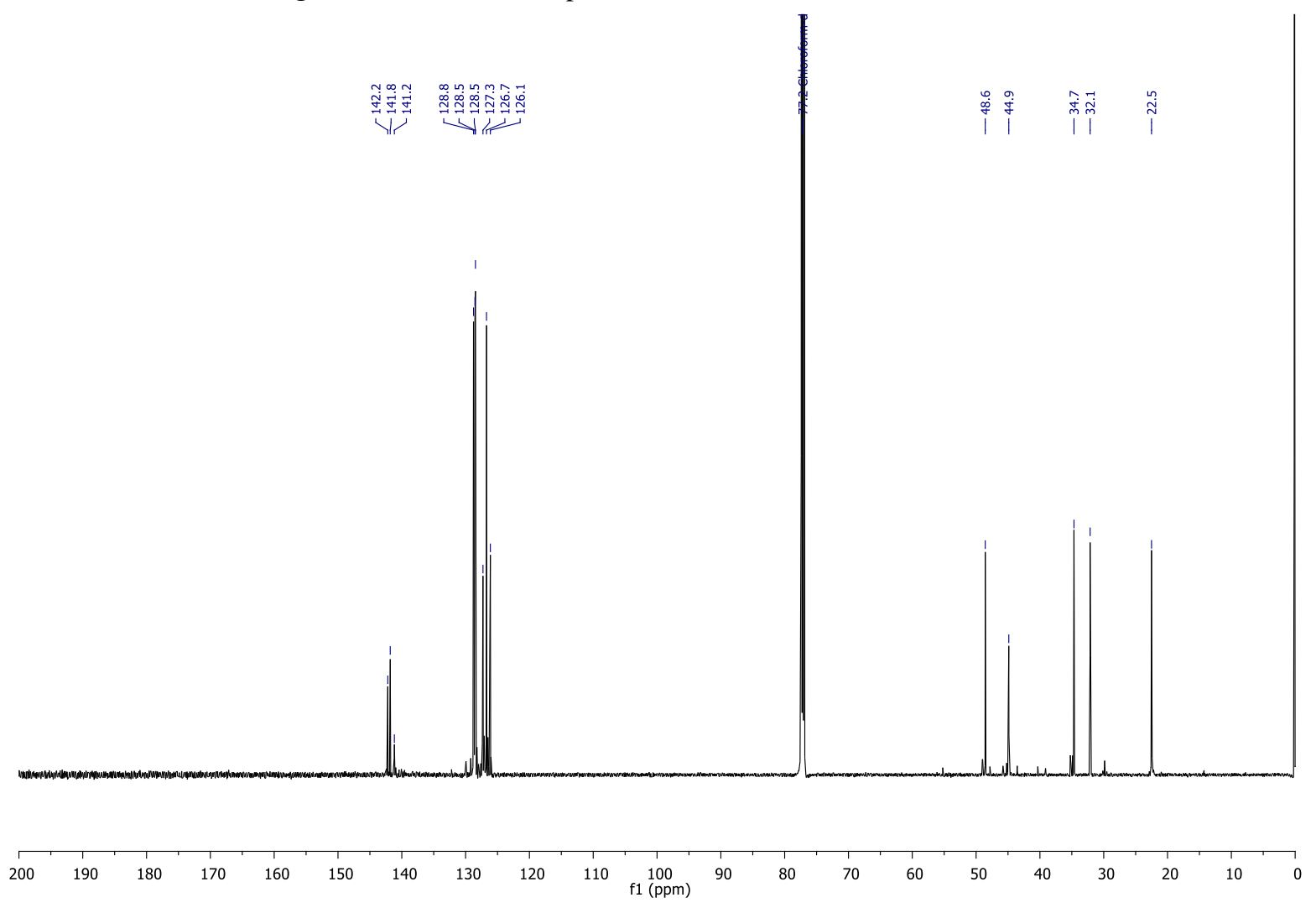

Figure S-238. ${ }^{13} \mathrm{C}$ NMR spectrum of $17\left(\mathrm{CDCl}_{3}, 151 \mathrm{MHz}, 298 \mathrm{~K}\right)$ 


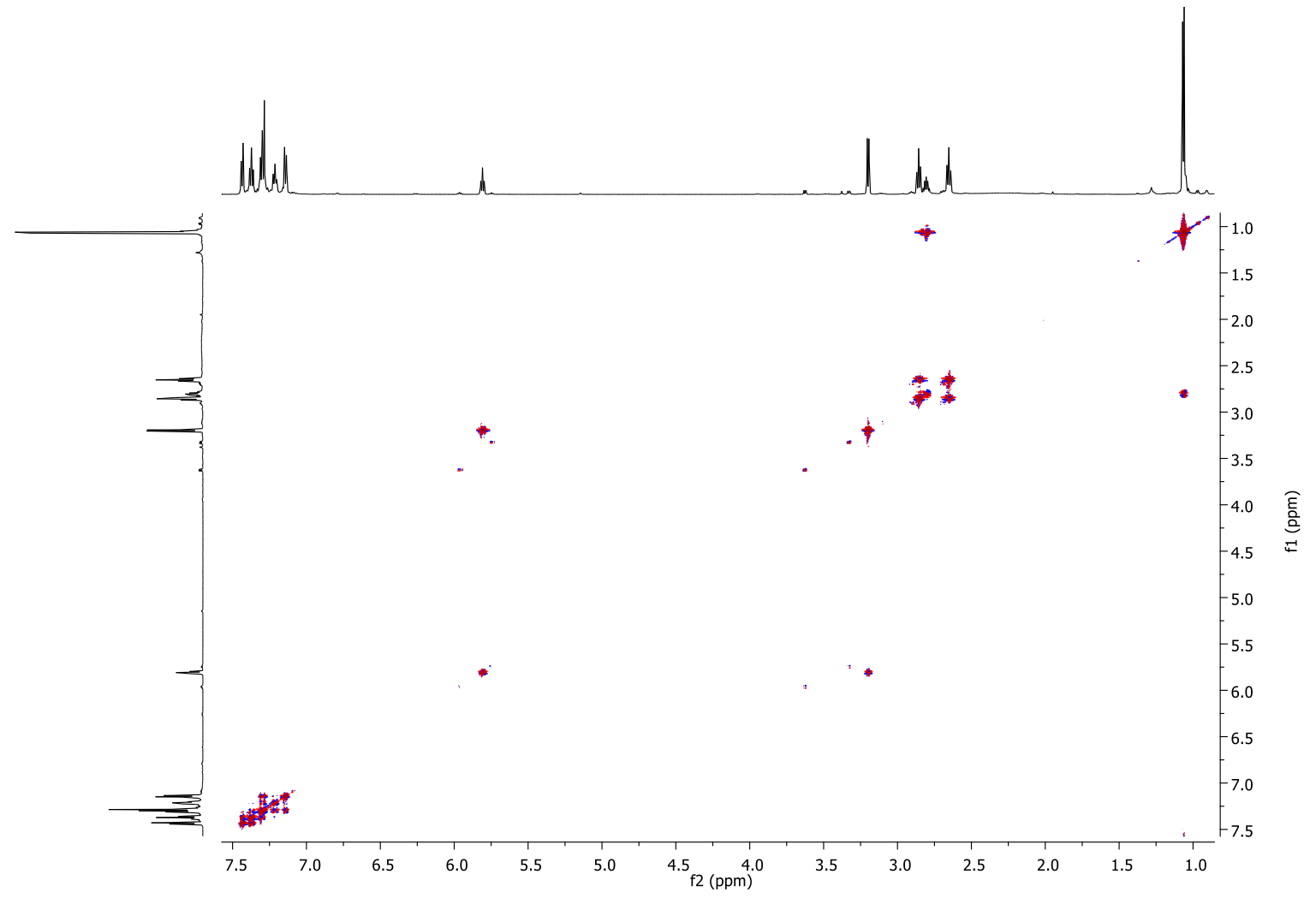

Figure S-239. Full COSY spectrum of $17\left(\mathrm{CDCl}_{3}, 600 \mathrm{MHz}, 298 \mathrm{~K}\right)$

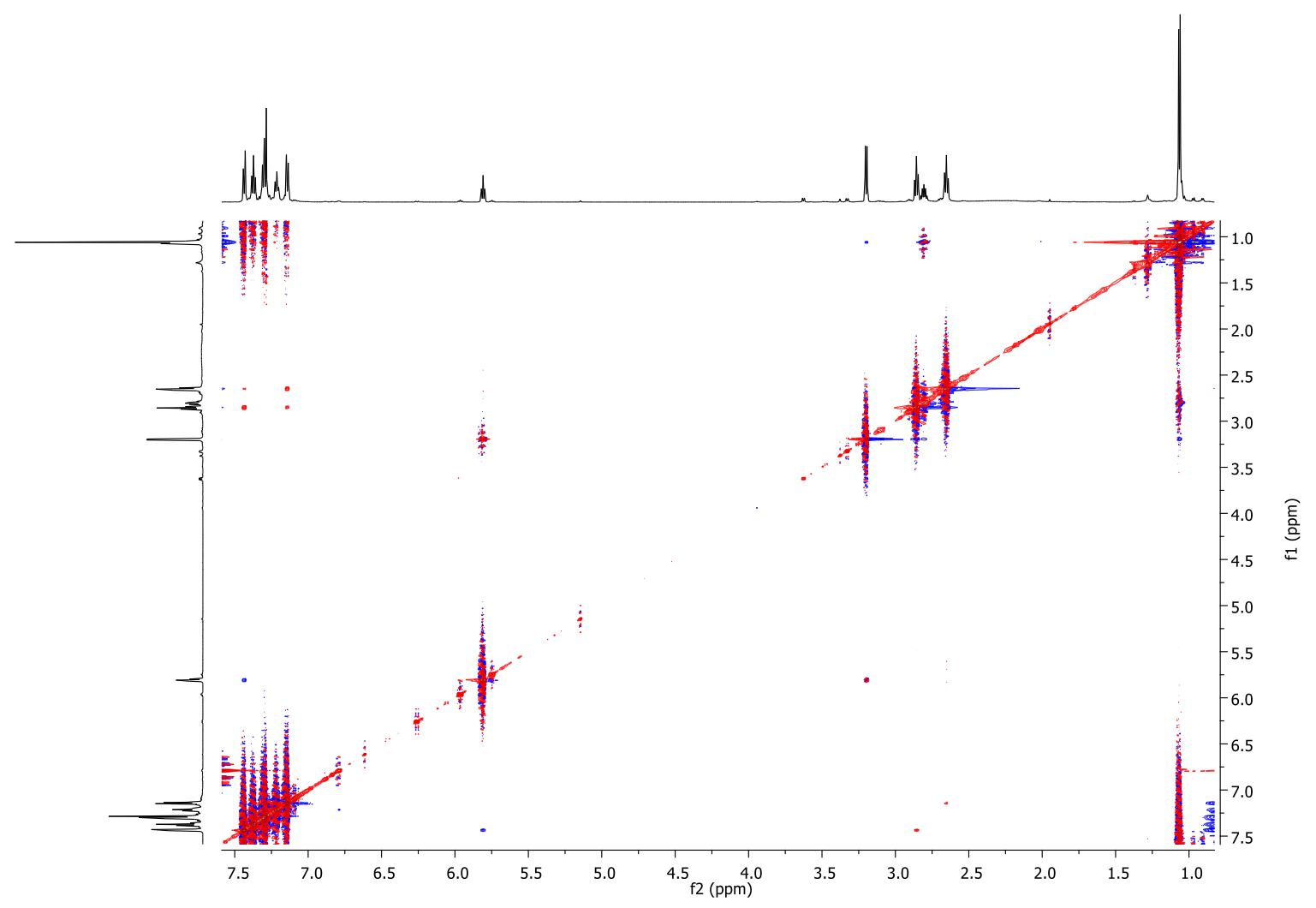

Figure S-240. Full NOESY spectrum of $17\left(\mathrm{CDCl}_{3}, 600 \mathrm{MHz}, 298 \mathrm{~K}\right)$ 


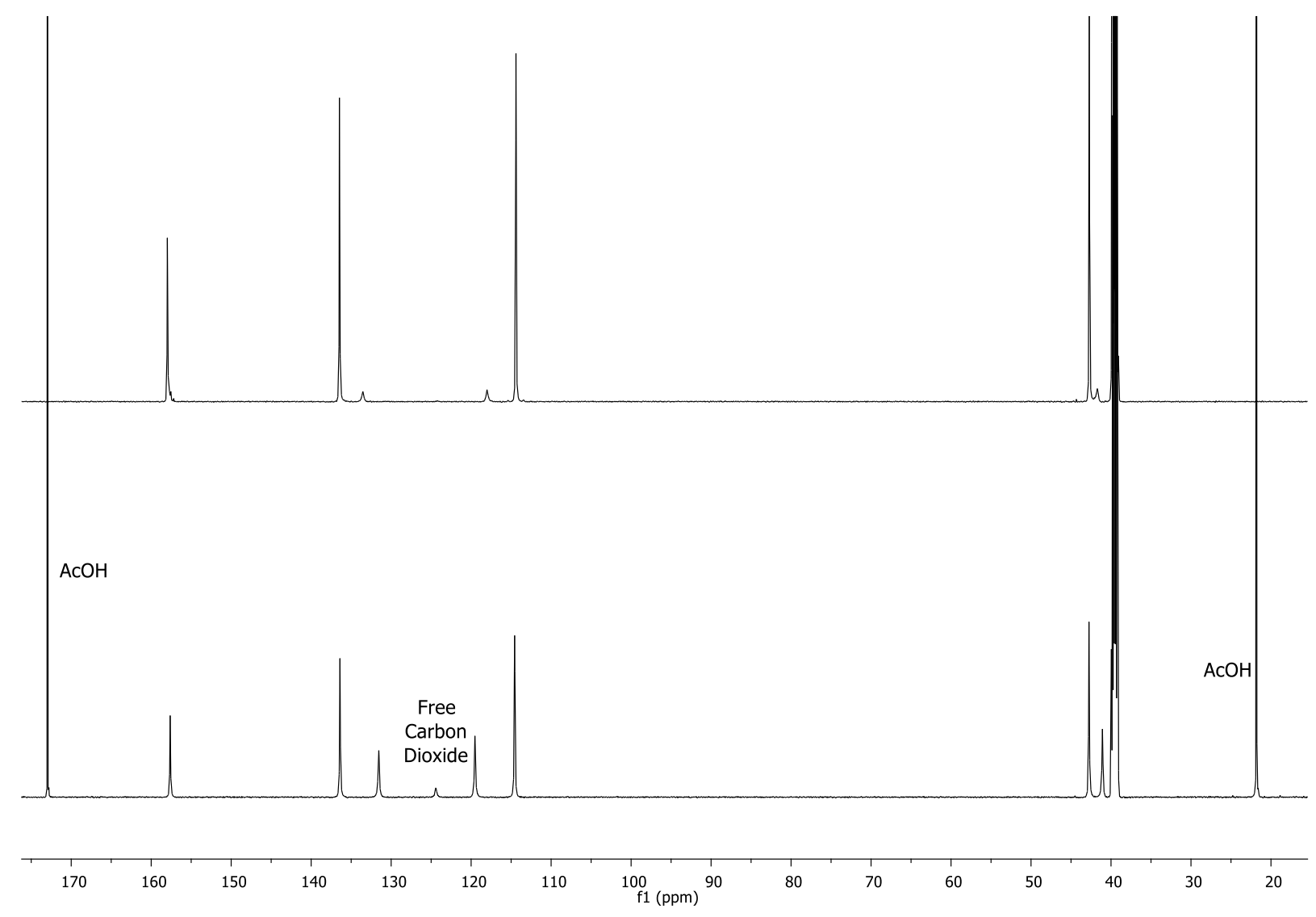

Figure S-241. ${ }^{13} \mathrm{C}$ NMR Spectra of Allylamine $+\mathrm{CO}_{2}$ without (top) and with (bottom) AcOH added (DMSO- $d_{6}, 600$ $\mathrm{MHz}, 298 \mathrm{~K}$ ) 


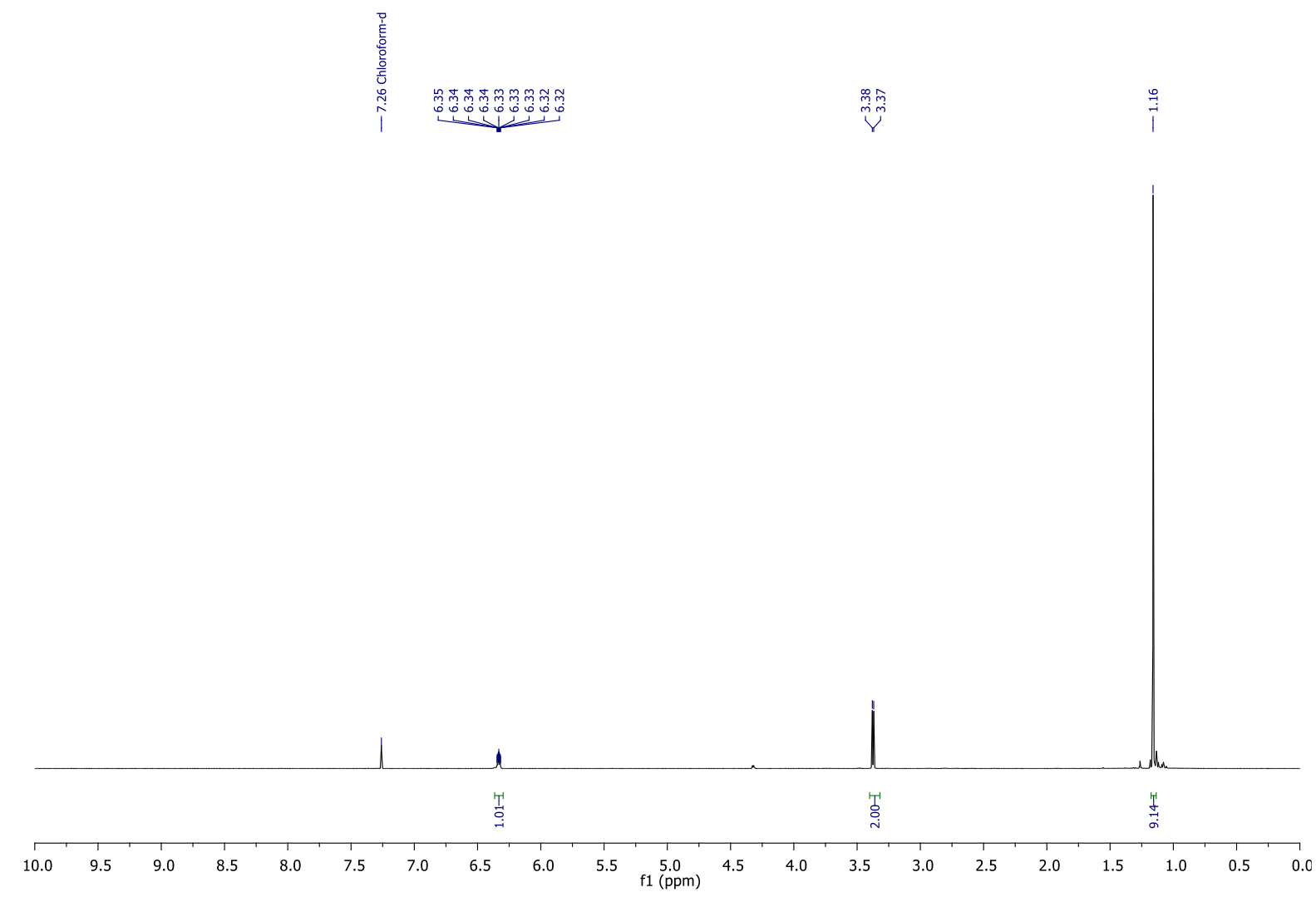

Figure $\boldsymbol{S - 2 4 2 .}{ }^{1} \mathrm{H}$ NMR spectrum of $\boldsymbol{S}-\mathbf{1 - 1} \boldsymbol{d}_{\mathbf{6}}\left(\mathrm{CDCl}_{3}, 600 \mathrm{MHz}, 298 \mathrm{~K}\right)$
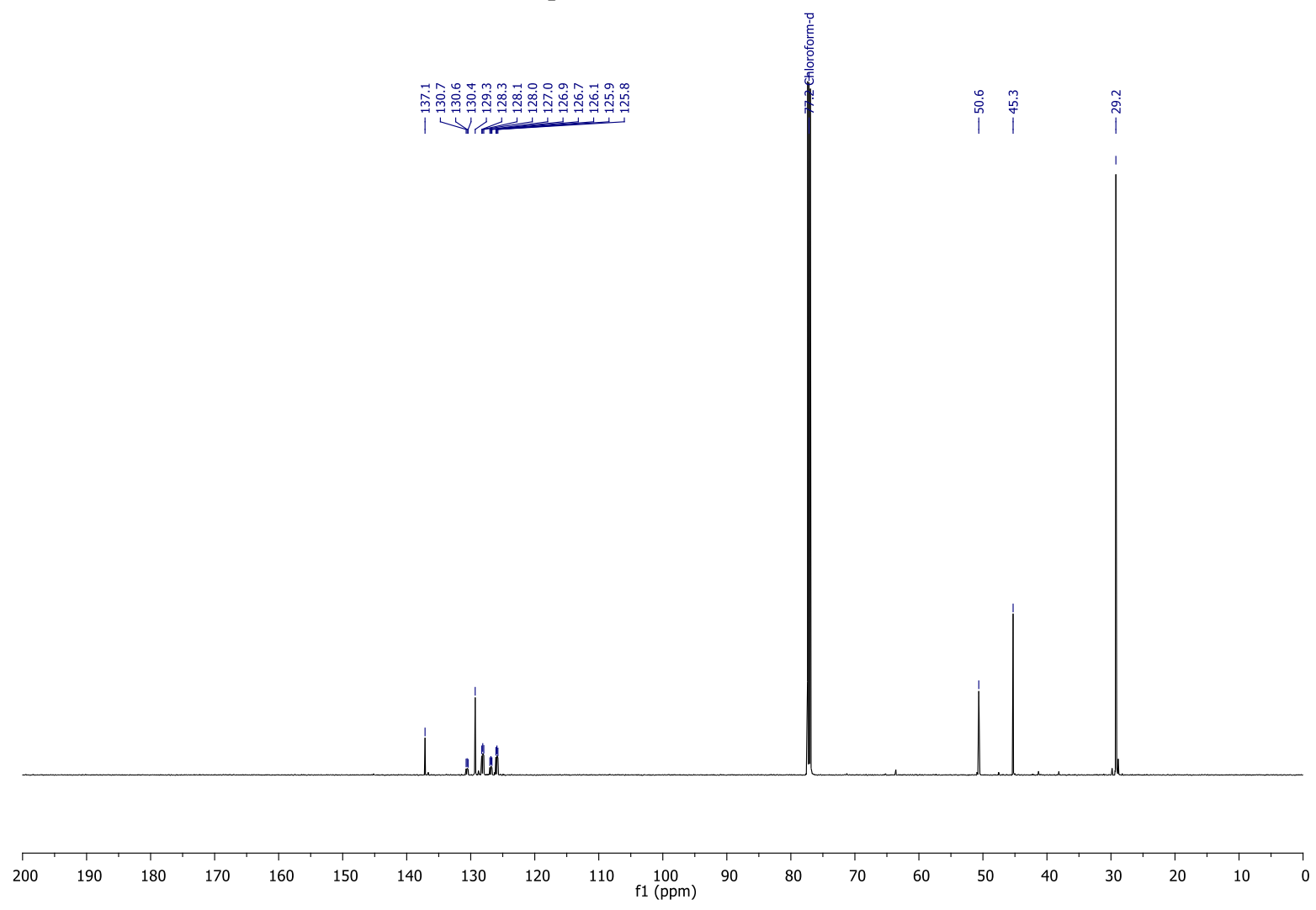

Figure $\boldsymbol{S - 2 4 3 .}{ }^{13} \mathrm{C}$ NMR spectrum of $\boldsymbol{S}-\mathbf{1}-\boldsymbol{d}_{\mathbf{6}}\left(\mathrm{CDCl}_{3}, 151 \mathrm{MHz}, 298 \mathrm{~K}\right)$ 


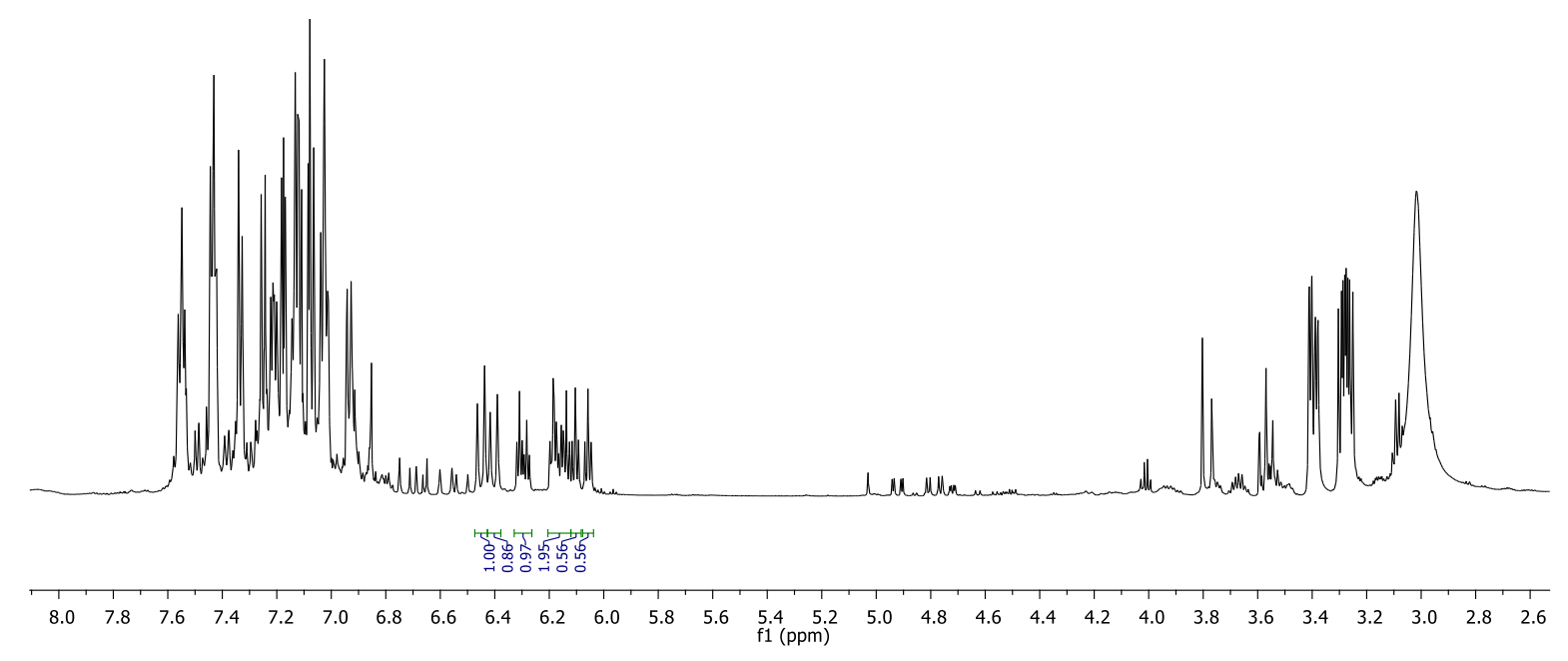

Figure S-244. ${ }^{1} \mathrm{H}$ NMR spectrum of Heterodiarylation via Synchronous Addition $\left(\mathrm{CDCl}_{3}, 600 \mathrm{MHz}, 298 \mathrm{~K}\right)$

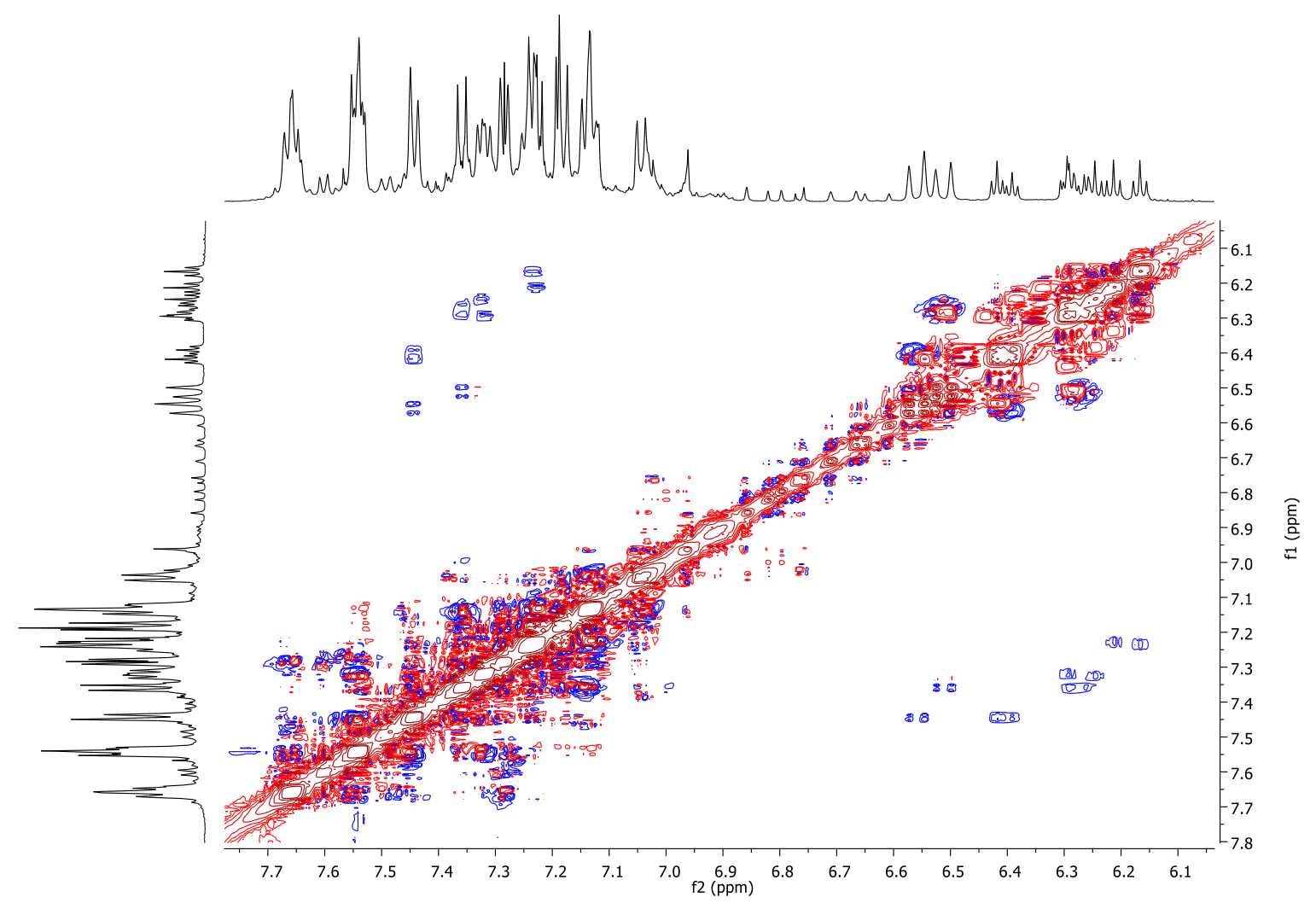

Figure S-245. Zoom NOESY spectrum of Heterodiarylation via Sequential Addition $\left(\mathrm{CDCl}_{3}, 600 \mathrm{MHz}, 298 \mathrm{~K}\right)$ 


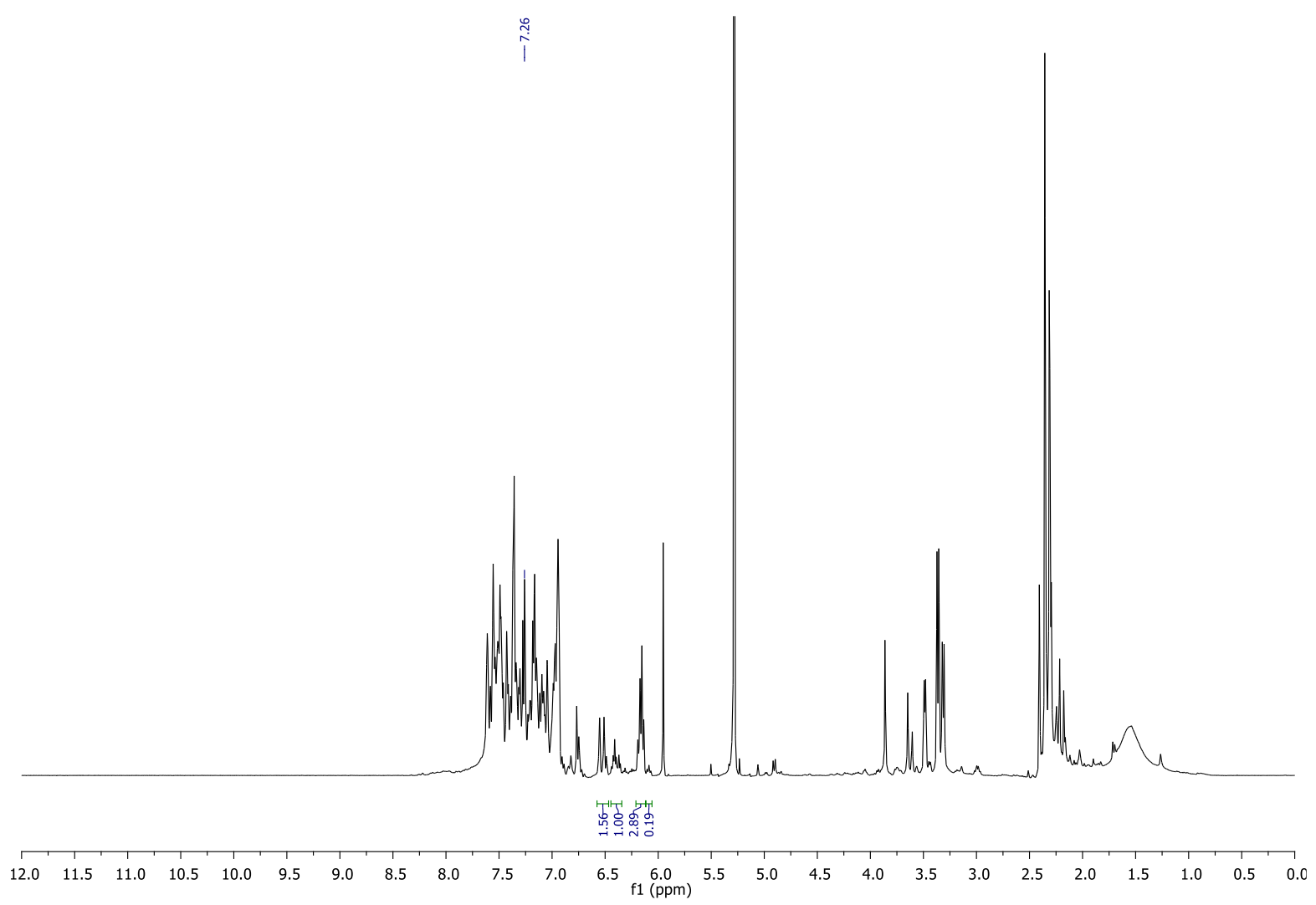

Figure S-246. ${ }^{1} \mathrm{H}$ NMR spectrum of Heterodiarylation via Sequential Addition $\left(\mathrm{CDCl}_{3}, 600 \mathrm{MHz}, 298 \mathrm{~K}\right)$ 


\section{X-Ray Data}

Table $S$-4. X-Ray Diffraction Data for $2 \mathrm{n} \cdot \mathrm{HOAc}$

CCDC Deposition \#
Chemical formula
Formula weight
Temperature
Wavelength
Crystal size
Crystal habit
Crystal system
Space group
Unit cell dimensions

Volume

$\mathbf{Z}$

Density (calculated)

Absorption coefficient

$\mathbf{F}(000)$

Theta range for data collection

Index ranges

Reflections collected

Independent reflections
1990723

$\mathrm{C}_{22} \mathrm{H}_{28} \mathrm{~N}_{2} \mathrm{O}_{3}$

$368.46 \mathrm{~g} / \mathrm{mol}$

$100(2) \mathrm{K}$

$1.54178 \AA$

$0.024 \times 0.065 \times 0.222 \mathrm{~mm}$

translucent yellow plate

triclinic

$\mathrm{P}-1$

$\mathrm{a}=9.6619(4) \AA \quad \alpha=101.631(2)^{\circ}$

$\mathrm{b}=10.3512(4) \AA \quad \beta=93.418(2)^{\circ}$

$\mathrm{c}=10.4500(4) \AA \quad \gamma=97.942(2)^{\circ}$

1009.72(7) $\AA^{3}$

2

$1.212 \mathrm{~g} / \mathrm{cm}^{3}$

$0.645 \mathrm{~mm}^{-1}$

396

4.34 to $69.76^{\circ}$

$-10<=\mathrm{h}<=11,-11<=\mathrm{k}<=12,-12<=1<=12$

8867

$3489[\mathrm{R}(\mathrm{int})=0.0167]$

Coverage of independent reflections $91.5 \%$

Absorption correction

Structure solution technique

Structure solution program

Refinement method

Refinement program

Function minimized

Data / restraints / parameters

Goodness-of-fit on $\mathbf{F}^{2}$

$\Delta / \sigma_{\max }$

Final R indices

Weighting scheme

Largest diff. peak and hole

R.M.S. deviation from mean
Multi-Scan

direct methods

SHELXT 2014/5 28

Full-matrix least-squares on $\mathrm{F}^{2}$

SHELXL-2018/3 ${ }^{29}$

$\Sigma \mathrm{w}\left(\mathrm{F}_{\mathrm{o}}^{2}-\mathrm{F}_{\mathrm{c}}^{2}\right)^{2}$

3489 / 0 / 248

1.636

0.002

3295 data; $\mathrm{I}>2 \sigma(\mathrm{I}) \mathrm{R} 1=0.0414, \mathrm{wR} 2=0.1715$

all data $\quad \mathrm{R} 1=0.0450, \mathrm{wR} 2=0.1787$

$\mathrm{w}=1 /\left[\sigma^{2}\left(\mathrm{~F}_{\mathrm{o}}^{2}\right)+(0.1000 \mathrm{P})^{2}\right]$

where $\mathrm{P}=\left(\mathrm{F}_{\mathrm{o}}^{2}+2 \mathrm{~F}_{\mathrm{c}}^{2}\right) / 3$

0.247 and $-0.278 \mathrm{e}^{-3}$

$0.088 \mathrm{e}^{-3}$ 


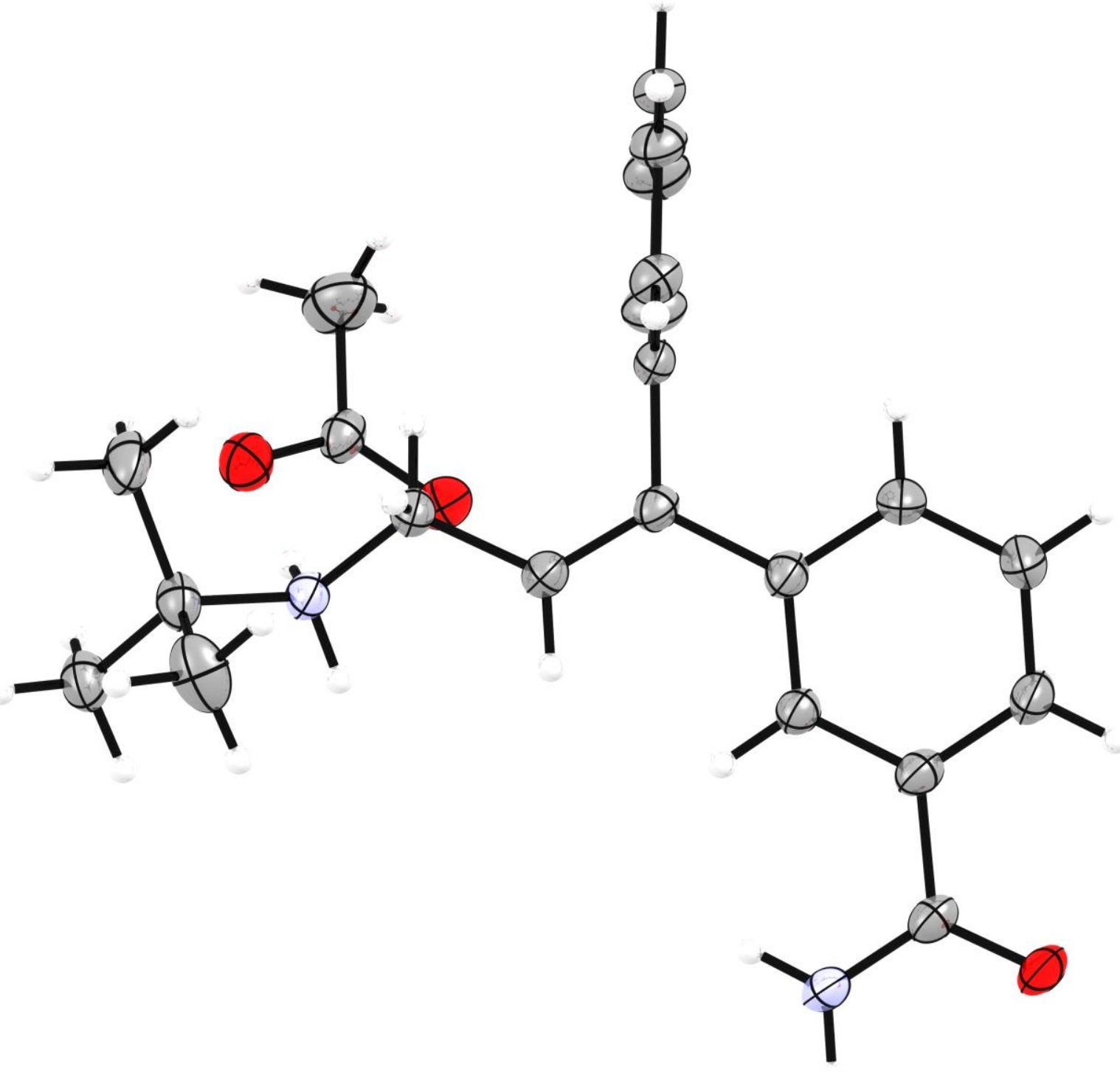

Figure S-247. X-Ray Diffraction ORTEP for $2 \mathrm{n} \cdot \mathrm{HOAc}$ 


\section{References}

${ }^{1}$ Fulmer, G. R.; Miller, A. J. M.; Sherden, N. H.; Gottlieb, H. E.; Nudelman, A.; Stoltz, B. M.; Bercaw, J. E.; Goldberg, K. I. NMR Chemical Shifts of Trace Impurities: Common Laboratory Solvents, Organics, and Gases in Deuterated Solvents Relevant to the Organometallic Chemist. Organometallics, 2010, 29, 2176-2179.

${ }^{2}$ Baldwin, B. W.; Kuntzleman, T. S.Liquid $\mathrm{CO}_{2}$ in Centrifuge Tubes: Separation of Chamazulene from Blue Tansy (Tanacetum annuum) Oil via Extraction and Thin-Layer Chromatography. J. Chem. Educ., 2018, 95, 620-624; b) Hudson, R.; Ackerman, H. M.; Gallo, L. K.; Gwinner, A. S.; Krauss, A.; Sears, J. D.; Bishop, A.; Esdale, K. N.; Katz, J. L. $\mathrm{CO}_{2}$ Dry Cleaning: A Benign Solvent Demonstration Accessible to K-8 Audiences. J. Chem. Educ., 2017, 94 , 480-482; c) Barcena, H.; Chen, P. An Anesthetic Drug Demonstration and an Introductory Antioxidant Activity Experiment with "Eugene, the Sleepy Fish". J. Chem. Educ., 2016, 93, 202-205; d) McKenzie, L. C.; Thompson, J. E.; Sullivan, R.; Hutchison, J. E. Green Chemical Processing in the Teaching Laboratory: AConvenient Liquid $\mathrm{CO}_{2}$ Extraction of Natural Products.Green Chem., 2004, 6, 355-358.

${ }^{3}$ Kapoor, M.; Chand-Thakuri, P.; Maxwell, J. M.; Young, M. C. Achieving Moderate Pressures in Sealed Vessels Using Dry Ice As a Solid $\mathrm{CO}_{2}$ Source. J. Vis. Exp., 2018, 138, e58281.

${ }^{4}$ De Kimpe, N.; Stanoeva, E.; Verhé, R.; Schamp, N. Synthesis of Secondary Allylic Amines. Synthesis, 1988, 587592.

${ }^{5}$ Ohshima, T.; Miyamoto, Y.; Ipposhi, J.; Nakahara, Y.; Utsunomiya, M.; Mashima, K. Platinum-Catalyzed Direct Amination of Allylic Alcohols under Mild Conditions: Ligand and Microwave Effects, Substrate Scope, and Mechanistic Study. J. Am. Chem. Soc., 2009, 131, 14317-14328.

${ }^{6}$ Takeuchi, R.; Ue, N.; Tanabe, K.; Yamashita, K.; Shiga, N. Iridium Complex-Catalyzed Allylic Amination of Allylic Esters. J. Am. Chem. Soc., 2001, 123, 9525-9534.

${ }^{7}$ Blanpain, A.; Clark, J. H.; Farmer, T. J.; Guo, Y.; Ingram, I. D. V.; Kendrick, J. E.; Lawrenson, S. B.; North, M.; Rodgers, G.; Whitwood, A. C. Rapid Ring-Opening Metathesis Polymerization of Monomers Obtained from Biomass-Derived Furfuryl Amines and Maleic Anhydride. ChemSusChem, 2019, 12, 2393-2401.

${ }^{8}$ Kerres, S.; Plut, E.; Malcherek, S.; Rehbein, J.; Reiser, O. Visible Light-Mediated Synthesis of Enantiopure $\gamma-$ Cyclobutane Amino and 3-(Aminomethyl)-5-phenylpentanoic Acids. Adv. Synth. Catal., 2019, 361, 1400-1407.

${ }^{9}$ Gracheva, I. A.; Svirschchevskaya, E. V.; Faerman, V. I.; Beletskaya, I. P.; Fedorov, A. Y. Synthesis and Antiproliferative Properties of Bifunctional Allocolchicine Derivatives. Synthesis, 2018, 50, 2753-2760.

${ }^{10}$ Wan, J.-H.; Hu, Y.; Liu, H.; Tu, Y.-H.; He, Z.-Y.; Sun, J.-S. The Catalytically Lignan-Activation-Based Approach for the Synthesis of (epi)-Podophyllotoxin Derivatives. J. Org. Chem., 2017, 82, 5652-5662.

${ }^{11}$ Mukherjee, S.; List, B. Chiral Counteranions in Asymmetric Transition-Metal Catalysis: Highly Enantioselective Pd/Brønsted Acid-Catalyzed Direct $\alpha$-Allylation of Aldehydes. J. Am. Chem. Soc., 2007, 129, 11336-11337.

${ }^{12}$ Shinde, G. B.; Niphade, N. C.; Deshmukh, S. P.; Toche, R. B.; Mathad, V. T. Industrial Application of the Forster Reaction: Novel One-Pot Synthesis of Cinacalcet Hydrochloride, a Calcimimetic Agent. Org. Process Res. Dev., 2011, 15, 455-461.

${ }^{13}$ Szabó, F.; Pethö, B.; Gonda, Z.; Novák, Z. Irong-Surfactant Nanocomposite-Catalyzed Benzylic Oxidation in Water. RSC Adv., 2013, 3, 4903-4908.

${ }^{14}$ Li, L.; Liu, Z.; Tang, S.; Li, J.; Ren, X.; Yang, G.; Li, H.; Yuan, B. Amphiphilic Ligands for Cu-Catalyzed Aerobic Oxidation to Synthesize 9-Fluorenones in Water. Catal. Commun., 2019, 127, 34-38. 
${ }^{15}$ Lidal, H. Carbon Dioxide Removal in Gas Treating Processes. 1992, Dr. Ing. Thesis, The University of Trondheim, The Norwegian Institute of Technology, Department of Chemical Engineering.

${ }^{16}$ Sugiura, H.; Yamazaki, S.; Go, K.; Ogawa, A. Intramolecular Cyclization of 3,3-Diarylpropenylamides of Electron-Deficient Alkenes: Stereoselective Synthesis of Functionalized Hexahydrobenzo[f]isoindoles. Eur. J. Org. Chem., 2019, 204-220.

${ }^{17}$ Hoegberg, T.; Ulff, B. Stereoconservative Reductive Methyl- and Dimethylamination of Isomeric 3,3Diarylpropenals. Synthetic and Mechanistic Studies on Control of the Stereochemistry. J. Org. Chem., 1984, 49, 4209-4214.

${ }^{18}$ van der Bent, A.; Bolmmaert, A. G. S.; Melman, C. T. M.; Ijzerman, A. P.; van Wijngaarden, I.; Soudijn, W. Hybrid Cholecystokinin-A Antagonists Based on Molecular Modeling of Lorglumide and L-364,718. J. Med. Chem., 1992, 35, 1042-1049.

${ }^{19}$ Chen, C.; Jin, S.; Zhang, Z.; Wei, B.; Wang, H.; Zhang, K.; Lv, H.; Dong, X.-D.; Zhang, X. Rhodium/YanphosCatalyzed Asymmetric Interrupted Intramolecular Hydroaminomethylation of trans-1,2-Disubstituted Alkenes. J. Am. Chem. Soc., 2016, 138, 9017-9020.

${ }^{20}$ Lei, Y.; Qui, R.; Zhang, L.; Xu, C.; Pan, Y.; Qin, X.; Li, H.; Xu, L.; Deng, Y. Palladium-Catalyzed Direct Arylation of Allylamines with Simple Arenes. ChemCatChem, 2015, 7, 1275-1279.

${ }^{21}$ Nazari, S. H.; Tiempos-Flores, N.; Forson, K. G.; Bourdeau, J. E.; Michaelis, D. J. C-N Bond Formation from Allylic Alcohols via Cooperative Nickel and Titanium Catalysis. J. Org. Chem., 2018, 83, 10646-10654.

${ }^{22}$ Senaweera, S.; Weaver, J. D. $\mathrm{S}_{\mathrm{N}}$ Ar Catalysis Enhanced by an Aromatic Donor-Acceptor Interaction; Facile Access to Chlorinated Polyfluoroarenes. Chem. Commun., 2017, 53, 7545-7548.

${ }^{23}$ Aggarwal, V. K.; Mereu, A. Superior amine catalysts for the Baylis-Hillman reaction: the use of DBU and its implications. Chem. Commun., 1999, 2311-2312.

${ }^{24}$ Geesala, R.; Gangasani, J. K.; Budde,M.; Balasubramanian, S.; Vaidya, J. R.; Das, A. 2-Azetidinones: Synthesis and biological evaluation as potential antibreast cancer agents. Eur. J. Med. Chem.,2016, 124, 544-558.

${ }^{25}$ Cho, M. Y.; Kwon, Y. B.; Yoo, B. W.; Yoon, C. M. Amination of the Baylis -Hillman Acetates in Ethanol. Synth. Commun., 2007, 37, 2677-2685.

26 Zhang, Y.; Li, Y.-X.; Liu, L.; Han, Z.-B. Palladium Nanoparticles Supported on UiO-66- $\mathrm{NH}_{2}$ as Heterogeneous Catalyst for Epoxidation of Styrene. Inorg. Chem. Commun., 2019, 100, 51-55.

${ }^{27}$ Katz, M. J.; Brown, Z. J.; Colón, Y. J.; Siu, P. W.; Scheidt, K. A.; Snurr, R. Q.; Hupp, J. T.; Farha, O. K. A Facile Synthesis of UiO-66, UiO-67 and Their Derivatives. Chem. Commun., 2013, 49, 9449-9451.

${ }^{28}$ Sheldrick, G. M. Crystal Structure Refinement with SHELXL. Acta. Cryst., 2015, C71, 3-8.

${ }^{29}$ Sheldrick, G. M. SHELXL2018/1. University of Gottingen, Germany. 